

\section{Panorama \\ des administrations \\ publiques \\ 2013}


Cet ouvrage est publié sous la responsabilité du Secrétaire général de l'OCDE. Les opinions et les interprétations exprimées ne reflètent pas nécessairement les vues officielles des pays membres de l'OCDE.

Ce document et toute carte qu'il peut comprendre sont sans préjudice du statut de tout territoire, de la souveraineté s'exerçant sur ce dernier, du tracé des frontières et limites internationales, et du nom de tout territoire, ville ou région.

\section{Merci de citer cet ouvrage comme suit :}

OCDE (2014), Panorama des administrations publiques 2013, Éditions OCDE.

http://dx.doi.org/10.1787/gov_glance-2013-fr

ISBN 978-92-64-20109-5 (imprimé)

ISBN 978-92-64-20650-2 (PDF)

Collection : Panorama des administrations publiques

ISSN 2221-4402 (imprimé)

ISSN 2221-4410 (en ligne)

Les données statistiques concernant Israël sont fournies par et sous la responsabilité des autorités israéliennes compétentes. L'utilisation de ces données par l'OCDE est sans préjudice du statut des hauteurs du Golan, de Jérusalem-Est et des colonies de peuplement israéliennes en Cisjordanie aux termes du droit international.

Crédits photo : Couverture @ Robert Adrian Hillman/Shutterstock.com ; $\odot$ tovovan/Shutterstock.com, chapitres 2 à 9 : Shutterstock/kentoh.

Les corrigenda des publications de l'OCDE sont disponibles sur : www.oecd.org/about/publishing/corrigenda.htm. (C) OCDE 2014

La copie, le téléchargement ou l'impression du contenu OCDE pour une utilisation personnelle sont autorisés. Il est possible d'inclure des extraits de publications, de bases de données et de produits multimédia de l'OCDE dans des documents, présentations, blogs, sites Internet et matériel pédagogique, sous réserve de faire mention de la source et du copyright. Toute demande en vue d'un usage public ou commercial ou concernant les droits de traduction devra être adressée à rights@oecd.org. Toute demande d'autorisation de photocopier une partie de ce contenu à des fins publiques ou commerciales devra être soumise au Copyright Clearance Center (CCC), info@copyright.com, ou au Centre français d'exploitation du droit de copie (CFC), contact@cfcopies.com. 


\section{Avant-propos}

L

a crise financière et économique qui a éclaté en 2008 et qui a touché la plupart des pays membres de l'OCDE a rouvert le débat sur le rôle de l'État et sur les modalités, le champ et la finalité de ses interventions. Le Panorama des administrations publiques 2013 présente des données quantitatives et qualitatives essentielles qui peuvent favoriser une prise de décision fondée sur des données probantes et aider les pouvoirs publics à préparer l'avenir. Ces données permettent de comparer les activités, les pratiques et les performances, des administrations selon un certain nombre de dimensions essentielles et de mettre en évidence les domaines qui méritent un examen plus approfondi. Par ailleurs, un chapitre thématique évoque les liens qui existent entre la confiance à l'égard des pouvoirs publics et les politiques et institutions de la gouvernance publique.

Ces travaux ont été conduits par Zsuzsanna Lonti, sous la direction de Rolf Alter et d'Edwin Lau, et rédigés par Natalia Nolan-Flecha, Santiago González, Jean-François Leruste et Alessandro Lupi. D'importantes contributions ont été rédigées et transmises par Mario Marcel et Stéphane Jacobzone (chapitre 1, "Confiance dans les pouvoirs publics, efficacité de l'action publique et stratégies en matière de gouvernance ") ; Catherine Gamper et Alice Lazzati (chapitre 2, "La gouvernance stratégique ») ; Monica Brezzi, Arthur Mickoleit et Camila Vammalle (chapitre 3, "Les finances et l'économie publiques ") ; Ronnie Downes, Ian Hawkesworth, Joung Jin Jang, Knut Klepvisk et Lisa Von Trapp (chapitre 4, "Les pratiques et les procédures budgétaires"); Robert Ball et Maya Beauvallet (chapitre 5, "L'emploi et la rémunération dans le secteur public "); Robert Ball, Michelle Marshalian et Tatyana Teplova (chapitre 6, "Les femmes dans la fonction publique "); Elodie Beth, Maria-Emma Cantera et Ulrika Kilnes (chapitre 7, "Les marchés publics »); Julio Bacio Terracino, Janos Bertok, Maria-Emma Cantera, Ronnie Downes, Ulrika Kilnes, Knut Klepvisk, Arthur Mickoleit, Adam Mollerup et Barbara Ubaldi (chapitre 8, "Une administration ouverte et inclusive ») ; et Filippo Cavassini, Alice Lazzati et Adam Mollerup (chapitre 9, "Éclairage - Au service des citoyens : l'accessibilité et la qualité des services publics"). Nous remercions Lia Beyeler, Laura Boutin, Kate Lancaster, Natasha Lawrance, Sophie Limoges, Jennifer Stein et Deirdre Wolfender, qui nous ont aidés à préparer le document en vue de sa publication.

Cette publication est le fruit de contributions d'un large éventail de sources et d'experts. Elle a bénéficié de travaux du Comité de la gouvernance publique de l'OCDE et du Groupe de direction chargé du Panorama des administrations publiques (voir annexe F) ; du Comité des statistiques de l'OCDE ; du Groupe de travail sur l'emploi et la gestion publics; du Groupe de travail des hauts responsables du budget ; du Groupe d'experts sur les conflits d'intérêts de l'OCDE ; du Réseau de hauts responsables de l'administration électronique; des Spécialistes de haut niveau des marchés publics et $d u$ Groupe d'experts sur l'administration ouverte et novatrice. De plus, des commentaires précieux ont été fournis par Peter Van de Ven et Catherine La Rosa-Elkaim (Direction des statistiques de l'OCDE), Richard Highfield, Mehmet Ceylan et Devi Thani (Centre de politique et d'administration fiscales), Gaétan Lafortune, Nicolaas Sieds Klazinga, Valérie Paris (Direction de l'emploi, du travail et des affaires sociales de l'OCDE), Corine Heckmann, Joris Ranchin (Direction de l'éducation et des compétences de l'OCDE), Peter Hoeller, Giussepe Nicoletti (Département des affaires économiques de l'OCDE), Messaoud Hammouya (Organisation internationale du travail, Genève) et Zoltan Mikolas (consultant). 



\section{Table des matières}

Préface - la gouvernance, ça compte $! \ldots \ldots \ldots \ldots \ldots \ldots \ldots \ldots \ldots \ldots \ldots$

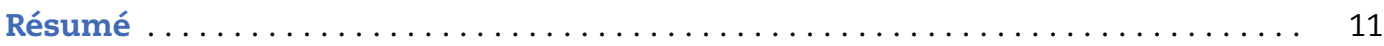

Guide du lecteur.................................... 14

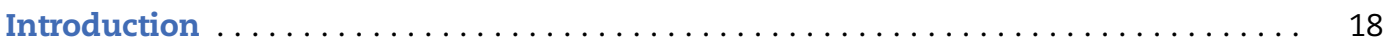

Chapitre 1. Confiance dans les pouvoirs publics, efficacité de l'action publique et stratégies en matière de gouvernance. . . . . . . . . . . . . . . 23

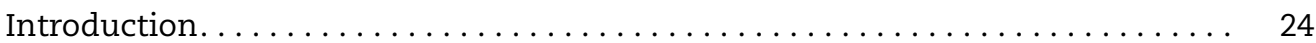

La confiance dans les pouvoirs publics, qu'est-ce que c'est ? ........... 25 Pourquoi la confiance dans les pouvoirs publics est-elle importante ? ........ 26 Mesurer la confiance à l'égard des pouvoirs publics.............. 28 Caractéristiques et évolution de la confiance à l'égard des pouvoirs publics

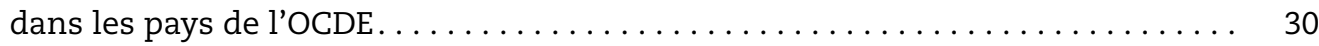
Les déterminants de la confiance dans les pouvoirs publics............ 34

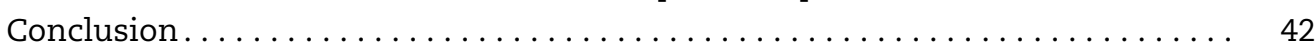

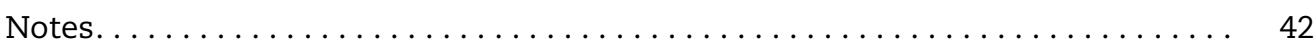

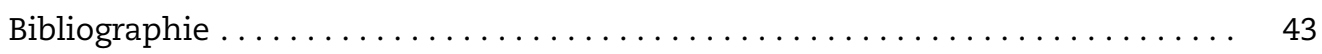

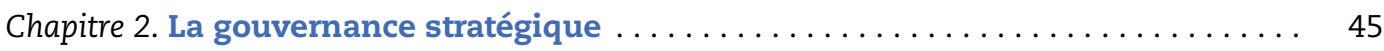

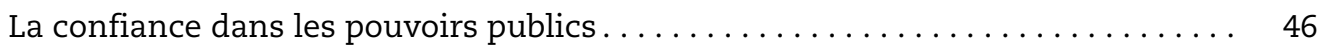

Renforcer l'équité grâce à certaines politiques publiques $\ldots \ldots \ldots \ldots \ldots \ldots$. 48

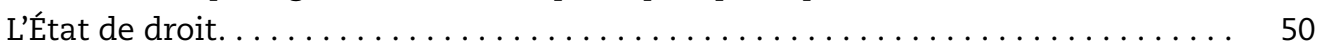

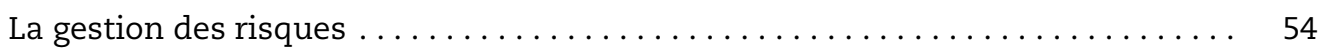

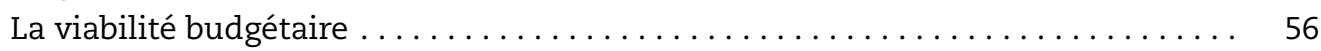

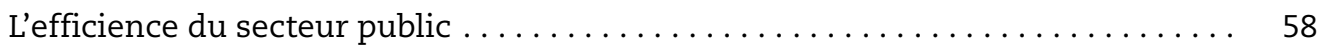

Le rapport coût-efficacité du secteur public ................. 62

Chapitre 3. Les finances et l'économie publiques.................... 67

Le solde budgétaire des administrations publiques .............. 68

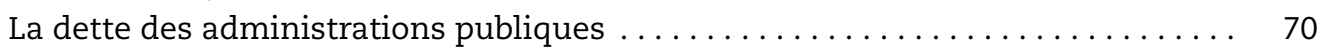

Le solde budgétaire et l'endettement par niveau d'administration $\ldots \ldots \ldots \ldots \quad 72$

Les recettes des administrations publiques ................... 74

La composition des recettes des administrations publiques . . . . . . . . 76

La répartition des recettes publiques entre les niveaux d'administration. . . . . 78

Les dépenses des administrations publiques................. 80

La ventilation par fonction des dépenses des administrations publiques

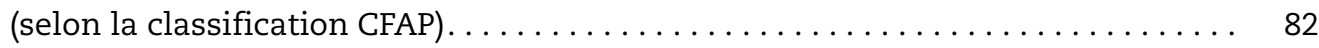

La ventilation des dépenses publiques selon les niveaux d'administration ..... 84

Les dépenses d'investissement des administrations publiques . . . . . . . . 87 
Les coûts de production et l'externalisation dans les administrations publiques . 90 Éclairage : les dépenses des administrations centrales en matière de TIC . . . . . 93

Chapitre 4. Les pratiques et les procédures budgétaires $\ldots \ldots \ldots \ldots \ldots \ldots \ldots \ldots . \ldots 7$

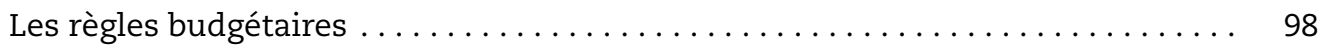

Les cadres de dépenses à moyen terme $\ldots \ldots \ldots \ldots \ldots \ldots \ldots \ldots \ldots \ldots \ldots$

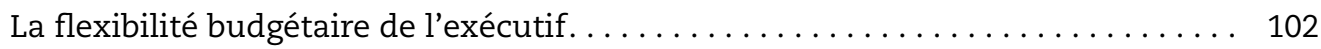

La budgétisation axée sur la performance ...................... 104

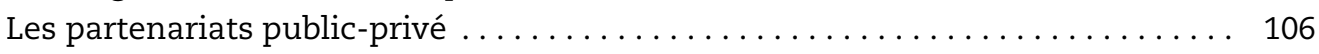

Les institutions budgétaires indépendantes. . . . . . . . . . . . . 108

Chapitre 5. L'emploi et la rémunération dans le secteur public ............. 111

L'emploi dans les administrations publiques et les sociétés publiques . . . . . . . 112

L'emploi au sein des différents niveaux d'administration . . . . . . . . . . . . 114

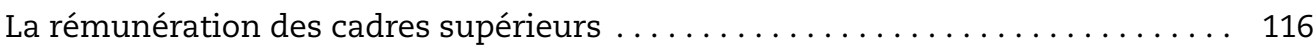

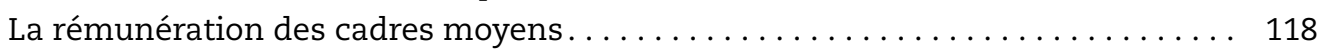

La rémunération des spécialistes dans l'administration centrale. . . . . . . . 120

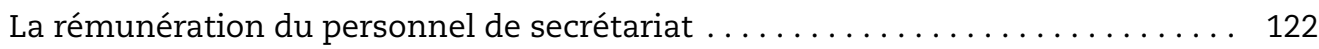

La rémunération dans certaines professions de service public............. 124

Les salaires des enseignants . . . . . . . . . . . . . . . . . . 126

Chapitre 6. Les femmes dans la fonction publique................... 129

Emploi des femmes dans les administrations publiques . . . . . . . . . . . 130

Emploi des femmes dans l'administration centrale . . . . . . . . . . . 132

Présence des femmes dans l'encadrement supérieur

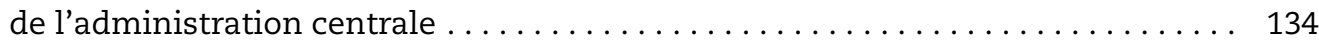

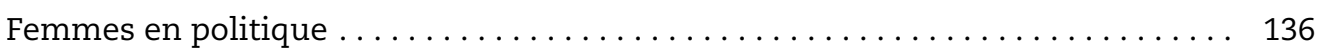

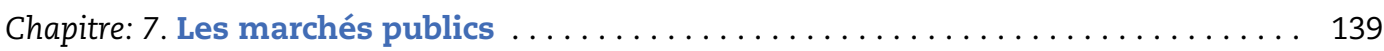

Les dépenses liées aux marchés publics .................. 140

Le recours à des instruments novateurs dans la passation

de marchés publics.................................. 142

L'utilisation stratégique des marchés publics $\ldots \ldots \ldots \ldots \ldots \ldots \ldots \ldots \ldots \ldots \ldots$

La concurrence loyale dans les marchés publics et les PME. . . . . . . . . 146

Chapitre 8. Une administration ouverte et inclusive . . . . . . . . . . . . . 149

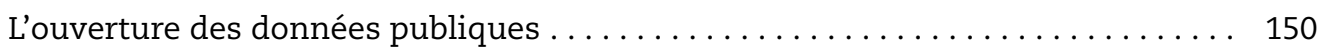

Les conflits d'intérêts et les déclarations de patrimoine .............. 152

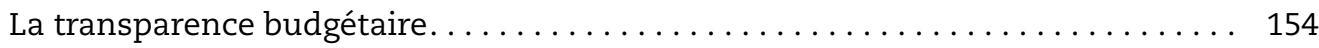

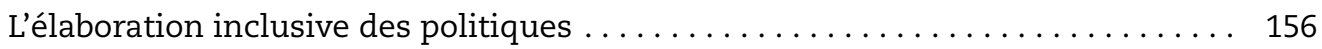

Chapitre: 9. Éclairage - au service des citoyens : l'accessibilité et la qualité

des services publics. . . . . . . . . . . . . . . . . . . . . 159

L'accès aux services publics : l'accessibilité financière ............. 160

La fréquentation des services publics en ligne . . . . . . . . . . . . . 164

La réactivité des services publics : la rapidité d'exécution. . . . . . . . . 168

La fiabilité des services publics : garantir les droits des citoyens . . . . . . . . . . 172

La satisfaction des citoyens à l'égard des services publics. . . . . . . . . . . 176

Annexe A. Méthodologie utilisée pour les agrégats relatifs aux recettes. . . . . . . 179

Annexe B. Classification des fonctions des administrations publiques

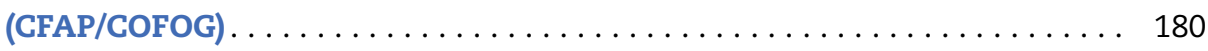

Annexe C. Indices composites sur les pratiques budgétaires $\ldots \ldots \ldots \ldots \ldots \ldots$. 
Annexe D. Méthodologie et notes supplémentaires concernant la rémunération

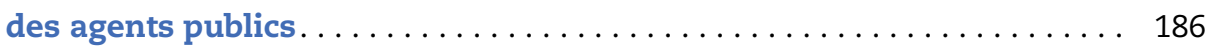

Annexe E. Données détaillées sur les déclarations de conflits d'intérêts . . . . . . . 196

Annexe F. Membres du Groupe de direction....................... 201

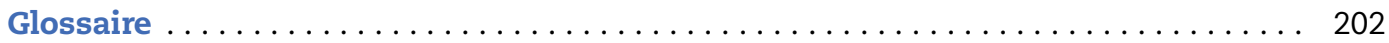

\section{Suivez les publications de l'OCDE sur:}

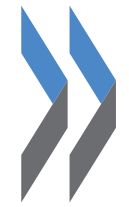

Yhttp://twitter.com/OECD_Pubs

f http://www.facebook.com/OECDPublications

in. http://www.linkedin.com/groups/OECD-Publications-4645871

Yibu http://www.youtube.com/oecdilibrary

http://www.oecd.org/oecddirect/

\section{Ce livre contient des...}

En bas des tableaux ou graphiques de cet ouvrage, vous trouverez des StatLinks. Pour télécharger le fichier Excel ${ }^{\circledR}$ correspondant, il vous suffit de retranscrire dans votre navigateur Internet le lien commençant par : $h t t p: / / d x . d o i . o r g$, ou de cliquer sur le lien depuis la version PDF de l'ouvrage. 


\section{Préface - la gouvernance, ça compte!}

$\mathrm{M}$ algré l'amélioration progressive de ses perspectives, l'économie mondiale continue de subir les conséquences de la crise financière, économique et sociale qui l'a frappée. Face à l'atonie de la croissance, au poids de la dette publique, à la persistance du chômage et au creusement des inégalités, une action déterminée s'impose. On attend des pouvoirs publics qu'ils remettent les économies sur les rails d'une croissance plus forte, plus verte et plus inclusive.

Les citoyens veulent que les autorités montrent la voie à suivre. En l'absence d'un leadership fort accompagné de politiques efficaces, la confiance à l'égard des pouvoirs publics s'érode facilement, et c'est ce qui s'est produit sous l'effet de la crise. Dans toute la zone OCDE, les citoyens ont perdu confiance dans l'aptitude des responsables publics à résoudre les problèmes économiques et à répondre à leurs besoins et à leurs exigences. Or, il est essentiel que les autorités retrouvent la confiance de la population pour pouvoir mener à bien les réformes nécessaires.

Pour reconquérir cette confiance, elles peuvent jouer sur un levier crucial : de bonnes performances. L'édition 2013 du Panorama des administrations publiques propose un tableau de bord visant à aider les décideurs et les citoyens à analyser et à comparer les performances des administrations et à recenser les domaines dans lesquels l'efficacité et l'efficience du secteur public peuvent être améliorées. Les 50 indicateurs présentés couvrent l'intégralité de la chaîne de production des biens et services publics (intrants, processus, produits et réalisations) et des aspects essentiels de la gestion et de la gouvernance publique tels que les pratiques budgétaires, l'intégrité et l'administration ouverte, l'administration électronique et les stratégies en matière de TIC.

Le Panorama des administrations publiques 2013 montre que, même si les gouvernements ont pris des mesures pour renforcer les institutions et optimiser la dépense publique, beaucoup reste à faire. Ainsi, malgré les efforts considérables déployés par de nombreux pays, leurs finances publiques doivent encore être assainies, et d'importantes inégalités hommes-femmes persistent. Par exemple, les femmes n'occupent que $40 \%$ des postes d'encadrement intermédiaire et $29 \%$ des postes d'encadrement supérieur au sein de la fonction publique. En outre, l'ouverture des données publiques gagne en importance en tant qu'outil de gouvernance - $56 \%$ des pays de l'OCDE se sont dotés d'une stratégie nationale en la matière - mais il faut aller plus loin pour garantir que les administrés puissent effectivement utiliser l'information disponible. Dans tous ces domaines et bien d'autres encore, la réforme du secteur public doit rester une priorité forte, à l'appui de nos objectifs économiques et sociaux.

Nous avons œuvré sans relâche à élargir le champ de nos indicateurs et de nos analyses dans le domaine de la gouvernance et à les fournir dans les meilleurs délais sur un large éventail de formats électroniques, afin d'assurer leur accessibilité. Nous ne 
doutons donc pas que le Panorama des administrations publiques 2013 constituera une ressource extrêmement précieuse au service des responsables publics, des citoyens et des chercheurs en quête de politiques meilleures pour une vie meilleure.

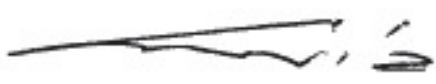

Angel Gurría

Secrétaire général de l'OCDE 



\section{Résumé}

L gouvernements de la zone OCDE à mettre en œuvre des plans d'ajustement structurel destinés à assainir leurs finances publiques. Toutefois, la confiance à l'égard des pouvoirs publics a considérablement décliné, à l'heure où les autorités peinent à répondre aux attentes croissantes des citoyens à partir de ressources publiques limitées. Entre 2007 et 2012, le taux de confiance dans les autorités nationales est passé, en moyenne, de $45 \%$ à $40 \%$, et les gouvernements ont donc du mal à mobiliser la population en faveur des réformes requises.

Une nouvelle approche de la gouvernance publique s'impose pour que les pouvoirs publics puissent répondre aux attentes des citoyens malgré leurs moyens limités. Cette démarche doit être fondée sur la création de capacités stratégiques, sur des institutions fortes, sur des instruments et processus efficaces et sur des réalisations claires et mesurables. Les indicateurs présentés dans le Panorama des administrations publiques 2013 illustrent le chemin déjà parcouru par les pays de l'OCDE sur la voie qui mène à cet état stratégique.

Principales conclusions

- Les finances publiques continuent de soulever des problèmes, malgré les importants efforts d'assainissement consentis par les pays. L'OCDE a estimé le redressement des soldes primaires sous-jacents qui serait nécessaire pour ramener la dette brute des administrations publiques à $60 \%$ du PIB d'ici 2030. En moyenne, dans les pays membres de l'OCDE, il faudrait que le PIB potentiel augmente d'environ $3 \%$ par rapport à la situation budgétaire de 2012. Or, le ratio dette publique/PIB continue d'augmenter au sein de plusieurs pays de l'OCDE et en 2011, en moyenne, les dépenses publiques ont dépassé les recettes publiques. Cette situation tient, pour partie, au coût des programmes de relance et à la stagnation des recettes provoquée par la crise, ainsi qu'à l'augmentation des dépenses liées au vieillissement de la population.

- Les pays ont adopté de nouvelles pratiques budgétaires et créé de nouvelles institutions de gouvernance. Les dispositifs préexistants s'étant avérés impuissants à assurer la discipline budgétaire, une évolution du cadre mondial de la gouvernance économique s'est imposée, ce qui conduit les pays à perfectionner les outils actuels et à mettre en œuvre de nouvelles stratégies. Par exemple, $97 \%$ des pays de l'OCDE ont mis en place des règles budgétaires, et le nombre moyen de règles budgétaires par pays a augmenté. Entre 2009 et 2013, huit pays se sont dotés d'une institution budgétaire indépendante chargée de favoriser la discipline budgétaire, de créer de l'information économique et de veiller à ce que les ressources soient affectées là où elles sont le plus nécessaires. 
- L'emploi public a tendance à rester à des niveaux stables à long terme. Entre 2001 et 2011, l'emploi dans les administrations publiques s'est maintenu à un niveau relativement inchangé, très légèrement inférieur à $16 \%$ de la main-d'œuvre totale. Ce pourcentage est relativement faible quand on le compare aux dépenses publiques moyennes, qui représentaient $45.4 \%$ du PIB en 2011, ce qui montre l'ampleur de l'externalisation. Bien que plusieurs pays de l'OCDE aient annoncé un gel des recrutements et des réductions d'effectifs dans le cadre de leurs plans d'assainissement budgétaire, une politique de forte réduction de l'emploi public est difficilement tenable à long terme, du fait de l'augmentation constante des exigences de la population.

- Des mécanismes supplémentaires sont nécessaires pour mieux assurer l'égalité hommes-femmes dans la fonction publique. Les pouvoirs publics ont pris diverses dispositions pour assurer l'égalité des chances des hommes et des femmes face à l'emploi public, y compris en formulant des objectifs de recrutement et de promotion et en instaurant des mesures destinées à favoriser un plus grand équilibre entre vie professionnelle et vie privée. Néanmoins, les chiffres montrent que les femmes occupent plus de $50 \%$ (et, dans certains cas, près de $90 \%$ ) des postes de secrétaire, mais qu'elles sont beaucoup moins bien représentées s'agissant des postes de niveau hiérarchique supérieur. Parmi les mesures visant à corriger ces disparités, on peut citer, par exemple, la budgétisation prenant en compte la problématique hommes-femmes, qui apporte un éclairage lié à cette problématique à toutes les étapes du cycle budgétaire. L'objectif consiste à éviter les dépenses « asexuées " et à améliorer l'efficacité des programmes publics en repérant les dépenses publiques produisant des effets disproportionnés selon le sexe. Toutefois, moins de la moitié des pays de l'OCDE ont institué cette budgétisation prenant en compte la problématique hommes-femmes.

- Les pays font désormais une utilisation plus stratégique des marchés publics. De nombreux pays de l'OCDE ont recours à des instruments novateurs de passation des marchés publics pour réaliser des économies d'échelle $(94 \%$ d'entre eux ont recours à des accords-cadre, par exemple), restructurant leurs approvisionnements, regroupant leurs achats et informatisant la passation de leurs marchés (97\% d'entre eux publient leurs appels d'offres sur un portail national en ligne). De plus, de nombreux pays de l'OCDE s'appuient sur leurs politiques de marchés publics non seulement pour optimiser la dépense publique, mais aussi pour servir d'autres objectifs tels que l'innovation, la croissance durable (73\% favorisent ainsi les marchés publics verts), le développement des petites et moyennes entreprises (70 \% favorisent le recours aux PME) et un accès plus égalitaire à la vie économique.

- La divulgation par les décideurs publics de leur patrimoine et de leurs intérêts privés reste un outil essentiel de gestion des conflits d'intérêts. La quasi-totalité des pays obligent leurs responsables publics à dévoiler leur patrimoine et leurs sources de revenus. En revanche, il est rare qu'un pays exige qu'ils déclarent leurs emplois antérieurs et leurs dettes.

- L'ouverture des données publiques (ODP) est en train de gagner en importance en tant qu'outil de gouvernance. Un peu plus de la moitié des pays de l'OCDE se sont dotés d'une stratégie nationale visant à assurer cette ouverture ; ils sont $12 \%$ à signaler l'existence de stratégies diverses au niveau des ministères sectoriels et $28 \%$ à déclarer avoir adopté à la fois une stratégie nationale et des stratégies à un niveau inférieur. Parmi les objectifs prioritaires poursuivis en la matière, on peut citer la transparence et l'ouverture, l'accroissement du volume d'activité du secteur privé et la création d'entreprises. De plus, les pays sont bien conscients que l'ouverture des données 
publiques peut permettre d'améliorer la prestation des services publics. En revanche, ils ne considèrent pas comme un objectif prioritaire la participation accrue des citoyens aux débats publics et aux processus de décision.

- Les citoyens font davantage confiance aux services publics dont ils sont usagers qu'à la notion abstraite d'autorités nationales. Malgré la baisse de la confiance dans les " pouvoirs publics ", les administrés se déclarent satisfaits des services que ceux-ci leur assurent. Ainsi, en moyenne, 72 \% disent faire confiance à leur police locale. Ils sont un pourcentage presque égal à se déclarer satisfaits de leur accès à des soins de santé de qualité, et $66 \%$ à être satisfaits du système éducatif et des établissements d'enseignement de leur ville ou de leur zone. Les niveaux de satisfaction sont restés relativement stables, en moyenne, pendant et juste après la crise financière et économique mondiale.

- Les pouvoirs publics des pays de l'OCDE s'emploient de plus en plus à mettre des biens et services publics de qualité à la disposition d'un large éventail de citoyens. De nombreux pays sont en train d'instaurer des normes en matière de niveau de service et de mettre en œuvre des dispositifs visant à mesurer les réactions des administrés et à les intégrer au processus. Pour la première fois, le Panorama des administrations publiques compare quatre dimensions de la qualité de service - l'accessibilité financière, la réactivité, la fiabilité et la satisfaction des citoyens - non seulement au niveau des pays, mais aussi au niveau des services publics essentiels que sont l'éducation, la santé, la justice et l'administration fiscale. 


\title{
Guide du lecteur
}

\begin{abstract}
A fin d'interpréter correctement les données figurant dans le Panorama des administrations publiques, les lecteurs doivent être au fait des considérations méthodologiques ci-après, qui valent pour un certain nombre d'indicateurs. Comme dans les éditions précédentes, les indicateurs sont normalement présentés sur deux pages, sauf pour quelques-uns d'entre eux, tels que les indicateurs relatifs à l'état de droit et à la qualité des services publics, qui sont présentés sur quatre pages. Pour les indicateurs présentés sur deux pages, la première page comporte un texte qui explique la pertinence du thème abordé et met en évidence certaines des grandes différences observées entre les pays de l'OCDE. Ce texte est suivi d'une rubrique "Méthodologie et définitions " qui précise les sources des données et fournit des informations indispensables à leur interprétation. En fin de première page, on trouve ensuite une rubrique " Pour en savoir plus " qui dresse la liste de travaux de référence permettant de replacer dans leur contexte les données fournies. La seconde page présente les données. Les graphiques illustrent les chiffres actuels et, dans la mesure du possible, leur évolution au fil du temps. On trouvera au dernier chapitre un glossaire des principaux termes employés dans la publication.
\end{abstract}

\section{Année civile/exercice budgétaire dans les statistiques de l’OCDE relatives aux comptes nationaux}

Sauf indication contraire, les données tirées des statistiques de l'OCDE relatives aux comptes nationaux portent sur l'année civile.

Les données relatives à l'Australie et à la Nouvelle-Zélande portent sur les exercices budgétaires suivants : du $1^{\mathrm{er}}$ juillet de l'année indiquée au 30 juin pour l'Australie et du $1^{\mathrm{er}}$ avril de l'année indiquée au 31 mars pour la Nouvelle-Zélande. Pour le Japon, les données relatives aux sous-secteurs des administrations publiques et des dépenses selon la CFAP/COFOG (Classification des fonctions des administrations publiques) portent sur l'exercice budgétaire.

Les données fondées sur le Système de comptabilité nationale $(\mathrm{SCN})$ ont été tirées des Statistiques de l'OCDE sur les comptes nationaux (base de données) le 12 août 2013.

\section{Portée géographique}

Le Panorama des administrations publiques 2013 présente des chiffres pour les 34 pays membres de l'OCDE, en fonction des données disponibles. Les données statistiques concernant Israël sont fournies par et sous la responsabilité des autorités israéliennes compétentes. L'utilisation de ces données par l'OCDE est sans préjudice du statut des hauteurs du Golan, de Jérusalem-Est et des colonies de peuplement israéliennes en Cisjordanie aux termes du droit international.

Des pays non membres tels que la Fédération de Russie, qui a entamé un processus d'adhésion à l'OCDE, et d'autres pays dotés du statut de participant auprès du Comité de la 
gouvernance publique de l'OCDE (Afrique du Sud, Brésil, Égypte, Ukraine) ont également fourni des données pour certains indicateurs. Les données relatives aux pays non membres sont présentées séparément, à la fin des tableaux et des graphiques.

\section{Codes des noms de pays (codes ISO)}

\begin{tabular}{llll}
\hline Pays membres de l'OCDE & & Pologne & POL \\
Allemagne & DEU & Portugal & PRT \\
Australie & AUS & République slovaque & SVK \\
Autriche & AUT & République tchèque & CZE \\
Belgique & BEL & Royaume-Uni & GBR \\
Canada & CAN & Slovénie & SVN \\
Chili & CHL & Suède & SWE \\
Corée & KOR & Suisse & CHE \\
Danemark & DNK & Turquie & TUR \\
Espagne & ESP & & \\
Estonie & EST & & RUS \\
États-Unis & USA & Pays candidats à l'adhésion à l'OCDE & \\
Finlande & FIN & Fédération de Russie & ZAF \\
France & FRA & & BRA \\
Grèce & GRC & Autres grandes économies & CHN \\
Hongrie & HUN & Afrique du Sud (observateur auprès du Comité de la gouvernance publique de l'OCDE) & IND \\
Irlande & IRL & Brésil (observateur auprès du Comité de la gouvernance publique de l'OCDE) & IDN \\
Islande & ISL & Chine & \\
Israël & ISR & Inde & \\
Italie & ITA & Indonésie & \\
Japon & JPN & & UKR \\
Luxembourg & LUX & & \\
Mexique & MEX & Autres observateurs auprès du Comité de la gouvernance publique de l'OCDE & \\
Norvège & NOR & Égypte & \\
Nouvelle-Zélande & NZL & Ukraine & \\
Pays-Bas & NLD & & EGY \\
\hline
\end{tabular}

\section{Moyennes et totaux OCDE}

\section{Moyennes}

Dans les graphiques et le texte, la moyenne OCDE désigne la moyenne arithmétique non pondérée des pays membres de l'OCDE pour lesquels des données sont disponibles. Elle exclut les données relatives aux pays non membres. Quand on ne dispose pas de données pour un pays membre de l'OCDE, c'est signalé dans les notes.

Quand un graphique présente des données portant sur une ou plusieurs années, la moyenne OCDE englobe tous les pays de l'OCDE pour lesquels on dispose de données. Par exemple, une moyenne OCDE pour 2009 englobe tous les pays actuellement membres de l'OCDE au sujet desquels on dispose de données pour l'année en question, même si, à l'époque, ils n'étaient pas membres de l'Organisation.

\section{Totaux}

Les totaux OCDE figurent généralement dans les tableaux et représentent la somme des données présentées dans la colonne correspondante au sujet des pays de l'OCDE pour lesquels des données sont disponibles. Les totaux excluent les données relatives aux pays non membres. Quand on ne dispose pas de données pour un pays membre de l'OCDE, c'est signalé dans les notes. 


\section{Suppléments en ligne}

Pour plusieurs indicateurs, on dispose de tableaux et de graphiques en ligne supplémentaires qui présentent des données par pays. Lorsque de tels suppléments sont disponibles, c'est signalé à la rubrique " Méthodologie et définitions " de l'indicateur. Le Panorama des administrations publiques 2013 offre également l'accès au service StatLinks, qui permet aux lecteurs de télécharger les fichiers Excel correspondant aux données présentées. Les liens StatLinks sont placés dans le coin inférieur droit des tableaux et des graphiques, et il est possible de les saisir dans un navigateur web ou, à partir d'une version électronique de la publication, de cliquer directement dessus.

Les supports complémentaires suivants sont également disponibles en ligne, à l'adresse www.oecd.org/gov/govataglance.htm:

- Des fiches par pays qui présentent un certain nombre de données essentielles pour chaque pays, avec une comparaison avec la moyenne OCDE.

- La base de données statistiques Panorama des administrations publiques, qui présente des données régulièrement actualisées pour une sélection d'indicateurs (via OECD.Stat).

- Des notes relatives au contexte de chaque pays qui fournissent des renseignements sur certaines grandes caractéristiques de la structure politique et administrative de chaque pays membre.

\section{Indicateurs par habitant}

Certains indicateurs (les indicateurs relatifs aux dépenses, aux recettes et à la dette publique, par exemple) sont présentés sur une base par habitant (c'est-à-dire par personne). Les estimations de la population de référence sont fondées sur la notion de résidence du Système de comptabilité nationale. Elles incluent les personnes qui sont résidentes dans un pays pendant un an ou plus, quelle que soit leur nationalité, et comprennent aussi les membres du personnel diplomatique et militaire étranger, ainsi que leurs familles, de même que les étudiants poursuivant des études à l'étranger et les patients suivant un traitement à l'étranger, même s'ils y restent pendant plus d'un an. La règle d'un an signifie que les résidents habituels vivant à l'étranger pendant moins d'un an sont inclus dans la population, tandis que les visiteurs étrangers (les vacanciers, par exemple) présents dans le pays pendant moins d'un an sont exclus. Point important à noter dans ce contexte: des individus peuvent être comptabilisés dans un pays comme employés (car ils contribuent au PIB de ce pays par le biais de la production) mais dans un autre pays comme résidents (leurs traitements et salaires étant pris en compte dans le revenu national brut de leur pays de résidence).

\section{Parités de pouvoir d'achat}

Les parités de pouvoir d'achat (PPA) sont les taux de change des monnaies qui égalisent le pouvoir d'achat de différents pays en éliminant les différences de niveaux des prix entre les pays. Lorsqu'on les convertit au moyen des PPA, les dépenses des différents pays sont, de fait, exprimées aux mêmes niveaux de prix. Cela signifie qu'un groupe équivalent de biens et de services aura le même coût dans les deux pays, ce qui permet d'effectuer des comparaisons internationales prenant uniquement en compte les différences au niveau du volume des biens et des services achetés.

2001 et 2009 : les PPA pour l'ensemble des pays européens correspondent aux résultats de référence annuels communiqués par Eurostat. Les PPA pour les pays non européens et la Fédération de Russie sont des estimations de l'OCDE. 
2011 : les PPA pour l'ensemble des pays membres de l'OCDE et la Fédération de Russie sont des résultats de référence préliminaires calculés par l'OCDE. Il convient d'interpréter avec prudence les estimations et les résultats préliminaires, du fait qu'ils sont susceptibles de faire l'objet de révisions.

Des informations supplémentaires sont disponibles sur le site Internet de l'OCDE consacré aux PPA : www.oecd.org/fr/std/prix-ppa/.

\section{Indices composites}

On trouvera dans cette publication plusieurs indices composites descriptifs portant sur des domaines étroitement définis liés aux conflits d'intérêts et aux pratiques et procédures budgétaires. Ces indices constituent un moyen commode de rassembler des informations d'ordre qualitatif éparses. Les indices composites présentés dans cette publication ont été créés selon les étapes définies dans le Handbook on Constructing Composite Indicators (Nardo et al., 2008).

On trouvera aux annexes $C$ et $E$, respectivement, des précisions sur les variables et les pondérations employées pour construire les indices composites relatifs, d'une part, aux pratiques et procédures budgétaires et, d'autre part, aux conflits d'intérêts. Les indices composites ont été élaborés en coopération avec les pays membres, à la lumière de bonnes pratiques et/ou de théories bien établies. En revanche, les variables composant les indices et leurs pondérations relatives reposent sur des jugements autorisés et, de ce fait, pourront évoluer par la suite. Il n'est pas possible de comparer les indices composites relatifs aux pratiques et procédures budgétaires à ceux qui figuraient dans l'édition 2009 du Panorama des administrations publiques, car les dernières enquêtes sur les pratiques et procédures budgétaires et sur la budgétisation axée sur la performance (2012) comportent des questions dont la formulation a légèrement évolué par rapport aux enquêtes de 2007. De plus, des questions ont été ajoutées, et certaines pondérations ont été redéfinies.

\section{Signes et abréviations}

$\begin{array}{ll}. . & \text { Valeur manquante ou non disponible } \\ \text { x/s.o. } & \text { Sans objet } \\ \text { EUR } & \text { Euros } \\ \text { USD } & \text { Dollars des États-Unis } \\ \text { p.p. } & \text { Points de pourcentage }\end{array}$




\section{Introduction}

\section{Objectifs}

La crise économique récente a mis en relief le rôle majeur des pouvoirs publics au sein de nos sociétés modernes. On attend d'eux qu'ils mettent en place les conditions nécessaires à la croissance économique qui améliorera le bien-être des citoyens, qu'ils régulent le comportement des entreprises et des individus au nom de l'intérêt général, qu'ils redistribuent les revenus dans un souci d'équité et qu'ils fournissent à la population des biens et des services publics, le tout en présence de contraintes budgétaires et de pressions démographiques. Or, l'aptitude des pouvoirs publics à remplir leur rôle avec efficacité et efficience dépend, pour partie, de leurs politiques et pratiques dans des domaines aussi divers que la budgétisation, la gestion des ressources humaines ou la passation des marchés publics, entre autres.

La série Panorama des administrations publiques a pour objet principal de fournir des données comparatives internationales fiables sur les activités des pouvoirs publics et sur leurs résultats, dans les pays de l'OCDE et dans le reste du monde. Les pays peuvent utiliser ces données pour comparer les performances de leurs administrations, suivre leurs évolutions internes et les évolutions internationales au fil du temps et fonder leur action sur des données probantes.

Les indicateurs du Panorama des administrations publiques sont en train de devenir une référence dans de nombreux domaines de la gouvernance publique. En plus des indicateurs de base qui constituent la marque de fabrique de la publication, cette troisième édition propose une sélection de nouveaux indicateurs et des sources de données supplémentaires, ce qui permet de dresser un panorama plus complet des administrations publiques dans l'ensemble des pays membres de l'OCDE.

\section{Les nouveautés du Panorama des administrations publiques 2013}

Cette édition 2013 propose plusieurs nouveautés par rapport au Panorama des administrations publiques 2011. Pour commencer, elle comporte trois nouveaux chapitres: "La gouvernance stratégique » (chapitre 2), qui rassemble des éléments issus des versions précédentes en y ajoutant de nouveaux indicateurs portant sur la confiance dans les pouvoirs publics, la gestion des risques et l'état de droit ; "Les femmes dans la fonction publique " (chapitre 6), qui analyse la place des femmes au sein de toutes les composantes de l'administration, du système judiciaire et du parlement ; et "Éclairage - Au service des citoyens : l'accessibilité et la qualité des services publics " (chapitre 9), qui présente un nouveau cadre d'évaluation axé sur des dimensions clés de la qualité de service (accessibilité, réactivité, fiabilité et satisfaction des usagers). Certaines caractéristiques cruciales de ces dimensions qualitatives sont mesurées pour les secteurs de l'éducation, de la santé, de la justice et de l'administration fiscale.

Les données relatives aux finances publiques sont présentées pour 2001, 2009 et 2011, c'est-à-dire pour des exercices qui ont respectivement précédé, coïncidé avec et suivi la 
crise économique. De nouveaux indicateurs relatifs à la dette, à l'équilibre budgétaire et à l'investissement sont fournis pour des niveaux d'administration infranationaux, afin de permettre une meilleure compréhension de la structure des finances publiques des pays membres et des responsabilités des administrations d'États fédérés et des municipalités.

Des enquêtes relatives aux pratiques et procédures budgétaires, à la budgétisation axée sur la performance, aux marchés publics et à la rémunération des fonctionnaires ont été réalisées en 2012, ce qui a permis d'intégrer un ensemble renouvelé d'indicateurs sur ces thèmes. Les versions actualisées des enquêtes ont permis de recueillir des données plus détaillées et de meilleure qualité. Ainsi, dans l'enquête sur la rémunération des fonctionnaires, des données relatives à des professions clés au contact direct de l'usager ont été recueillies pour la première fois, ce qui permet d'effectuer des comparaisons entre les divers pays de l'OCDE s'agissant des niveaux et de la structure de la rémunération des inspecteurs et enquêteurs de police, des agents de police, des agents de l'immigration, des inspecteurs des douanes et des inspecteurs des impôts. Pour cette édition, un indicateur relatif aux dépenses liées aux technologies de l'information et de la communication (TIC) est également présenté.

\section{Définition des administrations publiques}

Les données relatives aux finances publiques sont fondées sur la définition du secteur des « administrations publiques » qui figure dans le Système de comptabilité nationale (SCN). Les administrations publiques recouvrent donc les ministères/départements, organismes publics et services ainsi que certaines institutions sans but lucratif au niveau central, au niveau des États fédérés et au niveau local, ainsi que les administrations de sécurité sociale. Les données sur les recettes et les dépenses sont présentées pour les niveaux d'administration central et infranational (États fédérés et administrations locales) et, s'il y a lieu, pour les administrations de sécurité sociale. En revanche, les données relatives à l'emploi portent sur le secteur public, qui englobe les administrations publiques et les sociétés publiques telles que les banques, ports et aéroports à capitaux publics. Enfin, les données relatives aux pratiques et procédures de gestion publique portent uniquement sur les pratiques et procédures des administrations centrales.

\section{Portée}

La portée du Panorama des administrations publiques va au-delà des 34 pays membres de l'OCDE. Quand elles sont disponibles, des données sont également fournies au sujet des pays candidats à l'adhésion à l'OCDE - la Fédération de Russie, par exemple - ainsi que sur d'autres grandes économies telles que l'Afrique du Sud, la Chine, l'Inde et l'Indonésie. Pour certains indicateurs, des données relatives aux pays participant aux travaux du Comité de la gouvernance publique (Afrique du Sud, Brésil, Égypte et Ukraine) ont été prises en compte. Ces pays jouent un rôle important et grandissant dans l'économie mondiale et dans les structures politiques internationales.

Cette troisième édition du Panorama des administrations publiques comporte des indicateurs axés sur le contexte, les intrants, les processus, les produits et les réalisations. L'édition 2013 propose un éventail élargi d'indicateurs portant sur des aspects clés des performances des administrations liés aux produits et aux réalisations de certains secteurs - y compris, pour la première fois, celui de la justice - et sur certaines dimensions de la qualité de service en matière de santé, d'éducation, de justice et d'administration fiscale. On trouvera au graphique 0.1 le cadre conceptuel du Panorama des administrations publiques. 


\section{Graphique 0.1. Cadre conceptuel du Panorama des administrations publiques 2013}

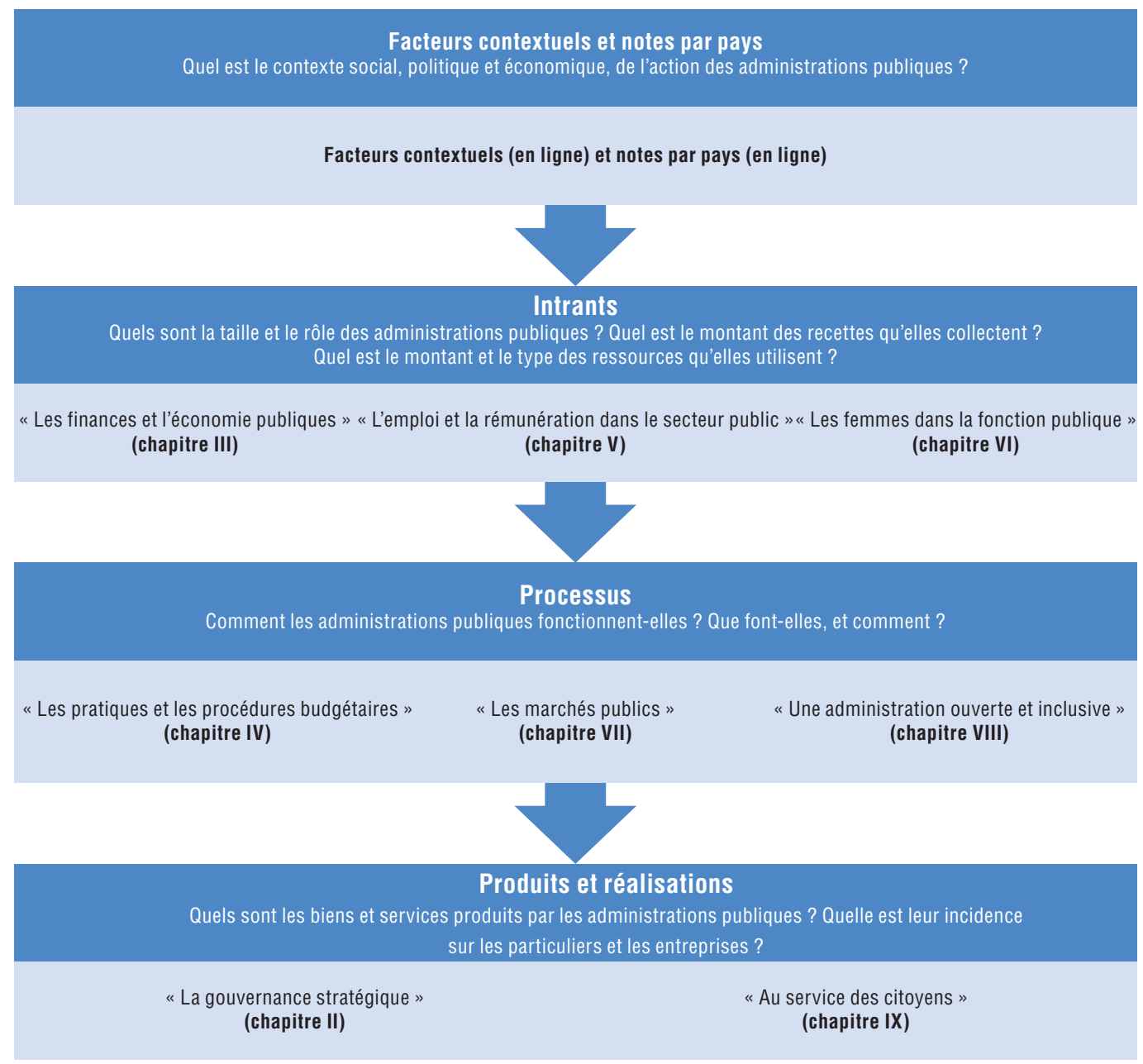

\section{Intrants}

Les intrants font référence aux ressources utilisées par les administrations publiques dans le cadre de leur fonction de production ainsi qu'à la façon dont ces ressources, qui correspondent au travail et au capital, sont combinées. Les chapitres qui décrivent ces intrants sont les suivants: "Les finances et l'économie publiques ", "L'emploi et la rémunération dans le secteur public » et «Les femmes dans la fonction publique ». Ils proposent des indicateurs relatifs aux dépenses publiques ainsi qu'aux coûts de production, à l'emploi et aux caractéristiques de la main-d'œuvre au sein du secteur public. Distinguer ainsi ces divers indicateurs aide à comprendre pourquoi les administrations publiques ont des capacités différentes à produire des biens publics et à les fournir aux citoyens.

\section{Processus}

Les processus font référence aux pratiques et aux procédures de gestion publique suivies par les pouvoirs publics pour mettre en œuvre leurs politiques. Il s'agit directement des moyens employés par les administrations publiques pour remplir leurs fonctions et pour atteindre leurs objectifs. Par conséquent, ils sont souvent essentiels pour assurer le respect de l'état de droit, la responsabilité et l'équité ainsi que la transparence de l'action 
des pouvoirs publics. Les réformes du secteur public ciblent généralement l'amélioration des processus ; par conséquent, la population leur accorde beaucoup d'attention. On trouvera dans cette édition des renseignements sur les pratiques et procédures budgétaires, sur les marchés publics et sur la mise en place d'une administration ouverte et inclusive.

\section{Produits et réalisations}

La frontière entre produits (" outputs") et réalisations (" outcomes") peut être floue ; les produits correspondent à la quantité de biens et de services produits par les administrations publiques, tandis que les réalisations correspondent aux effets des politiques et des pratiques sur les particuliers et les entreprises. Le succès d'une politique donnée devrait se mesurer à l'aune de ses produits dans un premier temps mais, au bout du compte, en fonction de ses réalisations. Un effort a été fait pour intégrer à cette édition un nombre accru d'indicateurs portant sur les produits et les réalisations. Les réalisations étant difficiles à mesurer, le cadre d'évaluation de la qualité précédemment évoqué a été conçu comme un outil visant à évaluer plusieurs dimensions de la prestation de services publics. On trouvera des exemples de ces indicateurs au chapitre 9, «Éclairage - Au service des citoyens : l'accessibilité et la qualité des services publics ».

\section{Structure}

Le Panorama des administrations publiques 2013 est structuré de la façon suivante : la publication s'ouvre par un chapitre spécial consacré à la confiance dans les pouvoirs publics, et notamment à l'état actuel de cette confiance et aux difficultés qui se profilent pour les pays membres de l'OCDE.

Les chapitres 2 à 9 présentent des données portant sur les aspects suivants de l'action publique : «La gouvernance stratégique », «Les finances et l'économie publiques », «Les pratiques et les procédures budgétaires ", "L’emploi et la rémunération dans le secteur public ", "Les femmes dans la fonction publique ", "Les marchés publics " et "Une administration ouverte et inclusive ", avec un chapitre spécial intitulé : "Éclairage - Au service des citoyens : l'accessibilité et la qualité des services publics ". Ces chapitres mettent en relief la nécessité de données plus probantes sur l'impact et l'utilité des divers outils de gestion publique adoptés. La publication se clôt sur un glossaire et sur plusieurs annexes méthodologiques.

\section{Les défis futurs}

Pour produire le Panorama des administrations publiques, l'OCDE travaille en étroite collaboration avec d'autres organisations, parmi lesquelles l'Organisation internationale du travail (OIT), le World Justice Project, la Commission européenne pour l'efficacité de la justice (CEPEJ), Gallup et la Commission européenne, afin de proposer une vue d'ensemble de la nature et des modalités de l'action publique tout en évitant les doublons au niveau de la collecte des données. Cette coopération permet d'obtenir des données comparables pour les pays couverts par la publication.

Pour les éditions futures, l'équipe chargée du Panorama des administrations publiques prévoit de mener des travaux dans les domaines suivants :

- une cartographie des organismes du secteur public et de leurs caractéristiques

- le recueil de nouvelles données sur les pratiques en matière de gestion réglementaire et sur leurs résultats 
- des indicateurs portant sur la structure, les fonctions, les pouvoirs, les responsabilités et les priorités des centres de gouvernement [c'est-à-dire du ou des services d'appui au travail collectif de l'exécutif et du Premier ministre ou du Président].

- éventuellement, une comparaison des niveaux et des structures de rémunération proposés dans le secteur public et le secteur privé

- de nouveaux indicateurs sur le lobbying et le financement de la vie politique

- une nouvelle édition de l'enquête sur les pratiques de gestion stratégique des ressources humaines. 


\section{Chapitre 1}

\section{Confiance dans les pouvoirs publics, efficacité de l'action publique et stratégies en matière de gouvernance}

Les données statistiques concernant Israël sont fournies par et sous la responsabilité des autorités israéliennes compétentes. L'utilisation de ces données par l'OCDE est sans préjudice du statut des hauteurs du Golan, de Jérusalem-Est et des colonies de peuplement israéliennes en Cisjordanie aux termes du droit international. 


\section{Introduction}

La crise économique et financière qui a éclaté en 2008 s'est soldée par une importante perte de confiance à l'égard des pouvoirs publics. En 2012, seuls 4 habitants de la zone OCDE sur 10 déclaraient leur faire confiance, en moyenne. À l'heure où les gouvernements cherchent la voie de la reprise économique, ils doivent relever un double défi : choisir les politiques à mener, mais aussi définir les modalités de leur mise en œuvre. Or, cette mise en œuvre implique la confiance. Si les citoyens n'ont pas confiance dans les pouvoirs publics, les marchés et les institutions, il est difficile de les faire adhérer aux réformes qui s'imposent, surtout quand elles impliquent des sacrifices de court terme, avec des gains de long terme peut-être moins tangibles. L'actuelle chute de la confiance à l'égard des pouvoirs publics est là pour le souligner : la confiance est un ingrédient essentiel, bien que trop souvent négligé, de la réussite de l'action publique.

Une baisse de confiance peut entraîner un moindre respect de la réglementation. Elle peut aussi rendre les particuliers et les entreprises plus frileux et les amener à reporter des décisions en matière d'investissement, d'innovation ou d'embauche qui seraient essentielles au retour de la compétitivité et à la relance. Nourrir la confiance, c'est donc investir dans la reprise économique et le bien-être social. La confiance fait partie des intrants des réformes du secteur public, puisqu'elle est nécessaire à leur mise en œuvre. Elle fait aussi partie de leurs réalisations, puisque les réformes influent sur les attitudes des individus et des organisations et sur des décisions qui jouent sur le bien-être économique et social. Par conséquent, la confiance que les particuliers et les entreprises accordent aux pouvoirs publics est essentielle pour une action publique efficace et efficiente, dans les périodes de conjoncture favorable comme dans les périodes de conjoncture défavorable. L'investissement dans la confiance doit être considéré comme une nouvelle démarche cruciale pour relancer la croissance économique et renforcer la cohésion sociale, et comme le signe que les pouvoirs publics sont en train de tirer les leçons de la crise.

Il est devenu plus difficile de maintenir la confiance, parce que l'information circule désormais plus rapidement et par des canaux plus diversifiés à travers l'ensemble de la société, notamment par le truchement de la société civile, d'Internet et des réseaux sociaux. Il semble donc que les pouvoirs publics désireux de conserver la confiance des parties prenantes ont affaire à un environnement plus complexe, dans lequel une action publique bien conçue et la reprise économique ne suffisent pas nécessairement à rétablir la confiance, si les citoyens se méfient du processus d'élaboration des politiques et trouvent injuste la répartition des coûts et des avantages. Il est essentiel de comprendre les déterminants de la confiance dans les pouvoirs publics pour enclencher un cycle vertueux propre à alimenter la croissance économique et le bien-être à moyen terme.

Le présent chapitre a pour objet d'étudier les rapports entre, d'un côté, la confiance dans les pouvoirs publics et, de l'autre, les politiques et les institutions de la gouvernance publique. Il évoque comment la confiance - et notamment la confiance à l'égard des pouvoirs publics - peut être définie, comment elle est mesurée et comment elle influe sur 
les attitudes des citoyens et sur leurs réactions face aux politiques publiques. À la lumière des données disponibles, il examine les déterminants de la confiance et cite un certain nombre de méthodes permettant de rendre les politiques plus fiables, plus réactives, plus ouvertes, plus inclusives et plus justes. Il s'agit d'une première exploration d'un thème qui, par le passé, a été abordé sous des angles relativement disparates par des experts de la gouvernance, de l'économie et de la sociologie. Même si d'autres travaux de recherche pourront être nécessaires en vue d'adopter un regard commun et de tirer des conclusions plus tranchées sur l'action à mener, le thème de la confiance permet d'ores et déjà d'aborder la gouvernance publique sous un angle nouveau, en prêtant une attention beaucoup plus grande aux perceptions de la population et à leur influence sur l'accueil réservé aux mesures et aux réformes lancées par les autorités. Comprendre la confiance pourrait donc permettre aux responsables publics et aux analystes de la gouvernance publique d'être plus sensibles aux attentes des citoyens et de mieux y répondre.

\section{La confiance dans les pouvoirs publics, qu'est-ce que c'est?}

Avoir confiance, c'est avoir une opinion positive de l'action d'un individu ou d'une organisation. Il s'agit d'un phénomène éminemment subjectif qui compte essentiellement dans la mesure où il influe sur le comportement. Il y a confiance dans les pouvoirs publics quand les citoyens se fient aux autorités pour mener une action judicieuse et juste (Easton, 1965). Cette confiance dépend de la congruence entre les préférences des citoyens - leur interprétation de ce qui est judicieux et juste - et leur perception du fonctionnement des pouvoirs publics (Bouckaert et Van de Walle, 2003). Les citoyens nourrissant des préférences diverses, ils se fondent sur une multitude de critères différents pour évaluer l'action des autorités et ses résultats. Ce qui est perçu comme judicieux et juste par un individu peut être jugé différemment par un autre. Pour analyser les déterminants de la confiance dans les pouvoirs publics, il faut comparer les préférences des citoyens à leur perception du fonctionnement des pouvoirs publics. Comme la confiance à l'égard des pouvoirs publics n'est pas dictée par leurs performances réelles, mais par leurs performances supposées, il faut également recenser les facteurs extérieurs aux performances des administrations qui jouent sur les perceptions des citoyens.

Globalement, la confiance dans les pouvoirs publics se fonde sur deux grandes composantes : 1) la confiance sociale, qui correspond à la confiance des citoyens à l'égard de la société dans laquelle ils vivent; et 2) la confiance politique, qui correspond au jugement que les citoyens portent sur le pouvoir et ses institutions. La confiance politique englobe à la fois la confiance au niveau supérieur, ou "macro ", qui est diffuse et fondée sur le système, et la confiance fondée sur les institutions. On a constaté que l'engagement civique et la confiance entre individus contribuaient à la confiance sociale globale (Putnam, 2000). Ce rapport n'est toutefois pas mécanique, et il peut être affecté par un certain nombre de facteurs d'ordre contextuel. Ainsi, il existe des pays où les individus n'ont pas confiance les uns en les autres - la confiance sociale est faible - et où ils comptent sur les institutions pour défendre leurs intérêts (Aghion et al., 2010).

Les attentes des citoyens jouent un rôle déterminant dans leur confiance à l'égard des pouvoirs publics. À mesure que le niveau d'instruction des citoyens s'élève, ils deviennent plus exigeants à l'égard des pouvoirs publics. Si les attentes des citoyens progressent plus vite que les performances des pouvoirs publics, la confiance et la satisfaction risquent de baisser. Cette évolution des attentes des citoyens pourrait peser d'un plus grand poids dans l'érosion du soutien politique que les performances effectives des administrations (Dalton, 2005), et elle pourrait surprendre des responsables publics restés fidèles à des diagnostics hérités du passé. 
En outre, la confiance des citoyens dans les pouvoirs publics est affectée de façon différente selon qu'ils ont connu une expérience positive ou négative en matière de prestation de services publics. Une expérience négative a une incidence bien plus forte sur la confiance à l'égard des pouvoirs publics qu'une expérience positive. Des politiques publiques ciblant les citoyens insatisfaits produiront donc un impact plus net sur cette confiance (Kampen et al., 2006).

Les analyses relatives à la confiance des citoyens valent aussi, dans une large mesure, pour la confiance des entreprises et même des fonctionnaires à l'égard des pouvoirs publics, mais il peut y avoir des différences au niveau de la formation des perceptions et des facteurs qui les influencent.

\section{Pourquoi la confiance dans les pouvoirs publics est-elle importante ?}

La confiance dans les pouvoirs publics a été identifiée comme l'un des principaux fondements de la légitimité et de la pérennité des systèmes politiques. La confiance est essentielle au bien-être et à la cohésion de la société, puisqu'elle influe sur l'aptitude des autorités à gouverner et leur permet d'agir sans recourir à la coercition. Elle représente donc un moyen efficient de réduire les coûts de transaction inhérents à toute relation sociale, économique et politique (Fukuyama, 1995). Un degré élevé de confiance à l'égard des pouvoirs publics peut accroître l'efficience et l'efficacité de leurs activités.

Un niveau minimum de confiance dans les pouvoirs publics est nécessaire en vue d'un fonctionnement juste et efficace des institutions publiques - notamment en termes de respect de l'état de droit, de fourniture de services publics de base et de mise à disposition d'infrastructures. L'état de droit et l'indépendance de la Justice sont particulièrement importants, car ils constituent des déterminants cruciaux de la confiance dans les pouvoirs publics, comme l'ont montré plusieurs études (Knack et Zak, 2003 ; Johnston, Krahn et Harrison, 2006 ; Blind, 2007). Dans la mesure où le bon fonctionnement des institutions publiques compte dans les décisions des investisseurs, la confiance dans les pouvoirs publics est un ingrédient indispensable en vue de la relance économique (Dasgupta, 2009 ; Algan et Cuha, 2013).

En même temps, la confiance dans les institutions publiques exerce sur le comportement des individus une influence qui peut aider l'action publique à atteindre ses objectifs, que ce soit dans le cadre de politiques et de programmes de portée relativement étroite (participation à des campagnes de vaccination, par exemple) ou de réformes plus vastes (protection de l'environnement ou réforme des retraites, par exemple). La confiance est importante, car de nombreux programmes publics ouvrent la voie à des comportements opportunistes. La confiance peut réduire le risque de voir apparaître de tels comportements, dans la mesure où les individus sont disposés à sacrifier certains avantages immédiats s'ils estiment que les politiques publiques auront des retombées positives à plus long terme, soit pour eux-mêmes (retraites), soit pour la collectivité (redistribution des revenus par la fiscalité).

La confiance dans les pouvoirs publics peut aider les gouvernements à mettre en œuvre des réformes structurelles dotées d'effets bénéfiques à long terme. De nombreuses réformes supposent d'accepter un sacrifice à court terme en échange de retombées positives à plus long terme, et nécessitent donc un large consensus social et politique pour être efficaces et viables. Dans un climat de forte confiance, de telles réformes peuvent être non seulement promulguées et mises en œuvre, mais aussi poursuivies suffisamment longtemps pour porter leurs fruits, ce qui permet d'inscrire les décisions dans un cadre temporel plus large. En revanche, dans un climat de faible confiance, les citoyens ont tendance à privilégier les 
avantages immédiats et partiels qu'ils peuvent s'approprier, ce qui incite les responsables politiques à jouer la carte du court terme et de l'opportunisme dans le cadre d'une démarche populiste (Gyorffy, 2013).

La confiance dans les pouvoirs publics peut favoriser un plus grand respect de la réglementation et réduire ses coûts de mise en application. Aussi aboutie et complète soit-elle, aucune règlementation n'est à l'abri de manquements. Un texte sera plus ou moins efficace selon que la population le considère comme suffisamment juste et légitime pour contrebalancer les avantages qu'elle tirerait de son non-respect. C'est particulièrement important quand l'écart est grand entre le coût lié au respect du texte et les avantages personnels tirés de son non-respect (en matière de fiscalité, par exemple), ou quand le respect du texte est difficile à contrôler (règles de circulation routière, par exemple). La confiance à l'égard du législateur peut conduire à un plus grand respect volontaire des textes (Murphy, 2004).

La confiance à l'égard des institutions publiques peut contribuer à renforcer la confiance dans l'économie en favorisant des décisions économiques - en matière d'investissement ou de consommation, par exemple - propices à la croissance. La confiance dans les institutions et la confiance entre individus peuvent atténuer le sentiment de risque associé aux décisions, dans des domaines allant de la consommation de biens durables à la mobilité professionnelle en passant par les embauches et l'investissement. Une hausse de la confiance entre individus accroît la productivité totale des facteurs, ce qui favorise le progrès économique (Dasgupta, 2009). Ce mécanisme favorise à son tour la croissance économique et élargit l'horizon de planification des agents économiques, ce qui accentue le dynamisme économique.

La confiance à l'égard des pouvoirs publics semble particulièrement cruciale pendant les situations de crise comme les catastrophes naturelles, les crises économiques ou les périodes d'agitation politique, qui ramènent aux fonctions fondamentales de la gouvernance publique. La capacité des autorités à gérer les crises et à mettre en œuvre des stratégies de sortie de crise efficaces conditionne souvent leur survie et la réélection des responsables politiques. Dans le cadre de catastrophes majeures, un manque de confiance peut entraver les procédures d'urgence et de relèvement, ce qui est très dommageable pour la société et pèse sur la capacité d'action des pouvoirs publics. De même, la crise économique actuelle révèlera peut-être des dimensions de la confiance que les années antérieures d'évolution graduelle des pays n'avaient pas mises en évidence.

La confiance n'est pas nécessairement à sens unique. La confiance des citoyens et des organisations à l'égard des pouvoirs publics n'est pas la seule à compter dans l'efficacité de l'action publique : la confiance des pouvoirs publics à l'égard des citoyens et des organisations, et la confiance au sein même des pouvoirs publics, peuvent également influer sur la conception des politiques et leurs retombées (Bouckaert, 2012). Le degré de confiance des pouvoirs publics à l'égard des particuliers et des entreprises se reflète dans le mode de fonctionnement des administrations, dans l'organisation des services publics et dans leur efficience et leur efficacité - par exemple, système fiscal et recours à l'autoréglementation et à l'autosurveillance. De plus, la confiance des individus et des entreprises à l'égard des pouvoirs publics et la confiance des pouvoirs publics à l'égard des individus et des entreprises se nourrissent l'une de l'autre. Des pouvoirs publics ouverts et réactifs offrent un environnement propice au renforcement de la confiance mutuelle entre les pouvoirs publics et les citoyens. Malheureusement, la confiance qui émane des pouvoirs publics et qui existe en leur sein a été beaucoup moins étudiée que la confiance à leur égard. 
La confiance est longue à conquérir, mais peut se perdre rapidement. Évoquer l'incidence de la confiance dont jouissent les pouvoirs publics sur leurs performances, sur l'économie et sur la société ne suffit pas. Il faut aussi décrire ce qui pourrait arriver en cas de montée en puissance de la méfiance à l'égard des pouvoirs publics. Une perte de confiance pourrait rendre les particuliers (et les entreprises) moins enclins à respecter la loi, à consentir des sacrifices en période de crise et à payer des impôts. Elle pourrait de ce fait alourdir les coûts pesant sur les administrations publiques - d'où une perte d'efficience - ou éroder leurs recettes. Elle pourrait aussi compliquer la tâche des administrations s'agissant d'attirer et de fidéliser des agents publics.

\section{Mesurer la confiance à l'égard des pouvoirs publics}

La confiance est une question de perception, et la mesurer présente de nombreuses difficultés. C'est vrai à l'échelle nationale, et encore plus au niveau international. La confiance étant une opinion positive, on mesure la confiance à l'égard des pouvoirs publics au moyen d'enquêtes d'opinion dans le cadre desquelles on demande à des particuliers, des entreprises ou des experts s'ils font confiance (certaines enquêtes emploient, en anglais, le terme de " confidence ", d'autres celui de "trust ") aux pouvoirs publics, à leurs dirigeants ou à des institutions officielles spécifiques (les autorités locales ou le système judiciaire, par exemple). On les interroge aussi souvent sur leur satisfaction à l'égard de services publics tels que la police locale, le système éducatif ou le système de santé, bien qu'il s'agisse d'une notion quelque peu différente de celle de confiance.

Plusieurs enquêtes internationales portent sur la confiance des citoyens à l'égard des pouvoirs publics (voir le tableau 1.1). L'enquête Gallup World Poll fournit des données pour l'ensemble des 34 pays de l'OCDE, avec une régularité suffisante pour refléter l'impact de la crise économique et financière mondiale sur la confiance dans les pouvoirs publics. C'est l'enquête World Values Survey qui mesure cette confiance depuis le plus longtemps, mais l'ensemble de données manque de cohésion, et les données ne sont disponibles que pour des groupes d'années dont le dernier remonte à 2005-07. L'Eurobaromètre de l'Union européenne propose l'ensemble de données le plus cohérent (et des points de données semestriels), mais il ne couvre que 23 pays membres de l'OCDE. Pour sa part, l'enquête Edelman Trust Barometer ne propose de séries chronologiques que pour un échantillon de population limité (la détention d'un diplôme universitaire et d'un revenu du ménage situé dans le quartile supérieur font partie des critères d'échantillonnage).

Tableau 1.1. Les enquêtes internationales sur la confiance à l'égard des pouvoirs publics

\begin{tabular}{|c|c|c|c|c|}
\hline Nom de l'enquête & $\begin{array}{l}\text { Nombre de pays } \\
\text { de l'OCDE couverts }\end{array}$ & $\begin{array}{c}\text { Années couvertes } \\
\text { et fréquence }\end{array}$ & Valeur mesurée & Réponses possibles \\
\hline World Gallup Poll & 34 & 2005-12 (annuelle) & $\begin{array}{c}\text { Confiance (“ confidence ») } \\
\text { dans les autorités nationales }\end{array}$ & 2 : oui/non \\
\hline World Values Survey & 25 & $\begin{array}{l}4 \text { groupes d'années : } \\
\text { 1989-93 ; 1994-98 ; } \\
\text { 1999-2004 ; 2005-08. }\end{array}$ & $\begin{array}{l}\text { Confiance (« confidence») } \\
\text { dans les pouvoirs publics }\end{array}$ & $\begin{array}{c}4 \text { : beaucoup/ } \\
\text { relativement beaucoup/ } \\
\text { pas beaucoup/pas du tout }\end{array}$ \\
\hline Eurobaromètre & 23 & 2003-13 (semestrielle) & $\begin{array}{c}\text { Confiance (« trust ») } \\
\text { dans les pouvoirs publics }\end{array}$ & $\begin{array}{l}2 \text { : plutôt confiance/ } \\
\text { plutôt pas confiance }\end{array}$ \\
\hline Edelman Trust Barometer & 15 & 2001-13 (annuelle) & $\begin{array}{c}\text { Confiance («trust }) \\
\text { dans les pouvoirs publics }\end{array}$ & $\begin{array}{l}9 \text { réponses possibles, } \\
\text { de } 1 \text { (aucune confiance) } \\
\text { à } 9 \text { (forte confiance) }\end{array}$ \\
\hline Latinobarómetro & 3 & 1995-2012 (annuelle) & $\begin{array}{c}\text { Confiance («trust») } \\
\text { dans les pouvoirs publics }\end{array}$ & $\begin{array}{l}4 \text { réponses : forte confiance/ } \\
\text { confiance modérée/confiance } \\
\text { restreinte/aucune confiance }\end{array}$ \\
\hline
\end{tabular}


Ces enquêtes étant conçues pour permettre des comparaisons internationales, leurs questions comportent une part d'ambiguïté et sont souvent assujetties à l'interprétation qu'en font les personnes interrogées, car la notion de "pouvoirs publics " n'y est généralement pas définie. Les enquêtes se fondent sur des méthodologies similaires en matière d'échantillonnage, mais divergent au niveau de la formulation des questions (par exemple, nuances entre une question portant sur la notion de " confidence » et une question portant sur la notion de " trust », échelles de notation différentes) et s'agissant des autres indicateurs de confiance susceptibles de servir d'éléments de comparaison (par exemple, confiance dans le parlement national, dans les institutions financières, dans le personnel politique, dans les fonctionnaires, dans les organisations internationales, dans les services publics tels que la santé ou l'éducation, dans les entreprises ou dans les institutions religieuses).

En raison de ces limites des enquêtes internationales, il est difficile d'analyser de façon approfondie l'évolution des niveaux de confiance des citoyens et les facteurs qui influent sur ces niveaux dans la zone OCDE et au-delà. Du fait de l'influence des facteurs culturels sur l'attitude de la population face aux institutions publiques, il est particulièrement ardu d'effectuer des comparaisons internationales pures des niveaux de confiance à l'égard des pouvoirs publics. Enfin - et peut-être surtout - les enquêtes existantes n'ont pas été conçues pour contribuer à l'analyse des politiques ou pour aboutir à des recommandations pratiques.

S'il n'est pas possible d'appuyer un exercice de comparaison internationale sur les enquêtes nationales relatives à la confiance à l'égard des pouvoirs publics, ces enquêtes nationales présentent plus d'intérêt en vue de l'analyse des politiques et, ce, pour de nombreuses raisons. Par rapport aux enquêtes internationales, elles éclairent mieux les déterminants de la confiance et peuvent être corrigées pour tenir compte des cycles électoraux. Par exemple, le baromètre de la confiance des citoyens réalisé par Metroscopia en Espagne propose chaque mois des données qui permettent de comparer la satisfaction à l'égard des pouvoirs publics à la perception de la situation économique. De plus, les enquêtes nationales couvrent de façon plus approfondie la confiance à l'égard de l'ensemble de la sphère publique. Ainsi, au Royaume-Uni, IPSOS Mori publie deux fois par an les grandes tendances de la confiance à l'égard des institutions publiques (par exemple, différents niveaux d'administration, parlement), des services publics, des politiques économiques (par exemple, en matière de croissance économique, de chômage, d'inflation et de pouvoir d'achat), des partis politiques et des responsables politiques (dirigeants de l'exécutif, personnel politique, parlementaires) ainsi que des données sur les perceptions relatives à la corruption au sein des pouvoirs publics. Les enquêtes nationales peuvent aussi permettre d'évaluer la confiance à l'égard des politiques en cours. Par exemple, en France, l'IFOP demande aux citoyens s'ils font confiance au gouvernement pour réaliser des objectifs spécifiques annoncés lors de son arrivée au pouvoir. Enfin, les enquêtes nationales portent généralement sur des séries temporelles beaucoup plus longues. Ainsi, aux États-Unis, le Pew Research Center propose des données sur la confiance à l'égard des pouvoirs publics depuis la fin des années cinquante.

Il semble donc qu'on pourrait en faire davantage pour renforcer la comparabilité des données tirées des enquêtes d'opinion et pour mieux alimenter le débat sur l'action à mener. Tout d'abord, on pourrait rendre les enquêtes plus représentatives. Les enquêtes actuelles portent sur des échantillons de petite taille et sont rarement géographiquement représentatives au sein du pays concerné. De plus, d'autres caractéristiques des personnes interrogées - leur âge, leur sexe, leur appartenance ethnique, leur niveau d'études, leur 
situation matrimoniale, leur niveau de revenu, leur expérience des services publics, etc. influent aussi sur leur perception des pouvoirs publics, et il serait utile que l'échantillon en tienne compte. Ensuite, les questions des enquêtes pourraient être améliorées. Les termes clés devraient être définis de façon précise. Par exemple, dans l'enquête Gallup World Poll, on demande aux personnes interrogées quelle confiance elles accordent aux autorités nationales (" national government »), sans préciser en quoi consistent ces autorités. Les répondants peuvent donc considérer, soit que cette expression désigne les dirigeants politiques, soit qu'elle désigne la bureaucratie. De plus, il faudrait formuler les questions et les choix de réponses de façon à permettre aux pouvoirs publics d'en tirer des conséquences concrètes (en modifiant leur comportement, par exemple). Enfin, une collecte régulière des données permettrait non seulement d'effectuer des comparaisons internationales ponctuelles, mais aussi de détecter les changements intervenant au fil du temps et les grandes tendances à l'œuvre, au sein de chaque pays comme à l'échelle internationale.

\section{Caractéristiques et évolution de la confiance à l'égard des pouvoirs publics dans les pays de l'OCDE}

Malgré les difficultés d'ordre méthodologique que soulève l'évaluation de la confiance dans les pouvoirs publics, les données disponibles révèlent l'existence de caractéristiques, de grandes tendances et de corrélations qui sont révélatrices du niveau de cette confiance dans les pays de l'OCDE et qui peuvent aider les responsables publics à approfondir la question.

En premier lieu, les données les plus récentes dont on dispose pour les pays de l'OCDE montrent que, quand on interroge les citoyens sur leur confiance à l'égard des autorités nationales, leurs réponses varient nettement selon les pays, avec un taux de confiance moyen nettement inférieur à $50 \%$ (graphique 1.1). En d'autres termes, quand on leur pose la question dans le cadre d'une enquête, moins de la moitié des citoyens des pays de l'OCDE déclarent faire confiance à leurs autorités nationales. Les moyennes nationales s'échelonnent entre près de $80 \%$ pour la Suisse et $12 \%$ pour la Grèce. Entre ces deux extrêmes, les niveaux de confiance constatés ne semblent pas refléter le niveau de vie, le PIB par habitant ou le taux de croissance. Alors que le Japon et la Corée - qui sont respectivement un pays à revenu élevé et un pays à forte croissance - présentent des scores de confiance inférieurs à la moyenne OCDE, la Turquie affiche un score largement supérieur à cette moyenne, malgré un PIB par habitant plus faible. Peut-être faut-il en déduire que la confiance à l'égard des pouvoirs publics tient davantage aux facteurs culturels, aux nouvelles attentes des citoyens et à l'actualité politique qu'aux évolutions économiques à long terme et aux niveaux de vie absolus. Les données relatives à certains pays émergents confortent cette analyse en mettant en relief l'influence des attentes des citoyens sur leur niveau de confiance (encadré 1.1).

En deuxième lieu, on constate un niveau moyen de confiance plus bas en 2012 qu'en 2007, avant la crise (partie inférieure du graphique 1.1). Le pourcentage moyen de personnes interrogées déclarant avoir confiance dans leurs autorités nationales a reculé de cinq points de pourcentage entre 2007 et 2012, passant de $45 \%$ à $40 \%$. Cette comparaison masque des variations beaucoup plus nettes au niveau des pays, la confiance ayant reculé dans plus des deux tiers des pays de l'OCDE entre 2007 et 2012. C'est dans les pays touchés par une crise de nature politique, budgétaire ou économique tels que la Grèce, la Slovénie, l'Irlande, l'Espagne, la Belgique et le Portugal que la confiance a le plus reculé. En revanche, la confiance à l'égard des pouvoirs publics s'est renforcée dans d'autres pays, notamment 


\section{Graphique 1.1. La confiance à l'égard des autorités nationales en 2012 et son évolution par rapport à 2007}

Par ordre décroissant d'évolution en points de pourcentage entre 2007 et 2012

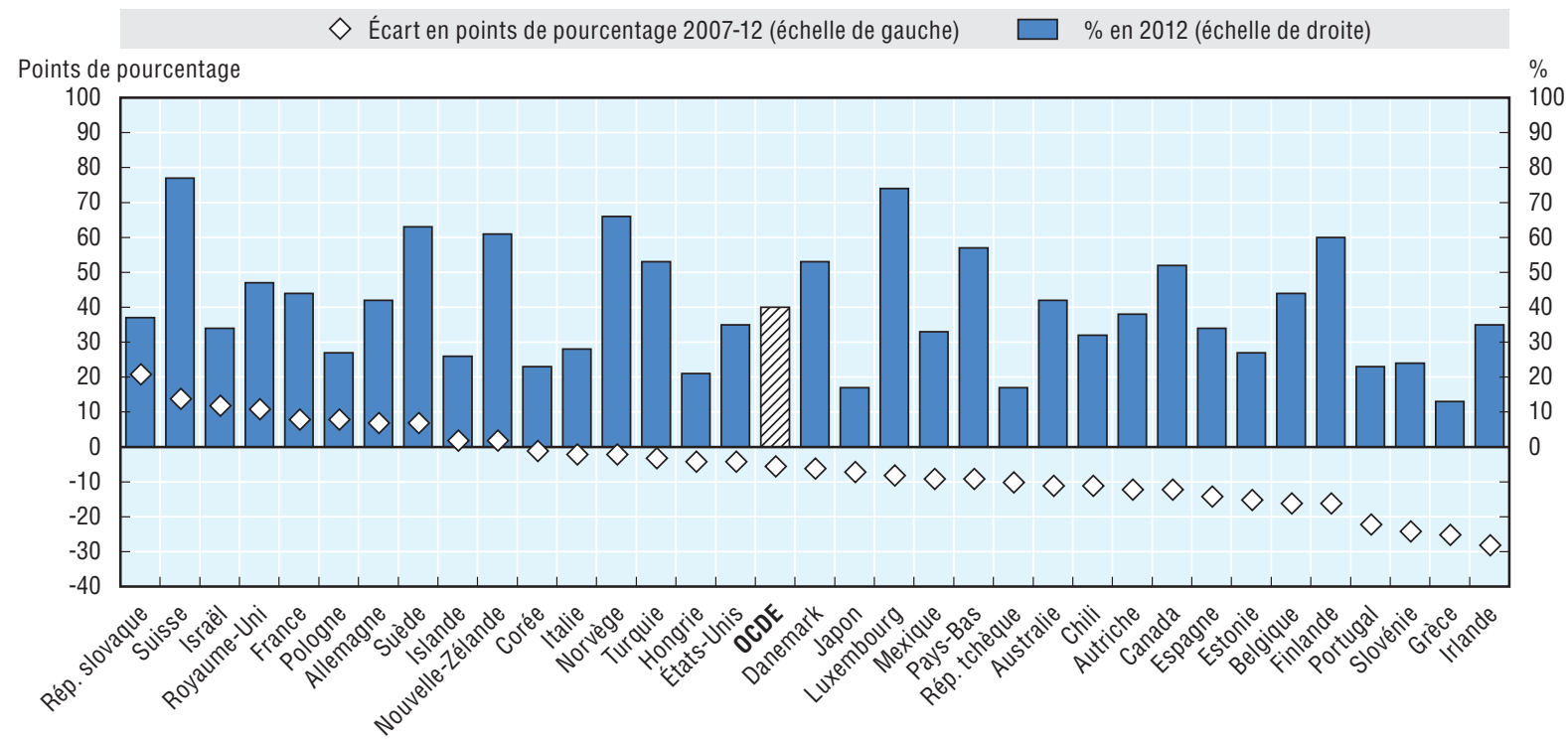

Note : Les données correspondent au pourcentage de réponses positives à la question "Avez-vous confiance dans les autorités nationales ?». Les données relatives à l'Allemagne, au Chili et au Royaume-Uni portent sur 2011 et non 2012. Les données relatives à l'Islande et au Luxembourg portent sur 2008 et non 2007. Les données relatives à l'Autriche, à la Finlande, à l'Irlande, à la Norvège, au Portugal, à la République slovaque, à la Slovénie et à la Suisse portent sur 2006 et non 2007.

Source: Gallup World Poll.

StatLink (ils http://dx.doi.org/10.1787/888933158503

\section{Encadré 1.1. La confiance à l'égard des autorités nationales dans les BRIICS (2012)}

Dans les BRIICS, en moyenne, la majorité des citoyens (54\%) déclarent faire confiance à leurs autorités nationales (chiffres de 2012). C'est en Indonésie et en Chine que ce niveau de confiance est le plus élevé (avec deux tiers de citoyens se déclarant confiants) et en Afrique du Sud, au Brésil et en Fédération de Russie qu'il est le plus faible (tous ces pays se situant dans une fourchette de $45 \%$ à $47 \%$ ). Dans tous les BRIICS, la confiance à l'égard des pouvoirs publics est plus élevée que la moyenne OCDE (40\%). Sur la période 2007-12, la confiance à l'égard des autorités nationales a baissé de trois points de pourcentage, en moyenne, dans l'ensemble des BRIICS (à l'exception de la Chine), ce qui représente un recul moins marqué que dans la zone $\mathrm{OCDE}$, où la baisse moyenne a été de cinq points de pourcentage. C'est en Indonésie que la confiance a le plus augmenté (de 15 points de pourcentage) et en Inde qu'elle a le plus diminué (de 27 points de pourcentage). L'existence, dans l'ensemble des BRIICS, de niveaux de confiance supérieurs à ceux constatés dans la plupart des pays de l'OCDE peut tenir à des facteurs d'ordre culturel et contextuel, mais aussi, peut-être, au fait que les citoyens nourrissent des attentes différentes à l'égard des services officiels et de leurs performances, parce que leur pays se situe à un stade de développement socio-économique différent. Les chercheurs s'accordent néanmoins à considérer qu'il est plus important d'atteindre le niveau optimal de confiance que le niveau maximal de confiance (Knack et Keefer, 1997 ; Nooteboom, 2006 ; Dasgupta, 2009 ; Bouckaert, 2012). 


\section{Encadré 1.1. La confiance à l'égard des autorités nationales dans les BRIICS (2012) (suite)}

\section{Dans les BRIICS, la confiance à l'égard des autorités nationales est plus élevée que dans la zone OCDE}

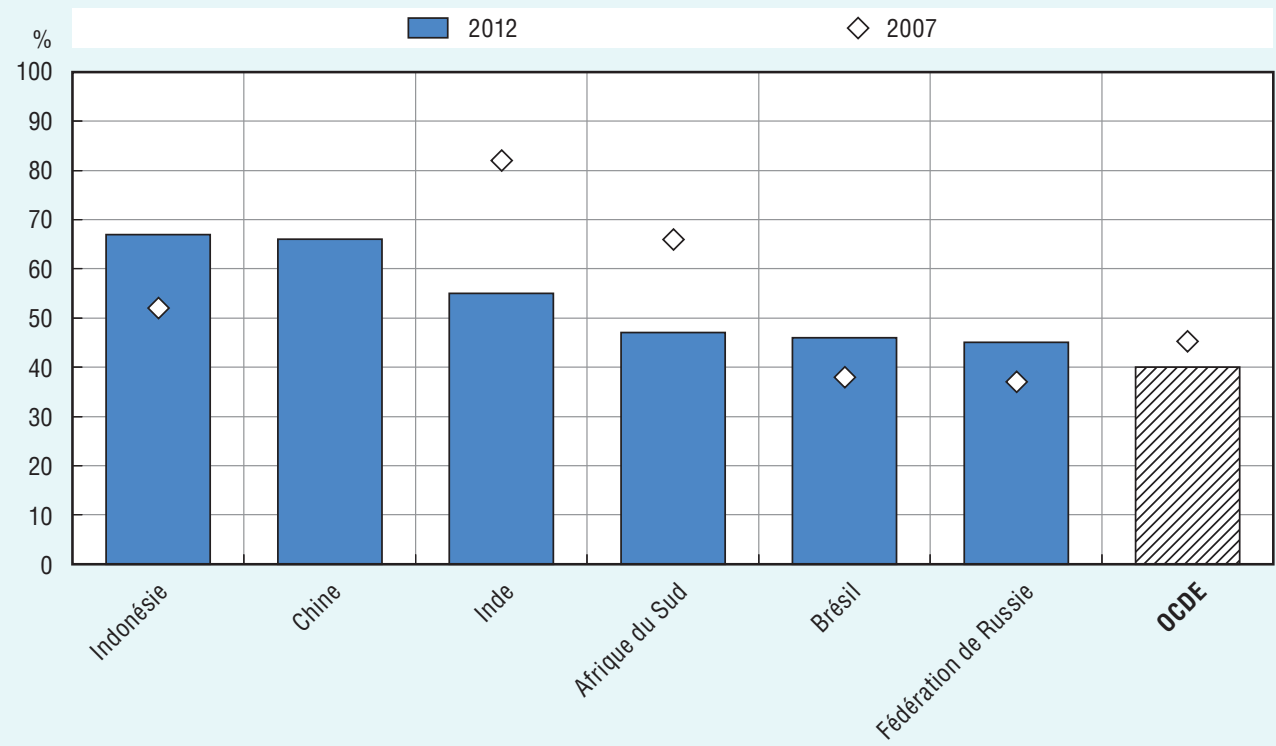

Note : Les données correspondent au pourcentage de réponses positives à la question " Dans ce pays, avezvous confiance dans chacune des entités suivantes ? Et dans les autorités nationales? ».

Source : Gallup World Poll.

en République slovaque, en Israël, au Royaume-Uni, en Pologne, en France, en Suisse, en Allemagne et en Suède.

En troisième lieu, on constate que la confiance à l'égard des pouvoirs publics se situe, en moyenne, à des niveaux similaires à la confiance dans deux institutions clés du secteur privé : a) les institutions financières et les banques ; et b) les médias. Toutefois, on note là aussi des variations importantes selon les pays de l'OCDE. Globalement, dans l'ensemble des pays de l'OCDE, les institutions financières et les banques bénéficient d'un niveau de confiance légèrement plus élevé ( $43 \%$ ) que les pouvoirs publics ( $40 \%$ ) (voir le graphique 1.2). Dans certains pays - ceux qui ont été les moins touchés par la crise financière de 2008 - les institutions financières et les banques jouissent d'un niveau de confiance élevé. C'est notamment le cas au Canada, en Pologne, en Finlande, en Norvège, au Mexique, en Australie et au Japon. À l'inverse, dans les pays les plus touchés par cette crise, la confiance tend à être relativement plus forte à l'égard des pouvoirs publics qu'à l'égard des institutions financières. C'est notamment le cas en Irlande, en Espagne et en Italie.

Les dernières données disponibles sur ce sujet montrent qu'en 2010, la confiance à l'égard des médias était nettement supérieure à la confiance à l'égard des pouvoirs publics en Irlande, en Espagne et au Portugal, alors qu'elle lui était sensiblement inférieure en Turquie, en Suède, aux Pays-Bas et au Luxembourg (voir le graphique 1.3). Dans la mesure où les pays du premier groupe sont ceux qui ont connu la plus forte dégradation de la confiance dans les pouvoirs publics au cours de la crise et où les pays du second groupe comptent parmi ceux qui affichent les niveaux de confiance les plus élevés et les plus stables, cette comparaison en dit peut-être plus long sur l'évolution de la confiance dans 


\section{Graphique 1.2. Confiance dans les institutions financières et confiance dans les pouvoirs publics}

Comparaison entre la confiance dans les institutions financières/les banques et la confiance dans les pouvoirs publics en 2012

Confiance dans les autorités nationales (\%)

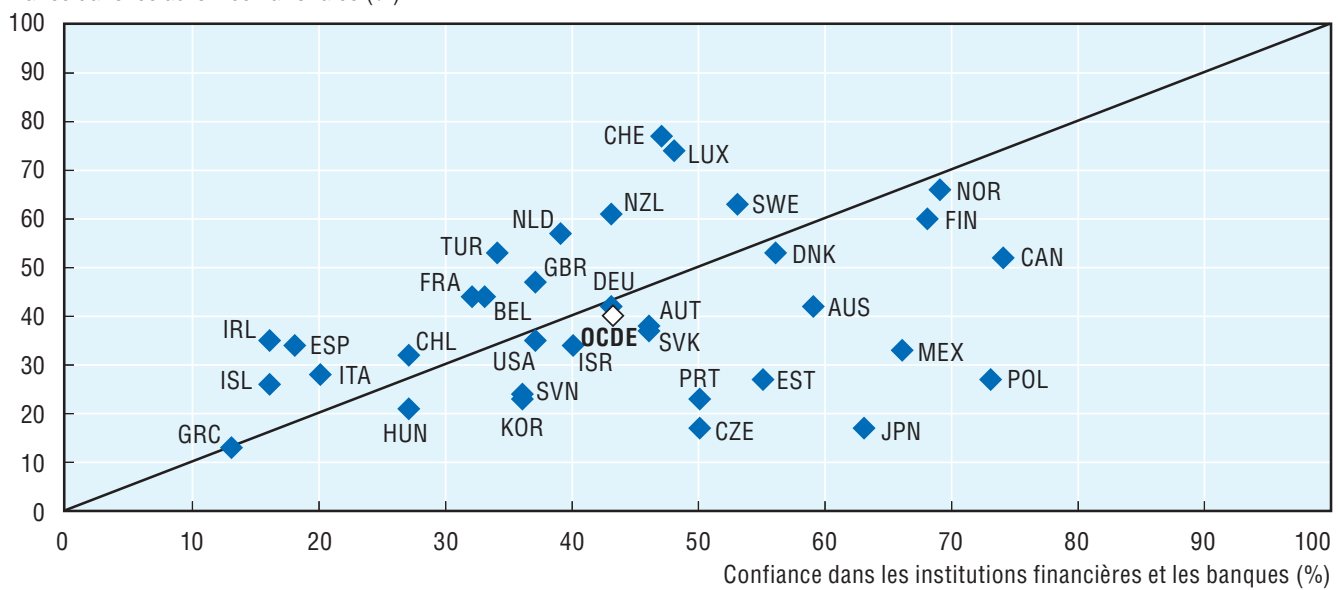

Note: Les données relatives à la confiance dans les autorités nationales correspondent au pourcentage de réponses positives à la question « Dans ce pays, avez-vous confiance dans chacune des entités suivantes ? Et dans les autorités nationales? ". Les données relatives à la confiance dans les institutions financières et les banques correspondent au pourcentage de réponses positives à la question «Dans ce pays, avez-vous confiance dans chacune des entités suivantes ? Et dans les institutions financières ou les banques ? ». Les données relatives au Chili, à l'Allemagne, et au Royaume-Uni portent sur 2010 et non 2011. Dans les pays situés en dessous de la ligne, la confiance dans les institutions financières et les banques est plus forte que la confiance dans les pouvoirs publics.

Informations sur les données concernant Israël : http://dx.doi.org/10.1787/888932315602.

Source : Gallup World Poll.

\section{Graphique 1.3. Confiance dans les médias et confiance dans les pouvoirs publics}

Comparaison entre la confiance dans les autorités nationales et la confiance dans les médias (2010)

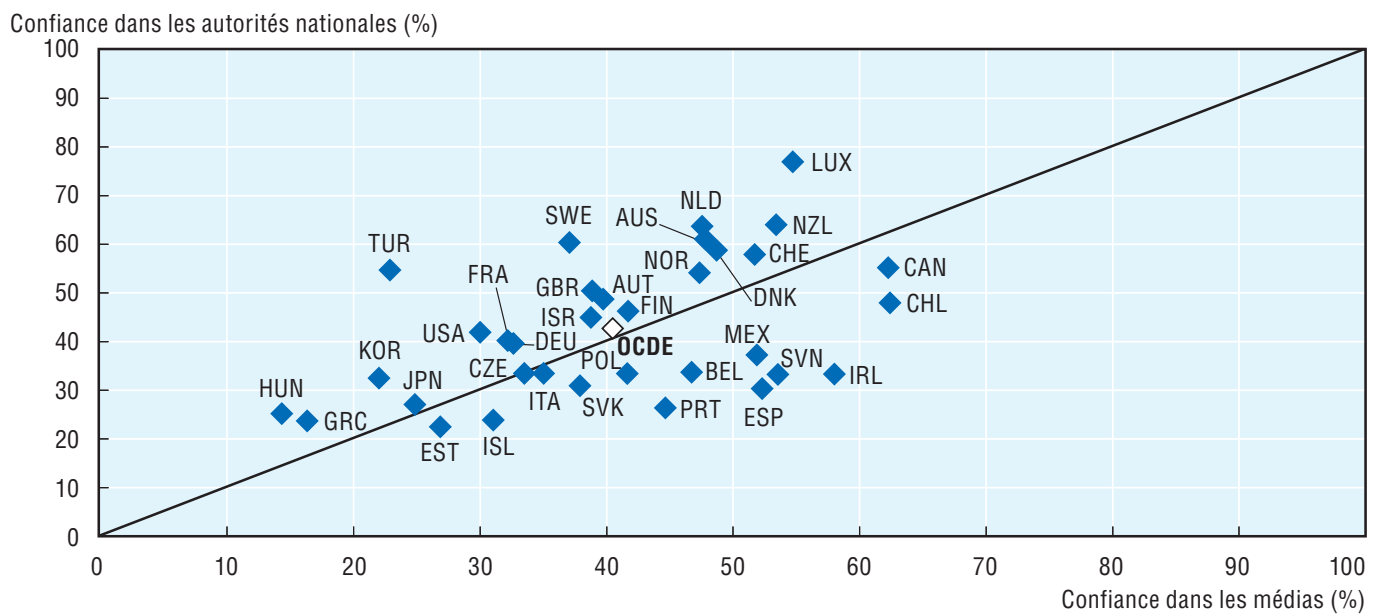

Note: Les données relatives à la confiance dans les autorités nationales correspondent au pourcentage de réponses positives à la question « Dans ce pays, avez-vous confiance dans chacune des entités suivantes ? Et dans les autorités nationales? ». Les données relatives à la confiance dans les médias correspondent au pourcentage de réponses positives à la question « Dans ce pays, avez-vous confiance dans chacune des entités suivantes ? Et dans la qualité et l'intégrité des médias ? ". Les données relatives à l'Islande et à la Norvège portent sur 2008 et non 2010 . Les données relatives à la Suisse et à l'Estonie portent sur 2009 et non 2010. Dans les pays situés en dessous de la ligne, la confiance dans les médias est plus forte que la confiance dans les pouvoirs publics.

Informations sur les données concernant Israël : http://dx.doi.org/10.1787/888932315602.

Source: Gallup World Poll. 
les pouvoirs publics que sur celle de la confiance à l'égard des médias. À l'inverse, lorsque l'on compare les niveaux de confiance dans les pouvoirs publics et dans les institutions financières, c'est peut-être la dynamique liée à ce deuxième type de confiance qui l'emporte.

\section{Les déterminants de la confiance dans les pouvoirs publics}

La confiance à l'égard des pouvoirs publics comporte de multiples facettes et repose sur un ensemble d'interactions économiques, sociales et politiques entre les citoyens et les administrations. De nombreux travaux empiriques ont été consacrés aux rapports entre cette confiance et des paramètres économiques, sociaux et liés à la gouvernance. Ils ont permis de recenser quatre grands types de déterminants de la confiance dans les pouvoirs publics : 1) la culture ; 2) le contexte institutionnel ; 3) les retombées économiques et sociales ; et 4) les performances des institutions. S'il y a plus ou moins consensus sur cet éventail de déterminants, les données sont contradictoires s'agissant de leur influence précise et de l'étroitesse de leurs liens réciproques avec la confiance. Néanmoins, on s'accorde généralement à considérer que la confiance et la plupart de ses déterminants sont interdépendants et créent les conditions de leur propre réalisation, et qu'ils sont donc complémentaires dans leur rapport à la gouvernance publique et au développement économique.

Selon Bouckaert (2012), la confiance à l'égard des pouvoirs publics peut s'analyser à trois niveaux. Au niveau supérieur (ou "macro »), la confiance a trait aux institutions politiques et au fonctionnement de la démocratie. Au niveau intermédiaire (ou "méso "), elle a trait à l'élaboration des politiques - c'est-à-dire à l'aptitude des pouvoirs publics à gérer les problématiques économiques et sociales et à susciter des attentes positives concernant le bien-être futur. Au niveau inférieur (ou « micro »), enfin, elle a trait à l'impact de l'action publique sur la vie quotidienne des citoyens par le biais de la prestation des services publics. Bien que distincts, ces trois niveaux interagissent, et un manque important de confiance à un niveau peut se répercuter sur la confiance aux autres niveaux, ainsi que sur les retombées des politiques. Par conséquent, les efforts visant à renforcer la confiance doivent amplifier les synergies entre ces diverses sphères.

La taxinomie proposée par Bouckaert est particulièrement utile pour deux raisons. Tout d'abord, elle semble indiquer que la confiance n'est pas simplement quelque chose qui arrive aux pouvoirs publics, mais un élément qu'ils peuvent influencer par leurs actes et leurs politiques. Deuxièmement, elle suggère que, s'agissant d'agir sur la confiance, ce n'est pas uniquement le fond des politiques qui compte, mais également leur forme, leurs destinataires et leurs partenaires. Par conséquent, ce ne sont pas seulement les résultats finals, mais aussi les processus suivis pour les obtenir, qui sont importants pour les particuliers et les entreprises.

Pour mieux comprendre la dimension de la confiance liée à la gouvernance publique le contexte institutionnel et ses performances - il peut être utile de décomposer ce concept en une série de composantes interdépendantes qui, ensemble, englobent toutes les attentes des citoyens. À cet égard, l'OCDE a recensé les composantes suivantes :

- La fiabilité : capacité des pouvoirs publics à réduire au minimum l'incertitude de l'environnement économique, social et politique et à agir de manière cohérente et prévisible.

- La réactivité : prestation de services publics accessibles, efficients, axés les usagers et répondant effectivement à leurs besoins et à leurs attentes. 
- L'ouverture et l'inclusivité : démarche systémique et exhaustive visant à institutionnaliser avec les parties prenantes une communication bilatérale dans le cadre de laquelle des informations pertinentes et exploitables sont fournies, le dialogue étant encouragé en tant que facteur de renforcement de la transparence, de la responsabilité et de la participation.

- L'intégrité : respect par l'État et les institutions publiques de normes de conduite et de principes d'ordre général qui contribuent à la préservation de l'intérêt général tout en prévenant la corruption.

- L'équité : au niveau des procédures, traitement égal des particuliers (et des entreprises) dans le cadre des processus d'élaboration et de mise en œuvre des politiques.

On s'est fondé, ci-après, sur les trois niveaux d'analyse de Bouckaert pour recenser les déterminants potentiels de la confiance dans le domaine de la gouvernance et pour évoquer les données tirées des enquêtes internationales qui semblent indiquer une corrélation statistique. L'exercice en est encore à un stade préliminaire et ne permet pas de tirer de conclusions quant à d'éventuels liens de causalité, mais il pourrait guider de futures recherches et discussions.

\section{Au niveau supérieur (" macro »)}

Au niveau supérieur ("macro»), ce sont les institutions politiques et le fonctionnement de la démocratie qui comptent pour la confiance à l'égard des pouvoirs publics. Pour devenir membre de l'OCDE, un pays doit remplir une condition préalable cruciale : être une démocratie dotée d'institutions politiques solides.

Pour ce qui est des institutions politiques, du moins dans les pays européens pour lesquels on dispose de données, les citoyens manifestent systématiquement une plus grande confiance dans les pouvoirs publics que dans les partis politiques (voir les graphiques 1.4 et 1.5). Selon des chiffres de 2013, parmi les pays européens membres de l'OCDE, un seul (le Danemark) présente des taux de confiance similaires à l'égard des

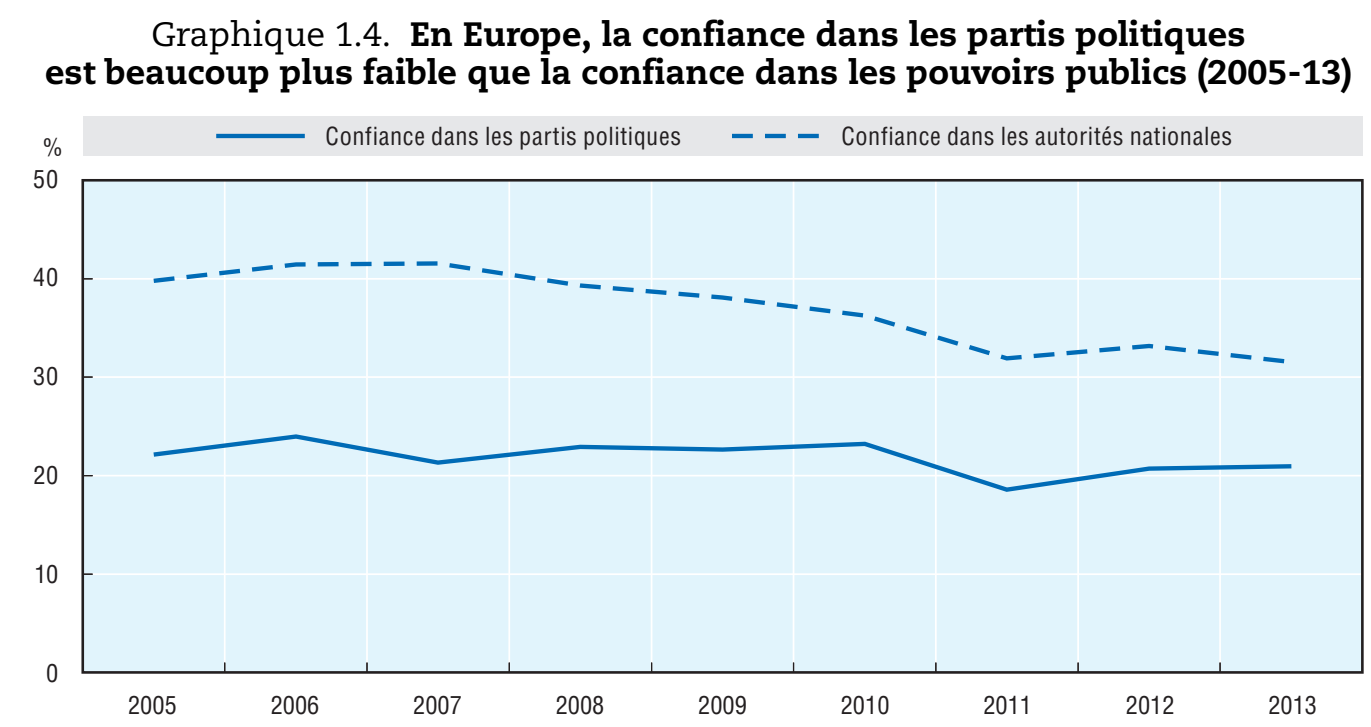

Note: Les données correspondent au pourcentage de réponses " plutôt confiance " à la question « Pour chacune des institutions suivantes, pourriez-vous me dire si vous avez plutôt confiance ou plutôt pas confiance en elle: le gouvernement (national) ; les partis politiques ". Les données correspondent à des moyennes annuelles pour 23 pays membres de l'OCDE ; on ne dispose pas de données pour l'Australie, le Canada, le Chili, Israël, le Japon, la Corée, le Mexique, la Nouvelle-Zélande, la Norvège la Suisse et les États-Unis.

Source: Eurobaromètre (base de données), calculs de l'OCDE. 


\section{Graphique 1.5. Confiance dans les pouvoirs publics et les partis politiques dans les pays européens membres de l'OCDE (2013)}

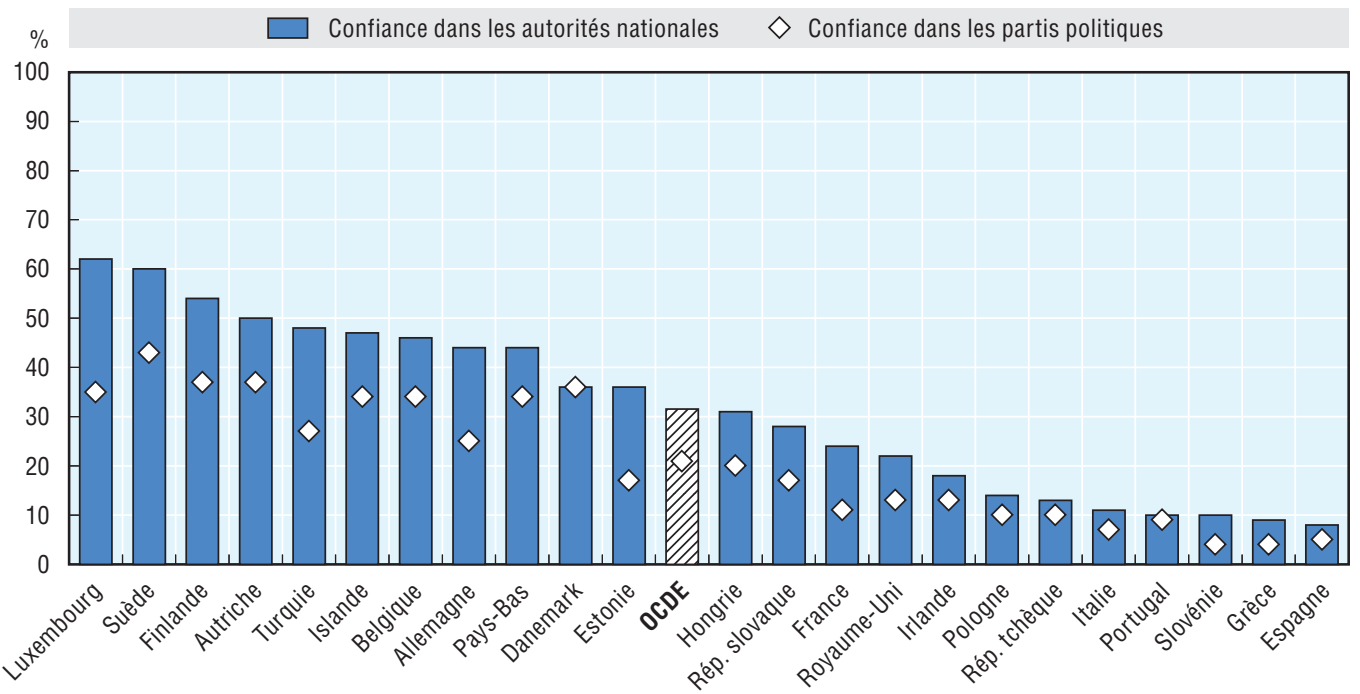

Note : Les données correspondent au pourcentage de réponses «plutôt confiance » à la question " Pour chacune des institutions suivantes, pourriez-vous me dire si vous avez plutôt confiance ou plutôt pas confiance en elle: le gouvernement (national) ; les partis politiques ". Les données correspondent à des moyennes annuelles pour 23 pays membres de l'OCDE ; on ne dispose pas de données pour l'Australie, le Canada, le Chili, Israël, le Japon, la Corée, le Mexique, la Nouvelle-Zélande, la Norvège, la Suisse et les États-Unis.

Source : Eurobaromètre (base de données).

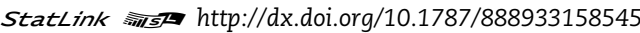

pouvoirs publics et des partis politiques; dans tous les autres, les partis politiques inspirent moins confiance que les pouvoirs publics. C'est dans les pays les plus touchés par les crises budgétaires, tels que la Slovénie, la Grèce, l'Espagne, l'Italie et le Portugal, que la confiance à l'égard des partis politiques est la plus faible, puisque moins de $10 \%$ des personnes interrogées déclarent leur faire confiance. C'est également dans ces pays que la confiance à l'égard des pouvoirs publics est la plus basse.

On trouve parmi les piliers de la démocratie, aux côtés d'élections libres et régulières, le principe du respect de l'état de droit, qui est à la fois une réalisation et un indicateur de performance du processus et qui signifie que nul n'est au-dessus des lois, y compris les pouvoirs publics, que les textes protègent les droits fondamentaux et que tout le monde a accès à la justice. C'est confirmé par la forte corrélation qui existe entre la confiance de la population dans les autorités nationales et dans le système judiciaire (voir le graphique 1.6). La confiance à l'égard du système judiciaire est tout à la fois une réalisation et une dimension clé de la gouvernance, dotée de rapports particulièrement étroits avec l'intégrité.

La participation électorale représente un autre indicateur supplétif traditionnel de la confiance à l'égard du système politique. Toutefois, on est en présence d'hypothèses contradictoires s'agissant des rapports entre cette participation et la confiance. Selon la première de ces hypothèses, une participation électorale élevée peut refléter une grande confiance dans le système politique; selon la deuxième, une confiance faible dans le gouvernement en place peut inciter les électeurs à se rendre aux urnes pour l'évincer. Toutefois, le coefficient de corrélation entre la confiance à l'égard des pouvoirs publics et la participation électorale est négligeable.

Quand ils se prononcent sur leur confiance à l'égard des autorités nationales, les citoyens formulent également un jugement sur les dirigeants de leur pays $\left(R^{2}: 0.9\right.$; voir le graphique 1.7). On peut se demander si les dirigeants en question correspondent 


\section{Graphique 1.6. La confiance dans le système judiciaire est importante pour la confiance dans les autorités nationales}

Corrélation entre la confiance dans les autorités nationales et la confiance dans le système judiciaire (2012)

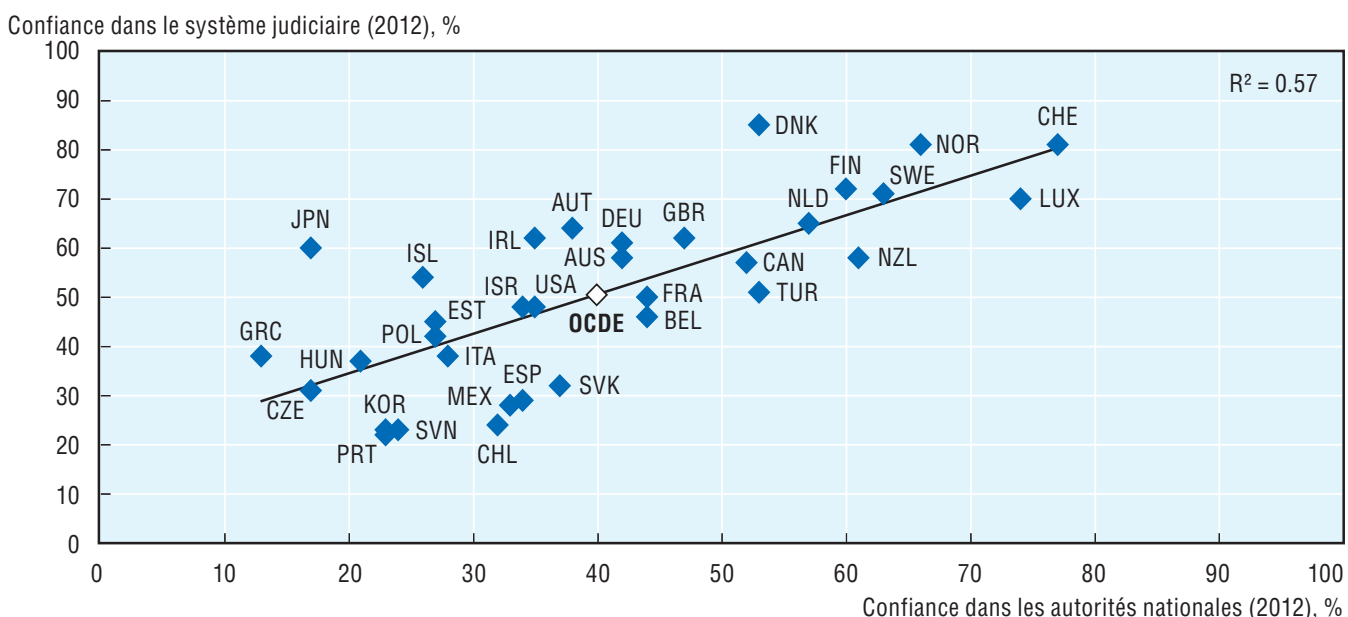

Note: Les données relatives à la confiance dans les autorités nationales correspondent au pourcentage de réponses positives à la question « Dans ce pays, avez-vous confiance dans chacune des entités suivantes ? Et dans les autorités nationales ? ". Les données relatives à la confiance dans le système judiciaire correspondent au pourcentage de réponses positives à la question «Dans ce pays, avez-vous confiance dans chacune des entités suivantes ? Et dans le système judiciaire et les tribunaux ? ". Les données relatives au Chili, à l'Allemagne et au Royaume-Uni portent sur 2011 et non 2012.

Informations sur les données concernant Israël : http://dx.doi.org/10.1787/888932315602.

Source : Gallup World Poll.

\section{Graphique 1.7. Les dirigeants jouent un rôle crucial dans la confiance à l'égard des autorités nationales}

Corrélation entre la confiance dans les autorités nationales et l'approbation à l'égard des dirigeants du pays (2012)

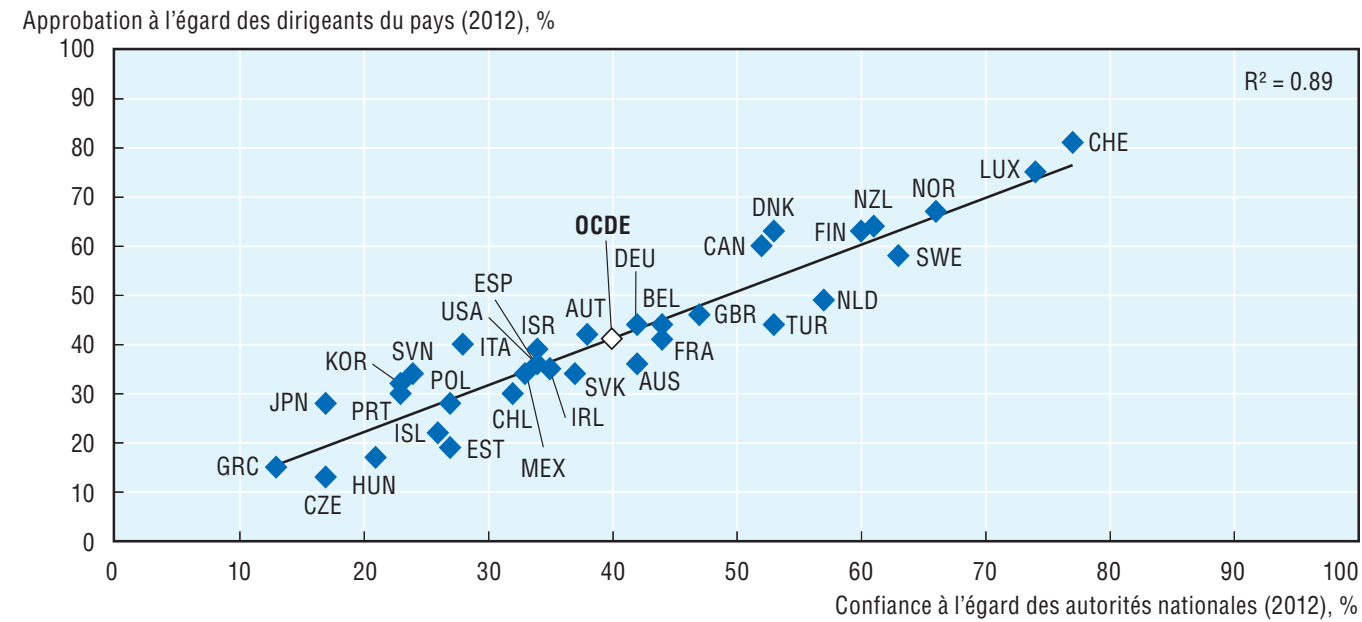

Note: Les données relatives à la confiance dans les autorités nationales correspondent au pourcentage de réponses positives à la question "Dans ce pays, avez-vous confiance dans chacune des entités suivantes ? Et dans les autorités nationales ? ". Les données relatives au niveau d'approbation à l'égard des dirigeants du pays correspondent au pourcentage de réponses "J'approuve " à la question "Approuvez-vous ou désapprouvez-vous le travail accompli par les dirigeants de ce pays ? ». Les données relatives au Chili, à l'Allemagne et au Royaume-Uni portent sur 2011 et non 2012.

Informations sur les données concernant Israël : http://dx.doi.org/10.1787/888932315602.

Source: Gallup World Poll. 
uniquement aux responsables politiques, ou s'il s'agit aussi des plus hauts responsables administratifs. Quoi qu'il en soit, ces résultats montrent que les dirigeants sont d'une importance déterminante pour la gouvernance publique, et que la stratégie, les performances et l'intégrité des autorités doivent pouvoir s'appuyer sur une interface politico-administrative efficace.

\section{Au niveau intermédiaire (" méso »)}

Au niveau intermédiaire (" méso »), la confiance peut être liée à l'élaboration stratégique des politiques - l'aptitude des pouvoirs publics à gérer les problématiques économiques et sociales et à susciter des attentes positives concernant le bien-être futur. L'édition 2013 de Panorama des administrations publiques comporte plusieurs indicateurs relatifs aux composantes et aux résultats de l'élaboration stratégique des politiques, tels que l'équité (chapitre 2), la gestion des risques (chapitre 2), la viabilité budgétaire (chapitre 2), les soldes budgétaires (chapitre 3), les niveaux d'endettement (chapitre 3) et les pratiques budgétaires (chapitre 4).

Quand on met ces indicateurs en rapport avec les niveaux de confiance à l'égard des pouvoirs publics et avec leur évolution, on s'aperçoit néanmoins qu'il n'existe pas de forte corrélation, à ceci près que le niveau de dépenses consacrées à la protection sociale (assurance chômage, pensions et prestations sociales) présente une corrélation modeste $\left(R^{2}: 0.44\right)$ avec le niveau de confiance à l'égard des pouvoirs publics. À l'heure où, dans un certain nombre de pays, les programmes sociaux sont devenus la cible de mesures d'assainissement budgétaire, la confiance à l'égard des pouvoirs publics risque de se dégrader encore sous l'effet des changements apportés à la teneur et aux conditions d'accès de ces programmes, si ces changements sont vus comme remettant en cause le contrat social qui lie l'État aux citoyens. L'impact sur la confiance pourrait néanmoins être atténué en fonction des processus suivis pour mener à bien les réformes, ce qui montre l'importance de l'équité non seulement au niveau des retombées (qui sera affecté, dans quelle mesure, et avec quelle justice dans le partage du fardeau), mais aussi au niveau du processus de décision (le processus de décision et les données de fait invoquées sont-ils transparents, et la population touchée par les décisions a-t-elle voix au chapitre). À cet égard, la confiance à l'égard des pouvoirs publics peut s'auto-entretenir en encourageant la participation des citoyens et en renforçant leur confiance dans les données de fait et les critères employés par les décideurs (et, par conséquent, dans la légitimité de leurs décisions).

Le rapport entre une gestion circonspecte des finances publiques et la confiance dans les pouvoirs publics n'est pas nécessairement simple. Il semble que, quand le budget de l'État est en ordre, ce rapport soit lointain et qu'en revanche, quand les pays connaissent d'importants problèmes budgétaires, cette préoccupation passe au premier plan. C'est ce qu'illustre clairement le graphique 1.8 , qui montre une forte corrélation négative $\left(R^{2}: 0.81\right)$ pour les cinq pays européens qui connaissent d'importants problèmes de dette publique (voir notamment l'encadré « Gros plan »). Des ratios dette publique/PIB élevés peuvent donc faire douter de la fiabilité des pouvoirs publics et de leur aptitude à limiter l'incertitude.

\section{Au niveau inférieur (" micro »)}

$\mathrm{Au}$ niveau inférieur ("micro»), on s'intéresse au vécu des citoyens face aux administrations dans le cadre de la prestation des services publics. La satisfaction à l'égard des services publics est beaucoup plus forte que la confiance dans les pouvoirs publics, mais une satisfaction plus élevée à l'égard des services publics ne se traduit pas nécessairement par une confiance accrue à l'égard des pouvoirs publics. 


\section{Graphique 1.8. La dette publique ne joue un rôle que dans les pays en crise budgétaire}

Corrélation entre la confiance dans les autorités nationales (2012) et la dette publique (2011)

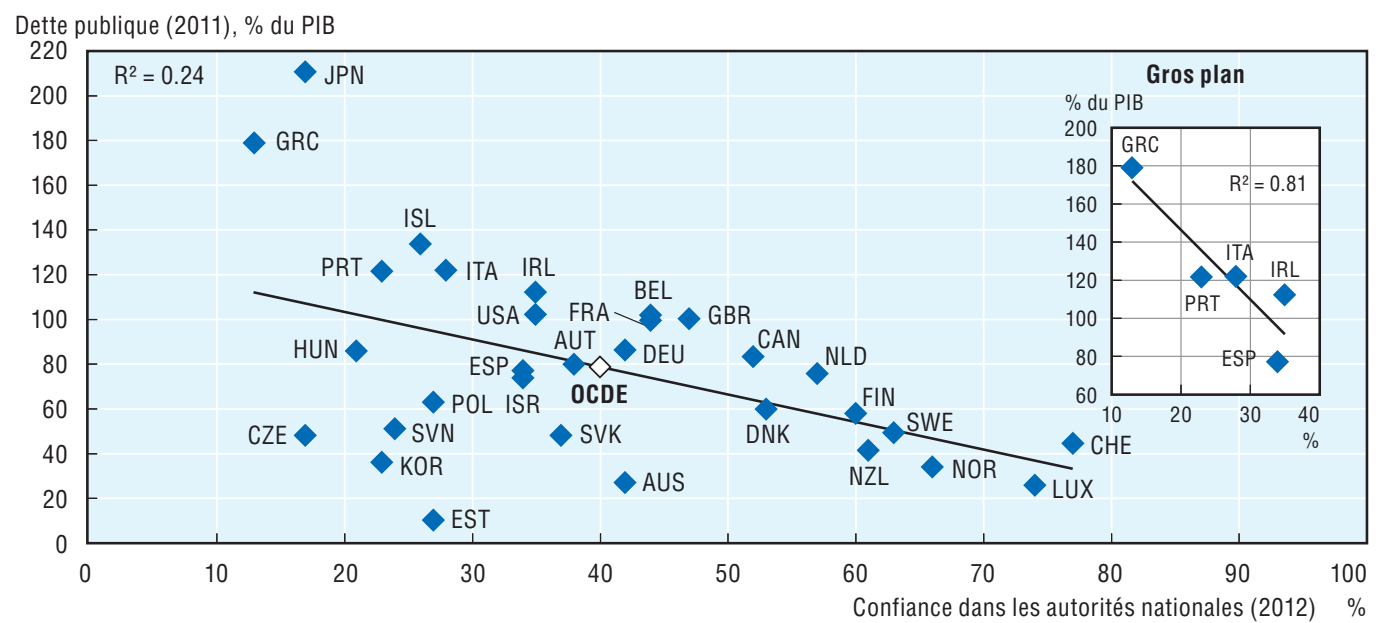

Note: Les données relatives à la confiance dans les autorités nationales correspondent au pourcentage de réponses positives à la question « Dans ce pays, avez-vous confiance dans chacune des entités suivantes ? Et dans les autorités nationales ? ". La dette publique correspond aux engagements financiers bruts des administrations publiques. Les données relatives à la confiance dans les autorités nationales au Chili, en Allemagne et au Royaume-Uni portent sur 2011 et non 2012.

Informations sur les données concernant Israël : http://dx.doi.org/10.1787/888932315602.

Source : Gallup World Poll et "Perspectives économiques de l'OCDE n 93 ", Perspectives économiques de l'OCDE : statistiques et projections (base de données), mai 2013, http://dx.doi.org/10.1787/data-00655-fr.

StatLink Aाst http://dx.doi.org/10.1787/888933158573

Les enquêtes montrent que les citoyens savent opérer des distinctions entre différents domaines et différentes composantes du secteur public quand on les interroge plus précisément (graphique 1.9). En 2012, pour la moyenne des pays de l'OCDE, c'était à l'égard de la police et du système de santé que la confiance était la plus forte (avec des taux de $72 \%$ et $71 \%$, respectivement). Venaient ensuite l'éducation (66\%), le système judiciaire (51 \%) et, au dernier rang, les autorités nationales (40\%). Ces chiffres montrent combien il est important de comprendre ce que l'on entend par " pouvoirs publics ": lorsque les citoyens expriment un niveau de confiance, à quelles composantes font-ils référence, au sein de ce qui constitue un vaste maillage d'acteurs, d'institutions, de textes et d'infrastructures de prestation de services publics?

Au-delà de cette vue d'ensemble, il existe d'importantes différences d'un pays à l'autre au niveau des rapports entre la confiance dans les autorités nationales et la satisfaction effective à l'égard des services publics. L'écart entre ces deux indicateurs est particulièrement net en Islande, au Japon, en Slovénie et en République tchèque, où la satisfaction à l'égard des services publics est élevée, alors qu'en Suisse, au Luxembourg et en Turquie, la confiance dans les autorités nationales et la satisfaction à l'égard des services publics se situent à des niveaux très proches. Cette constatation semble confirmer que les réponses actuellement fournies aux enquêtes sur la confiance dans les administrations nationales reflètent peutêtre davantage des opinions de court terme sur le système politique de certains pays que des opinions relatives aux pouvoirs publics en tant qu'institutions.

C'est généralement au niveau local, là où les services publics sont fournis et où le lien avec la performance des pouvoirs publics est le plus concret, que la confiance est la plus élevée. La confiance tend aussi à être plus forte chez les utilisateurs effectifs des services publics que chez les non-utilisateurs. Une analyse des écarts de confiance selon les niveaux d'administration et selon les différents types d'institutions publiques permettrait 


\section{Graphique 1.9. La satisfaction à l'égard des services publics est plus forte que la confiance dans les pouvoirs publics (2012)}

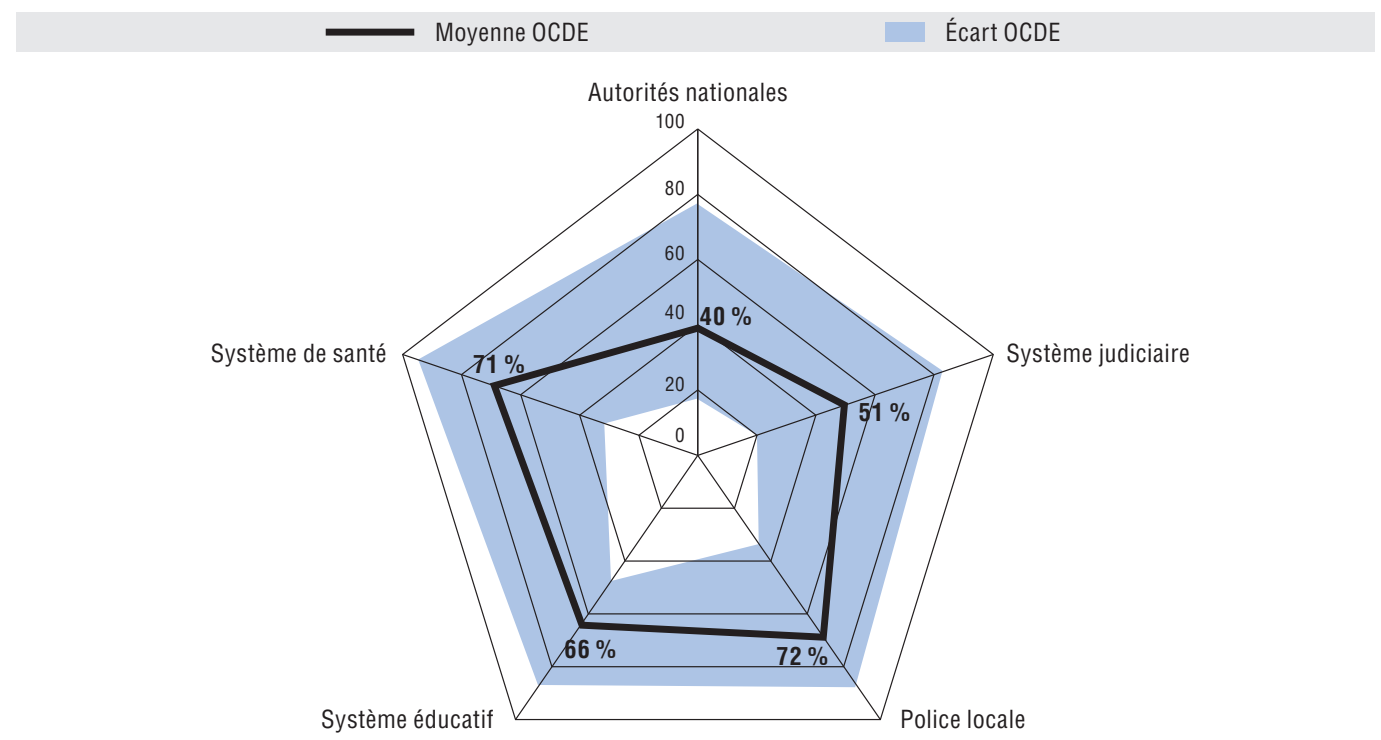

Note: Moyenne OCDE basée sur des données 2012 pour tous les pays sauf le Chili, l'Allemagne, le Japon, le Mexique, la Corée et le Royaume-Uni dont les données portent sur 2011 et non 2012. Les données relatives aux autorités nationales correspondent au pourcentage de réponses positives à la question « Dans ce pays, avez-vous confiance dans chacune des entités suivantes ? Et dans les autorités nationales ? ». Les données relatives au système judiciaire correspondent au pourcentage de réponses positives à la question « Dans ce pays, avez-vous confiance dans chacune des entités suivantes? Et dans le système judiciaire et les tribunaux ? ». Les données relatives à la police locale correspondent au pourcentage de réponses positives à la question « Dans la ville ou la zone que vous habitez, avezvous confiance dans la police locale ? ». Les données relatives au système éducatif correspondent au pourcentage de réponses "Satisfait(e) " à la question " Dans la ville ou la zone que vous habitez, êtes-vous satisfait(e) du système éducatif ou des établissements d'enseignement? ». Les données relatives au système de santé correspondent au pourcentage de réponses « Satisfait(e) » à la question « Dans la ville ou la zone que vous habitez, êtes-vous satisfait(e) de l'accès à des soins de santé de qualité ? ".

Source : Gallup World Poll.

StatLink क्ञाज http://dx.doi.org/10.1787/888933158580

d'éclairer les facteurs qui influent sur la perception des administrés et sur les différents leviers d'action susceptibles d'améliorer la perception de ces composantes de l'administration publique.

Le chapitre 9, consacré à la qualité des services publics, se fonde sur un cadre général d'évaluation de la qualité de ces services (tableau 1.2) et s'appuie sur des indicateurs existants de qualité des services présentés selon des dimensions clés : l'accessibilité, la rapidité d'exécution, la fiabilité et la satisfaction de l'usager. On y trouvera également des données sur l'utilisation des services d'administration électronique, car les administrations, les entreprises et les citoyens y ont de plus en plus recours. Ces dimensions de la qualité de service recoupent certaines des grandes dimensions de la gouvernance qui comptent aux yeux des citoyens, telles que l'inclusivité (accès), la réactivité et la fiabilité. Ces grandes dimensions qualitatives comportant de nombreuses facettes, nous avons présenté, à titre de première tentative, l'une de ces facettes pour chacun des quatre domaines pour lesquels on dispose de données : l'accessibilité financière, la rapidité d'exécution, l'exactitude et la satisfaction exprimée à l'égard des services.

\section{L'intégrité : une problématique transversale}

L'intégrité semble essentielle pour la confiance dans les pouvoirs publics, car il existe une forte corrélation entre la perception de la corruption et cette confiance (voir le graphique 1.10). Les outils et mécanismes visant à assurer l'intégrité, qui constituent des 
Tableau 1.2. Cadre d'évaluation de la qualité des services

\begin{tabular}{|c|c|c|c|}
\hline Accessibilité & Réactivité & Fiabilité & Satisfaction \\
\hline Accessibilité financière & Rapidité d'exécution & $\begin{array}{l}\text { Exactitude/compétence/droits de l'usager } \\
\text { (possibilité de formuler des réclamations ou } \\
\text { des suggestions, et de recevoir un soutien et/ou } \\
\text { un dédommagement) }\end{array}$ & $\begin{array}{l}\text { Satisfaction exprimée } \\
\text { (perception) }\end{array}$ \\
\hline Proximité géographique & $\begin{array}{l}\text { Concordance entre } \\
\text { le service et les besoins }\end{array}$ & Aspects tangibles (installations, machines, etc.) & $\begin{array}{l}\text { Confiance exprimée } \\
\text { (perception) }\end{array}$ \\
\hline $\begin{array}{l}\text { Adaptation aux besoins } \\
\text { des usagers handicapés }\end{array}$ & $\begin{array}{l}\text { Service clientèle } \\
\text { (courtoisie et traitement) }\end{array}$ & Cohérence/équité & \\
\hline $\begin{array}{l}\text { Adaptation aux besoins } \\
\text { des usagers de culture } \\
\text { différente (langue, etc.) }\end{array}$ & $\begin{array}{l}\text { Services intégrés (entre } \\
\text { différents modes } \\
\text { de prestation) }\end{array}$ & Sécurité (confidentialité, sûreté) & \\
\hline
\end{tabular}

\section{Graphique 1.10. Attention à la corruption !}

Corrélation entre la confiance dans les autorités nationales et la corruption perçue des pouvoirs publics (2012)

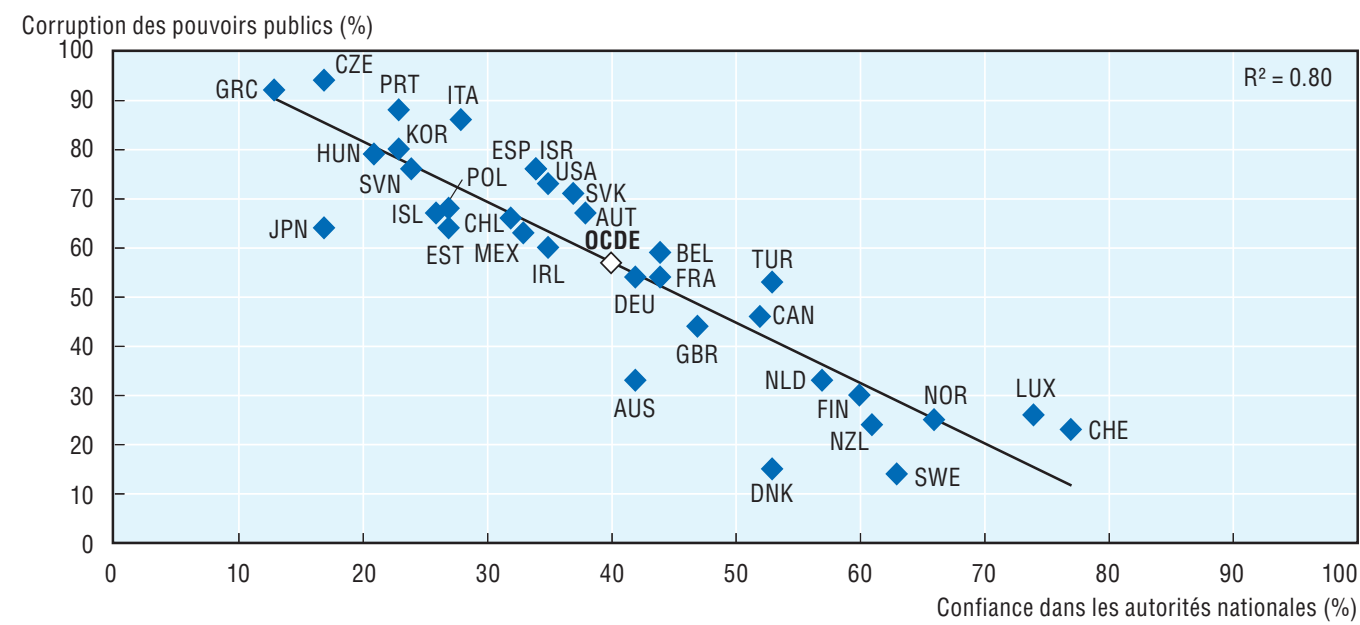

Note: Les données relatives à la confiance dans les autorités nationales correspondent au pourcentage de réponses positives à la question « Dans ce pays, avez-vous confiance dans chacune des entités suivantes ? Et dans les autorités nationales ? ». Les données relatives à la corruption perçue des pouvoirs publics correspondent au pourcentage de réponses positives à la question "La corruption est-elle répandue au sein des pouvoirs publics? ". Les données relatives au Chili, à l'Allemagne, et au Royaume-Uni portent sur 2011 et non 2012.

Source : Gallup World Poll.

StatLink 시내 $h t t p: / / d x . d o i . o r g / 10.1787 / 888933158594$

processus essentiels pour la gouvernance publique, ont pour objet de prévenir la corruption et de favoriser un comportement irréprochable. Ils contribuent ainsi à renforcer la crédibilité et la légitimité des acteurs de l'élaboration des politiques, à défendre l'intérêt général et à rétablir l'équité perçue des décisions adoptées. Des outils axés sur les zones sensibles de rencontre entre les secteurs public et privé - tels que des dispositifs efficaces de gestion des conflits d'intérêts, la formulation de règles de conduite strictes pour les fonctionnaires et une bonne réglementation du lobbying et du financement de la vie politique - peuvent permettre de limiter les influences indues et d'établir des garde-fous permettant de préserver l'intérêt général.

Cette publication présente des indicateurs relatifs à des pratiques de gestion publique visant à renforcer l'intégrité des administrations : le chapitre 8 évoque les conflits d'intérêts, les déclarations de patrimoine et la transparence budgétaire, et le chapitre 7 évoque la problématique d'une concurrence équitable pour les PME dans le cadre des marchés publics. 


\section{Conclusion}

Les défis institutionnels de la récente crise financière, économique et sociale ont contribué au lancement de vastes travaux de recherche sur le rôle des pouvoirs publics au sein des économies et des sociétés modernes. De plus en plus, les dirigeants et les analystes se demandent si la confiance n'est pas l'ingrédient manquant en vue d'une meilleure gestion des crises et de meilleurs résultats.

Pour comprendre et renforcer la confiance à l'égard des pouvoirs publics, il semble qu'il faille raisonner à moyen terme et lancer un programme global, plurisectoriel et associant des acteurs multiples. En premier lieu, il faudrait mesurer de façon plus exhaustive la confiance dans les pouvoirs publics et mieux cerner ses déterminants. Cela implique de mieux comprendre et connaître les notions de confiance et de confiance dans les pouvoirs publics. De plus, il faudrait effectuer des mesures régulières et internationalement comparables de la confiance des citoyens et des entreprises à l'égard des pouvoirs publics. On pourrait, pour cela, réaliser une ou plusieurs nouvelles enquêtes combinant des éléments des enquêtes existantes, ou améliorer ces dernières (au niveau de leur représentativité, de leur conception, de leur formulation et de leurs échelles de notation). À l'heure actuelle, aucun office statistique national (OSN) ne participe à l'évaluation de la confiance dans les pouvoirs publics.

En second lieu, il faudrait continuer de travailler à un cadre analytique accompagné de techniques économétriques plus sophistiquées visant à étudier de façon plus approfondie les rapports entre, d'une part, la confiance dans les pouvoirs publics et dans les diverses institutions et, d'autre part, les différentes dimensions de la performance des administrations, afin d'en tirer des conclusions quant aux domaines à cibler en priorité. Il est particulièrement important de comprendre le rôle et la responsabilité de chaque échelon administratif et de chaque acteur, qu'il s'agisse des dirigeants nationaux, des divers secteurs de l'action publique ou des agents chargés de la prestation de services à l'échelon administratif local, et d'examiner comment les décisions sont prises, transmises et mises en œuvre. L'OCDE pourrait contribuer à l'élaboration de comparaisons internationales et aider les pays à mutualiser les stratégies et les mesures qui ont fait leurs preuves en matière de rétablissement de la confiance. Ces travaux pourraient être précédés d'études de cas par pays réalisées, entre autres, dans le cadre des Examens de l'OCDE sur la gouvernance publique.

De nouveaux travaux axés sur la confiance dans les pouvoirs publics permettraient avant tout de jeter sur la gouvernance publique un nouvel éclairage mettant davantage en relief le rôle des citoyens. Au niveau institutionnel, cela devrait renforcer l'idée d'un contrat social entre l'État et le citoyen dans le cadre duquel ce dernier joue son rôle non seulement en payant ses impôts et en respectant la loi, mais aussi en étant réceptif à l'égard des politiques publiques et en collaborant à leur conception et à leur mise en œuvre. Pour obtenir cette adhésion du citoyen, les pouvoirs publics doivent, de leur côté, être plus inclusifs, plus transparents, plus réceptifs et plus efficients. S'ils reconnaissent et comprennent mieux le rôle crucial de la confiance dans l'efficacité des politiques, les pouvoirs publics seront mieux placés pour concevoir leurs programmes d'action et de réforme, avec de meilleures retombées pour tous.

\section{Notes}

1. Voir GOV/PGC(2013)1 (www2.oecd.org/oecdinfo/info.aspx?app=OLIScoteEN\&Ref=GOV/PGC(2013)1).

2. Cette publication (disponible en ligne dans l'annexe "Facteurs contextuels") comporte des renseignements d'ordre général sur les institutions politiques et sur la structure administrative de chaque pays membre. Le mode de fonctionnement de ces institutions est couvert par d'autres indicateurs de la publication. 


\section{Bibliographie}

Aghion, P., Y. Algan, P. Cahuc et A. Shleifer (2010), « Regulation and Distrust », The Quarterly Journal of Economics, vol. 125(3), Oxford, pp. 1015-1049.

Algan, Y. et P. Cahuc (2010), Handbook of Economic Growth, vol. 1, North-Holland Publishing, Amsterdam.

Blind, P.K. (2007), « Building Trust in Government in the Twenty-First Century: Review of Literature and Emerging Issues ", 7th Global Forum on Reinventing Government - Building Trust in Government, pp. 26-29.

Bouckaert, G. (2012), « Trust and Public Administration », Administration, vol. 60, n 1, pp. 91-115.

Bouckaert, G. et S. Van de Walle (2003), « Comparing Measures of Citizen Trust and User Satisfaction as Indicators of "Good Governance »: Difficulties in Linking Trust and Satisfaction Indicators ", International Review of Administrative Sciences, vol. 69(3), pp. 329-344.

Dalton, R.J. (2005), "The Social Transformation of Trust in Government ", International Review of Sociology, vol. 15, $\mathrm{n}^{\circ} 1$, mars, pp. 133-154.

Dasgupta, P. (2009), "A Matter of Trust: Social Capital and Economic Development ", document préparé en vue d'un exposé devant la Conférence annuelle de la Banque mondiale sur l'économie du développement (ABCDE), Séoul, juin.

Easton, D. (1965), A Systems Analysis of Political Life, John Wiley, New York.

Fukuyama, F. (1995), Trust: The Social Virtues and the Creation of Prosperity, Free Press, New York.

Gyorffy, D. (2013), Institutional Trust and Economic policy, Central European University Press, Budapest.

Johnston, W., H. Krahn et T. Harrison (2006), « Democracy, Political Institutions, and Trust: The Limits of Current Electoral Reform Proposals ", Cahiers canadiens de sociologie, vol. 31.2, pp. 165-182.

Kampen, J.K., S. Van de Walle et G. Bouckaert (2006), « Assessing the Relation Between Satisfaction with Public Service Delivery and Trust in Government: The Impact of the Predisposition of Citizens Toward Government on Evaluations of its Performance ", Public Performance and Management Review, vol. 29(4), pp. 387-404.

Knack, S. et P.J. Zak (2003), « Building Trust: Public Policy, Interpersonal Trust, and Economic Development ", Supreme Court Economic Review, n 10, pp. 91-107.

Knack, S. et P. Keefer (1997), "Does Social capital Have an economic payoff ? A cross-country investigation », The Quarterly Journal of Economics, vol. 112(4), Oxford, pp. 1251-1288.

Murphy, K. (2004), " The role of trust in nurturing compliance: A study of accused tax avoiders ", Law and Human Behavior, vol. 28(2), pp. 187-209.

Nooteboom, B. (2006), Social Capital, Institutions and Trust, Université de Tilburg.

Putnam, R. (2000), Bowling Alone: The Collapse and Revival of American Community, Simon and Schuster, New York.

\section{Enquêtes internationales mesurant la confiance à l'égard des pouvoirs publics : liens hypertexte}

World Gallup Poll : www.gallup.com/strategicconsulting/en-us/worldpoll.aspx.

World Values Survey : www.worldvaluessurvey.org.

Eurobaromètre : www.ec.europa.eu/public_opinion/index_fr.htm.

Edelman Trust Barometer : www.edelman.com/insights/intellectual-property/trust-2013.

Latinóbarometro : www.latinobarometro.org. 


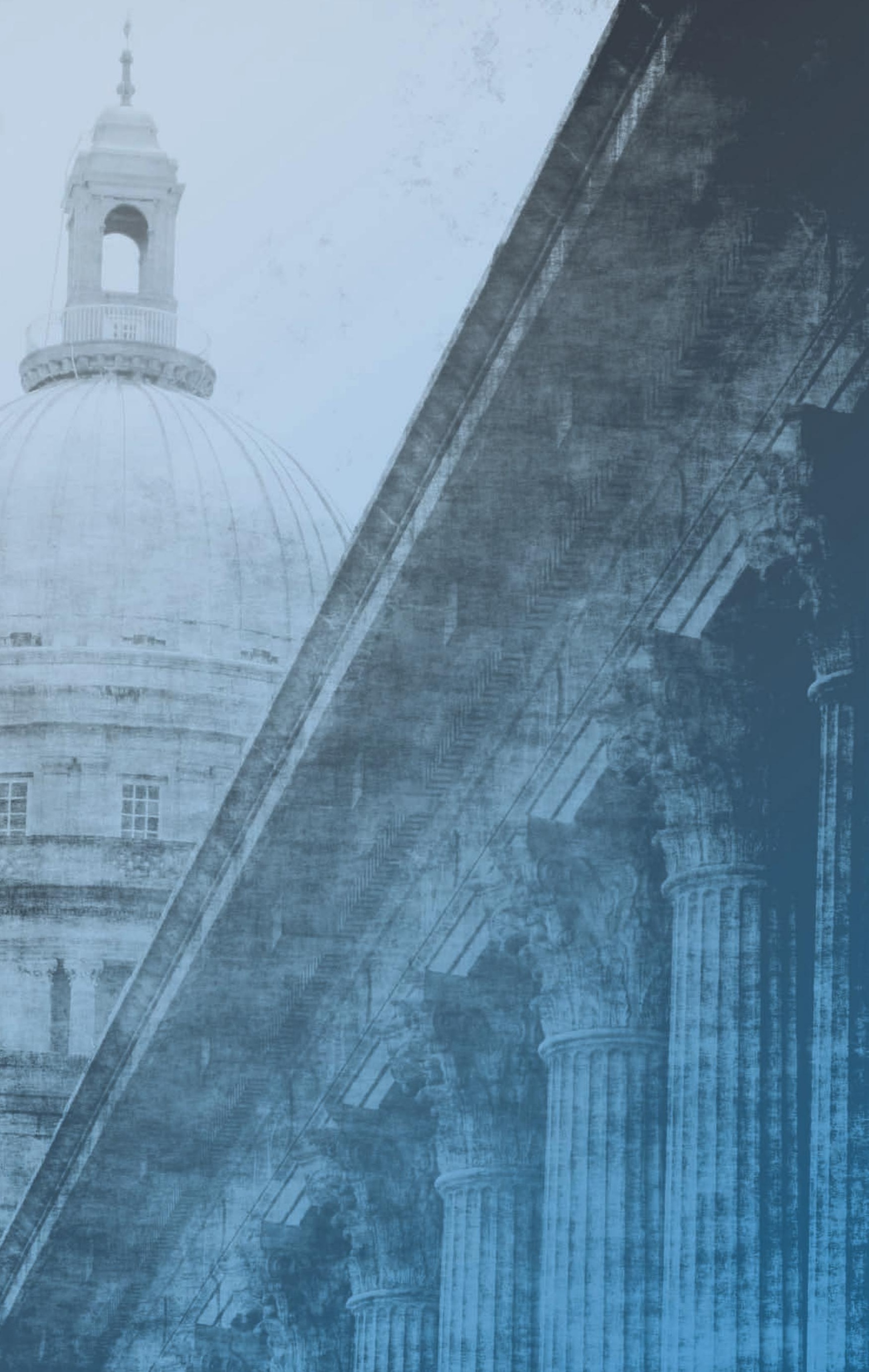




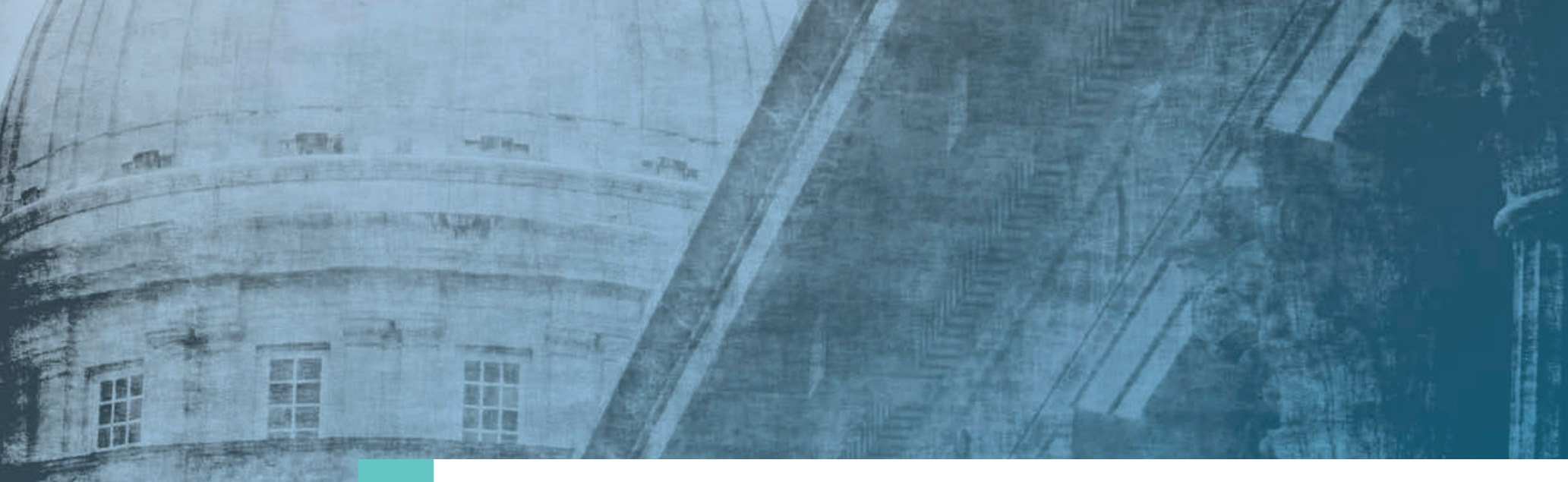

\section{LA GOUVERNANCE STRATÉGIQUE}

Ce chapitre présente des indicateurs clés portant sur des réalisations stratégiques des administrations publiques. Certains de ces indicateurs concernent les performances de domaines d'action et d'activités officielles spécifiques (des indicateurs d'efficience et d'efficacité pour les secteurs de la santé, de l'éducation et de la Justice ainsi que pour l'administration fiscale, par exemple), sur la base de conventions d'évaluation élaborées au sein même de ces domaines. D'autres portent sur les performances de l'ensemble des pouvoirs publics, comme les indicateurs sur l'État de droit, la gestion des risques ou la viabilité budgétaire. Certains indicateurs reflètent également des principes fondamentaux de la bonne gouvernance, comme l'État de droit ou l'équité - à travers le prisme du rôle des pouvoirs publics dans la réduction des inégalités de revenu - ou encore l'efficience et l'efficacité. La plupart des indicateurs portent sur un court laps de temps (les valeurs sont présentées pour quelques années spécifiques) mais, s'agissant de la viabilité budgétaire, la position budgétaire des administrations publiques fait l'objet de projections jusqu'en 2030. La diversité de ces indicateurs reflète les attentes variées des particuliers et des entreprises à l'égard des pouvoirs publics, ainsi que les vastes responsabilités qui pèsent sur ces derniers. Tous ces indicateurs sont stratégiques : ils sont importants pour le bien-être des sociétés et des économies. Nombre d'entre eux - les indicateurs relatifs à l'État de droit ou à la gestion des risques, par exemplereflètent l'exécution de fonctions qui ne peuvent pas être assurées par d'autres acteurs. 
Il y a confiance dans les pouvoirs publics quand les particuliers et les entreprises se fient aux autorités pour mener une action judicieuse et juste (Easton, 1965). C'est l'un des principaux fondements de la légitimité et de la pérennité des systèmes politiques. Cette confiance est essentielle au bien-être et à la cohésion de la société, puisqu'elle influe sur l'aptitude des autorités à gouverner et leur permet d'agir sans recourir à la coercition. Elle est donc nécessaire en vue d'un fonctionnement juste et efficace des institutions publiques.

La confiance à l'égard des institutions et pouvoirs publics dépend aussi de la congruence entre les préférences des particuliers et des entreprises, leur interprétation de ce qui est judicieux et juste, et leur perception du fonctionnement des pouvoirs publics. Par conséquent, la confiance dans les pouvoirs publics tient pour beaucoup à la culture et au contexte. Il existe des pays, tels que la Suisse, le Luxembourg, la Norvège et la Suède, où cette confiance est forte, et d'autres, tels que les pays d'Europe orientale, où elle est faible (graphique 2.1). De plus, son niveau peut être influencé par de nombreux facteurs d'ordre contextuel tels que la situation économique, les catastrophes naturelles ou l'ampleur de la corruption.

On mesure principalement la confiance dans les pouvoirs publics au moyen d'enquêtes d'opinion. En raison du poids de la culture locale et des autres facteurs d'ordre contextuel, il faut interpréter les comparaisons internationales avec beaucoup de précautions. À cet égard, il peut être plus instructif d'étudier l'évolution des niveaux de confiance au fil du temps que leurs niveaux en termes absolus. Entre 2007 et 2012, le taux moyen de confiance dans les autorités nationales est passé, sur l'ensemble de la zone OCDE, de $45 \%$ à $40 \%$, soit un recul de 5 points de pourcentage. Le déclin le plus net est intervenu en Slovénie, au Portugal, en Grèce et en Irlande autant de pays durement touchés par la crise financière, économique et budgétaire. Sur la même période, la confiance dans les pouvoirs publics progressait en République slovaque, en Suisse, en Israël, au Royaume-Uni et en France. À y regarder de plus près, il semble que, dans le cadre des enquêtes sur la confiance à l'égard des autorités nationales, les personnes interrogées expriment en réalité leur confiance à l'égard des responsables politiques.

Les pouvoirs publics sont également prestataires de services publics essentiels dans des domaines tels que l'éducation, la santé, la sûreté publique et la Justice. Dans la plupart des pays de l'OCDE, ces services sont principalement assurés à l'échelon local, sauf s'agissant de la Justice, qui est indépendante. Les citoyens manifestent plus de confiance - ou de satisfaction - à l'égard de ces services publics qu'à l'égard des autorités nationales, qui font figure de notion abstraite (graphique 2.2). En 2012, à l'échelle de l'ensemble des pays de l'OCDE, c'était la police qui bénéficiait du taux moyen de confiance/satisfaction le plus élevé (72 \%) ; elle était suivie de très près par le système de santé (71\%), puis par le système éducatif (66\%) et, enfin, le système judiciaire (50\%).

\section{Méthodologie et définitions}

Les données ont été recueillies dans le cadre de l'enquête Gallup World Poll, qui repose sur un échantillonnage probabiliste stratifié proportionnel, avec un échantillon de 1000 citoyens dans chaque pays.

On trouvera un complément d'information sur le site www.gallup.com/strategicconsulting/en-us/worldpoll.aspx.

\section{Pour en savoir plus}

OCDE (à paraître), Trust in government, assessing the evidence, understanding the policies (Confiance dans les pouvoirs publics : évaluer les données, comprendre les politiques), Éditions OCDE, Paris.

\section{Notes relatives aux graphiques}

Les données relatives à l'Allemagne, au Chili et au Royaume-Uni portent sur 2011 et non 2012. Les données relatives à l'Islande et au Luxembourg portent sur 2008 et non 2007. Les données relatives à l'Autriche, à la Finlande, à l'Irlande, à la Norvège, au Portugal, à la République slovaque, à la Slovénie et à la Suisse portent sur 2006 et non 2007.

2.1 : Les données correspondent au pourcentage de réponses positives à la question "Avez-vous confiance dans les autorités nationales ? ".

2.2 : Les données relatives à la Corée, au Japon et au Mexique portent sur 2011 et non 2012. Les données relatives au système judiciaire correspondent au pourcentage de réponses positives à la question "Dans ce pays, avez-vous confiance dans chacune des entités suivantes ? Et dans le système judiciaire et les tribunaux ? ". Les données relatives à la police locale correspondent au pourcentage de réponses positives à la question " Dans la ville ou la zone que vous habitez, avez-vous confiance dans la police locale? ». Les données relatives au système éducatif correspondent au pourcentage de réponses "Satisfait(e) " à la question " Dans la ville ou la zone que vous habitez, êtes-vous satisfait(e) du système éducatif ou des établissements d'enseignement? ". Les données relatives au système de santé correspondent au pourcentage de réponses "Satisfait(e) » à la question "Dans la ville ou la zone que vous habitez, êtes-vous satisfait(e) de l'accès à des soins de santé de qualité ? ".

Informations sur les données concernant Israël : http://dx.doi.org/10.1787/ 888932315602 . 


\subsection{La confiance à l'égard des autorités nationales en 2012 et son évolution par rapport à 2007}

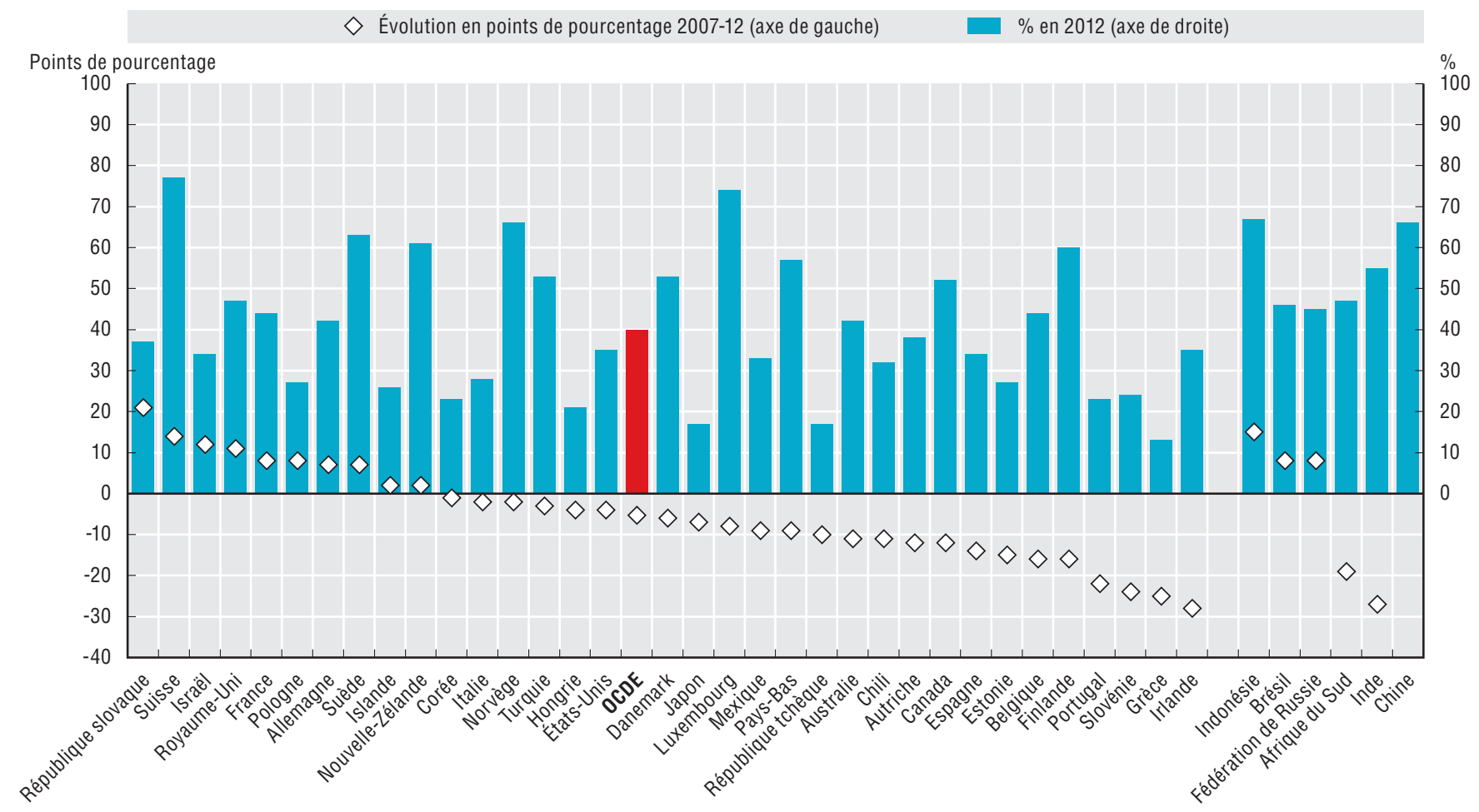

Source : Gallup World Poll.

StatLink काIsL http://dx.doi.org/10.1787/888933158503

\subsection{La confiance et la satisfaction selon les institutions publiques (2012)}

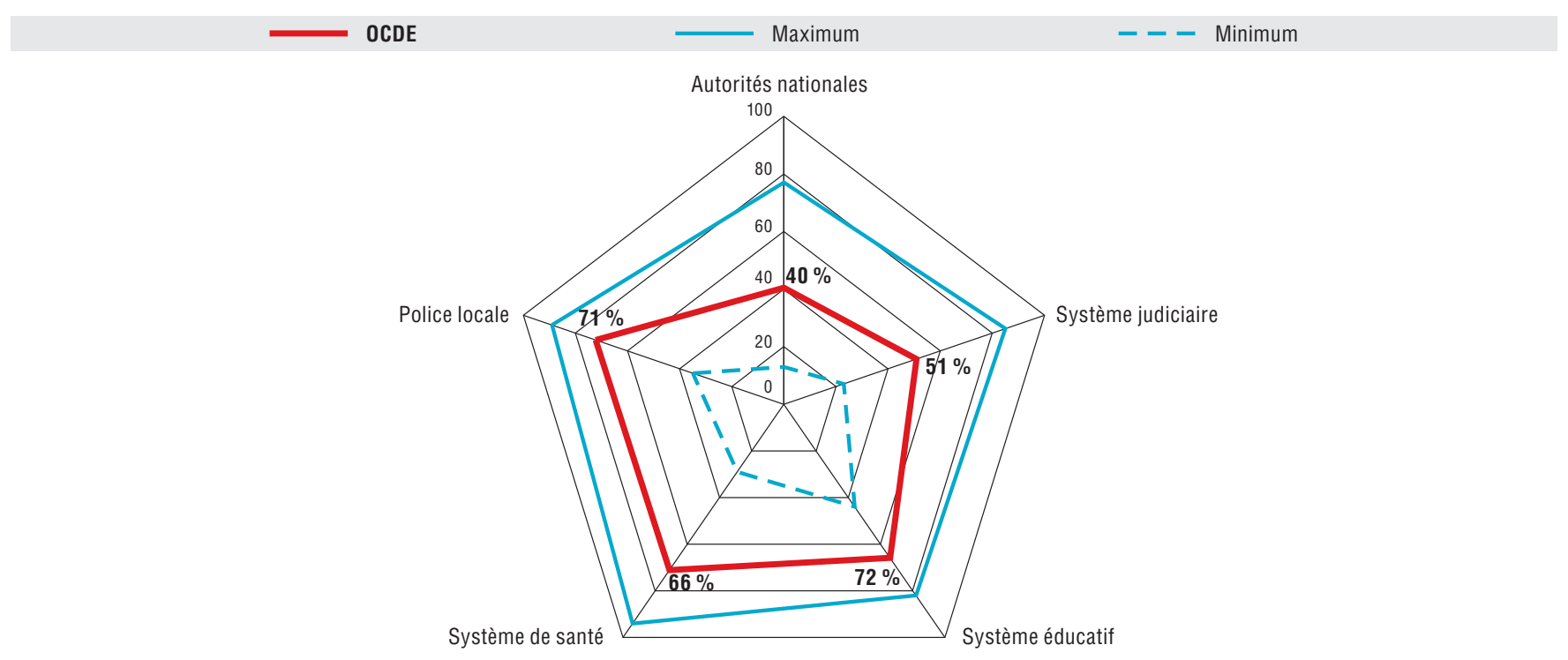

Source : Gallup World Poll. 
L'une des principales tâches de l'État est d'uniformiser les règles du jeu auxquelles sont soumis les citoyens en garantissant l'équité à la fois dans les procédures qu'il suit - la justice procédurale - et dans les résultats qu'il obtient - la justice distributive. Un élément clé de cette dernière est de permettre l'existence de différences de revenus, dans la mesure où elles reconnaissent et récompensent la performance, en acceptant que les individus contribuent différemment au bien-être économique et social. Parallèlement, l'État doit chercher à limiter les dommages économiques et sociaux qui peuvent découler des inégalités et tenir compte du consensus sociétal. Ce double objectif est à l'origine de la réduction des disparités de revenus grâce à un système d'imposition progressif et à des transferts monétaires (pensions et assurance chômage, par exemple).

Depuis les années 80, l'écart de revenu entre les riches et les pauvres s'accroît (OCDE 2011, Toujours plus d'inégalité : Pourquoi les écarts de revenus se creusent) dans la majorité des pays de l'OCDE, ce qui démontre que les fruits de la croissance économique n'ont pas été équitablement répartis entre les individus. On considère que la mondialisation, l'évolution des technologies et les réformes réglementaires et institutionnelles ont joué un rôle moteur dans l'accroissement des inégalités de revenus. La crise économique mondiale a encore intensifié cette tendance en touchant de manière inégale différents groupes de population et en pesant principalement sur les chômeurs et les travailleurs sousemployés.

La plupart des pays membres de l'OCDE ont adopté une panoplie de politiques publiques visant à réduire les inégalités de revenus et leurs coûts à long terme pour le développement économique. Les systèmes d'assurance et de protection sociale associent des transferts monétaires et un système d'imposition progressive des revenus. En outre, des programmes ciblés de relance budgétaire ont été adoptés pour stimuler la demande et amortir les effets de la crise sur les ménages les plus défavorisés. Ces mesures étaient destinées à lutter contre les inégalités de revenus par une redistribution entre les riches et les pauvres, mais également entre les générations, afin d'apporter un soutien aux groupes d'âge qui en avaient le plus besoin.

On considère que le coefficient de Gini est le principal indicateur permettant d'évaluer le niveau des inégalités de revenus dans un pays. L'impact du système de protection sociale mis en place par l'administration centrale grâce aux transferts et aux impôts peut être mesuré en comparant le coefficient avant et après ces transferts et impôts. En moyenne, le niveau des inégalités de revenus avant impôt dans un ensemble de pays membres de l'OCDE n'a pas évolué entre 2005 et 2010 (0.47). Néanmoins, certains pays tels que l'Irlande ont enregistré une hausse constante des inégalités avant impôts et transferts au cours de ces cinq dernières années. L'intervention publique s'est avérée primordiale dans ces cas, et a permis une réduction du coefficient de Gini d'environ 0.26 (contre une moyenne de 0.16). À l'opposé, le Chili reste le pays assurant la redistribution la plus faible pour ces deux années, avec un impact de 0.02 sur l'indice de Gini. Parallèlement, tous les pays semblent rester fidèles à un système d'imposition progressif, la Pologne et le Chili proposant moins de taux d'imposition différents que l'Irlande.

\section{Méthodologie et définitions}

La valeur du coefficient de Gini varie entre 0 , lorsque tous les individus d'une population perçoivent les mêmes revenus, et 1 , lorsque l'individu le plus riche capte la totalité des revenus. Les écarts entre les plus pauvres et les plus riches sont calculés comme étant le ratio entre le revenu moyen des $10 \%$ les plus pauvres et le revenu moyen des $10 \%$ les plus riches. La redistribution est mesurée en comparant les coefficients de Gini pour le revenu du marché (c'est-à-dire le revenu brut non corrigé pour tenir compte des transferts monétaires publics et des impôts sur les ménages) et le revenu disponible (c'està-dire le revenu net d'impôts et de transferts). La définition du revenu disponible des ménages ne tient pas compte des transferts en nature. Ces données sont issues de la base de données de l'OCDE sur la distribution des revenus, dont les informations sont collectées par un réseau de fournisseurs de données nationaux afin de comparer les performances des pays en matière de disparité de revenus.

Les données fiscales, issues de la publication de l'OCDE Les impôts sur les salaires, reprennent les taux d'imposition en vigueur pour l'exercice fiscal. En Australie, en Nouvelle-Zélande et au Royaume-Uni, l'exercice fiscal ne coïncide pas avec l'année civile. Les données illustrent l'écart entre deux scénarios : un célibataire sans personnes à charge dont la rémunération est égale à $67 \%$ du salaire moyen et un célibataire sans personnes à charge gagnant $167 \%$ du salaire moyen. Les taux moyens sont exprimés en pourcentage du salaire brut. Le salaire moyen mesure la rémunération brute annuelle moyenne des travailleurs manuels et non manuels adultes employés à temps plein dans l'un des secteurs couverts.

\section{Pour en savoir plus}

OCDE (2013), Les impôts sur les salaires 2013, Éditions OCDE, Paris, $h t t p: / / d x . d o i . o r g / 10.1787 /$ tax_wages-2013-fr.

OCDE (2011), Toujours plus d'inégalité : Pourquoi les écarts de revenus se creusent, Éditions OCDE, Paris, http://dx.doi.org/ 10.1787/9789264119550-fr.

\section{Notes relatives aux graphiques}

2.3 : On ne dispose pas de données pour la Hongrie, le Mexique et la Turquie. On ne dispose pas de données relatives à la Suisse pour 2005. 2005 : Les données relatives à l'Allemagne, à l'Australie, à la Finlande, à l'Italie, à la Norvège et à la Suède portent sur 2004. Les données relatives au Chili, à la Corée et au Japon portent sur 2006. Les données relatives à la Nouvelle-Zélande portent sur 2003. 2010 : Les données relatives au Chili, à l'Irlande, au Japon, à la NouvelleZélande et à la Suisse portent sur 2009.

2.4 : Les chiffres des salaires pour la Turquie se basent sur l'ancienne définition du travailleur moyen (CITI D, rév. 3). Les données renvoient à l'impôt sur le revenu, plus les cotisations de sécurité sociale acquittées par les salariés (en \% du salaire brut). Au Chili, les revenus moyens sont exonérés d'impôt sur le revenu, ce qui fait que ce dernier a une faible incidence sur le total des recettes fiscales.

Informations sur les données concernant Israël : http://dx.doi.org/10.1787/ 888932315602 . 

L'État de droit signifie que nul n'est au-dessus des lois, y compris les pouvoirs publics, que les textes protègent les droits fondamentaux et que tout le monde a accès à la justice. Il implique un ensemble de normes communes d'action définies par la loi et mises en application au moyen de procédures et de mécanismes de responsabilité visant à assurer la fiabilité, la prévisibilité et l'« administration par le droit ». On considère que l'État de droit constitue l'une des dimensions majeures qui déterminent la qualité et la bonne gouvernance d'un pays.

On distingue plusieurs interprétations de l'État de droit. Nous utilisons celle élaborée pour l'indice de l'État de droit du World Justice Project, que nous considérons comme l'une des approches les plus complètes et les plus systématiques. L'État de droit recouvre donc ici les quatre principes universels suivants : l'administration et ses représentants et agents doivent répondre de leurs actes devant la loi ; les lois sont claires, rendues publiques, stables et justes, et elles protègent les droits fondamentaux, y compris le droit à la sécurité des personnes et des biens; les procédures de promulgation, d'administration et d'application des lois sont accessibles, efficientes et justes ; la justice est assurée par des représentants et des intervenants neutres compétents, irréprochables sur le plan éthique et indépendants en nombre suffisant, dotés de ressources appropriées et représentatifs de la composition des populations au service desquelles ils sont placés.

À partir de ces quatre principes, le World Justice Project a défini neuf facteurs essentiels qui constituent la base de son indice de l'État de droit. Nous avons retenu ici les quatre facteurs les plus déterminants pour une bonne gouvernance. Il s'agit de la limitation des pouvoirs de l'administration, des droits fondamentaux, de la mise en œuvre de la réglementation et de la justice civile. La question de l'ouverture des données publiques sera abordée séparément. Les informations présentées cidessous relativement à ces facteurs correspondent à l'opinion exprimée par des experts et des citoyens.

\section{Limitation des pouvoirs de l'administration}

La limitation des pouvoirs de l'administration consiste en une combinaison de sept éléments clés (sous-facteurs) : les pouvoirs de l'administration sont définis dans la loi fondamentale; ils sont effectivement limités par le pouvoir législatif; ils sont effectivement limités par le pouvoir judiciaire; ils sont effectivement limités par des processus de vérification et d'examen indépendants; les représentants de l'administration sont sanctionnés en cas de faute ; les pouvoirs de l'administration font l'objet de contrôles extérieurs ; et la passation de pouvoirs est régie par la loi. Cet indicateur composite permet d'évaluer si l'autorité est répartie, selon des règles formelles ou par convention, d'une manière qui garantit qu'aucun organe officiel isolé n'ait la possibilité d'exercer un pouvoir sans entrave.

Même parmi les pays membres de l'OCDE, on relève des différences significatives dans la limitation des pouvoirs de l'administration. Les limites les plus strictes sont posées par les pays nordiques, suivis de l'Australie et de la Nouvelle-Zélande, et c'est en Turquie, au Mexique et en Grèce que le contrôle de ces pouvoirs est le moins prononcé. Comme on pouvait s'y attendre, le score moyen des pays membres de l'OCDE pour cet indicateur est élevé, ce qui montre qu'il existe un contrôle important des pouvoirs de l'administration. Pour ce qui est des pays partenaires, participants et candidats à l'adhésion, les contrôles exercés sur les pouvoirs de l'administration sont plus limités, notamment en Fédération de Russie, en Ukraine et en Chine.
L'analyse de la moyenne OCDE pour les sous-facteurs regroupés dans cet indicateur composite révèle que le sousfacteur qui obtient le meilleur score est celui qui porte sur les textes régissant la passation de pouvoirs (0.87) (1 signifiant un respect maximal de l'État de droit) ; que le sous-facteur le moins bien noté porte sur les sanctions infligées aux représentants de l'administration en cas de faute (0.67) ; et que le rôle des dispositifs indépendants de contrôle et d'examen mériterait d'être renforcé (0.73).

\section{Droits fondamentaux}

Cet indicateur composite mesure la protection des droits fondamentaux et, par conséquent, il s'agit d'une mesure normative. Il englobe l'évaluation de huit éléments clés : l'égalité de traitement et l'absence de discrimination; la garantie effective du droit à la vie et à la sécurité de la personne ; la régularité de la procédure et le respect des droits de la défense ; la garantie effective de la liberté d'opinion et d'expression; la garantie effective de la liberté de croyance et de religion ; le droit de ne pas subir d'ingérence arbitraire dans sa vie privée; la garantie effective des droits de réunion et d'association; et les droits fondamentaux des travailleurs. Il couvre un éventail relativement restreint de droits qui sont fermement établis par le droit international et qui relèvent essentiellement de préoccupations liées à l'État de droit et à la bonne gouvernance.

Le score moyen pour les pays membres de l'OCDE est élevé et atteint presque 0.8 , ce qui signifie que la garantie des droits fondamentaux est forte dans la plupart des pays. Comme ce qu'on a pu observer pour la limitation des pouvoirs de l'administration, les droits fondamentaux sont davantage garantis dans les pays nordiques (Suède, Danemark, Norvège et Finlande), suivis de la Nouvelle-Zélande et de l'Espagne, et le sont moins en Turquie, au Mexique et en Grèce. Cela indique une forte corrélation entre le fait de veiller à ce que les pouvoirs de l'administration soient limités et celui d'assurer le respect des droits fondamentaux $\left(R^{2}=0.81\right)$. En revanche, en moyenne, la mise en œuvre de la réglementation est moins bien assurée dans les pays membres de l'OCDE que le respect des droits fondamentaux. La situation est plus diversifiée dans les pays partenaires et participants : les droits fondamentaux sont bien garantis - bien qu'en dessous de la moyenne OCDE - au Brésil et en Afrique du Sud, alors que des améliorations pourraient être nécessaires dans les autres pays, en particulier la Chine et l'Égypte.

En moyenne, dans les pays membres de l'OCDE, c'est au niveau de l'égalité de traitement et de l'absence de discrimination que des progrès doivent être faits (0.7), alors que la garantie du droit à la vie et à la sécurité de la personne est le sous-domaine le plus abouti (0.86).

\section{Mise en œuvre de la réglementation}

Cet indicateur composite permet d'évaluer si les textes sont appliqués de manière équitable et effective. Il n'examine ni ce que les autorités réglementent, ni comment elles procèdent en la matière, mais uniquement les modalités de mise en œuvre et d'application des textes. Il prend en compte des domaines que tous les pays réglementent dans une certaine mesure, comme la santé publique, la sécurité au travail, la protection de l'environnement et l'activité commerciale. Ses éléments clés consistent à établir si les textes sont effectivement appliqués ; si les textes sont mis en œuvre et appliqués sans influence indue ; si les procédures administratives sont menées dans des 
délais raisonnables ; si la régularité de la procédure est respectée à cet égard ; et si les pouvoirs publics ne s'approprient pas des biens sans contrepartie raisonnable. Par conséquent, cet indicateur est différent des indicateurs de gestion de la qualité de la réglementation présentés dans Panorama des administrations publiques 2009, qui mettaient l'accent sur la consultation, l'analyse d'impact de la réglementation et la simplification réglementaire.

La mise en œuvre de la réglementation atteint son plus haut niveau en Suède, au Japon, au Danemark et en Autriche ; suivent de près l'Australie, la Norvège, les Pays-Bas, la Finlande et la Nouvelle-Zélande ; en revanche, elle mériterait d'être améliorée au Mexique, en Grèce, en Turquie et en Italie. Globalement, il existe une marge d'amélioration considérable dans de nombreux pays membres de l'OCDE, car la moyenne OCDE s'élève à 0.71 . Les pays partenaires et participants affichent tous des résultats inférieurs à la moyenne OCDE. Ceux qui obtiennent les scores les plus élevés sont le Brésil et l'Afrique du Sud, et c'est l'Ukraine qui obtient le score le plus faible.

L'examen des sous-facteurs de cette catégorie révèle qu'il y a rarement d'influence indue dans l'application et la mise en œuvre des textes, et que cet élément bénéficie du score le plus élevé (0.77), tandis que l'application effective des textes est l'aspect qui mériterait le plus d'être amélioré (0.67).

\section{Justice civile}

Cet indicateur composite mesure si les citoyens ordinaires peuvent obtenir un règlement efficace de leurs griefs par le biais du système de justice civile, ce qui requiert que le système soit accessible, abordable, efficace, impartial et respectueux des différences culturelles. Ses composantes permettent d'évaluer si les citoyens ont accès à la justice civile, et s'ils en ont les moyens financiers ; si la justice civile est exempte de discrimination ; si elle est exempte de corruption ; si elle n'est pas indûment influencée par les pouvoirs publics ; si elle n'est pas sujette à des délais déraisonnables ; si elle est effectivement mise en application; et si les modes alternatifs de règlement des différends sont accessibles, impartiaux et efficaces.

Les scores les plus élevés en matière de justice civile sont obtenus par les pays nordiques, ainsi que par les Pays-Bas et l'Allemagne. L'Italie, le Mexique et la Turquie sont les pays de l'OCDE qui affichent les plus faibles scores en la matière. Sur les quatre grands déterminants de l'État de droit examinés (limitation des pouvoirs de l'administration, droits fondamentaux, mise en œuvre de la réglementation et accès à la justice civile), c'est dans ce dernier domaine que la performance moyenne des pays membres de l'OCDE est la plus faible (0.69), avec un score situé juste en dessous de celui obtenu pour la mise en œuvre de la réglementation (0.71) ; ce sont le Brésil et l'Afrique du Sud qui obtiennent les meilleurs résultats parmi les pays partenaires.

Le principal problème concernant l'accès à la justice civile dans les pays membres de l'OCDE est la longueur des procédures (0.47), et c'est la composante liée à l'absence de corruption dans la justice civile qui obtient le meilleur résultat (0.8).

\section{Méthodologie et définitions}

Les données sont recueillies par le World Justice Project au moyen d'un ensemble de cinq questionnaires basés sur le cadre conceptuel de l'indice de l'État de droit. Les questionnaires sont utilisés pour sonder des experts et des membres du grand public dans chaque pays. En moyenne, chaque pays compte potentiellement plus de 300 experts locaux aptes à répondre aux questionnaires, et il est fait appel aux services d'instituts de sondage locaux pour réaliser l'enquête auprès du public. On dispose de données pour 28 pays de l'OCDE et pour 8 pays partenaires et participants. Toutes les variables utilisées pour noter chacun des indicateurs composites sont codées et normalisées pour aller de 0 à 1, 1 étant la meilleure note et 0 la plus basse. Des informations plus détaillées sur les facteurs sélectionnés (limitation des pouvoirs de l'administration (2.10), droits fondamentaux (2.11), mise en œuvre de la réglementation (2.12) et justice civile (2.13)) sont disponibles en ligne aux adresses $h t t p: / / d x . d o i . o r g / 10.1787 / 888932943172$, http:// dx.doi.org/10.1787/888932943191, http://dx.doi.org/10.1787/ 888932943210 et http://dx.doi.org/10.1787/888932943229, respectivement.

\section{Pour en savoir plus}

Aghast, M. et al. (2013), WJP Rule of Law Index 2012-2013, The World Justice Project, Washington.

\section{Notes relatives aux graphiques}

On ne dispose pas de données pour l'Irlande, l'Islande, Israël, le Luxembourg, la République slovaque et la Suisse. Les données pour la Hongrie ne sont pas présentées.

Pour l'Italie, les réformes législatives de 2013 ne sont pas prises en compte.

Informations sur les données concernant Israël : http://dx.doi.org/10.1787/ 888932315602 


\section{LA GOUVERNANCE STRATÉGIQUE}

\section{LṔtat de droit}

\subsection{Limitation des pouvoirs de l'administration (2012-13)}

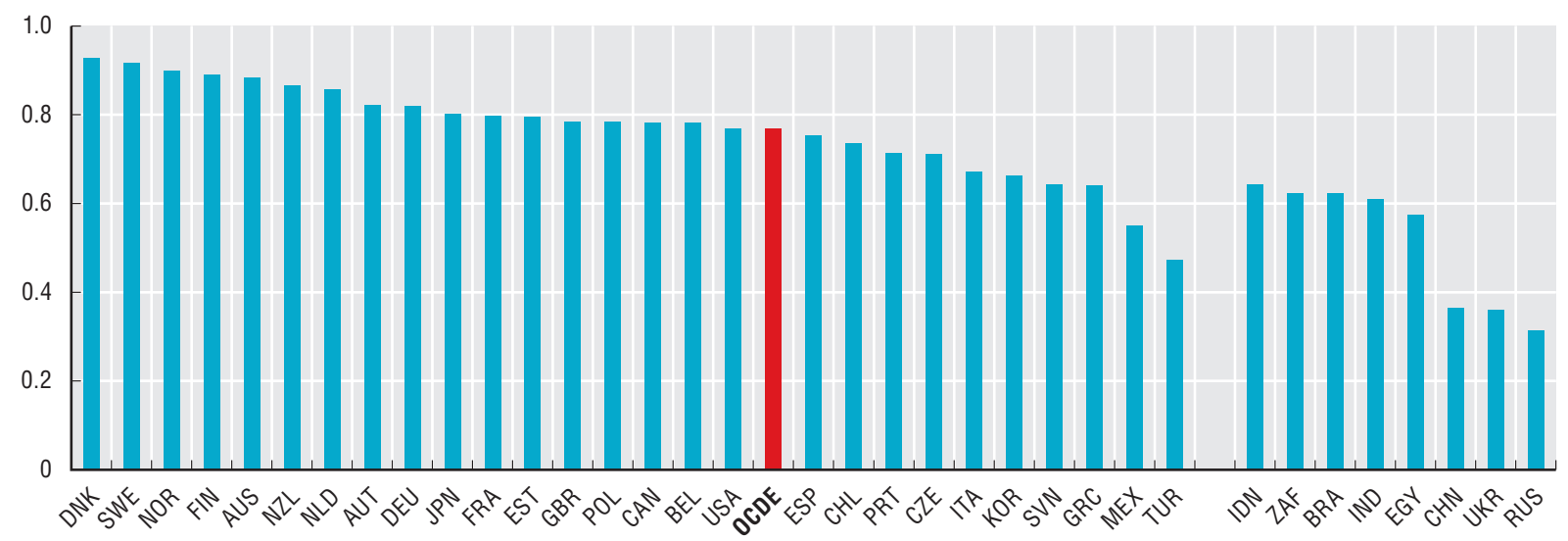

Source : World Justice Project.

StatLink ला1s< http://dx.doi.org/10.1787/888933158632

\subsection{Droits fondamentaux (2012-13)}

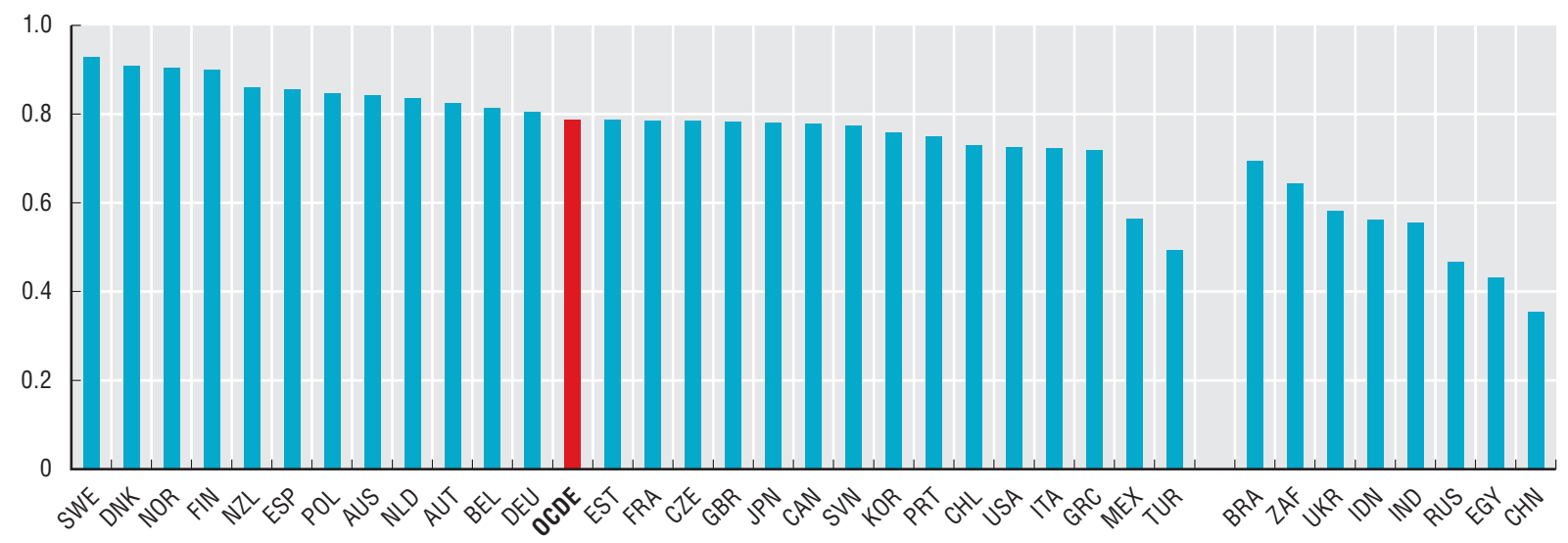

Source : World Justice Project,

StatLink काज़l http://dx.doi.org/10.1787/888933158641

\subsection{Limitation des pouvoirs de l'administration et droits fondamentaux (2012-13)}

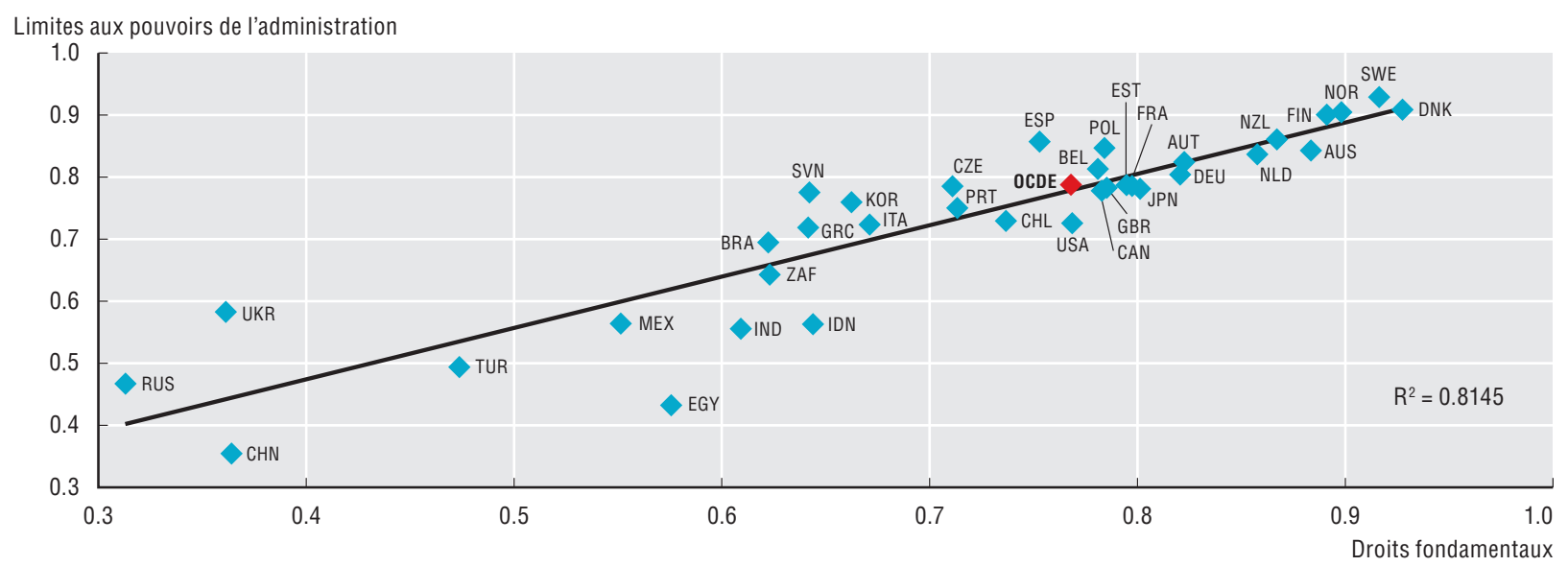

Source : World Justice Project. 

Les pays membres de l'OCDE ont été fortement touchés par des chocs perturbateurs au cours des dernières décennies, avec des retombées économiques croissantes.

Au cours des trente dernières années, le nombre de ces chocs est passé d'environ 100 par an à parfois plus de 300 par an sur l'ensemble des pays de l'OCDE, et ils ont provoqué des centaines de milliards de pertes annuelles. Ces chocs posent de multiples difficultés aux pouvoirs publics, mettent en péril de nombreuses vies et peuvent perturber l'activité des petites et moyennes entreprises et des sociétés transnationales. De vastes infrastructures d'importance vitale peuvent également être menacées, avec des effets dévastateurs, comme l'a démontré en 2011 le Grand séisme de l'est du Japon. Ces chocs perturbateurs à grande échelle ont conduit les pays à renforcer leurs politiques de gestion des risques, notamment sur le plan de l'identification et de l'évaluation des risques et de la mise en œuvre de mesures visant à renforcer la résilience.

La gestion des risques majeurs dans l'ensemble des pays de l'OCDE nécessite un appui au plus haut niveau politique, mais aussi un engagement en faveur de la réduction des risques à tous les niveaux de l'administration et du territoire, y compris les collectivités locales. Il faut pour cela instaurer des cadres stratégiques permettant de coordonner les grandes orientations, les capacités et la gouvernance afin que l'action publique puisse tenir compte des risques. La réduction des risques est supervisée par le centre de gouvernement (généralement le cabinet du Premier ministre) dans quatre pays de l'OCDE, et une coordination centrale est assurée dans la plupart des autres, souvent au niveau du service national chargé de la protection civile.

Par ailleurs, les politiques de gestion des risques ont été systématisées à l'échelle de l'ensemble des secteurs, à travers des stratégies, des plans et des outils. Parmi les pays de l'OCDE ayant lancé des examens interdisciplinaires de l'intégration de la gestion des risques aux politiques et investissements publics, la quasi-totalité prennent systématiquement en compte la gestion des risques de catastrophe dans leurs stratégies et leurs plans sectoriels en matière d'investissements publics. En revanche, seuls les deux tiers procèdent à des analyses coûts-avantages de la gestion des risques dans la conception et l'exploitation des investissements publics majeurs. L'importance attribuée à l'échelon local est illustrée par la mise en place, dans $86 \%$ des pays de l'OCDE, de cadres juridiques pour les responsabilités locales et, dans près des deux tiers d'entre eux, d'une réglementation tenant compte des risques pour l'occupation des sols et la promotion immobilière privée. Toutefois, la proportion d'autorités locales recevant des subventions régulières destinées à la réduction des risques de catastrophe est bien moins forte (62\%). Or, ce cadre juridique favorable restera dépourvu d'effet si les administrations locales ne bénéficient pas des ressources nécessaires pour mener à bien des activités de réduction des risques.

Pour les pouvoirs publics, la difficulté consiste à organiser une action intégrée permettant de relever des défis multidisciplinaires. À cet égard, les évaluations nationales du risque constituent un outil important qui peut permettre d'élaborer une stratégie de gestion intégrée multi-aléas en matière de gestion des risques. Toutefois, plus de la moitié des pays de l'OCDE effectuent ces évaluations de manière intégrée, en suivant une démarche multi-aléas et en incluant les risques futurs probables.

Par rapport aux défis auxquels doivent faire face les pays à faible revenu, le niveau atteint dans les pays de l'OCDE en matière de gestion des risques est élevé. Néanmoins, avec l'exposition grandissante aux risques et l'évolution des profils de risque, les pertes économiques continuent de croître, en dépit d'une tendance à la baisse des décès dus aux catastrophes. Les systèmes d'alerte précoce ont permis une diffusion efficace des messages d'alerte vers les populations menacées, qui, de leur côté, savent généralement comment y réagir. La sensibilisation au risque s'est également améliorée dans de nombreux pays, notamment grâce à des campagnes publiques efficaces et à l'inscription des grands principes de la gestion des risques aux programmes de l'enseignement primaire, secondaire et supérieur.

\section{Méthodologie et définitions}

Les données sur les catastrophes reposent sur la base de données OFDA/CRED sur les catastrophes internationales (EM-DAT) développée par l'Université catholique de Louvain-Bruxelles, Belgique. Les pertes sont fondées sur les estimations de SwissRe. La plateforme en ligne gérée dans le cadre de la Stratégie internationale des Nations Unies pour la prévention des catastrophes permet d'accéder à des rapports d'étape par pays sur les progrès réalisés au regard des objectifs du Cadre d'action de Hyogo (www.preventionweb.net/english/hyogo/). Les données présentées ici renvoient à la dernière période considérée (2011-13). Les rapports d'étape reposent sur une auto-évaluation réalisée à l'aide de processus associant de multiples parties prenantes. Enfin, des renseignements ont été obtenus au moyen d'un ensemble de questionnaires de l'OCDE, puis d'entretiens téléphoniques réalisés en 2012, en collaboration avec des agents publics et d'autres experts du Forum à haut niveau de l'OCDE sur les risques.

Le graphique 2.17, Nombre total de catastrophes par an (1980-2010), est disponible en ligne à l'adresse http:// dx.doi.org/10.1787/888932941101.

\section{Pour en savoir plus}

OCDE (2012), Disaster Risk Assessment and Risk Financing: A G20/ OECD Methodological Framework, OCDE, Paris, www.oecd.org/ gou/risk/G20disasterriskmanagement.pdf.

SwissRe (2011), "Closing the financial gap: New partnerships between the public and private sectors to finance disaster risks ", SwissRe Economic Research and Consulting, Zurich, http://media.swissre.com/documents/pub-closing-thefinancialgap_w1.pdf.

UNISDR (2013), "Réduction du risque de catastrophe : Bilan mondial - Du partage des risques aux bénéfices partagés: Analyse de la rentabilité de la réduction des risques de catastrophe ", Bureau des Nations Unies pour la prévention des catastrophes, Genève, www.preventionweb.net/english/ hyogo/gar/2013/en/gar-pdf/GAR2013_FR.pdf.

\section{Notes relatives aux tableaux}

On ne dispose pas de données pour l'Autriche, la Belgique, le Danemark, l'Estonie, l'Irlande, l'Islande, Israël, le Luxembourg et la République slovaque.

2.16 : Les informations sur le Canada sont issues des données du Cadre d'action de Hyogo pour la période 2009-11. 
2.14. Autorités locales des pays membres de l'OCDE disposant d'un mandat et d'un budget en matière de réduction des risques de catastrophe (RRC)

Les autorités locales disposent Les autorités locales reçoivent d'un mandat en matière de RRC des subventions régulières pour la RRC

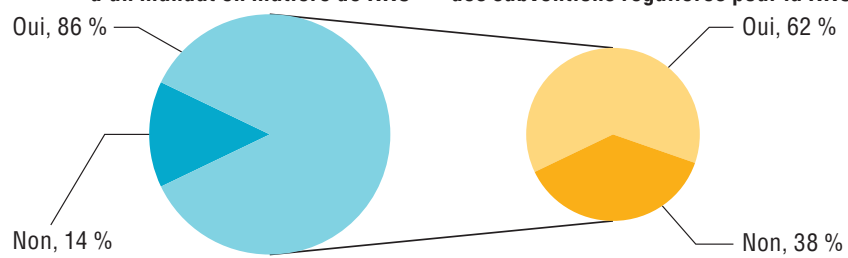

Source: Données tirées des rapports d'étape sur le Cadre d'action de Hyogo publiés à l'adresse : www.preventionweb.net/english/hyogo/progress/?pid:3\&pil:1. StatLink तiाs $h$ ttp://dx.doi.org/10.1787/888933158724

\subsection{Responsabilités en matière de coordination de la RRC dans les pays de l'OCDE}

\begin{tabular}{|c|c|}
\hline & Responsabilité de la coordination de la RRC: \\
\hline Cabinet du Premier ministre & Australie, France, Nouvelle-Zélande, Turquie \\
\hline $\begin{array}{l}\text { Unité centrale de planification } \\
\text { et/ou de coordination }\end{array}$ & $\begin{array}{l}\text { Chili, Corée, Égypte, Grèce, Japon, Norvège, } \\
\text { Pologne, Royaume-Uni, Suisse }\end{array}$ \\
\hline Service de la protection civile & $\begin{array}{l}\text { Australie, Finlande, France, Italie, Norvège, Portugal, } \\
\text { Slovénie, Suède }\end{array}$ \\
\hline $\begin{array}{l}\text { Organisme de planification } \\
\text { de l'environnement }\end{array}$ & France, Suisse \\
\hline Ministère des Finances & France \\
\hline
\end{tabular}

Source : Données tirées des rapports d'étape sur le Cadre d'action de Hyogo publiés à l'adresse : www.preventionweb.net/english/hyogo/progress/?pid:3\&pil:1. StatLink Aाst http://dx.doi.org/10.1787/888933158737

\subsection{Politiques nationales de gestion des risques des pays de l'OCDE}

\begin{tabular}{|c|c|c|c|c|c|c|c|c|c|c|c|c|c|c|c|}
\hline & \multicolumn{5}{|c|}{$\begin{array}{l}\text { Le risque dans la planification des politiques } \\
\text { nationales - prise en compte des risques dans : }\end{array}$} & \multicolumn{4}{|c|}{ Évaluation des risques } & \multicolumn{2}{|c|}{$\begin{array}{l}\text { Plans financiers } \\
\text { d'urgence }\end{array}$} & \multicolumn{3}{|c|}{ Alerte précoce } & 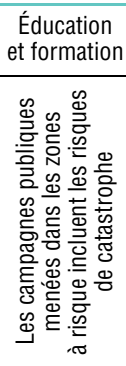 \\
\hline Allemagne & $\bullet$ & O & O & O & O & $\bullet$ & O & .. & O & O & O & $\bullet$ & $\bullet$ & $\bullet$ & $\bullet$ \\
\hline Australie & • & $\bullet$ & $\bullet$ & $\bullet$ & $\bullet$ & $\bullet$ & $\bullet$ & .. & $\bullet$ & $\bullet$ & $\bullet$ & $\bullet$ & $\bullet$ & $\bullet$ & $\bullet$ \\
\hline Canada &.. & .. & .. & .. & .. & $\bullet$ & $\bullet$ & $\bullet$ & .. & $\bullet$ & O & • & $\bullet$ & $\bullet$ & $\bullet$ \\
\hline Chili & $\bullet$ & $\bullet$ & $\bullet$ & $\bullet$ & - & .. & O & .. & O & $\bullet$ & $\bullet$ & $\bullet$ & $\bullet$ & $\bullet$ & $\bullet$ \\
\hline Corée & $\bullet$ & $\bullet$ & - & O & $\bullet$ & .. & $\bullet$ & .. & $\bullet$ & $\bullet$ & $\bullet$ & $\bullet$ & $\bullet$ & $\bullet$ & $\bullet$ \\
\hline Hongrie & $\bullet$ & $\bullet$ & $\bullet$ & $\bullet$ & $\bullet$ & $\bullet$ & O & $\bullet$ & $\bullet$ & $\bullet$ & O & $\bullet$ & $\bullet$ & $\bullet$ & $\bullet$ \\
\hline Italie & $\bullet$ & $\bullet$ & $\bullet$ & $\bullet$ & O & .. & $\bullet$ & .. & $\bullet$ & $\bullet$ & O & $\bullet$ & $\bullet$ & $\bullet$ & $\bullet$ \\
\hline Japon & • & $\bullet$ & - & O & $\bullet$ & .. & $\bullet$ & .. & O & $\bullet$ & $\bullet$ & $\bullet$ & $\bullet$ & $\bullet$ & $\bullet$ \\
\hline Mexique & - & - & - & - & • & - & - & .. & O & - & $\bullet$ & - & - & $\bullet$ & - \\
\hline Norvège & $\bullet$ & O & $\bullet$ & $\bullet$ & $\bullet$ & $\bullet$ & $\bullet$ & O & $\bullet$ & $\bullet$ & O & $\bullet$ & $\bullet$ & $\bullet$ & $\bullet$ \\
\hline Nouvelle-Zélande & $\bullet$ & O & $\bullet$ & $\bullet$ & $\bullet$ & $\bullet$ & $\bullet$ & .. & $\bullet$ & $\bullet$ & O & $\bullet$ & $\bullet$ & $\bullet$ & $\bullet$ \\
\hline Pays-Bas & • & O & $\bullet$ & $\bullet$ & $\bullet$ & $\bullet$ & $\bullet$ & - & $\bullet$ & $\bullet$ & $\mathrm{O}$ & $\bullet$ & $\bullet$ & $\bullet$ & $\bullet$ \\
\hline Pologne & - & $\bullet$ & - & - & O & .. & O & .. & O & $\bullet$ & $\bullet$ & • & $\bullet$ & • & .. \\
\hline Portugal & .. & $\bullet$ & $\bullet$ & $\bullet$ & O & .. & $\bullet$ & .. & • & $\bullet$ & O & O & O & O & $\bullet$ \\
\hline Rép. tchèque & - & $\bullet$ & - & O & O & .. & $\bullet$ & .. & O & $\bullet$ & O & $\bullet$ & $\bullet$ & $\bullet$ & • \\
\hline Royaume-Uni & • & O & - & $\bullet$ & O & - & $\bullet$ & $\bullet$ & • & $\bullet$ & O & $\bullet$ & $\bullet$ & $\bullet$ & • \\
\hline \multicolumn{16}{|l|}{ Total OCDE } \\
\hline - Oui & 22 & 16 & 22 & 18 & 17 & 14 & 16 & 8 & 15 & 22 & 8 & 23 & 21 & 23 & 23 \\
\hline O Non & 0 & 7 & 1 & 6 & 6 & 0 & 8 & 1 & 8 & 2 & 16 & 1 & 3 & 1 & 0 \\
\hline
\end{tabular}

Source: Données extraites des rapports d'étape sur le Cadre d'action de Hyogo publiés à l'adresse : www.preventionweb.net/english/hyogo/progress/ ?pid :3\&pil :1

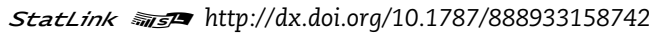


La viabilité budgétaire correspond à la capacité d'un État à maintenir les finances publiques dans une position crédible et fonctionnelle sur le long terme. Afin de garantir durablement cette viabilité, les pouvoirs publics doivent procéder en permanence à une anticipation stratégique des recettes et des engagements, des facteurs environnementaux et des tendances socio-économiques, pour adapter leur planification financière en conséquence. Des niveaux d'endettement élevés et croissants nuisent à la position budgétaire des États et peuvent enclencher un cercle vicieux où l'augmentation de la dette pèse sur le potentiel de croissance économique, car les fonds disponibles sont détournés de l'investissement productif. Depuis la crise financière et économique, de nombreux pays de l'OCDE restent confrontés à un ratio dette publique/PIB en hausse. Les coûts qu'ils supportent pour surmonter le ralentissement économique actuel, ainsi que l'augmentation prévue des dépenses liées au vieillissement de la population, constituent de sérieux obstacles pour la viabilité des finances publiques.

L'OCDE a produit des estimations relatives au redressement des soldes financiers primaires sous-jacents qui serait nécessaire pour ramener la dette brute des administrations publiques à $60 \%$ du PIB d'ici 2030. Il ressort de ce modèle que la Grèce, le Royaume-Uni et les États-Unis devraient, en moyenne, redresser leurs soldes primaires sous-jacents respectifs de 2012 de plus de $6 \%$ du PIB potentiel (économie fonctionnant à plein régime) pour ramener la dette publique à $60 \%$ du PIB dans ce délai. Le Japon aurait besoin de $13 \%$ du PIB potentiel pour atteindre ce ratio d'endettement, mais les efforts d'assainissement nécessaires seraient tellement importants qu'avec un tel scénario, ce pays ne devrait pas être capable d'atteindre cet objectif en 2030. En revanche, la situation actuelle des finances publiques (solde budgétaire et niveau d'endettement, par exemple) en Allemagne, en Corée, au Danemark, en Estonie et en Suisse est telle que ces pays ne devraient pas avoir besoin d'excédents pour ramener la dette à $60 \%$ du PIB.

En moyenne, les pays de l'OCDE ont annoncé ou mis en place des plans d'assainissement budgétaire équivalant à plus de $5.5 \%$ du PIB pour la période 2009-15, dont les deux tiers s'articulent autour de mesures portant sur les dépenses et le tiers restant autour de mesures portant sur les recettes. Toutefois, l'ampleur et la composition des plans d'assainissement cumulatifs varient fortement d'un pays à l'autre. Les pays qui connaissent les déséquilibres économiques les plus importants et la détérioration la plus rapide de leurs finances publiques ont besoin d'un assainissement budgétaire plus drastique. C'est pourquoi la Grèce, l'Irlande et le Portugal ont annoncé des mesures d'assainissement budgétaire atteignant plus de $12 \%$ du PIB. De leur côté, le Canada, la Suède et la Suisse ont annoncé ou mis en œuvre des plans d'assainissement représentant moins de $1.6 \%$ du PIB. La plupart des pays ont axé en priorité leur plan d'assainissement sur des mesures relatives aux dépenses. Seuls sept pays (Belgique, Danemark, Estonie, Italie, Pologne, République tchèque et Turquie) ont choisi de privilégier les mesures portant sur les recettes.

Pour de bonnes prévisions stratégiques, il faut tenir compte des coûts associés aux évolutions démographiques, d'autant plus que la plupart des pays membres de l'OCDE vont subir des pressions budgétaires croissantes sous l'effet de la hausse prévue des dépenses liées au vieillissement et de l'évolution des technologies (santé, dépendance, retraites). En moyenne, sans évolution des politiques, les dépenses publiques liées au vieillissement dans les pays de l'OCDE devraient augmenter de près de trois points de pourcentage du PIB entre 2014 et 2030.

\section{Méthodologie et définitions}

Les données des graphiques 2.18 et 2.20 sont issues des Perspectives économiques de l'OCDE, $\mathrm{n}^{\circ}$ 93. L'assainissement total requis pour atteindre un ratio engagements financiers bruts/PIB égal à $60 \%$ du PIB d'ici 2030 est mesuré sur deux périodes : entre 2012 et 2014 comme étant l'évolution du solde primaire sous-jacent, et de 2014 à 2030 comme étant la différence entre le niveau atteint en 2014 et sa moyenne sur la dernière période. Les hypothèses posées pour générer le solde primaire requis pour ramener le ratio dette/PIB à $60 \%$ sont disponibles dans Perspectives économiques de l'OCDE, $n^{\circ} 93$, dans l'encadré 4.5 et le tableau 4.2.

Les données du graphique 2.19 sont issues de l'enquête de l'OCDE sur l'assainissement budgétaire de 2012.

Pour la plupart des pays, les données sur la dette brute utilisées pour ces calculs se rapportent aux engagements (à court et long terme) des administrations publiques tels qu'ils sont définis dans le Système de comptabilité nationale (SCN). Cette définition diffère de celle que l'on trouve dans le traité de Maastricht et qui sert à évaluer les positions budgétaires dans l'UE.

\section{Pour en savoir plus}

OCDE (2013), « Perspectives économiques de l'OCDE n 93 ", Perspectives économiques de l'OCDE : statistiques et projections (base de données), http://dx.doi.org/10.1787/data-00655-fr.

OCDE (2012), Redresser les finances publiques, mise à jour 2012, Éditions OCDE, Paris, http://dx.doi.org/10.1787/9789264179608-fr.

\section{Notes relatives aux graphiques}

2.18 et 2.20 : On ne dispose pas de données pour le Chili, le Mexique et la Turquie. L'Allemagne, la Corée, le Danemark, l'Estonie, la Norvège et la Suisse n'ont besoin de prendre aucune mesure d'assainissement particulière pour parvenir à un ratio engagements financiers bruts/ PIB de $60 \%$ d'ici 2030. La moyenne OCDE n'est pas pondérée. Les projections budgétaires sont obtenues en appliquant un processus d'assainissement stylisé et ne doivent pas être interprétées comme des prévisions.

2.19 : Les données sont la somme des mesures annuelles d'assainissement allant de 2009-10 jusqu'en 2015 communiquées par les autorités nationales. Seuls les pays suivants ont fait état d'un assainissement en 2009 : Estonie, Hongrie, Irlande, Pologne et Slovénie. L'assainissement effectué en 2007-08 par la Hongrie n'est pas inclus. L'Autriche fait état d'un assainissement jusqu'en 2016. Les pays participants dont les noms suivent n'ont pas annoncé de plan concret d'assainissement et ne sont pas inclus dans le graphique : Corée, États-Unis et Japon. L'Australie signale un assainissement (en particulier pour 2013), mais applique une définition plus large du terme que le présent document. La NouvelleZélande et la Slovénie ont fait état de quelques mesures portant sur les recettes, mais ces dernières ne sont pas totalement quantifiées.

2.20 : Pour les dépenses liées au vieillissement, quand on ne disposait pas de projections pour la période 2014-30, une interpolation linéaire a été appliquée.

Informations sur les données concernant Israël : http://dx.doi.org/10.1787/ 888932315602 . 
2.18. Assainissement total requis entre 2012 et 2030 pour ramener les engagements financiers bruts des administrations publiques à $60 \%$ du PIB

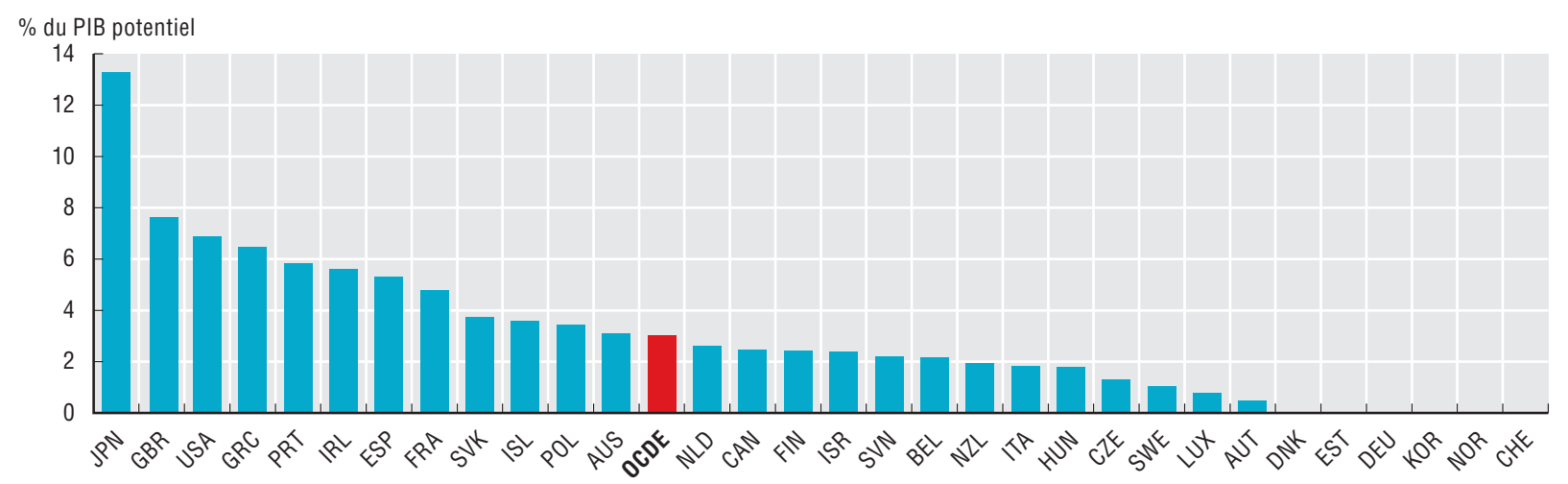

Source: Calculs de l'OCDE; OCDE (2013), “ Perspectives économiques de l'OCDE, n 93 ", Perspectives économiques de l’OCDE : statistiques et projections (base de données), mai 2013, http://dx.doi.org/10.1787/data-00655-fr.

\subsection{Assainissement budgétaire basé sur les dépenses et basé sur les recettes, en pourcentage du PIB (2009-15)}

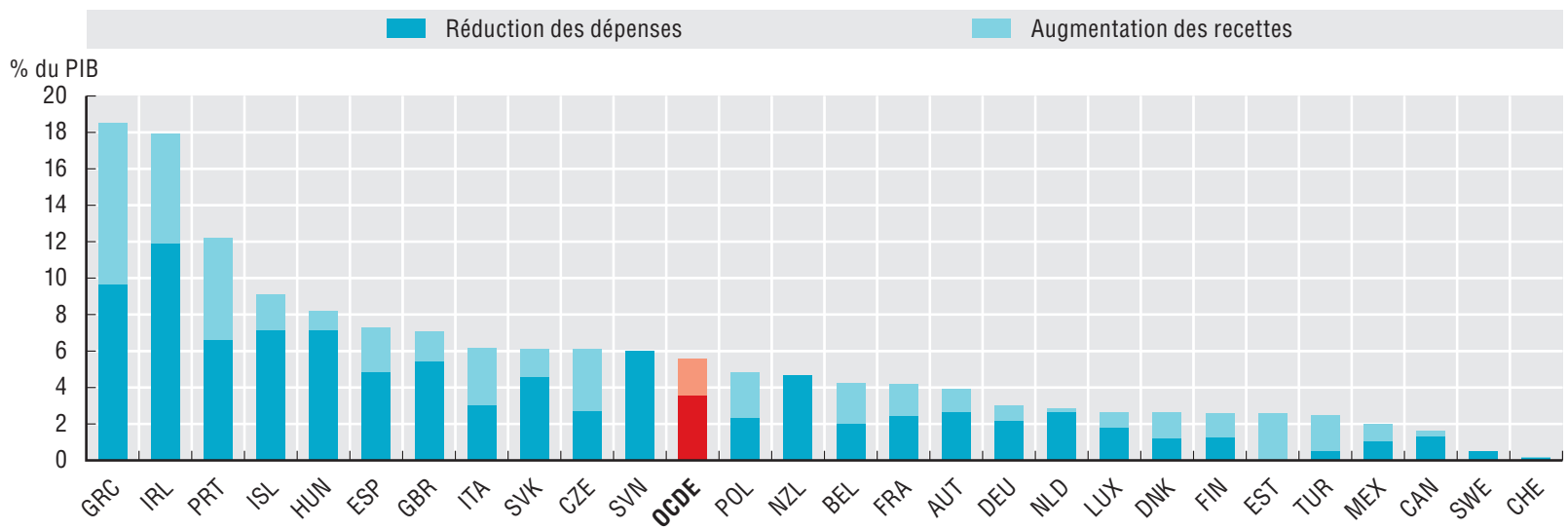

Source : Enquête de l'OCDE sur l'assainissement budgétaire 2012.

\subsection{Assainissement budgétaire requis et évolution prévue des dépenses liées au vieillissement (2014 à 2030)}

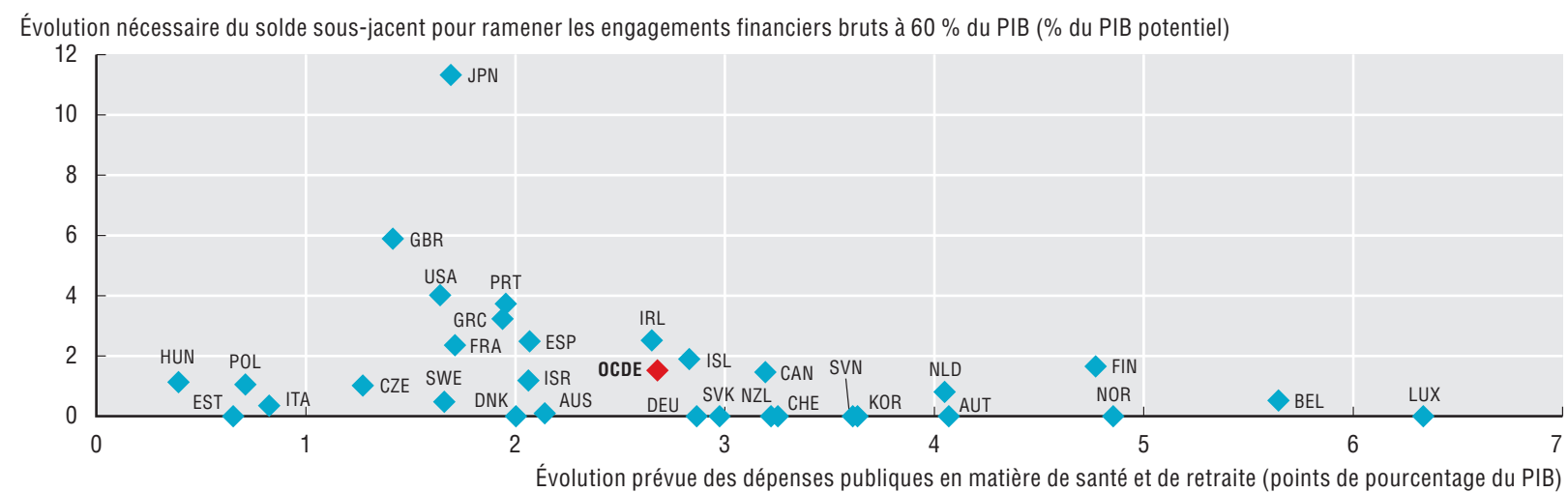

Source: Calculs de l'OCDE ; OCDE (2013), "Perspectives économiques de l'OCDE, n 93 ", Perspectives économiques de l’OCDE : statistiques et projections (base de données), mai 2013, http://dx.doi.org/10.1787/data-00655-fr. 
Estimer l'efficience consiste à évaluer le rapport entre les intrants investis et les extrants (ou produits) obtenus grâce à ces ressources. Améliorer cet indicateur afin de mieux maîtriser les dépenses est un objectif crucial pour les pays de l'OCDE. Les crises budgétaires auxquelles ont été confrontés de nombreux pays tant avant qu'après la grande récession économique et financière ont placé les performances du secteur public sur le devant de la scène.

Les indicateurs d'efficience comparent les évaluations relatives aux extrants aux évaluations relatives aux intrants. En les réunissant, ils permettent d'exprimer les deux dimensions de l'efficience, à savoir l'efficience technique (ou opérationnelle) et l'efficience allocative. L'évaluation des performances doit se baser sur l'efficience économique (ou efficience au regard du coût), qui est le produit de l'efficience opérationnelle et de l'efficience allocative.

Des indicateurs d'efficience sont ici présentés pour la santé, l'éducation, la justice et l'administration fiscale ; pour ces domaines, on dispose en effet de données sur les intrants et les extrants, et un consensus est en train de s'établir entre les pays sur des méthodes d'évaluation de l'efficience permettant des comparaisons internationales.

\section{La santé}

Il existe plusieurs indicateurs de l'efficience des soins de santé, au sein desquels la durée moyenne de séjour (DMS) à l'hôpital joue un rôle clé. Toutes choses égales par ailleurs, un séjour plus court est censé diminuer le coût par sortie et déplacer les soins du cadre hospitalier vers des cadres moins onéreux. Toutefois, un séjour plus court exige généralement une intensité de services plus élevée et coûte plus cher par journée d'hospitalisation. Un séjour trop court peut aussi compromettre les résultats obtenus sur le plan de la santé ou s'avérer préjudiciable au confort du patient ou à son rétablissement. En 2011, la durée moyenne de séjour à l'hôpital, toutes causes confondues, était de huit jours dans l'OCDE. C'est au Mexique et en Turquie que cette durée était la plus courte, puisqu'elle s'établissait à moins de la moitié de la moyenne OCDE. C'est au Japon qu'elle était la plus longue, avec près de 18 jours, soit plus du double de la moyenne OCDE. Au cours des dix dernières années, la DMS a baissé dans la plupart des pays, passant de 9.2 jours en 2000 à 8.0 jours en 2011. Au niveau du système, des facteurs comme des directives sur les pratiques ou les systèmes de paiement influent sur la durée de séjour. Au Japon, par exemple, l'offre abondante de lits et la structure des frais hospitaliers incitent les hôpitaux à garder les patients plus longtemps.

\section{Le secteur de la justice}

Des attentes fortes pèsent sur les pouvoirs publics afin qu'ils assurent une justice efficiente et réactive pour éviter aux citoyens qui la saisissent des délais et des coûts supplémentaires, notamment au niveau de leurs frais de représentation en justice. Un indicateur central de l'efficience des systèmes judiciaires civils peut être obtenu en associant le coût d'un procès, en pourcentage du montant de la demande (l'intrant), à la durée moyenne d'un procès en première instance (l'extrant) dans le pays considéré. Des tribunaux plus lents font baisser la confiance en la justice et, à long terme, peuvent faire grimper les coûts pour les entreprises et décourager les investissements privés. Des procès plus longs signifient également un fardeau économique plus lourd tant pour les citoyens que pour les pouvoirs publics. Dans les pays membres de l'OCDE, le coût d'un procès atteint en moyenne $19 \%$ du montant de la demande, et les premières instances durent en moyenne environ huit mois. La Corée, la NouvelleZélande et la Norvège obtiennent les meilleurs résultats, alors que les procès sont plus longs et plus coûteux en République slovaque. Par ailleurs, des frictions institutionnelles et une répartition géographique inégale des ressources judiciaires semblent constituer les principales raisons de la durée considérable des procès en première instance en Italie. Toutefois, les comparaisons de l'efficience dans ce secteur ne doivent pas être interprétées comme des indicateurs de la qualité de service, de la garantie d'une procédure régulière ou de la qualité des décisions des tribunaux.

\section{L'éducation}

Le développement et l'accumulation du capital humain sont essentiels pour assurer l'existence d'une main d'œuvre hautement qualifiée et bien équipée pour soutenir la concurrence sur le marché international du travail et pour exercer une citoyenneté active au sein de démocraties sachant répondre à ses besoins. L'acquis scolaire des individus est considéré comme un indicateur pertinent des résultats obtenus sur le plan de la production de capital humain. Lorsqu'il est comparé au montant de la dépense cumulée par élève au niveau national (c'est-à-dire les ressources affectées à l'enseignement), il peut jeter un éclairage sur les systèmes qui assurent les services les plus efficients. On constate une corrélation positive entre le niveau de compétence en lecture et en mathématiques mesuré par l'enquête PISA (Programme international pour le suivi des acquis des élèves) et les dépenses consacrées aux études primaires et secondaires, même si cette relation semble particulièrement avérée lorsque les niveaux de dépense cumulée par élève sont faibles (OCDE, PISA à la loupe 13). En plus du montant des dépenses, les résultats des élèves dépendent aussi, entre autres, de la qualité des enseignants, du milieu socio-économique individuel et des pratiques de gestion des établissements d'enseignement. Des pays comme la Corée, la Finlande et la Nouvelle-Zélande ont des dépenses par élève inférieures à la moyenne OCDE, mais obtiennent de meilleurs résultats. En revanche, l'Autriche et le Luxembourg affichent des résultats en dessous de la moyenne en dépit d'une dépense par élève plus élevée.

\section{L'administration fiscale}

Les impôts prélevés sur les citoyens et les entreprises représentent la principale ressource sur laquelle s'appuient les pouvoirs publics pour assurer la prestation des services publics. Le ratio relatif aux coûts de recouvrement est un indicateur d'efficience couramment employé par les administrations fiscales ; il compare les coûts d'administration annuels au montant total des recettes obtenues durant l'exercice. Toutes choses égales par ailleurs, une baisse de ce ratio peut dénoter une réduction des coûts relatifs (c'est-à-dire un gain d'efficience) ou une discipline fiscale accrue (c'est-àdire un gain d'efficacité). On observe pour la plupart des pays une tendance à la stabilité ou à la baisse entre 2005 et 2008, très probablement attribuable à une réduction des coûts. Certaines administrations fiscales ont en revanche connu une inversion de la tendance entre 2008 et 2011, avec une hausse du ratio vraisemblablement due à la réduction des recettes fiscales au lendemain de la crise économique. Il faut néanmoins comparer 
l'efficience des administrations fiscales avec prudence. En effet, les ratios d'efficience ici présentés sont influencés par les différences de taux d'imposition et la charge fiscale globale votée par le parlement; par des différences au niveau de l'éventail et de la nature des impôts prélevés; par la conjoncture macroéconomique, qui influe sur les recettes fiscales; et par des différences au niveau de la structure sousjacente des coûts pouvant être dues aux dispositifs institutionnels (par exemple, une administration des recettes partagée entre de multiples organismes, comme en Italie) et/ou à l'exercice de fonctions non fiscales (les douanes, par exemple).

\section{Méthodologie et définitions}

La durée moyenne de séjour (DMS) renvoie au nombre moyen de jours que les patients passent à l'hôpital. Elle est généralement obtenue en divisant le nombre total de jours passés par l'ensemble des patients hospitalisés au cours d'une année par le nombre des admissions ou sorties. Les admissions avec sortie le jour même ne sont pas prises en compte. Les données prennent en compte la totalité des hospitalisations (pas uniquement les soins curatifs/aigus).

Les données sur les procédures civiles sont issues de "Judicial Performance and its Determinants : A CrossCountry Perspective » (OCDE). La durée des procès est estimée (voir page 159). Le coût total du procès pour le justiciable (rapporté au montant de la demande), corrigé de la probabilité de bénéficier d'une aide juridictionnelle, fait référence à une affaire spécifique jugée au civil, du début à la fin. Il provient de la Base de données Doing Business de la Banque mondiale et englobe trois types de coûts engagés pour régler un litige commercial : frais de justice, frais d'exécution et frais d'avocats moyens.

Les chiffres sur la dépense par élève sont ceux de l'exercice 2009. La dépense par élève est égale à la dépense totale par établissement d'enseignement (public et privé confondus, si ce n'est pas précisé), divisée par le nombre d'élèves en équivalent temps plein inscrits, et elle inclut à la fois les services éducatifs et des services auxiliaires. Comme la durée des formations varie d'un pays à l'autre, la dépense annuelle par élève peut n'être qu'une mesure imparfaite de la dépense totale par élève. Les scores obtenus par les élèves sont issus d'une évaluation des compétences des jeunes de 15 ans effectuée dans le cadre du programme PISA.

Les données sur l'administration fiscale ont été fournies par les services fiscaux interrogés ou extraites de rapports officiels des pays. Il existe trois sortes de dépenses en matière d'administration fiscale : les coûts administratifs, salariaux et informatiques. Les coûts informatiques ont été définis comme le coût total de la fourniture d'un soutien informatique pour toutes les opérations administratives, qu'elles concernent les impôts ou non. Afin de faciliter les comparaisons, on s'est employé à distinguer les moyens utilisés et les coûts engagés pour les fonctions fiscales et non fiscales.

\section{Pour en savoir plus}

OCDE (2013a), Tax Administration 2013: Comparative Information on OECD and other Advanced and Emerging Economies, Éditions OCDE, Paris, http://dx.doi.org/10.1787/9789264200814-en.

OCDE (2013b, à paraître), Panorama de la santé 2013 : Les indicateurs de l'OCDE, Éditions OCDE, Paris.

OCDE (2012), Regards sur l'éducation 2012 : Les indicateurs de l'OCDE, Éditions OCDE, Paris, http://dx.doi.org/10.1787/eag2012-fr.

Palumbo, G. et al. (2013), "Judicial Performance and its Determinants: A Cross-Country Perspective "; Études de politique économique de l'OCDE, $\mathrm{n}^{\circ} 5$, Éditions OCDE, Paris, http://dx.doi.org/10.1787/5k44x00md5g8-en.

\section{Notes relatives aux graphiques}

2.21 : Pour le Canada, le Japon et les Pays-Bas, les données renvoient à la durée moyenne de séjour pour les soins curatifs (aigus), ce qui engendre une sous-estimation. Concernant les données indiquées pour 2011 : les données pour l'Australie, la Belgique, le Canada, le Chili, la Chine, la Fédération de Russie, la France et la Norvège sont celles de 2010 ; les données pour l'Islande sont celles de 2009 ; les données pour la Grèce et l'Indonésie sont celles de 2008. Concernant les données indiquées pour 2000 : les données pour la Chine et la Corée sont celles de 1999 ; les données pour l'Autriche et le Chili sont celles de 2001 ; les données pour le Luxembourg sont celles de 2002.

2.22 : Les données pour le Royaume-Uni ne couvrent que l'Angleterre et le Pays de Galles. Pour plus d'informations sur ces données, vous pouvez consulter la Base de données Doing Business.

2.23 : Les données relatives aux dépenses pour le Canada sont celles de 2008. Les données relatives aux dépenses pour le Chili sont celles de 2010. Les données relatives aux dépenses pour la Hongrie, l'Irlande, l'Italie, la Pologne, le Portugal, la Slovénie, la Suisse, le Brésil et la Fédération de Russie concernent uniquement les établissements publics.

2.24: Les cotisations de sécurité sociale (CSS) et les droits d'accise ne sont pas compris pour l'Allemagne, la France, la Pologne, le Portugal, la République slovaque et la République tchèque. Les CSS ne sont pas comprises pour l'Autriche, la Belgique, la Corée, l'Espagne, le Japon, le Luxembourg et le Mexique. Les droits d'accise ne sont pas compris pour la Finlande, l'Indonésie, la Nouvelle-Zélande et la Slovénie. Pour l'Afrique du Sud, le Brésil et l'Irlande, les coûts incluent les droits de douane. Pour l'Estonie, les coûts incluent les droits de douane pour 2005. Pour l'Espagne, les coûts incluent les droits de douane pour 2008 et 2011. Pour le Chili et la Suède, les coûts excluent le recouvrement des créances. Pour la Suisse, seule l'administration de la TVA est prise en compte. Pour l'Islande, les ratios calculés pour ces années sont sous-estimés car il semble que tous les coûts n'aient pas été quantifiés aux fins de l'enquête. Pour l'Italie, les ratios calculés pour ces années sont bien inférieurs aux vrais ratios, car ils ne tiennent pas compte des dépenses correspondant aux travaux relatifs à la fiscalité menés par d'autres organismes, qui n'ont pas été quantifiées. Pour les États-Unis, les ratios indiqués sont différents de ceux publiés par l'IRS, car on a utilisé en dénominateur le recouvrement "net ", et non "brut », de recettes. Les données pour l'Italie ne reflètent pas la réforme en cours visant à rationaliser le recouvrement des recettes.

Informations sur les données concernant Israël : http://dx.doi.org/ $10.1787 / 888932315602$. 


\section{LA GOUVERNANCE STRATÉGIQUE}

\section{L'efficience du secteur public}

\subsection{Durée moyenne de séjour toutes causes confondues (2000 et 2011)}

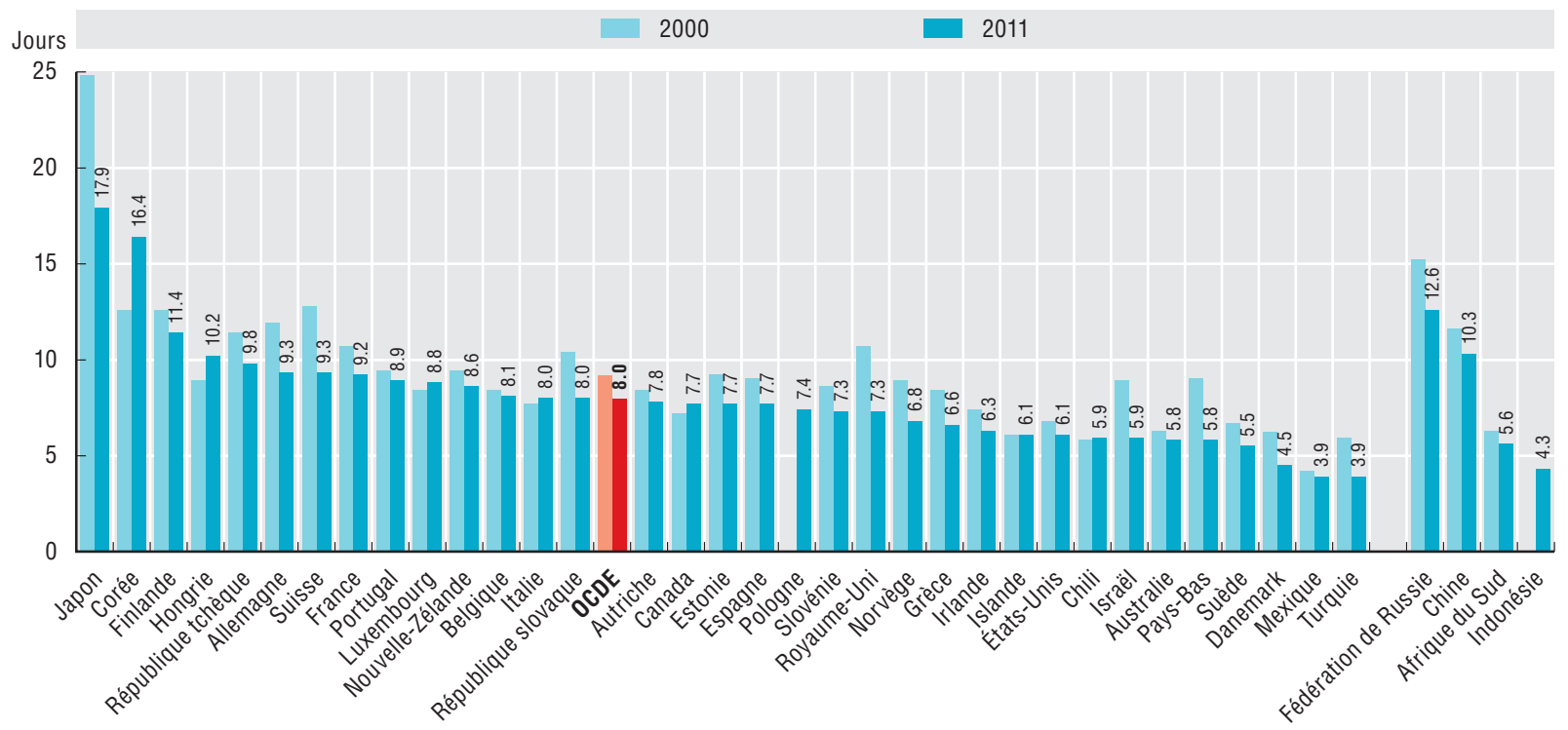

Source: Statistiques de l'OCDE sur la santé 2013.

StatLink काज्ञ http://dx.doi.org/10.1787/888933158791

\subsection{Durée d'un procès en première instance (en jours) et coût d'un procès}

(rapporté au montant de la demande, 2012)

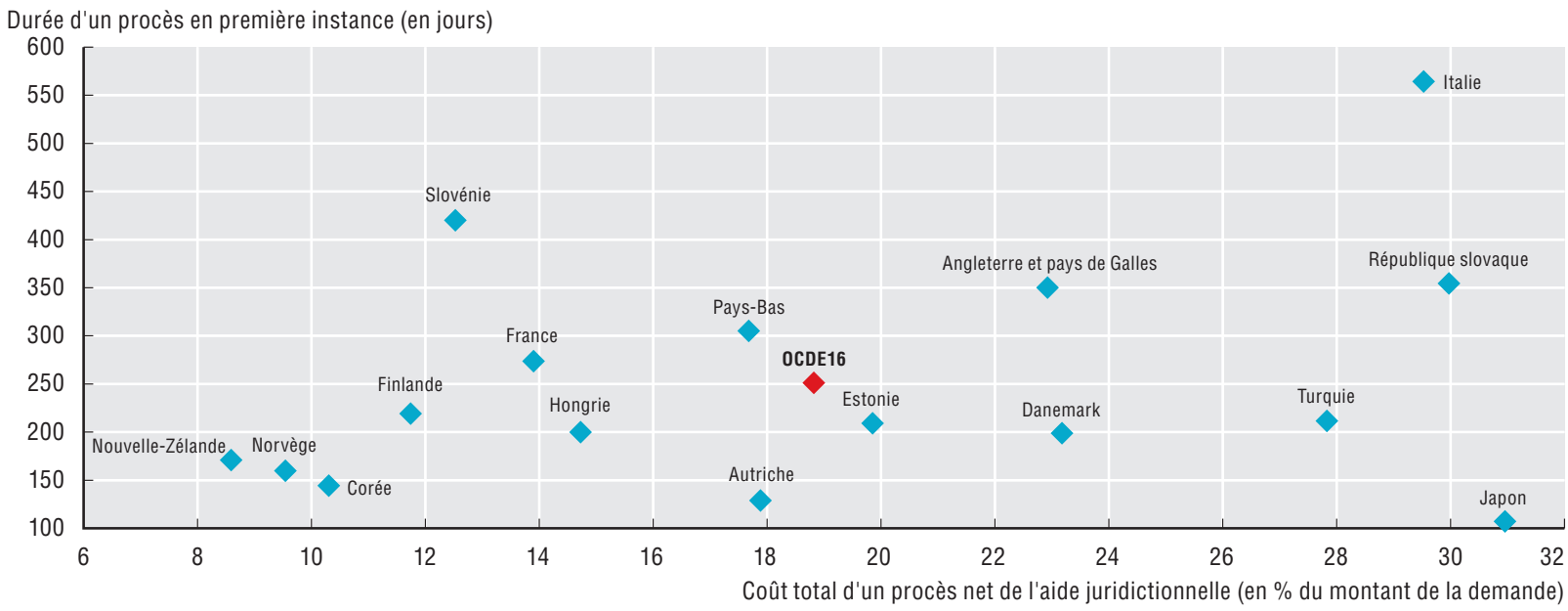

Source: Base de données Doing Business de la Banque mondiale et Palumbo, G. et al. (2013), "Judicial Performance and its Determinants : A Cross-Country Perspective ", Études de politique économique de l'OCDE, n 5, Éditions OCDE, Paris, http://dx.doi.org/10.1787/5k44x00md5g8-en. StatLink काजर http://dx.doi.org/10.1787/888933158807 
2.23. Scores PISA et dépense cumulée par élève entre 6 et 15 ans, en USD à PPA (2009)

Score en compréhension de l'écrit et dépense par élève
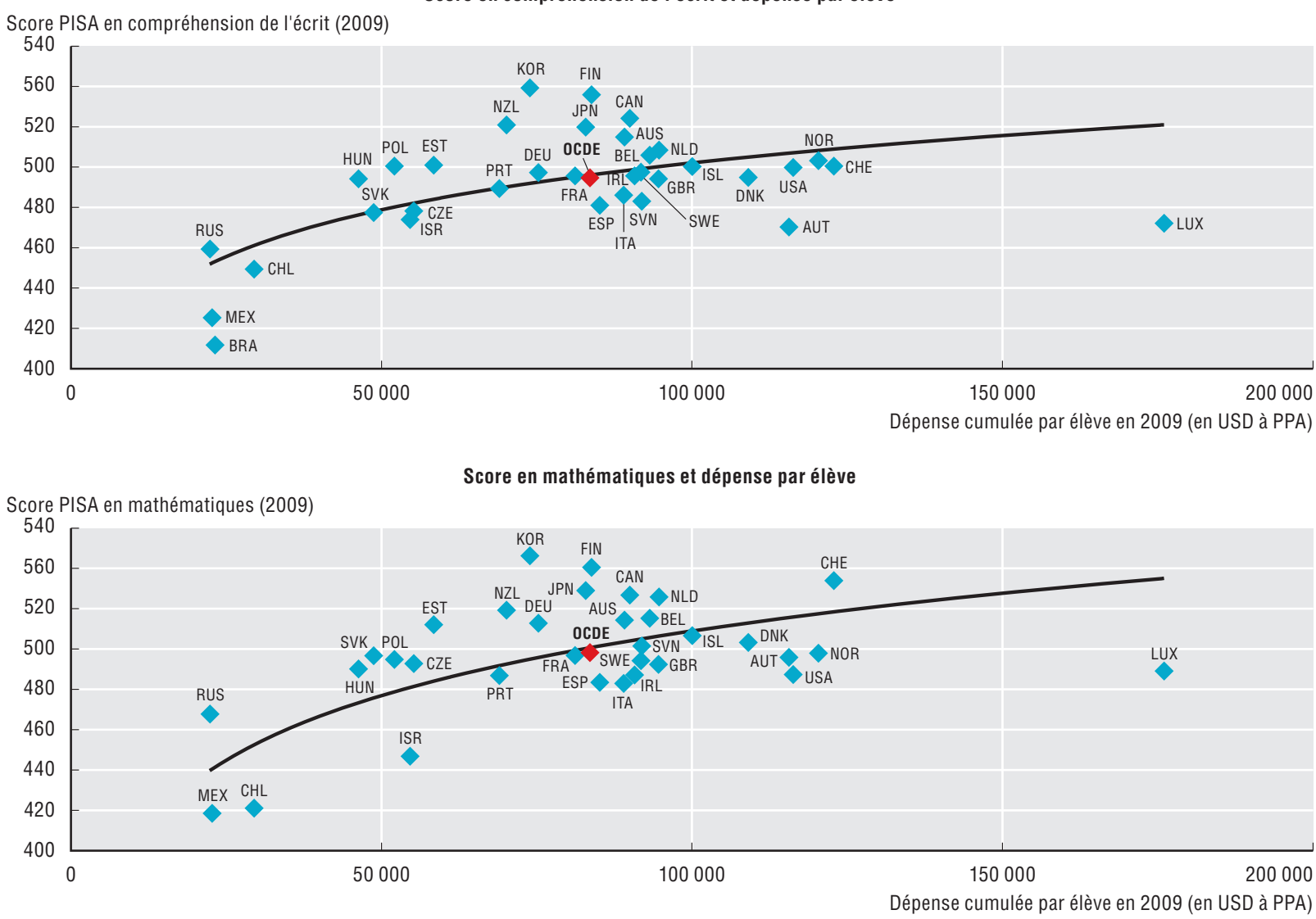

Source: OCDE (2012), Regards sur l'éducation 2012 : Les indicateurs de l'OCDE, Éditions OCDE, Paris, http://dx.doi.org/10.1787/eag-2012-fr, tableau B1.3b ; et OCDE (2010), Résultats du PISA 2009 : Savoirs et savoir-faire des élèves - Performance des élèves en compréhension de l'écrit, en mathématiques et en sciences (Volume I), PISA, Éditions OCDE, Paris, tableau I.2.3, http://dx.doi.org/10.1787/9789264097643-fr.

2.24. Ratios des coûts de recouvrement (coûts administratifs/recouvrement net de recettes) (2005, 2008 et 2011)

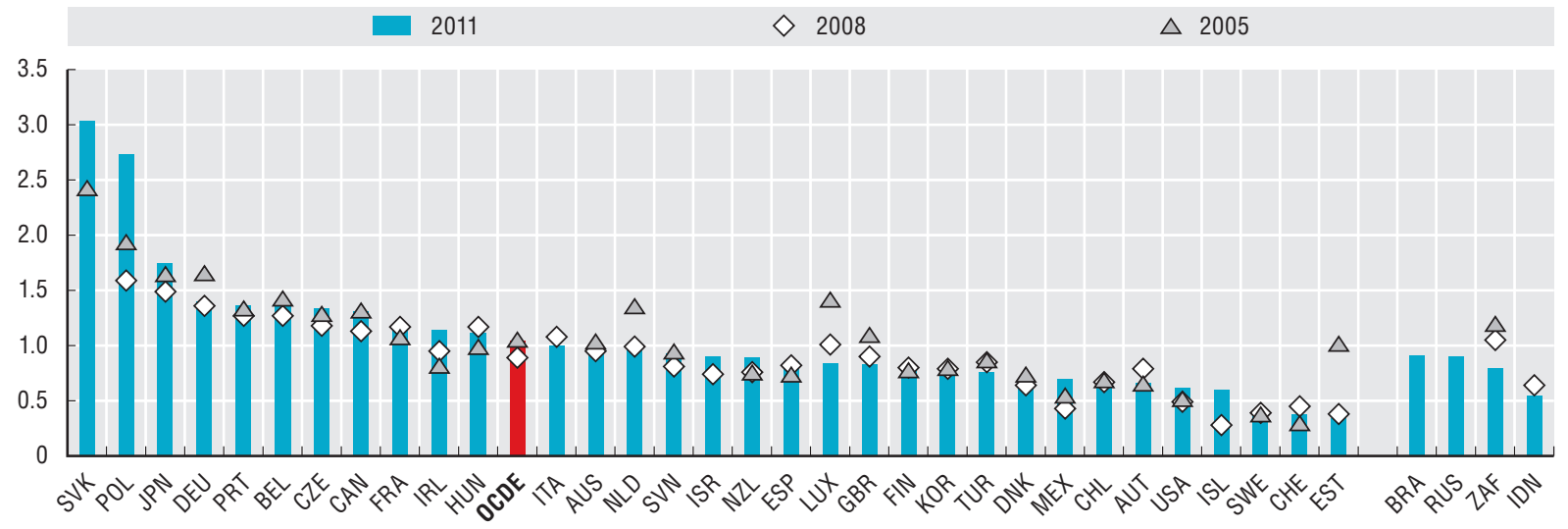

Source: OCDE (2013), Tax Administration 2013: Comparative Information on OECD and other Advanced and Emerging Economies, Éditions OCDE, Paris, http://dx.doi.org/10.1787/9789264200814-en. 
Alors que le concept d'efficience mesure la performance afin d'établir si les ressources investies permettent d'obtenir les produits souhaités de manière productive, le concept d'efficacité évalue dans quelle mesure une activité atteint les objectifs escomptés. Le rapport coût-efficacité, c'est-à-dire le rapport entre un intrant et un produit intermédiaire ou final, reflète le lien entre les ressources engagées et les résultats obtenus, et il est essentiel pour évaluer la réussite des politiques publiques.

L'évaluation de la performance des pouvoirs publics est particulièrement cruciale dans des secteurs comme l'éducation et la santé, qui sont essentiels au bien-être des citoyens et au développement économique et social des pays. Dans ces deux secteurs, l'évaluation des intrants et des réalisations est en outre suffisamment développée et normalisée à l'échelle internationale pour qu'il soit possible de comparer leur efficacité de façon pertinente.

\section{L'éducation}

Dans le secteur de l'éducation, le développement du capital humain et des compétences sont deux objectifs majeurs du secteur public, en raison de leurs effets bénéfiques sur les perspectives d'emploi et les revenus d'activité sur l'ensemble de la vie. De plus, une main d'œuvre mieux formée contribue à une meilleure croissance économique et, partant, à une société plus prospère. Ces effets influent également sur les ressources dont disposent les pouvoirs publics : des individus plus aptes à l'emploi et mieux formés sont susceptibles d'élargir l'assiette fiscale grâce à des contributions plus importantes, tout en pesant moins sur les dépenses au titre de l'assistance sociale.

L'un des principaux indicateurs permettant d'évaluer les réalisations obtenues dans le secteur de l'éducation est la Valeur actuelle nette (VAN) publique de l'éducation. La VAN mesure les retombées économiques des investissements publics dans ce secteur, après prise en compte de leurs coûts. Une scolarisation plus longue bénéficie à l'État par le biais des recettes fiscales supplémentaires permises par des rémunérations plus élevées et par de nouvelles arrivées sur le marché du travail. En moyenne, les pays membres de l'OCDE atteignent une VAN pour l'enseignement supérieur qui dépasse 100000 USD. Compte tenu du volume de l'investissement public, qui s'établit en moyenne à un tiers de la VAN, il est évident que les États ont tout intérêt à investir dans l'éducation. Les pays membres de l'OCDE où la VAN publique de l'investissement dans l'enseignement supérieur est la plus élevée sont les États-Unis, la Hongrie et l'Irlande ; en moyenne, les investissements dans le deuxième cycle du secondaire engendrent des gains plus faibles - en Estonie, les coûts dépassent même les gains.

\section{La santé}

De même, les États ont tout intérêt à évaluer le rapport coûtefficacité de leur système de santé, non seulement en raison de l'importance considérable des réalisations obtenues, mais aussi parce que les dépenses de ce secteur sont l'une des principales composantes des dépenses publiques, et qu'elles sont constamment poussées à la hausse par les évolutions technologiques et le vieillissement démographique.

Pour évaluer ce rapport coût-efficacité, les progrès de l'espérance de vie (la réalisation la plus facilement comparable et la plus largement adoptée) sont comparés aux dépenses totales de santé par habitant au sein des pays. Il faut cependant être prudent au moment de tirer des conclusions, car de nombreux facteurs ont une forte incidence sur l'espérance de vie; en outre, les dépenses totales de santé englobent les dépenses publiques et privées (la part des dépenses privées est particulièrement élevée dans des pays comme les États-Unis et le Mexique). Les résultats montrent qu'il existe un lien positif entre les dépenses totales de santé par habitant et l'espérance de vie, ce qui laisse penser que des dépenses de santé plus élevées permettent un meilleur état de santé des individus. L'Espagne, l'Italie et le Japon affichent une espérance de vie relativement élevée au regard de leurs dépenses de santé. À l'inverse, l'espérance de vie aux États-Unis, en Hongrie et au Mexique est relativement faible par rapport aux dépenses engagées. Des résultats similaires (voir le graphique en ligne) ont démontré que le lien positif global avec l'espérance de vie n'était pas affecté lorsque les dépenses publiques étaient seules prises en compte. Néanmoins, la faiblesse relative de l'espérance de vie aux États-Unis et au Mexique par rapport à la moyenne OCDE est légèrement moins marquée lorsque l'on prend uniquement en compte les dépenses publiques de santé.

\section{Méthodologie et définitions}

Le rendement économique public de l'éducation pour les personnes de sexe masculin est mesuré par la valeur actuelle nette (VAN). Les coûts publics incluent les recettes d'impôt sur le revenu perdues pendant les années de scolarisation. Les dépenses publiques sont liées au niveau de formation atteint, en tenant compte de la durée des études, et comprennent les dépenses directes et les transferts public-privé. Les avantages, pour le secteur public, sont les suivants : des individus aux revenus plus élevés permettent d'engranger plus de recettes au titre de l'impôt sur le revenu et plus de contributions sociales et d'économiser sur les prestations dues en deçà d'un certain seuil de revenus. Les valeurs des données utilisées pour calculer la VAN du deuxième cycle de l'enseignement secondaire reposent sur la différence entre les individus titulaires d'un diplôme du deuxième cycle du secondaire ou de l'enseignement post-secondaire non supérieur et ceux qui ne le sont pas. Les valeurs des données utilisées pour calculer la VAN de l'enseignement supérieur reposent sur la différence entre les individus titulaires d'un diplôme de l'enseignement supérieur et ceux titulaires d'un diplôme du deuxième cycle du secondaire.

L'espérance de vie mesure la durée moyenne pendant laquelle des personnes devraient vivre, compte tenu d'un ensemble donné de taux de mortalité liés à l'âge. L'espérance de vie à la naissance pour l'ensemble de la population est calculée en tant que moyenne non pondérée de l'espérance de vie des hommes et des femmes. Les dépenses totales de santé mesurent la consommation finale de biens et services de santé (c'està-dire les dépenses courantes), plus les investissements dans les infrastructures de santé. Elles incluent les dépenses des acteurs tant publics que privés consacrées aux biens et services médicaux, aux programmes de santé publique et de prévention et à l'administration de la santé. Le graphique 2.27, Espérance de vie à la naissance et dépenses publiques de santé par habitant (2011), est disponible en ligne à l'adresse http://dx.doi.org/10.1787/ 888932941291. 


\section{Pour en savoir plus}

OCDE (2013a), Regards sur l'éducation 2013 : Les indicateurs de l'OCDE, Éditions OCDE, Paris, http://dx.doi.org/10.1787/eag-2013-fr.

OCDE (2013b), Panorama de la santé 2013 : Les indicateurs de l'OCDE, Éditions OCDE, Paris, http://dx.doi.org/10.1787/health_glance2013-fr.

\section{Notes relatives aux graphiques}

2.25 : Les données pour le deuxième cycle de l'enseignement secondaire en Belgique et aux Pays-Bas ne sont pas comprises, car ces niveaux sont obligatoires. Les données pour le deuxième cycle du secondaire au Japon ne sont pas présentées, car les premier et deuxième cycles de l'enseignement secondaire ne sont pas séparés. Les données pour
l'Italie, les Pays-Bas et la Pologne sont celles de 2008. Les données pour le Japon sont celles de 2007. Les données pour la Turquie sont celles de 2005. Voir l'annexe 3 pour les notes (www.oecd.org/edu/eag.htm).

2.26: Les données relatives aux dépenses pour la Belgique et la Nouvelle-Zélande excluent les investissements. Celles pour les PaysBas renvoient aux dépenses courantes. Celles pour la Belgique, le Mexique et la Nouvelle-Zélande utilisent une méthodologie différente. Celles pour le Chili, Israël et le Mexique sont des estimations. Les données sur l'espérance de vie pour l'Australie, la Belgique, le Chili, les États-Unis, la France et l'Italie sont des estimations. Les données sur les dépenses pour l'Australie, Israël, le Japon et le Luxembourg sont celles de 2009. Les données sur les dépenses pour la Turquie sont celles de 2008. Les données sur l'espérance de vie pour le Canada sont celles de 2009.

Informations sur les données concernant Israël : http://dx.doi.org/10.1787/ 888932315602 


\section{LA GOUVERNANCE STRATÉGIQUE}

\section{Le rapport coût-efficacité du secteur public}

2.25. Valeur actuelle nette publique de l'obtention par une personne de sexe masculin, dans le cadre de sa formation initiale, d'un diplôme du deuxième cycle de l'enseignement secondaire ou de l'enseignement postsecondaire non supérieur et d'un diplôme de l'enseignement supérieur (2009 ou dernière année disponible)

En équivalents USD convertis sur la base des PPA pour le PIB

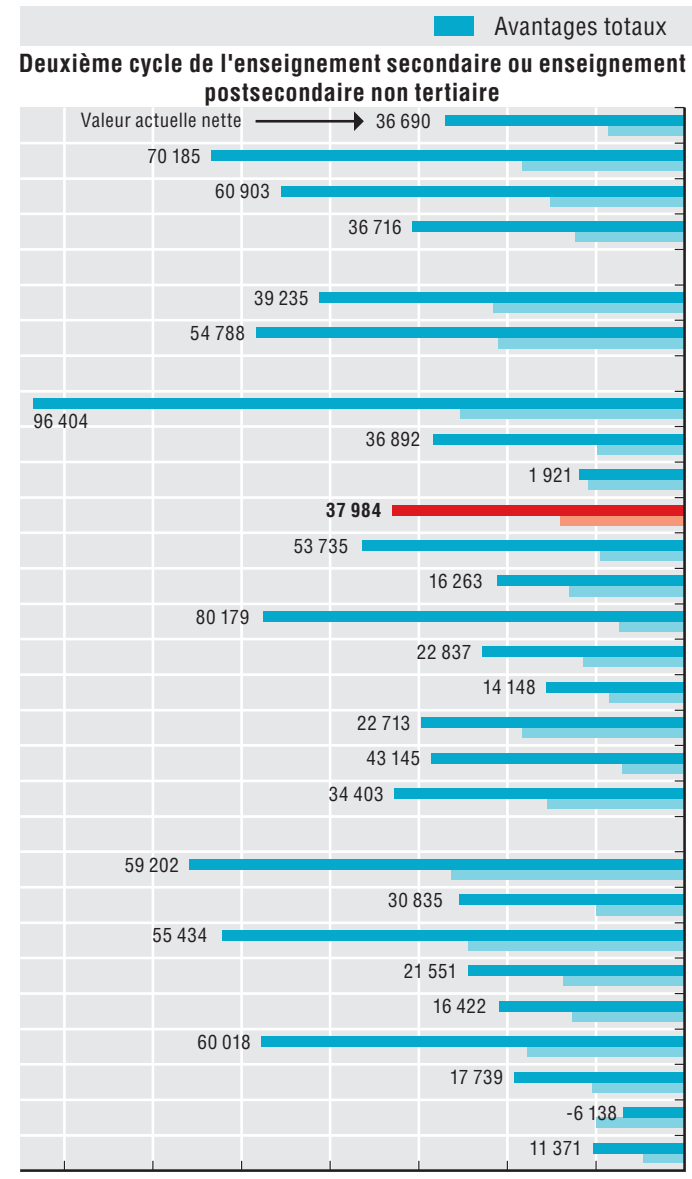

$1400001200001000008000060000 \quad 40000 \quad 20000 \quad 0$ USD PPA

Hongrie
États-Unis
Irlande
Slovénie
Belgique
Italie
Allemagne
Pays-Bas
Autriche
République tchèque
Pologne
OCDE
Australie
Finlande
Royaume-Uni
Portugal
Israël
France
République slovaque
Canada
Japon
Norvège
Grèce
Danemark
Corée
Nouvelle-Zélande
Suède
Espagne
Estonie
Turquie

Source : OCDE (2013), Regards sur l'éducation 2013 : Les indicateurs de l'OCDE, Éditions OCDE, Paris, tableau A7.2a et tableau A7.4a, http:// dx.doi.org/10.1787/eag-2013-fr. 


\section{LA GOUVERNANCE STRATÉGIQUE}

\section{Le rapport coût-efficacité du secteur public}

2.26. Espérance de vie à la naissance et dépenses totales de santé par habitant (2011)

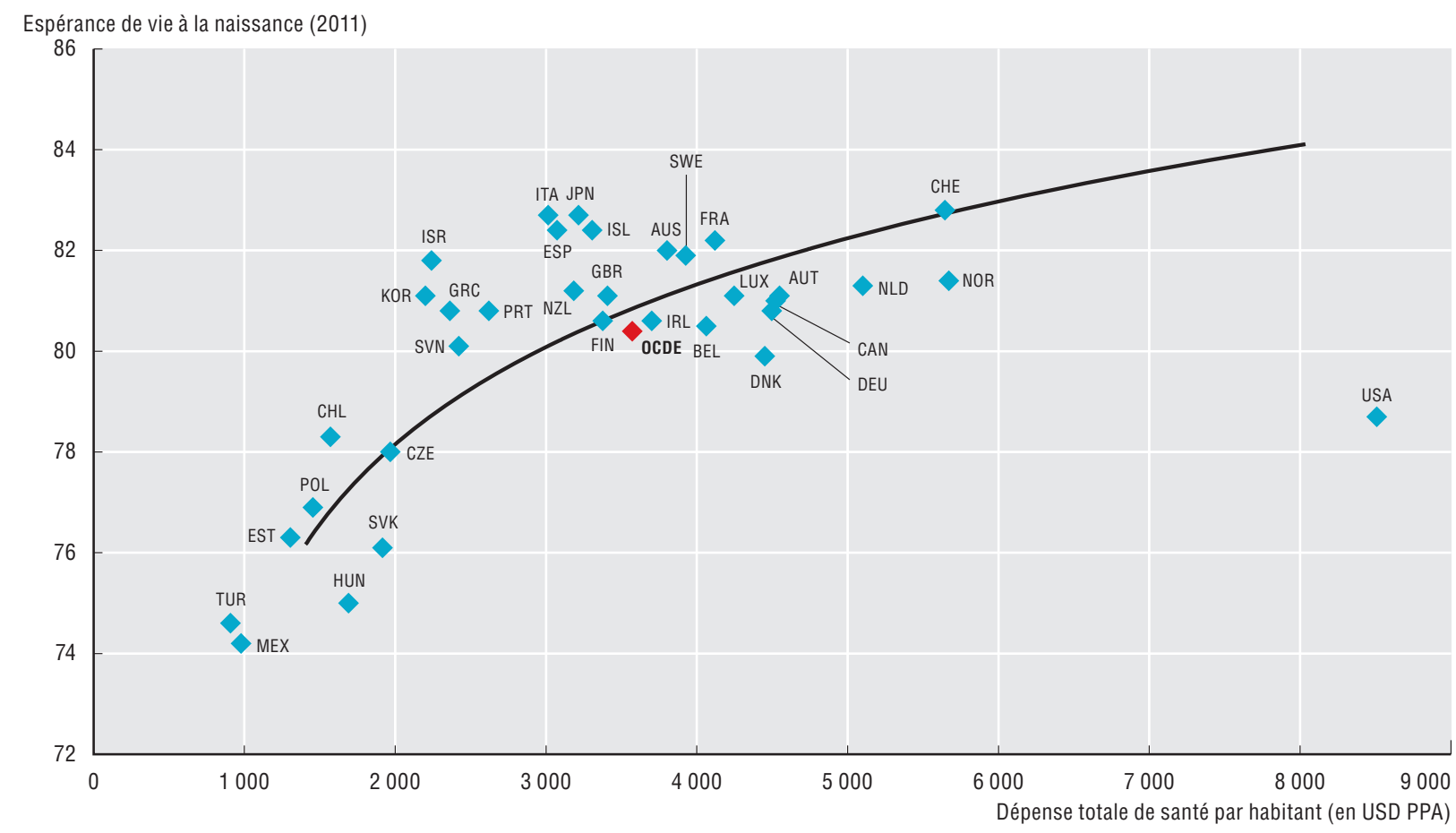

Source: Statistiques de l'OCDE sur la santé 2013.

StatLink नाडs http://dx.doi.org/10.1787/888933158845 


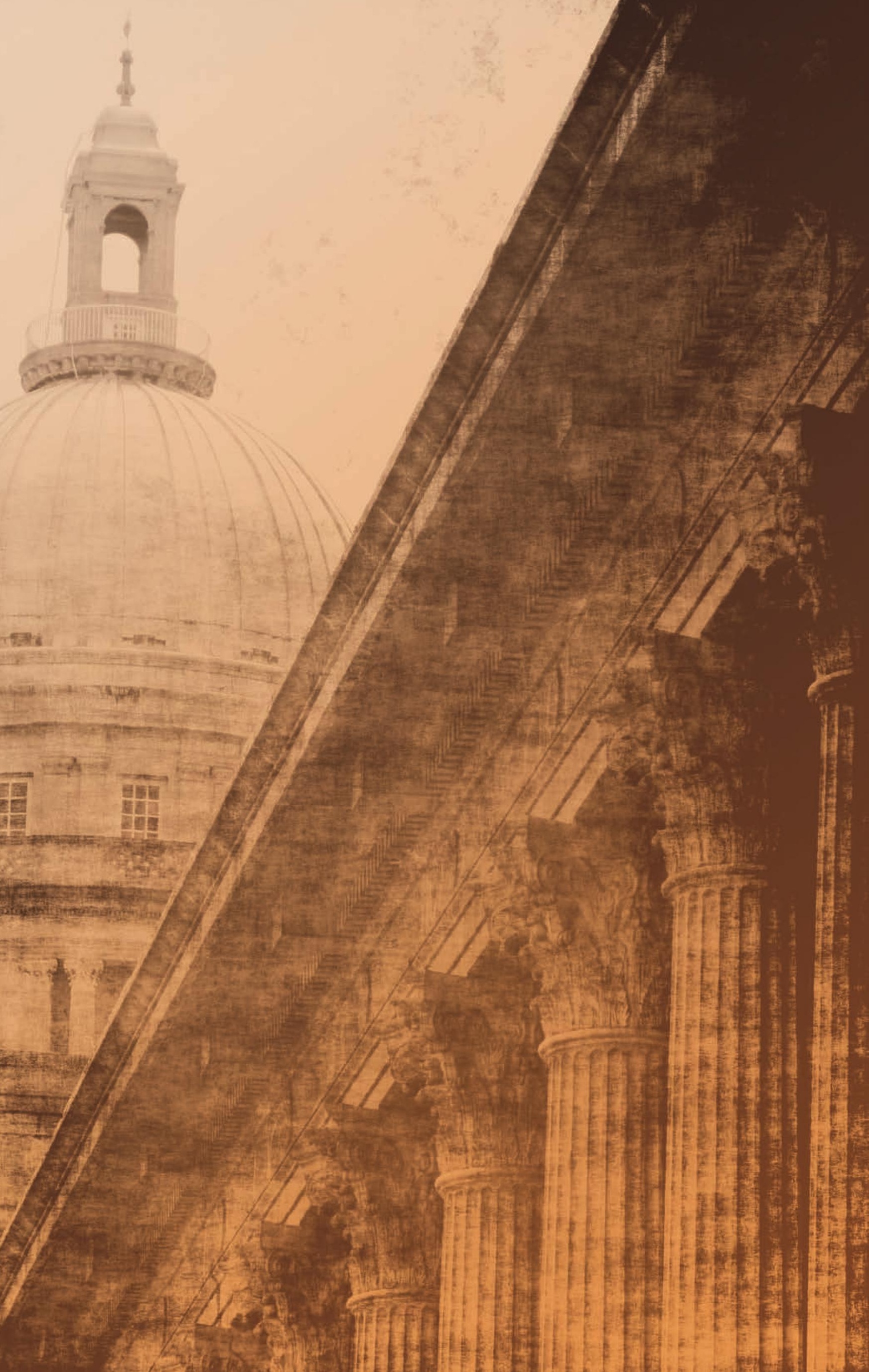



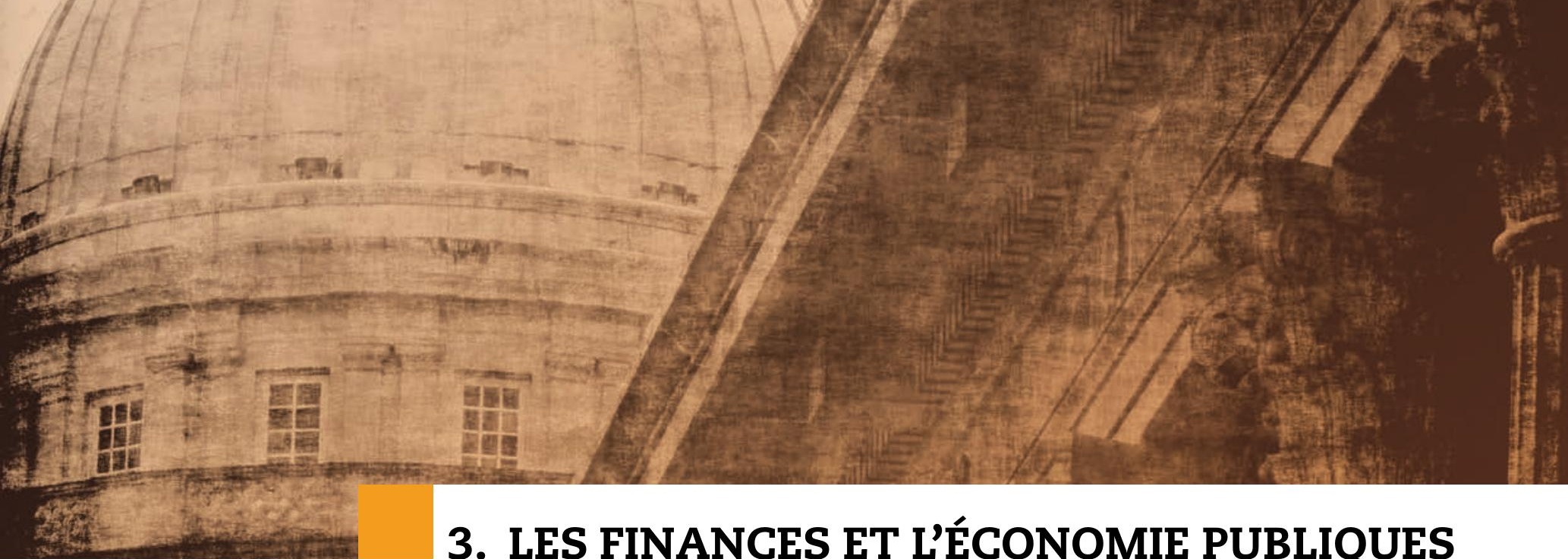

\section{LES FINANCES ET L'ÉCONOMIE PUBLIQUES}

Les pouvoirs publics ont deux responsabilités principales: fournir des biens et des services (par exemple en matière d'éducation et de santé) et assurer un rôle redistributif (par exemple au moyen de prestations sociales et de subventions). Parmi les nombreuses autres tâches qui leur incombent, ils doivent aussi gérer les risques, veiller à l'équité sociale, lutter contre la corruption et protéger l'environnement. Pour financer ces activités, ils mobilisent des ressources en levant des recettes (au moyen de l'impôt, par exemple) ou en recourant à l'emprunt.

Les finances publiques de la plupart des pays membres de l'OCDE se sont beaucoup dégradées à la suite de la crise financière et économique mondiale. Toutefois, dans la plupart des pays de l'OCDE, les soldes budgétaires s'améliorent depuis 2010 du fait des règles et nouvelles pratiques budgétaires et des plans d'assainissement mis en œuvre en réaction à la crise. Ces améliorations montrent que les gouvernements sont sur la bonne voie, mais les niveaux d'endettement public continuent à s'élever en raison de la faiblesse de la reprise de l'activité. La plupart des pays de l'OCDE sont donc encore confrontés au problème complexe qui consiste à trouver un équilibre entre le redressement budgétaire et la nécessité urgente de stimuler la croissance.

Ce chapitre décrit et analyse les écarts qui existent entre les pays membres au niveau d'indicateurs essentiels des finances et de l'économie publiques, éclairant ainsi comment les pouvoirs publics réagissent face aux tensions budgétaires. Il présente des données sur les déficits ou les excédents des administrations publiques et sur leur endettement. Il évalue les tendances observées en ce qui concerne le niveau et la composition des recettes et des dépenses publiques, les coûts de production des biens et services publics, le rôle des pouvoirs publics dans leur mise à disposition et l'ampleur des investissements publics. Compte tenu de l'importance des technologies de l'information et de la communication (TIC) pour l'innovation et la productivité des administrations publiques, ce chapitre présente aussi des données préliminaires relatives à leurs dépenses en ce domaine. Pour faire ressortir les tendances à plus long terme et l'incidence de la crise économique, il présente la plupart des indicateurs pour 2001 (année de base), pour 2009 (année de milieu de crise) et pour la dernière année pour laquelle des données sont disponibles (2011, le plus souvent). 
Le solde budgétaire des administrations publiques est la différence entre leurs recettes et leurs dépenses. Il y a déficit quand, pendant un exercice donné, les administrations publiques dépensent plus qu'elles n'encaissent de recettes. En revanche, le solde budgétaire est excédentaire lorsque les recettes sont supérieures aux dépenses. Une succession de lourds déficits est très préjudiciable à la viabilité des finances publiques, car ces déficits sont financés par un endettement supplémentaire. Lorsque l'encours de la dette est élevé, le coût de son service (charges d'intérêts et hausse des taux) accroît encore le déficit, ce qui fait obstacle à la viabilité budgétaire. Les gouvernements peuvent réduire le coût futur du service de la dette en améliorant le solde primaire, qui correspond au solde budgétaire hors les intérêts versés.

En 2011, les pays membres de l'OCDE enregistraient un déficit budgétaire représentant $3.5 \%$ de leur PIB. Les déficits les plus élevés étaient atteints en Irlande (13.3\%), aux États-Unis (10.1\%), en Grèce (9.6 \%), en Espagne (9.4 \%) et au Japon (8.9 \%). Seuls six pays membres réalisaient un excédent budgétaire : la Norvège (13.4\%), la Hongrie (4.2\%), la Corée (2\%), l'Estonie $(1.2 \%)$, la Suisse $(0.5 \%)$ et la Suède $(0.03 \%)$.

De 2001 à 2009, tous les pays de l'OCDE, sauf la Suisse et l'Allemagne, ont vu augmenter leur déficit, passé en moyenne de $0.7 \%$ à $5.5 \%$ du PIB. L'essentiel de la dégradation est intervenu en 2008 et 2009, sous l'effet de la crise financière et économique mondiale, qui a fait progresser les dépenses des administrations publiques plus vite que leurs recettes et que le PIB (en valeur nominale et dans tous les pays à l'exception d'Israël et de la Hongrie). La tendance s'est inversée après 2009, surtout du fait des règles et nouvelles pratiques budgétaires et des plans de redressement mis en œuvre en réaction à la crise. De 2009 à 2011, le taux de croissance des dépenses (en valeur nominale) a été inférieur à celui des recettes et du PIB dans la totalité des pays membres, à quatre exceptions près (le Japon, la Nouvelle-Zélande, la Slovénie et la Suisse). Par conséquent, le solde budgétaire exprimé en pourcentage du PIB s'est amélioré de deux points de pourcentage, en moyenne. Les contractions du déficit les plus marquées sont intervenues en Hongrie (contraction de 8.7 points de pourcentage et passage à un excédent de $4.2 \%$ ), en Grèce (6 points) et au Portugal (5.8 points). La nette amélioration du solde budgétaire hongrois est due à des transferts en capital des ménages vers les administrations publiques effectués en 2011 (pour un montant de 9.7 \% du PIB) au moyen de ponctions sur les caisses de retraite privées. Dans trois pays seulement, le solde budgétaire ne s'est pas amélioré entre 2009 et 2011 : le Japon, la Slovénie et la Suisse.

En 2011, le solde budgétaire primaire des pays membres de l'OCDE était déficitaire, en moyenne, à hauteur de $0.8 \%$ du PIB. Le service des intérêts de la dette représentait le reste du solde budgétaire total. Le solde primaire différait fortement selon les pays membres de l'OCDE : dans 19 d'entre eux, il était déficitaire, pour un montant allant de $10 \%$ du PIB en Irlande à $0.1 \%$ au Danemark; dans 14 pays, en revanche, il était excédentaire, pour un montant allant de $0.2 \%$ du PIB en Autriche à $14.5 \%$ en Norvège.

L'importance des charges d'intérêts au sein du solde budgétaire était aussi très variable selon les pays. Dans les 19 pays accusant un déficit primaire, ces charges représentaient en moyenne $2.9 \%$ du PIB, avec une fourchette allant de $1.4 \%$ en République tchèque à $7.2 \%$ en Grèce. À $2.4 \%$ du PIB, le déficit primaire de la Grèce n'était pas le plus élevé, mais les charges d'intérêts y étaient les plus lourdes parmi les pays membres de l'OCDE.

\section{Méthodologie et définitions}

Les données sur le solde budgétaire des administrations publiques sont tirées de la base de données des Statistiques de l'OCDE sur les comptes nationaux, qui sont établies conformément au Système de comptabilité nationale (SCN) ; ce dernier constitue un ensemble de concepts, de définitions, de nomenclatures et de règles de comptabilisation approuvés au plan international en matière de comptabilité nationale. Selon la terminologie du SCN, les administrations publiques se composent de l'administration centrale, des administrations d'États fédérés, des administrations locales et des administrations de sécurité sociale. On calcule le solde budgétaire des administrations publiques, également présenté comme leur capacité de financement (+) ou leur besoin de financement $(-)$, en soustrayant les dépenses totales des administrations publiques de leurs recettes totales. Les recettes englobent les cotisations sociales, les impôts hors cotisations sociales, les aides et d'autres recettes. Les dépenses comprennent la consommation intermédiaire, la rémunération des personnels, les subventions, les prestations sociales, les autres dépenses courantes (dont les intérêts de la dette), les transferts en capital et les autres dépenses en capital. Le solde primaire correspond au solde budgétaire hors les intérêts versés au titre des engagements des administrations publiques.

Le produit intérieur brut (PIB) est la mesure habituelle de la valeur des biens et services produits par un pays au cours d'une période donnée.

\section{Pour en savoir plus}

OCDE (2013a), Panorama des comptes nationaux 2013, Éditions OCDE, Paris, http://dx.doi.org/10.1787/na_glance-2013-fr.

OCDE (2013b), Perspectives économiques de l'OCDE, vol. 2013/1, Éditions OCDE, Paris, http://dx.doi.org/10.1787/eco_outlookv2013-1-fr.

\section{Notes relatives aux graphiques}

Les données relatives au Canada, à la Fédération de Russie et à la Nouvelle-Zélande portent sur 2010 et non 2011.

3.1 : On ne dispose pas de données relatives au Chili et à la Turquie pour 2001, et ces pays ne sont pas compris dans la moyenne OCDE. Les données relatives au Chili portent sur 2010 et non 2011 . Les données relatives au Japon et au Mexique pour 2001 sont des estimations. Les données relatives à la Fédération de Russie portent sur 2002 et non 2001.

3.2 : On ne dispose pas de données pour le Chili.

Informations sur les données concernant Israël : http://dx.doi.org/10.1787/ 888932315602 . 
3.1. Solde budgétaire des administrations publiques en pourcentage du PIB (2001, 2009 et 2011)

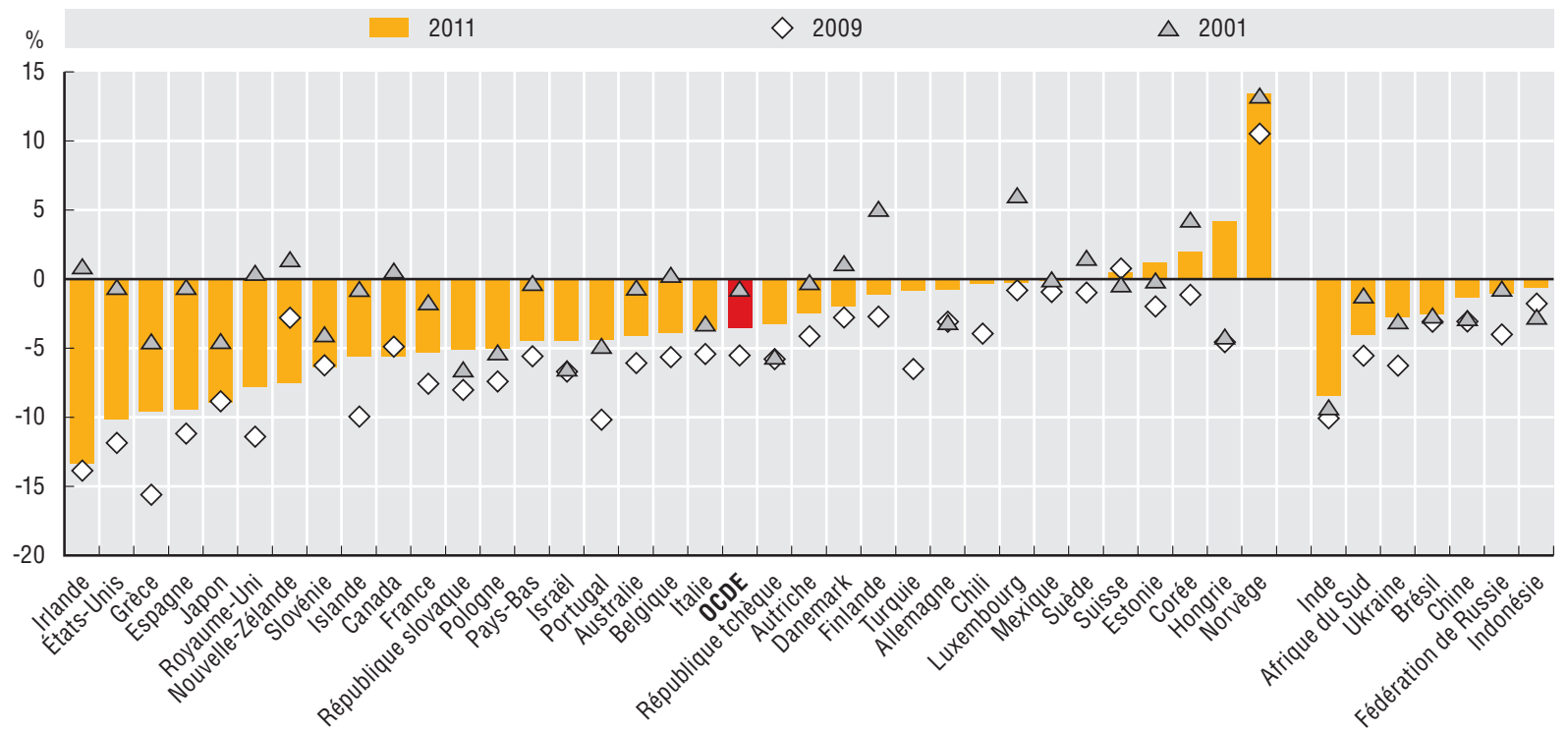

Source: Données sur les pays membres de l'OCDE : Statistiques de l'OCDE sur les comptes nationaux (base de données). Données sur les autres grandes économies (à l'exclusion de la Fédération de Russie) : Fonds monétaire international (2013), Perspectives de l'économie mondiale, avril 2013, FMI, Washington, DC.

3.2. Solde primaire des administrations publiques et charges d'intérêts en pourcentage du PIB (2011)

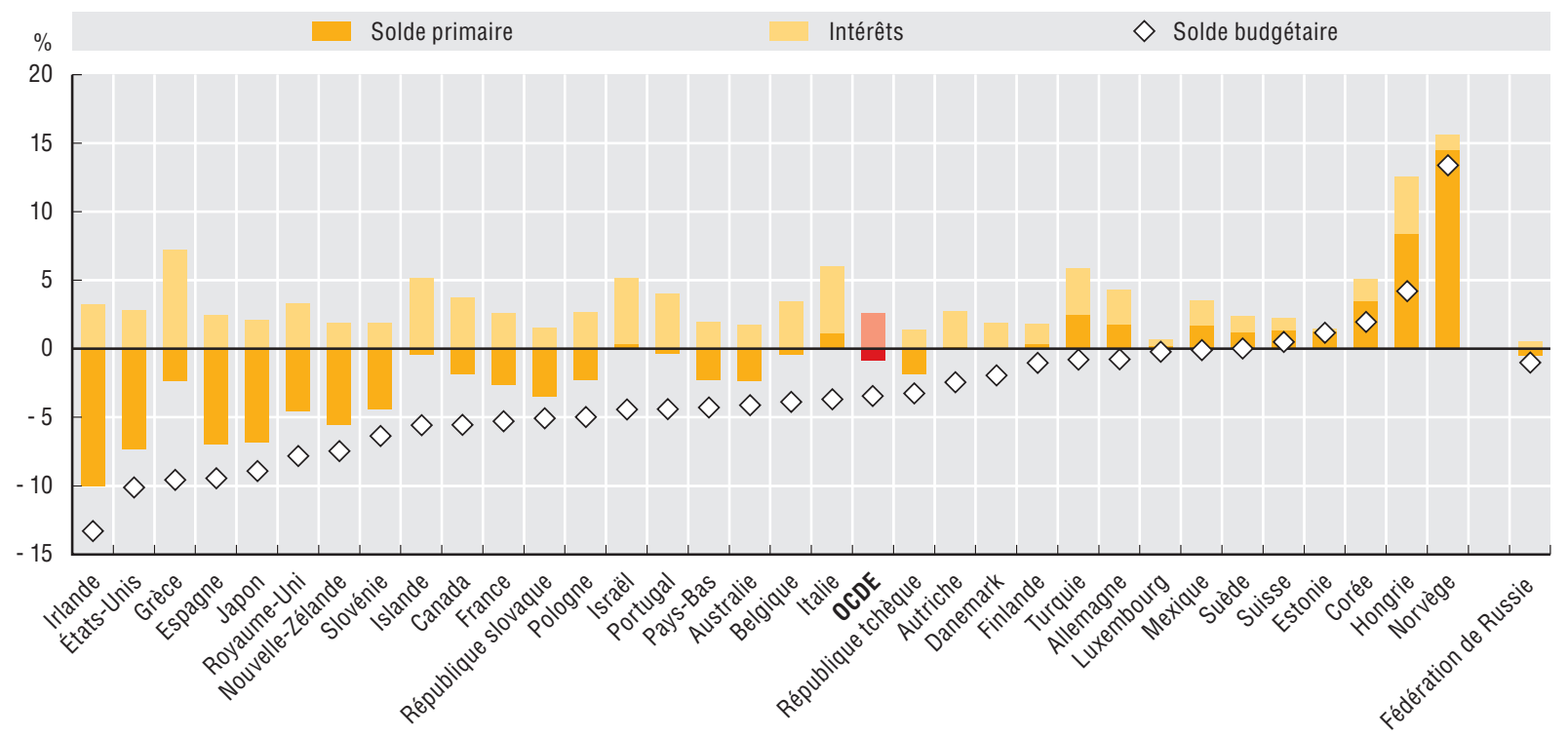

Source: Statistiques de l'OCDE sur les comptes nationaux (base de données). 
Lorsque leurs dépenses sont supérieures à leurs recettes, les administrations publiques ont besoin de ressources supplémentaires pour financer leur déficit, de sorte qu'elles empruntent et accroissent l'endettement public. L'évolution de la dette résulte des soldes budgétaires antérieurs ; la répétition de déficits la fait augmenter. À l'inverse, une succession d'excédents la réduit. Généralement, plus les engagements des administrations publiques sont élevés, plus les marchés envisagent la possibilité d'une défaillance sur leurs emprunts, et plus la prime de risque qu'ils exigent s'élève, ce qui alourdit le coût de la dette.

En 2011, la dette des administrations publiques représentait en moyenne $78.8 \%$ du PIB des pays membres de l'OCDE; ce pourcentage allait de $10 \%$ en Estonie à $228 \%$ au Japon. Dans la majorité des pays de l'OCDE, la dette était plus élevée en 2011 qu'en 2001. Toutefois, ce résultat s'explique par la conjonction de deux évolutions de sens contraire, puisque les ratios dette/PIB ont baissé jusqu'en 2007, surtout grâce à la croissance économique. Depuis lors, la dette augmente continuellement, principalement à cause de la crise financière mondiale et, plus précisément, de la baisse des recettes publiques, du recul de l'activité économique et/ou d'un surcroît de dépenses lié aux plans de relance et aux interventions destinées à aider les institutions financières. Les hausses de la dette les plus marquées ont eu lieu au Japon (76.6 points de pourcentage), en Irlande (67 points), au Royaume-Uni (55.1 points) et aux États-Unis (48.1 points).

Le poids de la dette par habitant varie considérablement, puisqu'il va de 2207 USD en Estonie à 77134 USD au Japon. Le chiffre moyen s'établit à 26774 USD dans les pays membres de l'OCDE. $\mathrm{Au}$ Japon, le montant est élevé mais, comme la plus grande partie de la dette publique est détenue par des ressortissants japonais, le risque de défaillance est jugé plus faible (d'où une moindre nécessité d'acquitter des primes de risque).

Sauf en Australie et en Estonie, les titres autres que les actions sont l'instrument de financement que privilégient les pays membres de l'OCDE. Quand ces titres occupent une grande place dans la structure de la dette, celle-ci ressent fortement les fluctuations du marché, ce qui influe sur son coût.

\section{Méthodologie et définitions}

Les données sont tirées de la base de données des Statistiques de l'OCDE sur les comptes nationaux, qui sont établies conformément au Système de comptabilité nationale (SCN) ; ce dernier constitue un ensemble de concepts, de définitions, de nomenclatures et de règles de comptabilisation approuvés au plan international en matière de comptabilité nationale. La dette est un concept communément utilisé, défini comme une souscatégorie spécifique de passifs distinguée en fonction des catégories d'instruments financiers comprises ou non comprises. En général, on définit la dette comme l'ensemble des passifs qui font obligation à un débiteur de verser à un créancier des intérêts ou du capital à une ou plusieurs dates futures. Tous les instruments de la dette sont donc des passifs, mais certains passifs, tels que les actions, les participations et les produits financiers dérivés, ne sont pas des dettes.

On calcule donc la dette en additionnant les catégories de passif suivantes (selon le Système de comptabilité nationale de 1993), chaque fois qu'elles sont disponibles ou applicables dans le bilan financier du secteur institutionnel : numéraire et dépôts ; titres autres que les actions, hors produits financiers dérivés ; crédits ; réserves techniques des entreprises d'assurance ; et autres comptes à payer. Avec le SCN, la plupart des instruments de la dette sont évalués aux prix du marché (mais certains pays peuvent ne pas appliquer ce mode d'évaluation, en particulier aux titres autres que les actions, hors produits financiers dérivés).

Ces données ne sont pas toujours comparables internationalement, à cause de différences de définition ou de traitement des composantes de la dette. En particulier, ces données incluent les systèmes publics de retraite non capitalisés pour certains pays de l'OCDE (par exemple l'Australie et le Canada) ainsi que dans le cas des pays pour lesquels les données sont tirées des Perspectives de l'économie mondiale du FMI. L'endettement de ces pays est donc surestimé relativement à celui de pays dotés de lourds engagements non financés au titre des retraites ne figurant pas dans les comptes principaux établis selon le SCN 1993.

La définition de la dette employée dans le SCN diffère de celle appliquée dans le cadre du traité de Maastricht, qui sert à évaluer les positions budgétaires dans l'Union européenne. Le graphique 3.7, « Dette des administrations publiques au sens de Maastricht, par détenteur de la dette (2011) ", est consultable en ligne à l'adresse http:// dx.doi.org/10.1787/888932941424.

On a calculé la dette des administrations publiques par habitant en la convertissant en USD de 2011 avec les parités de pouvoir d'achat (PPA) OCDE/Eurostat pour le PIB et en divisant le résultat obtenu par la population. Dans le cas des pays pour lesquels les données sont tirées des Perspectives de l'économie mondiale du FMI, on a utilisé un taux de change implicite basé sur les PPA. La PPA (parité de pouvoir d'achat) est le nombre d'unités monétaires du pays $B$ nécessaire pour acquérir une même quantité de biens et de services dans le pays A. Le graphique 3.6, intitulé « Taux annuel de progression de la dette publique par habitant (en termes réels, 2001-11) ", est consultable en ligne à l'adresse $h t t p: / / d x . d o i . o r g /$ $10.1787 / 888932941405$.

\section{Pour en savoir plus}

OCDE (2013), Panorama des statistiques de l'OCDE 2013 : Économie, environnement et société, Éditions OCDE, Paris, http:// dx.doi.org/10.1787/factbook-2013-fr.

\section{Notes relatives aux graphiques}

On ne dispose pas de données pour l'Islande, la Nouvelle-Zélande et la Turquie. Les données relatives au Chili, à la Corée, au Japon et au Royaume-Uni sont fournies sur une base non consolidée. Les données relatives à la Suisse portent sur 2010 et non 2011.

3.3 : On ne dispose pas de données relatives au Chili et au Luxembourg pour 2001, ni de données relatives au Mexique pour 2011, et ces pays ne sont pas compris dans la moyenne OCDE. Les données relatives à la Corée portent sur 2002 et non 2001. Les données relatives au Danemark portent sur 2003 et non 2001.

3.4 et 3.5 : On ne dispose pas de données pour le Mexique.

Informations sur les données concernant Israël : http://dx.doi.org/10.1787/ 888932315602 . 
3.3. Dette des administrations publiques en pourcentage du PIB (2001, 2009 et 2011)

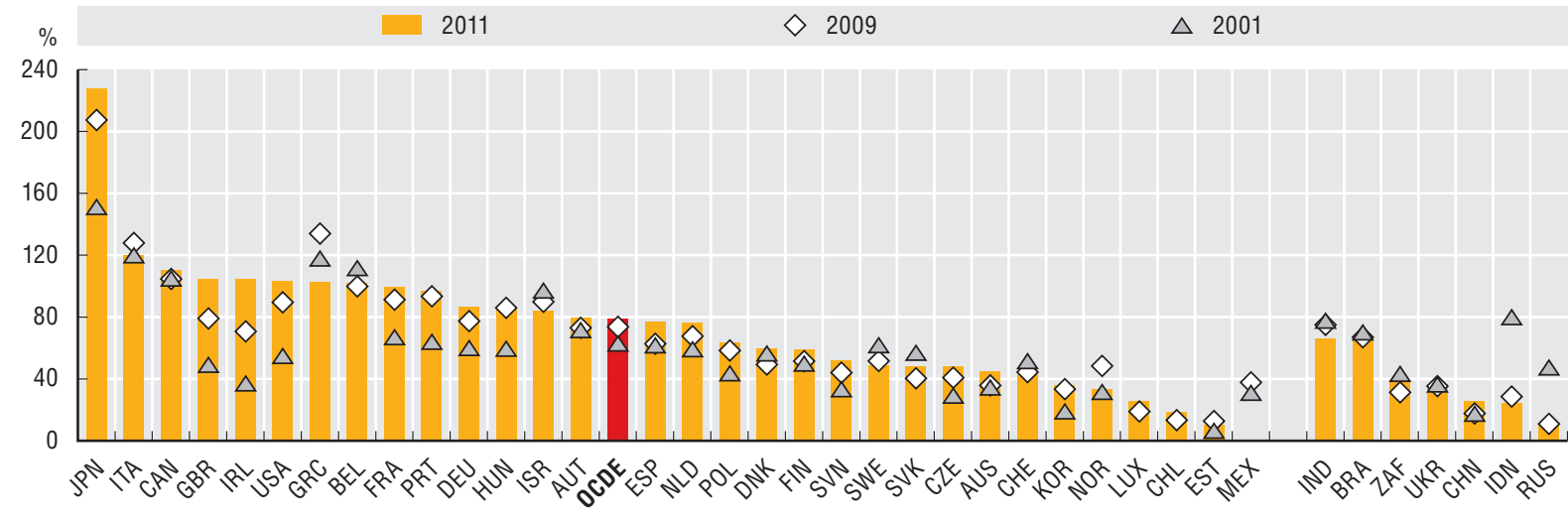

Source : Données sur les pays membres de l'OCDE : Statistiques de l'OCDE sur les comptes nationaux (base de données). Données sur les autres grandes économies (à l'exclusion de la Fédération de Russie) : Fonds monétaire international (2013), Perspectives de l'économie mondiale, avril 2013, FMI, Washington, DC.

\subsection{Dette des administrations publiques par habitant (2011)}

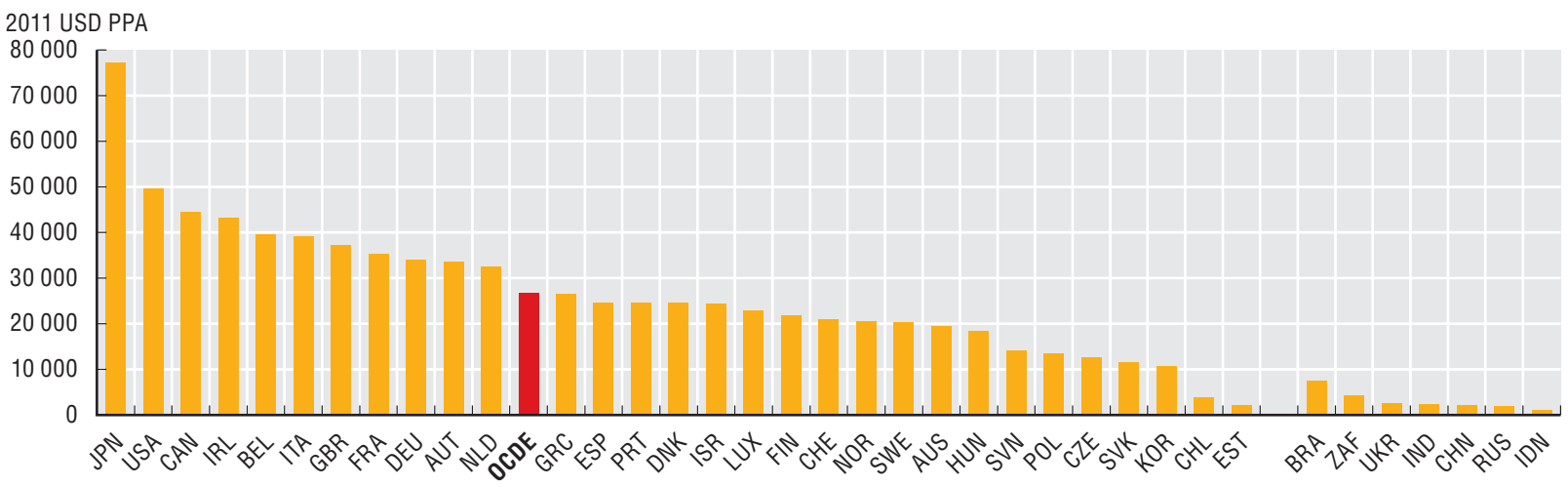

Source: Données sur les pays membres de l'OCDE : Statistiques de l'OCDE sur les comptes nationaux (base de données). Données sur les autres grandes économies (à l'exclusion de la Fédération de Russie) : Fonds monétaire international (2013), Perspectives de l'économie mondiale, avril 2013, FMI, Washington, DC.

\subsection{Structure de la dette publique par instruments financiers (2011)}

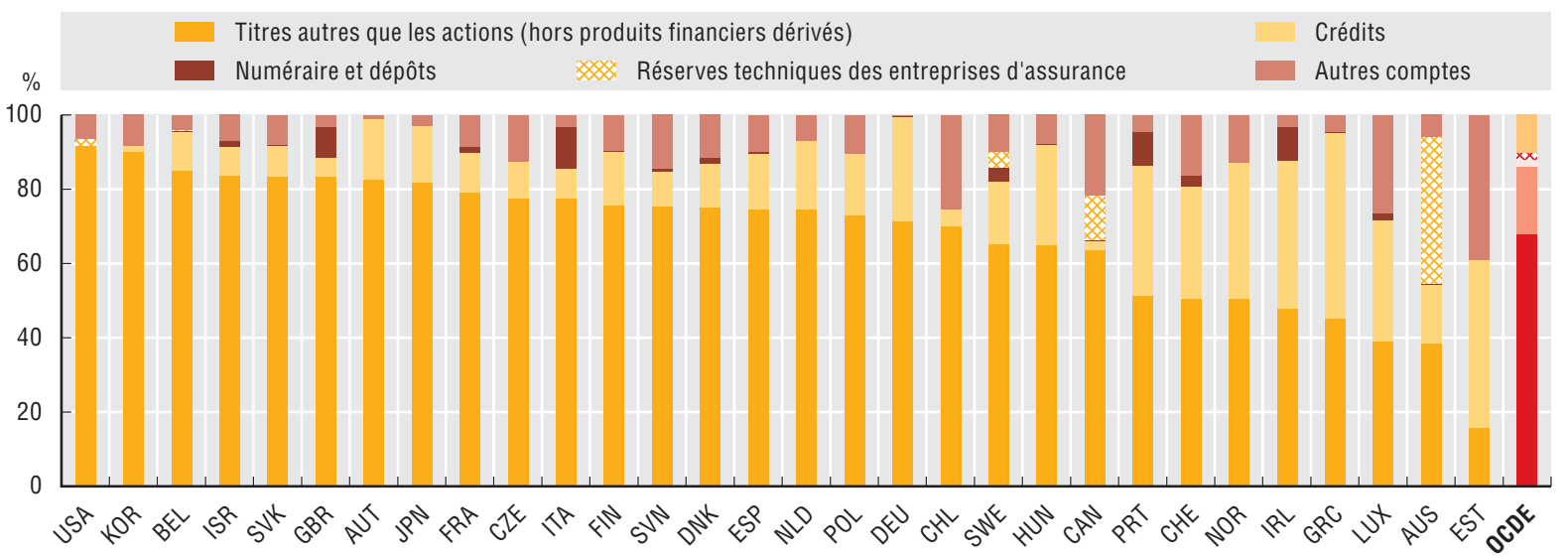

Source: Statistiques de l'OCDE sur les comptes nationaux (base de données). 
Les administrations centrales partagent à des degrés divers leur souveraineté avec les administrations infranationales. Ces dernières peuvent donc connaître des situations budgétaires diverses. Les différents systèmes politiques se caractérisent par des degrés variables d'autonomie des administrations d'États fédérés et des administrations locales en matière de souscription de dettes ; les administrations infranationales sont généralement soumises à des règles budgétaires strictes et, en particulier, leur capacité à s'endetter est souvent limitée. Les engagements en provenance des administrations infranationales résultant de la nécessité de financer les déficits par l'emprunt sont considérés comme de la dette de ces administrations. Des hausses même modestes de l'endettement d'un grand nombre d'entités administratives (des États fédérés ou des municipalités, par exemple) peuvent faire augmenter la dette des administrations publiques (tous échelons administratifs confondus), avec des conséquences sur les soldes budgétaires et, potentiellement, sur les taux d'intérêt de la dette publique.

Comparée à celle des administrations centrales, l'assiette des recettes des administrations infranationales est le plus souvent étroite. C'est pourquoi, dans les pays membres de l'OCDE, la plupart des administrations infranationales dépendent de transferts en provenance des autorités centrales. En 2011, et en moyenne des pays membres de l'OCDE, les administrations centrales accusaient un déficit budgétaire de $3.2 \%$ du PIB, soit seulement $0.2 \%$ point de pourcentage de moins que l'ensemble des administrations. En Allemagne, en Australie, au Canada et en Espagne, le solde au niveau des États fédérés représente plus du tiers du solde budgétaire des administrations publiques.

Globalement, dans les pays membres de l'OCDE, la dette infranationale n'est pas très significative par rapport à la dette des administrations publiques dans son ensemble, à quelques exceptions près (principalement des pays fédéraux et quasifédéraux). En 2011, elle représentait en moyenne $11.8 \%$ du PIB et s'échelonnait au niveau local entre $1.3 \%$ du PIB en Grèce et $38 \%$ au Japon. Au Canada, en Allemagne, aux États-Unis (administrations d'États fédérés et administrations locales confondues) et en Espagne, la dette des administrations d'États fédérés exprimée en pourcentage du PIB s'établissait respectivement à $53.3 \%, 26 \%, 24.7 \%$ et $18.7 \%$.

De 2001 à 2011, la répartition de la dette publique entre les niveaux d'administration n'a pas beaucoup évolué dans les pays membres de l'OCDE. Certes, au Royaume-Uni, la dette des administrations infranationales a diminué de 7.1 points de pourcentage, mais cette tendance va probablement s'inverser dans les prochaines années du fait d'une réforme du dispositif de financement du logement social (le «Housing Revenue Account ") qui pourrait amener les administrations locales à emprunter davantage. Aux États-Unis, au Japon et aux Pays-Bas, la part de la dette infranationale a aussi diminué de façon très nette. Toutefois, comme la dette publique totale a continué à s'élever dans ces pays, cette baisse peut être attribuée à une progression plus lente de la dette aux échelons infranationaux qu'à l'échelon central. En outre, les échelons infranationaux sont souvent soumis à des règles budgétaires strictes et contraints par les autorités centrales à participer à l'effort national d'assainissement des finances publiques.

\section{Méthodologie et définitions}

Les données sont tirées de la base de données des Statistiques de l'OCDE sur les comptes nationaux, qui sont établies conformément au Système de comptabilité nationale (SCN) ; ce dernier constitue un ensemble de définitions, de nomenclatures et de règles de comptabilisation approuvés au plan international en matière de comptabilité nationale. Selon la terminologie du SCN, les administrations publiques se composent de l'administration centrale, des administrations d'États fédérés (c'est-àdire les administrations au niveau des États fédérés ou des provinces), des administrations locales et des administrations de sécurité sociale. Les administrations classées dans la catégorie des administrations d'États fédérés n'existent que dans les neuf pays membres de l'OCDE dotés d'une structure fédérale : l'Allemagne, l'Australie, l'Autriche, la Belgique, le Canada, l'Espagne (pays considéré comme quasi-fédéral), les États-Unis, le Mexique et la Suisse.

On calcule le solde budgétaire des administrations publiques, également présenté comme leur capacité de financement (+) ou leur besoin de financement (-), en soustrayant les dépenses totales des administrations publiques de leurs recettes totales.

Pour plus de renseignements sur la dette, se reporter à la rubrique "Méthodologie et définitions " de l'indicateur précédent, " La dette des administrations publiques » à la page XX.

\section{Pour en savoir plus}

Ter-Minassian, T. (2007), "Fiscal Rules for Subnational Governments: Can They Promote Fiscal Discipline? ", OECD Journal on Budgeting, vol. 6, $n^{\circ}$ 3, Éditions OCDE, Paris, http:// dx.doi.org/10.1787/budget-v6-art17-en.

Vammalle, C. et C. Hulbert (2013), « Sub-National Finances and Fiscal Consolidation: Walking on Thin Ice ", OECD Regional Development Working Papers, $n^{\circ}$ 2013/02, Éditions OCDE, Paris, http://dx.doi.org/10.1787/5k49m8cqkcf3-en.

\section{Notes relatives aux graphiques}

On ne dispose pas de données pour le Chili. Les administrations locales sont comptabilisées dans la catégorie des " administrations d'États fédérés " pour l'Australie et les États-Unis. En Australie, il n'existe pas de système public d'assurance sociale. Les administrations de sécurité sociale sont comptabilisées dans la catégorie " administration centrale » aux États-Unis, en Norvège et au Royaume-Uni.

3.8 : Les données relatives au Canada et à la Nouvelle-Zélande portent sur 2010 et non 2011.

3.9: On ne dispose pas de données pour le Mexique, la NouvelleZélande, la Suisse et la Turquie. On ne dispose pas de données relatives à l'administration centrale pour l'Irlande et l'Islande, et ces pays ne sont pas compris dans la moyenne OCDE. Les données relatives à la Corée, aux États-Unis, au Japon et au Royaume-Uni sont fournies sur une base non consolidée. Les données relatives à la Suisse portent sur 2010 et non 2011.

3.10: On ne dispose pas de données pour l'Islande, le Mexique, la Nouvelle-Zélande et la Turquie. On ne dispose pas de données relatives à 2001 pour Israël et le Luxembourg, et ces pays ne sont pas compris dans la moyenne OCDE. Les données sont fournies sur une base non consolidée (sauf pour l'Australie). Les données relatives à la Suisse portent sur 2010 et non 2011. Les données relatives à la Corée portent sur 2002 et non 2001. Les données relatives au Danemark portent sur 2003 et non 2001.

Informations sur les données concernant Israël : http://dx.doi.org/10.1787/ 888932315602 . 
3.8. Solde budgétaire aux différents niveaux d'administration, en \% du PIB (2011)

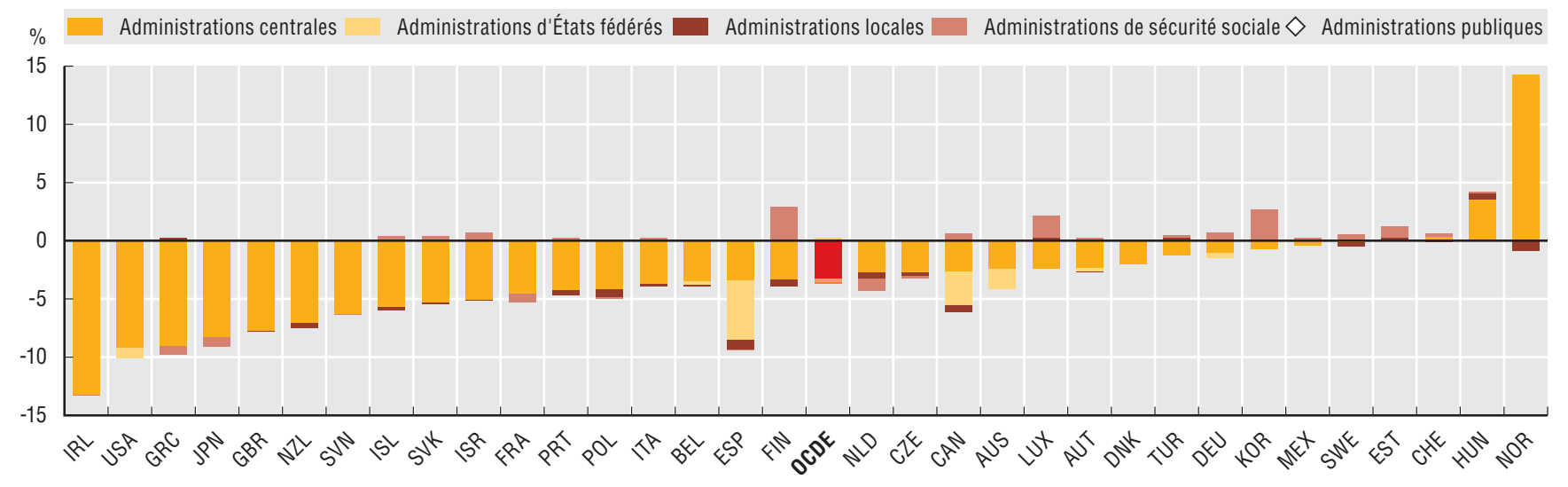

Source: Statistiques de l'OCDE sur les comptes nationaux (base de données).

\subsection{Dette des différents niveaux d'administration, en \% du PIB (2011)}

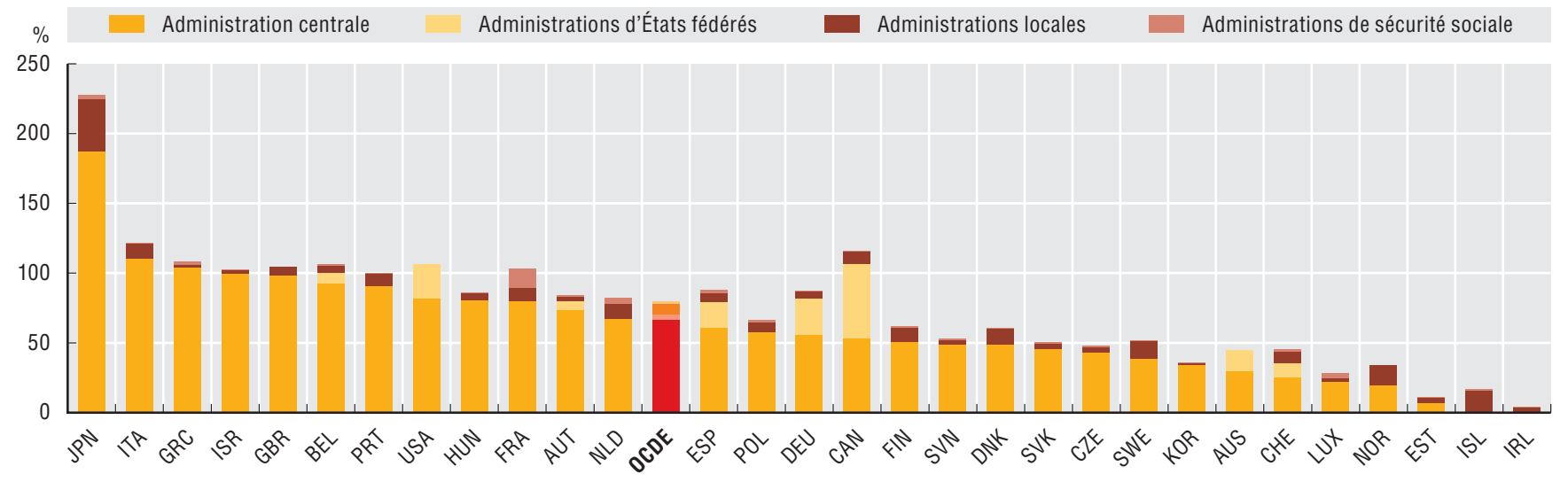

Source: Statistiques de l'OCDE sur les comptes nationaux (base de données).

3.10. Répartition de la dette publique entre les différents niveaux d'administration (2001 et 2011)

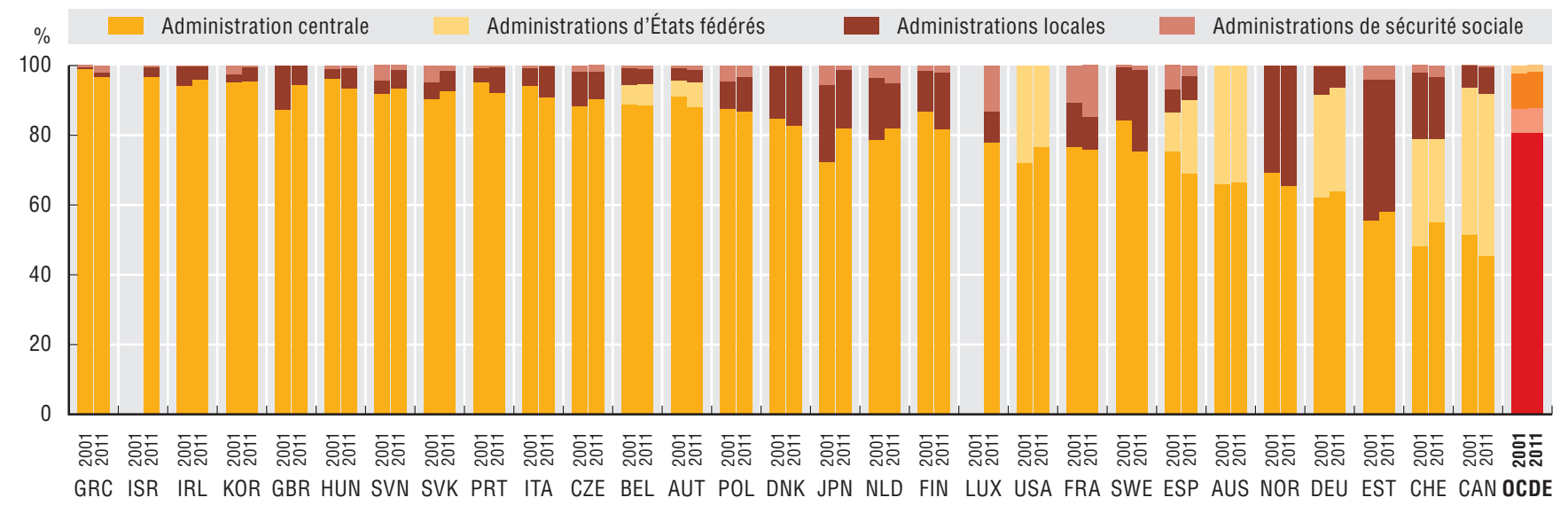

Source: Statistiques de l'OCDE sur les comptes nationaux (base de données). 
Les administrations publiques collectent des recettes pour deux finalités principales: financer les biens et les services qu'elles procurent aux particuliers et aux entreprises et remplir leur rôle redistributif. Les prélèvements fiscaux sur les ménages et les entreprises ainsi que les cotisations sociales sont leurs sources de recettes essentielles. Comparer les niveaux de recettes publiques en pourcentage du PIB ou par habitant entre les différents pays de l'OCDE donne une indication de l'importance du secteur public dans l'économie sous l'angle des ressources financières disponibles. Le montant total des recettes publiques collectées est déterminé par des décisions politiques passées et actuelles reposant elles-mêmes sur des attentes culturelles en termes de redistribution sociale, par les contraintes budgétaires, par les fluctuations conjoncturelles et par les performances de l'économie. Pour toutes ces raisons, le niveau des recettes publiques diffère fortement selon les pays membres de l'OCDE.

En 2011, les recettes des administrations publiques représentaient en moyenne $41.9 \%$ du PIB dans les pays de l'OCDE, soit seulement 0.2 point de pourcentage de plus que dix ans auparavant (41.7\% en 2001). La fourchette est très large, allant de $57.3 \%$ en Norvège à $22.7 \%$ au Mexique. Les pays nordiques tendent à prélever davantage de recettes que les autres groupes de pays, car la plupart des prestations sociales dont bénéficient les ménages y sont imposables. Bien que la part moyenne des recettes publiques dans le PIB soit restée stable de 2001 à 2011 sur l'ensemble de la zone OCDE, il y a eu des fluctuations significatives. C'est en Hongrie (10.1 points) et au Portugal (6.6 points) que cette part a le plus augmenté, même si, dans ces deux pays, la hausse est surtout intervenue depuis 2009, en réaction à la crise budgétaire qui les a touchés. De 2001 à 2011, la part des recettes publiques dans le PIB a surtout diminué en Israël (7.3 points de pourcentage) et en Suède (4.9 points), même si, en Israël, elle s'est élevée de 2009 à 2011. Les recettes publiques ont augmenté dans les deux tiers des pays de l'OCDE entre 2009 à 2011.

En 2011, en moyenne de la zone OCDE, les recettes publiques représentaient 15141 USD à PPA par habitant. Exprimé par rapport à la population, il existe un rapport de 1 à plus de 9 entre les pays de l'OCDE prélevant le plus et le moins de recettes publiques (36800 USD par habitant au Luxembourg contre près de 4000 USD au Mexique) ; exprimé en proportion $\mathrm{du}$ PIB, en revanche, ce rapport n'est que de 1 à 2.5 .

De 2001 à 2011, les recettes publiques par habitant ont augmenté en moyenne de $1.5 \%$ par an dans les pays membres de l'OCDE. Les progressions annuelles moyennes les plus fortes ont eu lieu en Estonie (5.3\%) et en Corée (5\%). Sur la même période, les recettes publiques n'ont diminué que dans quatre pays de l'OCDE, mais très modérément, dans une fourchette de $0.1 \%$ à $0.3 \%$ en moyenne annuelle; ces quatre pays sont l'Italie, les États-Unis, l'Espagne et le Canada.

\section{Méthodologie et définitions}

Les données sur les recettes publiques sont tirées de la base de données des Statistiques de l'OCDE sur les comptes nationaux, qui sont établies conformément au Système de comptabilité nationale (SCN) ; ce dernier constitue un ensemble de concepts, de définitions, de nomenclatures et de règles de comptabilisation approuvés au plan international en matière de comptabilité nationale. Selon la terminologie du SCN, les administrations publiques se composent de l'administration centrale, des administrations d'États fédérés (c'est-à-dire les administrations au niveau des États fédérés ou des provinces), des administrations locales et des administrations de sécurité sociale. Les recettes englobent les cotisations sociales, les impôts hors cotisations sociales, les aides et d'autres recettes. Le produit intérieur brut (PIB) est la mesure habituelle de la valeur des biens et services produits par un pays au cours d'une période donnée.

On a calculé les recettes publiques par habitant en convertissant les recettes totales en USD de 2011 avec les parités de pouvoir d'achat (PPA) OCDE/Eurostat pour le PIB et en divisant le résultat obtenu par la population. Dans le cas des pays pour lesquels les données sont tirées des Perspectives de l'économie mondiale du FMI, on a utilisé un taux de change implicite basé sur les PPA. La PPA (parité de pouvoir d'achat) est le nombre d'unités monétaires du pays $B$ nécessaire pour acquérir une même quantité de biens et de services dans le pays A.

\section{Pour en savoir plus}

OCDE (2013), Panorama des comptes nationaux 2013, Éditions OCDE, Paris, http://dx.doi.org/10.1787/na_glance-2013-fr.

\section{Notes relatives aux graphiques}

On ne dispose pas de données pour le Chili. Les données relatives au Canada, à la Fédération de Russie et à la Nouvelle-Zélande portent sur 2010 et non 2011. Les données relatives au Japon et au Mexique pour 2001 sont des estimations. Les données relatives à la Fédération de Russie portent sur 2002 et non 2001.

3.11 : On ne dispose pas de données relatives à la Turquie pour 2001, et ce pays n'est pas compris dans la moyenne OCDE.

3.12 : On ne dispose pas de données pour la Turquie.

Informations sur les données concernant Israël : http://dx.doi.org/10.1787/ 888932315602 . 
3.11. Recettes des administrations publiques en pourcentage du PIB (2001, 2009 et 2011)

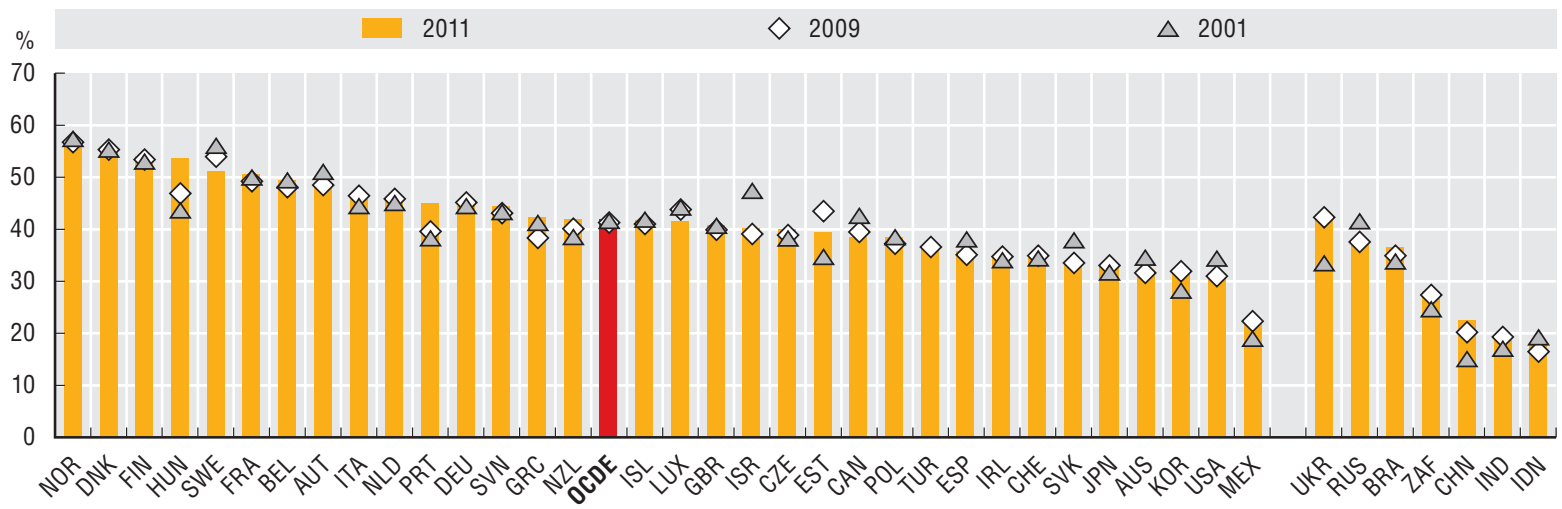

Source: Données sur les pays membres de l'OCDE : Statistiques de l'OCDE sur les comptes nationaux (base de données). Données sur les autres grandes économies (à l'exclusion de la Fédération de Russie) : Fonds monétaire international (2013), Perspectives de l'économie mondiale, avril 2013, FMI, Washington, DC.

\subsection{Recettes des administrations publiques par habitant (2011)}

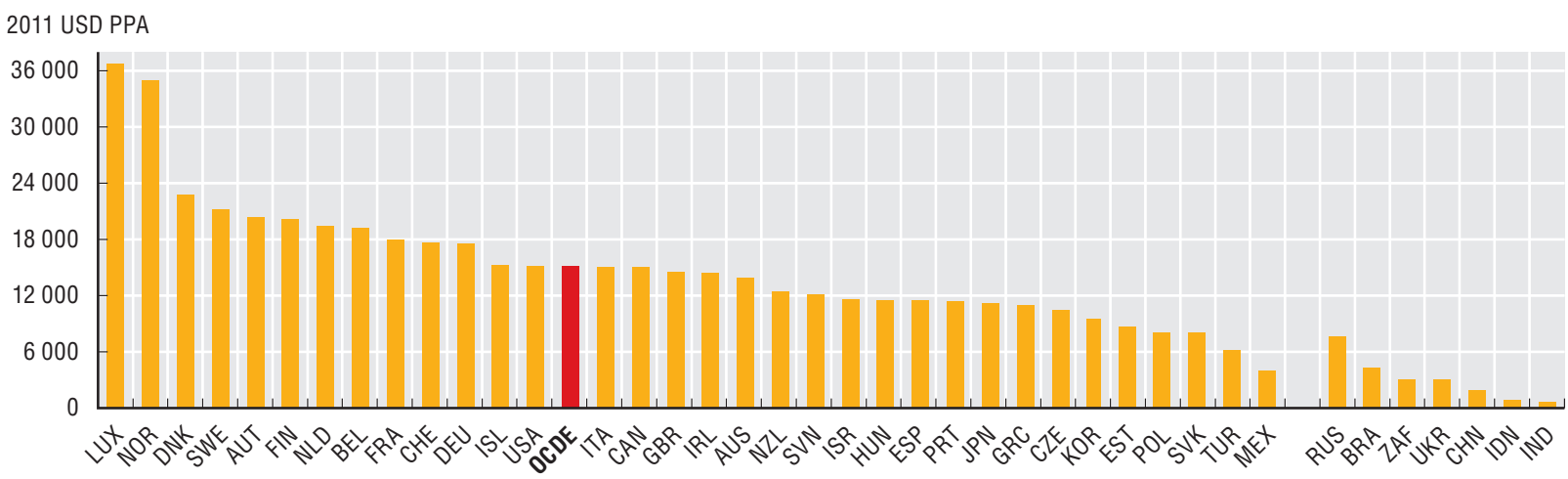

Source: Données sur les pays membres de l'OCDE : Statistiques de l'OCDE sur les comptes nationaux (base de données). Données sur les autres grandes économies (à l'exclusion de la Fédération de Russie) : Fonds monétaire international (2013), Perspectives de l'économie mondiale, avril 2013, FMI, Washington, DC.

3.13. Taux de croissance annuel moyen des recettes publiques par habitant en termes réels (de 2001 à 2011)

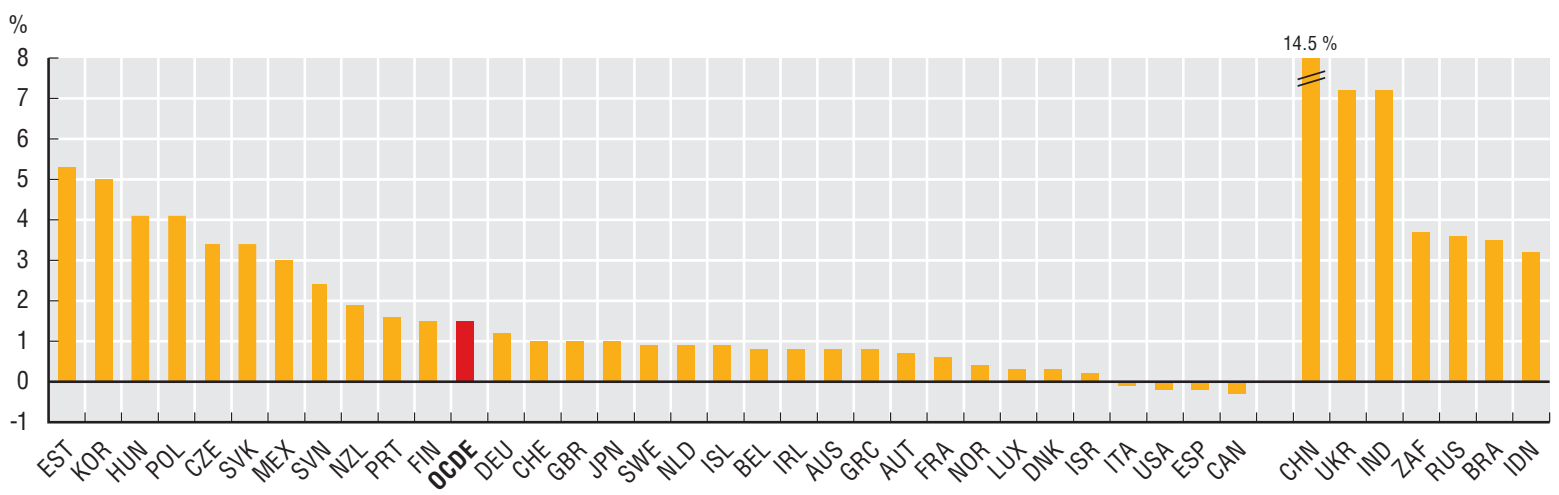

Source: Données sur les pays membres de l'OCDE : Statistiques de l'OCDE sur les comptes nationaux (base de données). Données sur les autres grandes économies (à l'exclusion de la Fédération de Russie) : Fonds monétaire international (2013), Perspectives de l'économie mondiale, avril 2013, FMI, Washington, DC. 
L'analyse de la composition des recettes des administrations publiques donne une indication de la contribution relative des citoyens et/ou des secteurs de l'économie au financement des dépenses publiques.

En 2011 et en moyenne des pays membres de l'OCDE, plus de $60 \%$ des recettes des administrations publiques ont été prélevées sous forme d'impôts hors cotisations sociales, et près de $25 \%$ sous forme de cotisations sociales, le reste provenant des aides et d'autres recettes. Les dépenses publiques sont financées différemment selon les pays de l'OCDE. Le Danemark et l'Australie, par exemple, dépendent relativement plus des impôts hors cotisations sociales (plus de 80 \% des recettes totales) et financent donc davantage la protection sociale par la fiscalité générale. En revanche, l'Allemagne, l'Espagne, la France, le Japon, la République tchèque et la République slovaque s'appuient relativement plus sur les cotisations sociales (près de $40 \%$ des recettes totales). La Norvège est le seul pays où les aides et les autres recettes représentent plus de $25 \%$ des recettes totales (cela s'explique surtout par les dividendes et intérêts touchés par le fonds souverain national, dont le capital augmente par accumulation des revenus nets en provenance du secteur pétrolier).

De 2009 à 2011, la composition des recettes publiques est restée assez stable en moyenne des pays membres de l'OCDE. La part des impôts hors cotisations sociales a augmenté de 0.4 point de pourcentage, celle des cotisations sociales a diminué de 0.6 point - la crise économique ayant réduit l'emploi et donc les rentrées de cotisations - et la part des aides et des autres recettes a progressé de 0.2 point. La composition des recettes publiques a surtout évolué en Hongrie (augmentation de 16.7 points de pourcentage de la part des aides et des autres recettes), au Mexique (hausse de 9.3 points de la part des impôts hors cotisations sociales) et au Portugal (progression de 6.8 points des aides et des autres recettes).

En 2010, en moyenne des pays membres de l'OCDE, un tiers des prélèvements obligatoires (impôts plus cotisations sociales) provenait des impôts sur le revenu et sur les bénéfices, un autre tiers des impôts sur les biens et les services (dont une part importante de taxe sur la valeur ajoutée), plus d'un quart des cotisations sociales et le reste des impôts sur le patrimoine (5.4\%), des impôts sur les salaires (1\%) et des autres impôts $(0.6 \%)$. La composition était très similaire en 2001 . Toutefois, les pays membres de l'OCDE recourent dans des proportions inégales aux différents prélèvements. Au Danemark, en Australie et en Nouvelle-Zélande, par exemple, la majorité des recettes fiscales a pour origine les impôts sur le revenu et sur les bénéfices.

\section{Méthodologie et définitions}

Les données sur les recettes publiques sont tirées de la base de données des Statistiques de l'OCDE sur les comptes nationaux, qui sont établies conformément au Système de comptabilité nationale (SCN) ; ce dernier constitue un ensemble de concepts, de définitions, de nomenclatures et de règles de comptabilisation approuvés au plan international en matière de comptabilité nationale. Selon la terminologie du SCN, les administrations publiques se composent de l'administration centrale, des administrations d'États fédérés (c'est-à-dire les administrations au niveau des états fédérés ou des provinces), des administrations locales et des administrations de sécurité sociale. Les recettes publiques englobent les impôts hors cotisations sociales (impôts sur la consommation, le revenu, le patrimoine, la propriété et le capital, par exemple), les cotisations sociales (par exemple les cotisations au titre des retraites, de la santé et d'autres risques), les aides (de gouvernements étrangers ou d'organisations internationales) et d'autres recettes (par exemples ventes, redevances, revenus du patrimoine et subventions). Ces agrégats ne sont pas directement disponibles dans les Statistiques de l'OCDE sur les comptes nationaux (base de données), et ils ont été construits à partir de lignes budgétaires de souscomptes (voir l'annexe A). Les données présentées dans le graphique 3.16 proviennent des Statistiques des recettes publiques de l'OCDE.

Les Statistiques des recettes publiques de l'OCDE et le SCN diffèrent sur la définition des recettes fiscales. Dans le SCN, les impôts sont des paiements obligatoires, sans contrepartie, en espèces ou en nature, effectués par des unités institutionnelles à des administrations publiques. Les cotisations sociales correspondent aux cotisations effectives ou imputées versées aux régimes d'assurance sociale afin de garantir le droit à des prestations sociales. Elles peuvent être obligatoires ou facultatives, et les régimes peuvent être capitalisés ou non. Les Statistiques des recettes publiques de l'OCDE traitent les cotisations de sécurité sociale obligatoires comme des impôts, alors que le SCN les traite comme des cotisations sociales, parce que le fait de recevoir une prestation sociale dépend dans la plupart des pays du versement de cotisations appropriées, même si le montant des prestations n'a pas nécessairement de rapport avec celui des cotisations.

\section{Pour en savoir plus}

OCDE (2012), Statistiques des recettes publiques 2012, Éditions OCDE, Paris, http://dx.doi.org/10.1787/rev_stats-2012-en-fr.

OECD (2010), Tax Policy Reform and Economic Growth, Études de politique fiscale de l'OCDE, $\mathrm{n}^{\circ} 20$, Éditions OCDE, Paris, http:// dx.doi.org/10.1787/9789264091085-en.

\section{Notes relatives aux graphiques}

3.14 et 3.15 : On ne dispose pas de données pour le Chili. On ne dispose pas de données relatives au Japon et à la Turquie pour 2001, et ces pays ne sont pas compris dans la moyenne OCDE. L'Australie ne perçoit pas de recettes sous forme de cotisations sociales, car il n'y a pas de système public d'assurance sociale. On ne dispose pas de données sur les impôts en capital pour la Fédération de Russie. Les données relatives au Canada, à la Fédération de Russie et à la Nouvelle-Zélande portent sur 2010 et non 2011. Les données relatives au Mexique portent sur 2003 et non 2001. Les données relatives à la Fédération de Russie portent sur 2002 et non 2001.

3.16: Pour les pays membres à la fois de l'OCDE et de l'Union européenne, l'imposition totale inclut les droits de douane perçus pour le compte de l'Union européenne.

Informations sur les données concernant Israël : http://dx.doi.org/10.1787/ 888932315602 . 
3.14. Composition des recettes des administrations publiques (2001 et 2011)

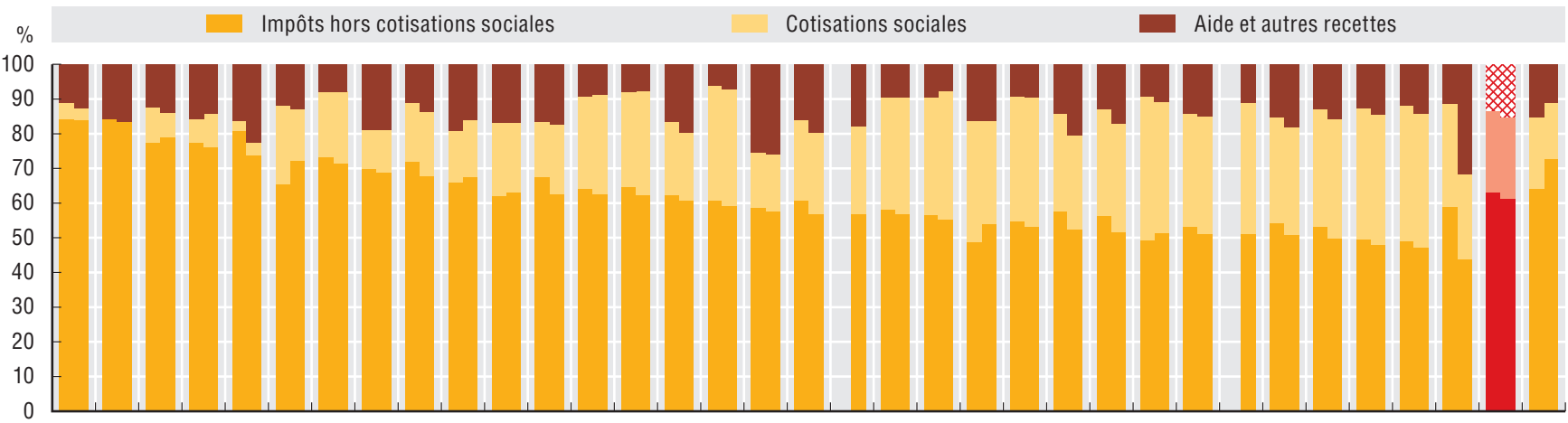

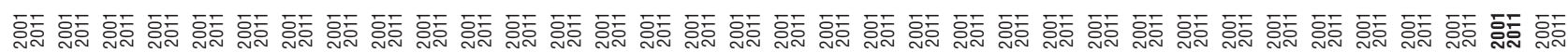

DNK AUS MEX ISL NZL SWE GBR CAN IRL ISR CHE KOR LUX ITA USA BEL NOR FIN TUR AUT ESP POL FRA PRT EST DEU NLD JPN GRC SVN SVK CZE HUN OCDE RUS Source : Statistiques de l'OCDE sur les comptes nationaux (base de données).

3.15. Évolution de la composition des recettes des administrations publiques (2009 à 2011)

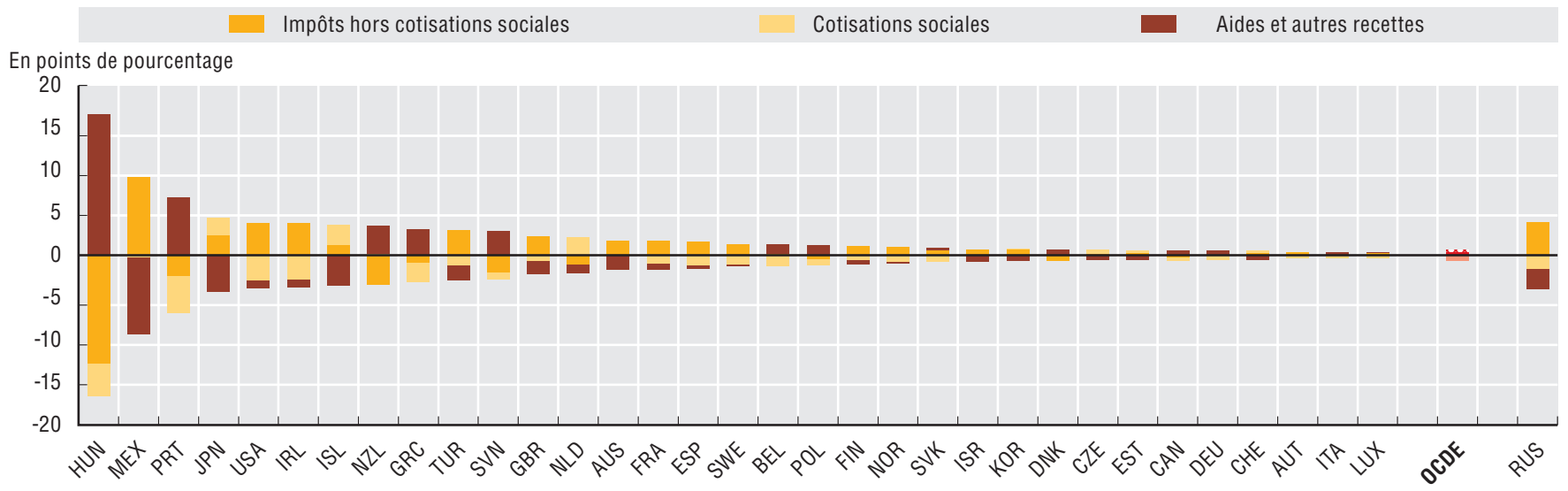

Source: Statistiques de l'OCDE sur les comptes nationaux (base de données).

\subsection{Composition des recettes fiscales en pourcentage de l'imposition totale (2001 et 2010)}

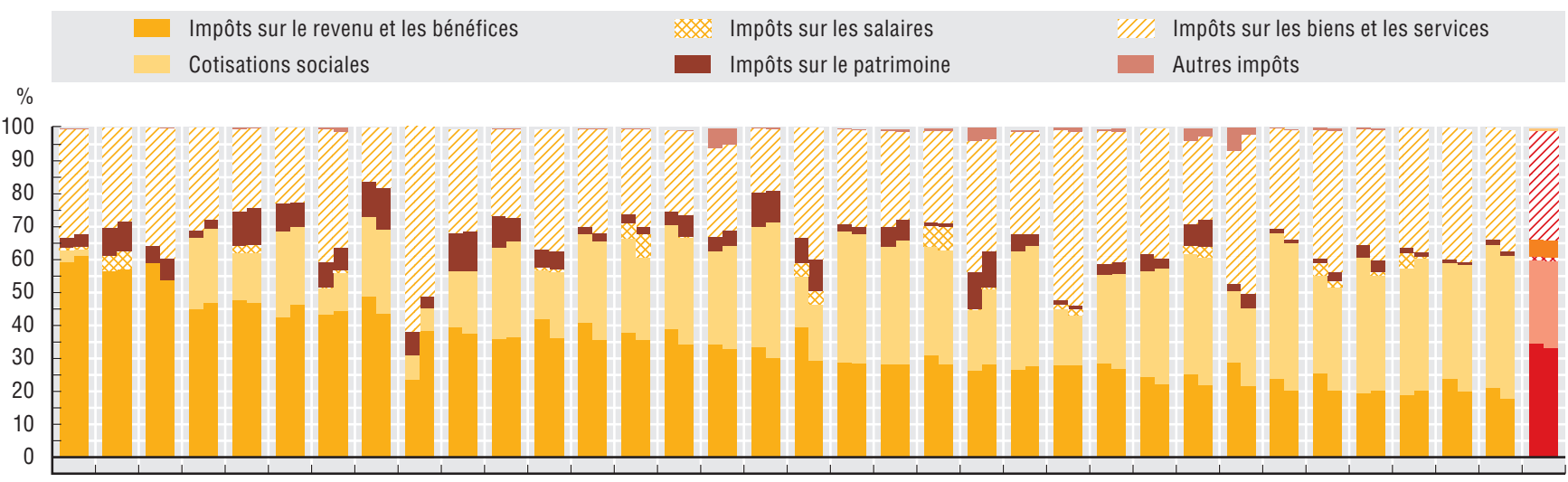

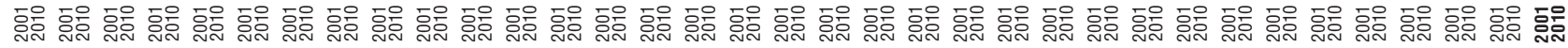
DNK AUS NZL NOR CAN CHE ISL USA CHL GBR LUX IRL FIN SWE BEL ITA JPN ISR DEU ESP AUT KOR NLD MEX PRT GRC FRA TUR CZE HUN POL SVN EST SVK OCDE

Source : OCDE (2012), Statistiques des recettes publiques 2012, Éditions OCDE, Paris, http://dx.doi.org/10.1787/rev_stats-2012-en-fr. 
Les recettes publiques sont collectées de façons différentes selon les niveaux d'administration, car l'échelon central, l'échelon des États fédérés/provinces et l'échelon local n'ont pas la même aptitude à lever des impôts et à prélever des cotisations sociales. Le degré de transfert de recettes entre les divers échelons donne une indication de leur interdépendance financière. Le montant des impôts perçus par les administrations infranationales peut être considéré comme représentatif de leur autonomie budgétaire.

En 2011, en moyenne des pays membres de l'OCDE, les administrations centrales percevaient la majeure partie des recettes des administrations publiques (60.3\%). Les administrations infranationales (échelon des États fédérés/ provinces et échelon local) percevaient en moyenne $21 \%$ du total, et les $18.7 \%$ restants étaient perçus par les administrations de sécurité sociale. La répartition des recettes publiques entre les niveaux d'administration varie énormément selon les pays membres de l'OCDE. Plus de $85 \%$ des recettes des administrations publiques sont perçues par l'administration centrale au Royaume-Uni (90.6\%), en Nouvelle-Zélande (89.6 \%) et en Norvège (86.2 \%). En revanche, l'administration centrale reçoit moins de la moitié des recettes publiques dans huit autres pays membres de l'OCDE, dont trois pays non fédéraux : la Finlande (42.9\%), la France (34.1\%) et le Japon $(24.1 \%)$. Au Japon et dans les pays nordiques, à l'exception de la Norvège, l'échelon local perçoit une part relativement élevée des recettes publiques totales : $31.2 \%$ en moyenne, contre une moyenne OCDE de $13.3 \%$. Dans les neuf pays fédéraux, c'est aux États-Unis (46\%), au Canada (43.2\%) et en Australie (38.8\%) que l'échelon des États fédérés/ provinces perçoit la proportion la plus forte des recettes publiques. En France, près de la moitié des recettes publiques est collectée par les administrations de sécurité sociale.

De 2001 à 2011, le pourcentage des recettes publiques encaissé par les administrations centrales a diminué de 1.7 point de pourcentage, en moyenne, dans les pays membres de l'OCDE. À l'inverse, la part des administrations infranationales a augmenté de 1.3 point. Dans sept pays seulement, la part des recettes publiques encaissée par les administrations centrales a progressé : le Danemark (9.4 points), la Hongrie (8.2 points), la Norvège (4 points), la Grèce (3.3 points), l'Allemagne (2.4 points), la Suisse (1.6 point) et le Portugal (0.1 point).

Les administrations centrales se financent surtout par les impôts hors cotisations sociales: en 2011, ces impôts représentaient en moyenne $77 \%$ de leurs recettes. Alors que les sources de recettes des administrations centrales sont relativement homogènes, celles des administrations infranationales sont très variables. Les recettes des administrations locales proviennent, en majorité, des transferts inter-administrations et de la catégorie des " autres recettes " (plus de 61 \% en moyenne en 2011). Les impôts sur le patrimoine représentent la plus grande part du solde de $39 \%$. Les limites imposées aux administrations infranationales en ce qui concerne la fixation de l'assiette, des taux et des allègements applicables aux impôts locaux pèsent peut-être sur leur aptitude à générer leurs propres sources de recettes et, potentiellement, à assurer des services publics davantage " sur mesure ".

\section{Méthodologie et définitions}

Les données sur les recettes publiques sont tirées de la base de données des Statistiques de l'OCDE sur les comptes nationaux, qui sont établies conformément au Système de comptabilité nationale (SCN) ; ce dernier constitue un ensemble de concepts, de définitions, de nomenclatures et de règles de comptabilisation approuvés au plan international en matière de comptabilité nationale. Selon la terminologie du SCN, les administrations publiques se composent de l'administration centrale, des administrations d'États fédérés (c'est-à-dire les administrations au niveau des États fédérés ou des provinces), des administrations locales et des administrations de sécurité sociale. Les administrations classées dans la catégorie des administrations d'États fédérés n'existent que dans les neuf pays membres de l'OCDE dotés d'une structure fédérale: l'Allemagne, l'Australie, l'Autriche, la Belgique, le Canada, l'Espagne (pays considéré comme quasi-fédéral), les ÉtatsUnis, le Mexique et la Suisse. Les données des graphiques 3.17 et 3.18 (consultables en ligne) ne prennent pas en compte les transferts entre niveaux d'administration, sauf pour l'Australie et le Japon. Le graphique 3.18, intitulé «Évolution de la répartition des recettes des administrations publiques entre les différents niveaux d'administration (2009-2011) ", ainsi que les graphiques $3.19,3.20$ et 3.21 (sur la composition des recettes des administrations centrales, des administrations d'États fédérés et des administrations locales) sont consultables en ligne aux adresses $h t t p: / / d x . d o i . o r g / 10.1787 / 888932941633$, http:// dx.doi.org/10.1787/888932941652, http://dx.doi.org/10.1787/ 888932941671 et $h t t p: / / d x$.doi.org/10.1787/888932941690.

Les recettes publiques englobent les impôts hors cotisations sociales (impôts sur la consommation, le revenu, le patrimoine, la propriété et le capital, par exemple), les cotisations sociales (par exemple les cotisations au titre des retraites, de la santé et d'autres risques), les aides et d'autres recettes. Les aides peuvent provenir de gouvernements étrangers, d'organisations internationales ou d'autres administrations publiques. Les autres recettes peuvent provenir de ventes, de redevances, de revenus du patrimoine ou de subventions. Ces agrégats ne sont pas directement disponibles dans les Statistiques de l'OCDE sur les comptes nationaux (base de données), et ils ont été construits à partir de lignes budgétaires de sous-comptes (voir l'annexe A).

\section{Pour en savoir plus}

Blöchliger, H. et al. (2010), «Fiscal Policy Across Levels of Government in Times of Crisis ", OECD Working Papers on Fiscal Federalism, $\mathrm{n}^{\circ} 12$, Éditions OCDE, Paris, http://dx.doi.org/ 10.1787/5k97b10wqn46-en.

\section{Notes relatives aux graphiques}

On ne dispose pas de données pour le Chili. On ne dispose pas de données relatives au Japon, à la Pologne et à la Turquie pour 2001, et ces pays ne sont pas compris dans la moyenne OCDE. Les transferts entre niveaux d'administration ne sont pas pris en compte (sauf pour l'Australie, le Japon et la Turquie). Les données relatives au Canada et à la NouvelleZélande portent sur 2010 et non 2011. Les données relatives au Mexique portent sur 2003 et non 2001. Les administrations locales sont comptabilisées dans la catégorie des " administrations d'États fédérés " pour l'Australie et les États-Unis. En Australie, il n'existe pas de système public d'assurance sociale. Les administrations de sécurité sociale sont comptabilisées dans la catégorie " administration centrale " pour les États-Unis, la Norvège, la Nouvelle-Zélande et le Royaume-Uni.

Informations sur les données concernant Israël : http://dx.doi.org/10.1787/ 888932315602 . 


\section{LES FINANCES ET L'ÉCONOMIE PUBLIQUES}

3.17. Répartition des recettes des administrations publiques entre les différents niveaux d'administration (2001 et 2011)

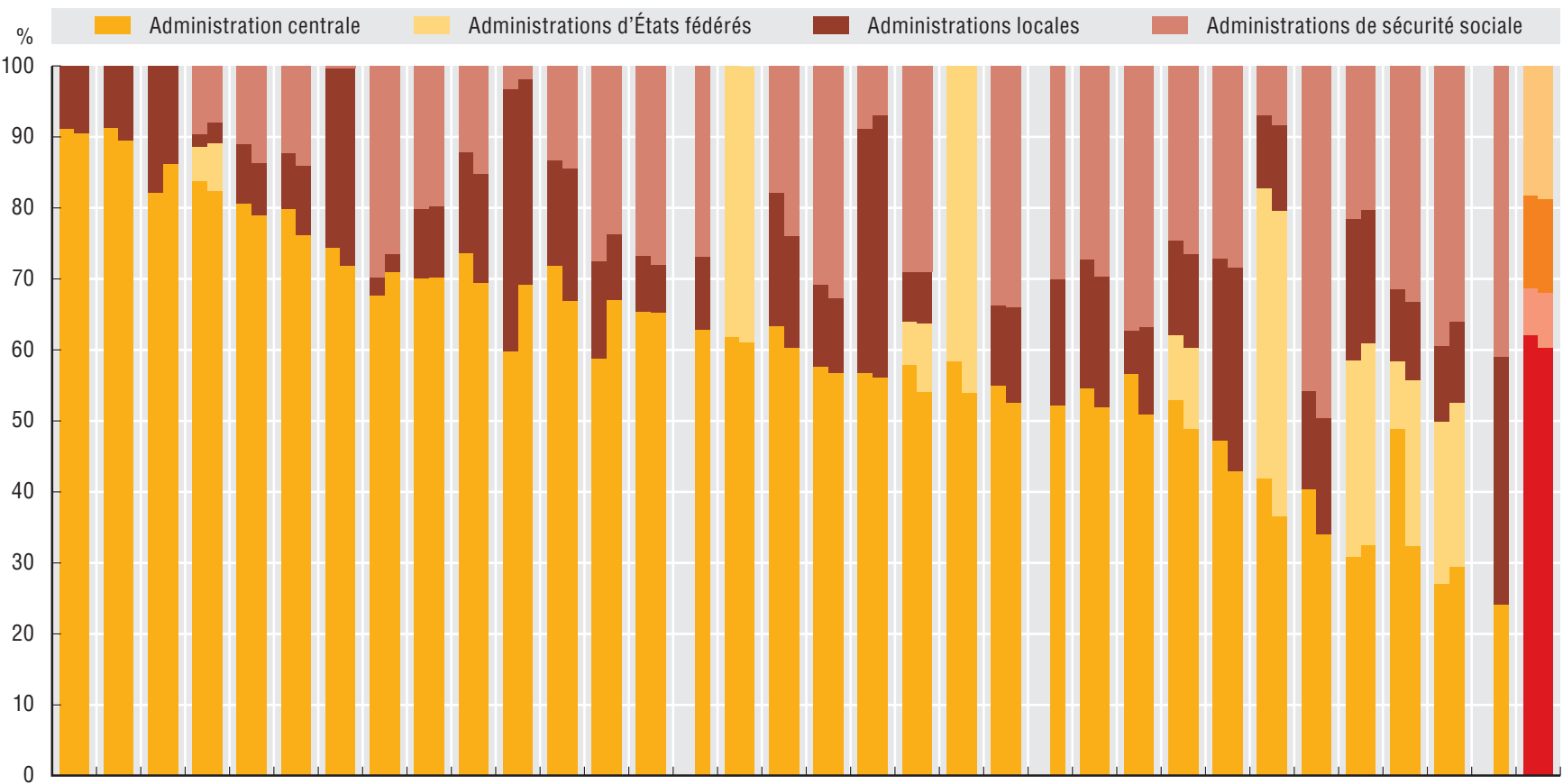

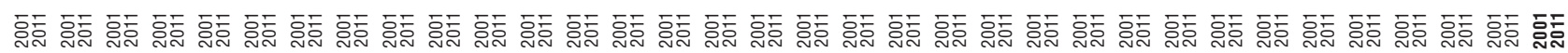
GBR NZL NOR MEX IRL ISR ISL GRC PRT EST DNK CZE HUN LUX TUR AUS KOR NLD SWE BEL USA SVN POL ITA SVK AUT FIN CAN FRA CHE ESP DEU JPN OCDE

Source: Statistiques de l'OCDE sur les comptes nationaux (base de données). 
Les administrations publiques dépensent pour deux finalités principales : pour produire et/ou financer des biens et services destinés aux particuliers et aux entreprises et pour remplir leur rôle redistributif. Comparer les niveaux de dépenses publiques en pourcentage du PIB ou par habitant entre les différents pays de l'OCDE donne une indication de l'importance du secteur public dans l'économie sous l'angle des ressources financières dépensées. Comme les recettes publiques, les dépenses publiques sont déterminées par des décisions politiques passées et actuelles reposant elles-mêmes sur des attentes culturelles en termes de redistribution sociale et de biens et services à fournir, par les contraintes budgétaires, par les fluctuations conjoncturelles et par les performances de l'économie. Le niveau des dépenses publiques varie donc fortement selon les pays de l'OCDE. Il est important de noter que le montant des dépenses n'est pas une indication de l'efficience ou de la productivité des administrations.

En 2011, les dépenses des administrations publiques représentaient en moyenne $45.4 \%$ du PIB des pays membres de l'OCDE. Les pourcentages s'échelonnaient entre $57.6 \%$ au Danemark et $22.8 \%$ au Mexique. En général, le pourcentage est supérieur dans les pays de l'OCDE membres de l'Union européenne. Le secteur public est particulièrement important au Danemark, en France et en Finlande, pays où les dépenses publiques atteignent ou dépassent $55 \%$ du PIB; il est particulièrement réduit en Corée et au Mexique, où la part des dépenses dans le PIB est de $30 \%$ et de $23 \%$, respectivement.

De 2001 à 2011, la part des dépenses publiques dans le PIB a connu d'importantes fluctuations dans les pays membres de l'OCDE. Elle a augmenté notablement de 2001 à 2009 (4.5 points de pourcentage en moyenne), surtout en Irlande (15.4 points), au Royaume-Uni (11.1 points) et en Estonie (10.7 points). Toutefois, l'essentiel de la hausse intervenue pendant cette période dans la zone de l'OCDE a été postérieure à 2007 : avec la contraction générale de l'activité, les stabilisateurs automatiques ont joué, et des dépenses discrétionnaires ont été engagées. De 2001 à 2009, trois pays seulement ont vu baisser la part des dépenses publiques dans leur PIB : Israël (8.1 points), la République slovaque (2.9 points) et la Suisse (0.7 point). De 2009 à 2011, la tendance générale s'est inversée et la part des dépenses publiques dans le PIB a diminué de 1.4 point en moyenne, en raison surtout du ralentissement de leur progression (ou dans certains cas de leur réduction) comparativement à la croissance $\mathrm{du}$ PIB. Les baisses les plus marquées ont eu lieu en Estonie (7.2 points), en Suède, en Islande et en République slovaque (baisse supérieure à 3 points dans ces trois pays). Dans le même temps, la part des dépenses publiques dans le PIB n'a augmenté que dans trois pays : la Nouvelle-Zélande (6.6 points de 2009 à 2010 sous l'effet de mesures de relance budgétaire), la Slovénie (1.4 point) et le Japon (0.1 point).

En moyenne des pays membres de l'OCDE, les dépenses publiques représentaient 16240 USD à PPA par habitant en 2011. Exprimé par rapport à la population, il existe un rapport de 1 à plus de 9 entre les pays de l'OCDE réalisant le plus et le moins de dépenses publiques (37 000 USD à PPA par habitant au Luxembourg contre près de 4000 USD à PPA au Mexique); exprimé en proportion du PIB, en revanche, ce rapport n'est que de 1 à 2.5 .

De 2001 à 2011, le taux annuel moyen de progression des dépenses publiques par habitant a été de $2.2 \%$ dans les pays membres de l'OCDE. Les plus fortes augmentations ont eu lieu en Corée (6.1\% en moyenne annuelle) et en Estonie (5\%) ; les dépenses publiques par habitant n'ont diminué qu'en Israël et, ce, modérément $(0.1 \%)$.

\section{Méthodologie et définitions}

Les données sur les dépenses publiques sont tirées de la base de données des Statistiques de l'OCDE sur les comptes nationaux, qui sont établies conformément au Système de comptabilité nationale (SCN) ; ce dernier constitue un ensemble de concepts, de définitions, de nomenclatures et de règles de comptabilisation approuvés au plan international en matière de comptabilité nationale. Selon la terminologie du SCN, les administrations publiques se composent de l'administration centrale, des administrations d'États fédérés, des administrations locales et des administrations de sécurité sociale. Les dépenses comprennent la consommation intermédiaire, la rémunération des personnels, les subventions, les prestations sociales, les autres dépenses courantes (dont les intérêts de la dette), les transferts en capital et les autres dépenses en capital.

Le produit intérieur brut (PIB) est la mesure habituelle de la valeur des biens et services produits par un pays au cours d'une période donnée. On a calculé la dépense publique par habitant en convertissant les dépenses publiques totales en USD de 2011 avec les parités de pouvoir d'achat (PPA) OCDE/Eurostat pour le PIB et en divisant le résultat obtenu par la population (dans le cas des pays pour lesquels les données sont tirées des Perspectives de l'économie mondiale du FMI, on a utilisé un taux de change implicite basé sur les PPA). La PPA (parité de pouvoir d'achat) est le nombre d'unités monétaires du pays $B$ nécessaire pour acquérir une même quantité de biens et de services dans le pays A.

\section{Pour en savoir plus}

OCDE (2013), Panorama des comptes nationaux 2013, Éditions OCDE, Paris, http://dx.doi.org/10.1787/na_glance-2013-fr.

\section{Notes relatives aux graphiques}

On ne dispose pas de données pour le Chili. Les données relatives au Canada, à la Fédération de Russie et à la Nouvelle-Zélande portent sur 2010 et non 2011. Les données relatives au Japon et au Mexique pour 2001 sont des estimations. Les données relatives à la Fédération de Russie portent sur 2002 et non 2001.

3.22 : On ne dispose pas de données relatives à la Turquie pour 2001, et ce pays n'est pas compris dans la moyenne OCDE.

3.24 : On ne dispose pas de données pour la Turquie.

Informations sur les données concernant Israël : http://dx.doi.org/10.1787/ 888932315602 . 
3.22. Dépenses des administrations publiques en pourcentage du PIB (2001, 2009 et 2011)

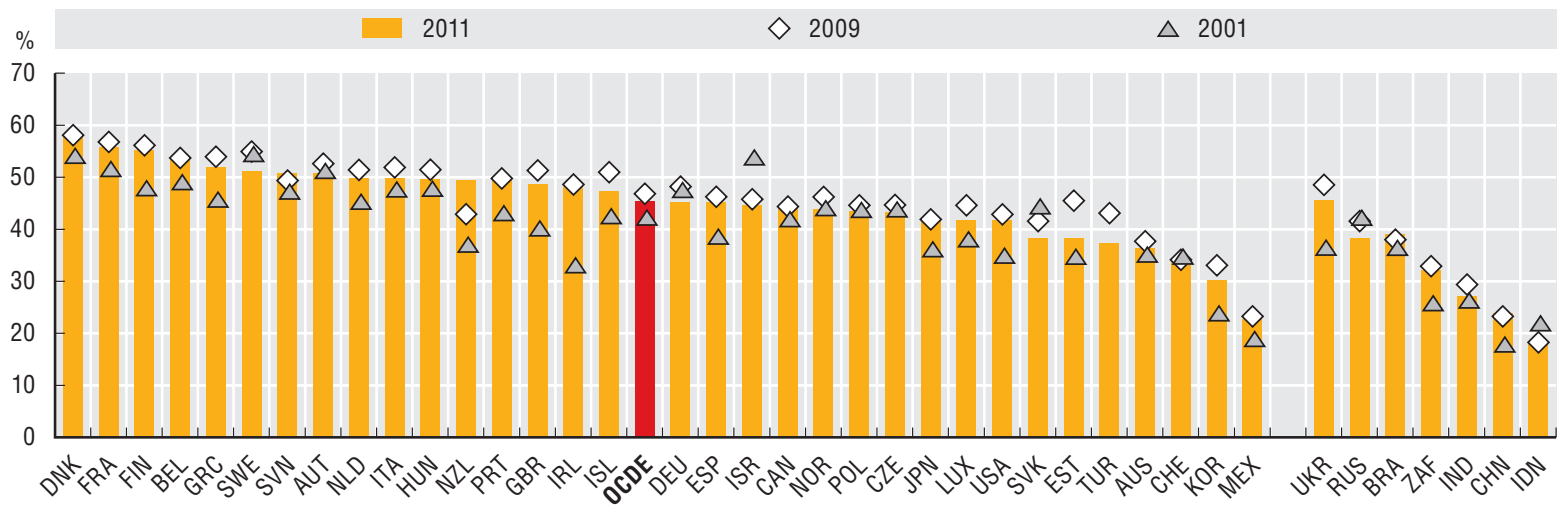

Source : Données sur les pays membres de l'OCDE : Statistiques de l'OCDE sur les comptes nationaux (base de données). Données sur les autres grandes économies (à l'exclusion de la Fédération de Russie) : Fonds monétaire international (2013), Perspectives de l'économie mondiale, avril 2013, FMI, Washington, DC.

\subsection{Dépenses des administrations publiques par habitant (2011)}

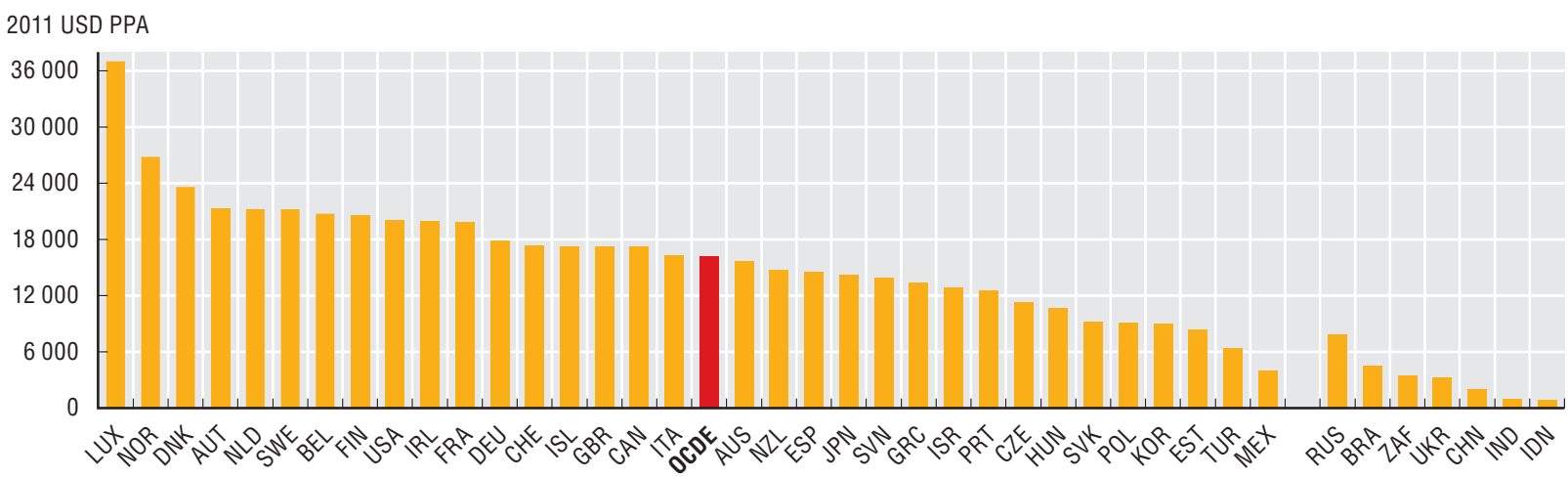

Source: Données sur les pays membres de l'OCDE : Statistiques de l'OCDE sur les comptes nationaux (base de données). Données sur les autres grandes économies (à l'exclusion de la Fédération de Russie) : Fonds monétaire international (2013), Perspectives de l'économie mondiale, avril 2013, FMI, Washington, DC.

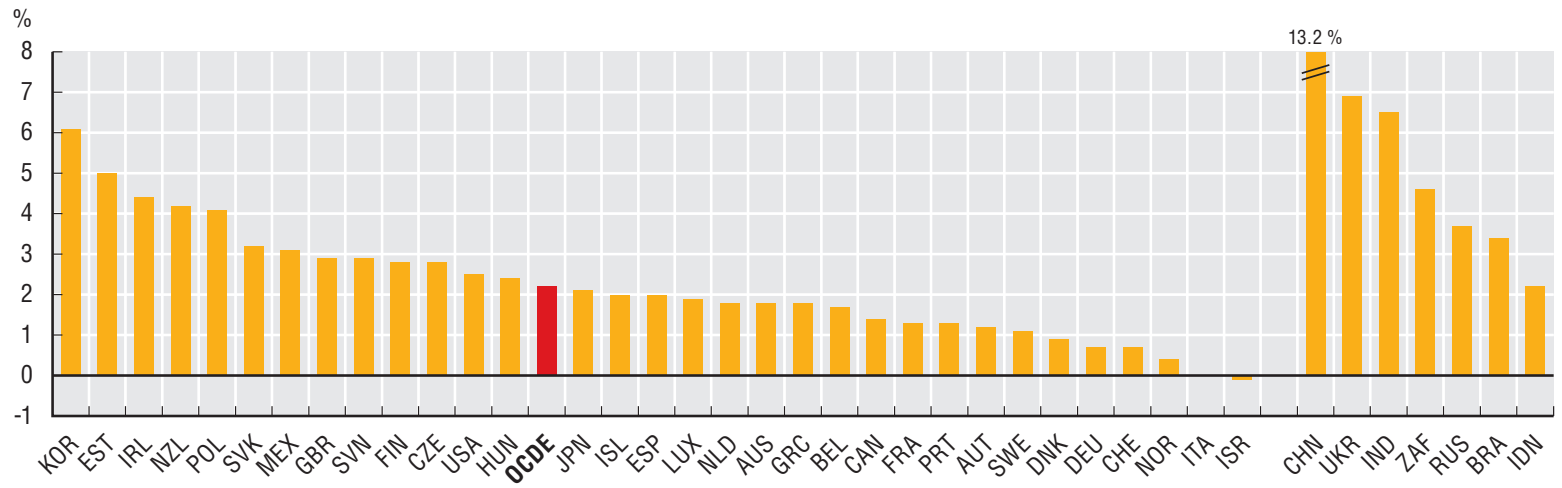

Source : Données sur les pays membres de l'OCDE : Statistiques de l'OCDE sur les comptes nationaux (base de données). Données sur les autres grandes économies (à l'exclusion de la Fédération de Russie) : Fonds monétaire international (2013), Perspectives de l'économie mondiale, avril 2013, FMI, Washington, DC. 
Les administrations consacrent l'argent public à une large gamme de biens et de services allant de la garde d'enfants à la construction de ponts ou aux subventions aux sources d'énergie alternative. Les engagements internationaux en faveur d'objectifs communs peuvent aussi influer sur la ventilation des dépenses; on peut citer le cas des pays de l'OCDE membres de l'Union européenne, qui poursuivent des objectifs communs concernant, entre autres, la croissance économique, l'agriculture, l'énergie, les infrastructures et la recherche-développement.

Sauf en Corée et aux États-Unis, c'est la fonction «protection sociale ", qui fait une grande place aux pensions de vieillesse mais qui englobe aussi les prestations de chômage et d'invalidité, qui constitue le principal poste de dépenses publiques dans l'ensemble des pays membres de l'OCDE : en moyenne, $35.6 \%$ du total en 2011. En Corée, c'est la fonction " affaires économiques" (qui englobe les aides sectorielles), tandis qu'aux États-Unis, c'est la fonction « santé », avec $21.4 \%$ du total des dépenses en 2011.

Bien que la ventilation des dépenses des administrations publiques reflète des préférences d'ordre social et stratégique, elle est aussi influencée par des facteurs comme le vieillissement de la population ou une dette publique élevée imposant le paiement d'intérêts substantiels. La répartition des ressources entre les différents secteurs de l'action publique a évolué de 2001 à 2011. Les pays membres de l'OCDE ont accru la proportion affectée à la protection sociale et à la santé de respectivement 2 et 1.2 points de pourcentage en moyenne. La fonction " loisirs, culture et culte " a également bénéficié d'une légère hausse (0.05 point). Le redéploiement des dépenses au profit de la protection sociale est surtout dû à l'incidence de la crise financière et économique (allocations de chômage et autres prestations sociales), tandis que le coût des nouvelles technologies médicales, l'innovation et le vieillissement de la population expliquent probablement le redéploiement vers les dépenses de santé. On observe, parallèlement, un recul de la part des autres fonctions: services généraux des administrations publiques (1.1 point) ; affaires économiques (0.6 point) ; défense et logement et équipements collectifs ( 0.5 point chacun); éducation ( 0.3 point) ; et, enfin, ordre et sécurité publics et protection de l'environnement (0.1 point chacun).

La santé est un bon exemple de variations du niveau des dépenses au sein d'un domaine de l'action publique. De 2001 à 2011, la part des dépenses de santé a augmenté dans tous les pays membres de l'OCDE, à l'exception de l'Irlande, de l'Islande, du Luxembourg, du Portugal et de la Slovénie. Toutefois, ces dernières années, cette tendance s'est ralentie ou inversée sous l'effet de la récession et de mesures visant à contenir activement la hausse des dépenses de santé. En Irlande, par exemple, l'essentiel du freinage a été obtenu grâce à des réductions de salaires, à la compression des effectifs du secteur ainsi qu'à la baisse des tarifs accordés aux professionnels de la santé et aux laboratoires pharmaceutiques. L'Islande a gelé les investissements dans les infrastructures de santé, tout en obtenant des gains d'efficience au moyen de la fusion d'hôpitaux.

\section{Méthodologie et définitions}

Les données sur les dépenses publiques sont tirées de la base de données des Statistiques de l'OCDE sur les comptes nationaux, qui sont établies conformément au Système de comptabilité nationale (SCN) ; ce dernier constitue un ensemble de concepts, de définitions, de nomenclatures et de règles de comptabilisation approuvés au plan international en matière de comptabilité nationale. Les données sur les dépenses sont ventilées selon la Classification des fonctions des administrations publiques (CFAP ou COFOG), qui classe les dépenses publiques selon dix fonctions : services généraux des administrations publiques ; défense ; ordre et sécurité publics; affaires économiques; protection de l'environnement; logement et équipements collectifs ; santé ; loisirs, culture et culte; enseignement; et protection sociale. On trouvera à l'annexe B des précisions sur la nature des dépenses comprises dans chaque catégorie. Les administrations publiques se composent de l'administration centrale, des administrations d'États fédérés (c'est-à-dire les administrations au niveau des États fédérés ou des provinces), des administrations locales et des administrations de sécurité sociale. Le tableau 3.27 (consultable en ligne à l'adresse http://dx.doi.org/10.1787/ 888932943324) et le tableau 3.28 (consultable en ligne à l'adresse http://dx.doi.org/10.1787/888932943343) présentent les dépenses des administrations publiques par fonction en pourcentage du PIB en 2011 et l'évolution intervenue entre 2001 et 2011.

La ventilation détaillée des dépenses publiques pour certaines fonctions de la CFAP est présentée au graphique 3.29 (services généraux des administrations publiques), au graphique 3.30 (ordre et sécurité publics), au graphique 3.31 (affaires économiques), au graphique 3.32 (santé), au graphique 3.33 (enseignement) et au graphique 3.34 (protection sociale). Ces graphiques sont consultables en ligne aux adresses http://dx.doi.org/ 10.1787/888932941766, http://dx.doi.org/10.1787/ 888932941785, http://dx.doi.org/10.1787/888932941804, http://dx.doi.org/10.1787/888932941823, http://dx.doi.org/ 10.1787/888932941842et http://dx.doi.org/10.1787/ 888932941861, respectivement.

\section{Pour en savoir plus}

OCDE (2012), Panorama de la santé : Europe 2012, Éditions OCDE, Paris, http://dx.doi.org/10.1787/9789264183896-en.

\section{Notes relatives aux tableaux}

On ne dispose pas de données pour le Canada, le Chili, le Mexique et la Nouvelle-Zélande.

3.26: On ne dispose pas de séries temporelles pour le Japon, la Suisse et la Turquie. Les données relatives à la Pologne portent sur 2002 et non 2001.

Informations sur les données concernant Israël : http://dx.doi.org/10.1787/ 888932315602 . 
3.25. Ventilation des dépenses des administrations publiques par fonction (2011)
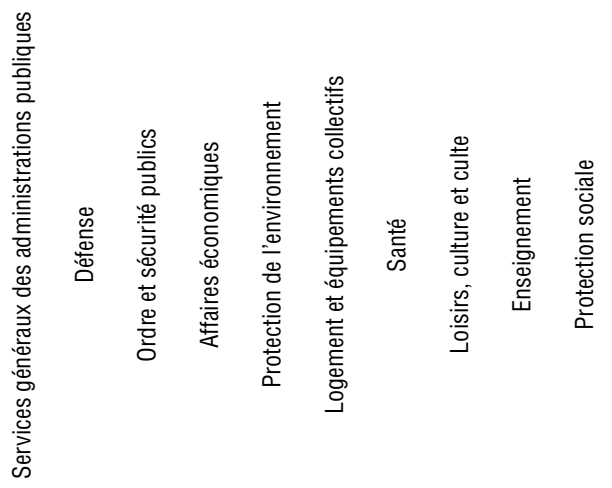

\begin{tabular}{|c|c|c|c|c|c|c|c|c|c|c|}
\hline Allemagne & 13.6 & 2.4 & 3.5 & 7.8 & 1.5 & 1.2 & 15.5 & 1.8 & 9.4 & 43.3 \\
\hline Australie & 12.5 & 4.1 & 4.8 & 11.4 & 2.6 & 1.8 & 19.2 & 2.1 & 14.5 & 27.1 \\
\hline Autriche & 13.1 & 1.4 & 2.9 & 10.5 & 1.0 & 1.2 & 15.3 & 2.0 & 11.0 & 41.6 \\
\hline Belgique & 15.0 & 1.8 & 3.4 & 12.3 & 1.4 & 0.7 & 14.8 & 2.4 & 11.6 & 36.6 \\
\hline Corée & 15.2 & 8.6 & 4.2 & 20.1 & 2.4 & 3.3 & 15.2 & 2.2 & 15.8 & 13.1 \\
\hline Danemark & 13.7 & 2.4 & 2.0 & 6.1 & 0.7 & 0.6 & 14.5 & 2.8 & 13.5 & 43.8 \\
\hline Espagne & 12.5 & 2.3 & 4.8 & 11.6 & 2.1 & 1.3 & 14.1 & 3.3 & 10.5 & 37.4 \\
\hline Estonie & 8.4 & 4.1 & 5.6 & 12.0 & -0.9 & 1.6 & 13.3 & 5.0 & 16.9 & 34.2 \\
\hline États-Unis & 12.4 & 11.7 & 5.5 & 9.4 & 0.0 & 2.1 & 21.4 & 0.7 & 15.5 & 21.3 \\
\hline Finlande & 13.3 & 2.6 & 2.7 & 8.8 & 0.5 & 1.0 & 14.2 & 2.2 & 11.6 & 43.1 \\
\hline France & 11.5 & 3.2 & 3.1 & 6.3 & 1.9 & 3.4 & 14.7 & 2.5 & 10.8 & 42.6 \\
\hline Grèce & 24.6 & 4.6 & 3.3 & 6.2 & 1.0 & 0.4 & 11.6 & 1.2 & 7.9 & 39.3 \\
\hline Hongrie & 17.5 & 2.3 & 3.9 & 14.4 & 1.5 & 1.6 & 10.4 & 3.5 & 10.5 & 34.5 \\
\hline Irlande & 11.4 & 0.9 & 3.7 & 16.4 & 2.1 & 1.3 & 15.6 & 1.8 & 10.9 & 35.9 \\
\hline Islande & 17.8 & 0.1 & 3.1 & 12.4 & 1.3 & 0.7 & 16.1 & 7.0 & 17.1 & 24.6 \\
\hline Israël & 14.7 & 14.7 & 3.8 & 5.8 & 1.5 & 1.0 & 12.3 & 3.9 & 16.5 & 25.9 \\
\hline Italie & 17.3 & 3.0 & 4.0 & 7.1 & 1.8 & 1.4 & 14.7 & 1.1 & 8.5 & 41.0 \\
\hline Japon & 11.0 & 2.2 & 3.1 & 9.8 & 2.9 & 1.8 & 17.3 & 0.8 & 8.4 & 42.7 \\
\hline Luxembourg & 11.4 & 1.0 & 2.5 & 9.9 & 2.8 & 1.8 & 11.4 & 4.0 & 12.1 & 43.2 \\
\hline Norvège & 9.7 & 3.6 & 2.2 & 9.6 & 1.5 & 1.6 & 16.5 & 2.9 & 12.6 & 39.8 \\
\hline Pays-Bas & 11.2 & 2.7 & 4.2 & 10.9 & 3.3 & 1.2 & 17.0 & 3.5 & 11.6 & 34.5 \\
\hline Pologne & 13.4 & 2.7 & 4.2 & 13.0 & 1.6 & 2.0 & 10.9 & 3.0 & 12.8 & 36.6 \\
\hline Portugal & 17.1 & 2.7 & 4.0 & 8.2 & 1.1 & 1.3 & 13.8 & 2.2 & 12.9 & 36.7 \\
\hline République slovaque & 15.4 & 2.7 & 6.4 & 9.8 & 2.7 & 2.6 & 15.5 & 3.0 & 10.6 & 31.3 \\
\hline République tchèque & 10.7 & 2.1 & 4.3 & 13.9 & 3.1 & 1.9 & 18.1 & 2.9 & 11.4 & 31.7 \\
\hline Royaume-Uni & 11.6 & 5.1 & 5.3 & 5.3 & 2.0 & 1.8 & 16.5 & 2.1 & 13.4 & 36.8 \\
\hline Slovénie & 12.4 & 2.3 & 3.3 & 11.4 & 1.6 & 1.3 & 13.5 & 3.7 & 13.2 & 37.3 \\
\hline Suède & 14.4 & 2.9 & 2.7 & 8.2 & 0.7 & 1.5 & 13.7 & 2.2 & 13.3 & 40.5 \\
\hline Suisse & 9.9 & 2.9 & 5.0 & 13.7 & 2.3 & 0.6 & 6.1 & 2.6 & 17.9 & 39.0 \\
\hline Turquie & 16.4 & 4.1 & 5.2 & 11.9 & 1.1 & 3.5 & 12.1 & 2.3 & 11.4 & 31.9 \\
\hline OCDE & 13.6 & 3.6 & 3.9 & 10.5 & 1.6 & 1.6 & 14.5 & 2.7 & 12.5 & 35.6 \\
\hline
\end{tabular}

Source: Statistiques de l'OCDE sur les comptes nationaux (base de données). Pour l'Australie, les données sont tirées des statistiques relatives aux finances publiques fournies par le Bureau australien des statistiques.

StatLink AाISL http://dx.doi.org/10.1787/888933159109
3.26. Évolution de la ventilation des dépenses des administrations publiques par fonction (2001-11)

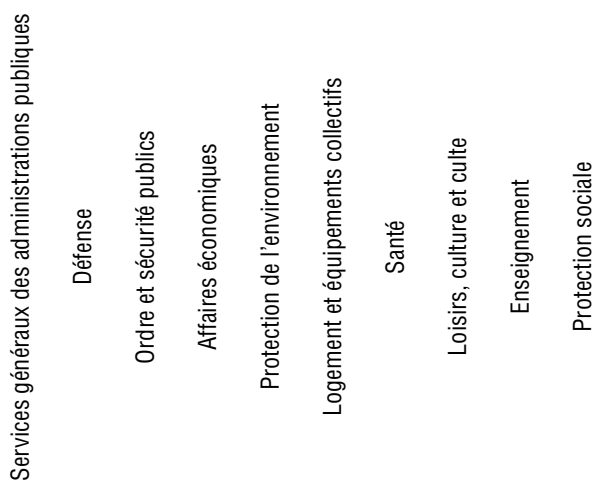

\begin{tabular}{lrrrrrrrrrr}
\hline Australie & 0.4 & -0.4 & 0.3 & -1.4 & 1.0 & -0.7 & 2.4 & -0.1 & 0.0 & -1.5 \\
Allemagne & 1.0 & 0.0 & 0.1 & -1.4 & 0.0 & -0.9 & 1.4 & 0.0 & 0.7 & -0.8 \\
Autriche & -2.5 & -0.3 & 0.0 & 0.3 & 0.0 & -0.3 & 1.7 & 0.1 & 0.2 & 0.8 \\
Belgique & -6.3 & -0.7 & 0.2 & 3.4 & -0.1 & 0.1 & 1.5 & 0.5 & -0.2 & 1.6 \\
Corée & 1.8 & -1.9 & -0.9 & -3.2 & -0.3 & -0.6 & 4.2 & -0.1 & -2.2 & 3.2 \\
Danemark & -1.6 & -0.6 & 0.2 & 0.1 & -0.4 & -0.7 & 1.9 & -0.3 & -0.1 & 1.5 \\
Espagne & -2.1 & -0.5 & 0.0 & -0.2 & -0.2 & -1.3 & 0.8 & -0.1 & -0.6 & 4.2 \\
Estonie & -0.9 & 0.2 & -1.4 & 1.4 & -3.0 & -0.2 & 1.6 & -0.7 & -2.1 & 5.0 \\
Etats-Unis & -2.5 & 2.3 & -0.4 & -1.7 & 0.0 & 0.5 & 2.2 & -0.2 & -2.0 & 1.7 \\
Finlande & -1.1 & -0.1 & -0.1 & -0.8 & -0.2 & 0.1 & 2.0 & -0.1 & -0.9 & 1.2 \\
France & -2.7 & -0.7 & 0.2 & -0.5 & 0.3 & -0.2 & 1.0 & 0.4 & -0.6 & 2.9 \\
Grèce & 1.1 & -2.8 & 0.7 & -3.8 & -0.2 & -0.5 & 0.3 & 0.4 & 1.8 & 2.8 \\
Hongrie & -4.6 & -0.3 & -0.5 & 1.9 & -0.1 & -0.2 & 0.1 & 0.2 & -0.6 & 4.0 \\
Irlande & 0.4 & -1.0 & -1.1 & 3.1 & -0.7 & -4.3 & -3.1 & -0.2 & -2.6 & 9.5 \\
Islande & 2.5 & 0.0 & -0.4 & -4.0 & -0.4 & -0.2 & -2.5 & -0.1 & -1.1 & 6.1 \\
Israël & -4.3 & -1.2 & 0.7 & 0.3 & 0.3 & -0.7 & 1.2 & 0.5 & 1.8 & 1.6 \\
Italie & -2.9 & 0.6 & 0.1 & -1.9 & 0.0 & -0.3 & 1.6 & -0.7 & -1.3 & 4.8 \\
Luxembourg & -0.6 & 0.2 & 0.1 & 2.7 & -0.5 & -0.3 & -1.3 & -0.4 & 0.1 & 0.0 \\
Norvège & -1.8 & -0.7 & -0.1 & -1.0 & 0.3 & 0.8 & 0.3 & 0.5 & -0.7 & 2.4 \\
Pays-Bas & -3.6 & -0.8 & 0.4 & -1.4 & 0.1 & -0.5 & 5.8 & -0.2 & 0.2 & 0.0 \\
Pologne & -0.1 & 0.0 & 0.8 & 5.0 & 0.2 & -1.7 & 1.0 & 0.6 & -1.0 & -4.9 \\
Portugal & 3.0 & -0.5 & 0.0 & -4.8 & -0.5 & -1.0 & -1.1 & -0.8 & -2.2 & 7.8 \\
République slovaque & -1.7 & -2.3 & 0.2 & -5.2 & 1.0 & 0.8 & 4.5 & 0.9 & 3.3 & -1.5 \\
République tchèque & 1.6 & -1.4 & -0.6 & -6.4 & 1.0 & -0.7 & 2.2 & 0.5 & 1.5 & 2.3 \\
Royaume-Uni & 1.0 & -0.6 & -0.4 & -1.0 & 0.3 & 0.0 & 1.8 & -0.4 & 0.3 & -1.0 \\
Slovénie & -2.0 & -0.4 & -0.7 & 2.0 & 0.0 & -0.1 & -0.4 & 1.1 & -0.6 & 1.1 \\
Suède & -0.5 & -1.0 & 0.2 & 1.0 & 0.1 & -0.3 & 1.8 & 0.2 & 0.1 & -1.6 \\
OCDE & $-\mathbf{1 . 1}$ & $-\mathbf{0 . 5}$ & $-\mathbf{0 . 1}$ & $-\mathbf{0 . 6}$ & $-\mathbf{0 . 1}$ & $-\mathbf{0 . 5}$ & $\mathbf{1 . 2}$ & $\mathbf{0 . 0}$ & $-\mathbf{0 . 3}$ & $\mathbf{2 . 0}$ \\
\hline Source: Statstan & & & & & & & & & & \\
\hline
\end{tabular}

Source: Statistiques de l'OCDE sur les comptes nationaux (base de données). Pour l'Australie, les données sont tirées des statistiques relatives aux finances publiques fournies par le Bureau australien des statistiques. StatLink Aiाst http://dx.doi.org/10.1787/888933159110 
Les différents niveaux d'administration se partagent la responsabilité de financer les biens et les services publics et de remplir un rôle redistributif. Leur degré de responsabilité en matière de dépenses dépend de l'organisation institutionnelle de chaque pays et de la répartition des prérogatives en matière de dépenses entre les échelons administratifs. Lorsque les échelons infranationaux sont plus autonomes, ils sont peutêtre mieux à même d'influer sur les politiques et les programmes publics.

En 2011 et en moyenne des pays de l'OCDE, l'administration centrale effectuait $46 \%$ des dépenses des administrations publiques. Les administrations infranationales (administrations d'États fédérés et administrations locales) en effectuaient $32 \%$, et les administrations de sécurité sociale représentaient le solde. Toutefois, le degré de décentralisation budgétaire est extrêmement variable selon les pays. En Irlande, par exemple, $76.4 \%$ du total des dépenses relèvent de l'administration centrale, soit une hausse de 27.1 points de pourcentage par rapport à 2001. En revanche, l'administration centrale procède à moins de $20 \%$ des dépenses publiques en Allemagne et en Suisse, pays qui ont tous deux une structure fédérale.

En général, l'administration centrale affecte une proportion relativement élevée de son budget à la protection sociale (pensions et allocations de chômage, par exemple), aux services généraux des administrations publiques (organes exécutifs et législatifs et opérations sur la dette publique, par exemple) et à la défense. Dans plus de la moitié des pays membres de l'OCDE, les dépenses de protection sociale constituent le principal poste budgétaire de l'administration centrale. En Belgique et en Espagne, l'administration centrale consacre plus de $60 \%$ de son budget aux services généraux des administrations publiques.

Sauf en Autriche, l'enseignement constitue le principal poste de dépenses au niveau des administrations d'États fédérés et des administrations locales. En outre, la protection de l'environnement, le logement et les équipements collectifs ainsi que les loisirs, la culture et le culte sont surtout financés par les administrations infranationales, ce qui illustre le lien plus immédiat et plus tangible de ces administrations avec les citoyens.

Dans tous les pays membres de l'OCDE, il y a une corrélation positive entre les dépenses et les recettes infranationales. Toutefois, l'autonomie fiscale des administrations infranationales est très variable. Les limites imposées à ces administrations en ce qui concerne la fixation de l'assiette, des taux et des allègements applicables aux impôts locaux pèsent sur leur aptitude à générer leurs propres sources de recettes, à s'adapter aux chocs économiques en relevant les taux d'imposition et, potentiellement, à assurer des services publics davantage "sur mesure ". C'est pourquoi les administrations locales ont tendance à beaucoup compter sur les transferts en provenance de l'administration centrale. Dans les pays membres de l'OCDE, on ne discerne pas de tendance claire à la décentralisation budgétaire.

\section{Méthodologie et définitions}

Les données sur les dépenses publiques sont tirées de la base de données des Statistiques de l'OCDE sur les comptes nationaux, qui sont établies conformément au Système de comptabilité nationale (SCN) ; ce dernier constitue un ensemble de concepts, de définitions, de nomenclatures et de règles de comptabilisation approuvés au plan international en matière de comptabilité nationale. Les données sur les dépenses sont ventilées selon la Classification des fonctions des administrations publiques (CFAP ou COFOG), qui classe les dépenses publiques selon dix fonctions : services généraux des administrations publiques ; défense ; ordre et sécurité publics; affaires économiques; protection de l'environnement; logement et équipements collectifs ; santé ; loisirs, culture et culte ; enseignement; et protection sociale. On trouvera à l'annexe B des précisions sur la nature des dépenses comprises dans chaque catégorie. Les administrations publiques se composent de l'administration centrale, des administrations d'États fédérés (c'est-à-dire les administrations au niveau des États fédérés ou des provinces), des administrations locales et des administrations de sécurité sociale. Les administrations classées dans la catégorie des administrations d'États fédérés n'existent que dans les neuf pays membres de l'OCDE dotés d'une structure fédérale: l'Allemagne, l'Australie, l'Autriche, la Belgique, le Canada, l'Espagne (pays considéré comme quasi-fédéral), les États-Unis, le Mexique et la Suisse.

Comme les données des graphiques 3.35 et 3.37 ("Évolution de la ventilation des dépenses des administrations publiques selon les niveaux d'administration (2009-2011) ") excluent les transferts entre niveaux d'administration, elles donnent une représentation approximative de la responsabilité globale de chaque niveau d'administration en matière de fourniture de biens et de services. En revanche, comme les données sur les dépenses au niveau des administrations centrales, des administrations d'États fédérés et des administrations locales (tableaux 3.38, 3.39 et 3.40) incluent les transferts entre les différents niveaux d'administration, elles montrent le montant dépensé pour chaque fonction à chaque niveau d'administration. Le graphique 3.37 (http://dx.doi.org/ 10.1787/888932941918) ainsi que les tableaux 3.38 (http:/ /dx.doi.org/10.1787/888932941937), 3.39 (http://dx.doi.org/ $10.1787 / 888932941956)$ et 3.40 (http://dx.doi.org/10.1787/ 888932941975) sont consultables en ligne. 


\section{Pour en savoir plus}

Blöchliger, H. et C. Vammalle (2012), Reforming Fiscal Federalism and Local Government: Beyond the Zero-Sum Game, OECD Fiscal Federalism Studies, Éditions OCDE, Paris, http://dx.doi.org/ 10.1787/9789264119970-en.

OCDE (2013), Panorama des comptes nationaux 2013, Éditions OCDE, Paris, http://dx.doi.org/10.1787/na_glance-2013-fr.

\section{Notes relatives aux graphiques}

On ne dispose pas de données pour le Chili. Les transferts entre niveaux d'administration ne sont pas pris en compte (sauf pour
l'Australie, le Japon et la Turquie). Les données relatives au Canada et à la Nouvelle-Zélande portent sur 2010 et non 2011.

3.35 : On ne dispose pas de données relatives au Japon, à la Pologne et à la Turquie pour 2001, et ces pays ne sont pas compris dans la moyenne OCDE. Les données relatives au Mexique portent sur 2003 et non 2001. Les administrations locales sont comptabilisées dans la catégorie des " administrations d'États fédérés " pour l'Australie et les États-Unis. En Australie, il n'existe pas de système public d'assurance sociale. Les administrations de sécurité sociale sont comptabilisées dans la catégorie " administration centrale " pour les États-Unis, la Norvège, la Nouvelle-Zélande et le Royaume-Uni.

Informations sur les données concernant Israël : http://dx.doi.org/10.1787/ 888932315602 


\section{LES FINANCES ET L'ÉCONOMIE PUBLIQUES}

La ventilation des dépenses publiques selon les niveaux d'administration

3.35. Ventilation des dépenses des administrations publiques selon les niveaux d'administration (2001 et 2011)

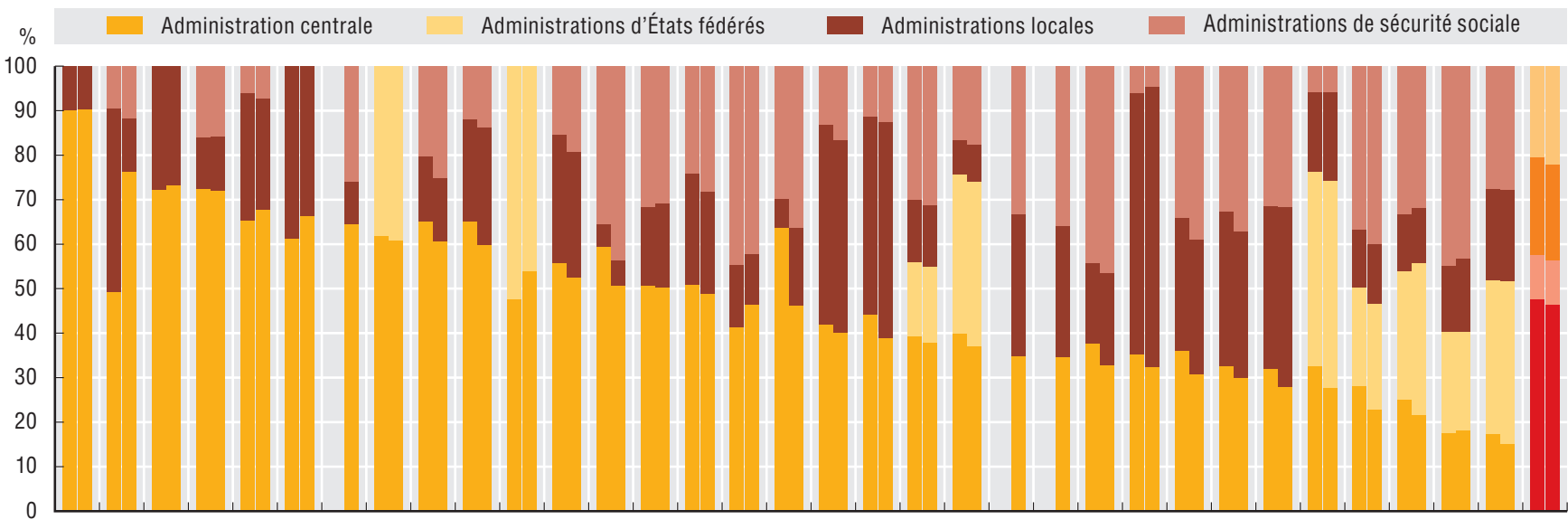

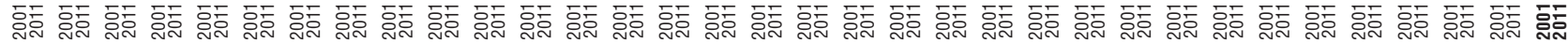

NZL IRL GBR ISR EST NOR TUR AUS PRT CZE USA ISL GRC SVN HUN LUX SVK KOR SWE AUT MEX POL JPN FRA DNK ITA NLD FIN CAN BEL ESP DEU CHE OCDE Source: Statistiques de l'OCDE sur les comptes nationaux (base de données).

3.36. Décentralisation budgétaire : part des administrations infranationales dans les recettes et les dépenses des administrations publiques (2011)

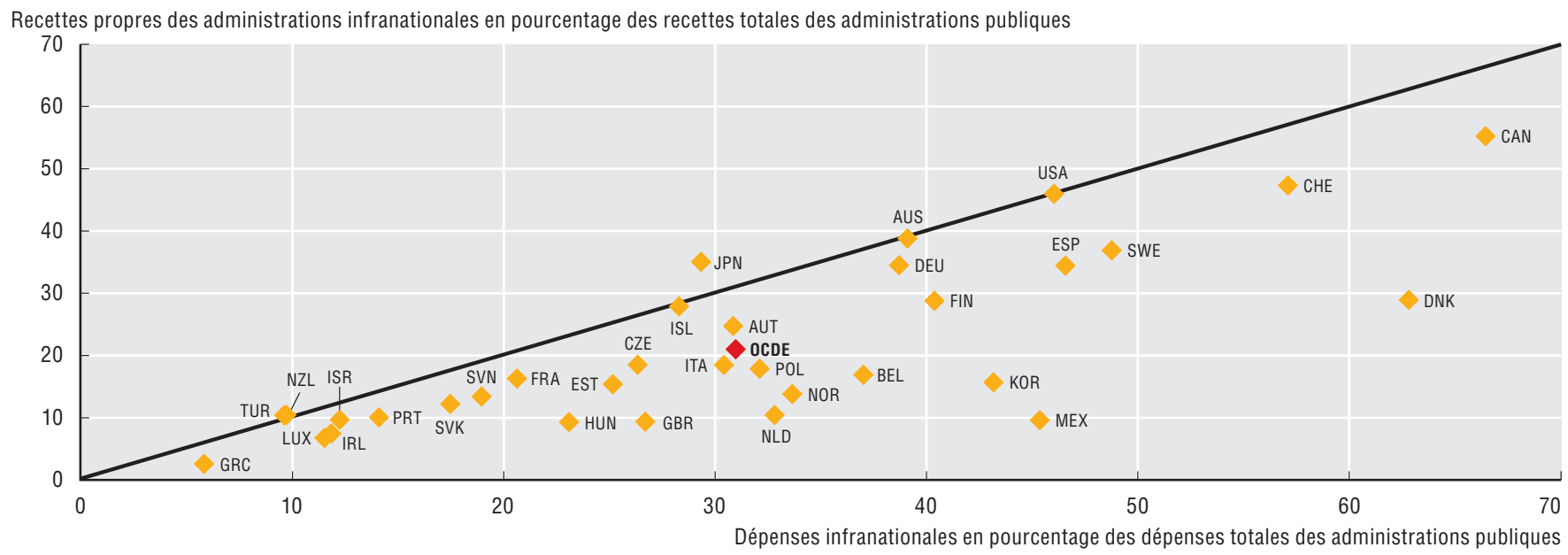

Source: Statistiques de l'OCDE sur les comptes nationaux (base de données). 
L'un des principaux objectifs de l'investissement public est de favoriser la croissance économique, notamment par le financement de projets d'infrastructures publiques (routes, logements, écoles, hôpitaux et réseaux de communication). En outre, certains projets ayant un rendement social souhaité (biens publics) ne sont pas rentables pour le secteur privé et sont donc réalisés par le secteur public. Les pouvoirs publics peuvent se servir de l'investissement public direct pour mener une action anticyclique. Ainsi, face à la récente récession économique, de nombreux gouvernements de l'OCDE ont adopté des plans de relance sous la forme d'une hausse des investissements dans les infrastructures immatérielles et matérielles.

En 2011, les dépenses d'investissement direct des administrations représentaient, en moyenne, $15.5 \%$ de l'investissement total au sein des pays membres de l'OCDE. Dans 23 d'entre eux, le pourcentage était inférieur à celui de 2009 en raison de la mise en œuvre de programmes d'austérité. De 2009 à 2011, la part de l'investissement public direct dans l'investissement total a continué à progresser en Pologne (3.6 points de pourcentage), en Irlande (1.9 point), au Danemark (1.8 point), en Hongrie (1.8 point), au Canada (1.3 point), en Australie (0.4 point), en Belgique et en Suisse (0.1 point chacune).

La part de l'investissement direct dans les dépenses des administrations publiques est très variable selon les pays. Les différences sont liées au stock existant d'infrastructures. En 2011, dans les pays membres de l'OCDE, l'investissement direct représentait en moyenne $6.7 \%$ des dépenses des administrations publiques. Dans quatre d'entre eux, cette part n'a cessé de s'élever, à la fois de 2001 à 2009 puis de 2009 à 2011. Il s'agit de la Pologne (5.4 points de pourcentage), du Canada (3.5 points), de la Suède (1.3 point) et du Danemark (0.3 point). Dans onze autres pays membres de l'OCDE, en revanche, elle a constamment diminué pendant ces deux périodes, les baisses les plus marquées étant observées en Irlande (7.5 points), en Corée (7.2 points) et en Islande (6.6 points). Dans les pays où la crise a été la plus forte, l'investissement direct des administrations publiques a subi les réductions les plus significatives pendant la phase ultérieure d'assainissement. Cela découle à la fois d'une hausse des dépenses affectées à d'autres postes (assurance chômage et autres dépenses sociales automatiques) et de mesures d'assainissement budgétaire ayant eu des retombées sur l'investissement.

La répartition des dépenses d'investissement direct entre les niveaux d'administration est étroitement liée à l'organisation politique des pays. Les investissements au niveau des administrations d'États fédérés ne concernent que les pays fédéraux, où ils représentent en moyenne plus du quart des investissements publics directs, mais atteignent des niveaux de $84 \%$ aux ÉtatsUnis et $66 \%$ en Australie. En moyenne des pays de l'OCDE, $62 \%$ environ des investissements publics directs sont réalisés par les échelons infranationaux, contre $37.3 \%$ par l'administration centrale. De 2001 à 2011, il n'y a pas eu de tendance générale à une décentralisation des investissements. Alors que dans certains pays, comme la République slovaque et la Hongrie, on a constaté un redéploiement significatif de l'administration centrale vers les administrations locales, dans d'autres, comme l'Irlande et la Pologne, la tendance a été inverse.

\section{Méthodologie et définitions}

Les données sont tirées de la base de données des Statistiques de l'OCDE sur les comptes nationaux, qui sont établies conformément au Système de comptabilité nationale (SCN); ce dernier constitue un ensemble de concepts, de définitions, de nomenclatures et de règles de comptabilisation approuvés au plan international en matière de comptabilité nationale. L'investissement des administrations publiques englobe l'investissement direct (mesuré par la formation brute de capital fixe) et l'investissement indirect (mesuré par les transferts en capital). La présente étude ne prend en compte que l'investissement direct. La formation brute de capital fixe porte surtout sur des infrastructures routières, mais englobe aussi d'autres infrastructures telles que des immeubles de bureaux, des logements, des écoles et des hôpitaux.

L'investissement total correspond aux dépenses d'investissement de l'ensemble de l'économie, c'est-àdire celles des administrations publiques, des sociétés non financières, des sociétés financières, des ménages et des institutions sans but lucratif.

Les administrations publiques se composent de l'administration centrale, des administrations d'États fédérés (c'est-à-dire les administrations au niveau des États fédérés ou des provinces), des administrations locales et des administrations de sécurité sociale. Les administrations classées dans la catégorie des administrations d'États fédérés n'existent que dans les neuf pays membres de l'OCDE dotés d'une structure fédérale : l'Allemagne, l'Australie, l'Autriche, la Belgique, le Canada, l'Espagne (pays considéré comme quasifédéral), les États-Unis, le Mexique et la Suisse. Le graphique 3.44, intitulé "Évolution de la répartition des dépenses d'investissement entre les différents niveaux d'administration (2001-2011) " est consultable en ligne à l'adresse $h t t p: / / d x . d o i . o r g / 10.1787 / 888932942051$.

\section{Pour en savoir plus}

OCDE (2013), Investir ensemble: Vers une gouvernance multiniveaux plus efficace, Éditions OCDE, Paris, http://dx.doi.org/ 10.1787/9789264200609-fr.

OCDE (2011), Making the Most of Public Investment in a Tight Fiscal Environment: Multi-Level Governance Lessons from the Crisis, Éditions OCDE, Paris, http://dx.doi.org/10.1787/ 9789264114470-en.

OCDE/Korea Institute of Public Finance (2012), Institutional and Financial Relations across Levels of Government, OECD Fiscal Federalism Studies, Éditions OCDE, Paris, http://dx.doi.org/ 10.1787/9789264167001-en. 


\section{LES FINANCES ET L'ÉCONOMIE PUBLIQUES}

\section{Les dépenses d'investissement des administrations publiques}

\section{Notes relatives aux graphiques}

Les données relatives au Canada et à la Fédération de Russie portent sur 2010 et non 2011. Les données relatives au Mexique portent sur 2003 et non 2001. Les données relatives à la Fédération de Russie portent sur 2002 et non 2001. Il y a des différences de disponibilité des données entre les graphiques 3.41 et 3.42 du fait de l'utilisation de tableaux de données différents au sein des Statistiques de l'OCDE sur les comptes nationaux (base de données).

3.41 : On ne dispose pas de données pour l'Islande. Le Chili (2001), la Grèce (2001), la Turquie (2001), Israël (2009-2011) et la NouvelleZélande (2009-2011) ne sont pas compris dans la moyenne de l'OCDE, faute de séries temporelles. Les données relatives à l'Australie et au Chili portent sur 2010 et non 2011. Les données relatives à l'Irlande portent sur 2002 et non 2001.
3.42 : On ne dispose pas de données pour le Chili. On ne dispose pas de données relatives au Japon et à la Turquie pour 2001, et ces pays ne sont pas compris dans la moyenne OCDE. Les données relatives à la Nouvelle-Zélande portent sur 2010 et non 2011.

3.43 : On ne dispose pas de données pour le Chili. Les données relatives à la Nouvelle-Zélande portent sur 2010 et non 2011. Les administrations locales sont comptabilisées dans la catégorie des " administrations d'États fédérés " pour l'Australie et les États-Unis. En Australie, il n'existe pas de système public d'assurance sociale. Les administrations de sécurité sociale sont comptabilisées dans la catégorie " administration centrale " pour les États-Unis, la Norvège, la Nouvelle-Zélande et le Royaume-Uni.

Informations sur les données concernant Israël : http://dx.doi.org/10.1787/ 888932315602 . 
3.41. Investissement public en pourcentage de l'investissement total (2001, 2009 et 2011)

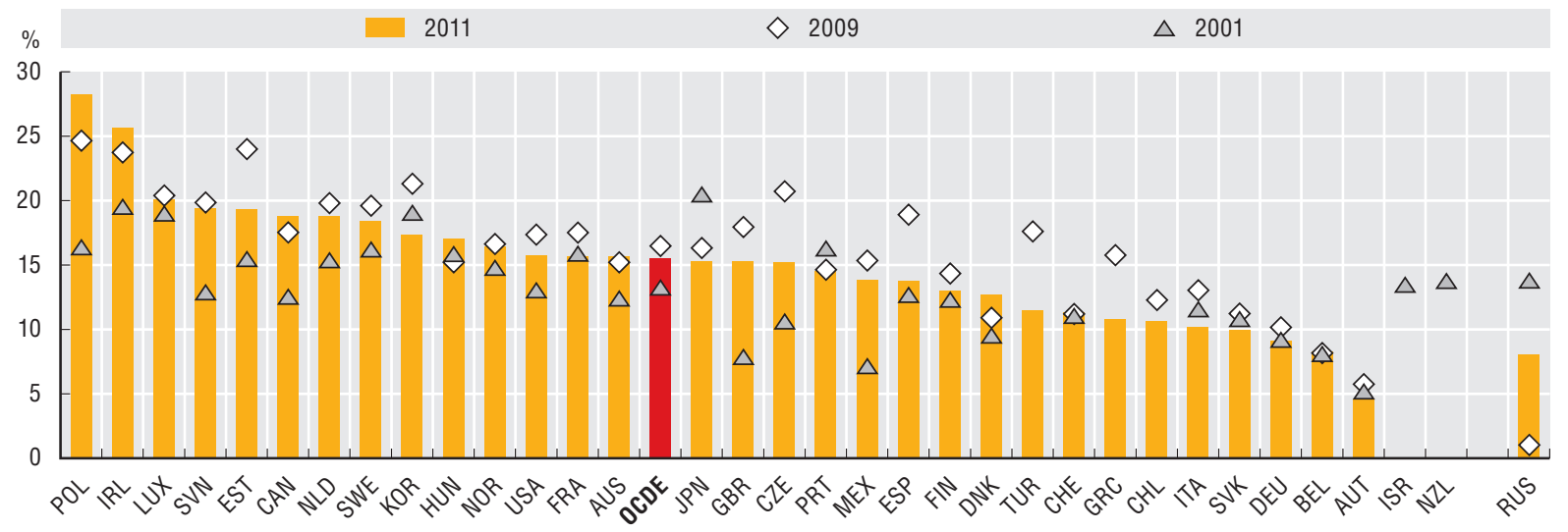

Source: Statistiques de l'OCDE sur les comptes nationaux (base de données).

3.42. Investissement public en pourcentage des dépenses publiques totales (2001, 2009 et 2011)

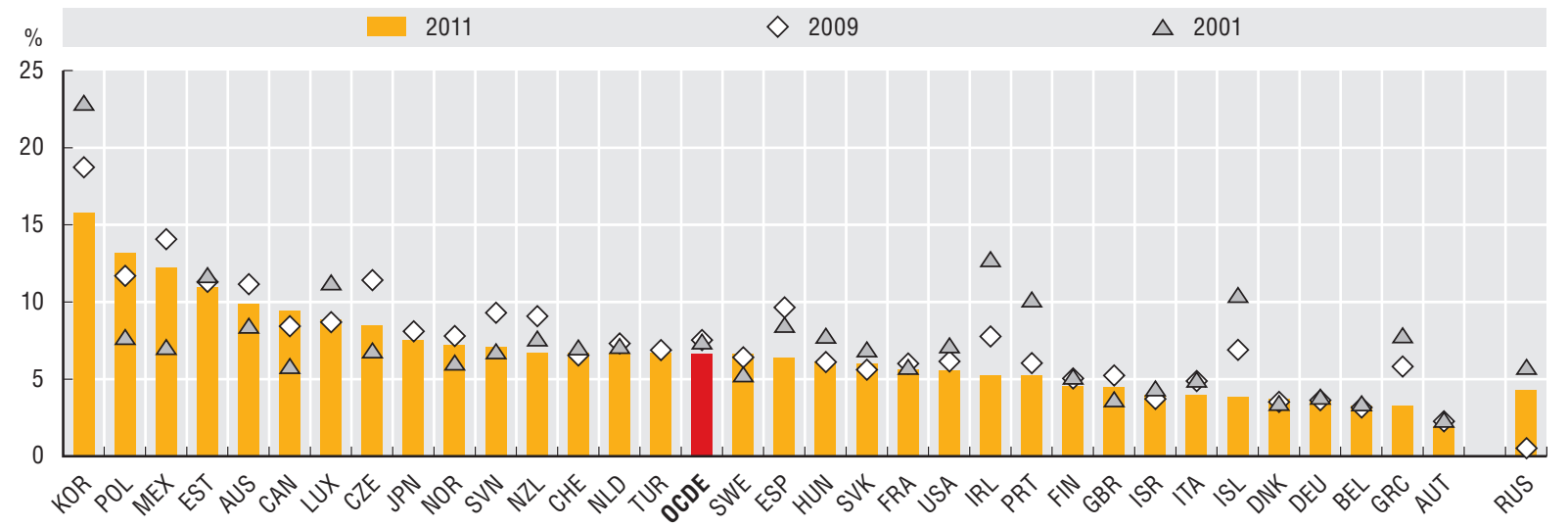

Source: Statistiques de l'OCDE sur les comptes nationaux (base de données).

StatLink क्ञाs http://dx.doi.org/10.1787/888933159275

3.43. Répartition des dépenses d'investissement entre les différents niveaux d'administration (2011)

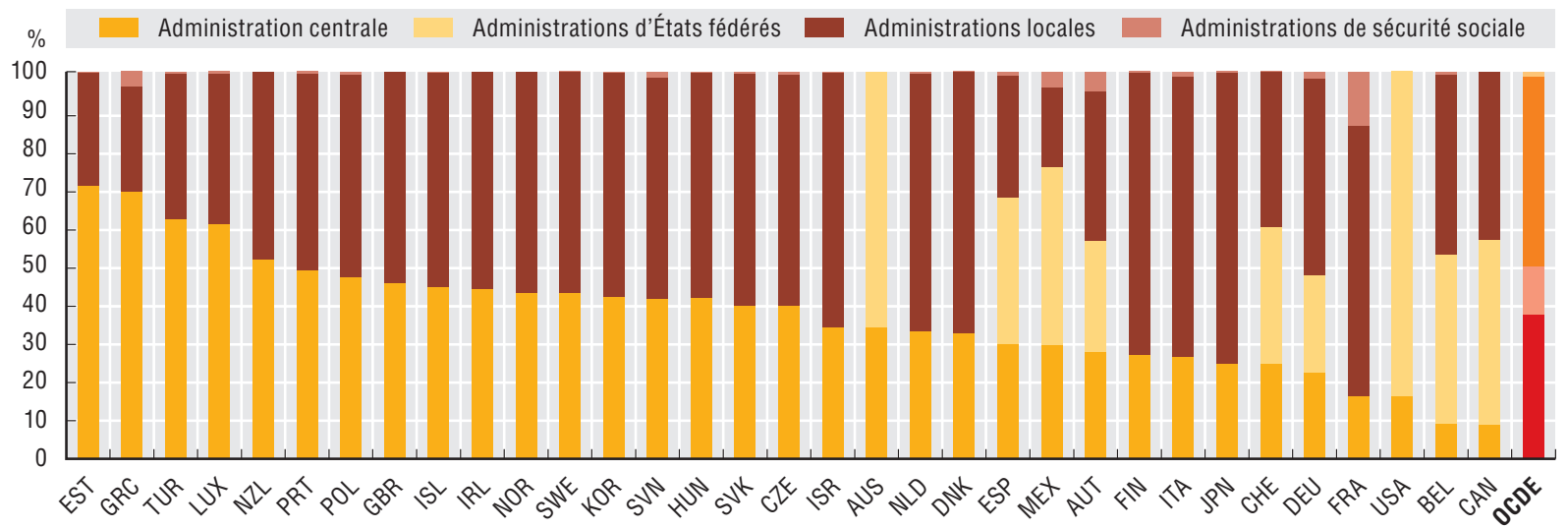

Source: Statistiques de l'OCDE sur les comptes nationaux (base de données). 
Les coûts de production correspondent à la part des dépenses publiques consacrée à la production de biens et de services. Si certaines administrations assurent elles-mêmes la production de la plupart des biens et des services publics, d'autres en confient une bonne part à des entités à but non lucratif ou privées. L'externalisation peut prendre deux formes. Les administrations peuvent, soit acquérir des biens et des services servant d'intrants (biens et services utilisés par elles, c'est-à-dire consommation intermédiaire), soit rémunérer une institution à but non lucratif ou privée pour qu'elle fournisse directement des biens et des services à l'utilisateur final (biens et services financés par les administrations, c'est-à-dire transferts sociaux en nature opérés par l'intermédiaire de producteurs marchands).

Les décisions des administrations publiques quant au volume et à la nature des biens et des services publics à produire et quant aux méthodes de production à privilégier influent sur les modalités de leur fourniture aux citoyens. On a recouru à l'externalisation pour bénéficier d'une expertise externe et pour fournir des biens et des services avec un meilleur rapport coût/efficience, bien que les résultats effectifs puissent varier. En outre, le recours à l'externalisation renforce le rôle des administrations publiques en tant que source de demande et d'emplois en dehors du secteur public. L'externalisation dans les administrations publiques est mesurée par le volume des achats de biens et de services effectués par l'administration centrale, les administrations d’États fédérés et les administrations locales.

En 2011, les coûts de production des biens et des services publics représentaient en moyenne près du quart du PIB dans les pays membres de l'OCDE, s'échelonnant de $32 \%$ au Danemark et aux Pays-Bas à $12 \%$ au Mexique. De 2001 à 2011, cette proportion a augmenté en moyenne de 1.6 point de pourcentage dans les pays de l'OCDE, mais la tendance s'est inversée après 2009, avec une contraction d'un point de pourcentage. Cet ajustement était dû, à hauteur d'environ $56 \%$, à la baisse de la part de la rémunération des personnels des administrations publiques dans le PIB.

S'agissant de la composition des coûts de production, la rémunération des personnels des administrations publiques représentait en 2011 près de la moitié des coûts (47\%), alors que l'externalisation (biens et services utilisés et financés par les administrations publiques) correspondait à une proportion moindre (44\%). La consommation de capital fixe absorbait les $9 \%$ restants.

En 2011, l'externalisation dans les administrations représentait en moyenne $10 \%$ du PIB des pays membres de l'OCDE. Toutefois, son importance était très variable, la fourchette allant de $2.8 \%$ au Mexique et de $5.4 \%$ en Suisse à $14.2 \%$ en Finlande et à $19 \%$ aux Pays-Bas. En Belgique, au Japon et en Allemagne, moins de $40 \%$ des dépenses correspondent à la consommation intermédiaire, ce qui signifie que les dépenses d'externalisation sont principalement liées à la délégation de fonctions à des tiers. En revanche, la Suisse, le Danemark, la Finlande et l'Estonie consacrent plus de $80 \%$ de leurs dépenses d'externalisation à la consommation intermédiaire, ce qui signifie que les administrations restent chargées dans une large mesure de la fourniture directe des biens et des services publics.

\section{Notes relatives aux graphiques}

Les données relatives au Canada, à la Fédération de Russie et à la

\section{Méthodologie et définitions}

Le concept et la méthodologie adoptés pour les coûts de production sont basés sur la classification existante des dépenses publiques du Système de comptabilité nationale (SCN). Selon la terminologie du SCN, les administrations publiques se composent de l'administration centrale, des administrations d'États fédérés (c'est-à-dire les administrations au niveau des États fédérés ou des provinces), des administrations locales et des administrations de sécurité sociale.

Dans le détail, les coûts de production des administrations publiques comprennent :

- la rémunération de leurs personnels, y compris la rémunération en espèces ou en nature, à quoi s'ajoute la totalité des cotisations obligatoires (et imputées) de l'employeur au régime d'assurance sociale et ses versements facultatifs pour leur compte.

- les biens et les services utilisés par les administrations publiques, qui sont la première composante de l'externalisation. Selon le SCN, il s'agit de la consommation intermédiaire (acquisition des produits intermédiaires nécessaires à la production des administrations, par exemple des prestations comptables ou informatiques).

- les biens et les services financés par les administrations publiques, qui sont la seconde composante de l'externalisation. Selon le SCN, cela inclut les transferts sociaux en nature effectués par le truchement de producteurs marchands et payés par les administrations publiques (dont ceux qui sont d'abord réglés par les administrés puis remboursés par les administrations, par exemple les traitements médicaux remboursés par le système public de sécurité sociale).

- la consommation de capital fixe (amortissement).

Les données incluent les emplois publics et la consommation intermédiaire affectés à des activités de production destinées à l'usage propre des administrations, par exemple dans le cadre de la construction de routes et d'autres projets d'investissement exécutés par des personnels publics. Les coûts de production présentés ici ne sont pas égaux à la valeur de la production indiquée dans le SCN. Le tableau 3.48, "Évolution des coûts de production en pourcentage du PIB (2009 à 2011) » est consultable en ligne à l'adresse $h t t p: / / d x . d o i . o r g / 10.1787 / 888932943362$. Le graphique 3.49, intitulé "Composition des dépenses d'externalisation des administrations publiques (2011) ", est consultable en ligne à l'adresse $h t t p: / / d x . d o i . o r g /$ $10.1787 / 888932942127$.

\section{Pour en savoir plus}

OCDE (2013), Panorama des comptes nationaux 2013, Éditions OCDE, Paris, http://dx.doi.org/10.1787/na_glance-2013-fr.

Nouvelle-Zélande portent sur 2010 et non 2011. Les données relatives au Mexique portent sur 2003 et non 2001. Les données relatives à la Fédération de Russie portent sur 2002 et non 2001. Le 
Canada, les États-Unis, l'Islande, Israël, le Mexique et le RoyaumeUni ne présentent pas séparément, dans leur comptabilité nationale, les biens et les services financés par les administrations publiques.

3.45 : On ne dispose pas de données relatives au Japon et à la Turquie pour 2001, et ces pays ne sont pas compris dans la moyenne OCDE. Les données relatives au Chili portent sur 2010 et non 2011, et concernent uniquement la rémunération des personnels (elles ne sont pas comprises dans la moyenne OCDE).

3.46 et 3.47 : On ne dispose pas de données pour le Chili.

Informations sur les données concernant Israël : http://dx.doi.org/10.1787/ 888932315602 . 


\section{LES FINANCES ET L'ÉCONOMIE PUBLIQUES}

Les coûts de production et l'externalisation dans les administrations publiques

3.45. Coûts de production en pourcentage du PIB (2001 et 2011)

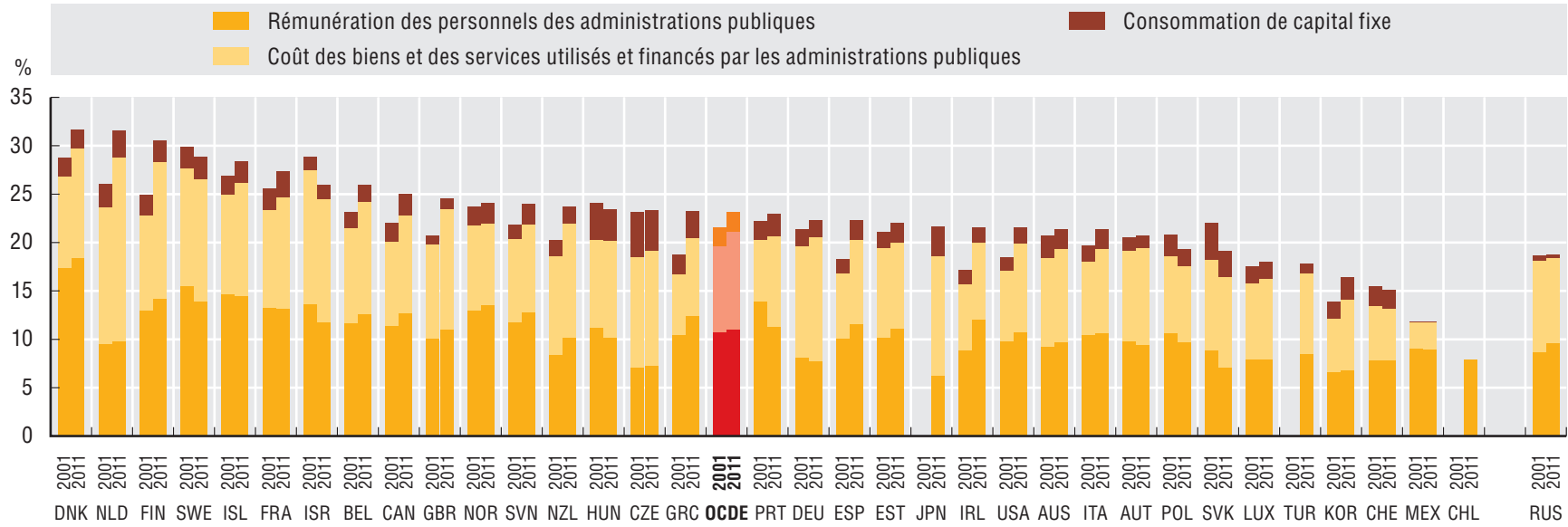

Source: Statistiques de l'OCDE sur les comptes nationaux (base de données). Les données relatives à l'Australie sont tirées des statistiques sur les finances publiques et sur les comptes nationaux fournies par le Bureau australien des statistiques.

StatLink (illst $h$ ttp://dx.doi.org/10.1787/888933159308

\subsection{Composition des coûts de production (2011)}

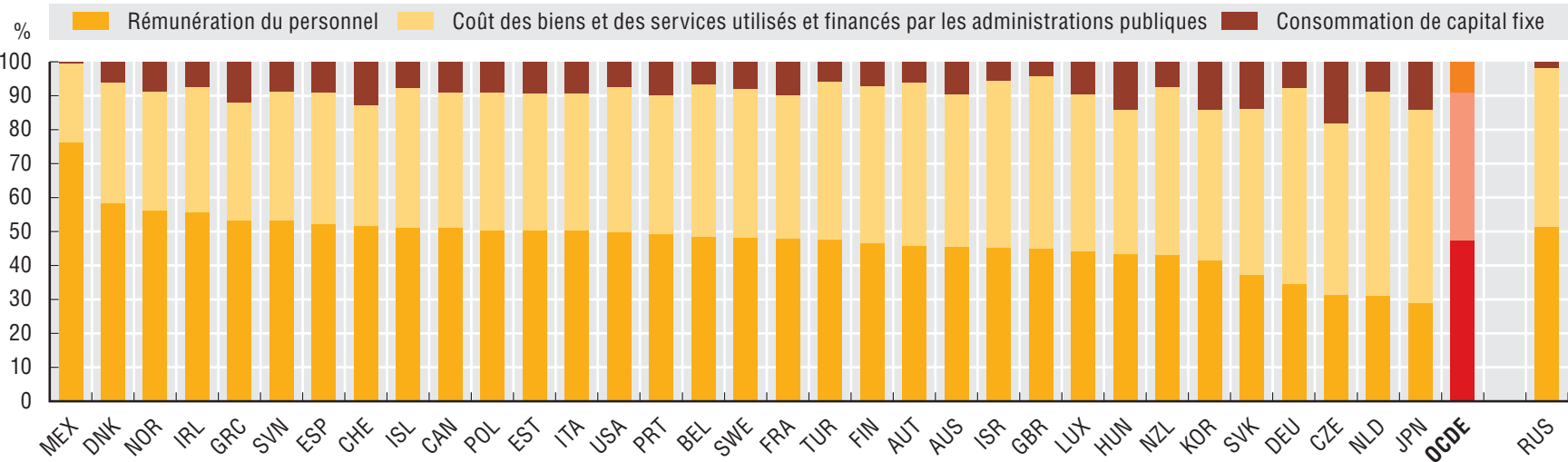

Source: Statistiques de l'OCDE sur les comptes nationaux (base de données). Les données relatives à l'Australie sont tirées des statistiques sur les finances publiques et sur les comptes nationaux fournies par le Bureau australien des statistiques.

StatLink ताIsL http://dx.doi.org/10.1787/888933159315

\subsection{Dépenses d'externalisation des administrations publiques en $\%$ du PIB (2011)}

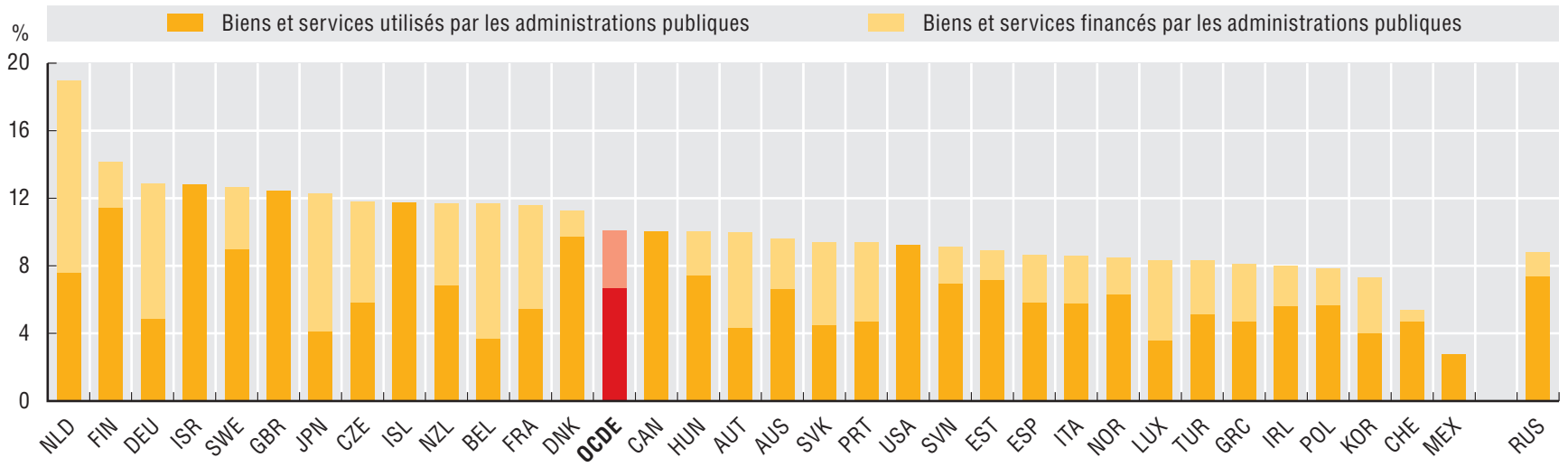

Source: Statistiques de l'OCDE sur les comptes nationaux (base de données). Les données relatives à l'Australie sont tirées des statistiques sur les finances publiques et sur les comptes nationaux fournies par le Bureau australien des statistiques. 
Les dépenses des administrations centrales en matière de TIC correspondent à la part de leur budget allouée aux technologies de l'information et des communications (par exemple, investissements en matériel et en logiciels, coûts de fonctionnement des infrastructures informatiques, rémunération des spécialistes et formation). Les pouvoirs publics voient dans le recours aux nouvelles technologies, et notamment à Internet, un moyen d'accroître l'efficience des opérations internes, d'élever la qualité des services publics et de mener une action plus optimisée et plus transparente.

Si les attentes sont fortes quant aux résultats pouvant être obtenus grâce aux TIC sur le plan de l'action publique, c'est aussi parce que les dépenses qui leur sont consacrées sont considérables. Leur montant en valeur absolue montre pourquoi il est important de comprendre les tendances sous-jacentes et d'optimiser l'emploi des TIC : aux États-Unis, ce montant s'établit à 75 milliards USD au niveau fédéral ; au Royaume-Uni, il est de 10 milliards USD ; au Canada, en France et en Australie, il se situe entre 4 et 5 milliards USD. Les dépenses consacrées aux TIC peuvent représenter plus de $2 \%$ du budget de l'administration centrale. Toutefois, il faut se garder d'interpréter une proportion élevée ou faible de dépenses de TIC comme un indicateur de priorités, de performances ou d'efficience. Il faut tenir compte d'importants facteurs d'ordre contextuel, bien que leur rôle ne soit pas encore parfaitement clair. Parmi les pays où l'administration centrale consacre plus de $1.5 \%$ de son budget aux TIC, on trouve à la fois des pays très centralisés (Nouvelle-Zélande, Finlande) et des pays fédéraux (États-Unis, Canada, Suisse) ainsi que, en termes de population, de petits pays comme de grands pays.

La dimension des administrations publiques peut aussi influer sur le profil des dépenses. En Slovénie et en Estonie, les administrations ont des effectifs similaires et dépensent un montant comparable par agent pour les infrastructures de TIC et leur fonctionnement; en Italie, en Espagne et en Allemagne, les administrations publiques sont de plus grande taille et dépensent davantage en TIC par agent (3 000 à 4000 USD à PPA environ). Les États-Unis se distinguent par une dépense moyenne par agent dépassant 26000 USD à PPA ; ils sont suivis de la Suisse, du Royaume-Uni, de la Corée et de l'Australie.

Pourtant, la dimension de l'administration publique n'explique pas tout. Les administrations centrales de l'Australie et de la Corée sont de taille similaire à celles du Chili et de la Finlande, mais dépensent à peu près trois fois plus par agent pour les TIC. En France et aux États-Unis, les effectifs employés au niveau central sont élevés comparativement à l'échantillon, mais la dépense TIC par agent est nettement supérieure aux États-Unis. On pourrait s'attendre à ce que des économies d'échelle réduisent la dépense moyenne par agent dans les administrations de dimension importante ; toutefois, la mise en œuvre des politiques publiques y est potentiellement plus complexe, ce qui tend à réduire les économies d'échelle.

Certains pays ont eu recours à des examens spécifiques de leurs dépenses en matière de TIC pour mieux analyser leurs caractéristiques, examiner les facteurs liés au contexte national et améliorer le rendement des TIC pour les administrations.

Des informations plus précises sur ces dépenses, et notamment des comparaisons de données ventilées, pourraient permettre d'améliorer les décisions prises dans ce domaine. L'analyse de telles données pourrait permettre de mieux comprendre l'évolution des différentes catégories de dépenses de TIC (coûts liés aux emplois publics par rapport aux coûts d'externalisation, par exemple) ; l'interaction entre les dépenses de TIC des administrations centrales et celles des administrations locales, qui fournissent la plupart des services publics; ou encore la contribution des nouvelles technologies à la réalisation des objectifs de l'action publique dans des domaines comme la santé, l'éducation ou la justice.

\section{Méthodologie et définitions}

Les données sur les dépenses en matière de TIC, qui sont disponibles dans 21 pays, portent sur les dépenses d'investissement, de fonctionnement et de personnel. Elles sont tirées d'une enquête de l'OCDE sur les dépenses des administrations publiques en matière de TIC menée en 2010 et 2011 auprès de responsables publics membres du Réseau sur l'administration électronique de l'OCDE. Des données supplémentaires ont été puisées dans des sources officielles librement accessibles. C'est la première fois que l'on recueille et que l'on harmonise des données pour un aussi grand nombre de pays. Les données présentées sont donc préliminaires, dans l'attente d'une harmonisation plus poussée.

\section{Pour en savoir plus}

OCDE (2010), OECD E-government Studies: Indicators Project, consultable sur www.oecd.org/governance/public-innovation.

\section{Notes relatives aux graphiques}

Les données relatives à l'Allemagne, à l'Australie, à la Corée, à l'Espagne, à l'Italie et à la Suisse portent sur 2010 et non 2011. Les données relatives à la Belgique et à la Slovénie portent sur 2009 et non 2011. Les données relatives à la France, à la Nouvelle-Zélande et au Royaume-Uni portent sur 2008 et non 2001.

3.50 : Les dépenses totales relatives aux TIC comprennent les dépenses d'investissement, de fonctionnement et de personnel, sauf dans les pays où les données sur les dépenses de personnel ne sont pas disponibles: Allemagne, Autriche, Belgique, Estonie, Islande, Royaume-Uni, Slovénie et Suisse. Les données relatives à l'Autriche et au Portugal portent sur 2010 et non 2011. Les données relatives à l'Islande portent sur 2008 et non 2011. Le Chili a communiqué des données détaillées sur les dépenses de TIC, mais elles ne sont pas incluses, faute de données relatives aux dépenses de l'administration centrale.

3.51 : Dans ce graphique, les dépenses de TIC comprennent seulement celles d'investissement et de fonctionnement, domaines on l'on peut s'attendre le plus à des économies d'échelle (les données relatives aux États-Unis, à la Nouvelle-Zélande et aux Pays-Bas incluent les dépenses de personnel). On ne dispose pas de données pour l'Autriche, l'Islande et le Portugal. Les données sur l'emploi dans les administrations centrales correspondent au nombre d'agents, sauf pour la Corée, la France, la Nouvelle-Zélande, le Royaume-Uni et la Suisse, où elles représentent des équivalents temps plein (ETP). De ce fait, la comparaison sous-estime le nombre d'emplois et surestime le total des dépenses TIC d'investissement et de fonctionnement par agent dans ces cinq pays. 
3.50. Dépenses totales en matière de TIC, en pourcentage des dépenses des administrations centrales (2011 ou dernière année disponible)

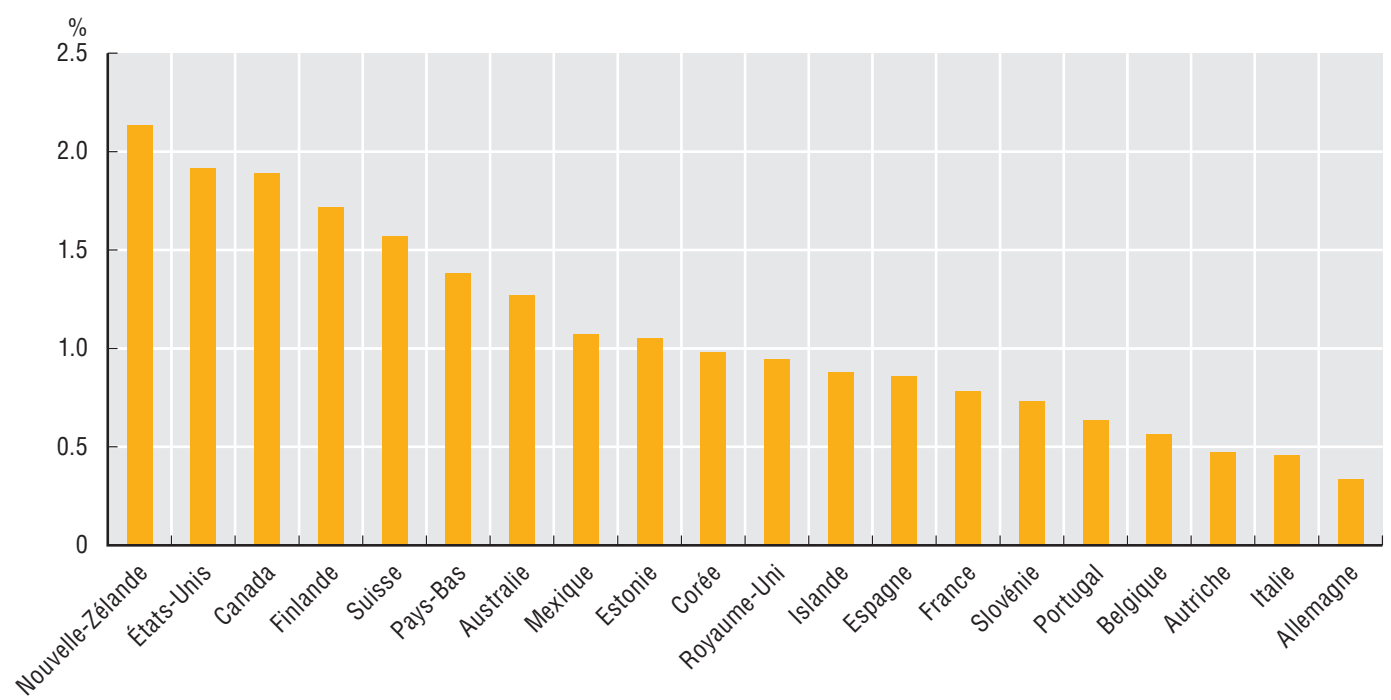

Source: Enquête de l'OCDE sur les dépenses en matière de TIC, 2010-2011; Statistiques de l'OCDE sur les comptes nationaux (base de données).

StatLink AाIs http://dx.doi.org/10.1787/888933159357 
3.51. Dépenses d'investissement et de fonctionnement relatives aux TIC par agent des administrations centrales, comparativement à l'emploi dans ces administrations (2011 ou dernière année disponible)

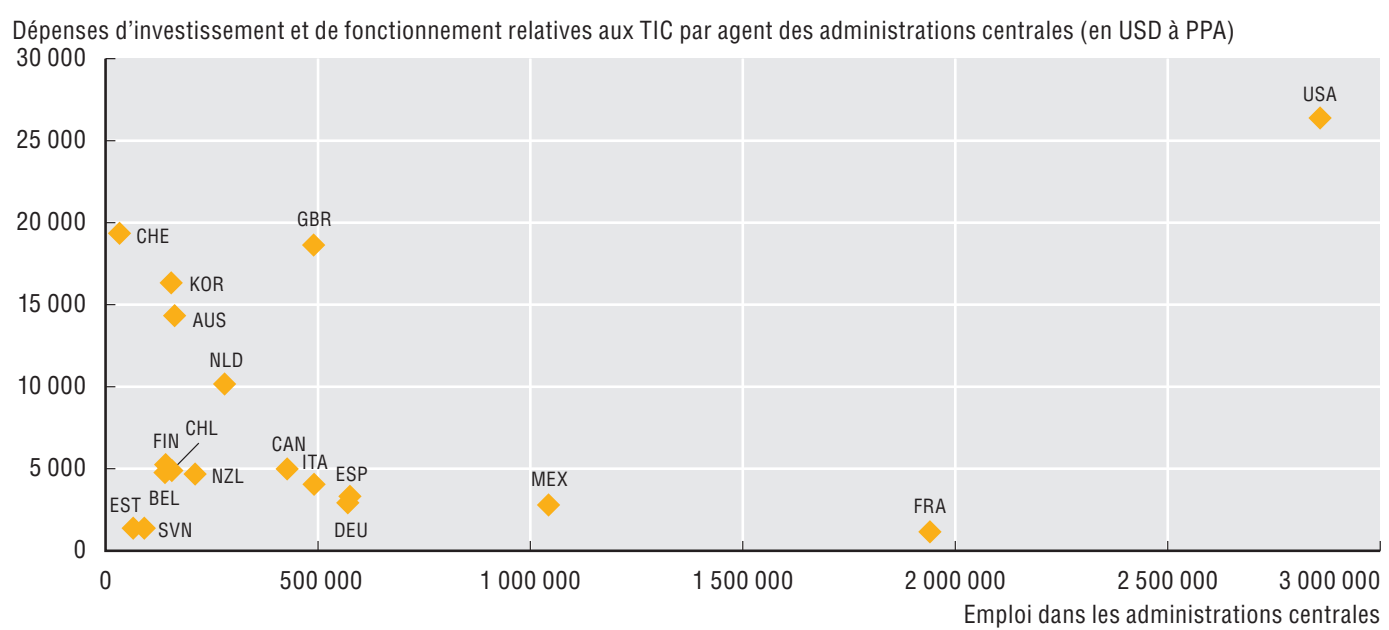

Source: Enquête de l'OCDE sur les dépenses en matière de TIC, 2010-11; Organisation internationale du travail (OIT), LABORSTA (base de données). Les données sur l'emploi pour le Chili, la Corée, la France, le Royaume-Uni et la Suisse sont tirées d'une enquête de l'OCDE de 2010 sur l'emploi public.

StatLink काज़ http://dx.doi.org/10.1787/888933159361 


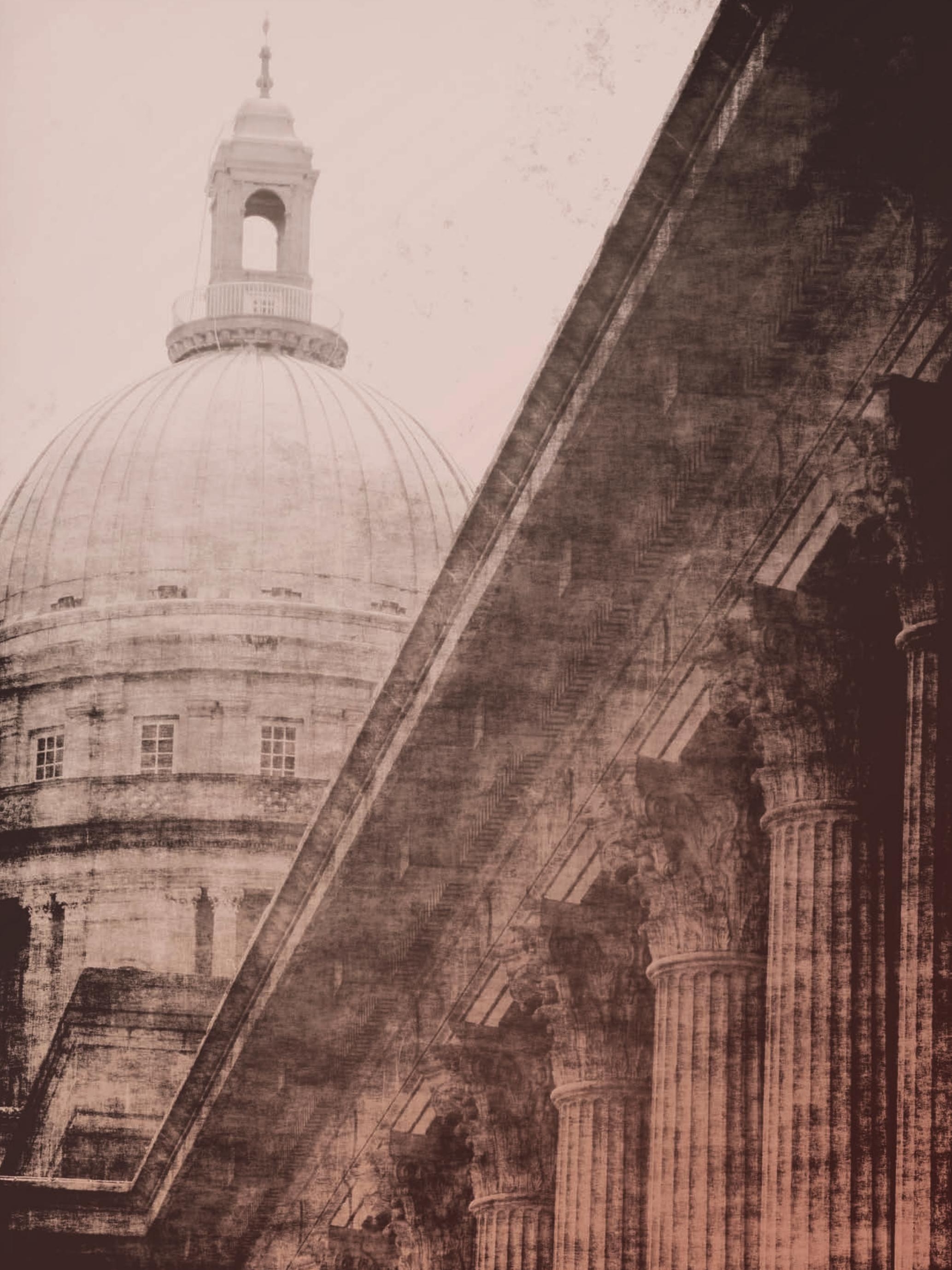




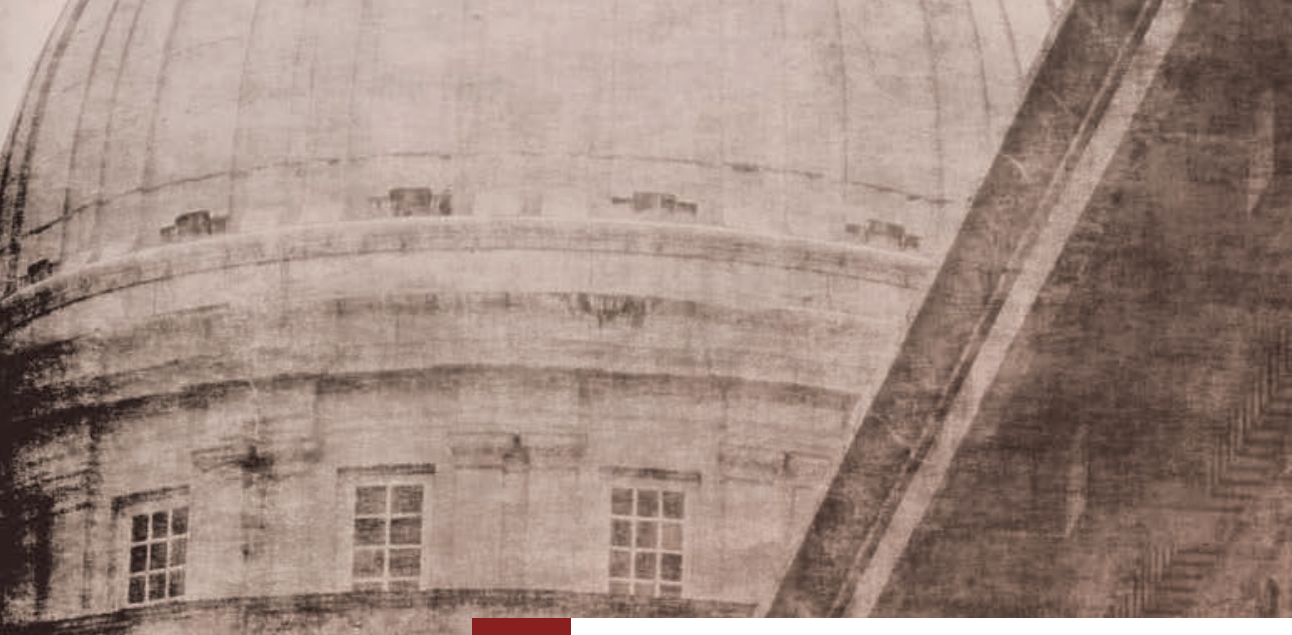

\section{LES PRATIQUES ET LES PROCÉDURES BUDGÉTAIRES}

Trois facteurs principaux déterminent la qualité des résultats budgétaires: le comportement général de l'économie, l'engagement politique en faveur de la discipline budgétaire et les dispositifs institutionnels en matière budgétaire. Le présent chapitre porte sur ce troisième facteur. Le bon fonctionnement des institutions budgétaires conditionne l'assainissement des finances publiques, la stabilité fiscale et l'équité intergénérationnelle. De plus, le dispositif national applicable aux dépenses publiques doit favoriser la discipline budgétaire, une affectation optimisée des ressources et un fonctionnement efficient des administrations.

On trouvera dans le présent chapitre des indicateurs relatifs à un certain nombre de grandes caractéristiques institutionnelles en matière budgétaire. Il s'agit de l'existence de règles budgétaires visant à assurer la viabilité par la fixation de contraintes, de l'intégration d'une perspective à moyen terme pour tenir compte des conséquences pluriannuelles des dépenses, du degré d'autonomie accordé aux administrations pour les décisions d'ordre budgétaire, de la prise en compte d'informations sur la performance pour l'établissement $d u$ budget, de l'évaluation des partenariats public-privé (par rapport à la passation de marchés d'infrastructure traditionnels) et enfin de l'existence d'une institution budgétaire indépendante chargée de contribuer à une gestion prudente des finances publiques. Bien que ces caractéristiques soient présentées comme des éléments distincts, elles se complètent et doivent être considérées comme formant un tout.

Les résultats présentés dans ce chapitre sont basés sur les réponses des pays à des enquêtes de l'OCDE et constituent des auto-évaluations. Les indices composites ont été calculés par l'OCDE dans le but de rassembler des informations d'ordre qualitatif éparses sur les pratiques budgétaires au sein d'indicateurs globaux plus faciles à interpréter que plusieurs variables distinctes; ces indices étant calculés dans le but de faire avancer le débat, ils sont susceptibles d'évoluer au fil du temps. 
Une règle budgétaire est une contrainte à long terme s'appliquant à la politique budgétaire sous la forme d'une limitation numérique des agrégats budgétaires. En l'absence de limites globales, la budgétisation par reconduction peut devenir un processus incontrôlable par lequel les pouvoirs publics accèdent aux demandes en dépensant au-delà de leurs moyens. Une règle budgétaire a deux caractéristiques fondamentales. D'abord, elle s'impose aux décisions politiques prises par le parlement et le pouvoir exécutif. Ensuite, c'est un indicateur concret de la gestion budgétaire de l'exécutif. Les règles budgétaires peuvent aider les pouvoirs publics à tenir leurs objectifs budgétaires et à faire preuve de discipline en la matière, mais il n'existe pas de règle unique pouvant convenir à tous les pays.

Les règles budgétaires peuvent porter sur différents aspects des performances des administrations publiques en la matière : les recettes, les dépenses, le solde budgétaire et la dette publique. Dans les pays de l'OCDE, les règles budgétaires les plus répandues portent sur le solde budgétaire (28 pays membres) et sur la dette (23), en raison des obligations imposées aux États membres de l'Union européenne. Les règles portant sur les recettes sont les moins courantes, puisque seulement cinq pays membres de l'OCDE en ont adopté (l'Australie, la France, la Grèce, les Pays-Bas et la République slovaque).

Les règles budgétaires peuvent avoir des fondements juridiques différents à l'échelon national ; elles peuvent être prévues soit par la constitution, soit par la législation, soit par la réglementation. Il arrive aussi qu'elles prennent la forme d'engagements politiques publics ou qu'elles soient édictées en interne par le ministère des Finances. L'Australie constitue un exemple intéressant, dans la mesure où on y trouve les quatre types de règles budgétaires. Le fondement juridique de trois d'entre elles est la Charte d'intégrité budgétaire ("Charter of Budget Honesty "), qui est un engagement politique fort; quant à la règle relative à la dette, elle est fixée par la législation. Le Japon et la Corée n'ont que des règles relatives aux dépenses qui, dans les deux cas, trouvent leur fondement dans des règlements et politiques internes.

Certains pays doivent aussi se plier à des règles budgétaires fixées à l'échelon supranational. S'agissant par exemple des pays de l'Union européenne, le Traité de Maastricht fixe une règle relative à la dette et deux règles relatives au solde budgétaire. Comme suite à l'adoption du nouveau Pacte budgétaire européen et d'un train de mesures d'assainissement budgétaire (le "Six-Pack "), les autorités de l'Union ont demandé aux États membres d'élever les règles budgétaires au rang de règles constitutionnelles, afin d'accroître le coût politique de tout non-respect. Dix pays (l'Allemagne, l'Autriche, la Belgique, l'Espagne, la Hongrie, l'Italie, la Pologne, la République slovaque, la République tchèque et la Suisse) ont inscrit des règles budgétaires dans leur constitution.

L'une des leçons les plus importantes du passé est que des règles trop rigides tendent à être inapplicables et à pécher par manque de réactivité face à la conjoncture économique ou politique. Pour des régimes budgétaires robustes, il vaut peutêtre mieux miser sur une volonté politique forte, sur un contrôle assuré par des institutions budgétaires et d'autres acteurs indépendants ainsi que sur des procédures claires et efficaces en cas de manquement aux règles. Sur ce dernier point, différentes mesures sont envisageables; elles vont de l'obligation de soumettre au parlement une proposition corrective à des mécanismes de correction automatique et de sanction. Les États membres de l'Union européenne sont assujettis à une procédure pour déficit excessif (PDE) qui consiste à examiner en plusieurs étapes la situation budgétaire d'un pays et peut aboutir à des sanctions. Les modifications récentes du cadre de gouvernance économique ont assoupli les conditions de lancement d'une PDE. Si l'on exclut la PDE, les mécanismes de correction automatique constituent, dans les pays membres de l'OCDE, l'instrument le plus répandu pour faire respecter les règles relatives au solde budgétaire; en ce qui concerne les règles relatives à la dette, l'obligation imposée à l'institution endettée de prendre des mesures est le mécanisme de correction le plus fréquent. Des sanctions automatiques en cas de manquement à des règles budgétaires ne sont appliquées que dans cinq pays membres de l'OCDE (les États-Unis, les Pays-Bas, la Pologne, la République slovaque et la Suisse).

\section{Méthodologie et définitions}

Les données, qui se réfèrent à 2012, sont tirées des réponses des pays à l'Enquête de l'OCDE sur les pratiques et procédures budgétaires (2012). Les répondants étaient principalement de hauts responsables budgétaires des pays membres de l'OCDE. Les réponses représentent l'évaluation par les pays eux-mêmes de leurs pratiques et procédures actuelles. Les données ne portent que sur l'administration centrale/fédérale et ne prennent pas en compte les pratiques budgétaires à l'échelon des administrations d'États fédérés et des administrations locales.

La législation est constituée des textes qui doivent être adoptés par le parlement: les textes de loi. La règlementation est constituée des textes qui peuvent être approuvés par le chef du gouvernement, un ministre ou le Conseil des ministres, c'est-à-dire par une autorité autre que le parlement: les textes réglementaires. Le parlement peut désavouer un texte réglementaire.

\section{Pour en savoir plus}

Anderson, B. et J. Sheppard (2010), « Fiscal futures, institutional budget reforms, and their effects: What can be learned? ", Revue de l'OCDE sur la gestion budgétaire, vol. 9, n 3, http:// dx.doi.org/10.1787/budget-9-5kmh6dnl056g.

Schick, A. (2010), « Post-Crisis Fiscal Rules: Stabilising Public Finance while Responding to Economic Aftershocks ", Revue de l'OCDE sur la gestion budgétaire, vol. 10, $\mathrm{n}^{\circ} 2$, http:// dx.doi.org/10.1787/budget-10-5km7rqpkqts1.

\section{Notes relatives aux tableaux}

4.1 et 4.2 : On ne dispose pas de données pour l'Islande. Les données reflètent la multiplicité des règles budgétaires nationales. La Nouvelle-Zélande et la Turquie, qui n'ont pas adopté de règles budgétaires, ne figurent pas dans le tableau.

4.1 : En Italie, la loi $n^{\circ} 243 / 2012$ a instauré une règle relative au budget structurel ainsi qu'une règle relative aux dépenses et une règle relative à la dette, conformément aux exigences européennes. Les deux premières entreront en vigueur en 2014 et la troisième en 2015.

Informations sur les données concernant Israël : http://dx.doi.org/ $10.1787 / 888932315602$. 
4.1. Catégories de règles budgétaires et fondement juridique (2012)

\begin{tabular}{|c|c|c|c|c|}
\hline & Règle(s) de dépenses & Règle(s) de solde (déficit/excédent) & Règle(s) de dette & Règle(s) de recettes \\
\hline Allemagne & & $\bullet$ & $\mathbf{\square}$ & \\
\hline Australie & O & O & $\square$ & O \\
\hline Autriche & $\square$ & $\boldsymbol{\bullet \bullet}$ & $\boldsymbol{\square \bullet}$ & \\
\hline Belgique & & $\bullet \diamond \square$ & - & \\
\hline Canada & & $\diamond$ & & \\
\hline Chili & $\square$ & $\square$ & & \\
\hline Corée & $\diamond$ & & & \\
\hline Danemark & ב & בם & [ & \\
\hline Espagne & $\square$ & $\bullet$ & • & \\
\hline Estonie & $\diamond$ & $\boldsymbol{\theta} \diamond$ & a & \\
\hline États-Unis & $\square$ & & $\square$ & \\
\hline Finlande & $\diamond$ & a & - & \\
\hline France & $\square$ & $\mathbf{\square}$ & घ & $\square$ \\
\hline Grèce & - & a & - & घ \\
\hline Hongrie & & 口a & $\bullet$ & \\
\hline Irlande & $\diamond$ & a & a & \\
\hline Israël & $\square$ & $\square$ & & \\
\hline Italie & & - & ש & \\
\hline Japon & $\diamond$ & & & \\
\hline Luxembourg & $\diamond$ & घ & 曰 & \\
\hline Mexique & & $\square$ & & \\
\hline Norvège & & $\diamond$ & & \\
\hline Pays-Bas & $\diamond$ & a & $\square$ व & $\diamond$ \\
\hline Pologne & $\square$ & - & ๑๑ロ & \\
\hline Portugal & $\square$ & 口 & 口 & \\
\hline République slovaque & & 口 & - & $\square$ \\
\hline République tchèque & $\square$ & $\bullet$ & 口 & \\
\hline Royaume-Uni & & 口曰 & 口曰 & \\
\hline Slovénie & $\square$ & 口 & ש & \\
\hline Suède & $\square$ & $\square$ & $\bar{\square}$ & \\
\hline Suisse & & $\bullet$ & & \\
\hline Fédération de Russie & $\square$ & & & $\square$ \\
\hline Total OCDE & 21 & 28 & 23 & 5 \\
\hline
\end{tabular}

- Constitution.

- Traité international

$\square$ Législation et/ou réglementation

$\diamond$ Règles ou politiques internes

O Engagement politique

Source : Enquête 2012 de l'OCDE sur les pratiques et procédures budgétaires.

4.2. Conséquences en cas d'entorse aux règles budgétaires (2012)

\begin{tabular}{|c|c|c|c|c|c|c|}
\hline $\begin{array}{l}\text { Type de règle/ } \\
\text { Dispositifs de } \\
\text { correction }\end{array}$ & $\begin{array}{l}\text { Dispositif de correction } \\
\text { automatique }\end{array}$ & $\begin{array}{l}\text { Présentation au parlement } \\
\text { d'une proposition de } \\
\text { mesures correctives }\end{array}$ & $\begin{array}{l}\text { Obligation de } \\
\text { mettre en œuvre } \\
\text { des mesures }\end{array}$ & $\begin{array}{l}\text { Sanctions } \\
\text { automatiques }\end{array}$ & $\begin{array}{l}\text { Procédure pour déficit excessif du } \\
\text { Pacte de stabilité et de croissance }\end{array}$ & Néant \\
\hline Dépenses & DNK, GRC, USA, ESP & $\begin{array}{l}\text { EST, FRA, ISR, NLD, } \\
\text { SWE, ESP }\end{array}$ & $\begin{array}{l}\text { AUT, GRC, NLD, } \\
\text { SWE, CHL, ESP }\end{array}$ & USA & & $\begin{array}{l}\text { AUS, CZE, FIN, FRA, } \\
\text { IRL, JPN, KOR, LUX, } \\
\text { POL, PRT, RUS, SVN }\end{array}$ \\
\hline Solde budgétaire & $\begin{array}{l}\text { AUT, BEL, DNK, FIN, FRA, } \\
\text { DEU, GRC, IRL, ITA, LUX, PRT, } \\
\text { SVK, SVN, ESP, CHE }\end{array}$ & ISR, ITA, MEX, ESP & GRC, ESP, CHL & NLD, CHE & $\begin{array}{l}\text { AUT, BEL, CZE, DNK, EST, FIN, FRA, } \\
\text { DEU, GRC, HUN, IRL, ITA, LUX, NLD, } \\
\text { POL, PRT, SVK, SVN, ESP, SWE, GBR }\end{array}$ & AUS, CAN, NOR \\
\hline Recettes & GRC & NLD & & & & AUS, FRA, RUS, SVK \\
\hline
\end{tabular}

Source : Enquête 2012 de l'OCDE sur les pratiques et procédures budgétaires. 
Les cadres de dépenses à moyen terme (CDMT) permettent aux administrations centrales ou fédérales de se placer dans une perspective budgétaire à moyen terme, et pas seulement annuelle. Les CDMT couvrent généralement une période de trois à cinq ans, dont l'exercice immédiatement à venir ; ils comprennent à la fois des plafonds annuels à caractère prescriptif et des estimations à terme à caractère descriptif.

Il existe un consensus international sur l'intérêt d'inscrire le budget dans une perspective à moyen terme. En premier lieu, les CDMT peuvent constituer, à l'usage des autorités budgétaires centrales, un instrument précieux pour gérer les dépenses de l'ensemble des administrations centrales et assurer la discipline budgétaire : les estimations de dépenses, qui contiennent des informations sur le coût des politiques et programmes en cours, constituent la référence pour établir les budgets des exercices suivants ; les plafonds de dépenses sont une contrainte imposée du sommet sur les dépenses des exercices futurs. En second lieu, les CDMT contribuent à la maîtrise des dépenses en permettant de prendre en compte des politiques pluriannuelles dont la mise en œuvre se situe dans un horizon assez lointain, telles que les grands projets d'investissement, les nouveaux programmes et les restructurations. En outre, cette perspective à moyen terme éclaire les gestionnaires des ministères sectoriels et des organismes publics sur l'orientation générale de l'action publique et sur les changements en matière de financement, ce qui leur donne le temps de s'adapter pour mieux planifier leurs activités.

Toutefois, l'effet de cette perspective à moyen terme dépend, en définitive, de la crédibilité des estimations et des plafonds de dépenses ainsi que de la manière dont ces informations sont utilisées par les décideurs et la société civile. La non-réalisation des objectifs budgétaires à moyen terme est souvent due à des déficiences au niveau de l'élaboration des cibles budgétaires, de leur formulation dans des textes et de leur mise en œuvre.

Tous les pays de l'OCDE, à quatre exceptions près (la Belgique, Israël, la Hongrie et le Luxembourg), fixent des plafonds de dépenses à moyen terme, qui couvrent le plus souvent une période de quatre ans. L'importance attachée à ces cadres varie fortement selon les pays, comme en témoigne le fait qu'ils peuvent être énoncés ou non dans des textes de loi, fixés par le pouvoir exécutif ou par le pouvoir législatif puis, en aval, faire ou non l'objet d'un contrôle assuré par des organes parlementaires ou indépendants. La plupart du temps, les plafonds de dépenses s'appliquent à des agrégats de dépenses. Dans certains pays (l'Allemagne, l'Autriche, la Corée, l'Italie, la Nouvelle-Zélande et les Pays-Bas), il existe des plafonds supplémentaires par programme, secteur et/ou organisation. Pour que les CDMT soient efficaces, il faut instituer des mécanismes de suivi et d'exécution dans le cadre desquels le pouvoir exécutif indique au parlement ou à une institution budgétaire indépendante s'ils sont respectés.

\section{Méthodologie et définitions}

Les données, qui se réfèrent à 2012, sont tirées des réponses des pays à l'Enquête de l'OCDE sur les pratiques et procédures budgétaires (2012). Les répondants étaient principalement de hauts responsables budgétaires des pays membres de l'OCDE. Les réponses représentent l'évaluation par les pays eux-mêmes de leurs pratiques et procédures actuelles. Les données ne portent que sur l'administration centrale/fédérale et ne prennent pas en compte les pratiques budgétaires à l'échelon des administrations d'États fédérés et des administrations locales.

Un CDMT a été défini comme un cadre d'intégration de la politique budgétaire et de budgétisation à moyen terme dans lequel la prévision des agrégats budgétaires est liée à un processus rigoureux d'établissement d'estimations budgétaires à moyen terme ventilées par ministère et fondées sur la politique des pouvoirs publics. Les estimations à terme des dépenses deviennent la base des négociations du budget des exercices suivants et elles sont rapprochées des résultats définitifs dans les rapports budgétaires.

L'indice composite du graphique 4.4 rassemble 10 variables relatives à l'intégration d'une perspective de moyen terme à la procédure budgétaire, au nombre d'années couvertes par les estimations, aux catégories de dépenses figurant dans les CDMT, à la possibilité de reporter d'une année sur l'autre les fonds inutilisés et aux modalités de suivi. Il convient de noter que l'indice n'a pas vocation à mesurer la qualité globale des CDMT, et qu'il est de nature descriptive. L'annexe C présente la méthodologie utilisée pour élaborer cet indice, y compris les pondérations attribuées à chaque variable.

\section{Pour en savoir plus}

Banque mondiale (2013), Beyond the Annual Budget - Global Experience with Medium-Term Expenditure Frameworks, Banque internationale pour la reconstruction et le développement, Banque mondiale, Washington, DC, http://dx.doi.org/10.1596/ 978-0-8213-9625-4.

OCDE (2013), Budgeting Practices and Procedures in OECD Countries, Éditions OCDE, Paris, http://dx.doi.org/10.1787/9789264 059696-en.

OCDE (2002), «Transparence budgétaire : les meilleures pratiques de l'OCDE ", Revue de l'OCDE sur la gestion budgétaire, vol. 1, $n^{\circ} 3$, pp. 7-15, http://dx.doi.org/10.1787/budget-v1-art14-fr.

\section{Notes relatives aux graphiques et aux tableaux}

On ne dispose pas de données pour l'Islande.

4.3 : En Allemagne, le fondement juridique du CDMT est le Finanzplan, qui est débattu en parallèle à l'examen du budget par le parlement ; il fixe les plafonds budgétaires. Aux Pays-Bas, les dépenses de fonctionnement sont intégrées aux dépenses totales et par secteur.

4.4 : L'indice est égal à 0 pour la Belgique, la Hongrie, Israël et le Luxembourg, car ces pays ont déclaré ne pas avoir de CDMT.

Informations sur les données concernant Israël : http://dx.doi.org/ $10.1787 / 888932315602$. 


\section{LES PRATIQUES ET LES PROCÉDURES BUDGÉTAIRES}

Les cadres de dépenses à moyen terme

4.3. Intégration d'une perspective de moyen terme à la procédure budgétaire au niveau de l'administration centrale (2012)

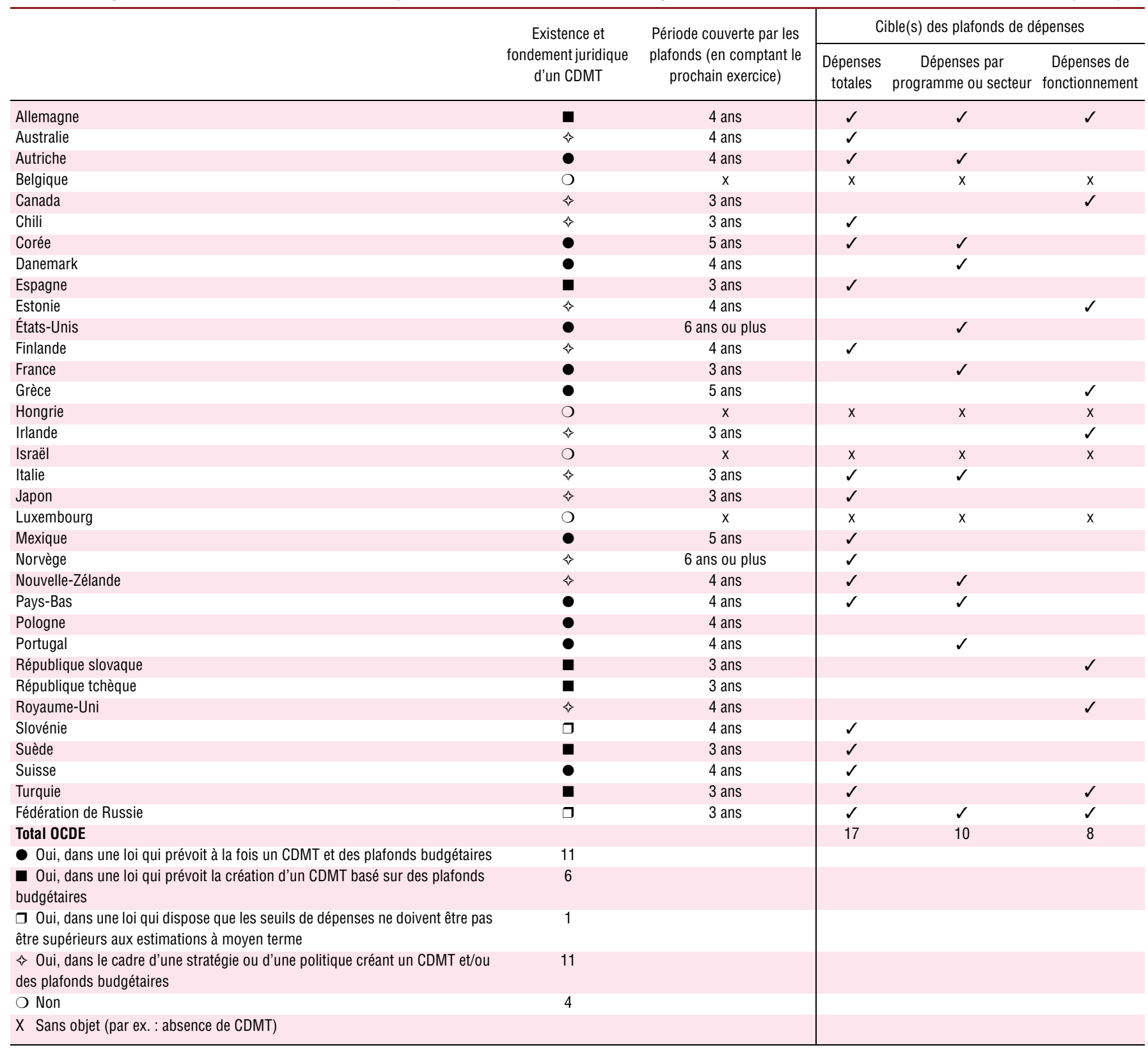

Source : Enquête 2012 de l'OCDE sur les pratiques et procédures budgétaires.

4.4. Intégration d'une perspective de moyen terme à la procédure budgétaire (2012)

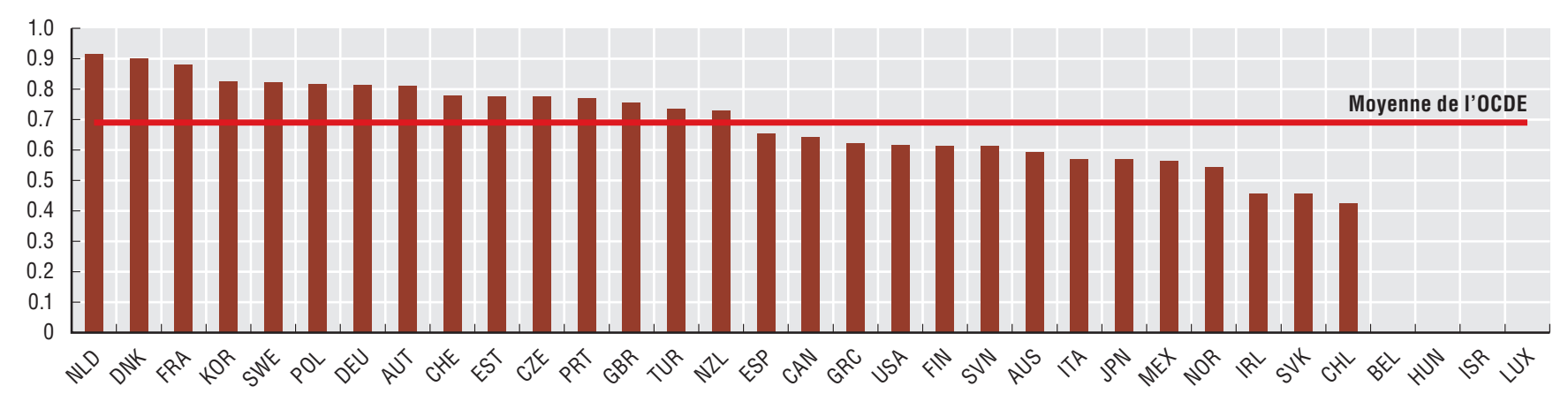

Source: Enquête 2012 de l'OCDE sur les pratiques et procédures budgétaires. 
Dans de nombreux pays de l'OCDE, les réformes budgétaires passent par un assouplissement du contrôle des intrants exercé par l'autorité budgétaire centrale, dans le but de donner aux entités administratives plus de flexibilité et d'autonomie pour atteindre leurs objectifs avec efficience et efficacité. Cette évolution part du principe que les dirigeants de ces entités sont les mieux placés pour réaliser leurs objectifs en termes de stratégie et de programmes (en d'autres termes, il faut laisser les gestionnaires gérer). De plus, même quand la procédure d'élaboration du budget est satisfaisante, il arrive que les hypothèses économiques changent, que les prix des intrants fluctuent et que l'évolution des priorités politiques nécessite un redéploiement des moyens. En donnant plus de flexibilité aux gestionnaires, on leur permet d'ajuster les dépenses en fonction des évolutions de cet ordre.

D'un autre côté, si on laisse aux gestionnaires une latitude totale et incontrôlée, la viabilité budgétaire peut être compromise. On s'expose alors à des risques d'abus de pouvoir par les gestionnaires, de creusement des déficits publics et de baisse de l'efficience. Une souplesse excessive peut aussi entraver la volonté du législateur et la finalité des crédits votés, car les ressources peuvent être réaffectées à des priorités différentes. C'est pourquoi, même si les modèles varient, la majorité des pays membres de l'OCDE opte pour un certain équilibre entre des directives et un contrôle de la performance venus d'en haut ("descendants") et des degrés divers de flexibilité au niveau des ministères. L'autorité budgétaire centrale ( $A B C$ ) définit les contraintes budgétaires descendantes sur la base des priorités politiques et de considérations liées aux dépenses à moyen terme, tandis que les ministères sectoriels, les organismes publics et les autres entités publiques sont chargés de répartir et d'utiliser ces fonds de manière à atteindre les objectifs fixés dans le cadre des programmes. Cette liberté s'accompagne toutefois de plus forts besoins en termes de transparence et de gestion de la performance (cf. l'indicateur relatif à la budgétisation axée sur la performance). Cette façon de procéder peut améliorer l'efficience et l'efficacité dans un contexte de priorités, de circonstances et de connaissances nouvelles. Elle incite aussi davantage les responsables politiques à s'intéresser aux produits et aux réalisations plutôt qu'aux seuls intrants.

L'un des éléments essentiels d'une flexibilité budgétaire de l'exécutif est le recours à des dotations forfaitaires, qui donne aux gestionnaires plus de marge pour affecter des fonds comme ils le souhaitent au sein de chaque programme et entre plusieurs programmes. La majorité des pays membres fixe des sous-limites à ces montants globaux, applicables le plus souvent aux dépenses de rémunération des personnels. De même, quand le nombre de crédits ouverts au niveau des lignes budgétaires est limité, cela offre plus de flexibilité à l'exécutif. Dans seulement le quart des pays membres de l'OCDE, il y a moins de 300 lignes budgétaires. La plupart des $A B C$ des pays membres permettent aussi aux ministères sectoriels et aux organismes publics de reporter sur l'exercice suivant les crédits non dépensés, bien qu'avec des restrictions telles que le respect de plafonds, la nécessité de demander l'autorisation ou les deux. En règle générale, il y a plus de souplesse pour les dépenses d'investissement que pour les dépenses de fonctionnement, car les projets d'investissement s'étalent souvent sur plusieurs années. En outre, certains pays autorisent l'exécutif à prélever par anticipation sur des crédits futurs. Toutefois, les sept pays qui suivent cette pratique plafonnent tous le montant du dépassement possible pendant l'exercice budgétaire en cours. Il arrive aussi que l'exécutif soit autorisé à accroître ou à diminuer les dépenses en cours d'exercice sans l'accord préalable du parlement. Cette flexibilité supplémentaire est souvent accordée dans l'idée qu'elle peut faciliter un usage optimal de l'argent public et inciter à améliorer l'efficience de la dépense publique.

\section{Méthodologie et définitions}

Les données, qui se réfèrent à 2012, sont tirées des réponses des pays à l'Enquête de l'OCDE sur les pratiques et procédures budgétaires (2012). Les répondants étaient principalement de hauts responsables budgétaires des pays membres de l'OCDE. Les réponses représentent l'évaluation par les pays eux-mêmes de leurs pratiques et procédures actuelles. Les données ne portent que sur l'administration centrale/fédérale et ne prennent pas en compte les pratiques budgétaires à l'échelon des administrations d’États fédérés et des administrations locales.

\section{Pour en savoir plus}

OCDE (2013), Budgeting Practices and Procedures in OECD Countries, Éditions OCDE, Paris, http://dx.doi.org/10.1787/9789264 059696-en.

\section{Notes relatives aux tableaux}

4.5: On ne dispose pas de données pour l'Islande. En Allemagne, les ministères sectoriels peuvent prélever par anticipation sur des dotations futures, à condition que le budget de l'exercice suivant contienne un poste de dépense ayant la même finalité et que le ministère ait l'intention d'équilibrer sa situation de trésorerie pendant l'exercice en cours. Si une dépense supplémentaire ne peut pas être considérée comme une dépense anticipée, elle doit être traitée comme un dépassement. En Espagne, bien que les ministères sectoriels ne reçoivent pas de dotations forfaitaires, ils sont autorisés à redéployer les fonds dans certaines limites. En Corée, en Finlande et en Slovénie, seules certaines catégories de dépenses peuvent être reportées sur l'exercice suivant. En Australie, les crédits annuels ne sont pas annulés en fin d'exercice et peuvent encore être utilisés dans la limite du montant disponible; en général, soit ils restent disponibles jusqu'à ce qu'ils aient été dépensés, soit ils sont réduits conformément aux dispositions correspondantes des lois annuelles de dotation qui les prévoient, à moins que la loi annuelle de dotation applicable soit abrogée par une autre loi. Aux États-Unis, les données ne portent que sur les grands départements et ministères sectoriels. En Turquie, certains crédits peuvent être exceptionnellement reportés ; cette possibilité est déterminée annuellement dans la loi de finances de l'administration centrale, et c'est le ministre des Finances qui décide d'autoriser ou non les reports.

Informations sur les données concernant Israël : http://dx.doi.org/ $10.1787 / 888932315602$. 
4.5. Possibilité pour les ministères sectoriels de reporter les crédits non utilisés et de prélever par anticipation sur les dotations futures (2012)

\begin{tabular}{|c|c|c|c|c|c|}
\hline & \multirow{2}{*}{$\begin{array}{l}\text { Nombre de sous-limites dans les dotations } \\
\text { forfaitaires des ministères sectoriels }\end{array}$} & \multicolumn{2}{|c|}{$\begin{array}{l}\text { Possibilité pour les ministères sectoriels de prélever } \\
\text { par anticipation sur des dotations futures }\end{array}$} & \multicolumn{2}{|c|}{$\begin{array}{l}\text { Possibilité pour les ministères sectoriels de reporter sur } \\
\text { l'exercice suivant les fonds ou les crédits non utilisés }\end{array}$} \\
\hline & & $\begin{array}{l}\text { Dépenses de } \\
\text { fonctionnement }\end{array}$ & $\begin{array}{l}\text { Dépenses } \\
\text { d'investissement }\end{array}$ & $\begin{array}{l}\text { Dépenses de } \\
\text { fonctionnement }\end{array}$ & Dépenses d'investissement \\
\hline Allemagne & Sans objet (pas de dotations forfaitaires) & $\mathbf{\square}$ & $\mathbf{\square}$ & $\bigcirc$ & O \\
\hline Australie & 0 & O & O & Sans objet & Sans objet \\
\hline Autriche & Sans objet (pas de dotations forfaitaires) & O & O & $\bullet$ & $\bullet$ \\
\hline Belgique & 2 & O & O & $\mathrm{O}$ & $O$ \\
\hline Canada & 2 & $\bigcirc$ & $\bigcirc$ & $\mathbf{a}$ & $\mathbf{\square}$ \\
\hline Chili & 3 ou plus & $\bigcirc$ & $\bigcirc$ & $\bigcirc$ & $O$ \\
\hline Corée & 3 ou plus & $\bigcirc$ & O & $\bullet$ & $\bullet$ \\
\hline Danemark & 1 & $\mathbf{\square}$ & O & $\bullet$ & $\bullet$ \\
\hline Espagne & Sans objet (pas de dotations forfaitaires) & O & O & $\mathbf{\square}$ & $\mathbf{\square}$ \\
\hline Estonie & 1 & O & O & $\mathbf{\square}$ & $\bullet$ \\
\hline États-Unis & 3 ou plus & O & O & $\mathbf{\square}$ & $\mathbf{\square}$ \\
\hline Finlande & 0 & $\bigcirc$ & O & $\bullet$ & $\bullet$ \\
\hline France & 0 & $\bigcirc$ & O & 口 & $\square$ \\
\hline Grèce & 3 ou plus & O & O & O & O \\
\hline Hongrie & 1 & $\bigcirc$ & 口 & - & - \\
\hline Irlande & 0 & O & O & O & $\mathbf{\square}$ \\
\hline Israël & 3 ou plus & O & O & $\bullet$ & $\bullet$ \\
\hline Italie & 1 & $\mathrm{O}$ & $\boldsymbol{\square}$ & $O$ & $\square$ \\
\hline Japon & 0 & $\bigcirc$ & O & - & - \\
\hline Luxembourg & 1 & $\bigcirc$ & $\mathbf{\square}$ & $\mathrm{O}$ & ○ \\
\hline Mexique & 3 ou plus & O & O & O & O \\
\hline Norvège & 0 & $\mathrm{O}$ & $O$ & 口 & 口 \\
\hline Nouvelle-Zélande & 0 & $\square$ & $\square$ & $\mathbf{\square}$ & $\square$ \\
\hline Pays-Bas & 0 & $\mathrm{O}$ & $\bigcirc$ & $\mathbf{\square}$ & $\boldsymbol{\square}$ \\
\hline Pologne & 0 & O & $\mathrm{O}$ & O & O \\
\hline Portugal & 1 & $\bigcirc$ & O & - & - \\
\hline République slovaque & 2 & ○ & O & O & - \\
\hline République tchèque & 3 ou plus & $\bigcirc$ & $\bigcirc$ & $\bullet$ & - \\
\hline Royaume-Uni & Sans objet (pas de dotations forfaitaires) & O & O & $\mathbf{\square}$ & $\mathbf{\square}$ \\
\hline Slovénie & 1 & $\mathrm{O}$ & $O$ & $\bullet$ & $\bullet$ \\
\hline Suède & 0 & $\square$ & $\square$ & 口 & $\square$ \\
\hline Suisse & 0 & $\mathrm{O}$ & O & $\bullet$ & $\bullet$ \\
\hline Turquie & Sans objet (pas de dotations forfaitaires) & $\bigcirc$ & $\bigcirc$ & $O$ & O \\
\hline Fédération de Russie & 3 ou plus & $\mathbf{\square}$ & $\mathbf{\square}$ & $\mathbf{\square}$ & $\bullet$ \\
\hline \multicolumn{6}{|l|}{ Total OCDE } \\
\hline Oui, sans limite & & 0 & 0 & 11 & 14 \\
\hline $\begin{array}{l}\text { Oui, jusqu'à une } \\
\text { certaine limite }\end{array}$ & & 4 & 6 & 10 & 11 \\
\hline Non, non autorisé & & 29 & 27 & 11 & 7 \\
\hline
\end{tabular}

Source: Enquête 2012 de l'OCDE sur les pratiques et procédures budgétaires. 
Les pouvoirs publics intègrent de plus en plus d'informations sur la performance aux processus de budgétisation et de gouvernance, dans le but d'obtenir de meilleurs résultats, d'optimiser la dépense publique et de donner plus de transparence aux décisions de dépenses. Une bonne information sur la performance peut contribuer à l'adoption de décisions plus judicieuses quant à l'emploi des ressources et à la gestion des programmes. Une transparence accrue au sujet de la performance et de l'affectation des ressources amène aussi les organismes publics à rendre davantage compte de leurs dépenses. De nombreux pays membres de l'OCDE pratiquent la budgétisation axée sur la performance, mais les méthodes utilisées sont très différentes, et il n'y a pas unanimité sur le système optimal à appliquer. Toutefois, l'OCDE a recensé trois grandes catégories de systèmes de budgétisation axée sur la performance : i) les systèmes consistant à présenter des informations sur la performance en marge des chiffres de financement, sans que ces informations soient nécessairement employées en vue des décisions de dépense ; ii) les systèmes dans lesquels les informations relatives à la performance influencent explicitement l'affectation des ressources; et iii) les systèmes de budgétisation directement axée sur la performance (budgétisation à base de formules), dans lesquels le financement est strictement lié aux produits et aux réalisations. La majorité des pays membres de l'OCDE relèvent de la première ou de la deuxième catégories, et quelques-uns ont adopté le troisième système pour certaines dépenses (relatives par exemple à l'enseignement supérieur ou aux hôpitaux).

L'enquête 2011 de l'OCDE sur la budgétisation axée sur la performance a montré que cette pratique était généralement décentralisée au sein de l'administration centrale/fédérale. En d'autres termes, elle est pratiquée, le plus souvent, au niveau des ministères sectoriels, qui s'appuient sur elle pour répartir leurs propres enveloppes budgétaires entre les organismes et les directions qui dépendent d'eux. Il y a une exception: celle des examens de dépenses, dans lesquels l'autorité budgétaire centrale et les directeurs généraux jouent un rôle plus important. Toutefois, certains pays recourent à une méthode plus centralisée, en mettant en place à l'échelle de l'ensemble des administrations un dispositif pour produire des informations sur la performance (évaluations et indicateurs), intégrer ces informations aux processus budgétaires et de responsabilité, s'en servir pour la prise de décisions et suivre les résultats et en rendre compte. La Corée, le Mexique et le Canada, par exemple, appliquent aux ministères sectoriels et aux organismes publics des systèmes normalisés et exhaustifs comportant notamment des principes directeurs, des modèles de rapports et des dispositifs de notation. Toutefois, dans la majorité des pays, la non-réalisation des objectifs de performance n'a, le plus souvent, pas de conséquences financières ; elle aboutit plutôt à la divulgation de cette performance médiocre et à un suivi renforcé par la suite.

Les informations relatives à la performance peuvent prendre de nombreuses formes, dont des données financières et opérationnelles, des évaluations, et même des statistiques et des rapports indépendants établis à l'extérieur des administrations. Les indicateurs relatifs aux intrants, tels que les données financières et opérationnelles, sont les informations les plus utilisées, de loin, dans les négociations budgétaires (tableau 4.6).

\section{Méthodologie et définitions}

Les données, qui se réfèrent à 2011, sont tirées des réponses des pays à l'Enquête de l'OCDE sur la budgétisation axée sur la performance (2011). Les répondants étaient principalement de hauts responsables budgétaires des pays membres de l'OCDE. Les réponses représentent l'évaluation par les pays eux-mêmes de leurs pratiques et procédures actuelles. Les données ne portent que sur l'administration centrale/fédérale et ne prennent pas en compte les pratiques de budgétisation axée sur la performance à l'échelon des administrations d'États fédérés et des administrations locales. S'agissant des pays membres de l'Union européenne, les résultats excluent tout financement émanant de l'UE.

Les examens de dépenses, qui constituent un mode particulier d'évaluation des administrations, sont lancés dans le but spécifique de trouver des possibilités d'économies dans l'ensemble des administrations. Le projet de l'OCDE sur l'optimisation de la dépense publique distingue ces examens des autres formes d'évaluation en fonction de trois caractéristiques principales :

1. Les examens de dépenses ne visent pas uniquement à analyser l'efficacité et l'efficience des programmes à leurs niveaux de financement existants ; ils visent aussi à examiner les conséquences pour les produits et les réalisations d'autres niveaux de financement possibles. Ils peuvent être de nature fonctionnelle (car axés sur l'efficience opérationnelle, par exemple) et/ ou stratégique (car axés à la fois sur l'efficience et sur la conformité des initiatives aux grandes priorités de l'action publique, par exemple).

2. La responsabilité des examens de dépenses incombe au ministère des Finances ou aux services du Premier ministre.

3. Les suites données aux examens de dépenses sont décidées lors de l'élaboration du budget.

L'indice composite du graphique 4.7 rassemble 11 variables portant sur la disponibilité et la teneur des informations obtenues en termes de performance, sur les procédures de suivi et de communication des résultats ainsi que sur la question de savoir si (et comment) les informations relatives à la performance sont utilisées dans les négociations budgétaires et à l'appui des décisions prises par l'autorité budgétaire centrale, les ministères sectoriels et les responsables politiques. Il convient de noter que l'indice n'a pas vocation à mesurer la qualité globale des systèmes de budgétisation axée sur la performance, et qu'il est de nature descriptive. L'annexe C présente la méthodologie utilisée pour élaborer cet indice, y compris les pondérations attribuées à chaque variable.

\section{Pour en savoir plus}

OECD (2008), « Performance Budgeting: A User's Guide », OECD Policy Brief, Éditions OCDE, Paris, www.oecd.org/governance/ budgeting/40357919.pdf.

OCDE (2007), La budgétisation axée sur la performance dans les pays de l'OCDE, Éditions OCDE, Paris, http://dx.doi.org/10.1787/ 9789264034075-fr.

\section{Notes relatives aux graphiques et aux tableaux}

On ne dispose pas de données pour l'Islande et Israël. En Autriche, les informations sur la performance ne sont pas encore utilisées pour les négociations budgétaires, mais pendant l'exécution du budget et pour améliorer l'efficience. 


\section{LES PRATIQUES ET LES PROCÉDURES BUDGÉTAIRES}

La budgétisation axée sur la performance

4.6. Pratiques de budgétisation axée sur la performance au niveau de l'administration centrale (2011)

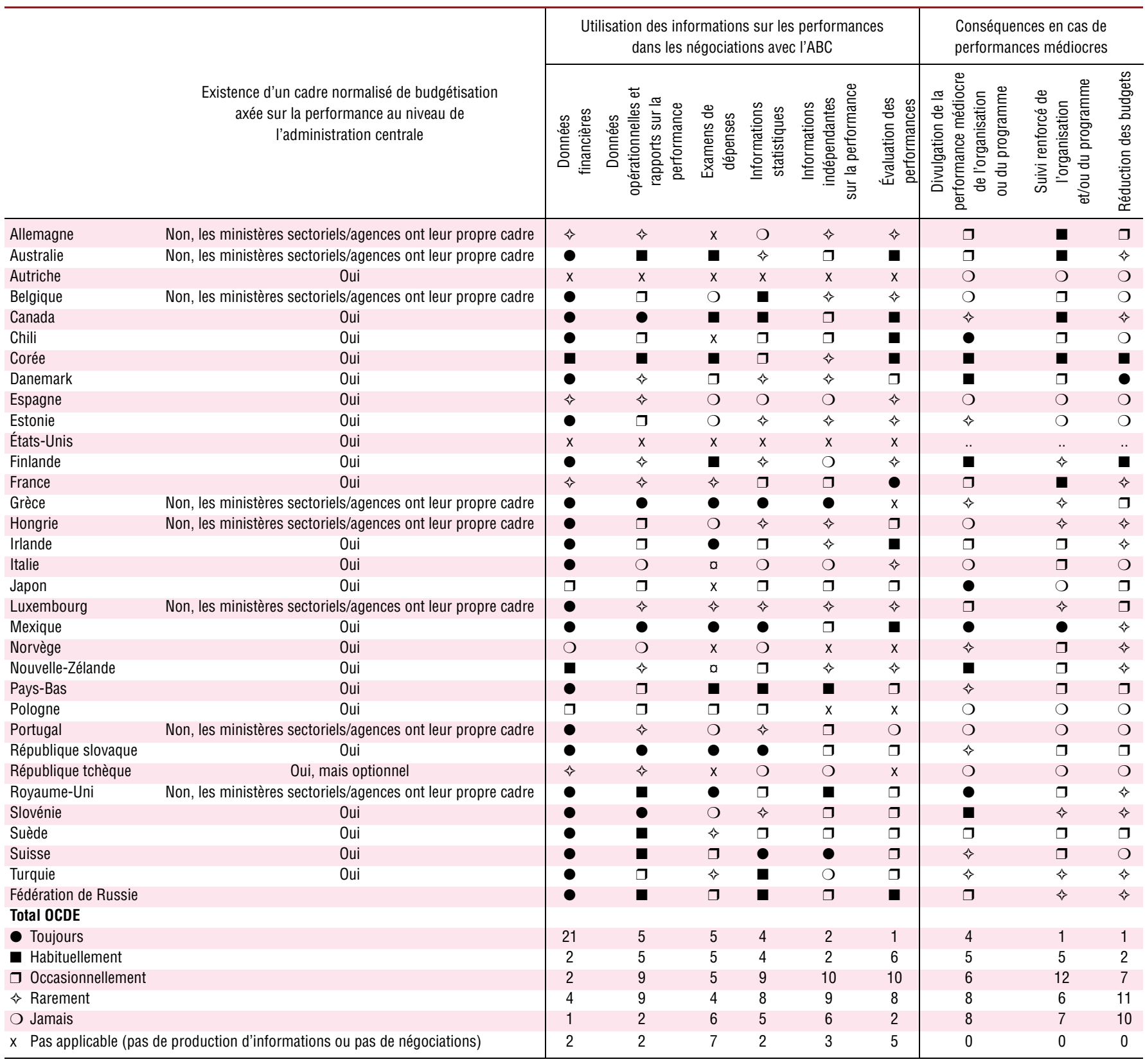

Source : Enquête 2011 de l'OCDE sur la budgétisation axée sur la performance.

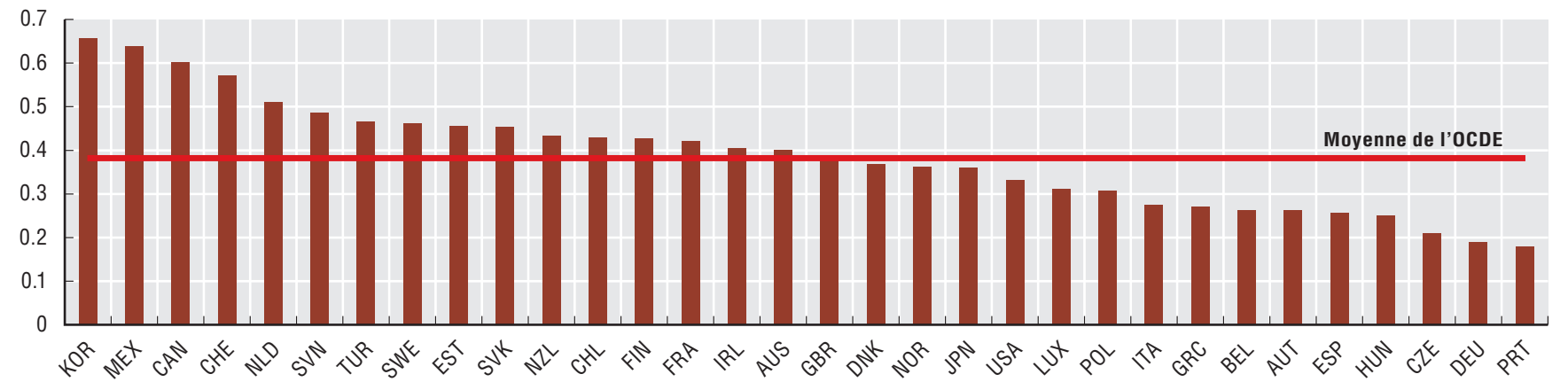

Source : Enquête 2011 de l'OCDE sur la budgétisation axée sur la performance. 
Un partenariat public-privé (PPP) est un accord contractuel de long terme entre une autorité publique et un partenaire privé dans le cadre duquel, en règle générale, ce partenaire finance et assure des services publics à partir d'un équipement (par exemple une infrastructure de transport ou de production d'énergie, ou encore un hôpital ou un établissement scolaire). Le partenaire privé peut se voir confier la conception, la construction, le financement, l'exploitation ou la gestion de l'actif et la fourniture du service correspondant pour une période prédéterminée ; il est rémunéré au moyen de versements unitaires fixes ou de redevances réglées par les usagers. Les PPP représentent moins de $15 \%$ des dépenses d'investissement annuelles des administrations centrales, et leur degré d'utilisation varie beaucoup selon les pays : le Royaume-Uni compte le plus de projets de cet ordre (648), devant la Corée (567) et l'Australie (127).

Les administrations publiques peuvent envisager la solution du PPP parce que, comparativement à des formes plus traditionnelles de passation de marchés publics d'infrastructures, cette formule peut leur permettre de mieux tirer parti de l'expertise du secteur privé pour combiner la conception et l'exploitation d'un équipement, ce qui permet d'assurer le service correspondant de façon plus efficiente. D'après les pays qui ont assez d'expérience et de données pour formuler un jugement à propos des PPP, ces dispositifs sont plus performants que les marchés d'infrastructure traditionnels (MIT) sur le plan du respect des délais, des coûts de construction et de la qualité (graphique 4.8). Il ressort toutefois de l'expérience de certains pays membres de l'OCDE que tous les PPP ne sont pas bien gérés et que tous ne procurent donc pas les avantages escomptés. Pour certains services, des contrats à long terme peuvent s'avérer trop rigides compte tenu de l'évolution des besoins du secteur public et de la technologie, tandis que la procédure de mise en place des PPP est souvent longue, complexe et coûteuse pour le secteur public comme pour le secteur privé. Des pays signalent aussi que les coûts de transaction pour les parties privées et publiques sont plus élevés avec les PPP qu'avec les MIT. Enfin, certains pays sont incités à recourir aux PPP pour financer des actifs en dehors du bilan du secteur public. Si un projet de PPP est conçu dans ce but, il peut ne pas figurer dans la dette nette du secteur public. Il en résulte alors un manque de transparence quant aux charges futures et aux risques budgétaires.

Pour répondre à ces défis, l'OCDE a défini des Principes applicables à la gouvernance publique des partenariats public-privé. Ces Principes formulent des orientations précises selon trois grands axes : i) il faut établir pour les PPP un cadre institutionnel clair, prévisible et légitime s'appuyant sur des autorités compétentes et dotées de ressources suffisantes; ii) il faut fonder le choix des PPP sur un objectif d'optimisation de la dépense publique ; iii) il faut utiliser de manière transparente la procédure budgétaire pour réduire au minimum les risques budgétaires et assurer l'intégrité de la procédure de passation du marché.

Il n'y a pas de réponse claire à la question de savoir si l'un des modes de passation des marchés publics permet systématiquement d'obtenir de meilleurs résultats que l'autre, quand on calcule sur la durée de vie totale de l'actif. Il est recommandé de recourir davantage à des évaluations de l'optimisation des ressources pour apprécier en amont si un projet convient bien à la formule PPP. L'optimisation des ressources doit être évaluée à toutes les étapes du projet et tenir compte des coûts de l'actif tout au long de son cycle de vie ainsi que des risques que le projet fait courir au secteur public. Or, si la majorité des pays membres de l'OCDE (21) évaluent l'optimisation des ressources en termes relatifs permise par les PPP par rapport aux marchés d'infrastructure traditionnels (MIT), ils ne le font, la plupart du temps, que pour certains projets (tableau 4.9). Les évaluations de l'optimisation des ressources en termes absolus sont plus courantes. Cer- tains pays ont mis en place des unités consacrées aux PPP afin de garantir une évaluation fiable de l'optimisation des ressources, de faire coïncider les objectifs déclarés et les objectifs de profit du secteur privé et de veiller à la transparence dans l'administration des PPP. En 2010, 17 pays de l'OCDE disposaient d'unités de ce type, et d'autres commençaient à suivre cette tendance.

\section{Méthodologie et définitions}

Les données sont tirées de l'Enquête de l'OCDE sur les pratiques et procédures budgétaires (2012). Les répondants étaient principalement de hauts responsables budgétaires des pays membres de l'OCDE. Les réponses représentent l'évaluation par les pays eux-mêmes de leurs pratiques et procédures actuelles. Les données ne portent que sur l'administration centrale/fédérale et ne prennent pas en compte les pratiques budgétaires à l'échelon des administrations d'États fédérés et des administrations locales.

Le concept de PPP englobe à la fois les PPP purs, c'est-à-dire les projets dans le cadre desquels le partenaire privé tire l'essentiel de ses recettes des pouvoirs publics (sous la forme de versements réguliers ou unitaires), et les concessions, dans le cadre desquelles le partenaire privé tire l'essentiel de ses recettes des redevances qu'il demande aux usagers du service.

Les évaluations de l'optimisation des ressources en termes relatifs consistent à comparer plusieurs formes d'obtention d'un actif pour déterminer laquelle optimise le mieux les ressources. Les évaluations de l'optimisation des ressources en termes absolus consistent à déterminer de manière générale si un projet (par exemple un barrage, un aéroport ou une autoroute) représente une utilisation optimale des ressources de la société. Les méthodes employées pour ces deux modes d'évaluation varient selon les pays.

\section{Pour en savoir plus}

Burger, P. et I. Hawkesworth (2013), " Capital budgeting and procurement practices - towards an integrated approach? ", rapport présenté à la réunion annuelle du Réseau des Hauts responsables du budget sur les partenariats public-privé, les 15 et 16 avril 2013.

OCDE (2012), Principes applicables à la gouvernance publique des partenariats public-privé, mai 2012, OCDE, Paris, http://acts.oecd.org/Instruments/ShowInstrumentView.aspx? InstrumentID $=275$ \&Lang $=$ fr\&Book=False.

OCDE (2010), Les unités consacrées aux partenariats public-privé : une étude des structures institutionnelles et de gouvernance, Éditions OCDE, Paris, http://dx.doi.org/10.1787/9789264083868-fr.

\section{Notes relatives aux graphiques et aux tableaux}

4.8 et 4.9 : On ne dispose pas de données pour l'Islande.

Informations sur les données concernant Israël : http://dx.doi.org/ $10.1787 / 888932315602$. 
4.8. Évaluations nationales des PPP par rapport aux MIT, selon divers critères

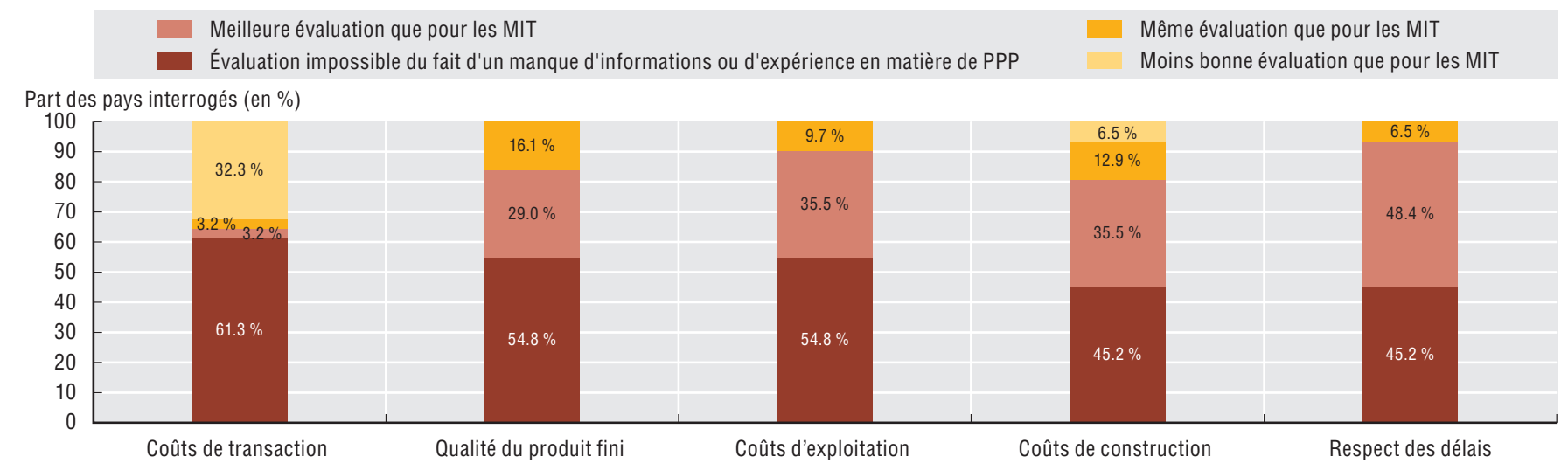

Source : Enquête 2012 de l'OCDE sur les pratiques et procédures budgétaires.

StatLink काis http://dx.doi.org/10.1787/888933159449

4.9. Évaluation des PPP et des MIT sur le plan de l'optimisation des ressources ; unités consacrées aux PPP

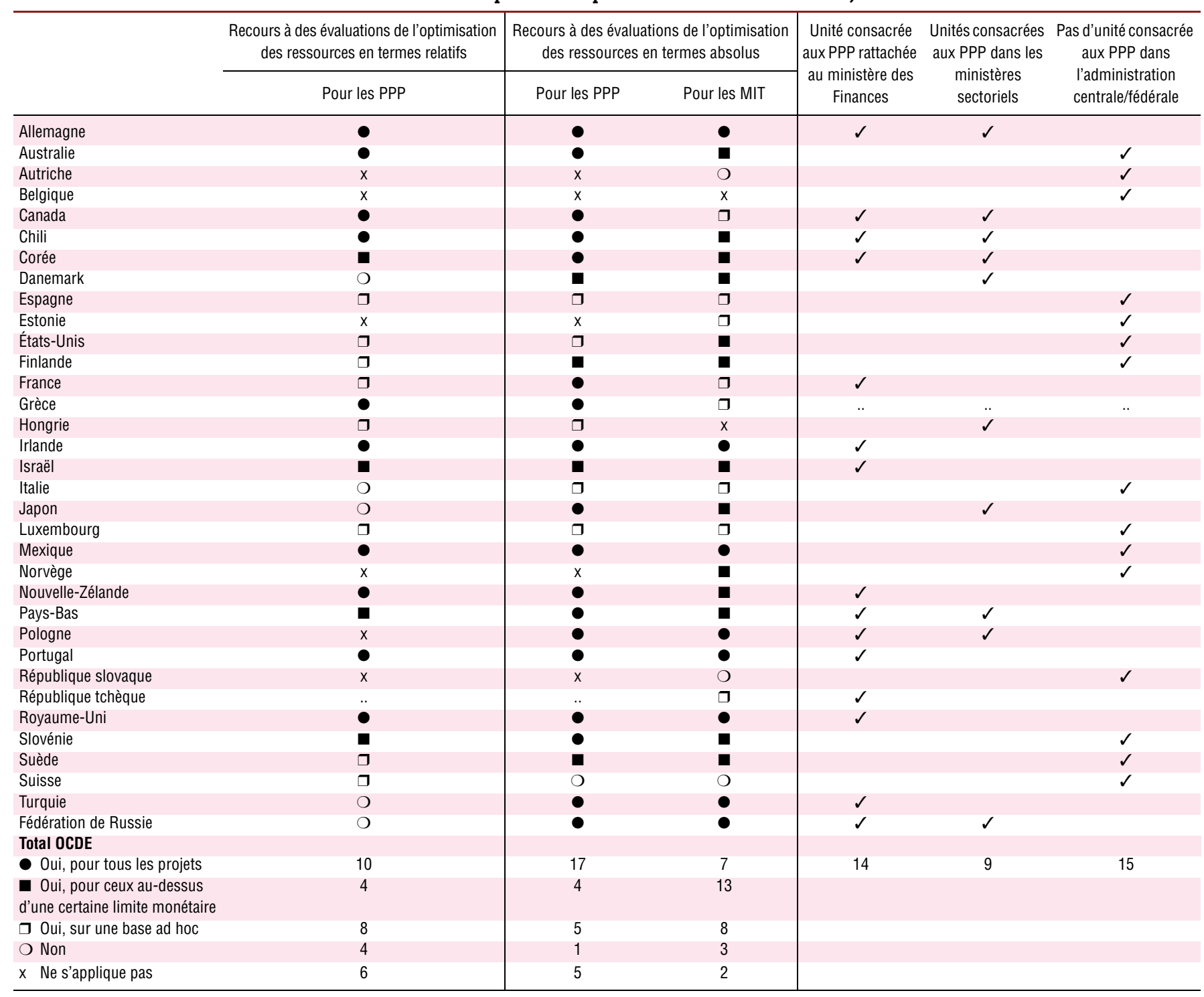

Source : Enquête 2012 de l'OCDE sur les pratiques et procédures budgétaires. 
$\mathrm{Au}$ milieu des années 90, des théoriciens de l'économie ont suggéré que les pays pourraient adapter à la sphère budgétaire certaines des expériences positives résultant de l'indépendance des banques centrales. Toutefois, c'est seulement après la poussée des déficits et de la dette publics qui a suivi la crise récente qu'un nombre croissant de pays ont décidé de créer des institutions budgétaires indépendantes (les IBI, généralement connues sous le nom de conseils budgétaires ou d'offices parlementaires du budget).

En créant ces institutions, les autorités cherchent à étayer des règles budgétaires qui se sont avérées insuffisantes pour assurer une gestion prudente des finances publiques, mais aussi à manifester leur volonté d'agir de façon vertueuse après la crise. Cela vaut particulièrement pour l'Union européenne, où de nouvelles règles demandent aux États membres de disposer d'instances indépendantes pour surveiller le respect des règles budgétaires et formuler ou entériner les prévisions macroéconomiques. Il y a d'autres exemples récents (Canada, Australie) de création d'instances destinées à améliorer la transparence budgétaire et à renforcer le rôle du parlement dans la procédure budgétaire. À cette nouvelle génération d'IBI s'ajoutent quelques institutions nettement plus anciennes, comme l'Office budgétaire du Congrès américain (CBO) ou le Bureau d'analyse de la politique économique néerlandais (CPB).

Actuellement, les institutions budgétaires indépendantes sont très diverses : leurs rôles, leurs ressources et leurs structures varient considérablement selon les pays. En dehors de l'analyse de la politique et des propositions budgétaires, elles exercent souvent des fonctions en matière de prévisions, de suivi du respect des règles budgétaires, d'examen de la viabilité budgétaire à long terme, de chiffrage des mesures proposées par les autorités et d'analyse de certains thèmes.

Les exigences découlant des nouvelles règles de l'Union européenne semblent indiquer qu'il existe un modèle dominant au sein de l'Union, bien que les besoins individuels des pays et leur contexte institutionnel propre continuent à déterminer nombre de choix. À l'exception du CPB néerlandais (et, jusqu'à une date récente, du Conseil économique danois), tous les conseils budgétaires des pays de l'OCDE membres de l'UE sont chargés de contrôler le respect des règles budgétaires. Dans les pays de l'OCDE extérieurs à l'UE, aucune des IBI n'est chargée de cette tâche.

Le rôle des IBI en matière de prévisions prend plusieurs formes. Le CPB néerlandais et l'Office de responsabilité budgétaire (OBR) britannique sont chargés d'établir les prévisions officielles. D'autres formulent des prévisions alternatives (aux États-Unis et au Canada, par exemple). D'autres encore donnent une opinion sur les prévisions des autorités (en France, en Irlande et en Suède, par exemple). La grande majorité des institutions effectue aussi des analyses de la viabilité budgétaire à long terme. Un peu moins de la moitié des IBI des pays membres de l'OCDE participe au chiffrage des mesures proposées par les autorités, et cette fonction est le plus souvent exercée par des offices parlementaires du budget. Le CPB néerlandais et le chef de l'Office parlementaire du budget (PBO) australien sont aussi chargés de chiffrer les programmes électoraux.

On trouve une diversité analogue dans la structure et les ressources des IBI. Certaines sont placées sous l'autorité de l'exécutif, tandis qu'un plus petit nombre dépend du parlement (en Australie, au Canada et en Italie - dans ce pays, une IBI a été instituée par une loi de décembre 2012 et devrait devenir opérationnelle en 2014 - et aux États-Unis). Deux ont été créées avec le statut d'unités autonomes au sein de l'institution nationale de contrôle des comptes publics (Finlande et France). Les IBI peuvent avoir une direction individuelle ou collégiale (conseil). Le CBO dispose du budget le plus important (45 millions USD) et d'environ 250 collaborateurs, alors que le Conseil budgétaire consultatif irlandais (IFAC) a un budget inférieur à 1 million EUR et un secrétariat comprenant moins de cinq personnes. Les institutions qui jouent un rôle dans le chiffrage des politiques publiques - l'une des fonctions exigeant le plus de ressources et un personnel ayant une connaissance spécifique des programmes publics - ont en général des effectifs plus nombreux. Trois institutions ont un budget alimenté par des engagements de financement pluriannuels, une pratique qui peut renforcer l'indépendance et protéger des pressions politiques (Australie, Pays-Bas et Royaume-Uni).

\section{Méthodologie et définitions}

Les données, recueillies au moyen d'une série de questions posées en 2012-2013 en vue de l'élaboration de notes approfondies par pays sur les institutions budgétaires indépendantes, portent sur les institutions de 17 pays membres de l'OCDE. Lors de la préparation des notes, le Secrétariat de l'OCDE a largement consulté des responsables des institutions budgétaires indépendantes retenues ainsi que des représentants des parlements et des administrations, des universitaires et, si nécessaire, d'autres parties prenantes. On rassemble actuellement des données supplémentaires sur de nouvelles institutions.

Le Chili a mis en place une instance budgétaire consultative au début de 2013. L'Espagne a déposé un projet de loi visant à créer une autorité budgétaire indépendante qui devrait être adopté à la fin de 2013.

\section{Pour en savoir plus}

Calmfors, L. et S. Wren-Lewis (2011), «What Should Fiscal Councils Do? ", Centre for Economic Studies and IFO Institute for Economic Research (CESifo), vol. 26, n 68, pp. 649-695, Londres.

Hagemann, R. (2011), " How Can Fiscal Councils Strengthen Fiscal Performance? ", OECD Journal: Economic Studies, vol. 2011/1, http://dx.doi.org/10.1787/eco_studies-2011$5 \mathrm{~kg} 2 \mathrm{~d} 3 \mathrm{~g} \times 4 \mathrm{~d} 5 \mathrm{c}$.

Kopits, G. (2011), « Independent Fiscal Institutions: Developing Good Practices ", Revue de l'OCDE sur la gestion budgétaire, vol. 11/3, pp. 35-52, http://dx.doi.org/10.1787/budget-11$5 \mathrm{~kg} 3 p d g c p n 42$

OCDE (2013, à paraître), Principles for Independent Fiscal Institutions and Country Notes, Éditions OCDE, Paris.

\section{Notes relatives aux tableaux}

On trouvera dans StatLinks des notes par pays. 
4.10. Rôles et ressources des institutions budgétaires indépendantes des pays de l'OCDE (2013)

\begin{tabular}{|c|c|c|c|c|c|c|c|c|c|}
\hline & Nom de l'institution & $\begin{array}{l}\text { Année } \\
\text { de } \\
\text { création }\end{array}$ & Budget & Effectifs & $\begin{array}{c}\text { Rôle dans } \\
\text { les prévisions } \\
\text { macro- } \\
\text { économiques }\end{array}$ & $\begin{array}{c}\text { Rôle dans l'analyse } \\
\text { de la viabilité } \\
\text { budgétaire } \\
\text { à long terme }\end{array}$ & $\begin{array}{c}\text { Rôle dans le } \\
\text { contrôle du respect } \\
\text { des règles } \\
\text { budgétaires }\end{array}$ & $\begin{array}{c}\text { Rôle dans } \\
\text { le chiffrage } \\
\text { des } \\
\text { politiques }\end{array}$ & $\begin{array}{c}\text { Rôle dans le } \\
\text { chiffrage des } \\
\text { programmes } \\
\text { électoraux }\end{array}$ \\
\hline Australie & $\begin{array}{l}\text { Office parlementaire } \\
\text { du budget (PBO) }\end{array}$ & 2011 & $\begin{array}{l}2.4 \text { millions AUD } \\
\text { sur quatre ans, plus } \\
\text { un financement } \\
\text { supplémentaire } \\
\text { de court terme en } \\
\text { période électorale }\end{array}$ & $\begin{array}{c}\text { 30-35 agents en cours } \\
\text { de recrutement }\end{array}$ & $\diamond$ & - & O & - & - \\
\hline Autriche & $\begin{array}{c}\text { Comité de la dette } \\
\text { publique (GDC) }\end{array}$ & 1970 & $\begin{array}{l}\text { Données non } \\
\text { disponibles }\end{array}$ & $\begin{array}{c}15 \text { membres du } \\
\text { Comité, secrétariat de } \\
3 \text { personnes }\end{array}$ & 口 & $\bullet$ & $\bullet$ & O & O \\
\hline Belgique & $\begin{array}{l}\text { Conseil supérieur des } \\
\text { finances (CSF) }\end{array}$ & 1936 & $\begin{array}{l}\text { Données non } \\
\text { disponibles }\end{array}$ & $\begin{array}{l}27 \text { membres du } \\
\text { Conseil, secrétariat de } \\
14 \text { personnes }\end{array}$ & 口 & $\bullet$ & $\bullet$ & O & O \\
\hline Canada & $\begin{array}{c}\text { Directeur } \\
\text { parlementaire du } \\
\text { budget (DPB) }\end{array}$ & 2008 & CAD $2.8 \mathrm{M}$ & 15 & $\square$ & 0 & O & $\bullet$ & O \\
\hline Corée & $\begin{array}{l}\text { Office du budget de } \\
\text { I'Assemblée nationale } \\
\text { (NABO) }\end{array}$ & 2003 & USD $12.7 \mathrm{M}$ & 125 & $\square$ & ○ & O & - & O \\
\hline Danemark & Conseil économique & 1962 & DKK $23.5 \mathrm{M}$ & $\begin{array}{l}25 \text { membres du } \\
\text { Conseil, secrétariat } \\
\text { de } 30 \text { personnes }\end{array}$ & $\square$ & - & ○ & O & O \\
\hline États-Unis & $\begin{array}{l}\text { Office budgétaire du } \\
\text { Congrès (CBO) }\end{array}$ & 1974 & USD $45.2 \mathrm{M}$ & 250 & $\square$ & - & O & - & O \\
\hline Finlande & $\begin{array}{l}\text { Service du contrôle } \\
\text { des politiques } \\
\text { budgétaires et du } \\
\text { secrétariat général }\end{array}$ & 2013 & EUR 1.4 M & $\begin{array}{l}7 \text { (plus des agents } \\
\text { chargés de l'appui } \\
\text { et de la } \\
\text { communication) }\end{array}$ & 口 & ○ & - & O & O \\
\hline France & $\begin{array}{l}\text { Haut Conseil des } \\
\text { finances publiques } \\
\text { (HCFP) }\end{array}$ & 2013 & $\begin{array}{l}\text { EUR } 782000 \\
\quad(\text { FY 2013) }\end{array}$ & $\begin{array}{l}10 \text { membres du } \\
\text { Conseil, } 5 \text { agents en } \\
\text { cours de recrutement }\end{array}$ & 口 & O & - & O & O \\
\hline Irlande & $\begin{array}{l}\text { Conseil consultatif } \\
\text { budgétaire }\end{array}$ & 2011 & $\begin{array}{l}\text { EUR } 800000 \\
\quad(\text { FY 2013) }\end{array}$ & $\begin{array}{c}5 \text { membres du Conseil, } \\
\text { secrétariat de } \\
3 \text { personnes }\end{array}$ & $\mathbf{\square}$ & O & - & O & O \\
\hline Mexique & $\begin{array}{l}\text { Centre d'étude des } \\
\text { finances publiques } \\
\text { (CEFP) }\end{array}$ & 1998 & $\begin{array}{l}50.9 \mathrm{M} \text { pesos } \\
\text { (FY 2009) }\end{array}$ & 59 & $\square$ & 0 & O & ○ & O \\
\hline Pays-Bas & $\begin{array}{l}\text { Bureau d'analyse de la } \\
\text { politique économique } \\
\text { (CPB) }\end{array}$ & 1945 & EUR $13.5 \mathrm{M}$ & 117 & 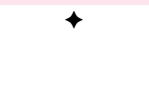 & 0 & O & ○ & 0 \\
\hline Portugal & $\begin{array}{l}\text { Conseil des finances } \\
\text { publiques (CFP) }\end{array}$ & 2011 & $\begin{array}{l}\text { EUR 2.65 M } \\
\text { (FY 2013) }\end{array}$ & $\begin{array}{c}5 \text { membres du Conseil, } \\
15-20 \text { agents en cours } \\
\text { de recrutement }\end{array}$ & 口 & - & - & O & O \\
\hline République slovaque & $\begin{array}{c}\text { Conseil de } \\
\text { responsabilité } \\
\text { budgétaire (CBR) }\end{array}$ & 2012 & EUR 2 M (FY 2013) & $\begin{array}{c}3 \text { membres du Conseil, } \\
15-20 \text { agents en cours } \\
\text { de recrutement }\end{array}$ & $\diamond$ & - & - & - & $\mathrm{O}$ \\
\hline Royaume-Uni & $\begin{array}{c}\text { Office de } \\
\text { responsabilité } \\
\text { budgétaire (OBR) }\end{array}$ & 2010 & GBP $1.75 \mathrm{M}$ & $\begin{array}{c}\text { Comité de responsabilité } \\
\text { budgétaire composé de } \\
3 \text { membres, } 2 \text { membres } \\
\text { non exécutifs, } \\
\text { secrétariat } \\
\text { de } 17 \text { personnes }\end{array}$ & $\hookrightarrow$ & $\bullet$ & - & $\bullet$ & O \\
\hline Slovénie & Conseil budgétaire & 2009 & $\begin{array}{l}\text { EUR } 100000 \\
\quad(F Y 2012)\end{array}$ & $\begin{array}{c}7 \text { membres du Conseil, } \\
\text { pas de secrétariat }\end{array}$ & 口 & - & - & O & O \\
\hline Suède & $\begin{array}{l}\text { Conseil de politique } \\
\text { budgétaire (FPC) }\end{array}$ & 2007 & SEK $7.55 \mathrm{M}$ & $\begin{array}{c}6 \text { membres du Conseil, } \\
\text { secrétariat } \\
\text { de } 5 \text { personnes }\end{array}$ & 口 & - & - & 0 & O \\
\hline
\end{tabular}

Oui.

Non.

$\diamond$ Aucun rôle.

\ Établissement des prévisions officielles.

- Simple évaluation des prévisions.

$\square$ Établissement de prévisions alternatives.

Source : OCDE (2013, à paraître), Principles for Independent Fiscal Institutions and Country Notes, Éditions OCDE, Paris. 


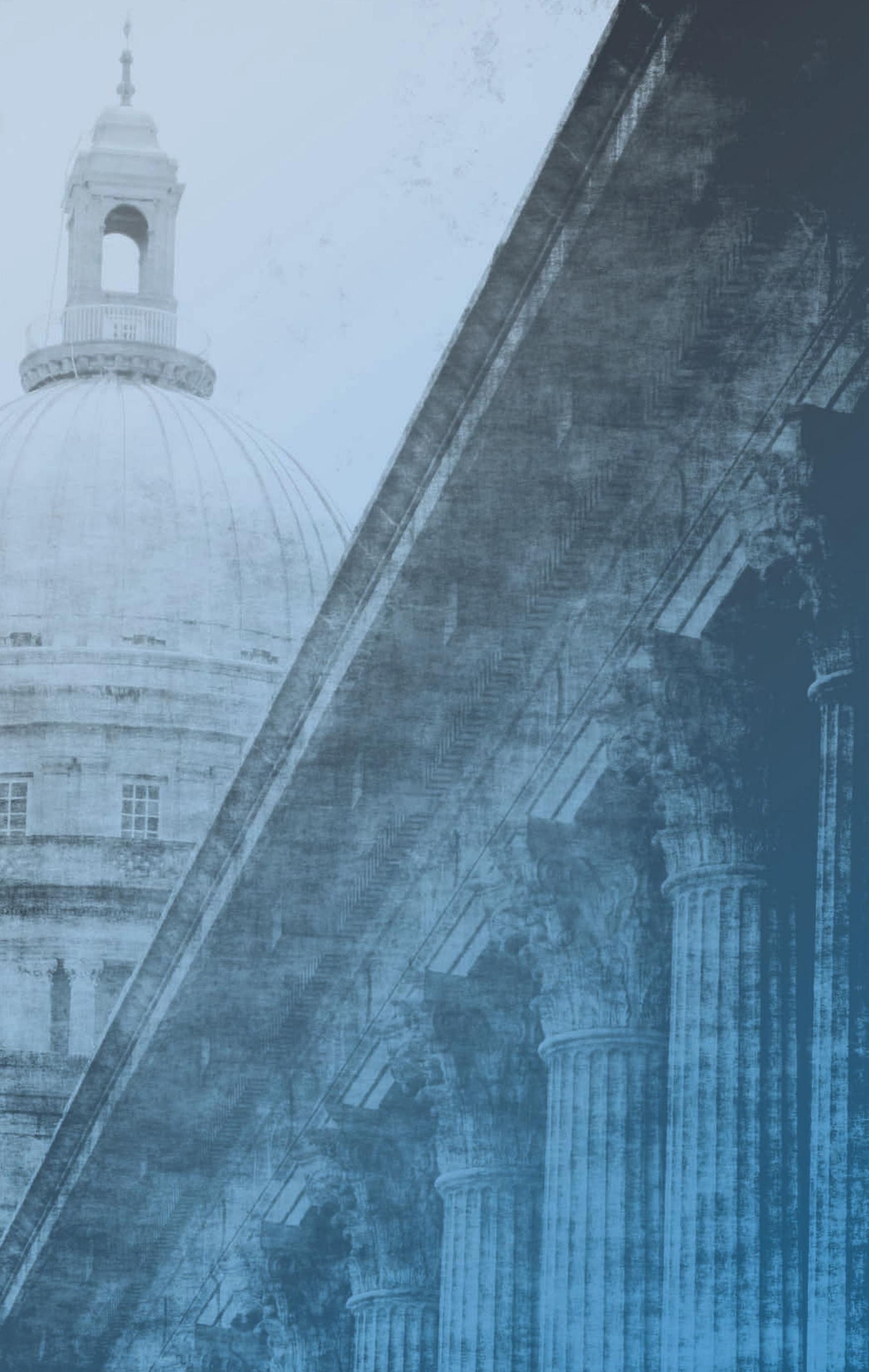



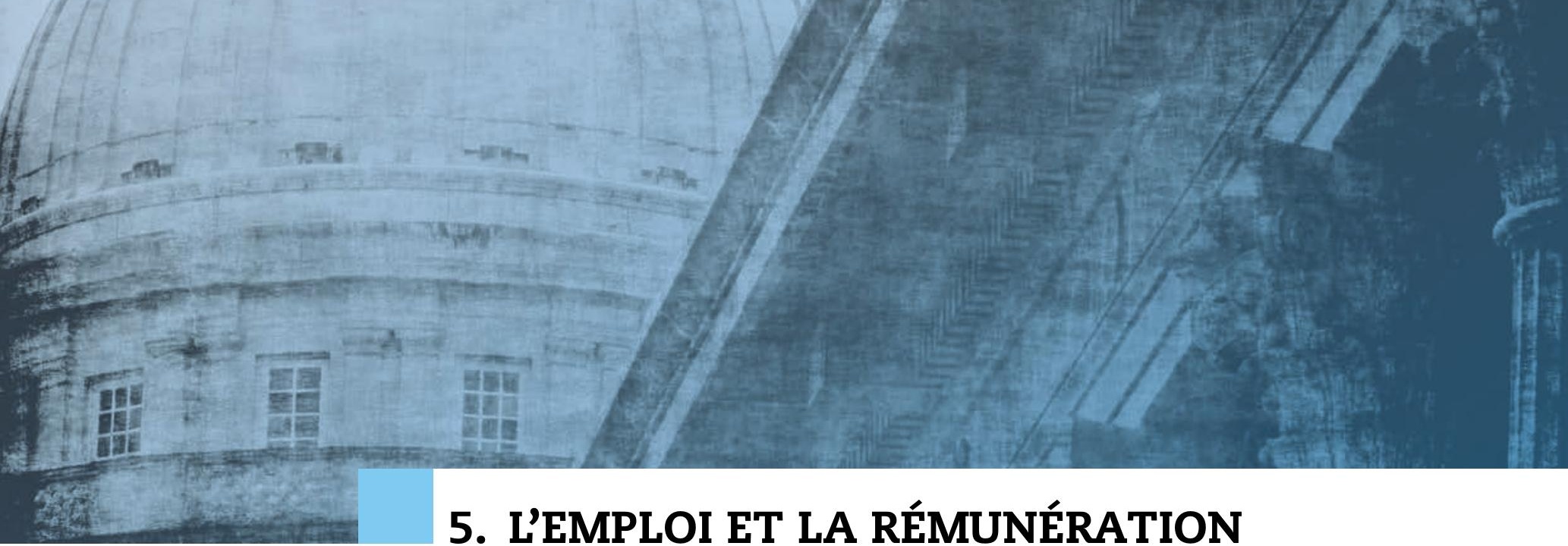

\section{L'EMPLOI ET LA RÉMUNÉRATION DANS LE SECTEUR PUBLIC}

On associe souvent l'importance des administrations publiques au nombre d'agents qu'elles emploient, même si, avec l'externalisation croissante des activités de service public, l'emploi dans la fonction publique devient un indicateur de moins en moins fiable du rôle des administrations. Cela étant, dans certains groupes professionnels clés - enseignants, personnel infirmier, médecins et policiers, par exemple - la fonction publique reste le principal employeur.

Dans ce chapitre, nous examinons l'évolution de l'emploi dans le secteur public entre 2001 et 2011. Les données dont nous disposons démontrent une nouvelle fois que l'emploi public est rigide. S'il est urai que bon nombre de pays membres de l'OCDE ont annoncé un gel des embauches et des réductions d'effectifs dans le cadre de plans d'assainissement des finances publiques, peu sont parvenus à réduire l'emploi public de façon durable et significative. Sachant que la demande de services publics ne diminue pas, il n'y a que deux moyens de réduire l'emploi à long terme: les gains de productivité, qui sont rarement quantifiables mais que l'on suppose relativement modestes; et l'externalisation, dans le cadre de laquelle les administrations continuent d'assumer la charge financière d'un service public, mais chargent le secteur privé de le fournir.

En dehors des données sur l'emploi public, peu de sujets suscitent autant d'intérêt que la rémunération des agents publics. Par rapport à l'édition de 2011, les données sur la rémunération présentées ici sont plus complètes et internationalement comparables. Les données ont été recueillies dans le cadre d'une d'enquête plus perfectionnée, pour un ensemble élargi d'emplois clés de l'administration centrale. À la rémunération des cadres supérieurs et des cadres moyens, des spécialistes et des secrétaires s'ajoute la rémunération d'agents assurant des services publics clés tels que les enseignants, les agents de l'immigration, les agents des douanes et des impôts et les agents de police. Le nombre de pays inclus dans la collecte des données est passé de 20 en 2011 à 26 en 2013. Toutefois, les pays participants n'ont pas tous pu fournir de données pour tous les emplois. 
Chaque État détermine les services publics qu'il souhaite fournir et la manière dont ils seront assurés. Certains pays s'appuient plus que d'autres, pour fournir ces services, sur des agents publics : ainsi, les enseignants, les professionnels de la santé, les personnels des services de sécurité et d'urgence et les administrateurs publics ont le statut de fonctionnaires dans de nombreux pays membres, et y assurent des services importants. D'autres pays ont davantage recours au secteur privé et au secteur non lucratif. La part de la population active qui travaille pour le secteur public rend compte de ce choix et joue un rôle dans le coût final des services publics pour le contribuable. L'importance relative de l'emploi public peut également avoir des répercussions sur le marché du travail et sur la productivité de l'économie.

Le volume de l'emploi public varie considérablement selon les pays membres de l'OCDE, les administrations des pays nordiques employant une part de la population active plus élevée que les autres. En 2011, par exemple, les administrations norvégienne et danoise employaient environ $30 \%$ de la population active, contre $9 \%$ au maximum en Corée, au Japon, en Grèce et au Mexique.

Sur l'ensemble des pays membres de l'OCDE, la part de l'emploi au sein des administrations publiques est restée relativement stable entre 2001 et 2011, à un peu moins de $16 \%$ de la population active en moyenne. Globalement, sur cette période, cette part n'a baissé que de manière minime. La République slovaque, la Suède, le Mexique et le Portugal ont enregistré les baisses les plus importantes: un peu plus de 2 points de pourcentage. L'emploi dans les sociétés publiques - telles que les postes et les chemins de fer de certains pays membres de l'OCDE - représente une part mineure de la population active, généralement inférieure à celle de l'emploi dans les administrations publiques. D'une façon générale, les sociétés publiques des pays de l'Europe centrale et orientale emploient une part de la population active plus élevée que pour les autres pays membres de l'OCDE, en dépit de la puissante vague de privatisations que ces pays ont connue au début des années 90. Globalement, l'emploi dans les sociétés publiques exprimé en pourcentage de la population active a baissé dans la majorité des pays membres de l'OCDE, passant en moyenne de 5.7 \% en 2001 à 4.7 \% en 2011.

\section{Méthodologie et définitions}

Les données portent sur 2001 et 2011, et elles ont été rassemblées par l'Organisation internationale du travail (OIT) et l'OCDE. Elles sont fondées sur les définitions du Système de comptabilité nationale (SCN) et recouvrent l'emploi dans les administrations publiques et les sociétés publiques. Le secteur des administrations publiques englobe tous les niveaux d'administration (administration centrale, administrations d'États fédérés, administrations locales et administrations de sécurité sociale) et comprend les ministères proprement dits ainsi que les organismes et départements publics et les institutions sans but lucratif contrôlées et principalement financées par les pouvoirs publics. Les sociétés publiques sont des entités juridiques principalement détenues ou contrôlées par l'État qui produisent des biens et des services destinés à la vente sur le marché. La catégorie des sociétés publiques englobe les quasi-sociétés.
Les données correspondent au nombre d'agents, sauf pour l'Autriche, l'Italie, la Nouvelle-Zélande, les Pays-Bas et la République tchèque, où elles représentent des équivalents temps plein (ETP). De ce fait, le nombre d'emplois pour ces cinq pays est comparativement sous-évalué. La main-d'œuvre, ou population active, comprend toutes les personnes répondant aux critères pour être incluses parmi les personnes employées ou au chômage. À des fins de comparaison internationale, la population en âge de travailler est généralement définie comme les personnes âgées de 15 ans et plus, même si ce seuil peut varier selon les pays. La population active désigne toutes les personnes en âge de travailler qui fournissent la main-d'œuvre nécessaire à la production de biens et de services au cours d'une période de référence déterminée.

\section{Pour en savoir plus}

OCDE (2011, version française à paraître), Les fonctionnaires, partenaires de la croissance: Pour une fonction publique plus forte, plus resserrée et plus équitable, Éditions OCDE, Paris, http:// dx.doi.org/10.1787/9789264166707-en.

Pilichowski, E. et E. Turkisch (2008), « L'emploi dans le secteur des administrations publiques sous l'angle des coûts de production des biens et des services dans le domaine public ", Documents de travail de l'OCDE sur la gouvernance publique, $n^{\circ} 8$, Éditions OCDE, Paris, http://dx.doi.org/10.1787/ 242053865825 .

\section{Notes relatives aux graphiques}

On ne dispose pas de données pour l'Islande. Les données pour l'Australie et le Chili se rapportent au secteur public (administrations publiques et sociétés publiques). Les données pour l'Allemagne, l'Irlande, le Royaume-Uni, la Suède et l'Ukraine portent sur 2010 et non 2011. Les données pour le Brésil, la Fédération de Russie, la Hongrie, le Japon et le Mexique portent sur 2009 et non 2011. Les données pour le Chili, la Grèce, le Portugal et la Suisse portent sur 2008 et non 2011. Les données pour la France portent sur 2006 et non 2011. Les données pour l'Ukraine portent sur 2002 et non 2001.

5.1 : On ne dispose pas de données relatives à la Corée et à la Turquie pour 2001, et ces pays ne sont pas inclus dans la moyenne OCDE. Les données pour la Norvège portent sur 2010 et non 2011. Les données pour l'Afrique du Sud portent sur 2006 et non 2011.

5.2 : Les données sur les sociétés publiques manquant pour l'Autriche, la Belgique, la Corée, les États-Unis, la Hongrie, Israël, l'Italie, le Japon et le Portugal, les données relatives à ces pays ne sont pas présentées. Les données pour la République tchèque portent sur 2010 et non 2011. Les données pour la Finlande portent sur 2008 et non 2011. Les données pour la Norvège portent sur 2007 et non 2011. Les données pour les Pays-Bas portent sur 2005 et non 2011.

Informations sur les données concernant Israël : http://dx.doi.org/10.1787/ 888932315602 . 
5.1. Emploi dans les administrations publiques, en pourcentage de la population active (2001 et 2011)

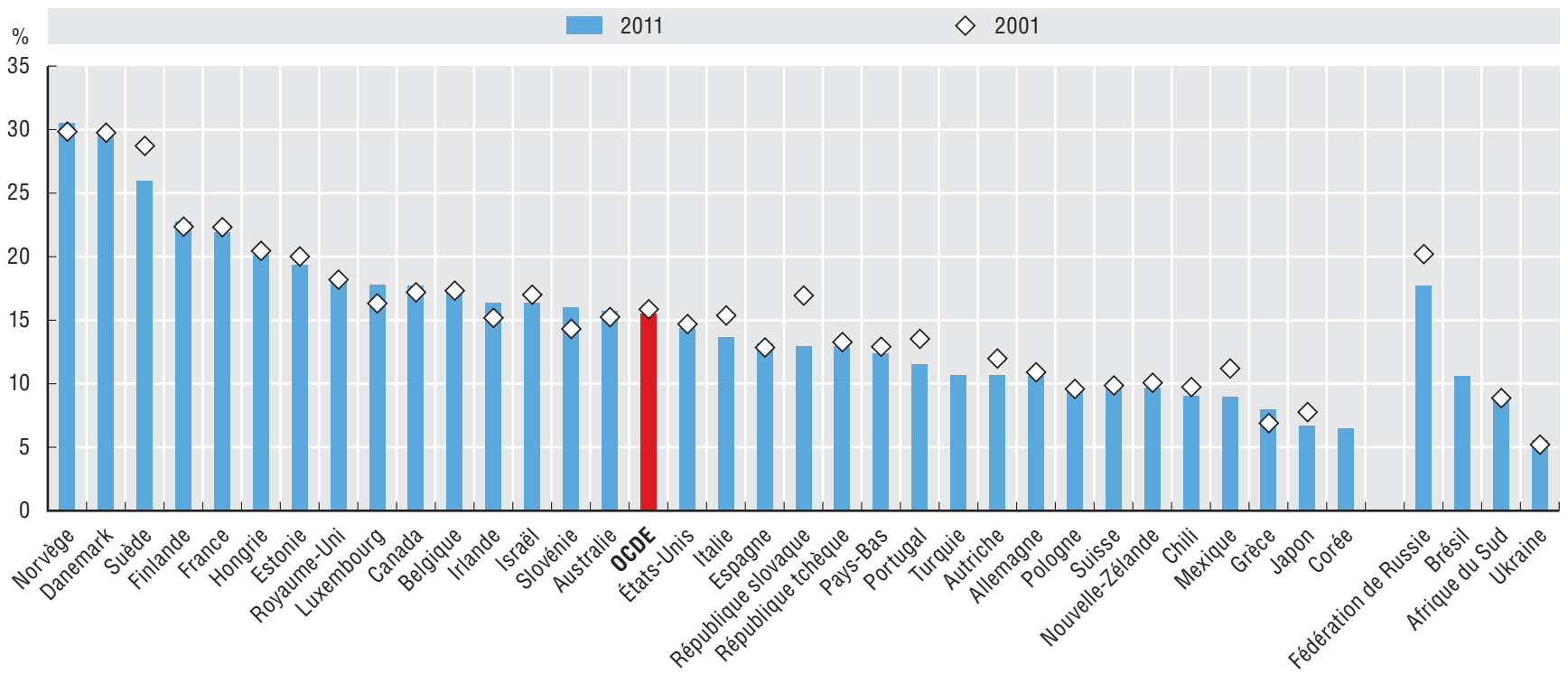

Source: Organisation internationale du travail (OIT), base de données LABORSTA ; Statistiques de la population active de l'OCDE (base de données). Les données relatives à la Corée ont été fournies par des représentants officiels.

\subsection{Emploi dans les administrations et les sociétés publiques, en pourcentage de la population active (2001 et 2011)}

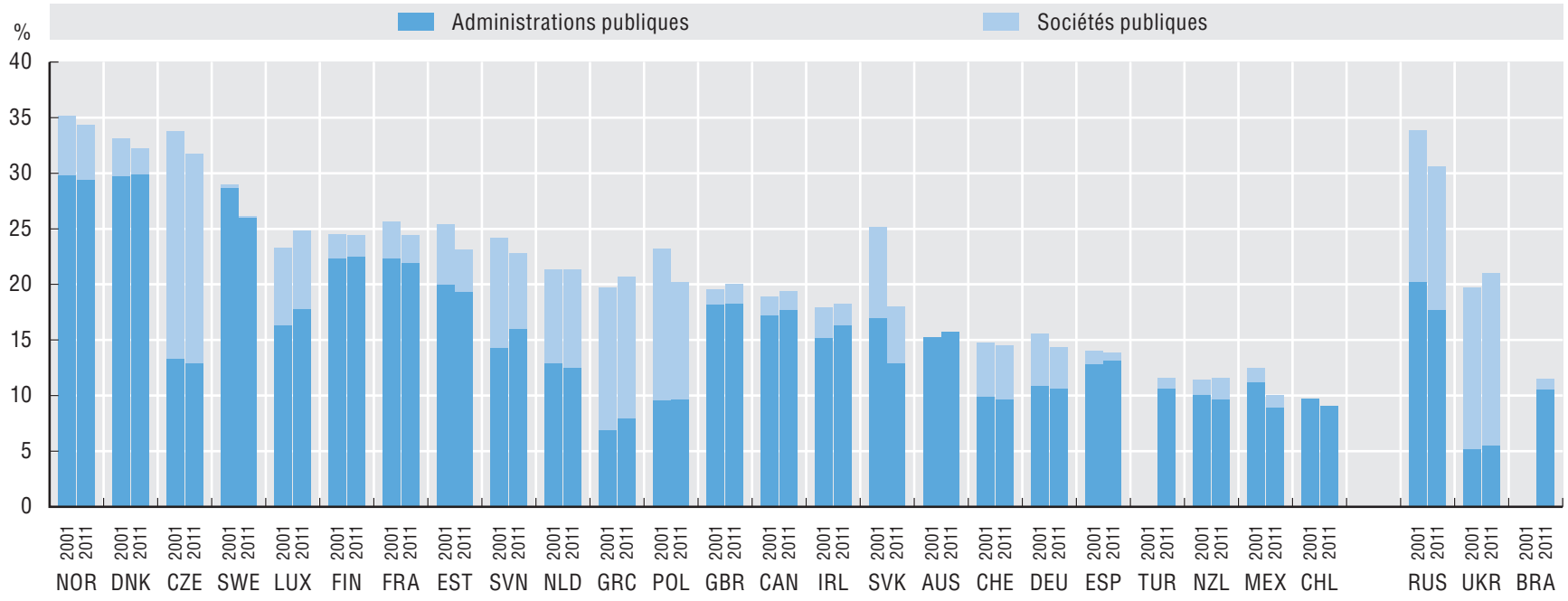

Source : Organisation internationale du travail (OIT), base de données LABORSTA ; Statistiques de la population active de l'OCDE (base de données). 
La part de personnel employé aux échelons administratifs infranationaux constitue un indicateur du niveau de décentralisation des administrations publiques. En général, plus les administrations infranationales concentrent une part importante des effectifs, plus cela signifie que des responsabilités en matière de prestation de services publics ont été déléguées aux administrations régionales et locales. S'il est vrai que la décentralisation peut accroître la réactivité des administrations face aux besoins et aux priorités d'ordre local, elle peut aussi entraîner des disparités dans la prestation des services publics au sein d'un même pays.

En 2011, la majorité des pays employait plus d'agents au niveau infranational qu'au niveau central. Dans les États fédéraux, moins d'un tiers de l'ensemble des fonctionnaires travaillent à l'échelon central, ce qui est le signe d'un niveau élevé de décentralisation. Dans les États unitaires, la proportion de fonctionnaires employés au niveau de l'administration centrale est beaucoup plus variable, puisqu'elle va de moins de $20 \%$ au Japon et en Suède à environ $90 \%$ en Irlande, en Turquie et en Nouvelle-Zélande.

Entre 2001 et 2011, le pourcentage d'agents publics employés au niveau central est resté relativement stable, avec une légère évolution vers une plus grande décentralisation dans la majorité des pays membres de l'OCDE. Quelques pays ont connu une forte décentralisation au cours de cette période, notamment l'Espagne, la République tchèque et le Japon, où la part des agents publics employés au niveau infranational a augmenté d'au moins 10 points de pourcentage. Dans le cas de l'Espagne, cette augmentation est la conséquence d'une délégation de responsabilités vers le niveau infranational associée à une réduction des effectifs depuis 2010. Seul un pays - la Norvège - a connu une centralisation notable des agents publics au cours de cette période, avec une augmentation de 13 points de pourcentage de la part des agents employés au niveau central, même si près des deux tiers des fonctionnaires restent employés à l'échelon infranational.

\section{Méthodologie et définitions}

Les données ont été rassemblées par l'Organisation internationale du travail (OIT) et l'OCDE, et elles portent sur 2001 et 2011, sauf indication contraire. Elles sont fondées sur les définitions du Système de comptabilité nationale (SCN) et recouvrent l'emploi public à l'échelon central et aux échelons infranationaux. L'administration infranationale englobe les administrations d'États fédérés et les administrations locales, y compris les régions, les provinces et les municipalités. Ensemble, le niveau central et les niveaux infranationaux forment les administrations publiques. Les pays ont également fourni des données sur l'emploi dans les administrations de sécurité sociale, qui font partie des administrations publiques et englobent toutes les unités institutionnelles à l'échelon central, à l'échelon des États fédérés et à l'échelon local dont l'activité principale consiste à fournir des prestations sociales. Dans la mesure où les administrations de sécurité sociale relèvent de divers niveaux d'administration, l'emploi dans cette catégorie a été comptabilisé séparément, sauf indication contraire. Cela étant, dans la plupart des pays, à l'exception de la France, du Mexique et de l'Allemagne, les administrations de sécurité sociale emploient un nombre restreint d'agents et représentent un faible pourcentage des effectifs totaux. Les pays suivants sont considérés comme des États fédéraux dans l'ensemble de données: l'Allemagne, la Belgique, le Brésil, le Canada, l'Espagne (considérée comme un pays quasi fédéral), les États-Unis, la Fédération de Russie, le Mexique et la Suisse.

Les données correspondent au nombre d'agents, sauf pour l'Italie, la Nouvelle-Zélande et les Pays-Bas, où elles représentent des équivalents temps plein (ETP). De ce fait, le nombre d'emplois pour ces trois pays est comparativement sous-évalué.

\section{Pour en savoir plus}

Charbit, C. et M. Michalun (2009), " Mind the Gaps: Managing Mutual Dependence in Relations among Levels of Government ", OECD Working Papers on Public Governance, $\mathrm{n}^{\circ} 14$, Éditions OCDE, Paris, http://dx.doi.org/10.1787/22125 3707200 .

OCDE (2011, version française à paraître), Les fonctionnaires, partenaires de la croissance: Pour une fonction publique plus forte, plus resserrée et plus équitable, Éditions OCDE, Paris, http:// dx.doi.org/10.1787/9789264166707-en.

\section{Notes relatives aux graphiques}

On ne dispose pas de données pour l'Australie, l'Autriche, le Chili, la Corée, l'Islande, la Pologne, la République slovaque et le Royaume-Uni. L'emploi au sein des administrations de sécurité sociale n'a pas été identifié séparément pour le Canada, l'Espagne, l'Estonie, les États-Unis, l'Irlande, le Japon, la Norvège, la Nouvelle-Zélande et la Suisse (c'est-à-dire qu'il est comptabilisé dans l'administration centrale ou dans les administrations infranationales). Les données pour l'Allemagne, l'Irlande, la Norvège et la Suède portent sur 2010 et non 2011. Les données pour la Fédération de Russie, le Japon et le Mexique portent sur 2009 et non 2011. Les données pour la Grèce, la Hongrie et la Suisse portent sur 2008 et non 2011. Les données pour la République tchèque portent sur 2007 et non 2011. Les données pour l'Afrique du Sud et la France portent sur 2006 et non 2011.

5.3 : Les données pour le Brésil portent sur 2009. Les données pour le Portugal portent sur 2008.

5.4 : On ne dispose pas de données pour le Portugal.

Informations sur les données concernant Israël : http://dx.doi.org/10.1787/ 888932315602. 
Le niveau de rémunération globale des cadres supérieurs du secteur public est un indicateur de l'attractivité du secteur public et de sa capacité à fidéliser ses recrues pour des fonctions impliquant de hauts niveaux de responsabilité. La rémunération de ces postes représente une part minime des dépenses publiques, mais elle est dotée d'une valeur symbolique, car elle concerne des personnels qui jouent un rôle de premier plan dans l'élaboration et la mise en œuvre des politiques publiques, et dont la nomination est souvent discrétionnaire.

Les cadres de niveau D1 sont des fonctionnaires de très haut rang situés juste en dessous du ministre ou du secrétaire d'État, et les cadres de niveau D2 sont situés immédiatement en dessous des cadres D1 (voir l'annexe D pour plus de précisions). Les cadres D1 gagnent en moyenne $32 \%$ de plus que les cadres D2, mais peuvent parfois gagner moins qu'eux lorsqu'ils ont moins d'ancienneté, c'est-à-dire lorsqu'ils occupent leur poste depuis moins longtemps.

En moyenne, le niveau de rémunération des cadres D1 avoisine les 230000 USD à PPA, dont environ 36000 USD à PPA de cotisations sociales de l'employeur et 31000 USD à PPA d'ajustement lié au temps de travail. La rémunération globale des cadres D2 s'établit à environ 175000 USD à PPA (en comptant les cotisations sociales de l'employeur et les congés). Les différences de rémunération d'un pays à l'autre résultent de disparités dans la part d'employés hautement qualifiés, dans les niveaux d'ancienneté et dans la proportion de femmes occupant des postes à responsabilités. Elles peuvent également s'expliquer par des structures d'organisation différentes. Par rapport au PIB par habitant (qui rend compte des différents niveaux de développement économique des pays), c'est en Italie, en Nouvelle-Zélande et au Chili que le niveau de rémunération des cadres D1 est le plus élevé, et en Islande, en République slovaque et en Norvège qu'il est le plus faible.

Ces disparités de rémunération peuvent aussi être dues à des différences sur les marchés du travail nationaux, s'agissant en particulier des rémunérations pratiquées dans le secteur privé pour des compétences comparables. Comparer la rémunération moyenne des cadres supérieurs à la rémunération des diplômés de l'enseignement supérieur permet d'évaluer en partie cet effet. En moyenne, la rémunération d'un cadre D1 est 3.4 fois supérieure à la rémunération d'un diplômé de l'enseignement supérieur.

\section{Méthodologie et définitions}

Les données se rapportent à 2011 et ont été rassemblées dans le cadre d'une enquête de l'OCDE sur la rémunération des fonctionnaires des administrations centrales/ fédérales (2012). Des représentants de ministères et d'organismes de l'administration centrale y ont répondu par l'intermédiaire du Réseau de l'OCDE sur l'emploi et la gestion publics.

Les données portent sur six ministères/départements de l'administration centrale (Intérieur, Finances, Justice, Éducation, Santé et Environnement ou leurs équivalents). La classification et la définition des professions sont une adaptation de la Classification internationale type des professions (CITP) établie par l'Organisation internationale du travail (OIT). Les niveaux de rémunération sont calculés en établissant la moyenne des rémunérations du personnel en fonction.
La rémunération totale comprend les salaires et traitements ainsi que les cotisations sociales de l'employeur, que le régime soit capitalisé ou non. Les seules cotisations sociales prises en compte sont les cotisations aux régimes de santé et de retraite, afin d'obtenir des données cohérentes à travers l'ensemble des pays.

On a converti les rémunérations en USD en utilisant les PPA pour le PIB tirées de la Base de données des Statistiques de l'OCDE sur les comptes nationaux. Les données sont ajustées pour tenir compte du nombre moyen de jours de congé mais pas du nombre d'heures travaillées par semaine, puisque les cadres sont censés - officiellement ou non - avoir des journées de travail plus longues.

Voir l'annexe D pour une présentation complète de la méthodologie suivie.

\section{Pour en savoir plus}

OCDE (2012), Public Sector Compensation in Times of Austerity, Éditions OCDE, Paris, http://dx.doi.org/10.1787/9789264177758-en.

\section{Notes relatives aux graphiques}

On ne dispose pas de données pour le Canada, la Hongrie, l'Irlande, le Luxembourg, le Mexique, la République tchèque, la Suisse et la Turquie.

Il n'y a pas de distinction entre les données portant sur la rémunération des postes $\mathrm{D} 2$ et les données relatives à la rémunération des postes D1 pour la Finlande et la Slovénie. Belgique : les ministères de l'Éducation et de l'Environnement ne sont pas pris en compte, parce qu'ils ne relèvent pas de l'administration fédérale. Danemark : le ministère de l'Éducation n'est pas pris en compte, parce que beaucoup de ses missions ont été transférées à d'autres ministères. Estonie : on ne dispose pas de données relatives aux ministères de l'Éducation et de l'Environnement. Grèce, Nouvelle-Zélande : on ne dispose que de données sur les rémunérations plancher et les rémunérations plafond; on ne présente donc pas ici la moyenne effective, mais la moyenne entre le salaire plancher et le salaire plafond. Islande : le ministère de la Justice relève du ministère de l'Intérieur. Italie : une loi de 2011 plafonne la rémunération des cadres supérieurs à 370000 USD à PPA à partir de 2012. Japon : les données sont présentées en termes de niveaux plancher et plafond de la rémunération totale ; la moyenne arithmétique a été prise en compte dans la moyenne de l'OCDE. République slovaque : seule la moitié des agents du ministère de la Justice est prise en compte, pour des raisons statistiques (cohérence avec les codes CITP). Le directeur général (poste D1 ; un directeur général par ministère) peut demander chaque mois la prise en charge de ses frais de service obligatoires et d'autres dépenses personnelles, sous forme d'une rémunération forfaitaire non imposable représentant $121 \%$ du salaire de référence le plus élevé (celui-ci étant de 935.50 EUR). Ce montant n'est pas pris en compte dans les données. Espagne : les données relatives aux postes D1 ne tiennent pas compte des primes. Suède : le ministère de l'Intérieur relève du cabinet du Premier ministre et n'est pas pris en compte dans les données. Royaume-Uni : les données portent sur 2012 (sur la base des PPA 2012) et la moyenne représente une médiane, et non la moyenne arithmétique.

Voir l'annexe D pour des notes supplémentaires.

Informations sur les données concernant Israël : http://dx.doi.org/10.1787/ 888932315602 . 
5.5. Rémunération annuelle moyenne des cadres supérieurs de l'administration centrale (2011)

Ajustée pour tenir compte des différences de congés

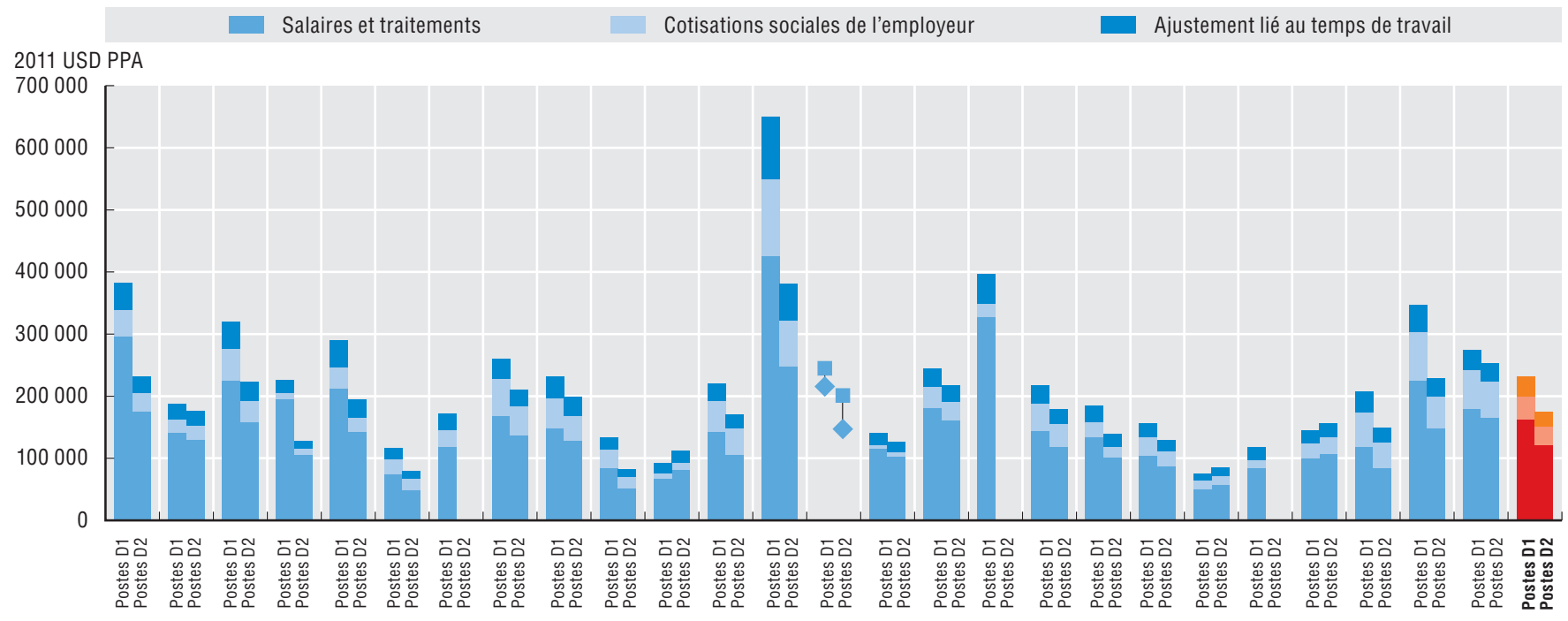

AUS AUT BEL CHL DNK EST FIN FRA DEU GRC ISL ISR ITA JPN KOR NLD NZL NOR POL PRT SVK SVN ESP SWE GBR USA OCDE

Source: Enquête de l'OCDE sur la rémunération des fonctionnaires des administrations centrales/fédérales (2012) ; OCDE, STAN/Statistiques sur les comptes nationaux (base de données).

StatLink काist http://dx.doi.org/10.1787/888933159516

\subsection{Rémunération annuelle moyenne des cadres supérieurs de l'administration centrale par rapport au PIB} par habitant et à la rémunération des diplômés de l'enseignement supérieur

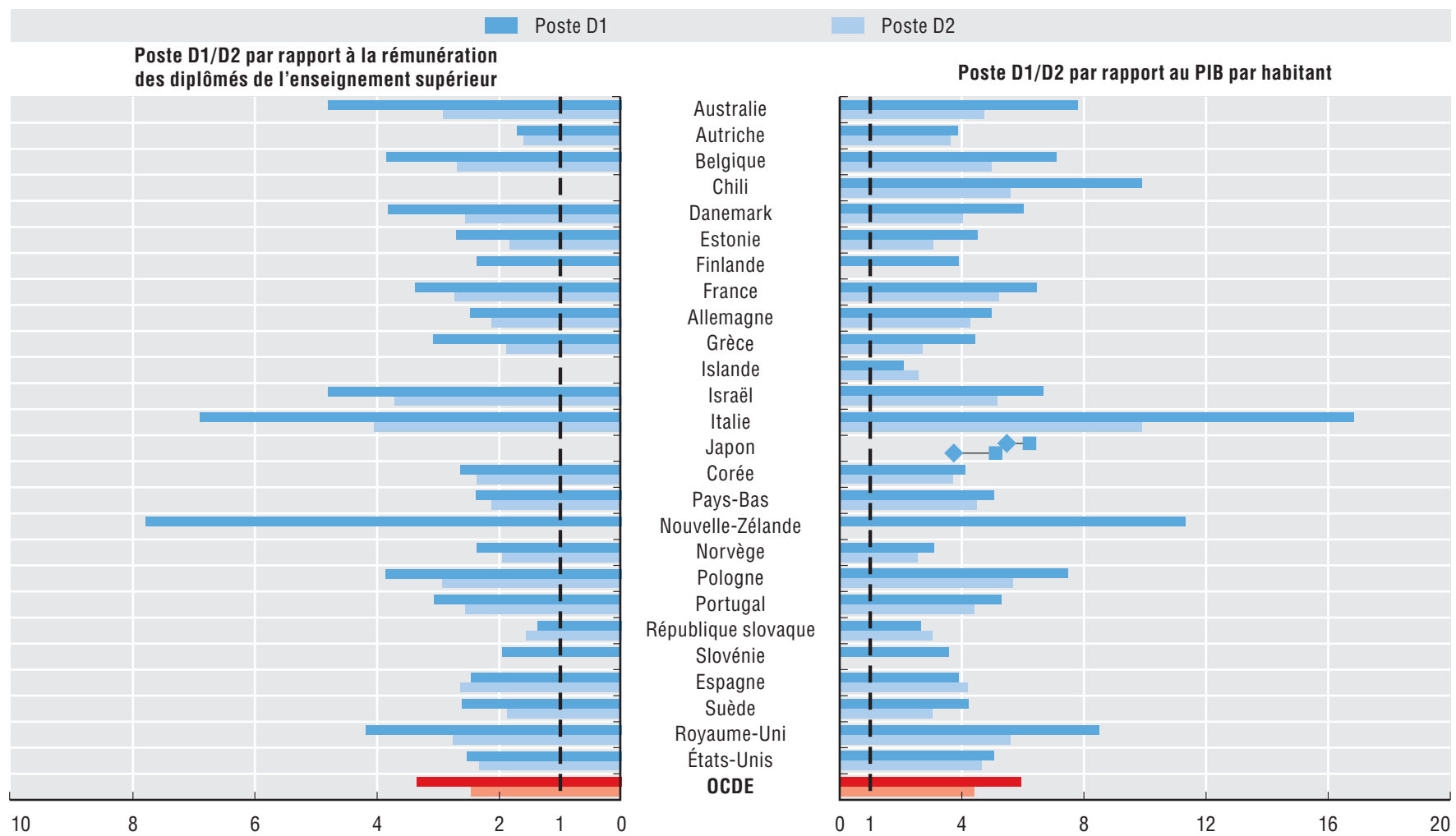

Source: Enquête de l'OCDE sur la rémunération des fonctionnaires des administrations centrales/fédérales (2012); OCDE, STAN/Statistiques sur les comptes nationaux (base de données) ; OCDE (2013), Regards sur l'éducation 2013 : Les indicateurs de l'OCDE, Éditions OCDE, Paris, http://dx.doi.org/10.1787/ eag-2013-fr. 
Les cadres moyens sont situés entre les cadres supérieurs et les spécialistes dans la hiérarchie des effectifs de l'administration centrale. Ils occupent par conséquent une position intermédiaire déterminante pour mener à bien les réformes de la gestion des ressources humaines et pour dialoguer avec tous les agents de la fonction publique et instaurer un climat de confiance et de dialogue social.

En moyenne, la rémunération des cadres moyens de niveau D3 s'élève à environ 126000 USD à PPA, dont $17 \%$ correspondent aux cotisations sociales de l'employeur et $14 \%$ à l'ajustement lié au temps de travail. La rémunération globale des cadres moyens de niveau D4 atteint environ 113000 USD à PPA (après prise en compte des cotisations sociales de l'employeur et des congés).

Les cadres D3 planifient, dirigent et coordonnent le fonctionnement d'une direction/unité administrative au sein d'un ministère, et les cadres $\mathrm{D} 4$ se situent immédiatement en dessous des D3 (voir l'annexe D pour plus de précisions). La différence de rémunération entre les postes $\mathrm{D} 3$ et $\mathrm{D} 4$ est plus faible qu'entre les postes D2 et D1. Les cadres D3 gagnent en moyenne $10 \%$ de plus que les cadres D4. En outre, la rémunération des cadres moyens est nettement inférieure à celle des cadres supérieurs - les cadres supérieurs de niveau D1 gagnent $84 \%$ de plus que les cadres moyens de niveau D3, et presque deux fois plus que les cadres de niveau D4. C'est en Italie, en Australie, au Royaume-Uni et en Israël que cette différence entre les postes D1 et D4 est la plus marquée, et aux États-Unis et en Corée qu'elle est la plus faible.

Par rapport au PIB par habitant, c'est en Pologne et au Chili que le niveau de rémunération des cadres moyens de niveau D3 est le plus élevé, et en Norvège et en Estonie qu'il est le plus faible. De même, c'est aux États-Unis que la rémunération des cadres moyens de niveau D4 est la plus élevée, et en Norvège, en Israël et en Grèce qu'elle est la plus faible.

Ces disparités de rémunération peuvent aussi être dues à des différences sur les marchés du travail nationaux. En moyenne, la rémunération d'un cadre moyen de niveau D3 est 1.8 fois supérieure à la rémunération d'un diplômé de l'enseignement supérieur. Les postes D3 semblent relativement mieux rémunérés en Pologne et, à l'inverse, l'attrait de ces postes est moindre en Autriche, en Estonie et en République slovaque. La comparaison avec la rémunération des diplômés de l'enseignement supérieur montre que les postes D4 sont compétitifs par rapport au secteur privé aux États-Unis et en Belgique et, au contraire, peu attractifs en Autriche, en Israël et en Norvège.

\section{Méthodologie et définitions}

Les données se rapportent à 2011 et ont été rassemblées dans le cadre d'une enquête de l'OCDE sur la rémunération des fonctionnaires des administrations centrales/fédérales (2012). Des représentants de ministères et d'organismes de l'administration centrale y ont répondu par l'intermédiaire du Réseau de l'OCDE sur l'emploi et la gestion publics.

Les données portent sur six ministères/départements de l'administration centrale (Intérieur, Finances, Justice, Éducation, Santé et Environnement ou leurs équivalents). La classification et la définition des professions sont une adaptation de la Classification internationale type des professions (CITP) établie par l'Organisation internationale du travail (OIT). Les niveaux de rémunération sont calculés en établissant la moyenne des rémunérations du personnel en fonction.
La rémunération totale comprend les salaires et traitements bruts ainsi que les cotisations sociales de l'employeur, que le régime soit capitalisé ou non. Les seules cotisations sociales prises en compte sont les cotisations aux régimes de santé et de retraite, afin d'obtenir des données cohérentes à travers l'ensemble des pays.

On a converti les rémunérations en USD en utilisant les PPA pour le PIB tirées de la Base de données des Statistiques de l'OCDE sur les comptes nationaux. Les données sont ajustées pour tenir compte du nombre moyen de jours de congé mais pas du nombre d'heures travaillées par semaine, puisque les cadres sont censés - officiellement ou non - avoir des journées de travail plus longues.

Voir l'annexe D pour une présentation complète de la méthodologie suivie.

\section{Pour en savoir plus}

OCDE (2012), Public Sector Compensation in Times of Austerity, Éditions OCDE, Paris, http://dx.doi.org/10.1787/9789264177758-en.

\section{Notes relatives aux graphiques}

On ne dispose pas de données pour le Canada, la Hongrie, l'Irlande, le Luxembourg, le Mexique, la Nouvelle-Zélande, la République tchèque, la Suisse et la Turquie.

Il n'y a pas de distinction entre les données portant sur la rémunération des postes $\mathrm{D} 4$ et les données relatives à la rémunération des postes D3 pour l'Estonie, la Finlande, le Japon et la Slovénie. Belgique : les ministères de l'Éducation et de l'Environnement ne sont pas pris en compte, parce qu'ils ne relèvent pas de l'administration fédérale. Danemark : le ministère de l'Éducation n'est pas pris en compte, parce que beaucoup de ses missions ont été transférées à d'autres ministères. Estonie : on ne dispose pas de données relatives aux ministères de l'Éducation et de l'Environnement. Grèce : on ne dispose que de données sur les rémunérations plancher et les rémunérations plafond; on ne présente donc pas ici la moyenne effective, mais la moyenne entre le salaire plancher et le salaire plafond. Islande : le ministère de la Justice relève du ministère de l'Intérieur. Japon: les données relatives aux postes D3 sont présentées en termes de niveaux plancher et plafond de la rémunération totale ; la moyenne arithmétique a été prise en compte dans la moyenne de l'OCDE. République slovaque : seule la moitié des agents du ministère de la Justice est prise en compte, pour des raisons statistiques (cohérence avec les codes CITP). Suède : le ministère de l'Intérieur relève du cabinet du Premier ministre et n'est pas pris en compte dans les données. Royaume-Uni : les données portent sur 2012 (sur la base des PPA 2012) et la moyenne représente une médiane, et non la moyenne arithmétique.

Voir l'annexe D pour des notes supplémentaires.

Informations sur les données concernant Israël : http://dx.doi.org/10.1787/ 888932315602 . 


\subsection{Rémunération annuelle moyenne des cadres moyens de l'administration centrale (2011)}

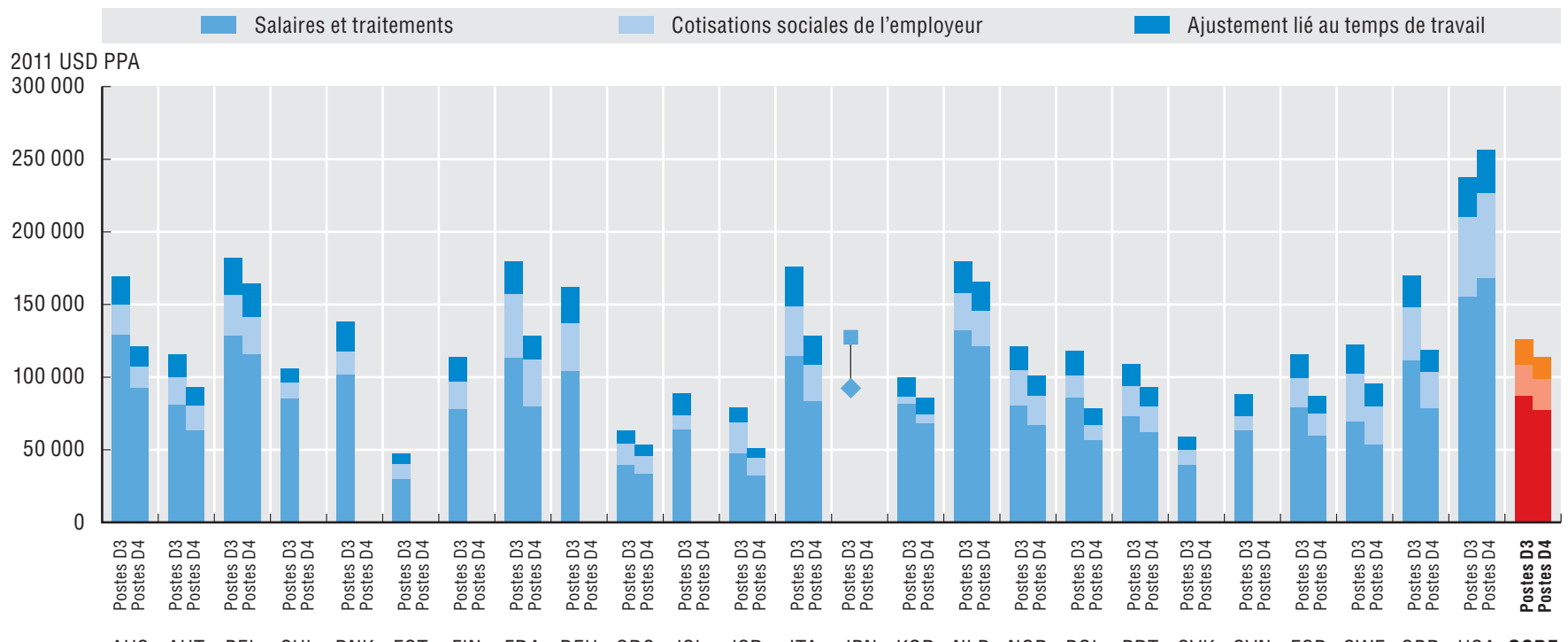

Source: Enquête de l'OCDE sur la rémunération des fonctionnaires des administrations centrales/fédérales (2012) ; OCDE, STAN/Statistiques sur les comptes nationaux (base de données).

5.8. Rémunération annuelle moyenne des cadres moyens de l'administration centrale par rapport au PIB par habitant et à la rémunération des diplômés de l'enseignement supérieur

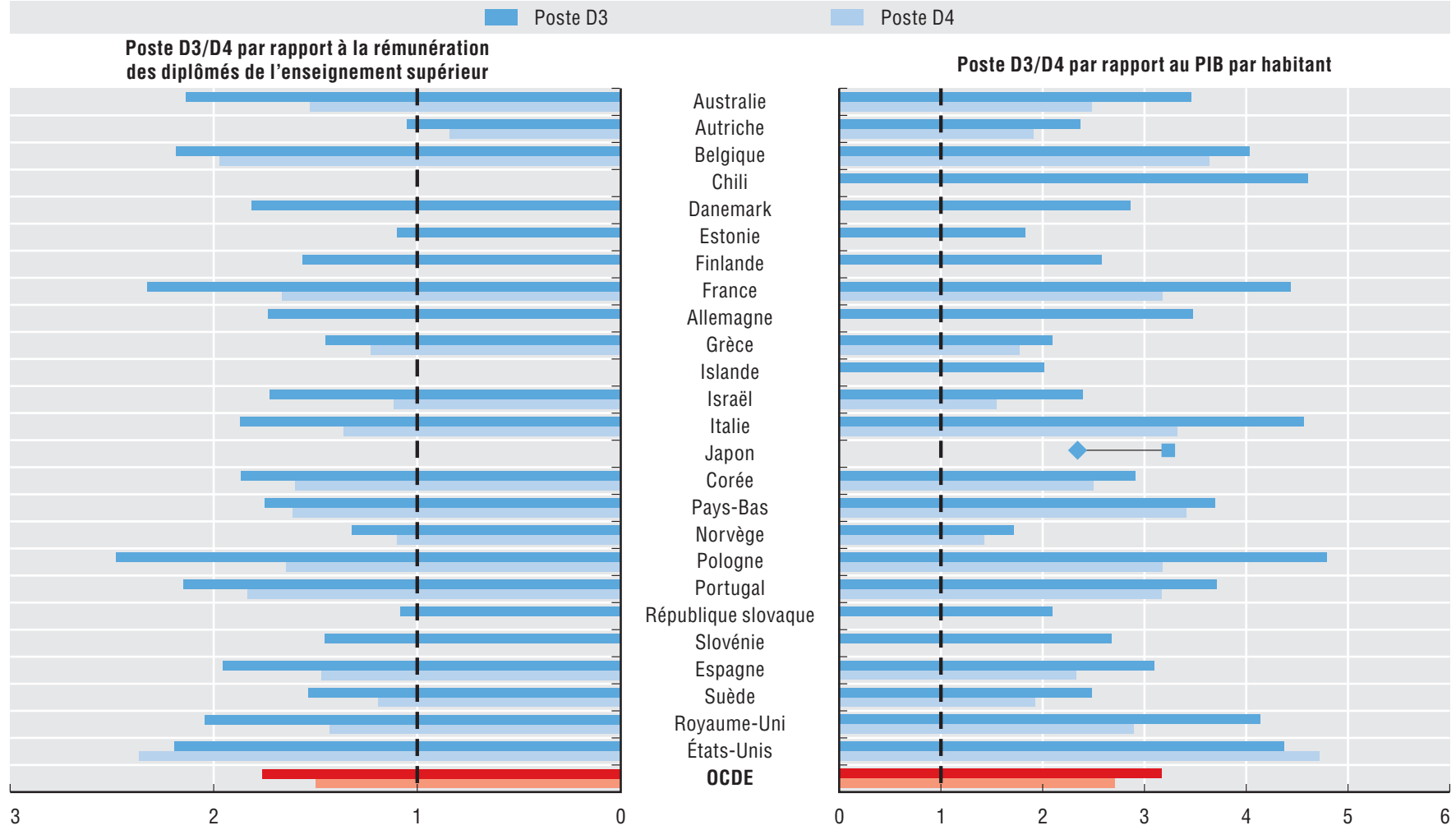

Source: Enquête de l'OCDE sur la rémunération des fonctionnaires des administrations centrales/fédérales (2012); OCDE, STAN/Statistiques sur les comptes nationaux (base de données) ; OCDE (2013), Regards sur l'éducation 2013 : Les indicateurs de l'OCDE, Éditions OCDE, Paris, http://dx.doi.org/10.1787/ eag-2013-fr. 
Les capacités d'analyse des économistes et des analystes des politiques sont essentielles pour améliorer la capacité des administrations à prendre des décisions fondées sur des données probantes. Les spécialistes n'exercent pas de fonctions de direction (ils encadrent trois personnes au maximum) et occupent un rang supérieur au personnel administratif/de secrétariat (voir l'annexe D pour plus de précisions). Les données sont présentées pour les spécialistes seniors et juniors.

En moyenne, le niveau de rémunération des spécialistes seniors s'élève à environ 89000 USD à PPA, dont $17 \%$ correspondent aux cotisations sociales de l'employeur et à l'ajustement lié au temps de travail. La rémunération des spécialistes juniors s'élève à près de 72000 USD à PPA (après prise en compte des cotisations sociales de l'employeur, des heures de travail et des congés).

Les spécialistes seniors gagnent en moyenne $24 \%$ de plus que les juniors. C'est en Belgique, au Chili et au Danemark que la différence entre les deux niveaux de rétribution est la plus élevée, et en Corée qu'elle est la plus faible. Les cadres supérieurs de niveau D1 gagnent en moyenne 2.6 fois plus que les spécialistes seniors. C'est en Italie et au Royaume-Uni que la différence entre les cadres D1 et les spécialistes seniors est la plus forte, et en République slovaque, en Espagne et en Islande qu'elle est la plus faible. L'enquête semble montrer que les rémunérations varient moins selon les pays pour ces deux groupes professionnels que pour les cadres supérieurs et les cadres moyens.

Les différences de niveaux de rémunération peuvent être dues à divers facteurs qui ne sont pas pris en compte. Par rapport au PIB par habitant, c'est au Chili que le niveau de rémunération des spécialistes est le plus élevé, et en Grèce, en Estonie et en Islande qu'il est le plus faible. En moyenne, les spécialistes juniors perçoivent une rémunération presque identique à celle des diplômés de l'enseignement supérieur.

\section{Méthodologie et définitions}

Les données se rapportent à 2011 et ont été rassemblées dans le cadre d'une enquête de l'OCDE sur la rémunération des fonctionnaires des administrations centrales/fédérales (2012). Des représentants de ministères et d'organismes de l'administration centrale y ont répondu par l'intermédiaire du Réseau de l'OCDE sur l'emploi et la gestion publics.

Les données portent sur six ministères/départements de l'administration centrale (Intérieur, Finances, Justice, Éducation, Santé et Environnement ou leurs équivalents). La classification et la définition des professions sont une adaptation de la Classification internationale type des professions (CITP) établie par l'Organisation internationale du travail (OIT). Les niveaux de rémunération sont calculés en établissant la moyenne des rémunérations du personnel en fonction.

La rémunération totale comprend les salaires et traitements bruts ainsi que les cotisations sociales de l'employeur, que le régime soit capitalisé ou non. Les seules cotisations sociales prises en compte sont les cotisations aux régimes de santé et de retraite, afin d'obtenir des données cohérentes à travers l'ensemble des pays.
On a converti les rémunérations en USD en utilisant les PPA pour le PIB tirées de la Base de données des Statistiques de l'OCDE sur les comptes nationaux. L'ajustement lié au temps de travail compense les différences au niveau de la durée du travail, en tenant compte à la fois du nombre moyen de jours/d'heures de travail et du nombre moyen de jours de congé.

Voir l'annexe D pour une présentation complète de la méthodologie suivie.

\section{Pour en savoir plus}

OCDE (2012), Public Sector Compensation in Times of Austerity, Éditions OCDE, Paris, $h$ ttp://dx.doi.org/10.1787/9789264177758-en.

\section{Notes relatives aux graphiques}

On ne dispose pas de données pour l'Australie, le Canada, la Hongrie, l'Irlande, le Japon, le Luxembourg, le Mexique, la Norvège, la Nouvelle-Zélande, la République tchèque, la Suisse et la Turquie.

Il n'y a pas de distinction entre les spécialistes seniors et les spécialistes juniors pour l'Autriche, l'Espagne, l'Estonie, les États-Unis, la Grèce, l'Islande, Israël, l'Italie, le Portugal et la République slovaque. Allemagne : la distinction entre les deux catégories se fonde sur le niveau de formation, et non sur le degré d'expérience. Belgique : les ministères de l'Éducation et de l'Environnement ne sont pas pris en compte, parce qu'ils ne relèvent pas de l'administration fédérale. Danemark : le ministère de l'Éducation n'est pas pris en compte, parce que beaucoup de ses missions ont été transférées à d'autres ministères. Estonie : on ne dispose pas de données relatives aux ministères de l'Éducation et de l'Environnement. Grèce : on ne dispose que de données sur les rémunérations plancher et les rémunérations plafond; on ne présente donc pas ici la moyenne effective, mais la moyenne entre le salaire plancher et le salaire plafond. Islande : le ministère de la Justice relève du ministère de l'Intérieur. Italie : le nombre d'agents englobe les agents à temps plein comme les agents à temps partiel. République slovaque : seule la moitié des agents du ministère de la Justice est prise en compte, pour des raisons statistiques (cohérence avec les codes CITP). Suède : le ministère de l'Intérieur relève du cabinet du Premier ministre et n'est pas pris en compte dans les données. Royaume-Uni : les données portent sur 2012 (sur la base des PPA 2012) et la moyenne représente une médiane, et non la moyenne arithmétique.

Voir l'annexe D pour des notes supplémentaires.

Informations sur les données concernant Israël : http://dx.doi.org/10.1787/ 888932315602 . 
5.9. Rémunération annuelle moyenne des spécialistes seniors et juniors dans l'administration centrale (2011) Ajustée pour tenir compte des différences d'heures de travail et de congés

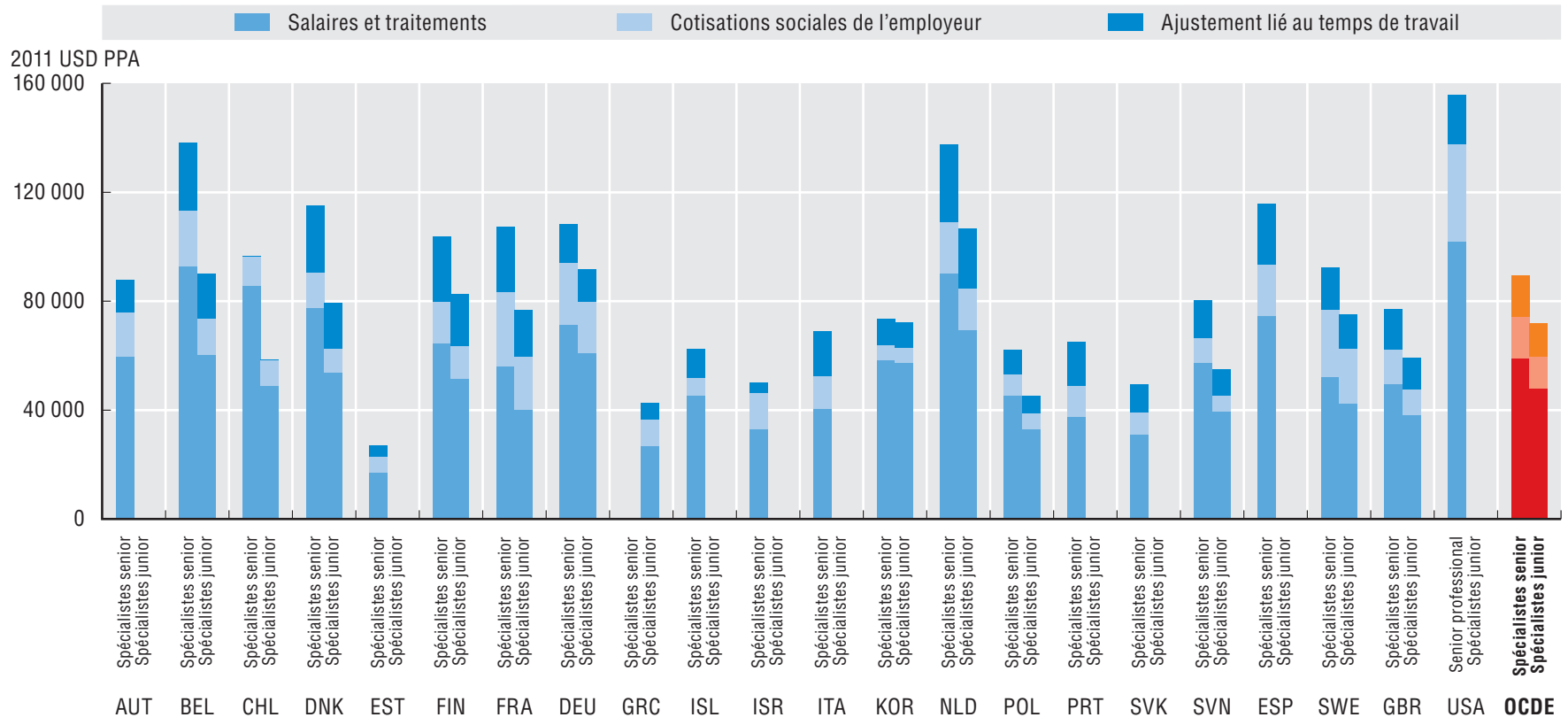

Source: Enquête de l'OCDE sur la rémunération des fonctionnaires des administrations centrales/fédérales (2012); OCDE, STAN/Statistiques sur les comptes nationaux (base de données).

5.10. Rémunération annuelle moyenne des spécialistes seniors et juniors dans l'administration centrale par rapport au PIB par habitant et à la rémunération des diplômés de l'enseignement supérieur

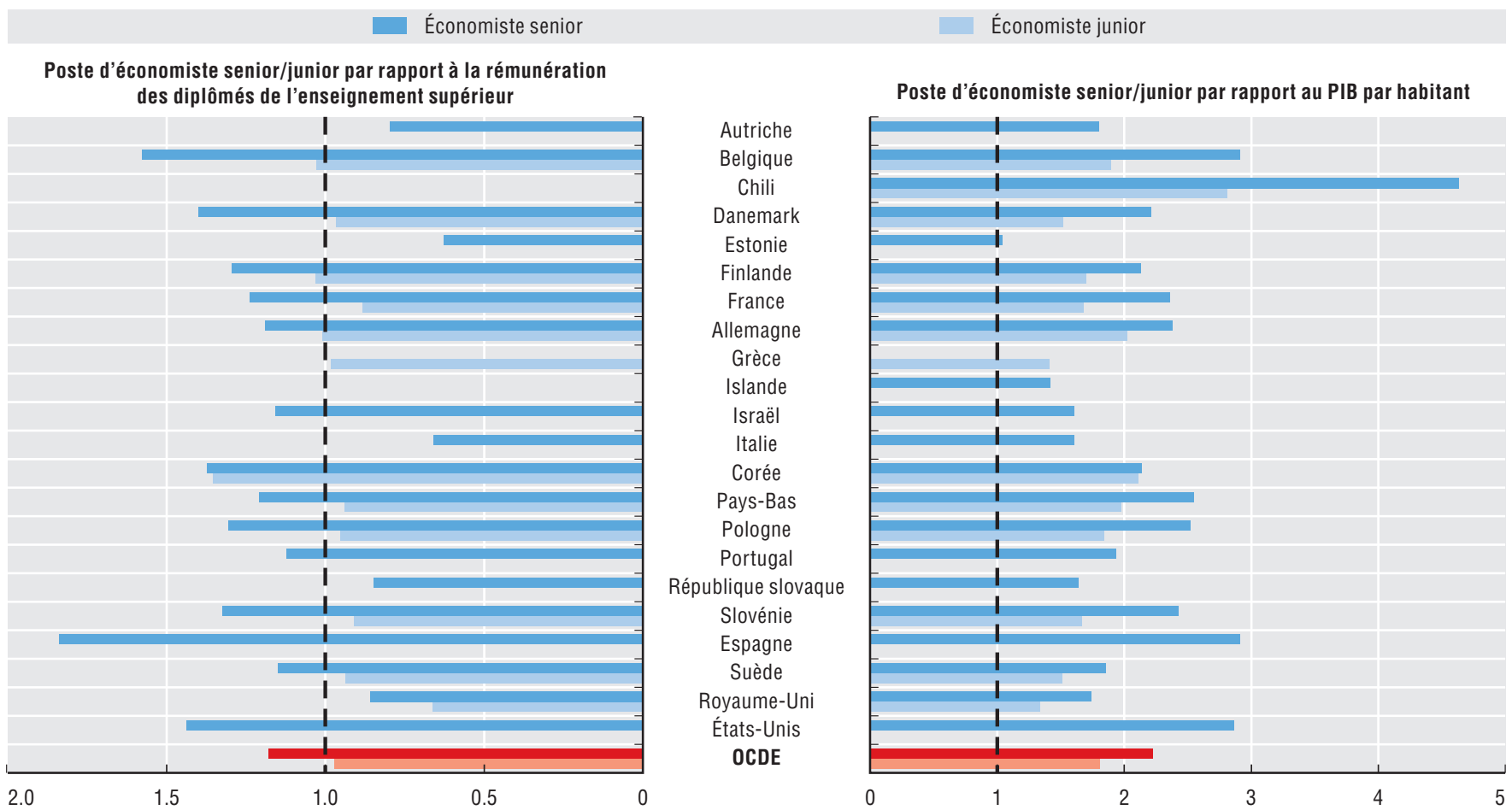

Source : Enquête de l'OCDE sur la rémunération des fonctionnaires des administrations centrales/fédérales (2012); OCDE, STAN/Statistiques sur les comptes nationaux (base de données) ; OCDE (2013), Regards sur l'éducation 2013 : Les indicateurs de l'OCDE, Éditions OCDE, Paris, http://dx.doi.org/10.1787/ eag-2013-fr. 
Par rapport aux autres emplois de l'administration centrale, la rémunération des emplois de secrétaire est celle qui, au sein de la zone OCDE, semble varier le moins selon les pays. En moyenne, le niveau de rémunération des secrétaires s'élève à environ 50000 USD à PPA, dont environ 8600 USD à PPA de cotisations sociales de l'employeur et 8500 USD à PPA d'ajustement lié au temps de travail. Par conséquent, le salaire brut représente $66 \%$ de leur rémunération globale. Pour rendre compte du montant total des cotisations aux systèmes de sécurité sociale, il convient d'ajouter les cotisations sociales de l'employé qui sont comprises dans le salaire brut. Comme pour les autres professions, la structure de la rémunération globale (c'est-à-dire les parts respectives des salaires et des cotisations sociales de l'employeur) diffère d'un pays à l'autre. Ces différences sont liées au consensus historique, culturel et politique sur la manière de financer le système de sécurité sociale. Les cadres supérieurs de niveau D1 gagnent en moyenne 4.6 fois plus que les secrétaires. C'est en Italie, en Australie et au Royaume-Uni que ces différences sont les plus marquées, et en Islande, en République slovaque et en Espagne qu'elles le sont le moins.

Après correction par le PIB par habitant, c'est en Pologne et aux Pays-Bas que la rémunération des secrétaires est la plus élevée, et en République slovaque et en Estonie qu'elle est la plus faible.

Ces disparités de rémunération peuvent aussi être dues à des différences sur les marchés du travail nationaux, s'agissant en particulier des rémunérations pratiquées dans le secteur privé pour des compétences comparables. La comparaison entre la rémunération des secrétaires et celle des diplômés de l'enseignement supérieur montre que les secrétaires sont mieux rémunérés en Corée et en Espagne. À l'inverse, les postes de secrétaire paraissent moins attractifs en République slovaque. Il est important de relever que le rapport entre la rémunération des secrétaires et la rémunération moyenne des diplômés de l'enseignement supérieur est inférieur à un pour l'ensemble des pays et s'élève à 0.7 en moyenne. Cela peut traduire le fait qu'il n'est pas nécessaire d'être diplômé de l'enseignement supérieur pour obtenir un poste de secrétaire, mais cela peut aussi être révélateur de différences sur les marchés du travail nationaux, s'agissant en particulier des rémunérations pratiquées dans le secteur privé pour des postes comparables.

\section{Méthodologie et définitions}

Les données se rapportent à 2011 et ont été rassemblées dans le cadre d'une enquête de l'OCDE sur la rémunération des fonctionnaires des administrations centrales/ fédérales (2012). Des représentants de ministères et d'organismes de l'administration centrale y ont répondu par l'intermédiaire du Réseau de l'OCDE sur l'emploi et la gestion publics.
Les données portent sur six ministères/départements de l'administration centrale (Intérieur, Finances, Justice, Éducation, Santé et Environnement ou leurs équivalents). La classification et la définition des professions sont une adaptation de la Classification internationale type des professions (CITP) établie par l'Organisation internationale du travail (OIT). Les niveaux de rémunération sont calculés en établissant la moyenne des rémunérations du personnel en fonction.

La rémunération totale comprend les salaires et traitements bruts ainsi que les cotisations sociales de l'employeur, que le régime soit capitalisé ou non. Les seules cotisations sociales prises en compte sont les cotisations aux régimes de santé et de retraite, afin d'obtenir des données cohérentes à travers l'ensemble des pays.

On a converti les rémunérations en USD en utilisant les PPA pour le PIB tirées de la Base de données des Statistiques de l'OCDE sur les comptes nationaux. L'ajustement lié au temps de travail compense les différences au niveau de la durée du travail, en tenant compte à la fois du nombre moyen de jours/d'heures de travail et du nombre moyen de jours de congé.

Voir l'annexe D pour une présentation complète de la méthodologie suivie.

\section{Pour en savoir plus}

OCDE (2012), Public Sector Compensation in Times of Austerity, Éditions OCDE, Paris, http://dx.doi.org/10.1787/9789264177758-en.

\section{Notes relatives aux graphiques}

On ne dispose pas de données pour le Canada, la Grèce, la Hongrie, l'Irlande, le Japon, le Luxembourg, le Mexique, la Nouvelle-Zélande, la République tchèque, la Suisse et la Turquie.

Belgique : les ministères de l'Éducation et de l'Environnement ne sont pas pris en compte, parce qu'ils ne relèvent pas de l'administration fédérale. Danemark : le ministère de l'Éducation n'est pas pris en compte, parce que beaucoup de ses missions ont été transférées à d'autres ministères. Estonie : on ne dispose pas de données relatives aux ministères de l'Éducation et de l'Environnement. Islande : le ministère de la Justice relève du ministère de l'Intérieur. Italie : le nombre d'agents englobe les agents à temps plein comme les agents à temps partiel. République slovaque : seule la moitié des agents du ministère de la Justice est prise en compte, pour des raisons statistiques (cohérence avec les codes CITP). Suède : le ministère de l'Intérieur relève du cabinet du Premier ministre et n'est pas pris en compte dans les données. Royaume-Uni : les données portent sur 2012 (sur la base des PPA 2012) et la moyenne représente une médiane, et non la moyenne arithmétique.

Voir l'annexe D pour des notes supplémentaires.

Informations sur les données concernant Israël : http://dx.doi.org/10.1787/ 888932315602 . 
5.11. Rémunération annuelle moyenne des fonctionnaires assurant des fonctions de secrétariat (2011)

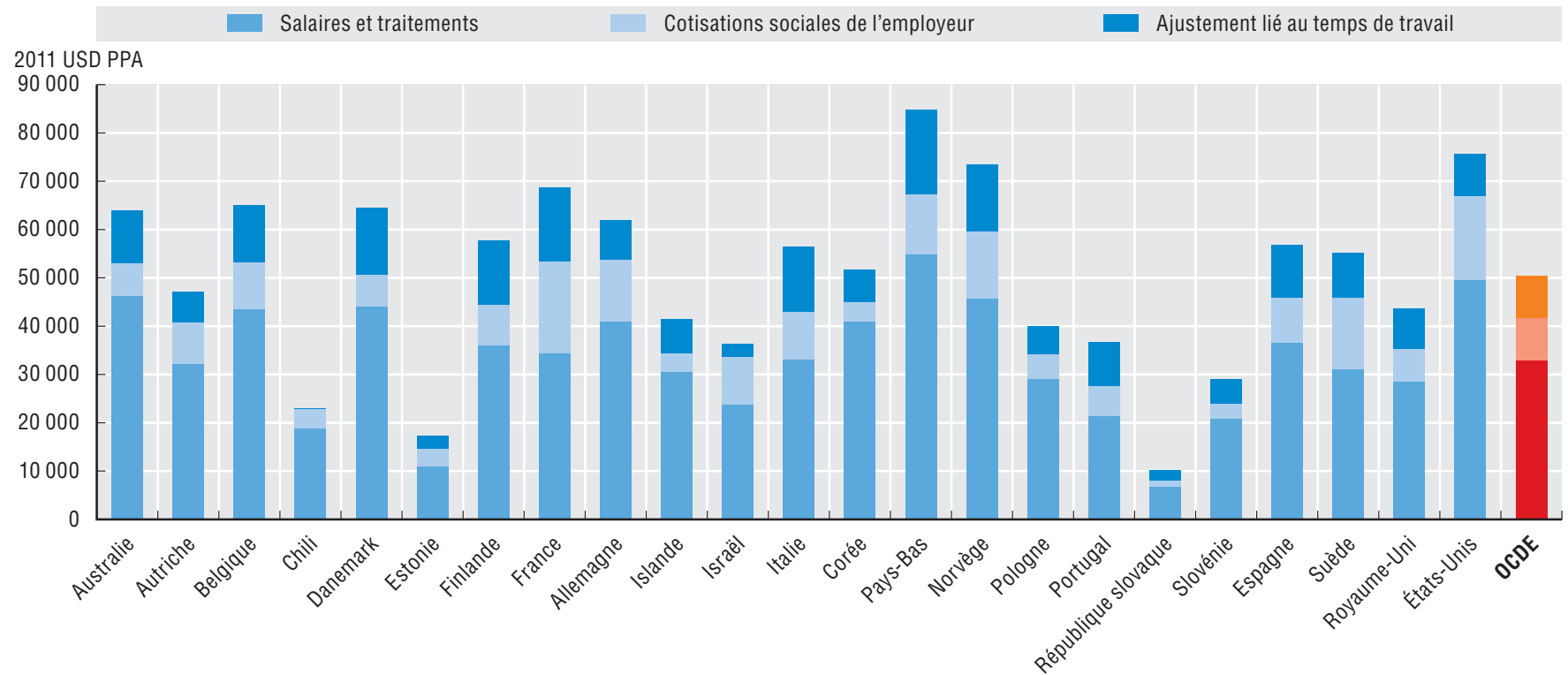

Source : Enquête de l'OCDE sur la rémunération des fonctionnaires des administrations centrales/fédérales (2012); OCDE, STAN/Statistiques sur les comptes nationaux (base de données).

\subsection{Rémunération annuelle moyenne des fonctionnaires assurant des fonctions de secrétariat dans l'administration centrale par rapport au PIB par habitant et à la rémunération des diplômés de l'enseignement supérieur}

Poste de secrétaire par rapport à la rémunération des diplômés de l'enseignement supérieur

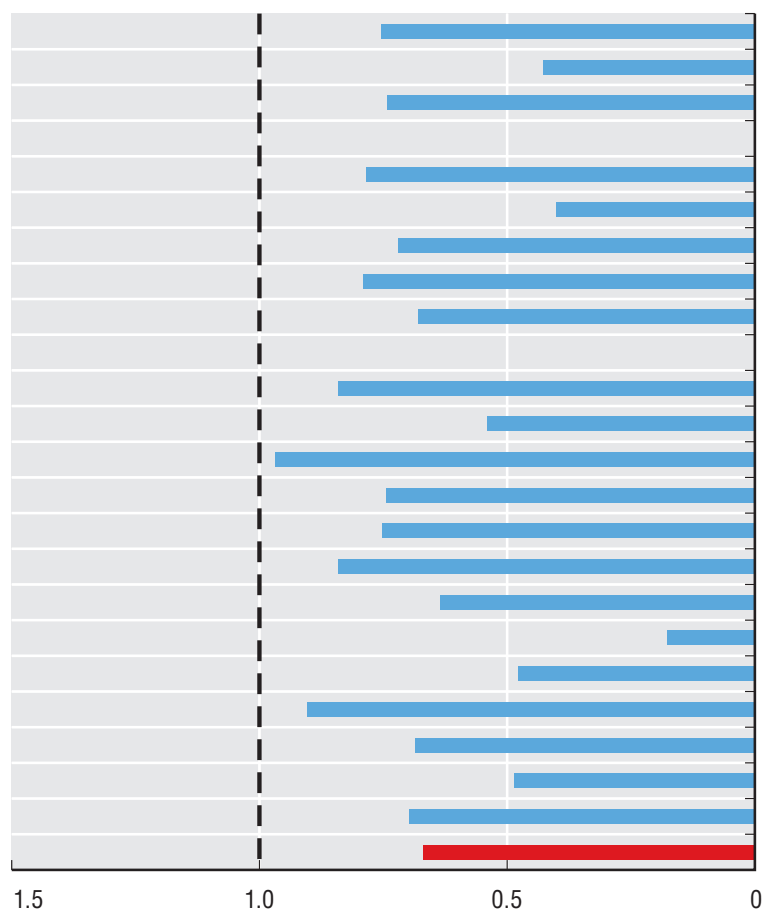

Poste de secrétaire par rapport au PIB par habitant

Australie
Autriche
Belgique
Chili
Danemark
Estonie
Finlande
France
Allemagne
Islande
Israël
Italie
Corée
Pays-Bas
Norvège
Pologne
Portugal
Elique slovaque
Slovénie
Espagne
Suède
Royaume-Uni
États-Unis
ocDE

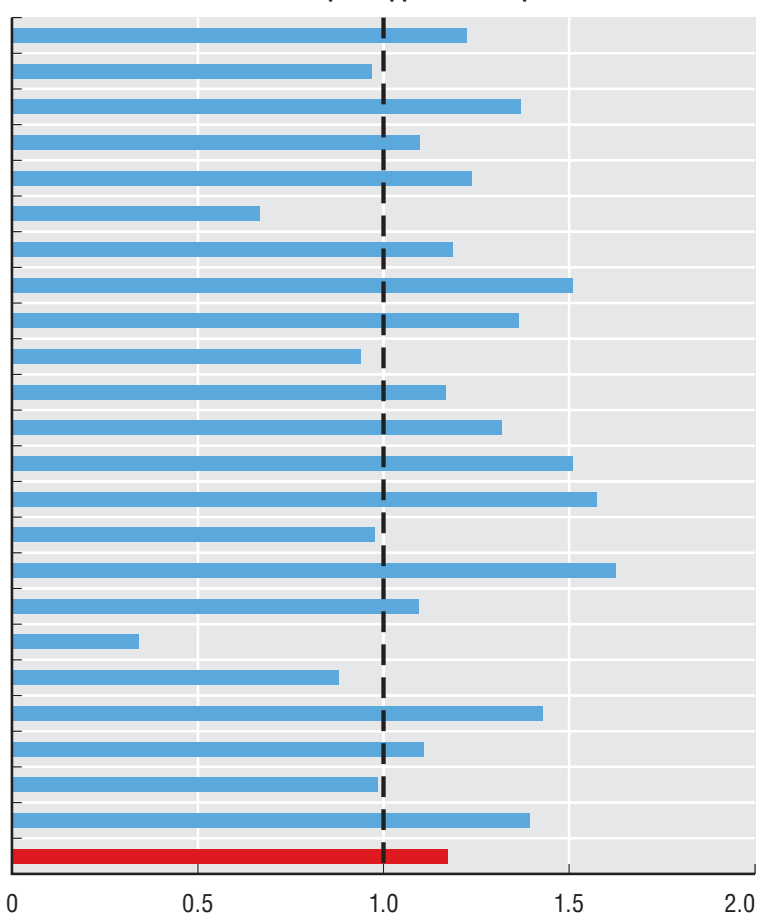

Source: Enquête de l'OCDE sur la rémunération des fonctionnaires des administrations centrales/fédérales (2012); OCDE, STAN/Statistiques sur les comptes nationaux (base de données) ; OCDE (2013), Regards sur l'éducation 2013 : Les indicateurs de l'OCDE, Éditions OCDE, Paris, http://dx.doi.org/10.1787/ eag-2013-fr. 
Les agents des administrations centrales appartenant à des professions de service public travaillent au contact direct des usagers. On trouvera ici des données pour des professions liées au maintien de l'ordre et à l'administration fiscale : inspecteurs et enquêteurs de police (ci-après: "inspecteurs de police "), agents de police, agents de l'immigration, inspecteurs des douanes et inspecteurs des impôts. Bien que toutes les administrations centrales emploient des fonctionnaires à la réalisation de ces missions, certaines de ces fonctions sont également assumées par les administrations infranationales. Dans certains pays, quelques fonctions (immigration ou fiscalité) ne peuvent pas être distinguées.

En moyenne, la rémunération des inspecteurs de police s'élève à environ 83000 USD à PPA, dont $18 \%$ correspondent aux cotisations sociales de l'employeur et $19 \%$ à l'ajustement lié au temps de travail. La rémunération des agents de police s'élève à environ 63000 USD à PPA (après prise en compte des cotisations sociales de l'employeur, des heures de travail et des congés).

Les différences de rémunération sont relativement faibles entre ces cinq professions. Un inspecteur de police gagne en moyenne 33 \% de plus qu'un agent de police. Le budget consacré aux inspecteurs de police par rapport aux agents de police est proportionnellement plus important aux États-Unis et au Royaume-Uni que dans les autres pays. À l'inverse, les agents de police sont presque rémunérés au même niveau que les inspecteurs de police en Islande, aux Pays-Bas et en Norvège.

Les administrations centrales semblent mieux rémunérer les inspecteurs des impôts que les agents de police, surtout aux Pays-Bas, en Slovénie, au Portugal et en Espagne. Par ailleurs, le Portugal, le Royaume-Uni et les États-Unis semblent, dans une large mesure, consacrer un budget plus important aux inspecteurs des douanes qu'aux agents de police. Dans ce même groupe de pays et aux Pays-Bas, les agents de l'immigration semblent mieux rémunérés que les agents de police.

Comme pour les autres professions, la structure de la rémunération globale (c'est-à-dire les parts respectives des salaires et traitements et des cotisations sociales de l'employeur) diffère d'un pays à l'autre. Ces différences sont liées au consensus historique, culturel et politique sur la manière de financer le système de sécurité sociale.

Deux professions ont été examinées par rapport au PIB par habitant et au salaire moyen d'un diplômé de l'enseignement supérieur : celles d'inspecteur de police et d'agent de police. En moyenne, la rémunération des inspecteurs de police est presque égale à la rémunération moyenne des diplômés de l'enseignement supérieur, tandis que la rémunération des agents de police est légèrement inférieure à cette moyenne de référence. Cela signifie que la rémunération de ces postes est aussi attractive, ou légèrement moins attractive, que la rémunération moyenne dans l'ensemble de l'économie.

\section{Méthodologie et définitions}

Les données se rapportent à 2011 et ont été rassemblées dans le cadre d'une enquête de l'OCDE sur la rémunération des fonctionnaires des administrations centrales/ fédérales (2012). Des représentants de ministères et d'organismes de l'administration centrale y ont répondu par l'intermédiaire du Réseau de l'OCDE sur l'emploi et la gestion publics.
Les données portent sur certains agents de service public au contact direct des usagers (enquêteurs/inspecteurs de police, agents de police, agents de l'immigration, inspecteurs des douanes et inspecteurs des impôts). La classification et la définition des professions sont une adaptation de la Classification internationale type des professions (CITP) établie par l'Organisation internationale du travail (OIT).

La rémunération totale comprend les salaires et traitements bruts ainsi que les cotisations sociales de l'employeur, que le régime soit capitalisé ou non, y compris les cotisations de retraite versées par le truchement du budget de l'État et non par celui de cotisations sociales de l'employeur (principalement pour certains systèmes par répartition). Les seules cotisations sociales prises en compte sont les cotisations aux régimes de santé et de retraite, afin d'obtenir des données cohérentes à travers l'ensemble des pays.

On a converti les rémunérations en USD en utilisant les PPA pour le PIB tirées de la Base de données des Statistiques de l'OCDE sur les comptes nationaux. L'ajustement lié au temps de travail compense les différences au niveau de la durée du travail, en tenant compte à la fois du nombre moyen de jours/d'heures de travail et du nombre moyen de jours de congé.

Voir l'annexe D pour une présentation complète de la méthodologie suivie.

\section{Pour en savoir plus}

OCDE (2012), Public Sector Compensation in Times of Austerity, Éditions OCDE, Paris, http://dx.doi.org/10.1787/9789264177758-en.

\section{Notes relatives aux graphiques}

On ne dispose pas de données pour l'Allemagne, le Canada, le Chili, la Grèce, la Hongrie, l'Irlande, le Japon, le Luxembourg, le Mexique, la Nouvelle-Zélande, la République tchèque, la Suisse et la Turquie.

Les agents de l'immigration sont comptabilisés avec les agents de police en Autriche, au Danemark, en Espagne, en Islande, en Italie, en Norvège et en République slovaque. Il n'y a pas de distinction entre les données portant sur les inspecteurs de police et sur les agents de police pour la Corée, Israël et la Suède. Les agents des douanes sont comptabilisés avec les agents de police pour la République slovaque. Les inspecteurs des impôts et les agents de l'immigration sont comptabilisés avec les agents de police en Islande. Australie : les agents et inspecteurs des polices municipales et locales représentent la majeure partie des fonctionnaires de police et ne sont pas pris en compte ici. Autriche : seuls les agents contractuels sont pris en compte, puisqu'ils représentent la majorité des agents exerçant des professions de service public. Pologne : les professions de service public ne relèvent pas de la fonction publique, sauf dans le cas des inspecteurs des impôts. Royaume-Uni : les données portent sur 2012 (sur la base des PPA 2012). On ne dispose que de données sur les rémunérations plancher et les rémunérations plafond; on ne présente donc pas ici la moyenne effective, mais la moyenne entre le salaire plancher et le salaire plafond.

Voir l'annexe D pour des notes supplémentaires.

Informations sur les données concernant Israël : http://dx.doi.org/10.1787/ 888932315602 . 
5.13. Rémunération annuelle moyenne dans certaines professions de service public (2011)

Ajustée pour tenir compte des différences d'heures de travail et de congés

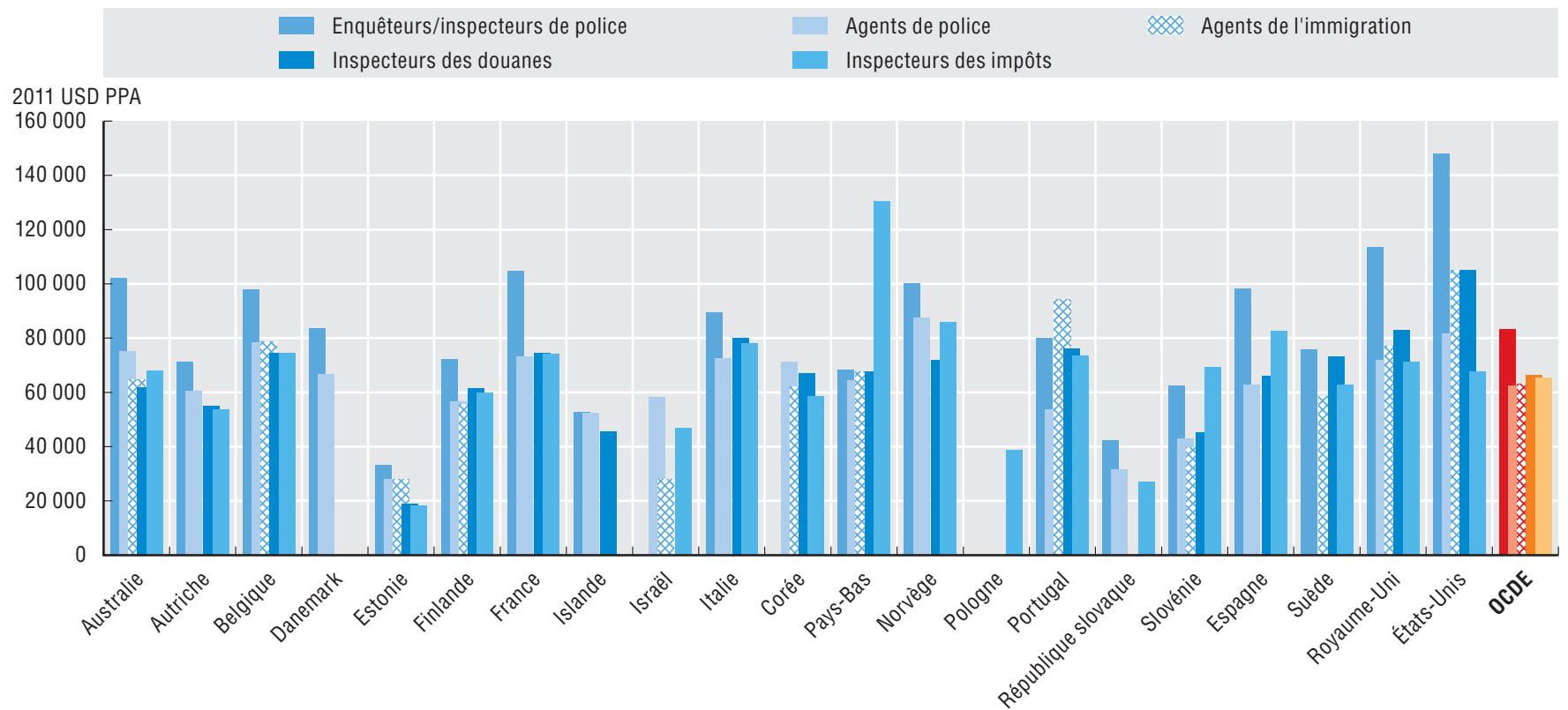

Source: Enquête de l'OCDE sur la rémunération des fonctionnaires des administrations centrales/fédérales (2012) ; OCDE, STAN/Statistiques sur les comptes nationaux (base de données).

StatLink काIsL http://dx.doi.org/10.1787/888933159591

\subsection{Rémunération annuelle moyenne des inspecteurs et des agents de police de l'administration centrale par rapport au PIB par habitant et à la rémunération des diplômés de l'enseignement supérieur}

Ratio en 2011

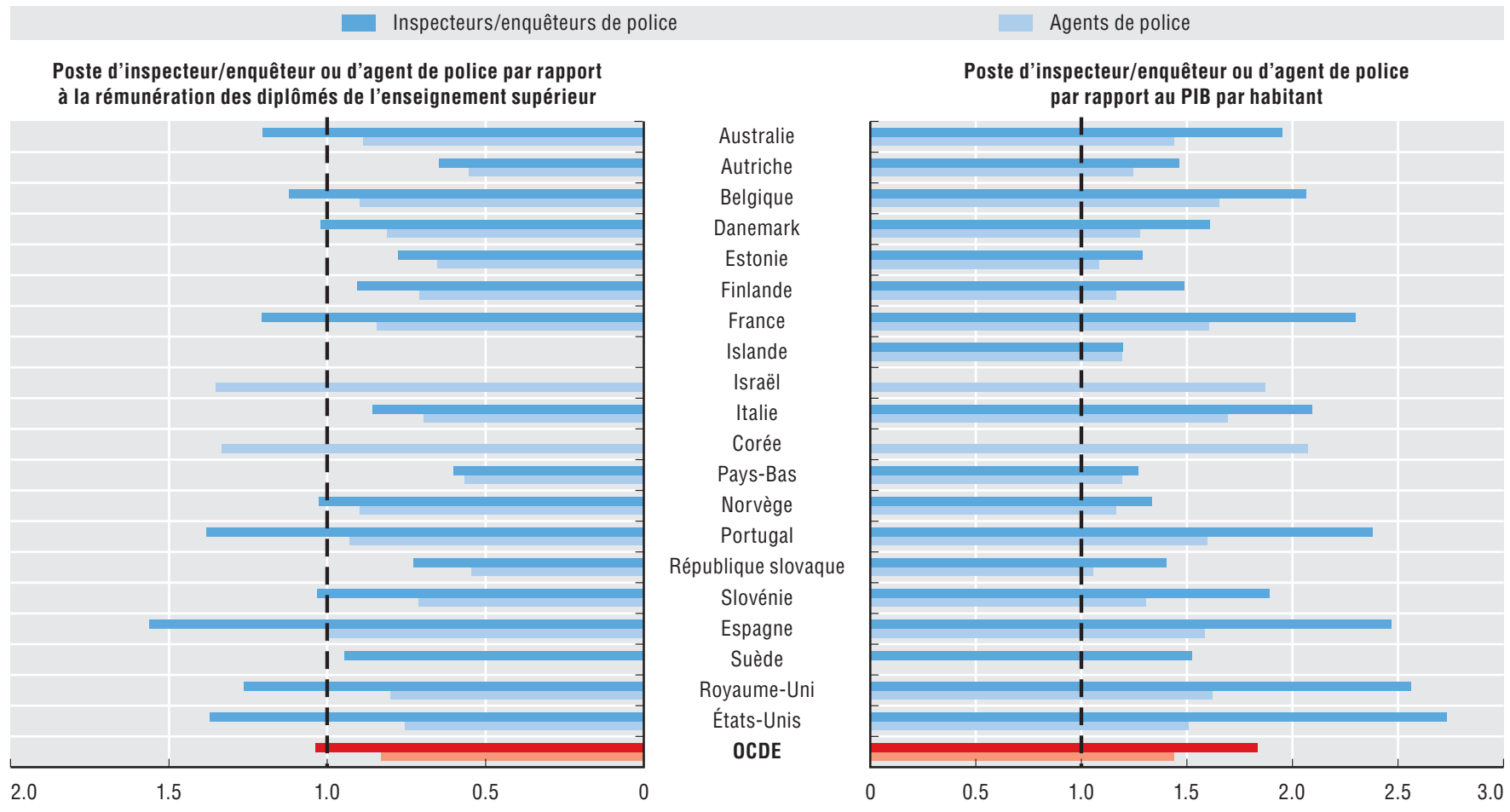

Source : Enquête de l'OCDE sur la rémunération des fonctionnaires des administrations centrales/fédérales (2012); OCDE, STAN/Statistiques sur les comptes nationaux (base de données) ; OCDE (2013), Regards sur l'éducation 2013 : Les indicateurs de l'OCDE, Éditions OCDE, Paris, http://dx.doi.org/10.1787/ eag-2013-fr. 
Les enseignants sont l'épine dorsale du secteur de l'éducation, lequel est un facteur déterminant de productivité et de croissance. Leurs salaires constituent le premier poste de dépenses du système éducatif, qui est à forte intensité de main-d'œuvre. Les salaires et les conditions de travail jouent un rôle important pour attirer, motiver et fidéliser des enseignants qualifiés.

Il existe dans la plupart des pays trois catégories d'enseignants : ceux du primaire, ceux du premier cycle du secondaire et ceux du deuxième cycle du secondaire. Leur salaire augmente avec leur niveau de qualification, avec leur niveau d'expérience et avec le niveau d'enseignement au sein duquel ils exercent. Les données présentées ici permettent de comparer les salaires bruts en début de carrière, en milieu de carrière et au niveau maximum des enseignants du premier cycle du secondaire ayant la formation minimale nécessaire et exerçant dans le secteur public. Pour effectuer des comparaisons internationales, il faut toutefois tenir compte du fait que les traitements statutaires ne sont qu'une composante, certes importante, de la rémunération des enseignants. Des écarts entre les pays au niveau des avantages sociaux, des cotisations sociales de l'employeur et de l'employé et des primes et allocations peuvent induire des différences dans la rémunération totale. De plus, les salaires des enseignants n'ont pas été corrigés des différences au niveau du nombre d'heures de travail contractuel et du nombre de jours de congé, qui peuvent être pertinentes pour des comparaisons nationales et internationales. Ces données peuvent toutefois fournir des indications sur les différences qui existent entre les pays de l'OCDE au niveau du rendement de l'expérience des enseignants.

Les salaires de base annuels statutaires bruts des enseignants du premier cycle de l'enseignement secondaire ayant 15 ans d'expérience varient de moins de 15000 USD à PPA en Estonie, en République slovaque et en Hongrie à plus de 60000 USD à PPA au Luxembourg, en Allemagne et aux Pays-Bas en 2011. La moyenne pour les pays membres de l'OCDE atteint près de 40000 USD à PPA. En Corée, au Japon et au Mexique, les salaires du dernier échelon sont deux fois supérieurs aux salaires de début de carrière. Les salaires du dernier échelon sont, en moyenne, supérieurs d'environ $60 \%$ aux salaires de début de carrière.

En général, les salaires bruts des enseignants sont inférieurs aux salaires annuels moyens bruts des employés possédant un niveau d'éducation équivalent (diplômés de l'enseignement supérieur âgés de 25 à 64 ans travaillant à temps plein toute l'année). En Espagne, en Corée, au Luxembourg et au Portugal, les enseignants ont un niveau de rémunération supérieur au salaire moyen des diplômés de l'enseignement supérieur. En Nouvelle-Zélande, au Canada, en Allemagne et en Finlande, ils ont un niveau de rémunération presque égal à ce salaire moyen. En revanche, en République slovaque, en Islande, en République tchèque, en Hongrie, en Italie et en Autriche, ils ont un niveau de rémunération nettement inférieur à ce salaire moyen.

\section{Méthodologie et définitions}

Les salaires statutaires correspondent aux salaires prévus par les barèmes de traitement officiels. Les salaires indiqués sont les salaires bruts (somme totale versée par l'employeur, avant impôts), moins la contribution de l'employeur aux régimes de sécurité sociale et de retraite (en fonction des barèmes de traitement existants). Ils sont fournis ici pour un enseignant exerçant à pleintemps et ayant la formation minimale nécessaire pour être pleinement qualifié, à trois stades : en début de carrière, au bout de 15 ans d'expérience et au niveau maximum (dernier échelon).

Il convient d'effectuer une distinction entre les salaires statutaires mentionnés dans cet indicateur, d'une part, et les dépenses salariales des administrations et les salaires moyens des enseignants, d'autre part.

Les salaires bruts des enseignants ont été convertis en USD en utilisant les PPA de la base de données des Statistiques de l'OCDE sur les comptes nationaux.

L'indicateur de salaire relatif est calculé pour la dernière année pour laquelle on dispose de données sur les revenus. Dans le cas présent, les chiffres représentent les salaires des enseignants effectivement versés après 15 ans d'exercice de la profession. Les revenus des diplômés de l'enseignement supérieur sont les revenus moyens pour un emploi exercé à temps plein et toute l'année, dans le groupe d'âge de 25 à 64 ans, avec une éducation de niveau 5A/5B/6 selon la CITE.

\section{Pour en savoir plus}

OCDE (2013), Regards sur l'éducation 2013 : Les indicateurs de l'OCDE, Éditions OCDE, Paris, http://dx.doi.org/10.1787/eag2013-fr.

\section{Notes relatives aux graphiques}

La Belgique figure sous "Belgique (Fr.) » et "Belgique (Fl.) 》. Le Royaume-Uni figure sous " Angleterre » et "Écosse ».

5.15 : On ne dispose pas de données pour la Suisse et la Turquie.

5.16 : On ne dispose pas de données pour la Grèce, le Japon, le Mexique, la Suisse et la Turquie. Les données pour l'Australie, le Canada, l'Espagne, la Finlande, l'Irlande, l'Italie, le Luxembourg, la Norvège, les Pays-Bas, la Pologne et le Portugal portent sur 2010. Les données pour la France portent sur 2009. Les données pour l'Islande portent sur 2006.

Informations sur les données concernant Israël : http://dx.doi.org/10.1787/ 888932315602. 
5.15. Salaires des enseignants du premier cycle du secondaire dans les établissements publics (2011)

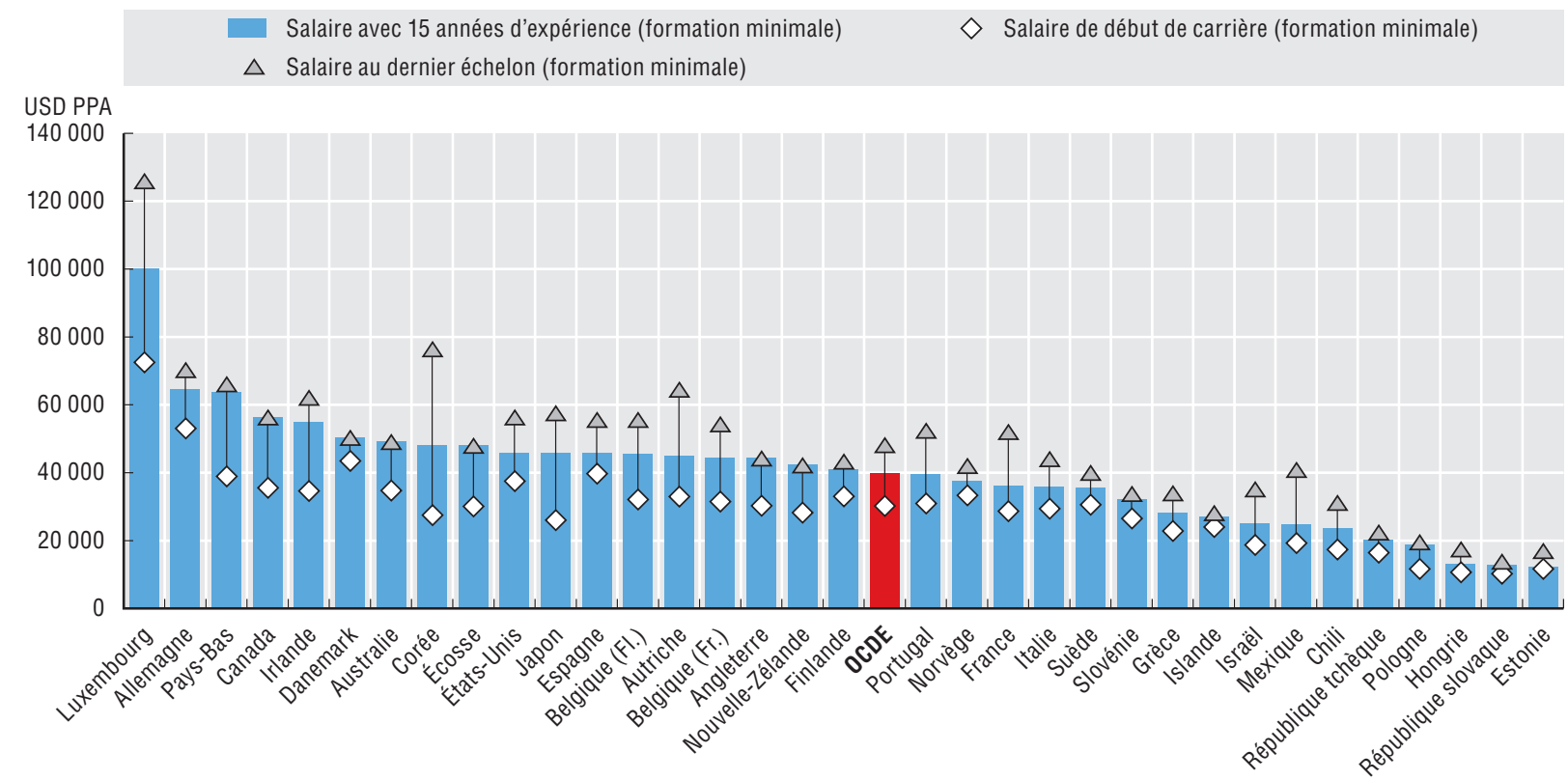

Source : OCDE (2013), Regards sur l'éducation 2013 : Les indicateurs de l'OCDE, Éditions OCDE, Paris, http://dx.doi.org/10.1787/eag-2013-fr.

StatLink AाIs $h t t p: / / d x . d o i . o r g / 10.1787 / 888933159615$

5.16. Rapport entre les salaires des enseignants et les revenus des diplômés de l'enseignement supérieur (2011)

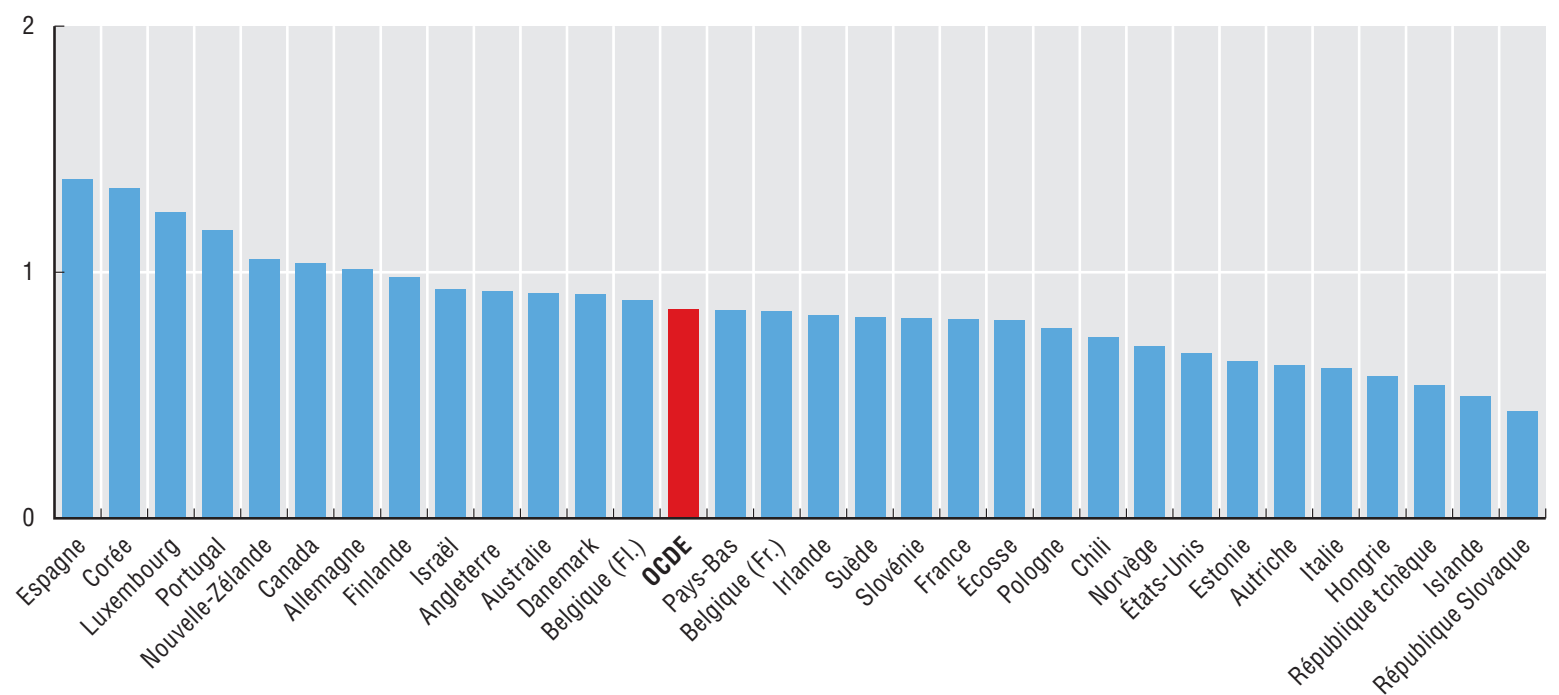

Source : OCDE (2013), Regards sur l'éducation 2013 : Les indicateurs de l'OCDE, Éditions OCDE, Paris, http://dx.doi.org/10.1787/eag-2013-fr.

StatLink क्ञाs http://dx.doi.org/10.1787/888933159629 


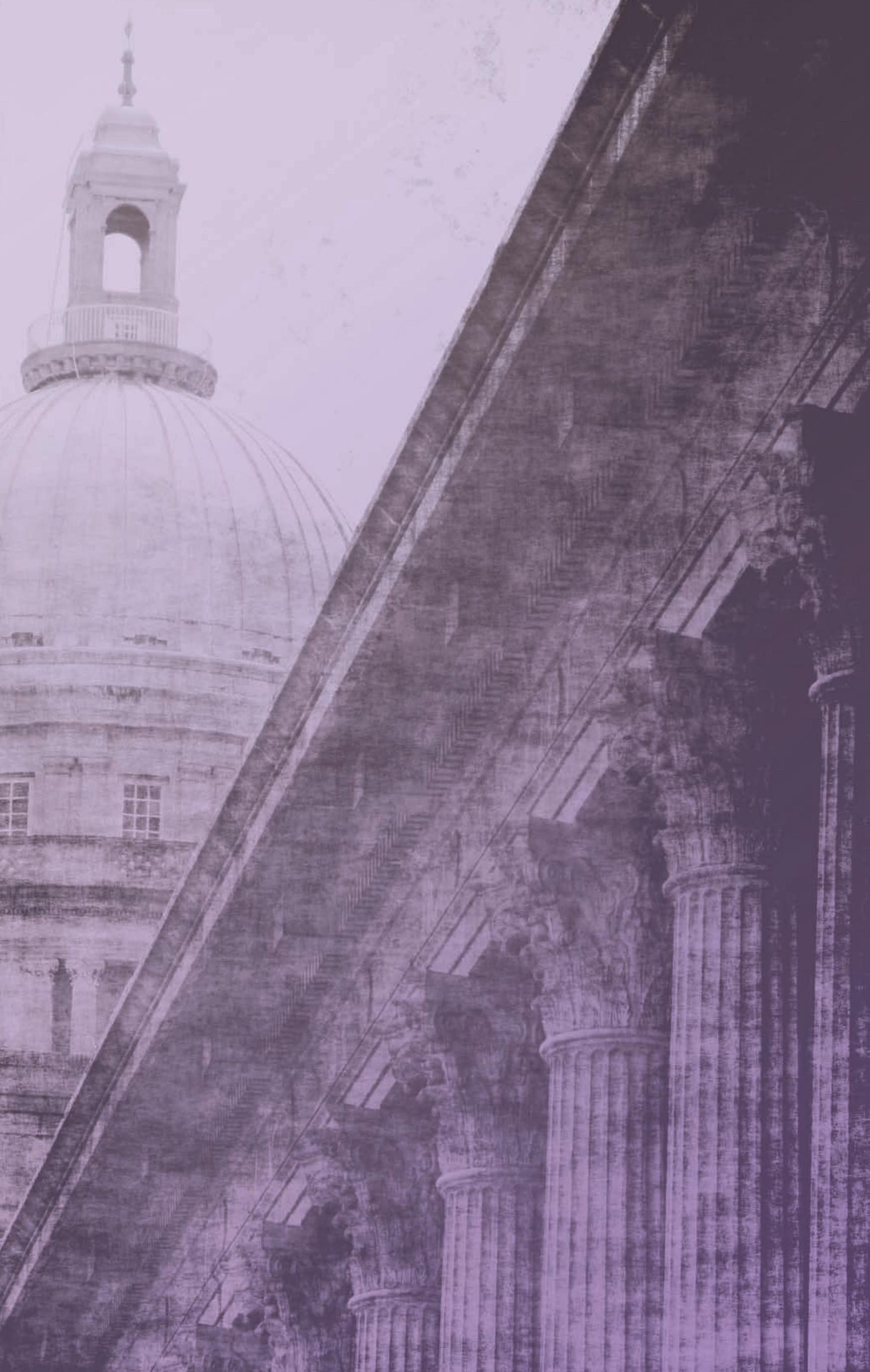




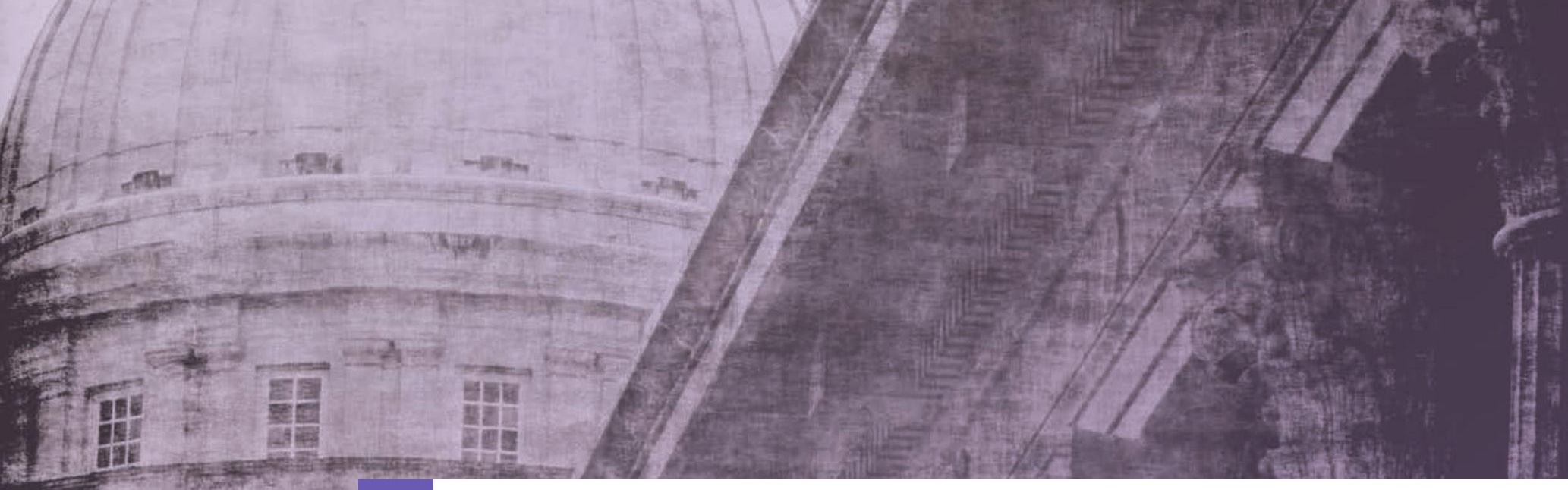

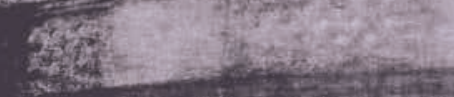 \\ 6. LES FEMMES DANS LA FONCTION PUBLIQUE}

Dans la majorité des pays membres de l'OCDE, les femmes constituent une part importante et croissante des effectifs de la fonction publique. Les autorités des pays ont pris en fait tout un éventail de mesures pour garantir l'égalité des chances entre les salariés féminins et masculins de l'administration publique, comme l'instauration d'objectifs de recrutement et de promotion, ou la mise en place de dispositifs qui permettent de concilier plus facilement activité professionnelle et vie privée. Il subsiste néanmoins de fortes disparités entre les catégories professionnelles quant à la place faite à chaque sexe, et les femmes se heurtent toujours à des obstacles lorsqu'elles tentent d'accéder aux postes d'encadrement supérieur de la fonction publique. Le déséquilibre qui en résulte entre elles et les hommes aux échelons élevés de l'administration a pour effet d'amoindrir leur rôle dans la prise de décision, notamment au sein du système judiciaire où leur présence contribue à préserver l'égalité des droits entre tous les citoyens. Par conséquent, il est nécessaire d'intensifier les efforts pour combler les écarts entre les sexes et donner aux autorités davantage de moyens d'intégrer la problématique hommes-femmes dans la conception et la mise en œuvre des politiques et des programmes, afin de garantir l'équité et de renforcer la réactivité de l'action gouvernementale et des services publics.

Ce chapitre présente des indicateurs sur la proportion de femmes au niveau des pouvoirs exécutif, judiciaire et législatif dans les pays membres de l'OCDE. Il contient des données sur les femmes et le travail à temps partiel et sur leur présence à diverses fonctions de l'administration, ainsi qu'une comparaison entre la part de l'emploi des femmes dans le secteur public et leur part de la population active totale. Ces données montrent une persistance de la ségrégation professionnelle des femmes, qui n'est pas sans effet à long terme sur leur rémunération et leurs perspectives de progression professionnelle. 
La proportion de femmes dans les effectifs de la fonction publique constitue un important indicateur du degré d'ouverture et d'équité des institutions publiques. Poursuivant leurs efforts pour renforcer la place des valeurs fondamentales de la fonction publique, comme le mérite et la transparence, les autorités des pays sont de plus en plus conscientes de l'importance des mesures de promotion de la diversité, notamment de la représentation des deux sexes. Faire en sorte que le secteur public soit représentatif de la population qu'il sert contribue de surcroît à améliorer la qualité des politiques et à renforcer la réactivité des services publics en assurant une meilleure compréhension des besoins des citoyens. C'est aussi une bonne pratique à suivre pour stimuler la productivité dans le secteur public car l'administration peut ainsi exploiter au mieux la réserve de talents disponible. Enfin, œuvrer au renforcement de la présence des femmes dans les effectifs de la fonction publique peut être un moyen d'accroître la mobilité sociale.

En moyenne dans les pays membres de l'OCDE, la proportion de femmes dans les effectifs des administrations publiques (56\%) est supérieure à leur part de la population active totale, qui se situe entre $40 \%$ et $50 \%$. Les politiques concernant l'emploi dans la fonction publique peuvent susciter beaucoup d'intérêt chez les femmes dans certains pays car le secteur public offre dans bien des cas, par rapport au secteur privé, des conditions de travail plus souples, un congé parental rémunéré plus long et/ou des prestations plus élevées pour la garde des enfants ou d'autres services. Il existe néanmoins des disparités entre les pays membres : c'est dans les pays nordiques, en Estonie et en Slovénie que la différence est la plus importante, la proportion de femmes dans les administrations publiques y étant supérieure de 20 points de pourcentage à leur part de l'emploi total. À l'inverse, en Grèce, au Japon, aux Pays-Bas et en Turquie, la représentation des femmes dans la population active est légèrement plus forte que dans les effectifs des administrations publiques.

La proportion de femmes travaillant dans les administrations publiques a augmenté entre 2001 et 2010 dans les 21 pays membres de l'OCDE sur lesquels des données sont disponibles. C'est l'Estonie qui a enregistré l'augmentation la plus forte de l'emploi des femmes au cours de cette période, et celles-ci constituaient trois quarts des effectifs des administrations publiques en 2010, ce qui est supérieur à la proportion relevée dans tous les autres pays membres de l'OCDE. En revanche, selon les chiffres les plus récents, les femmes représentaient seulement $24 \%$ et $36 \%$ des salariés des administrations publiques respectivement en Turquie et en Grèce.

\section{Méthodologie et définitions}

Les données proviennent de l'Organisation internationale du travail (OIT) et se rapportent à 2001 et 2010. Le secteur des administrations publiques comprend tous les niveaux d'administration, notamment: a) toutes les unités de l'administration centrale, de l'administration des États fédérés ou des administrations locales ; b) toutes les administrations de sécurité sociale à chaque niveau d'administration; et c) toutes les institutions sans but lucratif non marchandes qui sont contrôlées et principalement financées par des administrations publiques. La population active est constituée de toutes les personnes qui remplissent les conditions requises pour être classées dans la population occupant un emploi ou au chômage.

Les données se rapportent au nombre de salariés, sauf dans le cas des Pays-Bas et de la Nouvelle-Zélande pour lesquels elles représentent des équivalents temps plein (ETP). Par conséquent, les chiffres de l'emploi sont sousévalués pour ces deux pays par comparaison avec les autres. Les données concernant le Canada, le Chili, la Finlande, la Pologne et la Turquie se rapportent au secteur public (administrations publiques et entreprises publiques) et non aux administrations publiques.

\section{Pour en savoir plus}

OCDE (2012), Inégalités hommes-femmes : il est temps d'agir, Éditions OCDE, Paris, http://dx.doi.org/10.1787/978926417 9660-fr.

OCDE (2011, version française à paraître), Les fonctionnaires, partenaires de la croissance: pour une fonction publique plus forte, plus resserrée et plus équitable, Éditions OCDE, Paris, http://dx.doi.org/10.1787/9789264166707-en.

\section{Notes relatives aux graphiques}

Les données sur le Japon se rapportent à 2009 et non à 2010. Les données sur le Canada, le Chili, la Finlande, la Grèce, la Pologne et la Suisse se rapportent à 2008 et non à 2010. Les données sur la Norvège et la Suède se rapportent à 2007 et non à 2010. Les données sur la Turquie se rapportent à 2006 et non à 2010. Les données sur les Pays-Bas se rapportent à 2005 et non à 2010.

6.1 : Les données relatives à la proportion de femmes dans les administrations publiques proviennent de la base de données LABORSTA de l'Organisation internationale du travail (OIT). Les données concernant la proportion de femmes dans la population active sont issues de la base de données de l'OCDE sur les statistiques de la population active. 


\section{LES FEMMES DANS LA FONCTION PUBLIQUE}

Emploi des femmes dans les administrations publiques

6.1. Proportion de femmes dans les administrations publiques et dans la population active (2010)

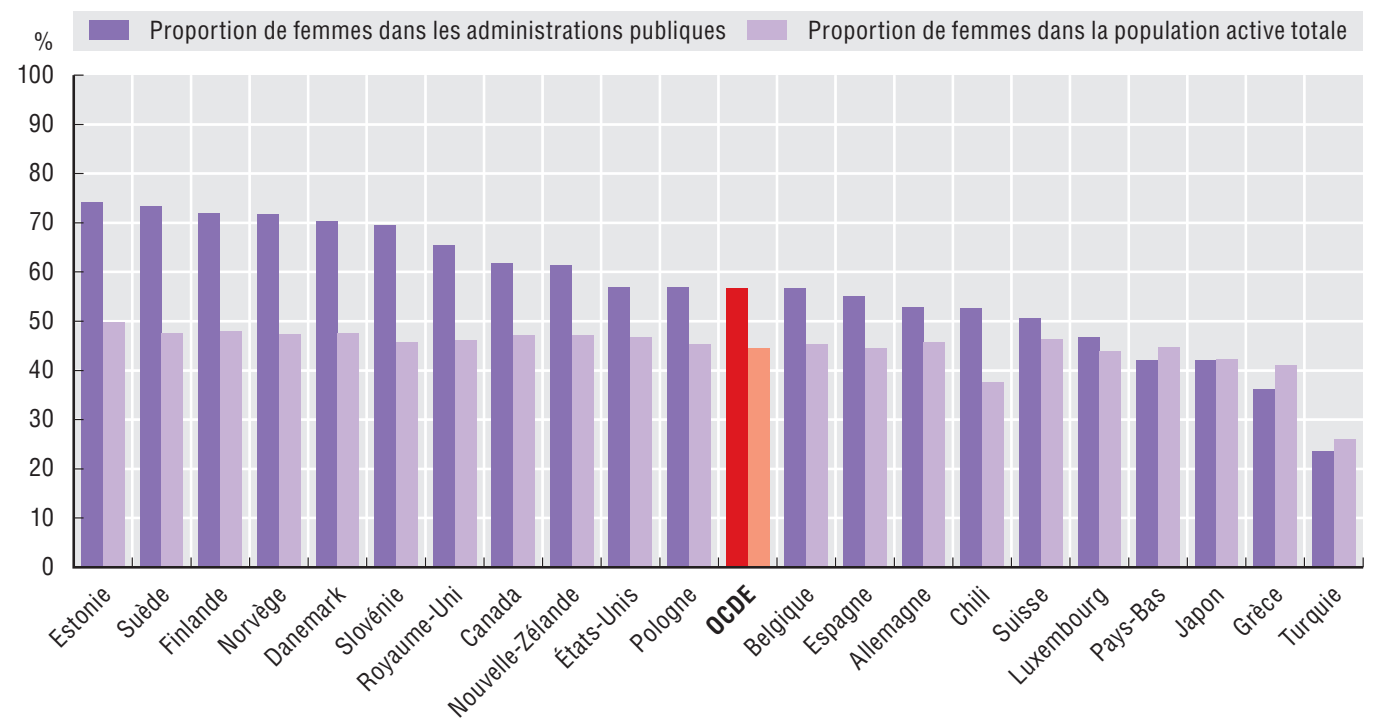

Source: Organisation internationale du travail (OIT), base de données LABORSTA; et OCDE, Base de données sur les statistiques de la population active.

StatLink काISL http://dx.doi.org/10.1787/888933159633

\subsection{Proportion de femmes dans les administrations publiques (2001 et 2010)}

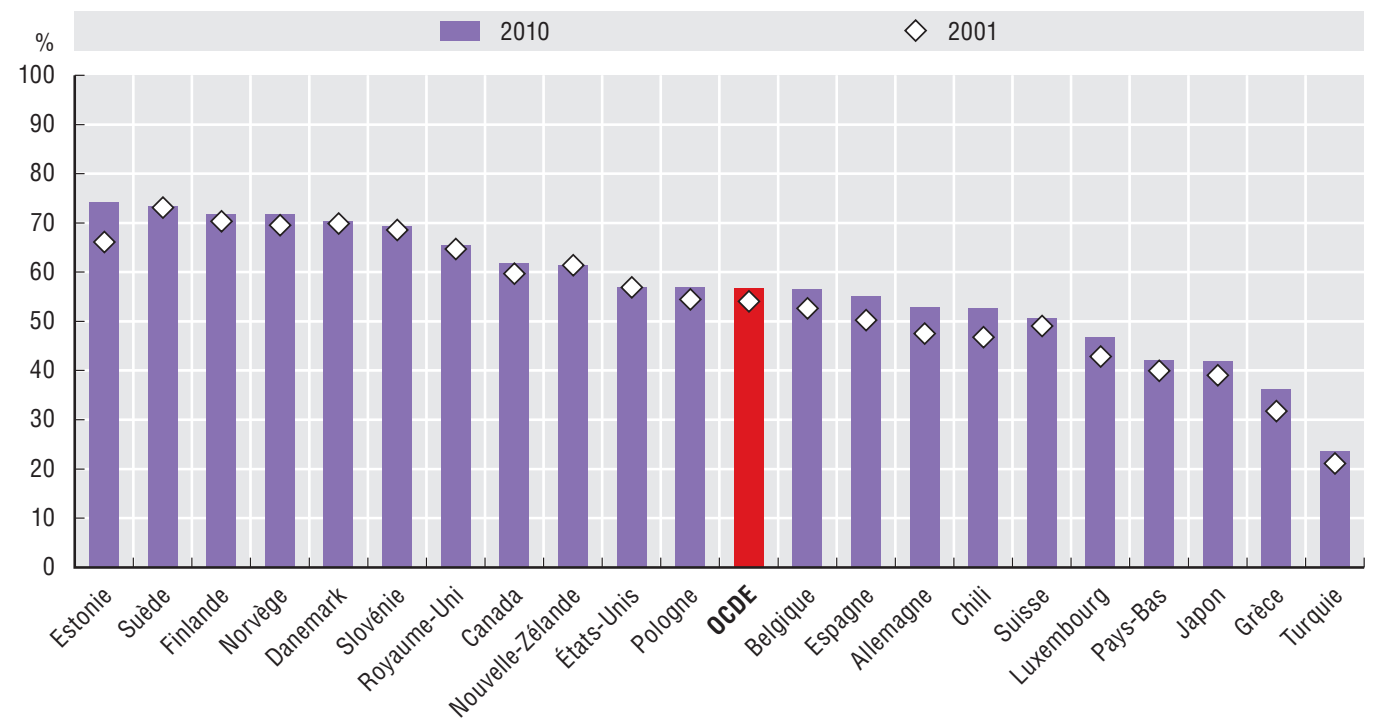

Source : Organisation internationale du travail (OIT), base de données LABORSTA. 
L'emploi dans l'administration centrale, sous-ensemble de l'emploi total dans les administrations publiques, recouvre tout un éventail de postes de cadre, de technicien et de secrétaire situés dans les ministères et autres services de l'administration centrale. Par conséquent, la présence des femmes dans l'administration centrale, notamment aux hautes fonctions administratives, constitue un important indicateur du rôle qu'elles jouent dans l'élaboration et la mise en œuvre des politiques des pays membres de l'OCDE.

En moyenne, les femmes représentaient un peu plus de $50 \%$ de l'ensemble des salariés de l'administration centrale en 2010 dans les 22 pays sur lesquels des données sont disponibles. Ce chiffre est légèrement inférieur à la proportion de femmes dans les effectifs des administrations publiques (57\%, voir graphique 6.2), qui tient compte des professions majoritairement exercées par les femmes, comme celles d'enseignant et d'infirmière, que l'on recense plus souvent au niveau des États fédérés et à l'échelon local. Le Chili, l'Italie et la Pologne continuent d'enregistrer les pourcentages les plus élevés de femmes dans les effectifs de l'administration centrale, lesquels se situent aux alentours de $70 \%$, cette proportion étant la plus faible au Japon (16\%), suivi de l'Allemagne (39\%). La présence des femmes dans l'administration centrale reste légèrement supérieure à leur représentation dans la population active, ce qui est dû dans beaucoup de pays à des politiques consistant, par exemple, à offrir des conditions de travail plus souples et un congé parental rémunéré.

La proportion de femmes dans les effectifs de l'administration centrale a augmenté entre 2000 et 2010 dans la quasi-totalité des 15 pays membres de l'OCDE sur lesquels des données sont disponibles (dans deux pays, à savoir l'Estonie et le Japon, elle a diminué de moins d'un point de pourcentage). Toutefois, malgré la progression que l'emploi des femmes dans l'administration centrale a enregistrée au cours de la dernière décennie, celles-ci continuent d'être surreprésentées aux postes subalternes. Dans 15 pays sur 19, les femmes occupent plus de $50 \%$ des postes de secrétaire, proportion proche de $90 \%$ en Slovénie et en Autriche. Elles sont beaucoup moins nombreuses aux niveaux plus élevés, où elles ne représentent que $40 \%$ des cadres moyens et $29 \%$ des cadres supérieurs (voir graphique 6.4).

En outre, les femmes représentent une part disproportionnée des travailleurs à temps partiel dans l'administration centrale. Dans les 16 pays membres de l'OCDE qui ont fourni des données, deux tiers ou plus des travailleurs à temps partiel étaient des femmes en 2010, proportion qui dépassait $85 \%$ en Allemagne, au Luxembourg et en France. Si le travail à temps partiel peut être une solution intéressante pour les salariés désireux de pouvoir concilier plus facilement activité professionnelle et obligations familiales, il donne généralement lieu à une rémunération plus faible et réduit les possibilités de progression professionnelle sur le long terme. La mise en place d'une voie de passage de l'activité à temps partiel au travail à temps plein constitue pour les pays un moyen d'offrir à tous les salariés une marge de manœuvre sans compromettre leurs perspectives d'évolution professionnelle à long terme.

\section{Méthodologie et définitions}

Les données ont été recueillies au moyen de l'Enquête 2011 de l'OCDE sur la parité dans l'emploi public. Les répondants étaient principalement des hauts fonctionnaires des services de gestion des ressources humaines de l'administration centrale.

L'administration centrale (également dénommée administration nationale ou fédérale) désigne les ministères, services et organismes contrôlés et financés à l'échelon central de l'administration. Les données sur l'emploi se rapportent aux personnes employées selon le cadre général d'emploi, qui définit les conditions d'emploi de la plupart des salariés de l'administration, ainsi que de la majorité des agents statutaires. L'emploi à temps partiel recouvre les postes assortis d'une durée hebdomadaire d'activité inférieure à celle d'un emploi à temps complet. Sa définition diffère selon les pays mais il correspond généralement à un temps de travail de moins de 30 ou 35 heures par semaine.

Les postes d'encadrement supérieur se situent au-dessous des fonctions de ministre ou de vice-ministre. Immédiatement en deçà de l'encadrement supérieur se trouve l'encadrement intermédiaire qui correspond aux postes comportant la gestion d'au moins trois personnes. Il s'agit généralement des chefs de division, de département et autres services d'un ministère. Les techniciens se situent entre les cadres et le personnel de soutien administratif et de bureau. Les postes de secrétaire sont occupés par les agents chargés des tâches administratives et des fonctions d’appui administratif général.

\section{Pour en savoir plus}

OCDE (2012), Inégalités hommes-femmes : il est temps d’agir, Éditions OCDE, Paris, http://dx.doi.org/10.1787/9789264179660-fr.

OCDE (2011, version française à paraître), Les fonctionnaires, partenaires de la croissance : pour une fonction publique plus forte, plus resserrée et plus équitable, Éditions OCDE, Paris, http:// dx.doi.org/10.1787/9789264166707-en.

\section{Notes relatives aux graphiques}

Les données sur le Luxembourg, la Slovénie et la Suède se rapportent à 2011 et non à 2010. Les données sur la France se rapportent à 2009 et non à 2010. Les données sur les Pays-Bas représentent des équivalents temps plein.

6.3 : Les données sur l'Estonie, le Japon et l'Espagne se rapportent uniquement aux salariés à temps plein. Il n'y a pas de données disponibles sur la Finlande pour 2010.

6.4 : Les données sur la Suisse concernant les postes de secrétaire comprennent aussi les postes de technicien. 
6.3. Proportion de femmes dans les effectifs de l'administration centrale (2000 et 2010)

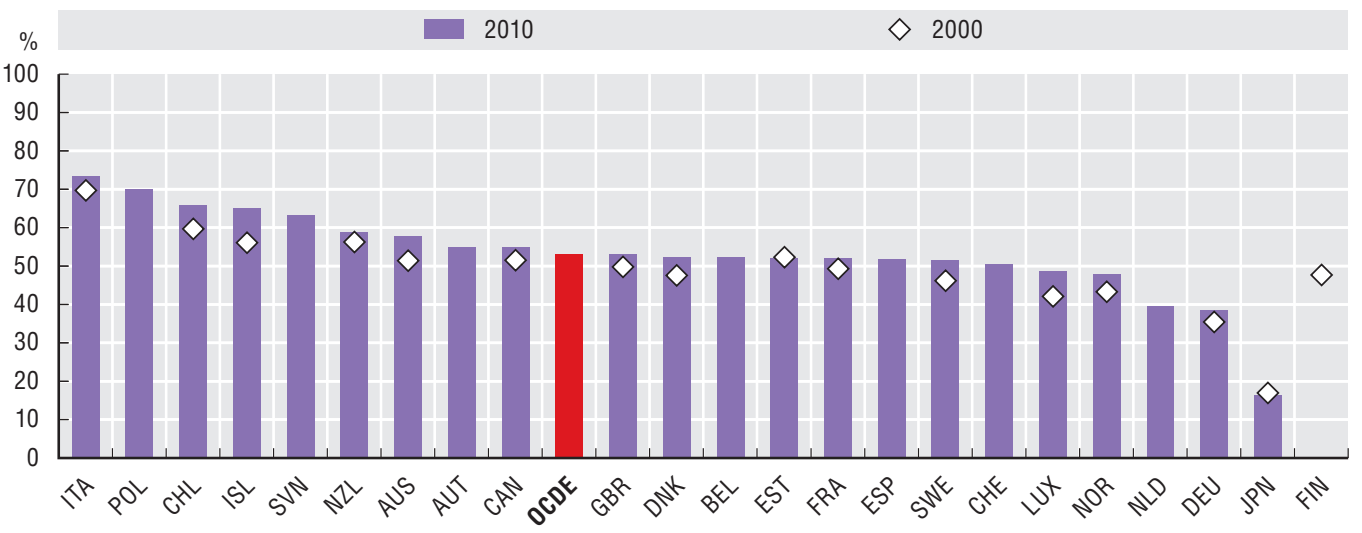

Source : Enquête 2011 de l'OCDE sur la parité dans l'emploi public.

StatLink AाIst http://dx.doi.org/10.1787/888933159654

6.4. Proportion de femmes dans les effectifs de l'administration centrale par catégorie professionnelle (2010)

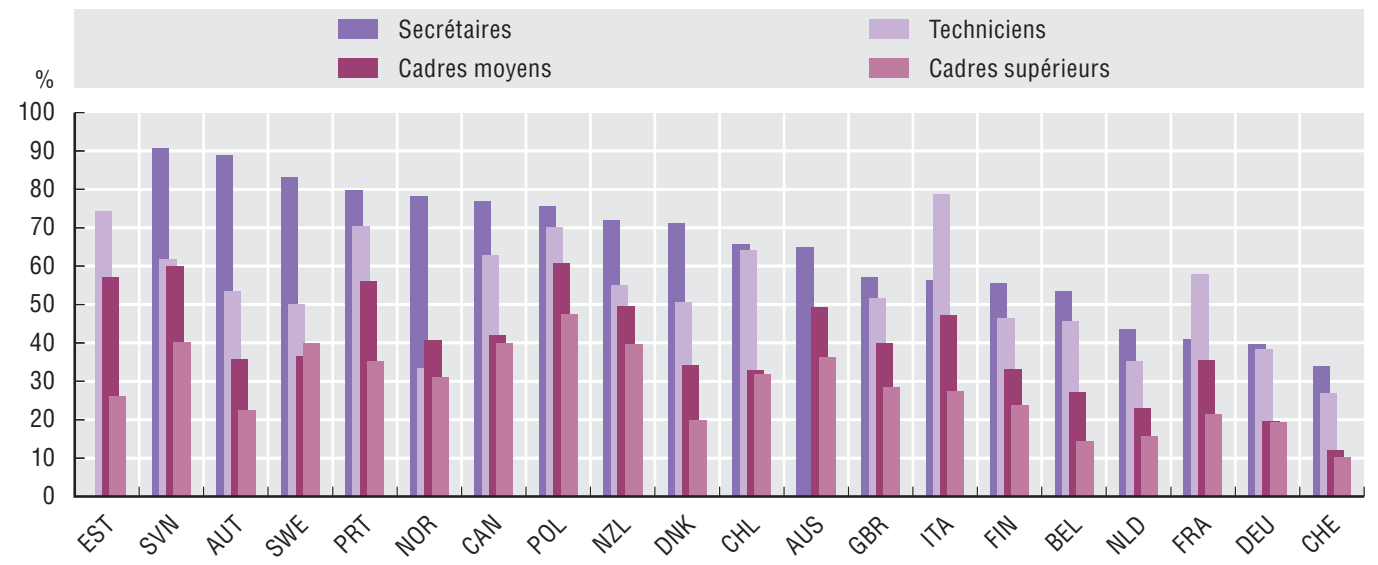

Source : Enquête 2011 de l'OCDE sur la parité dans l'emploi public.

StatLink जाञाय http://dx.doi.org/10.1787/888933159663

6.5. Part des femmes dans les effectifs à temps partiel de l'administration centrale (2010)

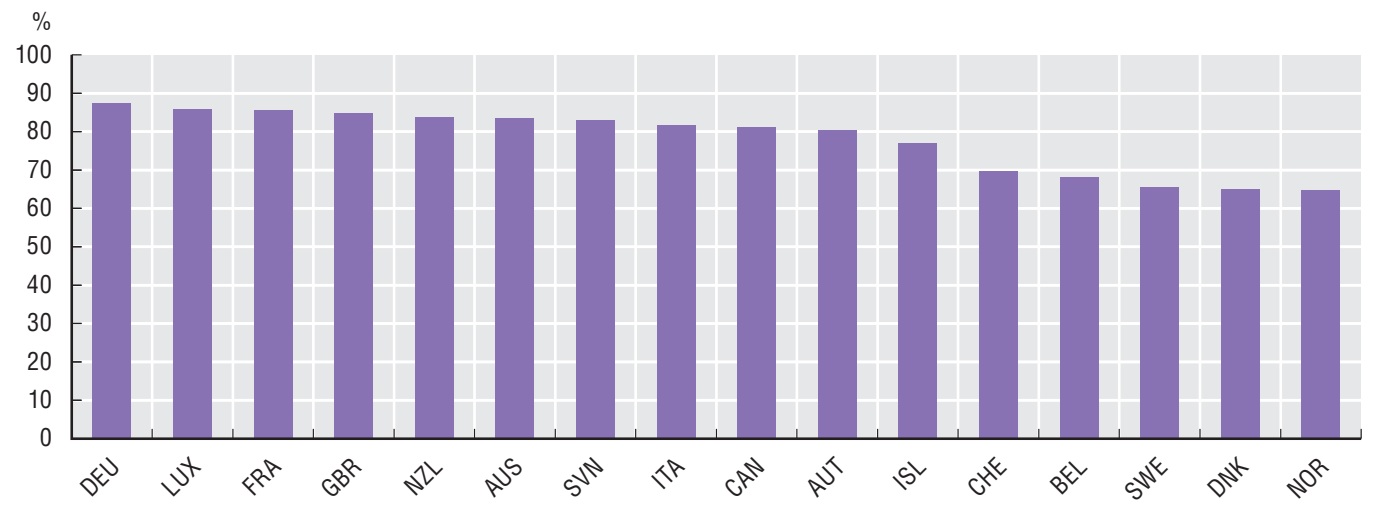

Source: Enquête 2011 de l'OCDE sur la parité dans l'emploi public. 
Malgré la proportion croissante de femmes dans les effectifs de l'administration centrale (voir aussi le graphique 6.3), celles-ci demeurent sous-représentées dans les hautes fonctions administratives ou dirigeantes. Le déséquilibre ainsi observé entre les sexes aux échelons élevés de l'administration centrale a pour effet de limiter le rôle des femmes dans le processus de décision, ce qui nuit à l'équité des politiques publiques et des textes de loi. Citons, par exemple, l'important problème de la représentation des femmes dans le système judiciaire, qui est déterminante pour assurer l'égalité des droits et éliminer la discrimination fondée sur le sexe dans les décisions de justice.

En moyenne dans les pays membres de l'OCDE, les femmes détiennent plus de $50 \%$ des postes de l'administration centrale, mais seulement $29 \%$ des postes d'encadrement supérieur dans les 18 pays sur lesquels des données sont disponibles. La proportion de femmes aux postes de niveau supérieur de l'administration centrale diffère sensiblement selon les pays membres. En Pologne, près de $50 \%$ des postes d'encadrement supérieur sont occupés par des femmes, mais ce chiffre est plus que divisé par deux dans des pays tels que la Belgique ou la Suisse. L'écart le plus grand entre la proportion de femmes dans les effectifs de l'administration centrale et leur présence aux postes de niveau supérieur est relevé en Autriche, en Belgique et en Italie.

On observe une tendance analogue dans le système judiciaire de beaucoup de pays membres de l'OCDE. En 2010, les femmes représentaient un peu moins de la moitié des juges professionnels dans les pays membres sur lesquels des données sont disponibles mais, la même année, elles occupaient seulement $29 \%$ des postes de président de tribunaux de première et deuxième instance. Les femmes sont aussi relativement moins présentes dans les cours suprêmes ( $28 \%$ des juges). Elles exerçaient la fonction de présidente de cour suprême dans seulement cinq des pays membres sur lesquels des données sont disponibles (République tchèque, Irlande, Grèce, Finlande et Suède).

Afin de corriger ces déséquilibres, certains pays prennent des mesures volontaristes pour assurer aux femmes des possibilités d'emploi égales. Parmi elles figurent l'instauration de quotas en faveur de la diversité ou de la parité femmes-hommes, la définition de règles ou lignes directrices pour l'égalité des salaires, la mise en place de dispositifs de formation et de parrainage pour les fonctions de direction, ainsi que l'application de mesures visant à faciliter la conciliation entre vie privée et vie professionnelle, comme les horaires flexibles. Des pays tels que le Royaume-Uni et le Canada ont constitué des commissions judiciaires spéciales qui œuvrent au renforcement de la parité entre femmes et hommes dans la réserve des candidats parmi lesquels seront choisis les juges.

\section{Méthodologie et définitions}

Les données sur le nombre de femmes aux postes d'encadrement supérieur de l'administration centrale ont été obtenues au moyen de l'Enquête 2011 de l'OCDE sur la parité dans l'emploi public. Les réponses ont été recueillies auprès de fonctionnaires chargés de la gestion des ressources humaines et des politiques de l'emploi au niveau central/fédéral.

Les données concernant le nombre de femmes juges professionnelles et de femmes présidentes de tribunaux ont été recueillies auprès de la Commission européenne pour l'efficacité de la justice (CEPEJ) du Conseil de l'Europe. Sauf indication contraire, il s'agit dans le second cas des tribunaux de première et deuxième instance. Les données relatives aux femmes juges de cour suprême proviennent de la Base de données de la Commission européenne sur les femmes et les hommes dans la prise de décision. Selon la définition adoptée par la Commission européenne, la cour suprême est la juridiction la plus élevée d'un pays ayant compétence en matière civile et pénale. Cependant, dans certains pays, la cour suprême peut aussi détenir une compétence en matière administrative et/ou constitutionnelle. Les juges comprennent les présidents de cours suprêmes.

\section{Pour en savoir plus}

CEPEJ (2012), Rapport d'évaluation des systèmes judiciaires européens - Édition 2012 (données 2010), Études de la CEPEJ $\mathrm{n}^{\circ} 18$, Éditions du Conseil de l'Europe, Strasbourg.

OCDE (2012), Inégalités hommes-femmes: il est temps d'agir, Éditions OCDE, Paris, http://dx.doi.org/10.1787/978926 4179660-fr.

\section{Notes relatives aux graphiques}

6.6 : Les données sur la proportion de femmes dans les effectifs de l'administration centrale ne sont pas disponibles pour le Portugal et la Finlande. Les données sur le Luxembourg, la Slovénie et la Suède se rapportent à 2011 et non à 2010. Les données sur la France se rapportent à 2009 et non à 2010. Les données sur l'Estonie, le Japon et l'Espagne se rapportent uniquement aux salariés à temps complet. Les données sur les Pays-Bas représentent des équivalents temps plein.

6.7 : Les données sur la proportion de femmes parmi les présidents de tribunaux en Islande, en Irlande, en Suisse et en Turquie se rapportent aux tribunaux de première instance seulement. Les données sur l'Espagne se rapportent aux tribunaux de deuxième instance seulement.

6.8 : Les données sur l'Australie, le Canada, la Corée, le Mexique, la Nouvelle-Zélande, la Suisse et l'Ukraine se rapportent à 2010. Les données sur le Chili, la Norvège et Israël se rapportent à 2011. Les données sur les États-Unis et le Japon se rapportent à 2013.

Informations sur les données concernant Israël : http://dx.doi.org/ 10.1787/888932315602. 


\section{LES FEMMES DANS LA FONCTION PUBLIQUE}

\section{Présence des femmes dans l'encadrement supérieur de l'administration centrale}

6.6. Proportion de femmes aux postes d'encadrement supérieur comparée à leur part des effectifs de l'administration centrale (2010)

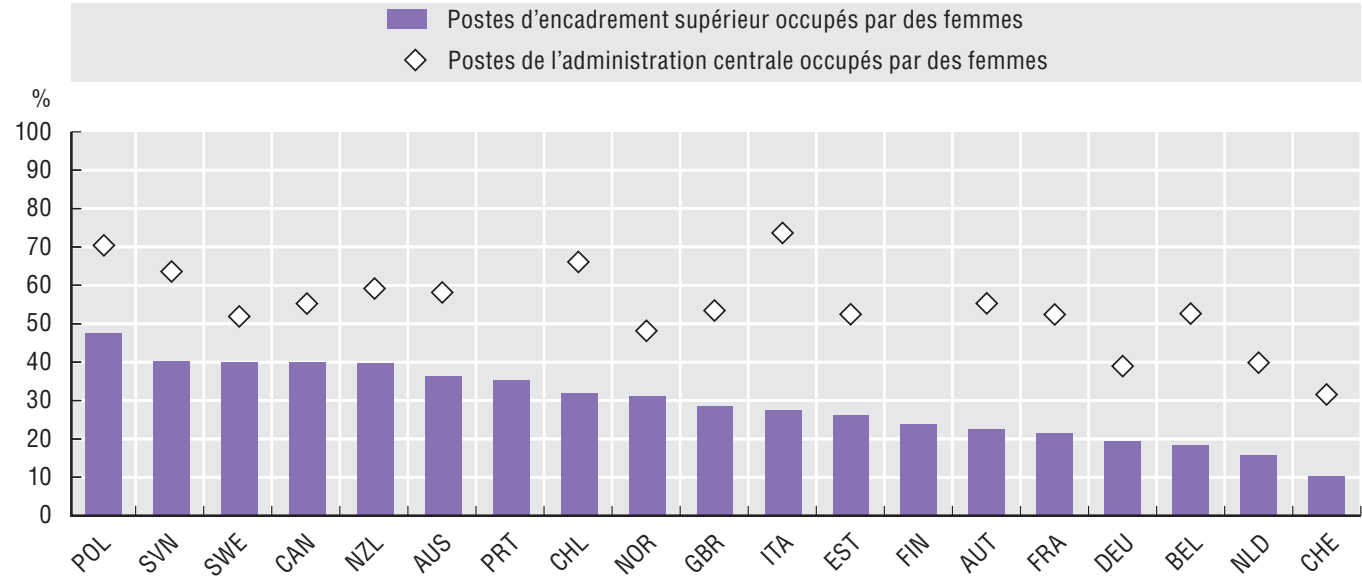

Source : Enquête 2011 de l'OCDE sur la parité dans l'emploi public.

StatLink तiाs $h$ ttp://dx.doi.org/10.1787/888933159685

\subsection{Proportion de femmes parmi les présidents de tribunaux comparée à leur part des effectifs de juges professionnels (2010)}

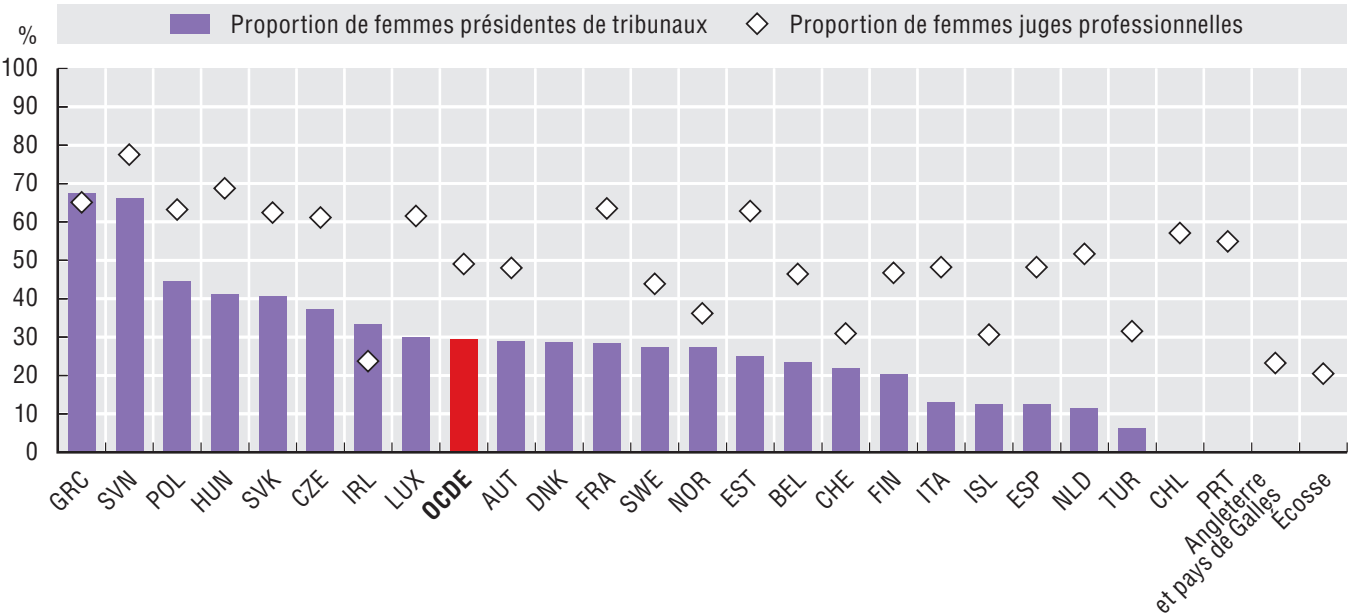

Source : CEPEJ (2012), Rapport d'évaluation des systèmes judiciaires européens - Édition 2012 (données 2010) - Études de la CEPEJ n 18 , Éditions du Conseil de l’Europe, Strasbourg. Les données sur le Chili ont été communiquées par les autorités nationales. StatLink (ints http://dx.doi.org/10.1787/888933159695

\subsection{Proportion de femmes juges de cours suprêmes (2012 ou année disponible la plus récente)}

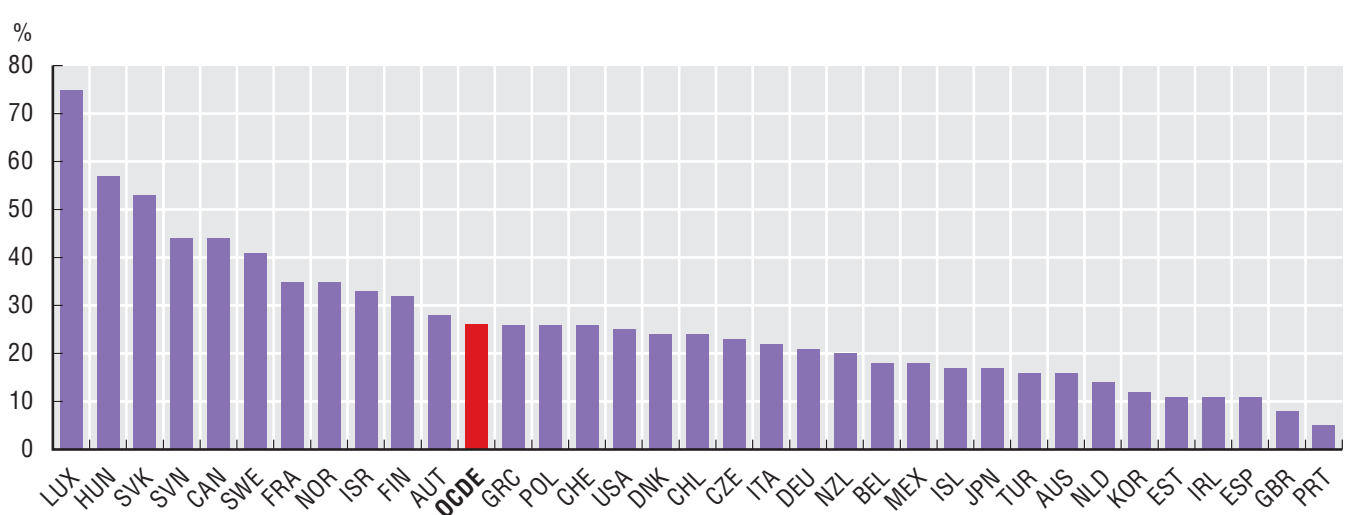

Source: Pays de l'UE : Base de données de la Commission européenne sur les femmes et les hommes dans la prise de décision. Pays non membres de l'UE : Enquête 2011 de l'OCDE sur les femmes et les emplois de direction de la fonction publique.

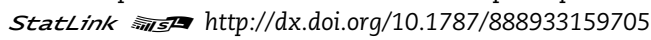


La représentation des femmes dans la vie politique est importante non seulement pour assurer l'égalité des sexes dans le processus politique, mais aussi pour diriger l'attention vers des problèmes d'ordre socio-économique essentiels, comme le développement humain, la violence fondée sur le sexe, les politiques favorables à la famille, l'égalité des salaires, les pensions de retraite, la réforme électorale et la fourniture des services. Or, les femmes se heurtent toujours à un " plafond de verre " qui les empêche de prendre pleinement part à la vie politique au niveau du pouvoir législatif et du pouvoir exécutif politique, et elles restent en général sous-représentées dans la sphère politique.

En moyenne dans les pays de l'OCDE, les femmes occupaient, au début de 2012, un peu plus du quart des sièges de la Chambre basse ou de la Chambre unique, 12 pays membres seulement ayant atteint ou dépassé le seuil de $30 \%$ recommandé par les Nations unies et l'Union interparlementaire pour la représentation des femmes. Il s'agit là d'une légère progression par rapport à 2002, soit six points de pourcentage de plus en moyenne. Dans les pays nordiques, la représentation des femmes au sein des parlements est généralement la plus forte des pays membres de l'OCDE, et celles-ci occupaient ainsi $40 \%$ ou plus des sièges de ces assemblées en Suède, en Finlande, en Islande et en Norvège. En Turquie, au Japon, au Chili et en Hongrie, les femmes détenaient moins d'un quart des sièges.

Les pays dotés d'un système électoral proportionnel comptent en général davantage de femmes dans leur assemblée législative, peut-être en raison de la pratique consistant pour les partis politiques à constituer des listes de candidats plus paritaires. Dans la zone de l'OCDE, neuf des douze pays qui respectent le seuil de $30 \%$ utilisent une forme ou une autre de représentation proportionnelle pour l'élection des parlementaires. Outre la nature du système électoral en vigueur, la représentation politique des femmes peut être déterminée par des obstacles d'ordre culturel et financier, ainsi que par la difficulté de concilier les obligations imposées par la vie politique avec celles qui sont liées à la vie privée. Face à cette situation, neuf pays membres de l'OCDE ont instauré des quotas de femmes pour faire progresser l'égalité des sexes au sein des parlements. L'application de ces quotas prend toutefois des formes variables et peut ainsi se faire au cours du processus de désignation des candidats (par exemple, règle concernant l'inscription de femmes sur les listes des partis politiques ou leur désignation comme candidates pour des circonscriptions électorales données), ou être fondée sur les résultats, un certain pourcentage des sièges du parlement étant réservé aux femmes.

En ce qui concerne le pouvoir exécutif politique dans les pays membres de l'OCDE, le pourcentage de femmes ministres est passé de $21 \%$ en 2005 à $25 \%$ en 2012. Selon les données pour 2012, la proportion de femmes ministres va de plus de $50 \%$ en Norvège, en Suède, en Finlande et en Islande à moins de $10 \%$ en Hongrie, en Estonie, en Slovénie, en Grèce et en Turquie. Les femmes se voient souvent confier les portefeuilles relatifs aux politiques sociales et culturelles. Le processus de nomination des ministres dépend certes du système politique du pays (vote ou nomination par les parlementaires, ou bien nomination par la présidence avec ou sans l'approbation du parlement), mais dans aucun système la représentation des femmes n'est égale à celle des hommes.

\section{Méthodologie et définitions}

Les données concernant les femmes parlementaires se rapportent à la Chambre basse ou à la Chambre unique et proviennent de la base de données PARLINE de l'Union interparlementaire. Elles représentent la proportion de femmes parlementaires relevée aux dates du 31 octobre 2012 et du 25 octobre 2002.

Les barres de couleur pâle dans le graphique ci-dessous représentent les pays où des quotas légaux de candidates étaient en vigueur à la date de janvier 2013 pour la Chambre basse ou la Chambre unique. Les quotas légaux sont inscrits dans la loi électorale du pays, sa législation relative aux partis politiques ou d'autres textes de loi comparables. Le fait que les deux formes de quotas reposent par définition sur des dispositions légales oblige toutes les formations politiques qui prennent part aux élections à les appliquer, sans distinction. Celles qui ne respectent pas les quotas légaux s'exposent à des sanctions. Les données relatives aux quotas de femmes sont issues de la Base de données mondiale des quotas de femmes de l'Institute for Democracy and Electoral Assistance (IDEA).

Les données sur les femmes ministres proviennent des affiches de l'Union interparlementaire sur "Les femmes en politique ". Elles se rapportent aux femmes qui occupaient des postes de ministre au 1er janvier 2012 et au 1er janvier 2005. Elles indiquent la part des femmes dans l'effectif total des ministres, y compris les vicepremiers ministres et les vice-ministres. Les Premiers ministres/chefs de gouvernement ont aussi été pris en compte lorsqu'ils détenaient un portefeuille ministériel. Les vice-présidents et les dirigeants d'instances gouvernementales ou d'organismes publics n'ont pas été comptabilisés dans le total.

\section{Pour en savoir plus}

International IDEA (2007), Designing for Equality, Stockholm, Suède.

OCDE (2012), Inégalités hommes-femmes : il est temps d'agir, Éditions OCDE, Paris, http://dx.doi.org/10.1787/97892 64179660-fr.

\section{Notes relatives aux graphiques}

6.9 : Les données se rapportent à la proportion de femmes parlementaires relevée aux dates du 31 octobre 2012 et du 25 octobre 2002. Les pourcentages représentent le nombre de femmes parlementaires en proportion du total des sièges pourvus. Il n'y a pas de données disponibles sur la République slovaque pour 2002.

6.10 : Les données se rapportent aux femmes qui exerçaient la fonction de ministre aux dates du $1^{\mathrm{er}}$ janvier 2012 et du $1^{\mathrm{er}}$ janvier 2005 . Le total comprend les vice-premiers ministres et les vice-ministres. Les Premiers ministres/chefs de gouvernement ont aussi été inclus lorsqu'ils détenaient un portefeuille ministériel. Les vice-présidents et les dirigeants d'instances gouvernementales ou d'organismes publics n'ont pas été pris en compte.

Informations sur les données concernant Israël : http://dx.doi.org/ $10.1787 / 888932315602$. 
6.9. Proportion de femmes parlementaires et quotas légaux (2012 et 2002)

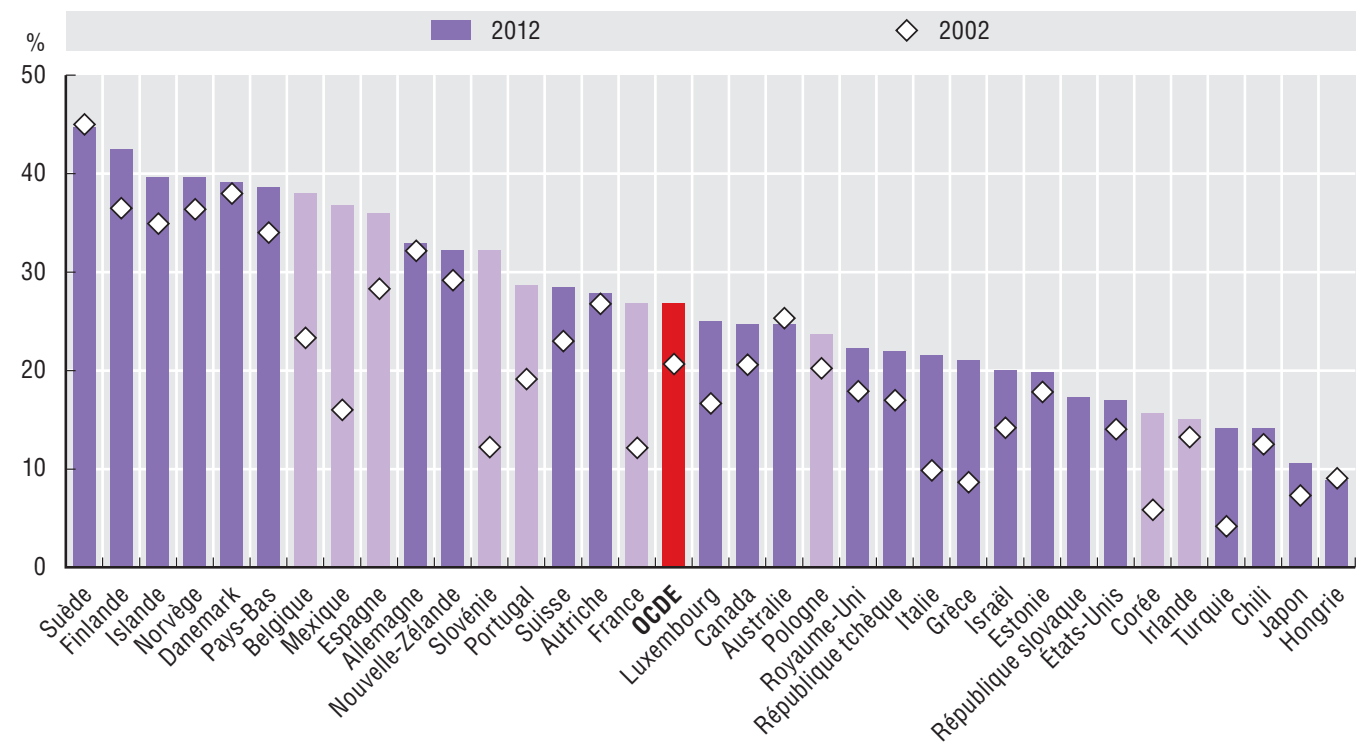

Note: Les barres de couleur pâle représentent les pays où des quotas légaux de candidates étaient en vigueur à la date du 21 janvier 2013 pour la Chambre basse ou la Chambre unique.

Source: Union interparlementaire (UIP), base de données PARLINE ; et IDEA, Quota Project (base de données mondiale des quotas de femmes).

StatLink ताIs $h$ ttp://dx.doi.org/10.1787/888933159717

\subsection{Proportion de femmes ministres (2005 et 2012)}

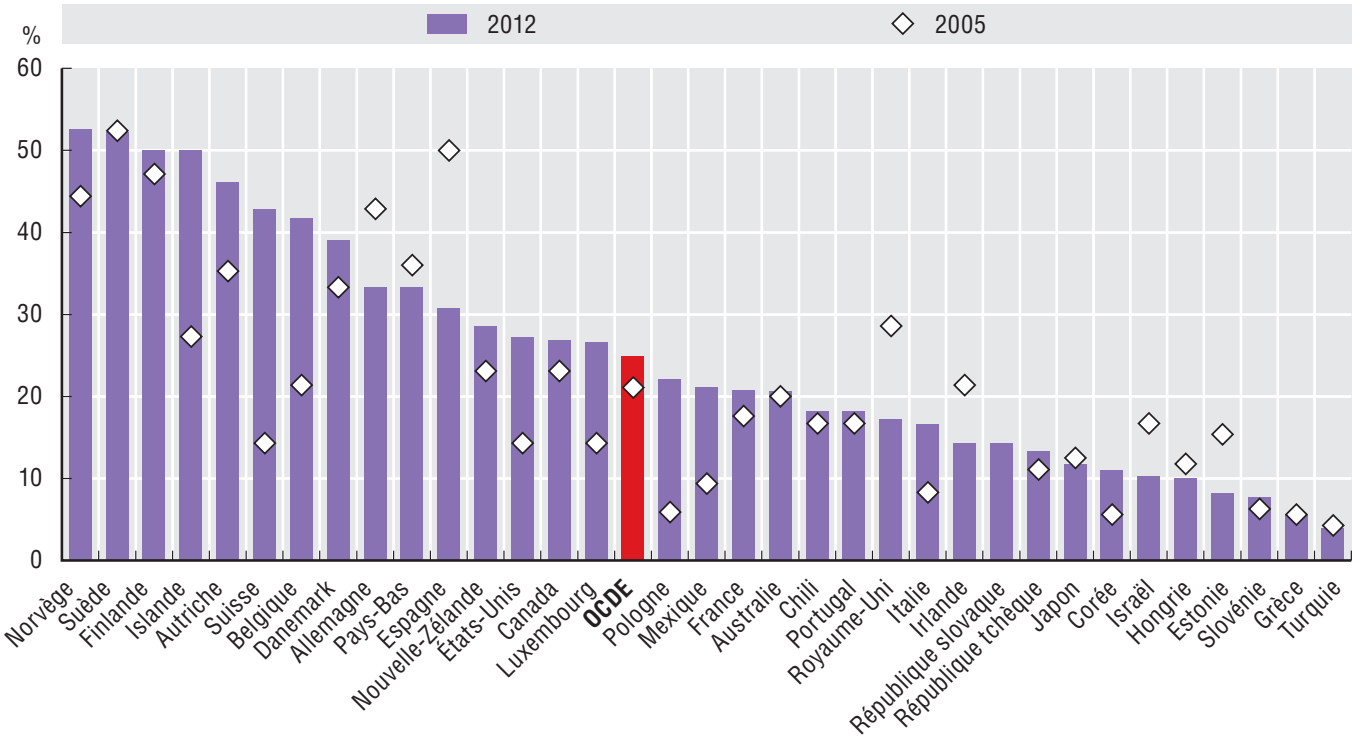

Source: Union interparlementaire (UIP), affiches sur " Les femmes en politique », 2012 et 2005.

StatLink AाISL $h$ ttp://dx.doi.org/10.1787/888933159724 


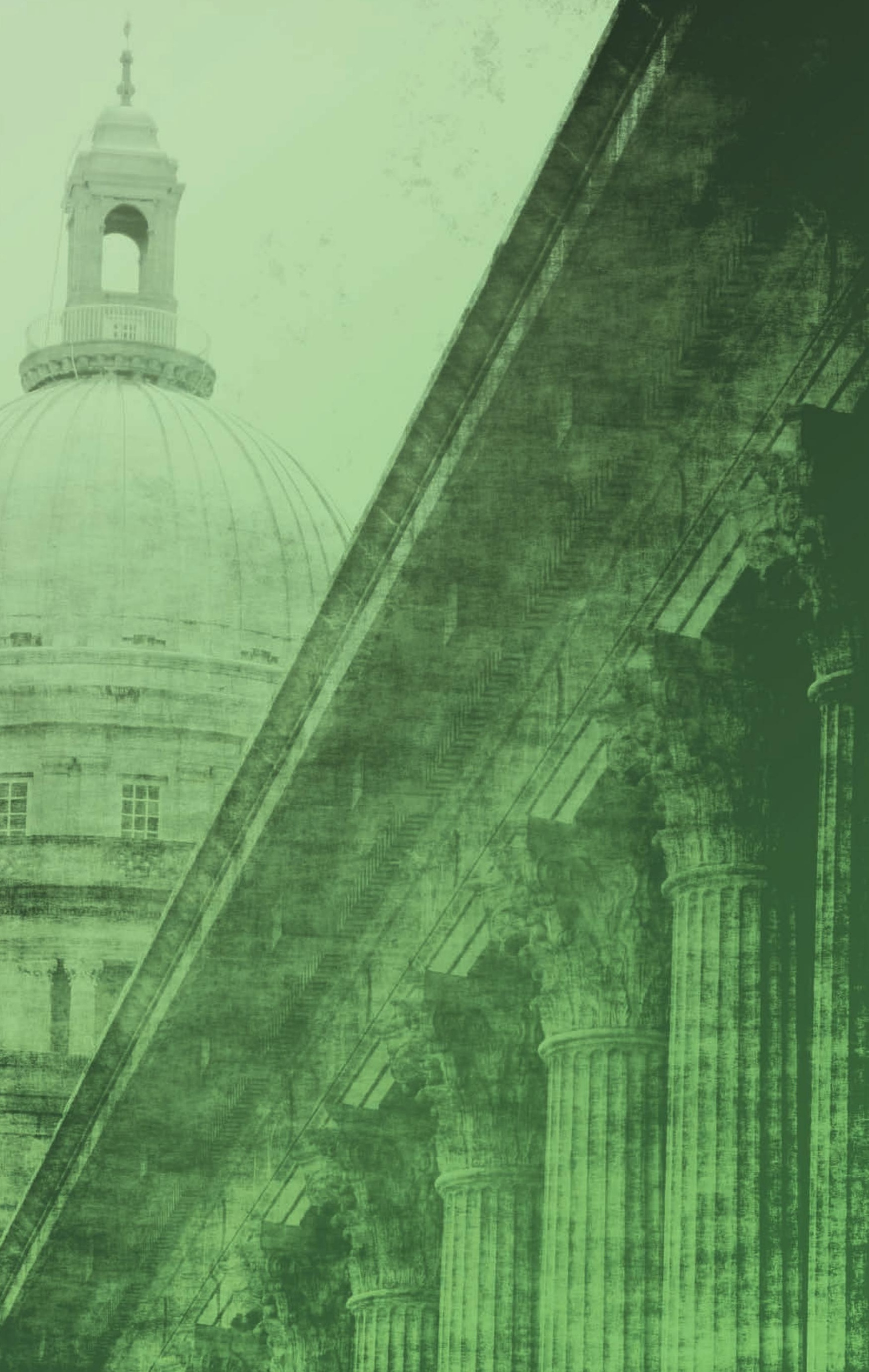




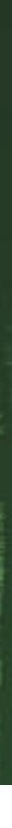

\section{LES MARCHÉS PUBLICS}

Les pays de l'OCDE consacrent en moyenne $13 \%$ de leur PIB et $29 \%$ de leurs dépenses publiques aux marchés publics, qui représentent donc une activité très importante. Ce chapitre fournit des données estimatives sur le pouvoir d'achat associé aux marchés publics et met en évidence leur capacité à servir de levier à la réalisation d'objectifs économiques, sociaux et environnementaux tout en assurant l'optimisation des ressources et l'efficience des dépenses.

Les gouvernements des pays membres de l'OCDE sont aujourd'hui particulièrement soucieux de réaliser des économies afin d'assainir leurs finances publiques et de dégager une marge de manœuvre budgétaire pour d'autres politiques. Bon nombre d'entre eux ont donc recours à des instruments novateurs pour réaliser des économies d'échelle, restructurant leurs approvisionnements, regroupant leurs achats et informatisant la passation de leurs marchés.

Ils sont par ailleurs nombreux à s'appuyer sur les marchés publics non seulement pour optimiser la dépense publique, mais aussi pour poursuivre d'autres objectifs consistant à stimuler l'innovation, à promouvoir une croissance durable, à encourager le développement des PME et à assurer un accès plus égalitaire à la vie économique. On trouvera dans ce chapitre des précisions sur ces démarches ainsi que sur d'autres dimensions d'ordre stratégique liées à la passation de marchés publics.

Dans les années à venir, les pouvoirs publics seront de plus en plus confrontés à la difficile tâche de concilier les divers objectifs poursuivis à travers les marchés publics, de renforcer le suivi et l'évaluation des dépenses et des résultats liés à ces marchés et de professionnaliser leur passation. 
Les marchés publics correspondent aux achats de biens, de services et de travaux effectués par les administrations et les entreprises publiques. Ils constituent une part importante des dépenses totales des administrations publiques. En 2011, les dépenses des administrations publiques liées aux marchés publics représentaient en moyenne $29 \%$ de leurs dépenses totales (et $13 \%$ du PIB).

Compte tenu des montants en jeu, les pays qui parviendraient à réaliser des gains d'efficience au niveau de leurs marchés publics pourraient espérer réaliser des économies considérables et dégager ainsi une marge de manœuvre budgétaire pour leurs politiques économiques et sociales. Par exemple, si les pays membres de l'OCDE réussissaient à réduire de $10 \%$ les dépenses liées à leurs marchés publics grâce à des gains d'efficience (en conservant le même panier de biens et de services), les dépenses totales de leurs administrations publiques seraient allégées de $2.9 \%$ en moyenne, ce qui représente $1.3 \%$ du PIB en 2011. Différents outils permettent de réaliser des gains d'efficience, comme ceux consistant à recourir aux TIC et à regrouper les achats pour réaliser des économies d'échelle.

Il faudrait associer les administrations infranationales aux efforts visant à améliorer l'efficience des marchés publics, étant donné que les dépenses liées aux marchés publics passés par les administrations d'États fédérés et les administrations locales représentent, en moyenne des pays de l'OCDE, $55 \%$ du total des dépenses liées aux marchés publics. Tout cela est particulièrement important pour les pays à structure fédérale Allemagne, Autriche, Belgique, Canada, Espagne, États-Unis, Mexique et Suisse - où les marchés publics des administrations d'États fédérés et des administrations locales représentent, en moyenne, $76 \%$ du total des dépenses liées aux marchés publics. Cela étant, les États unitaires devraient également faire des efforts à l'échelon infranational, qui représente en moyenne $48 \%$ des dépenses liées aux marchés publics, avec des pourcentages particulièrement élevés en Italie $(80 \%)$, en Finlande (72\%), au Danemark (69\%), au Japon (69\%) et en Suède $(69 \%)$.

\section{Méthodologie et définitions}

Le montant des dépenses liées aux marchés publics a été estimé à partir de données tirées de la base de données des Statistiques de l'OCDE sur les comptes nationaux, qui sont établies conformément au Système de comptabilité nationale (SCN). Les marchés publics sont définis comme la somme de la consommation intermédiaire (biens et services achetés par les administrations publiques pour leur propre usage, comme des prestations comptables ou informatiques), de la formation brute de capital fixe (acquisition d'équipements moins ventes d'actifs fixes, par ex. construction de nouvelles routes) et des transferts sociaux en nature opérés par l'intermédiaire de producteurs marchands (achat par les administrations publiques de biens et services produits par des producteurs marchands et fournis aux ménages). Le graphique 7.3, " Marchés publics en pourcentage du PIB (2011) ", est consultable en ligne à l'adresse http:// dx.doi.org/10.1787/888932942773.

Les marchés publics englobent ici ceux de l'administration centrale, des administrations d'États fédérés et des administrations locales. L'échelon infranational correspond aux administrations d'États fédérés et aux administrations locales. Sauf indication contraire dans les notes, les administrations de sécurité sociale sont exclues de l'analyse (toutefois, le graphique 7.4, "Ventilation des marchés publics par niveau d'administration, administrations de sécurité sociale comprises ", est consultable en ligne à l'adresse http://dx.doi.org/10.1787/888932942792). Les administrations classées dans la catégorie des administrations d'États fédérés n'existent que dans les neuf pays membres de l'OCDE dotés d'une structure fédérale : l'Allemagne, l'Australie, l'Autriche, la Belgique, le Canada, l'Espagne (pays considéré comme quasi fédéral), les États-Unis, le Mexique et la Suisse. Les entreprises publiques ont elles aussi été exclues de l'estimation des dépenses liées aux marchés publics.

\section{Pour en savoir plus}

OCDE (2013, à paraître), Principles for Integrity in Public Procurement : Progress in OECD Countries, Éditions OCDE, Paris.

OCDE (2011), Panorama des comptes nationaux 2011, Éditions OCDE. Paris, http://dx.doi.org/10.1787/na_glance-2011-fr.

\section{Notes relatives aux graphiques}

On ne dispose pas de données pour le Chili. Les données relatives au Canada et à la Nouvelle-Zélande portent sur 2010 et non 2011.

7.2: On ne dispose pas de données pour l'Australie. Aux États-Unis, en Norvège, en Nouvelle-Zélande et au Royaume-Uni, les administrations de sécurité sociale sont comptabilisées dans l'administration centrale. Les données concernant les administrations infranationales au Japon se rapportent aux exercices budgétaires.

Informations sur les données concernant Israël : http://dx.doi.org/ 10.1787/888932315602. 
7.1. Part des marchés publics dans les dépenses totales des administrations publiques (2011)

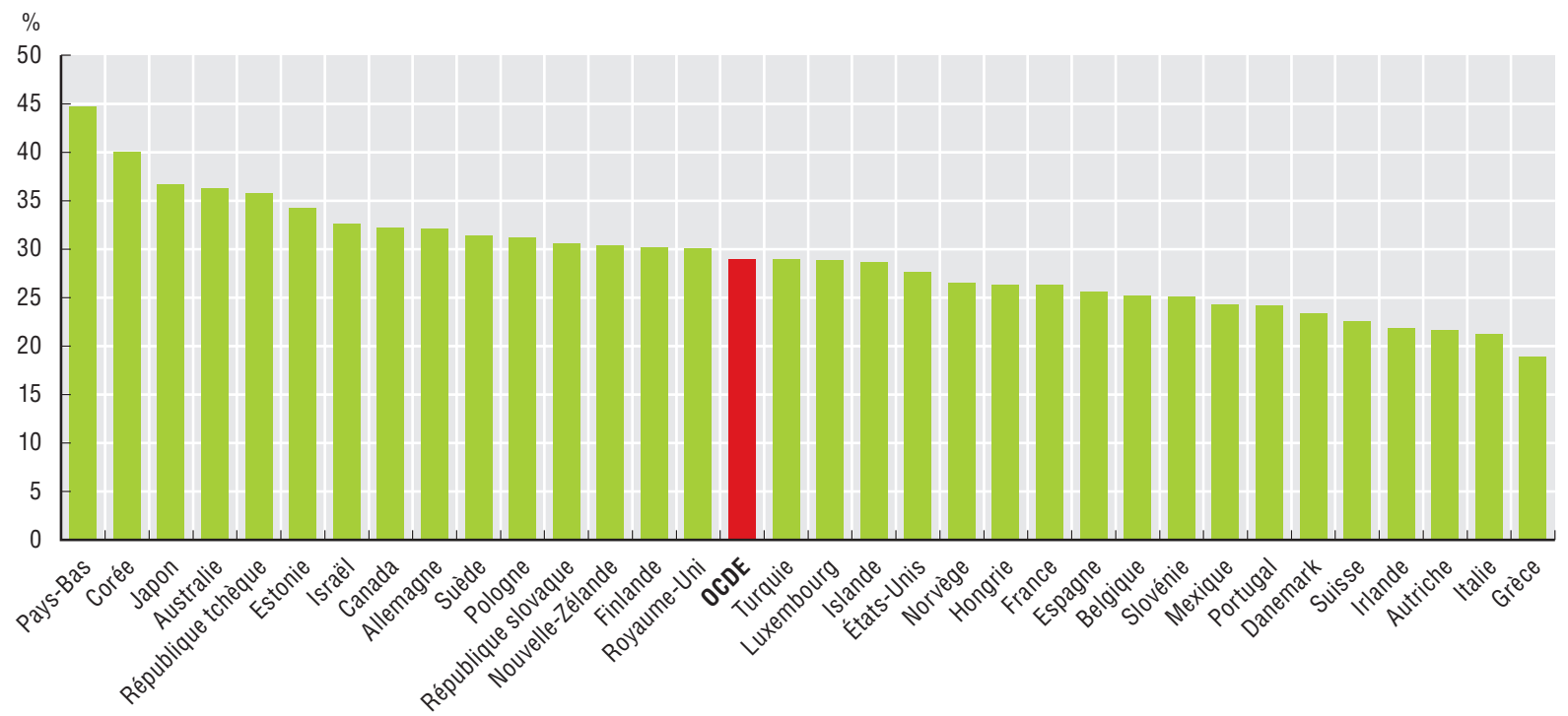

Source: Statistiques de l'OCDE sur les comptes nationaux (base de données). Les données relatives à l'Australie sont tirées des statistiques sur les finances publiques et sur les comptes nationaux fournies par le Bureau australien des statistiques.

\subsection{Ventilation des marchés publics par niveau d'administration, hors administrations de sécurité sociale (2011)}

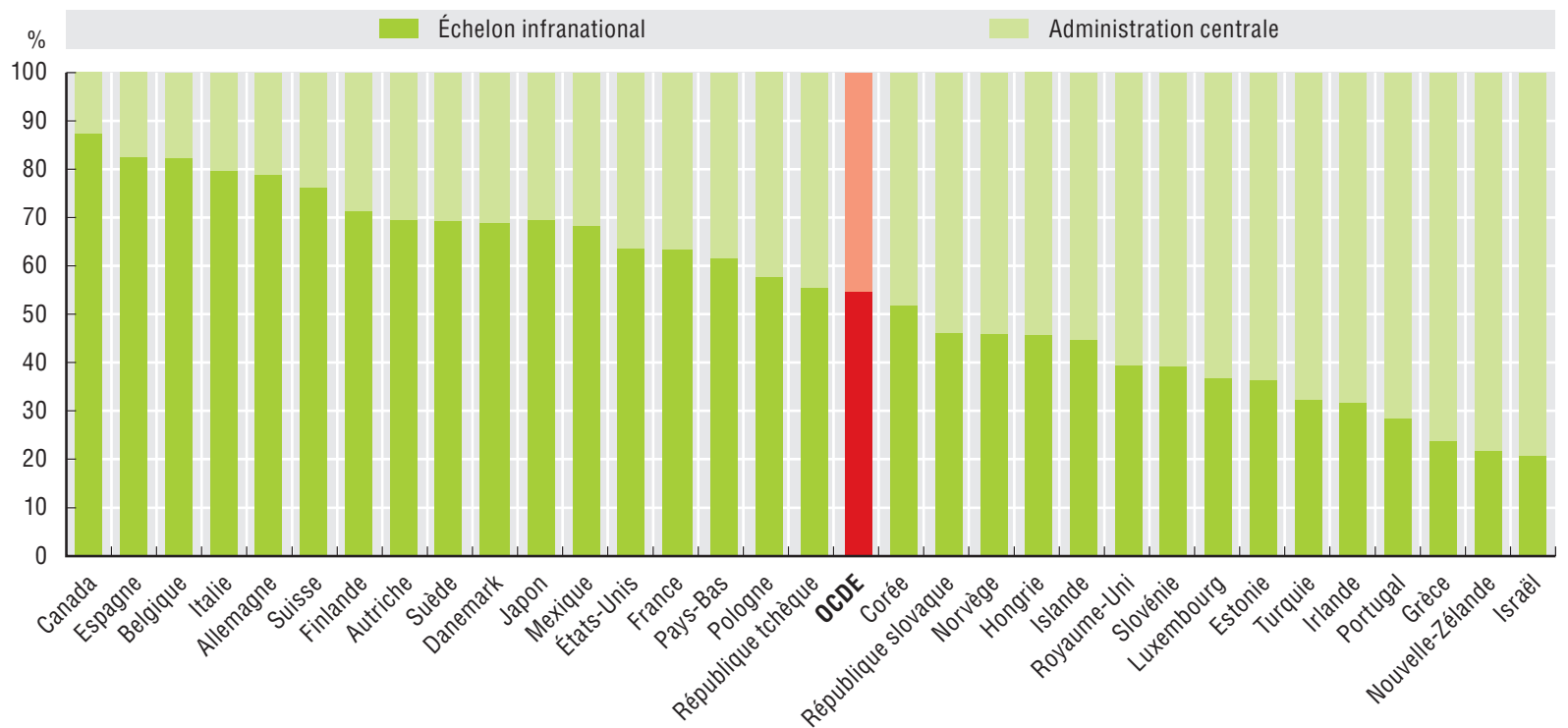

Source: Statistiques de l'OCDE sur les comptes nationaux (base de données). 
Mus par la nécessité d'améliorer la productivité en période d'austérité, de nombreux pays membres de l'OCDE investissent dans des outils novateurs visant à rationaliser les procédures de passation des marchés publics et à optimiser les dépenses correspondantes. Ils ont ainsi de plus en plus recours à des plateformes électroniques de passation des marchés, à des accords-cadre, à des systèmes de présélection, à des enchères électroniques inversées et à des contrats assortis d'options.

La passation électronique de marchés publics - c'est-à-dire le recours aux technologies de l'information et de la communication dans la passation des marchés publics - peut faciliter l'accès aux marchés publics et renforcer la concurrence. Les outils liés à ces marchés publics en ligne peuvent aussi permettre aux administrations de comprimer leurs coûts en réduisant les charges administratives, en raccourcissant le cycle en matière de passation des marchés et en assurant un meilleur respect des règles. De nombreuses administrations centrales ont investi dans des systèmes de passation électronique de marchés publics, mais les pouvoirs publics ne tirent pas encore pleinement profit des avantages potentiels de ces outils. Ainsi, dans les pays de l'OCDE, ces systèmes continuent d'être utilisés principalement comme des plateformes de publication d'informations, et non comme des outils de dialogue avec les fournisseurs. Si presque tous les pays de l'OCDE $(97 \%)$ publient leurs appels d'offres sur un portail national, $48 \%$ seulement offrent aux fournisseurs potentiels la possibilité de présenter leurs soumissions par voie électronique. Le Service coréen de la passation des marchés publics a fait exception à la règle en lançant en 2011 un nouveau service permettant aux fournisseurs de présenter leurs soumissions par téléphone portable, grâce à des jetons de sécurité et des applications de création récente.

Des pays mettent également en œuvre de nouvelles procédures de passation des marchés publics dans une optique de réduction des coûts. Afin de réaliser des économies d'échelle, presque tous les pays membres de l'OCDE (94\%) recourent à des accords-cadres. Cependant, ils ne sont qu'un tiers environ à calculer les économies résultant de l'utilisation de ces mécanismes, pour vérifier si des économies d'échelle ont été réalisées. Par ailleurs, moins de la moitié des pays membres de l'OCDE interrogés (42 \%) recourent régulièrement aux enchères électroniques inversées. L'utilisation de cet outil exige de prendre en compte à la fois les conditions requises pour que l'opération réussisse et les risques d'impact négatif. Cette opération peut permettre de réaliser des économies si la concurrence s'intensifie, mais elle peut aussi placer en position défavorable les petites et moyennes entreprises, qui ont souvent des volumes de production et des marges bénéficiaires moins élevés.

\section{Méthodologie et définitions}

Les données ont été rassemblées dans le cadre de l'Enquête 2011 de l'OCDE sur la mise en œuvre de la Recommandation de 2008 sur les marchés publics (à laquelle 29 pays membres ont répondu) et de l'Enquête 2012 de l'OCDE sur les marchés publics (à laquelle 33 pays'membres ont répondu). Pour ces deux enquêtes, les réponses ont été fournies par des délégués nationaux chargés des politiques de passation des marchés publics au sein de l'administration centrale.
Dans le graphique 7.6, les soumissions électroniques font référence à la pratique consistant, pour les entreprises, à passer par Internet pour proposer des biens ou des services dans le cadre des systèmes de passation électronique de marchés publics de leur pays.

Un contrat assorti d'options est un contrat offrant à l'entité adjudicatrice la possibilité de demander un nombre prédéterminé de biens ou de services supplémentaires ou de prolonger la durée de validité du marché pour une période prédéterminée, selon des modalités précisées dans le contrat.Une enchère électronique inversée est une technique d'achat en ligne et en temps réel que l'entité adjudicatrice utilise pour sélectionner la soumission à retenir et dans laquelle les fournisseurs ou entrepreneurs présentent au cours d'une période déterminée des offres de plus en plus basses faisant l'objet d'une évaluation automatique.

La nature des accords-cadres varie selon les pays, mais généralement il s'agit d'accords entre l'entité adjudicatrice et les fournisseurs qui établissent certains termes et peuvent faciliter l'attribution des contrats futurs. La conclusion d'accords-cadre se déroule en deux étapes, la première étant la sélection du ou des fournisseurs ou entrepreneurs qui seront parties à un accord-cadre avec une entité adjudicatrice, la deuxième étant l'attribution d'un marché au titre de l'accord-cadre à un fournisseur ou entrepreneur partie à l'accord.

Un système de présélection vise à identifier en amont les fournisseurs ou entrepreneurs qui ont les qualifications requises pour exécuter le marché.

\section{Pour en savoir plus}

Commission européenne (2011), Evaluation Report: Impact and Effectiveness of EU Public Procurement Legislation Part 1, Document de travail des services de la Commission, SEC(2011)853 Final, Bruxelles.

Commission européenne (2010), Livre vert sur le développement des marchés publics électroniques dans l'Union européenne, $\operatorname{COM}(2010) 571$ Final, Bruxelles.

OCDE (2013, à paraître), Principles for Integrity in Public Procurement: Progress in OECD Countries, Éditions OCDE, Paris.

\section{Notes relatives aux graphiques}

7.5 : On ne dispose pas de données pour la Grèce. Tous les pays de l'Union européenne doivent publier le planning de leurs achats sur le site de l'Union européenne consacré aux marchés publics (www.ted.europa/eu). Pour le Japon, la réponse tient compte des fonctionnalités d'un système de passation électronique de marchés publics qui devrait être opérationnel en 2013.

7.6 : Les entreprises emploient toutes au moins 10 personnes (sauf pour le secteur financier). On ne dispose pas de données pour l'Australie, le Canada, le Chili, la Corée, les États-Unis, Israël, le Japon, le Mexique, la Nouvelle-Zélande, la Suisse et la Turquie. Les données relatives à la Turquie portent sur 2011 et non 2012.

Informations sur les données concernant Israël : http://dx.doi.org/ 10.1787/888932315602. 
7.5. Recours à des instruments novateurs dans la passation de marchés publics au niveau de l'administration centrale (2012)

\begin{tabular}{|c|c|c|c|c|c|c|c|c|}
\hline & \multicolumn{4}{|c|}{$\begin{array}{c}\text { Fonctionnalités des systèmes } \\
\text { de passation électronique de } \\
\text { marchés publics }\end{array}$} & \multicolumn{4}{|c|}{$\begin{array}{l}\text { Autres instruments et } \\
\text { mécanismes de passation } \\
\text { des marchés publics }\end{array}$} \\
\hline & 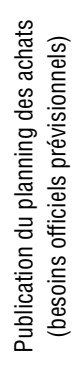 & 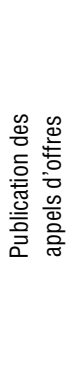 & 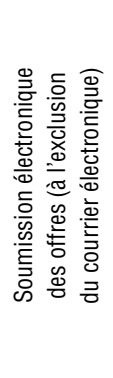 & 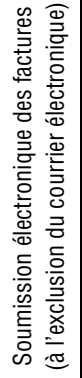 & 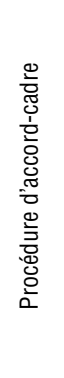 & 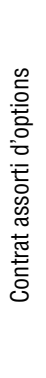 & 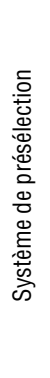 & 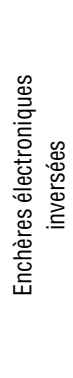 \\
\hline Allemagne & O & O & $\mathbf{\square}$ & O & $\square$ & $\square$ & $\hookrightarrow$ & $\diamond$ \\
\hline Australie & $\bullet$ & $\bullet$ & $\bullet$ & $\mathbf{\square}$ & $\hookrightarrow$ & 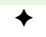 & $\hookrightarrow$ & 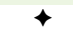 \\
\hline Autriche & 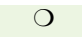 & $\bullet$ & O & $O$ & $\square$ & $\square$ & $\hookrightarrow$ & $\diamond$ \\
\hline Belgique & - & - & $\bullet$ & $O$ & $\diamond$ & $\diamond$ & $\diamond$ & $\diamond$ \\
\hline Canada & O & $\bullet$ & $\mathbf{\square}$ & O & $\square$ & $\square$ & $\square$ & $\diamond$ \\
\hline Chili & $\bullet$ & $\bullet$ & $\bullet$ & O & $\square$ & $\diamond$ & $\hookrightarrow$ & $\diamond$ \\
\hline Corée & - & $\bullet$ & $\bullet$ & $\bullet$ & $\hookrightarrow$ & $\hookrightarrow$ & $\square$ & $\diamond$ \\
\hline Danemark & 口 & $\bullet$ & - & - & $\square$ & $\square$ & $\square$ & $\hookrightarrow$ \\
\hline Espagne & $\bullet$ & $\bullet$ & $\mathbf{\square}$ & $\mathbf{\square}$ & $\square$ & $\hookrightarrow$ & $\downarrow$ & $\diamond$ \\
\hline Estonie & O & ○ & ○ & O & $\square$ & $\diamond$ & $\diamond$ & $\diamond$ \\
\hline États-Unis & 0 & $\bullet$ & $\mathbf{\square}$ & $\mathbf{\square}$ & $\square$ & $\square$ & $\square$ & $\hookrightarrow$ \\
\hline Finlande & $O$ & - & $\mathbf{\square}$ & - & $\hookrightarrow$ & $\hookrightarrow$ & $\hookrightarrow$ & $\hookrightarrow$ \\
\hline France & 0 & 무 & 0 & $\mathbf{\square}$ & $\hookrightarrow$ & $\hookrightarrow$ & $\diamond$ & $\diamond$ \\
\hline Hongrie & $\mathbf{\square}$ & $\bullet$ & $\mathbf{\square}$ & $\mathbf{\square}$ & $\hookrightarrow$ & $\hookrightarrow$ & $\downarrow$ & $\hookrightarrow$ \\
\hline Irlande & 0 & $\bullet$ & $\bullet$ & $O$ & $\square$ & $\diamond$ & $\diamond$ & $\diamond$ \\
\hline Islande & $\mathbf{\square}$ & $\mathbf{\square}$ & O & $\bullet$ & $\square$ & $\hookrightarrow$ & $\hookrightarrow$ & $\diamond$ \\
\hline Israël & 0 & 0 & 口 & 口 & $\square$ & $\square$ & $\hookrightarrow$ & $\square$ \\
\hline Italie & $\bullet$ & $\bullet$ & $\bullet$ & O & $\square$ & $\square$ & $\hookrightarrow$ & $\diamond$ \\
\hline Japon & $\bullet$ & $\bullet$ & $\mathbf{\square}$ & $\bigcirc$ & $\diamond$ & $\hookrightarrow$ & $\square$ & $\hookrightarrow$ \\
\hline Luxembourg & $O$ & $\bullet$ & $\bullet$ & $O$ & $\hookrightarrow$ & $\diamond$ & $\diamond$ & $\diamond$ \\
\hline Mexique & - & - & $\bullet$ & $O$ & $\square$ & $\diamond$ & $\diamond$ & - \\
\hline Norvège & $\bullet$ & $\bullet$ & $\mathbf{\square}$ & $\bullet$ & $\square$ & $\square$ & $\hookrightarrow$ & $\diamond$ \\
\hline Nouvelle-Zélande & $\bullet$ & $\bullet$ & O & $\bullet$ & $\hookrightarrow$ & $\hookrightarrow$ & $\hookrightarrow$ & $\diamond$ \\
\hline Pays-Bas & $\mathbf{\square}$ & 무 & 0 & $\mathbf{\square}$ & $\square$ & $\square$ & $\diamond$ & $\diamond$ \\
\hline Pologne & O & $\bullet$ & O & O & $\hookrightarrow$ & $\hookrightarrow$ & $\diamond$ & $\hookrightarrow$ \\
\hline Portugal & $\bullet$ & $\bullet$ & $\bullet$ & O & $\square$ & $\diamond$ & $\square$ & $\hookrightarrow$ \\
\hline $\begin{array}{l}\text { République } \\
\text { slovaque }\end{array}$ & $\bigcirc$ & $\bullet$ & $\bullet$ & $\bigcirc$ & $\square$ & $\hookrightarrow$ & $\hookrightarrow$ & $\square$ \\
\hline République tchèque & - & - & ○ & $\square$ & $\square$ & $\hookrightarrow$ & $\square$ & $\hookrightarrow$ \\
\hline Royaume-Uni & $\bullet$ & $\bullet$ & $\mathbf{\square}$ & $\mathbf{\square}$ & $\square$ & $\hookrightarrow$ & $\hookrightarrow$ & $\hookrightarrow$ \\
\hline Slovénie & - & - & $\mathbf{\square}$ & $\mathbf{\square}$ & $\square$ & $\diamond$ & $\square$ & $\hookrightarrow$ \\
\hline Suède & - & $\bullet$ & - & $\bullet$ & $\hookrightarrow$ & $\hookrightarrow$ & $\hookrightarrow$ & $\diamond$ \\
\hline Suisse & - & 0 & $O$ & - & $\square$ & $\square$ & $\square$ & $\diamond$ \\
\hline Turquie & 0 & $\bullet$ & O & O & $\square$ & $\diamond$ & $\square$ & $\diamond$ \\
\hline \multicolumn{9}{|l|}{ Total OCDE } \\
\hline & - 20 & 32 & 16 & 8 & $\square 22$ & 10 & 10 & 3 \\
\hline & ㅁ 8 & 7 & 13 & 10 & $\downarrow 9$ & 14 & 15 & 11 \\
\hline & ○ 10 & 0 & 6 & 15 & $\diamond 2$ & 9 & 8 & 19 \\
\hline
\end{tabular}

- Oui, dans un système national central de passation électronique de marchés publics

- Oui, dans le système de passation électronique de marchés publics de certaines entités adjudicatrices

O Non

$\square$ L'instrument est régulièrement utilisé par tous les pouvoirs adjudicateurs

\ L'instrument est régulièrement utilisé par certaines autorités adjudicatrices

$\diamond$ L'instrument n'est pas utilisé régulièrement

Source: Enquête 2012 de l'OCDE sur les marchés publics.

StatLink Ailst http://dx.doi.org/10.1787/888933159779

\subsection{Pourcentage d'entreprises utilisant des systèmes} de passation électronique de marchés publics (2012)

Pour accéder au dossier d'appel d'offres et au cahier des charges

$\diamond$ Pour présenter des soumissions électroniques dans leur pays

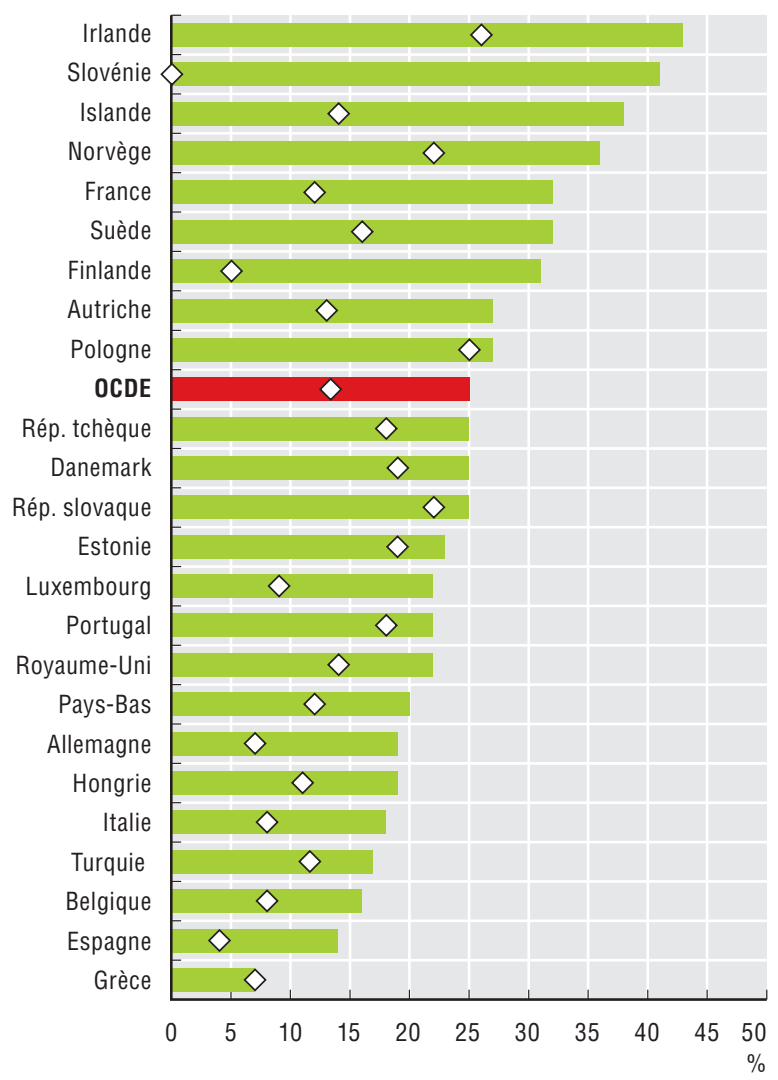

Source : Eurostat.

StatLink AाISL http://dx.doi.org/10.1787/888933159785 
Les pays membres de l'OCDE ne voient plus l'optimisation de la dépense publique, au sens strict du rapport qualité/prix, comme le seul objectif des marchés publics. Ils intègrent peu à peu des objectifs plus stratégiques tels que le soutien aux petites et moyennes entreprises (PME), l'innovation et les considérations environnementales.

La hiérarchisation des objectifs représente un nouveau défi pour les pouvoirs publics. Près de $70 \%$ des pays membres de l'OCDE (23 pays sur 33) ont élaboré au niveau de l'administration centrale une stratégie ou une politique visant à faire des marchés publics un instrument de soutien aux PME. La moitié des pays concernés se sont dotés de règles obligatoires sur le soutien aux PME dans le cadre des marchés publics, et le tiers d'entre eux de règles non obligatoires, mais avec des objectifs librement consentis. De plus, $76 \%$ des pays de l'OCDE ont élaboré au niveau de l'administration centrale une stratégie ou une politique visant à favoriser des marchés publics respectueux de l'environnement, dits "écologiques " ou « verts".

Cela étant, la plupart des pays membres de l'OCDE n'évaluent pas systématiquement le coût d'opportunité de la poursuite d'objectifs socio-économiques et environnementaux, si bien que les gouvernements manquent parfois des outils nécessaires pour établir des priorités entre des objectifs concurrents (l'optimisation de la dépense publique par opposition au soutien à des objectifs socio-économiques et environnementaux, par exemple). Les pouvoirs publics n'optimisent donc peut-être pas toujours l'utilisation de leurs ressources dans les marchés publics. De plus, alors que les pays membres de l'OCDE ont, dans leur vaste majorité, mis en place une stratégie en faveur des PME, ils sont $61 \%$ à ne pas tenir de statistiques du nombre et du montant des marchés attribués à des PME. Or, sans ces informations, il est très difficile de mesurer l'efficacité de cette stratégie.

Dans le contexte actuel, les acheteurs publics sont censés se conformer à des règles de plus en plus complexes et poursuivre des objectifs d'optimisation de la dépense publique tout en tenant compte de considérations stratégiques. Or, selon près de la moitié des pays de l'OCDE interrogés, la principale faiblesse des systèmes de passation des marchés publics tient à l'insuffisance des capacités, avec un manque d'acheteurs publics mais aussi un déficit de connaissances spécialisées sur les technologies disponibles, les innovations et les évolutions du marché.

Dans de nombreux pays, la passation des marchés publics reste gérée comme une fonction administrative, plus d'un tiers des pays (39\%) indiquant que la fonction d'acheteur public n'est même pas reconnue comme une profession spécifique. Sur les 18 pays membres de l'OCDE où la profession d'acheteur public est reconnue en tant que telle, 11 disposent de descriptions de poste formelles pour les acheteurs publics et 8 ont mis en place des programmes de certification ou de licence professionnelle (Australie, Canada, Chili, États-Unis, Irlande, Nouvelle-Zélande, République slovaque et Suisse). Cependant, seuls cinq pays ont adopté des lignes directrices en matière d'intégrité (codes de conduite, par exemple) spécifiquement applicables aux acheteurs publics.

\section{Méthodologie et définitions}

Les données ont été rassemblées dans le cadre de l'Enquête 2011 de l'OCDE sur la mise en œuvre de la Recommandation de 2008 sur les marchés publics (à laquelle 29 pays membres ont répondu) et de l'Enquête 2012 de l'OCDE sur les marchés publics (à laquelle 33 pays membres ont répondu). Pour ces deux enquêtes, les réponses ont été fournies par des délégués nationaux chargés des politiques de passation des marchés publics au sein de l'administration centrale.

On considère que la fonction d'acheteur public est reconnue comme une profession spécifique si elle fait l'objet d'un programme de certification ou de licence professionnelle, d'un cadrage précis (par exemple description de poste/rôle formels) et/ou des lignes directrices en matière d'intégrité (par exemple codes de conduite spécifiques aux acheteurs publics).

La Commission européenne définit les marchés publics verts, ou écologiques, comme « un processus de passation de marchés dans le cadre duquel les pouvoirs publics cherchent à obtenir des biens, des services et des travaux dont l'incidence sur l'environnement sur toute leur durée de vie sera moindre que dans le cas de biens, services et travaux à vocation identique mais ayant fait l'objet de procédures de passation de marchés différentes. "

\section{Pour en savoir plus}

OCDE (2013, à paraître), Principles for Integrity in Public Procurement: Progress in OECD Countries, Éditions OCDE, Paris.

OCDE (2011), Making the Most of Public Investment in a Tight Fiscal Environment: Multi-level Governance Lessons from the Crisis, Éditions OCDE, Paris, http://dx.doi.org/10.1787/978926 4114470-en.

OCDE (2007), «Improving the Environmental Performance of Public Procurement: Report on Implementation of the Council Recommendation ", Éditions OCDE, Paris, vol. 7/9, http://dx.doi.org/10.1787/oecd_papers-v7-art26-en.

\section{Notes relatives aux graphiques et aux tableaux}

7.7 : On ne dispose pas de données pour la Corée, le Danemark et la Grèce.

7.8 : On ne dispose pas de données pour la Grèce. L'Australie a adopté une politique en faveur d'une plus grande parité hommes/femmes au sein des entreprises répondant aux appels d'offres publics. En Allemagne, les réponses rendent compte de la situation au niveau de l'administration fédérale. Cela étant, il est important de souligner que les Länder allemands ont adopté une multitude de mesures visant à promouvoir les marchés publics verts et à soutenir les PME. Le Danemark lancera en 2013 une stratégie visant à relever des défis stratégiques tels que l'innovation, le respect de l'environnement et le soutien aux PME. Les données pour la Turquie ont été fournies par l'Institut de statistique turc.

Informations sur les données concernant Israël : http://dx.doi.org/ $10.1787 / 888932315602$. 
7.7. Reconnaissance de la fonction d'acheteur public comme une profession spécifique (2010)

Non : $\mathbf{3 9} \%$

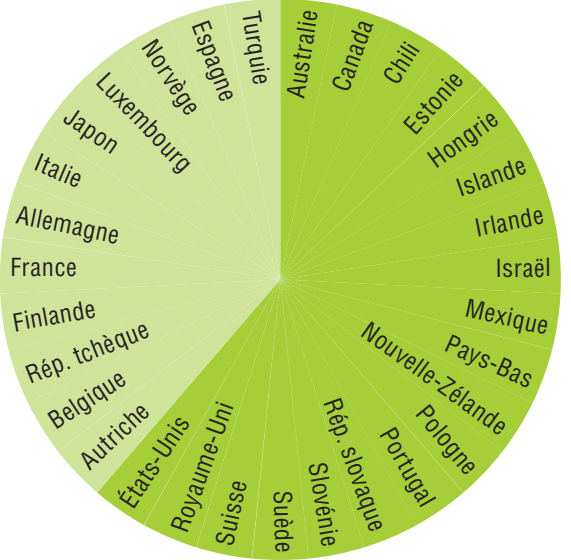

Oui : $61 \%$

Source: Enquête 2011 de l'OCDE sur la mise en œuvre de la Recommandation de 2008 sur les marchés publics.

StatLink काजा http://dx.doi.org/10.1787/888933159799
7.8. Politiques et stratégies mettant les marchés publics au service d'objectifs socio-économiques et/ou environnementaux (2011)

\begin{tabular}{|c|c|c|c|c|c|c|}
\hline & \multicolumn{3}{|c|}{$\begin{array}{l}\text { Politiques ou stratégies en } \\
\text { place pour mettre les marchés } \\
\text { publics au service d'objectifs } \\
\text { socio-économiques et/ou } \\
\text { environnementaux }\end{array}$} & \multicolumn{3}{|c|}{$\begin{array}{l}\text { Évaluation des résultats } \\
\text { des politiques ou stratégies } \\
\text { mettant les marchés publics } \\
\text { au service d'objectifs } \\
\text { socio-économiques et/ou } \\
\text { environnementaux }\end{array}$} \\
\hline & 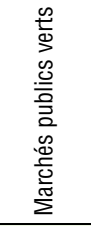 & 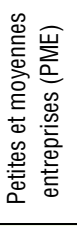 & 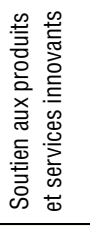 & 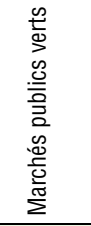 & 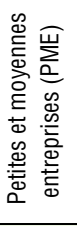 & 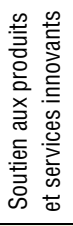 \\
\hline Allemagne & - & - & - & $\diamond$ & $\diamond$ & $\diamond$ \\
\hline Australie & $\bullet$ & $\bullet$ & $\bullet$ & $\diamond$ & $\diamond$ & $\diamond$ \\
\hline Autriche & 0 & - & $\bullet$ & $*$ & $\mathbf{\square}$ & $\diamond$ \\
\hline Belgique & $\bullet$ & O & O & $*$ & $X$ & $X$ \\
\hline Canada & 0 & - & $\bullet$ & $\mathbf{\square}$ & 口 & $\mathbf{\square}$ \\
\hline Chili & $\square$ & 0 & $\square$ & $X$ & $\mathbf{\square}$ & $X$ \\
\hline Corée & $\bullet$ & $\bullet$ & $\bullet$ & 口 & 口 & 口 \\
\hline Danemark & 0 & $\bullet$ & O & $\diamond$ & $\diamond$ & $x$ \\
\hline Espagne & 0 & - & • & $\diamond$ & $\diamond$ & $\diamond$ \\
\hline Estonie & $O$ & O & O & $x$ & $X$ & $X$ \\
\hline États-Unis & 0 & - & - & $\mathbf{\square}$ & $\mathbf{\square}$ & $\mathbf{\square}$ \\
\hline Finlande & $\bullet$ & $\square$ & 0 & 口 & $X$ & $*$ \\
\hline France & $\square$ & $\bullet$ & • & 口 & 口 & $\diamond$ \\
\hline Hongrie & $\square$ & - & $O$ & $X$ & 口 & $X$ \\
\hline Irlande & 0 & - & 0 & $\downarrow$ & $\hookrightarrow$ & $\hookrightarrow$ \\
\hline Islande & • & $O$ & O & $\diamond$ & $x$ & $x$ \\
\hline Israël & 0 & - & $O$ & 口 & 口 & $*$ \\
\hline Italie & 0 & 0 & $\bullet$ & $\mathbf{\square}$ & $\mathbf{\square}$ & $\mathbf{\square}$ \\
\hline Japon & $\bullet$ & 0 & $O$ & $\mathbf{\square}$ & $\mathbf{\square}$ & $x$ \\
\hline Luxembourg & $\square$ & $\square$ & $O$ & $X$ & $X$ & $X$ \\
\hline Mexique & - & - & $\mathrm{O}$ & $\diamond$ & $\mathbf{\square}$ & $x$ \\
\hline Norvège & $\bullet$ & - & • & $\mathbf{\square}$ & $\diamond$ & $\diamond$ \\
\hline Nouvelle-Zélande & $\bigcirc$ & O & O & $X$ & $X$ & $X$ \\
\hline Pays-Bas & - & $\bullet$ & $\bullet$ & $\mathbf{\square}$ & $\mathbf{\square}$ & $*$ \\
\hline Pologne & - & - & $\bullet$ & $\mathbf{\square}$ & $\mathbf{\square}$ & $\mathbf{\square}$ \\
\hline Portugal & - & - & $\neg$ & $\mathbf{\square}$ & $*$ & $X$ \\
\hline République slovaque & $\square$ & O & $\mathrm{O}$ & $x$ & $x$ & $X$ \\
\hline République tchèque & - & $\square$ & $\square$ & $\downarrow$ & $X$ & $X$ \\
\hline Royaume-Uni & $\square$ & • & ・ロ & $\mathbf{\square}$ & $\mathbf{\square}$ & $X$ \\
\hline Slovénie & $\bullet$ & •ロ & $O$ & $\mathbf{\square}$ & $\mathbf{\square}$ & $X$ \\
\hline Suède & $\square$ & $\square$ & $\square$ & $X$ & $X$ & $X$ \\
\hline Suisse & $\bullet$ & ○ & - & $\mathbf{\square}$ & $\hookrightarrow$ & $\mathbf{\square}$ \\
\hline Turquie & O & O & O & $X$ & $x$ & $X$ \\
\hline \multirow[t]{5}{*}{ Total OCDE } & - 25 & 23 & 16 & घ 15 & 15 & 6 \\
\hline & $\square \quad 10$ & 7 & 6 & $* \quad 2$ & 1 & 3 \\
\hline & ○ 3 & 6 & 13 & $\downarrow 2$ & 2 & 2 \\
\hline & & & & $\diamond \quad 6$ & 5 & 5 \\
\hline & & & & X 8 & 10 & 17 \\
\hline
\end{tabular}

- Oui, une stratégie/politique a été mise au point au niveau central

$\diamond$ Oui, régulièrement

$\square$ Oui, certaines entités adjudicatrices ont mis au point une politique interne

* Oui, ponctuellement

Non, aucune stratégie/politique n'a été mise en place

$\downarrow$ Ne sait pas

- Non

$\mathrm{X}$ Sans objet

Source : Enquête 2012 de l'OCDE sur les marchés publics.

StatLink ताIS http://dx.doi.org/10.1787/888933159804 
Il reste très difficile d'assurer des règles du jeu équitables aux fournisseurs potentiels en matière d'accès aux marchés publics, surtout à l'échelon international. Les marchés publics internationaux dans un espace intégré tel que celui de l'Union européenne représentent moins de $4 \%$ de la valeur totale des marchés attribués.

Au plan national, le recours à des exceptions aux appels d'offres concurrentiels limite la concurrence. Dans le cadre des mesures de relance qui ont suivi la crise financière, le recours à ces exceptions s'est accru de $18 \%$ entre 2008 et 2011 dans les pays membres de l'OCDE, principalement en raison de l'utilisation de procédures accélérées. Pour garantir une concurrence maximale tout en assurant l'efficience du processus de passation des marchés publics, il est essentiel que les pays limitent strictement les exceptions à la mise en concurrence à un nombre restreint de circonstances, car cette pratique peut donner lieu à des abus nuisant à l'efficience administrative de la passation des marchés.

Alors que les PME représentent une part importante de l'économie et du marché du travail à l'échelle mondiale, leur part dans les marchés publics est loin d'être aussi significative. Afin de favoriser des règles du jeu équitables, $85 \%$ des pays membres de l'OCDE ont adopté des mesures visant directement les PME, qui souffrent d'un désavantage comparatif face aux appels d'offres. Les mesures les plus courantes sont l'organisation d'activités de formation et d'ateliers pour les PME (58\% des pays de l'OCDE) et la mise en ligne d'une documentation ou d'orientations ciblant les PME (51\%). Moins d'un tiers des pays membres de l'OCDE (30\%) ont simplifié leurs procédures administratives pour faciliter la participation des PME aux appels d'offres.

Un tiers des pays membres de l'OCDE (33\%) ont mis en place des dispositions législatives ou des politiques spécifiques (marchés réservés, par exemple) pour inciter les PME à répondre aux appels d'offres publics. Ce traitement préférentiel existe, par exemple, en Australie, en Corée, aux États-Unis et en France. En dehors de ces mesures réglementaires, les PME bénéficient d'un traitement financier préférentiel (sous forme de dispense de participation financière, par exemple) dans $6 \%$ seulement des pays membres de l'OCDE.

\section{Méthodologie et définitions}

Les données ont été rassemblées dans le cadre de deux enquêtes portant sur la passation des marchés publics au niveau de l'administration centrale. Le Brésil, l'Égypte, la Fédération de Russie, le Maroc et 29 pays membres de l'OCDE ont répondu à l'Enquête 2011 de l'OCDE sur la mise en œuvre de la Recommandation de 2008 sur les marchés publics. On ne dispose pas de données pour la Corée, le Danemark, l'Espagne et la Grèce. Le Brésil, la Colombie et 33 pays'membres de l'OCDE ont répondu à l'Enquête' 2012 de l'OCDE sur les marchés publics. On ne dispose pas de données pour la Grèce. Pour ces deux enquêtes, les réponses ont été fournies par des délégués nationaux chargés des politiques de passation des marchés publics au sein de l'administration centrale. Le tableau 7.11, «Passation de marchés publics au niveau de l'administration centrale, par procédure : disponibilité des données sur le nombre et le montant des marchés ", est consultable en ligne à l'adresse $h t t p: / / d x$.doi.org/ 10.1787/888932943571.

\section{Pour en savoir plus}

Commission européenne (2010) L'évaluation de l'incidence et de l'efficacité de la législation de l'Union européenne en matière de marchés publics, Office "Infrastructures et logistique ", Bruxelles.

OCDE (à paraître), OECD Review of the United States Federal Public Procurement, Éditions OCDE, Paris.

OCDE (2013, à paraître), Principles for Integrity in Public Procurement: Progress in OECD Countries, Éditions OCDE, Paris.

OCDE (2009), Principes de l'OCDE pour renforcer l'intégrité dans les marchés publics, Éditions OCDE, Paris, http://dx.doi.org/ 10.1787/9789264056527-fr.

OCDE (2008), OECD Framework for the Evaluation of SME and Entrepreneurship Policies and Programmes, Éditions OCDE, Paris, http://dx.doi.org/10.1787/9789264040090-en.

\section{Notes relatives aux graphiques et aux tableaux}

7.9 : Au Danemark, l'Autorité de la concurrence et de la protection des consommateurs a publié un guide présentant, étape par étape, des informations sur les règles, les procédures et les questions essentielles concernant l'établissement de consortiums de PME. En Espagne, l'organisme central chargé de l'évaluation des marchés publics (le Conseil consultatif des marchés publics) est en contact avec les PME et les associations de PME afin d'être à l'écoute de leurs exigences en la matière. En Estonie, il n'existe pas de dispositifs spécifiques pour aider les PME, puisque la majorité des entreprises estoniennes sont rangées dans cette catégorie. En Finlande, le service central des marchés publics planifie les appels d'offres de manière à encourager les PME à y répondre. En Nouvelle-Zélande, la majorité des entreprises sont rangées dans la catégorie des PME. Bien qu'il n'existe pas de politique spécifique en faveur des PME, un soutien leur est apporté sous forme de mesures visant à réduire les coûts pour les fournisseurs (grâce notamment à une simplification des procédures, à l'élaboration de guides et de modèles en ligne et à l'organisation d'activités de formation et d'ateliers, tant pour les fournisseurs que pour les professionnels des marchés publics). En République tchèque, les autorités adjudicatrices doivent obligatoirement fixer des conditions non discriminatoires de participation aux appels d'offres. Au Royaume-Uni, il existe un programme de travail visant à passer plus de marchés avec des PME lorsque c'est avantageux pour le contribuable. On peut citer comme exemple de documentation destinée aux PME le document « Winning the Contract " proposé sur le site Web LearnDirect. Le processus de passation des marchés publics a également été simplifié : par exemple, les services officiels ont supprimé les questionnaires de présélection pour la plupart des marchés inférieurs au seuil européen d'environ 100000 livres sterling.

7.10 : On ne dispose pas de données pour la Belgique et la Grèce.

Informations sur les données concernant Israël : http://dx.doi.org/ $10.1787 / 888932315602$. 
7.9. Dispositifs en place pour favoriser un accès équitable des PME aux marchés publics au niveau de l'administration centrale
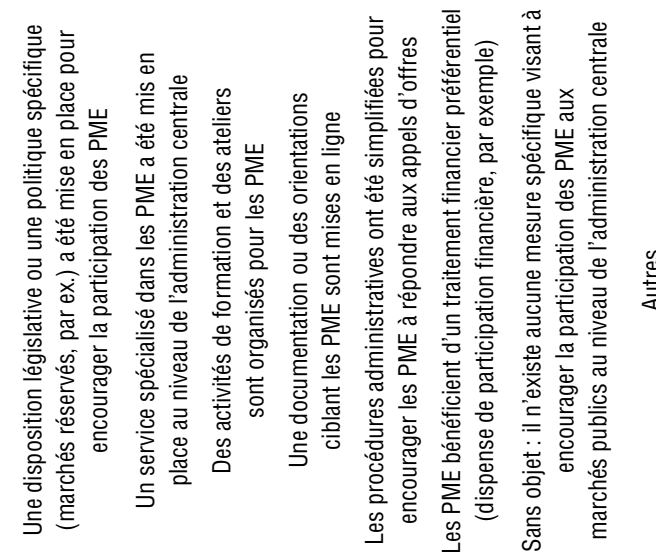

\begin{tabular}{|c|c|c|c|c|c|c|c|c|}
\hline Allemagne & $\bullet$ & O & O & 0 & $O$ & O & 0 & O \\
\hline Australie & O & - & O & 0 & O & 0 & O & O \\
\hline Autriche & O & $\bullet$ & - & $\bullet$ & O & O & O & O \\
\hline Belgique & O & $\bullet$ & - & O & $\bullet$ & O & O & O \\
\hline Canada & O & $\bullet$ & - & $\bullet$ & $O$ & O & O & O \\
\hline Chili & O & O & - & - & O & O & O & 0 \\
\hline Corée & $\bullet$ & $\bullet$ & - & $\bullet$ & $O$ & - & O & O \\
\hline Danemark & O & - & - & - & O & O & O & $\bullet$ \\
\hline Espagne & $\bullet$ & $\bullet$ & O & $\bullet$ & $\bullet$ & O & O & $\bullet$ \\
\hline Estonie & O & O & O & $O$ & O & O & 0 & $\bullet$ \\
\hline États-Unis & $\bullet$ & $\bullet$ & - & $\bullet$ & $\bullet$ & O & O & O \\
\hline Finlande & O & O & O & $O$ & O & O & $O$ & $\bullet$ \\
\hline France & $\bullet$ & O & $\bullet$ & $\bullet$ & O & O & $O$ & $\bullet$ \\
\hline Hongrie & - & O & - & - & - & O & 0 & 0 \\
\hline Irlande & 0 & O & - & - & - & 0 & 0 & 0 \\
\hline Islande & O & O & O & O & O & $O$ & $\bullet$ & O \\
\hline Israël & O & - & O & - & O & O & 0 & 0 \\
\hline Italie & O & O & - & $\bullet$ & $O$ & $O$ & O & O \\
\hline Japon & $\bullet$ & $\bullet$ & O & $\bullet$ & $O$ & O & 0 & O \\
\hline Luxembourg & O & O & O & O & $\bullet$ & $O$ & $O$ & O \\
\hline Mexique & - & - & - & - & O & - & 0 & O \\
\hline Norvège & O & - & O & 0 & O & O & O & 0 \\
\hline Nouvelle-Zélande & O & O & - & $O$ & $\bullet$ & O & O & O \\
\hline Pays-Bas & $\bullet$ & O & - & O & $\bullet$ & O & O & O \\
\hline Pologne & $\bullet$ & $\bullet$ & - & $\bullet$ & $O$ & O & O & O \\
\hline Portugal & O & O & $\bullet$ & O & O & O & O & O \\
\hline $\begin{array}{l}\text { République } \\
\text { slovaque }\end{array}$ & O & O & O & $O$ & O & $O$ & $\bullet$ & O \\
\hline République tchèque & O & O & O & O & O & O & $\bullet$ & O \\
\hline Royaume-Uni & O & $\bullet$ & - & $\bullet$ & $\bullet$ & O & O & $\bullet$ \\
\hline Slovénie & $\bullet$ & O & O & O & $\bullet$ & O & $O$ & O \\
\hline Suède & O & O & O & O & O & O & $\bullet$ & O \\
\hline Suisse & $\bullet$ & O & - & $\bullet$ & $\bullet$ & O & O & $\mathrm{O}$ \\
\hline Turquie & O & $\bullet$ & - & O & O & O & $O$ & O \\
\hline \multicolumn{9}{|l|}{ Total OCDE } \\
\hline - Oui & 12 & 15 & 19 & 17 & 11 & 2 & 4 & 6 \\
\hline O Non & 21 & 18 & 14 & 16 & 22 & 31 & 29 & 27 \\
\hline
\end{tabular}

Source : Enquête 2012 de l'OCDE sur les marchés publics.

StatLink क्ञाs http://dx.doi.org/10.1787/888933159814
7.10. Est-il obligatoire d'effectuer des évaluations ou audits a posteriori des situations dans lesquelles des exceptions ont été invoquées pour procéder à l'attribution directe d'un marché de l'administration centrale?

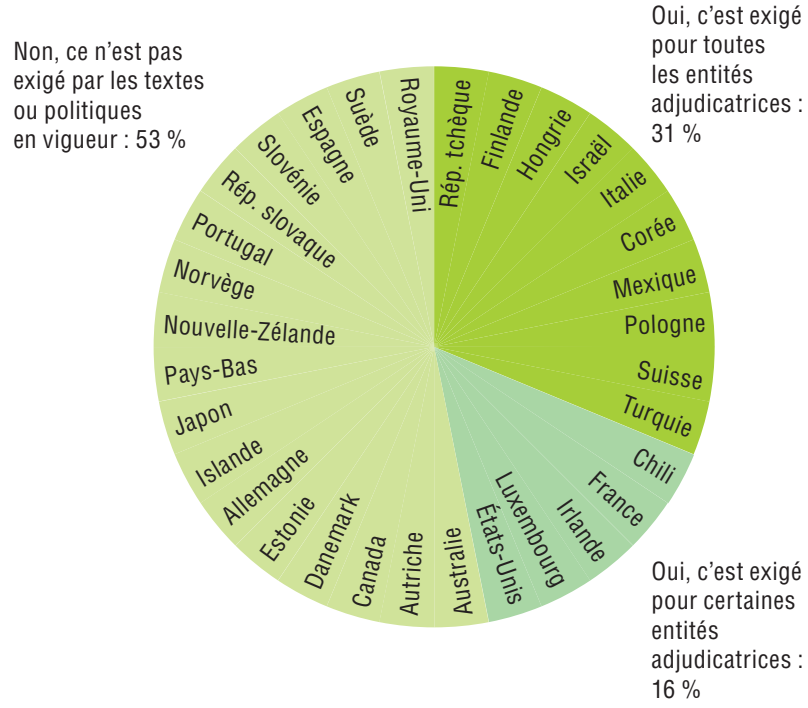

Source : Enquête 2012 de l'OCDE sur les marchés publics. StatLink तiाst http://dx.doi.org/10.1787/888933159825 


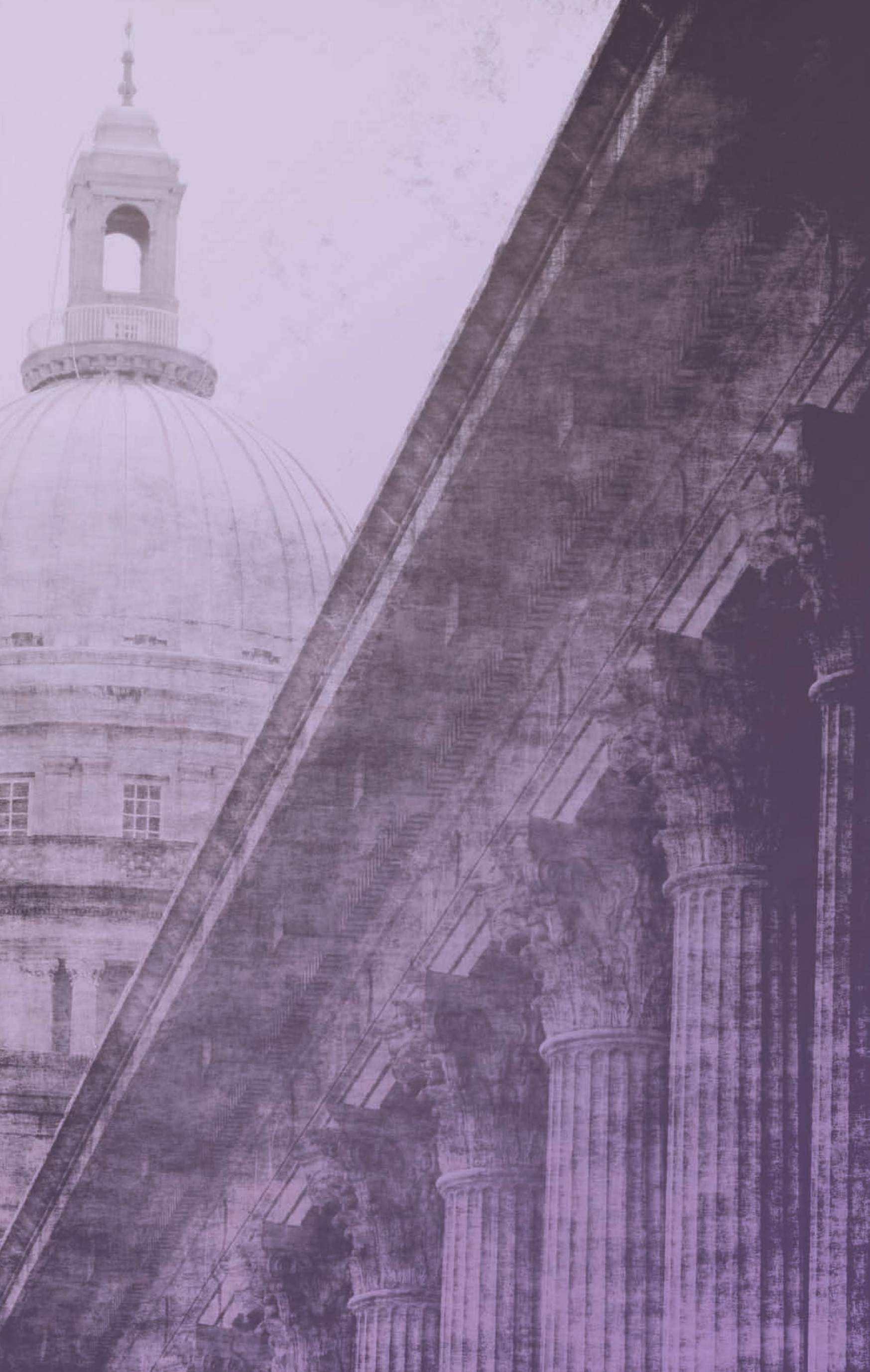




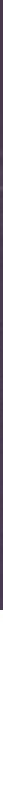

Les citoyens attendent des administrations publiques de l'ouverture et de l'inclusivité, c'est-à-dire l'institutionnalisation systémique et globale d'une communication bilatérale avec les parties prenantes dans le cadre de laquelle des informations pertinentes et exploitables sont fournies et où le dialogue est encouragé en tant que facteur de renforcement de la transparence, de la responsabilité et de la participation. Des processus plus ouverts et plus inclusifs d'élaboration des politiques permettent d'adopter des politiques plus éclairées et mieux adaptées aux besoins des citoyens. En favorisant la participation des administrés, on peut améliorer la participation citoyenne, renforcer la confiance dans les institutions et mettre à profit des formes productives de responsabilité, y compris au niveau de la prestation des services publics. Les politiques d'accès à l'information et d'ouverture des données publiques sont des piliers cruciaux pour l'ouverture de l'administration. La transparence budgétaire constitue elle aussi un facteur d'ouverture important, car c'est au sein du budget que les objectifs de l'action publique sont conciliés et mis en œuure en termes financiers. Toutefois, plus de transparence ne rime pas nécessairement avec plus de responsabilité. Une administration peut être transparente même si elle n'a pas adopté les nouvelles technologies, et une autre peut donner accès à ses données tout en restant profondément opaque et imperméable à toute responsabilité. Enfin, l'élaboration inclusive des politiques publiques a pour objet d'évaluer l'impact de ces politiques sur certains groupes sociaux clés qu'elles risqueraient de désavantager. 
Les organismes publics produisent et recueillent un large éventail de données afin de mener à bien leurs travaux. Partager ces données avec le grand public permet d'assurer une plus grande transparence et de mieux faire connaître les activités officielles. L'ouverture des données publiques peut aussi permettre de trouver de nouvelles pistes pour améliorer les performances des administrations et les placer face à leurs responsabilités. Elle permet également d'instaurer une véritable démarche de participation citoyenne et de collaboration en vue de la mise en place de services et de politiques novateurs, à forte valeur ajoutée et sachant répondre aux attentes des administrés. Elle devrait aussi, à terme, permettre aux administrations comme aux administrés de prendre de meilleures décisions. En effet, les citoyens pourront se fonder sur les données publiques pour prendre des décisions plus éclairées propres à améliorer leur qualité de vie, tandis que les administrations pourront accéder plus facilement à des ensembles de données plus vastes, ce qui les aidera à prendre des décisions fondées sur des données probantes. Enfin, on voit dans l'ouverture des données publiques une source potentielle de croissance économique et un tremplin pour de nouvelles formes d'entrepreneuriat et d'innovation sociale.

Toutefois, l'ouverture des données publiques en est encore à ses débuts, et il est difficile de comparer les performances des différents pays s'agissant de l'accessibilité et de la qualité des données fournies. Premièrement, les stratégies et politiques d'ouverture des données publiques sont en constante évolution. Deuxièmement, l'administration et la production des données en question sont souvent déléguées aux échelons administratifs régionaux et locaux. Troisièmement, il n'existe pas de définitions internationales communes - d'un ensemble de données, par exemple.

Au sein de la zone OCDE, on assiste à une montée en puissance des démarches stratégiques en matière d'ouverture des données publiques : $56 \%$ des pays de l'OCDE se sont dotés d'une stratégie nationale, $12 \%$ signalent l'existence de stratégies différentes selon les ministères sectoriels et $28 \%$ indiquent que ces deux types de stratégies coexistent. Seuls $4 \%$ des pays interrogés sont dépourvus de toute stratégie en la matière.

Les grands objectifs stratégiques de l'ouverture des données publiques varient selon les pays de l'OCDE. La plupart des pays semblent rechercher en priorité la transparence et l'ouverture, l'augmentation des volumes pour les entreprises du secteur privé et la création d'entreprises. Les pays semblent aussi conscients des possibilités qu'offre l'ouverture des données publiques en termes d'amélioration des services publics. En revanche, la participation des citoyens aux débats publics et aux processus de décision ne figure pas parmi les priorités. Il est essentiel de bien cerner les principaux objectifs poursuivis afin de déterminer les types de données à diffuser en priorité et le format à adopter. En effet, selon le but recherché, les ensembles de données à ouvrir sont différents. S'il est possible de servir des objectifs de responsabilité et de transparence en diffusant des données agrégées, des ensembles de données plus détaillés peuvent être nécessaires si l'objectif consiste à favoriser la croissance économique.

Dans une optique de création de valeur, la disponibilité des données, leur accessibilité et leur pertinence pour les usagers sont essentielles. En termes de disponibilité, il semble que ce soit au Canada, aux États-Unis, aux Pays-Bas et en Slovénie que l'on trouve les "guichets uniques " centralisés d'accès aux données publiques proposant le plus grand nombre d'ensembles de données. Toute comparaison internationale des ensembles de données fournis doit cependant être prudente, en raison de différences au niveau des ensembles de données et des modes de diffusion choisis : certains pays optent pour un guichet unique à l'échelon national, d'autres pour des portails gérés par les autorités locales.

\section{Méthodologie et définitions}

Les données sont tirées de l'Enquête 2013 de l'OCDE sur l'ouverture des données publiques, qui est axée sur la disponibilité des données publiques à l'échelon central/ fédéral. L'Égypte, la Fédération de Russie, l'Ukraine et 25 pays de l'OCDE ont participé à cette enquête. Les réponses ont été fournies par des délégués nationaux chargés de l'administration électronique et des données publiques.

On entend par "données publiques » l'ensemble des données ou des informations produites ou commandées par des organismes officiels. On entend par « ouverture des données " la possibilité offerte à quiconque d'utiliser, de réutiliser et de distribuer les données, avec pour seule obligation éventuelle celle d'en citer l'origine et de les partager à son tour selon les mêmes conditions. Un ensemble de données est un recueil de données généralement présentées sous forme de tableau.

Les objectifs évoqués sont ceux de la politique ou stratégie d'ouverture des données publiques mise en place à l'échelon central ou fédéral. Les pays interrogés ayant été invités à citer les cinq principaux objectifs de leur politique, il est possible que la liste complète de ces objectifs soit plus longue. La réutilisation des données publiques consiste à intégrer des données et informations rendues publiques dans des contextes différents de celui dont elles ont été extraites (pour le développement de logiciels ou des communiqués de presse, par exemple). Elle peut être interdite par des textes relatifs au droit d'auteur ou autorisée aux termes d'une mention explicite en ce sens.

Le tableau 8.3, "Disponibilité de données en format ouvert sur les sites web des ministères sectoriels (2013) ", est consultable en ligne à l'adresse $h t t p: / / d x$.doi.org/ $10.1787 / 888932943609$.

\section{Pour en savoir plus}

Ubaldi, B. (2013), « Open Government Data: Towards Empirical Analysis of Open Government Data Initiatives ", OECD Working Papers on Public Governance, $\mathrm{n}^{\circ} 22$, Éditions OCDE, Paris, http://dx.doi.org/10.1787/5k46bj4f03s7-en.

\section{Notes relatives aux graphiques}

8.1: On ne dispose pas de données pour les pays non dotés de guichets uniques centralisés d'accès aux données publiques : l'Estonie, la Finlande, l'Irlande, le Japon et la Suisse. On ne dispose pas de données pour le Danemark et la France. Les données relatives à l'Australie portent uniquement sur un programme lancé par les autorités fédérales (http://data.gov.au). Les données relatives à l'Espagne portent uniquement sur un programme lancé par les autorités centrales (http:// datos.gob.es).

8.2 : En Égypte et en Suisse, les autorités centrales/fédérales n'ont pas, pour l'heure, lancé de politique ou de stratégie d'ouverture des données publiques.

Informations sur les données concernant Israël : http://dx.doi.org/ $10.1787 / 888932315602$. 
8.1. Nombre d'ensembles de données proposés dans les guichets uniques centralisés d'accès aux données publiques (2013)

Nombre d'ensembles de données

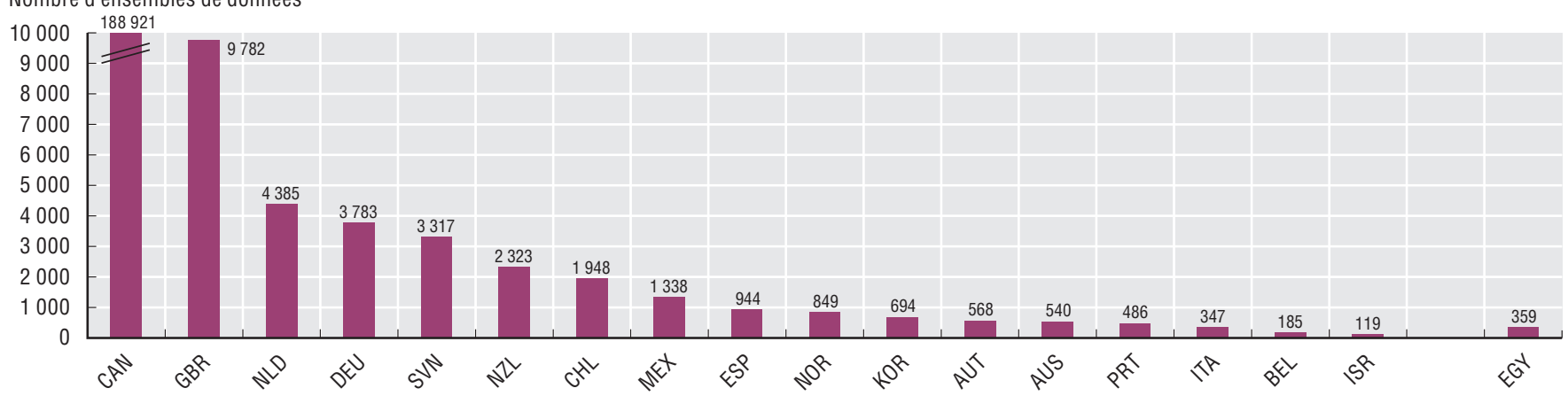

Source : Enquête 2013 de l'OCDE sur l'ouverture des données publiques.

8.2. Cinq principaux objectifs de la stratégie d'ouverture des données publiques des autorités centrales/fédérales (2013)

\begin{tabular}{|c|c|c|c|c|c|c|c|c|c|c|}
\hline & $\begin{array}{l}\text { Accroître la } \\
\text { transparence I }\end{array}$ & $\begin{array}{l}\text { Accroître } \\
\text { l'ouverture }\end{array}$ & $\begin{array}{l}\text { Améliorer les } \\
\text { performances du } \\
\text { secteur public en } \\
\text { rendant les } \\
\text { intervenants plus } \\
\text { redevables des } \\
\text { travaux/résultats }\end{array}$ & $\begin{array}{l}\text { Assurer les services } \\
\text { publics avec plus } \\
\text { d'efficacité et } \\
\text { d'efficience en } \\
\text { améliorant le } \\
\text { fonctionnement interne } \\
\text { et la collaboration }\end{array}$ & $\begin{array}{l}\text { Assurer les services publics } \\
\text { avec plus d'efficacité et } \\
\text { d'efficience en offrant au } \\
\text { secteur privé la possibilité de } \\
\text { fournir des services fondés } \\
\text { sur une réutilisation des } \\
\text { données publiques }\end{array}$ & $\begin{array}{l}\text { Créer de la } \\
\text { valeur } \\
\text { économique } \\
\text { pour le } \\
\text { secteur } \\
\text { public }\end{array}$ & $\begin{array}{l}\text { Créer de la valeur } \\
\text { économique pour } \\
\text { le secteur privé/ } \\
\text { accroître le volume } \\
\text { d'activité du } \\
\text { secteur privé }\end{array}$ & $\begin{array}{l}\text { Favoriser } \\
\text { lacréation } \\
\text { d'entre- } \\
\text { prises }\end{array}$ & $\begin{array}{l}\text { Favoriser la } \\
\text { participation } \\
\text { des citoyens } \\
\text { aux débats- } \\
\text { publics }\end{array}$ & $\begin{array}{l}\text { Permettre } \\
\text { aux citoyens } \\
\text { de participer } \\
\text { aux } \\
\text { processus } \\
\text { de décision }\end{array}$ \\
\hline Allemagne & $\bullet$ & O & 0 & $\bullet$ & $O$ & O & O & $\bullet$ & $\bullet$ & $\bullet$ \\
\hline Australie & O & $\bullet$ & O & $\bullet$ & $\bullet$ & O & $\bullet$ & O & O & $\bullet$ \\
\hline Autriche & $\bullet$ & $\bullet$ & $O$ & $O$ & $\bullet$ & O & $\bullet$ & O & $\bullet$ & $\bigcirc$ \\
\hline Belgique & O & - & O & - & - & O & - & 0 & O & $\mathrm{O}$ \\
\hline Canada & $O$ & - & $\bullet$ & $\bullet$ & $O$ & $O$ & $\bullet$ & $O$ & $\bullet$ & $O$ \\
\hline Chili & $\bullet$ & O & $\bullet$ & O & $\bullet$ & O & O & O & $\bullet$ & $\bullet$ \\
\hline Corée & - & - & $O$ & $O$ & $\bullet$ & O & - & $\bullet$ & $O$ & $\bigcirc$ \\
\hline Danemark & O & O & O & - & ○ & O & ○ & - & O & O \\
\hline Espagne & $\bullet$ & $\bullet$ & $\bullet$ & 0 & $O$ & 0 & $\bullet$ & $\bullet$ & O & O \\
\hline Estonie & - & ○ & - & $O$ & ○ & O & - & $\mathrm{O}$ & O & $\mathrm{O}$ \\
\hline Finlande & - & $O$ & 0 & $O$ & $\bullet$ & O & $\bullet$ & $\bullet$ & $\bigcirc$ & $O$ \\
\hline France & - & O & - & 0 & O & O & ○ & $\bullet$ & O & $\bigcirc$ \\
\hline Irlande & $\bullet$ & $\bullet$ & O & $\bullet$ & $\bullet$ & O & O & $\bullet$ & 0 & O \\
\hline Israël & $\bullet$ & $\bullet$ & O & - & $\bullet$ & O & O & 0 & O & O \\
\hline Italie & $O$ & $\bullet$ & $\bullet$ & $\bullet$ & $O$ & 0 & $\bullet$ & $\bullet$ & O & $\bigcirc$ \\
\hline Japon & $\bullet$ & $\bullet$ & O & O & $\bullet$ & O & $\bullet$ & $\bullet$ & O & O \\
\hline Mexique & $\bullet$ & $\bullet$ & - & $O$ & $O$ & 0 & $O$ & 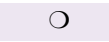 & $\bullet$ & $\bigcirc$ \\
\hline Norvège & - & $\mathrm{O}$ & $\mathrm{O}$ & - & - & O & - & - & $\mathrm{O}$ & $\mathrm{O}$ \\
\hline Nouvelle-Zélande & $\bullet$ & 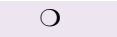 & O & $\bullet$ & $O$ & O & $\bullet$ & $O$ & $\bullet$ & 0 \\
\hline Pays-Bas & O & $\bullet$ & $\bullet$ & O & $O$ & O & $\bullet$ & O & $\bullet$ & O \\
\hline Portugal & $O$ & $\bullet$ & O & $\bullet$ & $\bigcirc$ & O & $\bigcirc$ & $\bullet$ & O & $\bullet$ \\
\hline Royaume-Uni & $\bullet$ & O & $\bullet$ & $\bullet$ & $O$ & O & $\bullet$ & O & O & $\bullet$ \\
\hline Slovénie & $\bullet$ & $\bullet$ & 0 & O & $\bullet$ & O & O & $\bullet$ & O & $\bullet$ \\
\hline Suède & $\bullet$ & ○ & $\bullet$ & ○ & $O$ & $O$ & $O$ & 0 & $O$ & $\bigcirc$ \\
\hline Suisse & $x$ & $x$ & $x$ & $x$ & $\mathrm{x}$ & $x$ & $\mathrm{x}$ & $x$ & $x$ & $x$ \\
\hline Égypte & $x$ & $\mathrm{x}$ & $x$ & $x$ & $x$ & $x$ & $x$ & $x$ & $x$ & $x$ \\
\hline $\begin{array}{l}\text { Fédération } \\
\text { de Russie }\end{array}$ & $\bullet$ & $\bullet$ & O & $O$ & $O$ & - & $O$ & $\bullet$ & - & $O$ \\
\hline Ukraine & $\bullet$ & $\bigcirc$ & $\bigcirc$ & $\bullet$ & $O$ & $\bullet$ & $\bullet$ & $\bigcirc$ & $\bullet$ & O \\
\hline Total OCDE & 17 & 16 & 11 & 14 & 13 & 0 & 16 & 15 & 7 & 7 \\
\hline
\end{tabular}

Oui

O Non

$\mathrm{x}$ Sans objet

Source : Enquête 2013 de l'OCDE sur l'ouverture des données publiques. 


\section{UNE ADMINISTRATION OUVERTE ET INCLUSIVE}

Les conflits d'intérêts et les déclarations de patrimoine

De plus en plus, et surtout depuis la crise économique et financière, on attend des pouvoirs publics une prise de décision ouverte et équitable. Des pressions croissantes s'exercent donc sur les administrations pour qu'elles évitent de laisser des intérêts privés influencer indûment leurs décisions. Dans le même temps, de nouvelles formes de partenariat entre les pouvoirs publics et les secteurs privé et associatif compliquent la tâche des décideurs et gestionnaires publics chargés d'assurer l'intégrité des opérations en cause. Il est donc essentiel de préserver l'intégrité du processus d'adoption des décisions publiques afin de rétablir la confiance dans les pouvoirs publics.

Il y a conflit d'intérêts quand les intérêts privés d'un agent public risquent de compromettre la bonne exécution de ses fonctions. Si elles ne sont pas dûment repérées et gérées, les situations de conflit d'intérêts peuvent conduire à la corruption. À l'inverse, une démarche excessivement stricte peut être coûteuse et impraticable, et risque de dissuader des candidats potentiels expérimentés et compétents d'accepter un poste ou un mandat public.

L'expérience montre que la divulgation du patrimoine et des intérêts privés des décideurs publics reste un instrument essentiel de gestion des conflits d'intérêts. Le graphique 8.4 présente de façon synthétique, pour les décideurs de haut niveau des trois pouvoirs (exécutif, législatif et judiciaire), le niveau de divulgation exigé et le niveau d'accessibilité des informations fournies. Ces niveaux dépendent de l'obligation faite aux décideurs de haut niveau de déclarer des intérêts privés tels que leurs actifs, leurs dettes, la source et le montant de leurs revenus, leurs postes extérieurs, rémunérés ou non, les cadeaux qu'ils ont reçus et leurs emplois antérieurs.

S'il est courant que les décideurs publics des pays de l'OCDE doivent déclarer leurs actifs et leurs intérêts privés, les niveaux de divulgation varient d'un pouvoir à l'autre. Les obligations de divulgation sont considérablement plus lourdes au sein des pouvoirs exécutif et législatif qu'au sein du pouvoir judiciaire. Par exemple, aucune obligation de divulgation ne pèse sur les juges et les procureurs en France, au Luxembourg, en Nouvelle-Zélande et en République tchèque. Au Luxembourg, aucune obligation de divulgation ne pèse sur les décideurs des trois pouvoirs. S'agissant des intérêts privés concernés, les pays s'intéressent principalement aux postes extérieurs rémunérés et aux cadeaux, soit en les interdisant, soit en exigeant qu'ils soient déclarés.

On constate par ailleurs d'importants écarts entre les pays de l'OCDE s'agissant de l'accès du public aux informations fournies par les décideurs. Dans la majorité des pays, l'information n'est que partiellement mise à la disposition du public. Dans certains pays, cela s'explique par l'importance accordée au respect de la vie privée. Dans la grande majorité des cas, l'information relative aux actifs et aux sources de revenus est rendue publique. En revanche, seuls quelques pays rendent publique l'information relative aux emplois antérieurs et aux dettes.

Parmi les pays de l'OCDE à avoir instauré une déclaration obligatoire, un peu plus de $80 \%$ vérifient que le formulaire de déclaration a été rempli (tableau 8.5). En revanche, moins de la moitié d'entre eux réalisent des audits internes des informations fournies pour vérifier leur exactitude. En Irlande, en Italie, en Suisse et en Turquie, la remise des formulaires de déclaration n'est suivie d'aucune autre étape. Toutefois, en Irlande et en Italie, la plupart des informations fournies sont mises à la disposition du public, ce qui permet aux citoyens eux-mêmes de les examiner.

\section{Méthodologie et définitions}

Les données portent sur 2012 et sont tirées d'une enquête de l'OCDE sur la gestion des conflits d'intérêts. Les réponses ont été fournies par des délégués nationaux chargés des politiques d'intégrité au sein de l'administration centrale ou fédérale.

L'expression "agent public " est définie comme englobant toute personne qui détient un mandat législatif, exécutif, administratif ou judiciaire d'un pays, qu'elle ait été nommée ou élue, à titre permanent ou temporaire, qu'elle soit rémunérée ou non rémunérée, et quel que soit son niveau hiérarchique; et toute autre personne qui exerce une fonction publique, y compris pour un organisme public ou une entreprise publique, ou qui fournit un service public, tels que ces termes sont définis dans le droit interne du pays. Pour les décideurs de haut niveau, l'expression " pouvoir exécutif » fait référence aux présidents, premiers ministres et ministres; l'expression "pouvoir législatif» fait référence aux parlementaires des chambres hautes et basses des parlements; et l'expression "pouvoir judiciaire " fait référence aux juges et aux procureurs.

Pour le calcul des agrégats de données nationales, tous les intérêts privés et toutes les positions ont été considérés comme d'importance égale et, par conséquent, ont reçu la même pondération. On trouvera à l'annexe E des précisions sur les déclarations de conflits d'intérêts.

\section{Pour en savoir plus}

OCDE (2010), L'emploi d'après-mandat : Bonnes pratiques en matière de prévention des conflits d'intérêts, Éditions OCDE, Paris, http:// dx.doi.org/10.1787/9789264056725-fr.

OCDE (2007), OECD Guidelines for Managing Conflict of Interest in the Public Service: Report on Implementation, Éditions OCDE, Paris.

OCDE (2003), Recommandation du Conseil sur les Lignes directrices de l'OCDE pour la gestion des conflits d'intérêts dans le service public, Éditions OCDE, Paris.

\section{Notes relatives aux graphiques}

8.4 : Les données relatives au Brésil, à la Fédération de Russie, à la Grèce, à Israël et à la République tchèque portent sur 2010 et non 2012. Les données reflètent les pratiques des pays membres. On trouvera à l'annexe $\mathrm{E}$ des données et des notes relatives aux divers pays examinés.

8.5 : On ne dispose pas de données pour la Grèce, Israël et la République tchèque. Pour l'Allemagne, l'Australie, l'Autriche, le Canada, le Chili, la Corée, le Danemark, l'Espagne, l'Estonie, les États-Unis, la Hongrie, l'Italie, le Japon, le Luxembourg, le Mexique, la Norvège, la République slovaque, la Suisse et la Turquie, l'information fournie porte uniquement sur l'exécutif.

Informations sur les données concernant Israël : http://dx.doi.org/ $10.1787 / 888932315602$. 
8.4. Déclarations de patrimoine : niveau de divulgation des intérêts privés et d'accessibilité des informations fournies (2012)

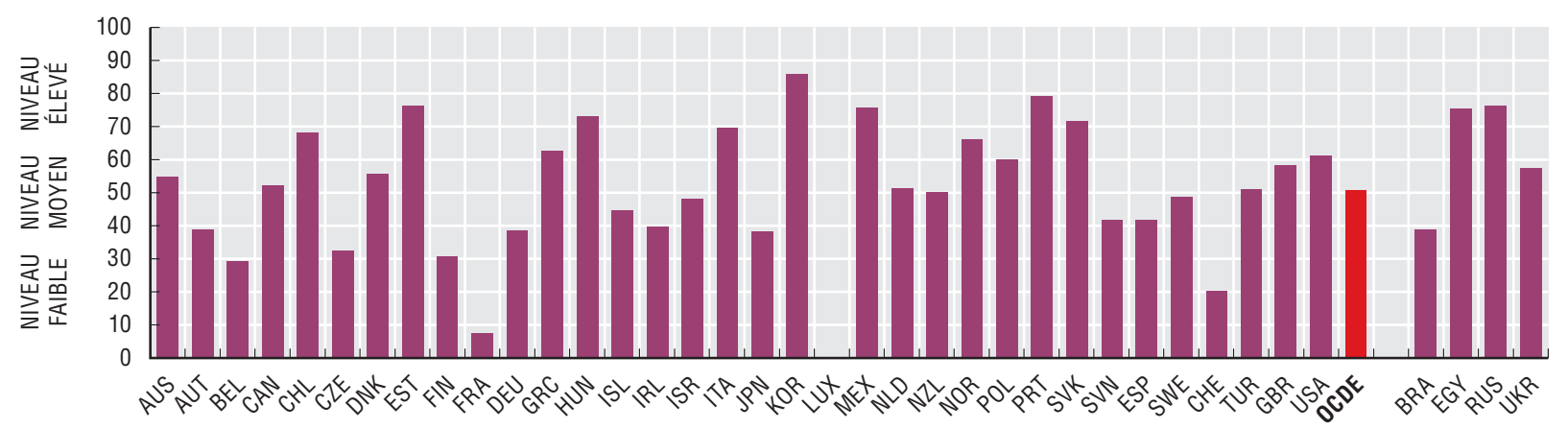

Source: Enquête de l'OCDE sur la gestion des conflits d'intérêts (2012).

StatLink काISL http://dx.doi.org/10.1787/888933159874

8.5. Suite donnée aux déclarations d'intérêts privés des agents publics (2012)

\begin{tabular}{|c|c|c|c|}
\hline & $\begin{array}{l}\text { Vérification de la remise } \\
\text { du formulaire } \\
\text { de déclaration }\end{array}$ & $\begin{array}{l}\text { Vérification de la fourniture } \\
\text { de tous les } \\
\text { renseignements demandés }\end{array}$ & $\begin{array}{c}\text { Audit interne visant à vérifier } \\
\text { l'exactitude } \\
\text { des informations fournies }\end{array}$ \\
\hline Allemagne & - & $\bullet$ & $\square$ \\
\hline Australie & 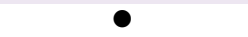 & O & O \\
\hline Autriche & 0 & $\bullet$ & 0 \\
\hline Belgique & - & $\bullet$ & O \\
\hline Canada & $\bullet$ & $\bullet$ & O \\
\hline Chili & 0 & • & $\mathrm{O}$ \\
\hline Corée & - & $\bullet$ & 0 \\
\hline Danemark & 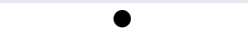 & $\bullet$ & O \\
\hline Espagne & $\bullet$ & $\bullet$ & $\bullet$ \\
\hline Estonie & - & $\square$ & $\square$ \\
\hline États-Unis & 0 & - & O \\
\hline Finlande & ○ & $\bullet$ & $\bigcirc$ \\
\hline France & $\bullet$ & $\square$ & $\square$ \\
\hline Hongrie & 0 & $\mathrm{O}$ & O \\
\hline Irlande & 0 & O & O \\
\hline Islande & $\bullet$ & O & $\bigcirc$ \\
\hline Italie & $O$ & $\mathrm{O}$ & $O$ \\
\hline Japon & $O$ & $\bullet$ & $\bullet$ \\
\hline Luxembourg & $x$ & $x$ & $x$ \\
\hline Mexique & $\bullet$ & $\bullet$ & $\square$ \\
\hline Norvège & 0 & $\square$ & 0 \\
\hline Nouvelle-Zélande & 0 & - & $\square$ \\
\hline Pays-Bas & $\bullet$ & - & 0 \\
\hline Pologne & $\bullet$ & $\bullet$ & $\square$ \\
\hline Portugal & 0 & - & 0 \\
\hline République slovaque & 0 & - & O \\
\hline Royaume-Uni & $\bullet$ & $\bullet$ & - \\
\hline Slovénie & ○ & $\square$ & $\square$ \\
\hline Suède & 0 & - & $\square$ \\
\hline Suisse & O & O & O \\
\hline Turquie & O & O & O \\
\hline Égypte & $\bullet$ & - & $\bigcirc$ \\
\hline Ukraine & $\square$ & $\square$ & O \\
\hline \multicolumn{4}{|l|}{ Total OCDE } \\
\hline $\begin{array}{l}\text { Procédure suivie pour tous les agents soumis à une obligation } \\
\text { de déclaration }\end{array}$ & 25 & 19 & 6 \\
\hline $\begin{array}{l}\square \text { Procédure suivie uniquement pour certains des agents soumis } \\
\text { à une obligation de déclaration }\end{array}$ & 0 & 4 & 8 \\
\hline Procédure non suivie & 5 & 7 & 16 \\
\hline
\end{tabular}

Source : Enquête de l'OCDE sur la gestion des conflits d'intérêts (2012). 
Le budget national est l'un des principaux documents stratégiques du gouvernement, puisqu'il reflète ses objectifs d'action et ses priorités en matière de dépenses. La transparence budgétaire - c'est-à-dire la publication et l'accessibilité de l'information essentielle en matière de finances publiques et de budget - est donc au cœur d'une bonne gouvernance. La crise économique et sociale a souligné la nécessité d'une plus grande transparence budgétaire, et c'est devenu l'un des piliers des stratégies nationales d'ouverture de l'administration. Dans son guide "Transparence budgétaire: les meilleures pratiques de l'OCDE ", l'OCDE reconnaît explicitement qu'il est important de faire connaître l'information budgétaire officielle en temps opportun et de façon systématique et qu'il est nécessaire d'assurer la qualité, l'intégrité et - point très important - l'accessibilité de cette information, afin d'informer les citoyens et les parlementaires et de placer l'exécutif face à ses responsabilités.

Une documentation budgétaire publiquement disponible et exhaustive peut aider le grand public à comprendre les politiques menées en matière de finances publiques et les priorités gouvernementales. La publication de l'information budgétaire peut contribuer à la discipline budgétaire, à la bonne affectation des ressources et à l'efficience opérationnelle. Elle peut aussi permettre d'inciter le gouvernement à assumer ses responsabilités en termes de réalisme et de viabilité des budgets et d'impact socio-économique des mesures prévues. Parce que la présence d'informations au sein des documents budgétaires ne garantit pas nécessairement leur exactitude, la transparence budgétaire permet aussi aux citoyens, aux parlementaires et aux organisations de la société civile de s'appuyer sur les chiffres fournis pour placer le gouvernement face à ses responsabilités en termes de résultats budgétaires. Par ces biais, la transparence budgétaire contribue aussi à la confiance dans les pouvoirs publics.

La fourniture, dans le budget de l'exécutif, d'une information sur le cadre budgétaire et sur les politiques et priorités du gouvernement représente un élément crucial de la transparence budgétaire. La quantité et le type de renseignements fournis varient selon les pays. Si les objectifs de la politique budgétaire, les hypothèses macroéconomiques et les perspectives à moyen terme (voir l'indicateur relatif aux cadres de dépenses à moyen terme) sont généralement mentionnés dans le budget des pays de l'OCDE, il est plus rare d'y voir figurer des informations sur les dépenses fiscales, sur les performances et sur les projections budgétaires à long terme. On peut sans doute considérer que le principal obstacle au respect des objectifs budgétaires et des prévisions de déficit ou d'excédent tient au recours à des hypothèses macroéconomiques peu fiables. D'après une enquête de l'OCDE sur les pratiques et procédures budgétaires, environ $95 \%$ des pays de l'OCDE publient les objectifs à moyen terme de leur politique budgétaire et les budgets proposés et adoptés. La transparence au niveau des dépenses hors budget ou extrabudgétaires est également importante, car les autorités peuvent être tentées de garder ces dépenses en dehors des bilans. Dans les pays de l'OCDE, les dépenses hors budget les plus courantes sont les dépenses liées aux administrations de sécurité sociale (13 pays), aux fonds publics de financement des dépenses de santé (10 pays) et aux garanties de crédits (9 pays). La majorité des pays membres intègrent ces dépenses à leur budget, même s'il y a des exceptions (voir le tableau 8.8, en ligne).

La publication d'informations relatives aux finances publiques et au budget de l'État est essentielle et prend de plus en plus d'ampleur, mais elle peut aussi s'avérer complexe et, sans que ce soit le but recherché, réduire la transparence et la responsabilité. De fait, les non-experts peuvent facilement être intimidés par le jargon technique employé et par le volume d'informations budgétaires présenté aux parlementaires, ou encore déconcertés par le rôle et l'ampleur des activités extrabudgétaires. Certains pays de l'OCDE (14) publient des " budgets du citoyen ". Il s'agit de synthèses en langage simple des principaux volets du budget annuel présenté au parlement, avec des explications et des définitions des termes techniques.

\section{Méthodologie et définitions}

Les données, qui se réfèrent à 2012, sont tirées des réponses des pays à l'Enquête de l'OCDE sur les pratiques et procédures budgétaires (2012). Les répondants étaient principalement de hauts responsables budgétaires des pays membres de l'OCDE. Les réponses représentent l'évaluation par les pays eux-mêmes de leurs pratiques et procédures actuelles. Les données ne portent que sur l'administration centrale/fédérale et ne prennent pas en compte les pratiques budgétaires à l'échelon des administrations d'États fédérés et des administrations locales.

Les fonds hors budget sont des fonds publics spéciaux qui ne font pas partie du budget et dont les recettes proviennent de prélèvements affectés, éventuellement en complément d'autres sources de recettes telles que des redevances ou des contributions tirées d'un fonds général des impôts. Les prélèvements affectés se distinguent des redevances dans la mesure où ils ne reflètent pas la valeur marchande des services que les recettes servent à financer. Ils peuvent notamment être inférieurs ou supérieurs à cette valeur marchande en fonction de considérations d'ordre social.

Un «budget du citoyen " est défini ici comme une synthèse en langage simple des principaux volets du budget annuel présenté au parlement. Ce document devrait se suffire à lui-même et expliquer la teneur du projet annuel de budget et ses effets escomptés. Même si ce guide comporte des liens vers ou des références à des documents plus détaillés, le lecteur devrait pouvoir le comprendre sans être obligé de se référer à ces autres documents ou d'en connaître la teneur.

Le tableau 8.8, "Intégration des dépenses extrabudgétaires aux documents budgétaires ", est disponible en ligne à l'adresse http://dx.doi.org/10.1787/888932943666.

\section{Pour en savoir plus}

FMI (2001, 2007), Manuel sur la transparence des finances publiques, Fonds monétaire international, Washington, DC.

OCDE (2013, à paraître), Budgeting Practices and Procedures in OECD Countries, Éditions OCDE, Paris.

OCDE (2002), «Transparence budgétaire: les meilleures pratiques de l'OCDE ", Revue de l'OCDE sur la gestion budgétaire, vol. 1/3, pp. 7-15, http://dx.doi.org/10.1787/budget-v1-art14-fr.

\section{Notes relatives aux graphiques}

On ne dispose pas de données pour l'Islande.

Informations sur les données concernant Israël : http://dx.doi.org/ $10.1787 / 888932315602$. 
La transparence budgétaire

8.6. Publication de " budgets du citoyen " dans les pays de l'OCDE (2012)

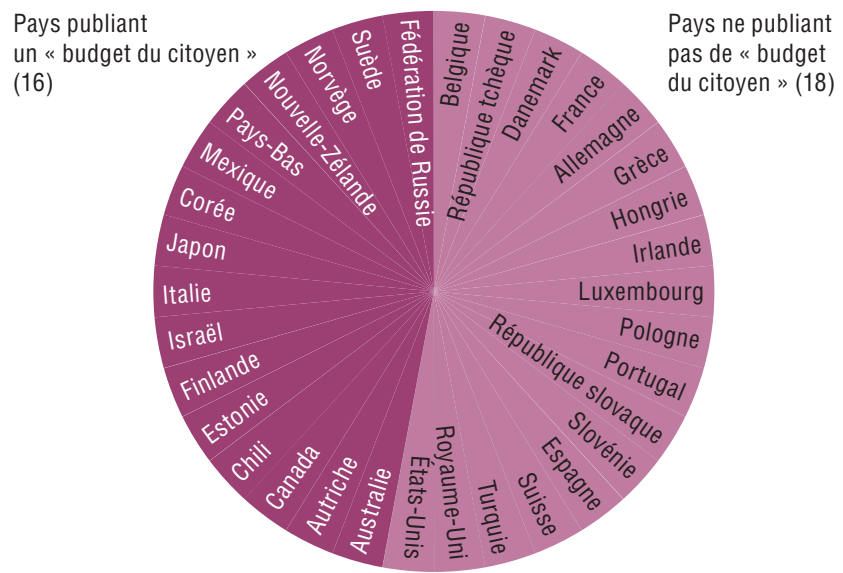

Source : Enquête de l'OCDE sur les pratiques et procédures budgétaires (2012).

StatLink काजा http://dx.doi.org/10.1787/888933159891

\subsection{Informations budgétaires rendues publiques (2012)}

\begin{tabular}{|c|c|c|c|c|c|c|c|c|c|}
\hline & 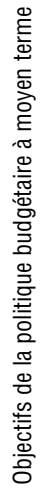 & 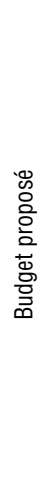 & 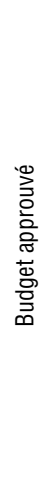 & 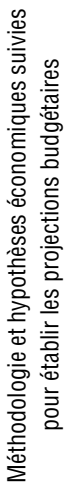 & 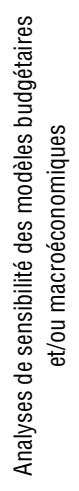 & 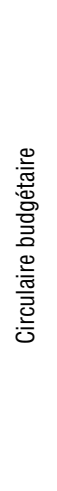 & 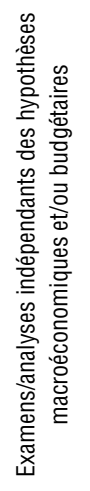 & 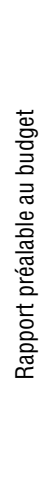 & 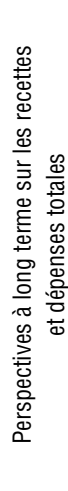 \\
\hline Allemagne & $\bullet$ & - & $\bullet$ & $\bullet$ & - & O & $\bullet$ & $x$ & $\bullet$ \\
\hline Australie & $\bullet$ & - & $\bullet$ & $\bullet$ & $\bullet$ & O & $O$ & $x$ & $\bullet$ \\
\hline Autriche & - & • & • & $\bullet$ & O & O & - & $x$ & • \\
\hline Belgique & $\bullet$ & - & $\bullet$ & $\bullet$ & $x$ & - & $\bullet$ & $x$ & $x$ \\
\hline Canada & $\bullet$ & - & $\bullet$ & $\bullet$ & $\bullet$ & $x$ & $\bullet$ & $x$ & $\bullet$ \\
\hline Chili & $\bullet$ & - & $\bullet$ & $\bullet$ & $O$ & $\bullet$ & $x$ & $\bullet$ & $x$ \\
\hline Corée & $\bullet$ & - & $\bullet$ & O & O & $\bullet$ & - & $x$ & $x$ \\
\hline Danemark & $\bullet$ & - & $\bullet$ & $\bullet$ & O & O & O & $x$ & $x$ \\
\hline Espagne & $\bullet$ & $\bullet$ & $\bullet$ & $O$ & 0 & $\bullet$ & $\bullet$ & O & $x$ \\
\hline Estonie & $\bullet$ & - & $\bullet$ & $\bullet$ & • & O & $O$ & $\bullet$ & $x$ \\
\hline États-Unis & $\bullet$ & - & $\bullet$ & $\bullet$ & $\bullet$ & $\bullet$ & $x$ & $x$ & $\bullet$ \\
\hline Finlande & $\bullet$ & - & $\bullet$ & $\bullet$ & O & - & $x$ & $x$ & $\bullet$ \\
\hline France & $\bullet$ & - & $\bullet$ & $\bullet$ & $\bullet$ & - & $\bullet$ & $\bullet$ & O \\
\hline Grèce & $\bullet$ & - & $x$ & O & $x$ & O & $x$ & $\bullet$ & $x$ \\
\hline Hongrie & $\bullet$ & - & $\bullet$ & $\bullet$ & O & $\bullet$ & O & O & $x$ \\
\hline Irlande & $\bullet$ & $\bullet$ & $\bullet$ & $\bullet$ & $\bullet$ & $\bullet$ & $\bullet$ & $\bullet$ & $x$ \\
\hline Israël & $\bullet$ & - & $\bullet$ & - & $O$ & O & O & $\bullet$ & $\mathrm{x}$ \\
\hline Italie & $x$ & $\bullet$ & $\bullet$ & $\bullet$ & $\bullet$ & $\bullet$ & $\bullet$ & $x$ & $x$ \\
\hline Japon & $\bullet$ & $\bullet$ & $\bullet$ & $\bullet$ & $\bullet$ & $\bullet$ & $x$ & $\bullet$ & $\bullet$ \\
\hline Luxembourg & $\bullet$ & $\bullet$ & $\bullet$ & $\bullet$ & $\bullet$ & O & $\bullet$ & $\bullet$ & $\bullet$ \\
\hline Mexique & $\bullet$ & - & $\bullet$ & $\bullet$ & $\bullet$ & O & $x$ & $\bullet$ & $x$ \\
\hline Norvège & $\bullet$ & $\bullet$ & $\bullet$ & O & O & $\bullet$ & O & $x$ & $x$ \\
\hline Nouvelle-Zélande & $\bullet$ & - & $\bullet$ & $\bullet$ & $\bullet$ & O & $O$ & $\bullet$ & $\bullet$ \\
\hline Pays-Bas & $\bullet$ & - & $\bullet$ & $\bullet$ & $\bullet$ & O & $\bullet$ & $x$ & $\bullet$ \\
\hline Pologne & $\bullet$ & $\bullet$ & $\bullet$ & $\bullet$ & $\bullet$ & $\bullet$ & O & $x$ & $x$ \\
\hline Portugal & $\bullet$ & - & $\bullet$ & O & $\bullet$ & $\bullet$ & $\bullet$ & $\bullet$ & $x$ \\
\hline République slovaque & $\bullet$ & - & $\bullet$ & O & O & $\bullet$ & $\bullet$ & $x$ & $x$ \\
\hline République tchèque & $\bullet$ & $\bullet$ & $\bullet$ & $\bullet$ & $\bullet$ & O & $\bullet$ & $x$ & O \\
\hline Royaume-Uni & $\bullet$ & O & $\bullet$ & $\bullet$ & $\bullet$ & $x$ & $\bullet$ & $\bullet$ & $\bullet$ \\
\hline Slovénie & O & $\bullet$ & $\bullet$ & $\bullet$ & $\bullet$ & $\bullet$ & $x$ & O & $\bullet$ \\
\hline Suède & $\bullet$ & $\bullet$ & $\bullet$ & $\bullet$ & $\bullet$ & O & $\bullet$ & $\bullet$ & $\bullet$ \\
\hline Suisse & $\bullet$ & - & $\bullet$ & $\bullet$ & $\bullet$ & O & $x$ & $x$ & $\bullet$ \\
\hline Turquie & $\bullet$ & - & $\bullet$ & $\bullet$ & O & $\bullet$ & O & $\bullet$ & $O$ \\
\hline Fédération de Russie & $\bullet$ & $\bullet$ & $\bullet$ & $\bullet$ & $\bullet$ & $\bullet$ & $\bullet$ & $\bullet$ & $\bullet$ \\
\hline \multicolumn{10}{|l|}{ Total OCDE } \\
\hline - Rendu public & 31 & 32 & 32 & 27 & 20 & 17 & 16 & 14 & 14 \\
\hline O Pas rendu public & 1 & 0 & 0 & 6 & 11 & 14 & 9 & 3 & 3 \\
\hline x Sans objet & 1 & 1 & 1 & 0 & 2 & 2 & 8 & 16 & 16 \\
\hline
\end{tabular}

Source: Enquête de l'OCDE sur les pratiques et procédures budgétaires (2012).

StatLink AाISL $h t t p: / / d x . d o i . o r g / 10.1787 / 888933159908$ 
Pour être ouverte et inclusive, l'élaboration des politiques doit être transparente, fondée sur des données probantes, accessible et sensible aux attentes d'un éventail aussi large que possible de citoyens. Elle doit avoir pour objectif d'ouvrir le processus d'élaboration des politiques à des voix et des opinions diverses, y compris celles des représentants des cultures traditionnelles. Pour que le processus soit couronné de succès, ces éléments doivent être présents à toutes les étapes de la conception et de la mise en œuvre des politiques et services publics. Si l'élaboration inclusive des politiques renforce la transparence, la responsabilité, la participation des citoyens et leurs compétences civiques, elle permet aussi aux pouvoirs publics de mener des politiques plus efficaces en collaborant avec les particuliers, les organisations de la société civile, les entreprises et les autres parties prenantes afin de produire de réelles améliorations au niveau des retombées de l'action menée et de la qualité des services publics.

Les évaluations de l'impact selon le sexe font partie des instruments que les décideurs publics peuvent employer pour évaluer, en fonction de critères pertinents au regard de la problématique hommes-femmes, l'impact spécifique que de nouveaux textes ou de nouvelles politiques peuvent avoir sur les femmes. Il est crucial de sensibiliser les décideurs aux effets différents que les orientations choisies peuvent avoir sur les hommes et sur les femmes si l'on souhaite une élaboration inclusive des politiques dans différents domaines. Néanmoins, même des décisions apparemment neutres sur le plan de l'égalité des sexes peuvent influer, de façon intentionnelle ou non, sur l'aptitude des femmes à jouer un rôle égal au sein de la société. Sous l'effet de certaines mesures, les femmes peuvent avoir plus de difficultés à trouver un emploi, à suivre des études, à créer une entreprise, à subvenir aux besoins de leur famille ou à faire valoir leurs droits fondamentaux. Par exemple, un texte permettant aux deux parents de prendre un congé pour rester au chevet d'un enfant malade a des chances de concerner avant tout les femmes, qui assument la plus grande part des tâches liées aux enfants. Les évaluations de l'impact selon le sexe peuvent être effectuées en amont (avant que la loi ou politique concernée ne soit adoptée ou n'entre en vigueur) et en aval (après son entrée en vigueur). D'après une enquête de l'OCDE sur la parité dans les cadres nationaux, les politiques publiques et l'accès aux responsabilités, les évaluations en amont sont plus courantes. S'agissant des textes législatifs, par exemple, $84 \%$ des pays de l'OCDE interrogés (soit 16 pays) déclarent exiger une évaluation en amont de l'impact selon le sexe, alors qu'ils ne sont que $37 \%$ (soit 7 pays) à exiger une évaluation en aval de cet impact. Toutefois, de manière générale, ces évaluations ne semblent pas être pleinement entrées dans les mœurs : la majorité des pays interrogés les réservent aux textes législatifs et réglementaires, sans y recourir pour les politiques et les programmes.

La budgétisation prenant en compte la problématique hommes-femmes est probablement la forme la plus connue d'évaluation de l'impact selon le sexe. Elle tient compte de cette problématique à toutes les étapes du cycle d'élaboration des budgets. L'objectif consiste à éviter les dépenses " asexuées " et à améliorer l'efficacité des programmes publics en repérant les dépenses publiques produisant des effets disproportionnés selon le sexe. À peine plus de la moitié des pays interrogés (10) déclarent avoir instauré des obligations en la matière. La Belgique, la Corée, l'Espagne, la Finlande, la France, Israël, le Mexique, la Norvège, la République slovaque et la Suède indiquent procéder systématiquement à de telles évaluations pour les budgets centraux/fédéraux.

La consultation des citoyens offre aux pouvoirs publics un deuxième moyen d'ouvrir le processus d'élaboration des politiques. Selon les Principes directeurs pour l'élaboration transparente et participative des politiques formulés par l'OCDE, tous les citoyens doivent bénéficier des mêmes opportunités et avoir à leur disposition des canaux multiples pour accéder à l'information, être consultés et participer, et il convient de mettre en œuvre tous les efforts nécessaires pour faire participer un public aussi divers que possible. Dans cette optique, les autorités des pays de l'OCDE s'appuient sur les technologies de l'information et des communications (TIC) afin de renforcer la sensibilisation et la participation. Le recours aux consultations basées sur les TIC varie considérablement selon les pays et, en moyenne, les citoyens utilisent peu ces technologies dans les pays de l'Union européenne. D'après les Statistiques sur la société de l'information d'Eurostat (base de données), en moyenne, moins de $10 \%$ des particuliers déclarent avoir utilisé Internet pour participer à des consultations en ligne ou pour voter sur des sujets civiques ou politiques (par ex. urbanisme ou signature de pétitions). C'est dans les pays nordiques que la propension à recourir à des outils de consultation ou de vote en ligne est la plus forte.

\section{Méthodologie et définitions}

Les données portent sur 2012 et sont tirées des réponses fournies par les pays à une enquête de 2011 de l'OCDE sur la parité dans les cadres nationaux, les politiques publiques et l'accès aux responsabilités. Les réponses ont été fournies, pour l'essentiel, par de hauts responsables budgétaires des pays de l'OCDE. Elles correspondent à l'évaluation que les pays font eux-mêmes de leurs pratiques et procédures actuelles. Les données portent uniquement sur les administrations centrales et fédérales.

Les indicateurs relatifs aux particuliers et aux entreprises sont tirés des Statistiques sur la société de l'information d'Eurostat (base de données), qui évaluent la proportion des particuliers et des entreprises qui utilisent Internet pour participer à des consultations en ligne ou pour voter sur des sujets civiques ou politiques (par ex. urbanisme ou signature de pétitions). Les données sont recueillies par les offices statistiques nationaux à partir de l'enquête type annuelle d'Eurostat sur l'utilisation des TIC et le commerce électronique dans les entreprises et de l'enquête communautaire annuelle d'Eurostat sur l'utilisation des TIC par les ménages et les particuliers.

\section{Pour en savoir plus}

OCDE (2012), Inégalités hommes-femmes: Il est temps d'agir, Éditions OCDE, Paris, 10.1787/9789264179660-fr.

OCDE (2009), Focus on Citizens: Public Engagement for Better Policy and Services, OECD Studies on Public Engagement, Éditions OCDE, Paris, http://dx.doi.org/10.1787/9789264048874-en.

\section{Notes relatives aux graphiques}

8.9: On ne dispose pas de données pour l'Autriche, le Canada, le Danemark, l'Estonie, les États-Unis, la Hongrie, l'Islande, l'Italie, le Japon, les Pays-Bas, la Pologne, le Portugal, le Royaume-Uni, la Slovénie et la Turquie.

8.10: On ne dispose pas de données pour l'Australie, le Canada, le Chili, la Corée, les États-Unis, Israël, le Japon, le Mexique, la NouvelleZélande, la Suisse et la Turquie.

Informations sur les données concernant Israël : http://dx.doi.org/ $10.1787 / 888932315602$. 
8.9. Obligation de procéder à des évaluations de l’impact selon le sexe au niveau de l'administration centrale/fédérale (2011)

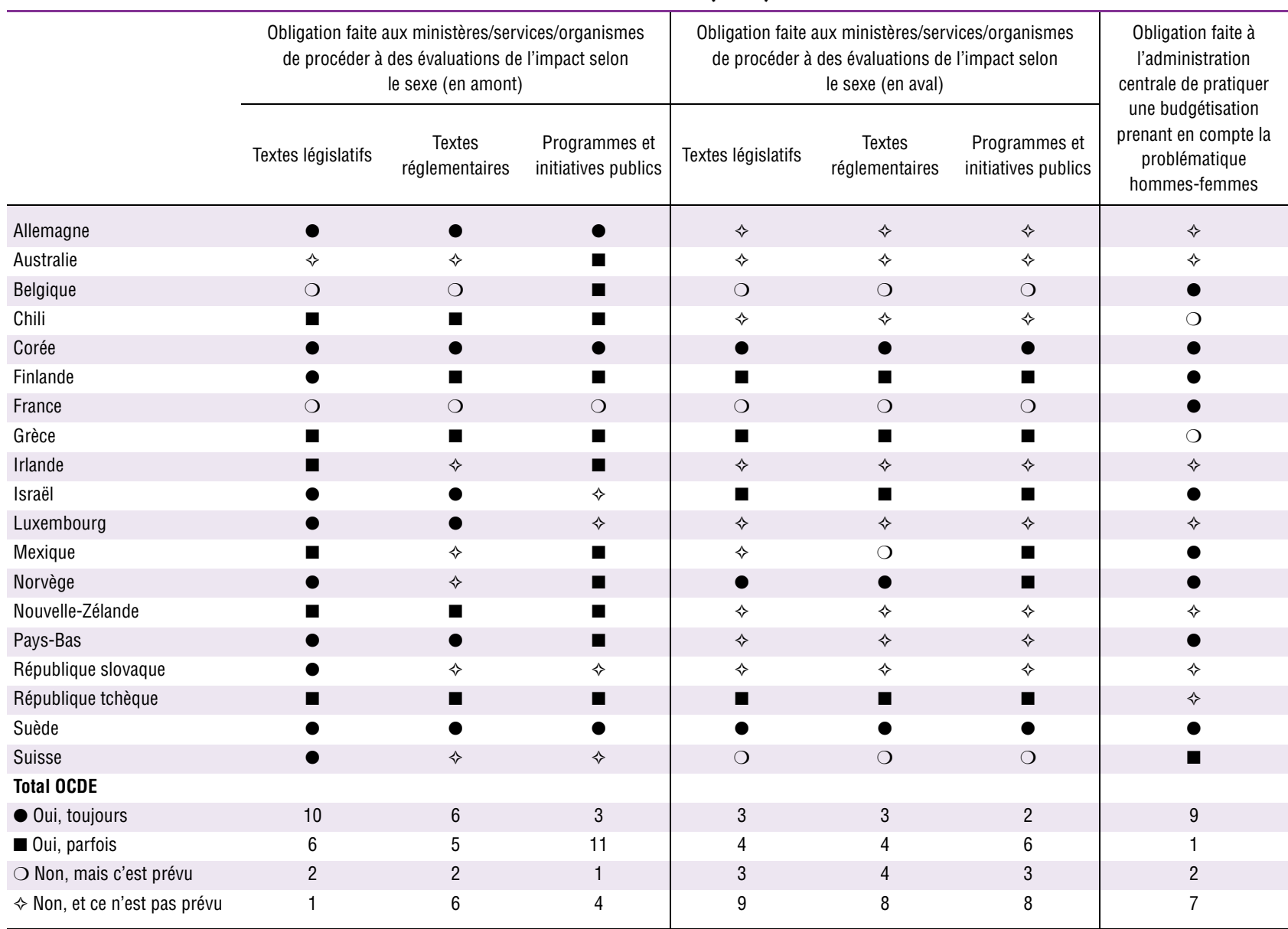

Source: Enquête de l'OCDE sur la parité dans les cadres nationaux, les politiques publiques et l'accès aux responsabilités (2011).

\subsection{Pourcentage de particuliers ayant participé à des consultations ou des votes en ligne}

Pourcentage des particuliers

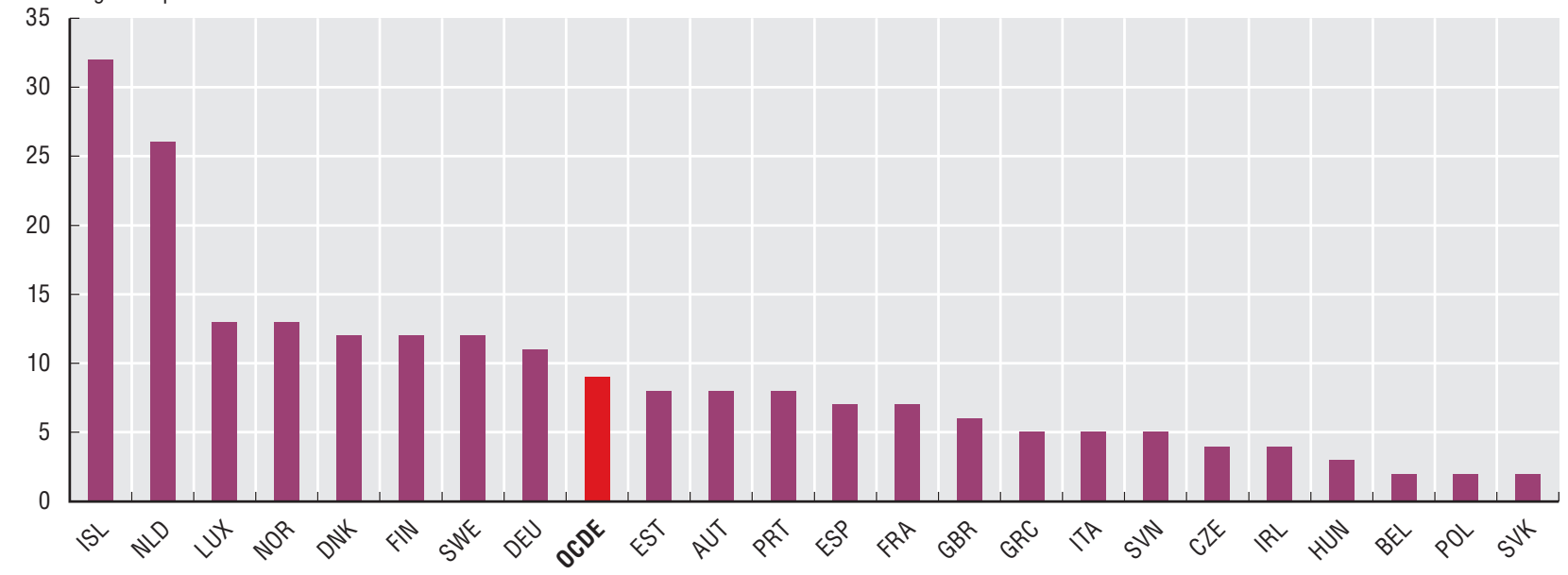

Source: Statistiques sur la société de l'information d'Eurostat (base de données). 


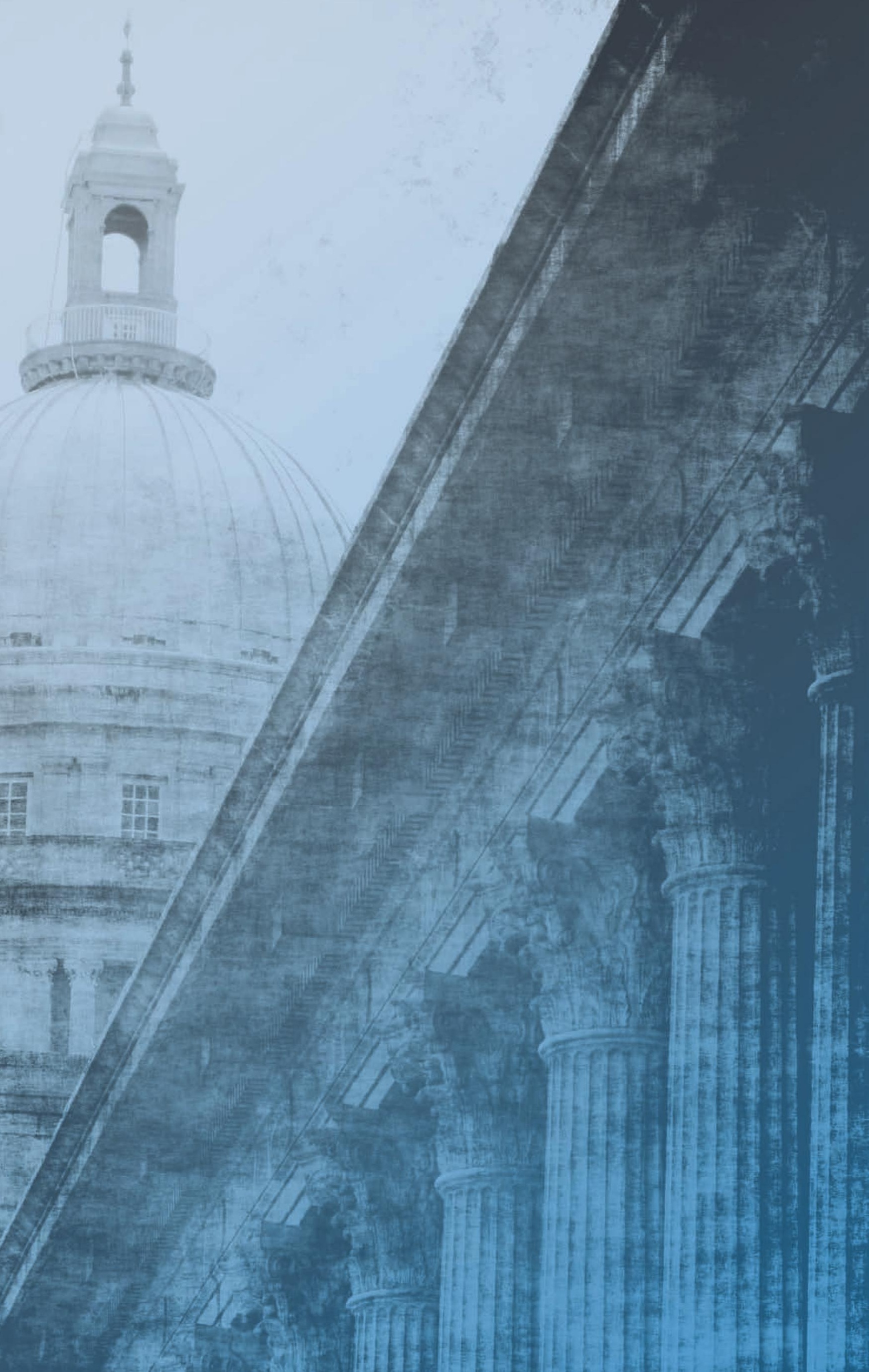




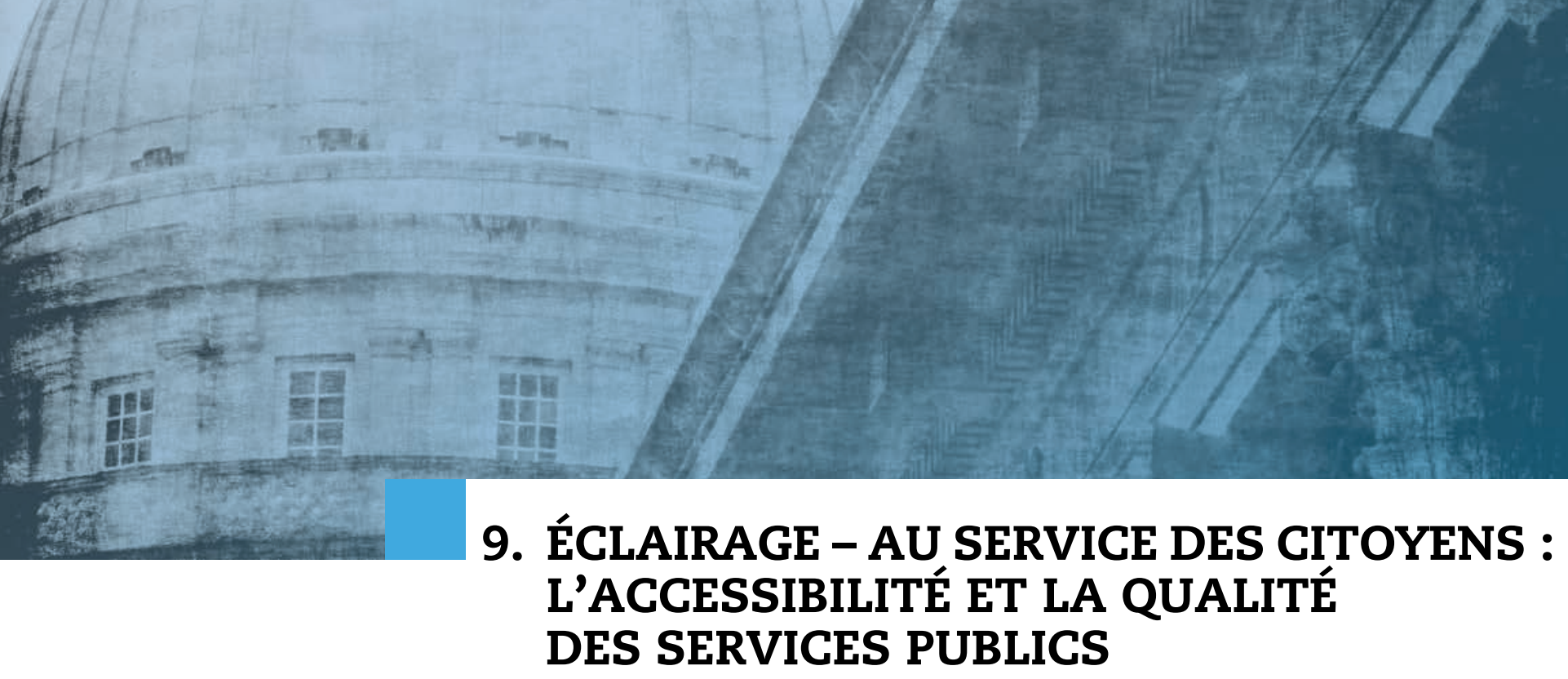

Les administrations des pays membres de l'OCDE s'emploient de plus en plus à mettre des biens et services publics de qualité à la disposition d'un éventail de citoyens aussi large que possible, tout en veillant à mieux répondre à la diversité des besoins individuels. De nombreux pays sont en train d'instaurer des exigences plus rigoureuses en matière de niveau de service et de performance et de mettre en œuure des dispositifs visant à mesurer les réactions des citoyens et à les prendre en compte dans le cadre de la prestation des services publics.

Plusieurs raisons expliquent ce passage à des stratégies plus axées sur les citoyens. Ces derniers, plus instruits et mieux informés, jugent les administrations publiques sur leurs performances, et notamment sur la qualité de service. De fait, les expériences vécues par les citoyens dans leurs rapports avec les organismes publics - et leur degré de satisfaction concernant l'accueil, les biens et les services dont ils ont bénéficié - peuvent influer sur leur perception des capacités et de l'équité des institutions publiques et déterminer s'ils considèrent qu'il est fait bon usage de leurs impôts et autres contributions financières. De plus, en période d'austérité fiscale, il est également nécessaire de mesurer la qualité de service afin d'évaluer l'impact des restrictions budgétaires sur les services assurés et de déterminer si les administrations et organismes publics parviennent à réaliser des gains d'efficience sans porter préjudice aux aspects auxquels les citoyens accordent le plus d'importance.

Le présent chapitre constitue une première tentative visant à comparer certaines dimensions de la qualité de service non seulement au niveau des pays, mais aussi au niveau des services publics essentiels que sont l'éducation, la santé, la justice et l'administration fiscale. Il présente un choix d'indicateurs axés sur les citoyens et relatifs à différentes dimensions de l'accessibilité et de la qualité des services publics : l'accessibilité financière, l'existence de services en ligne, la rapidité d'exécution et la fiabilité. Des données d'opinion relatives au niveau de satisfaction affiché par les citoyens à l'égard de ces services sont également présentées. Chacune de ces dimensions de la qualité de service comporte différentes facettes. Ainsi, la notion « d'accessibilité » est susceptible de renvoyer à plusieurs facteurs, allant de la proximité géographique avec les prestataires des services à l'adaptation des installations ou des modes de prestation à différents besoins. Ce chapitre ne présente cependant qu'un sous-ensemble d'indicateurs, choisis en fonction de la disponibilité des données et de leur comparabilité d'un pays membre à l'autre. 
L'impact des biens et services publics de base tels que l'éducation, les soins de santé ou la justice sur la vie des citoyens dépend largement de la mesure dans laquelle les destinataires de ces services peuvent y avoir accès et en bénéficier effectivement. L'accessibilité des services publics peut être considérée comme un critère de performance des administrations publiques, car elle reflète leur capacité à prendre en compte la diversité et la nature des différents besoins, à concevoir des modes de prestation et de communication adaptés et à garantir l'équité et l'impartialité au niveau de la fourniture et de la répartition des prestations assurées.

Les obstacles entravant l'accès aux services publics peuvent prendre différentes formes : éloignement géographique, installations inadaptées aux usagers ayant besoin d'arrangements spéciaux, choix trop restreint de modes de prestation des services (Internet, téléphone, face-à-face, etc.), insuffisance des informations disponibles ou emploi d'un langage complexe, manque d'interprètes ou de traducteurs pour les usagers ne maîtrisant pas la langue du pays, heures d'ouverture peu pratiques ou encore formalités administratives excessives. De tels obstacles peuvent empêcher les usagers de savoir qu'ils ont droit à certains services ou que ces services existent, ou encore les dissuader d'essayer d'en bénéficier. L'accessibilité financière, c'est-à-dire les coûts économiques de l'acquisition d'un bien ou d'un service par rapport aux revenus des personnes concernées, peut par ailleurs représenter un obstacle majeur à l'accès aux services. Ce critère financier peut non seulement déterminer si un bien ou un service sera consommé, mais aussi avoir un impact sur la confiance des citoyens dans le caractère équitable des institutions publiques.

Si les administrations ont tout intérêt à ce que les citoyens, en particulier les personnes à faibles revenus ou d'autres groupes vulnérables, puissent s'offrir les services de base, elles doivent aussi trouver le juste équilibre entre les préoccupations relatives à l'équité et au bien-être et la volonté de laisser des choix à l'usager et d'inciter les prestataires de services publics à améliorer leurs performances. En effet, subventionner ou fournir des services à des prix inférieurs à ceux du marché peut avoir pour effet d'inciter les usagers à consommer des biens dont ils n'ont pas besoin ou encore d'affaiblir la concurrence, réduisant ainsi le nombre de fournisseurs et les incitations à améliorer la qualité.

\section{Services de santé}

Si la plupart des pays membres de l'OCDE offrent une couverture maladie universelle pour un panier de services essentiels, il se peut que les citoyens doivent payer certains services ou médicaments. Ces coûts peuvent conduire certains d'entre eux à renoncer à consulter ou à entreprendre un traitement, ou à tarder à le faire, ce qui a pour effet de limiter de manière générale l'accès aux soins de santé et peut entraîner des coûts accrus liés à une hospitalisation ultérieure. En outre, les citoyens ayant davantage besoin des services de santé peuvent voir peser sur eux une part plus lourde de ces coûts s'ils ne sont pas exemptés de certains d'entre eux ou en l'absence de plafonnement des dépenses restant à leur charge. Au sein de la zone OCDE, il existe de forts écarts au niveau de la part des dépenses de santé à la charge des patients dans la consommation finale des ménages. Ces dépenses représentent $1.5 \%$ de la consommation finale des ménages en France, aux Pays-Bas, au Royaume-Uni et en Turquie, mais plus de $4 \%$ au Chili, en Corée, au Mexique et au Portugal (graphique 9.1). Il est surtout important de s'intéresser à la répartition de ces dépenses selon les niveaux de revenus. Ainsi, de nombreux pays prévoient des exemptions et des plafonnements des dépenses à la charge des patients ayant les revenus les plus faibles, de manière à préserver leur accès aux soins.

\section{Justice}

Les citoyens subissent également des délais et des coûts lorsqu'ils doivent faire appel à la justice. Dans des cas où ils sont défendeurs ou accusés, ils doivent en outre supporter des frais d'avocat. La fourniture d'une aide judiciaire peut faciliter l'accès à la justice, en permettant même à ceux qui n'auraient pas les ressources financières nécessaires d'exercer leur droit à un procès équitable. L'aide judiciaire telle qu'elle est entendue ici peut prendre la forme d'une représentation légale gratuite ou subventionnée, de conseils juridiques et d'exemptions de frais. La proportion des affaires dans lesquelles l'aide judiciaire est accordée peut donner une idée de l'ampleur des soutiens financiers publics mis à la disposition des citoyens. Sur les 11 pays membres de l'OCDE pour lesquels des données sont disponibles, quatre - la Finlande, la France, la Norvège et les Pays-Bas - assurent aux citoyens une aide judiciaire dans plus de $10 \%$ des affaires de première instance. Dans les autres pays, l'aide judiciaire est accordée pour un pourcentage inférieur ou égal à $6 \%$ des affaires de première instance (graphique 9.2). Dans certains pays, tels que l'Autriche, les affaires de première instance sans contestation sur l'objet du litige ne donnent pas toujours lieu à l'octroi de l'aide judiciaire, ce qui peut fausser les chiffres relatifs à la proportion d'affaires bénéficiant du soutien de l'État.

\section{Éducation}

En plus des coûts directs, les citoyens doivent quelquefois faire face à des frais indirects pour accéder aux services publics. Par exemple, en sus des droits universitaires, ils devront prendre en considération les frais de subsistance, ainsi que les revenus potentiels auxquels ils renoncent en décidant d'entamer des études supérieures. Dans cinq pays - le Chili, les États-Unis, le Japon, la Nouvelle-Zélande et le Royaume-Uni - les frais de scolarité exigés par les établissements publics d'enseignement supérieur représentent au moins $14 \%$ du revenu disponible par personne (graphique 9.3). Dans cinq autres pays - l'Autriche, l'Espagne, l'Italie, les Pays-Bas et la Suisse - ces frais pèsent beaucoup moins sur le revenu des citoyens, puisqu'ils représentent de $3 \%$ à $7 \%$ du revenu disponible par personne. Enfin, dans cinq autres pays - le Danemark, la Finlande, le Mexique, la Norvège et la Suède - les établissements publics d'enseignement supérieur n'exigent pas de frais de scolarité.

Dans dix pays, la moitié des étudiants au moins bénéficie d'un soutien financier. Parmi ces pays, on retrouve cinq des six pays pratiquant des frais de scolarité supérieurs à la moyenne (Australie, Chili, États-Unis, Nouvelle-Zélande et Royaume-Uni), un pays où les frais de scolarité rapportés au revenu disponible par personne sont relativement modérés (Pays-Bas) et la majorité des pays n'exigeant pas de frais de scolarité (Danemark, Finlande, Norvège et Suède). Dans ces pays, en moyenne, environ $71 \%$ des citoyens de la classe d'âge concernée entreprennent des études supérieures, alors qu'ils ne sont que $45 \%$ environ, en moyenne, dans les pays où moins de la moitié des étudiants bénéficie d'un soutien financier. 


\section{Méthodologie et définitions}

Les données sur les dépenses de santé à la charge des patients proviennent des Statistiques de l'OCDE sur la santé 2013. Les dépenses de santé à la charge des patients sont celles supportées directement par les patients lorsque les systèmes d'assurance publics et privés ne couvrent pas en totalité le coût d'un produit ou d'un service de santé. Elles incluent la participation des patients aux coûts et les autres dépenses payées directement par les ménages, et englobent également, pour certains pays, les estimations des paiements informels aux prestataires de soins. Seules les dépenses médicales (à savoir les dépenses en soins de santé, moins les dépenses pour le volet sanitaire des soins de longue durée) sont présentées ici, car la capacité des pays à évaluer les dépenses privées afférentes aux soins de longue durée est très variable.

Les données sur l'aide judiciaire et les affaires de première instance proviennent de l'évaluation des systèmes judiciaires menée par la Commission européenne pour l'efficacité de la justice (CEPEJ). L'aide judiciaire consiste en l'affectation de fonds publics visant à subventionner ou à financer les frais d'avocat. Elle peut aussi consister en la fourniture de conseils juridiques gratuits ou en l'exemption de certains frais. Les critères d'octroi de l'aide judiciaire varient selon les pays et peuvent aussi bien porter sur les caractéristiques du justiciable concerné que sur celles de son affaire. Les données sur les coûts estimatifs des affaires proviennent de la base de données Doing Business du Groupe de la Banque mondiale. Les coûts sont rapportés au montant du litige, considéré comme équivalant à $200 \%$ du revenu par habitant. La corruption n'est pas prise en compte. Trois types de coûts sont pris en compte : les frais de justice, les frais d'exécution et les honoraires moyens des avocats.

Les données relatives aux frais de scolarité, aux aides financières et aux taux d'accès à l'enseignement supérieur de type A proviennent de Regards sur l'éducation 2013 : Les indicateurs de l'OCDE (OCDE, 2013). Les frais de scolarité ne concernent que les programmes de type A du premier degré des établissements publics (en équivalents USD convertis sur la base des PPA) pour l'année universitaire 2010-2011. Le revenu disponible ajusté désigne le montant maximal qu'une unité peut se permettre de dépenser pour l'acquisition de biens ou de services de consommation sans avoir à entamer ses actifs financiers ou non financiers ni à accroître son endettement, ajusté pour tenir compte des transferts publics. Les taux d'accès désignent le pourcentage estimé des individus appartenant à une classe d'âge accédant pour la première fois à l'enseignement supérieur de type A.

\section{Pour en savoir plus}

CEPEJ (2012), Systèmes judiciaires européens - Édition 2012. Efficacité et qualité de la justice, Éditions du Conseil de l'Europe, Strasbourg.

OCDE (2013a), Regards sur l'éducation 2013 : Les indicateurs de l'OCDE, Éditions OCDE, Paris, http://dx.doi.org/10.1787/eag2013-fr.

OCDE (2013b, à paraître), Panorama de la santé 2013 : Les indicateurs de l'OCDE, Éditions OCDE, Paris.

\section{Notes relatives aux graphiques}

9.1 : Les données relatives à l'Australie, à l'Autriche, au Canada, au Japon, au Mexique, à la Norvège et à la Nouvelle-Zélande portent sur 2010. Les données relatives à Israël portent sur 2009. Les données relatives à la Turquie portent sur 2008.

9.2 : Affaires pénales et autres que pénales confondues pour tous les pays, à l'exception de l'Autriche et de la France, pour lesquelles les données concernent uniquement les affaires autres que pénales.

9.3: Pour les États-Unis, le taux d'accès à l'enseignement et le pourcentage des étudiants bénéficiant de prêts ou de bourses publics concernent tous les étudiants (ressortissants nationaux scolarisés à temps plein et étudiants non ressortissants nationaux/étrangers scolarisés à temps plein), et les données relatives aux taux d'accès concernent tant les programmes de type A que les programmes de type B. Pour la France, les frais de scolarité moyens, qui vont de 200 à 1402 USD selon les programmes universitaires, dépendent du ministère de l'Éducation. Pour le Japon, les frais de scolarité indiqués concernent les établissements publics; or, plus des deux tiers des étudiants sont inscrits dans des établissements privés. Pour le Chili, si seuls les établissements publics sont pris en compte, la proportion des étudiants bénéficiant de prêts et/ou bourses/allocations publics s'élève à $68 \%$. Données sur les prêts/bourses : les données relatives à l'Australie ne tiennent pas compte des étudiants étrangers. Les données relatives au Mexique et au Royaume-Uni portent sur l'année universitaire 2008-2009. Les données relatives au Danemark, aux États-Unis, à la France, au Mexique et aux Pays-Bas ne concernent que les universités publiques, y compris l'enseignement supérieur de type B en France. Voir l'annexe 3 de Regards sur l'éducation 2013 : Les indicateurs de l'OCDE (OCDE, 2013) pour de plus amples informations (www.oecd.org/fr/edu/rse.htm).

Informations sur les données concernant Israël : $h t t p: / / d x . d o i . o r g / 10.1787 /$ 888932315602. 


\section{9. ÉCLAIRAGE - AU SERVICE DES CITOYENS : L'ACCESSIBILITÉ ET LA QUALITÉ}

\section{L'accès aux services publics : l'accessibilité financière}

9.1. Dépenses de santé à la charge des patients, en pourcentage de la consommation finale des ménages (2011 ou dernière année disponible)

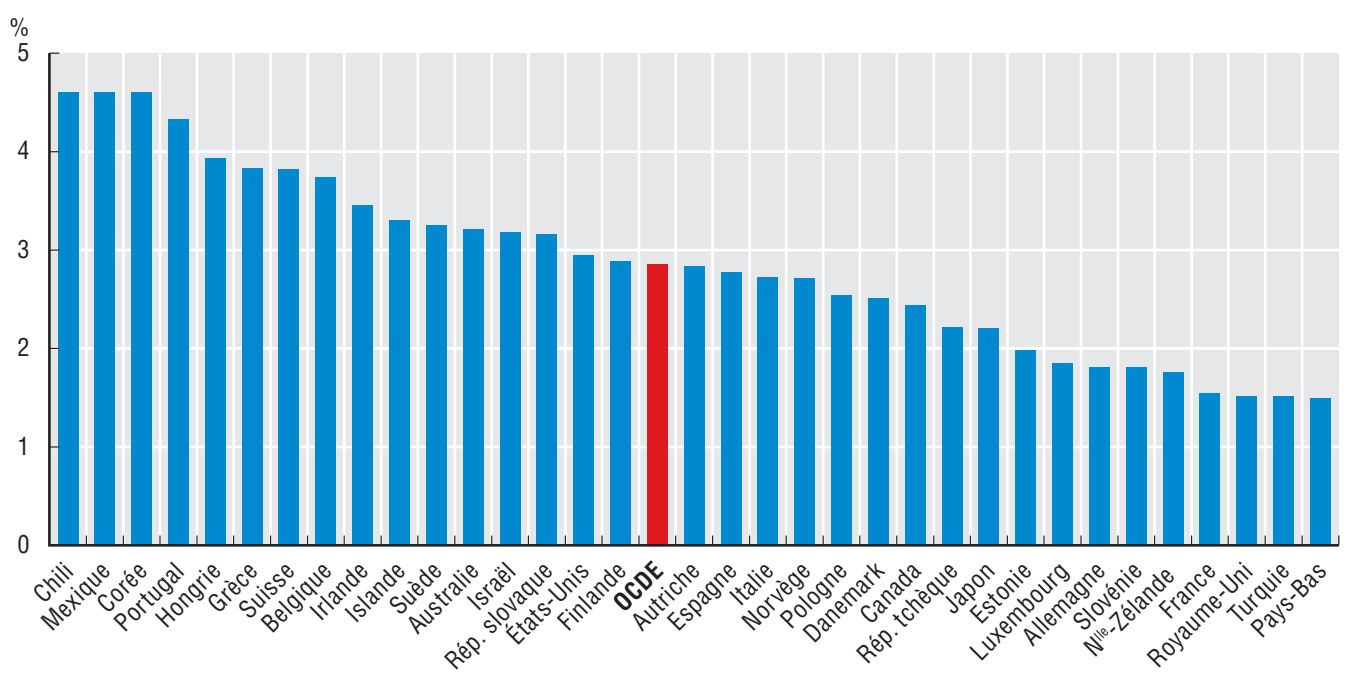

Source: Statistiques de l'OCDE sur la santé 2013.

StatLink ins $h$ ittp://dx.doi.org/10.1787/888933159948

9.2. Affaires ayant bénéficié de l'aide judiciaire (2010)

En pourcentage des affaires de première instance

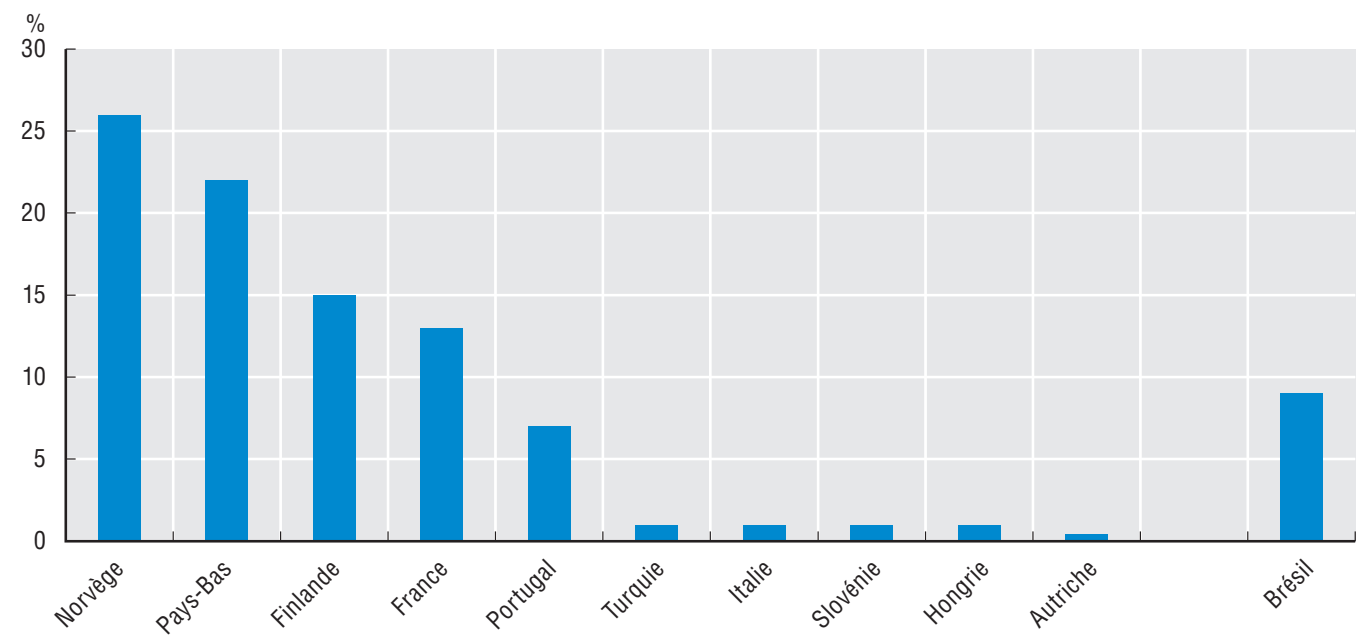

Source : CEPEJ (2012), Systèmes judiciaires européens (base de données). Calculs de l'OCDE basés sur les questions 91, 94, 97, 98, 99 et 100. Les données relatives au Brésil ont été fournies par les autorités nationales.

StatLink Aाsस http://dx.doi.org/10.1787/888933159953 
9.3. Frais de scolarité et aides financières (2010-11)

O Taux d'accès à l'enseignement supérieur de type $\mathrm{A}$

Frais de scolarité en \% du revenu disponible

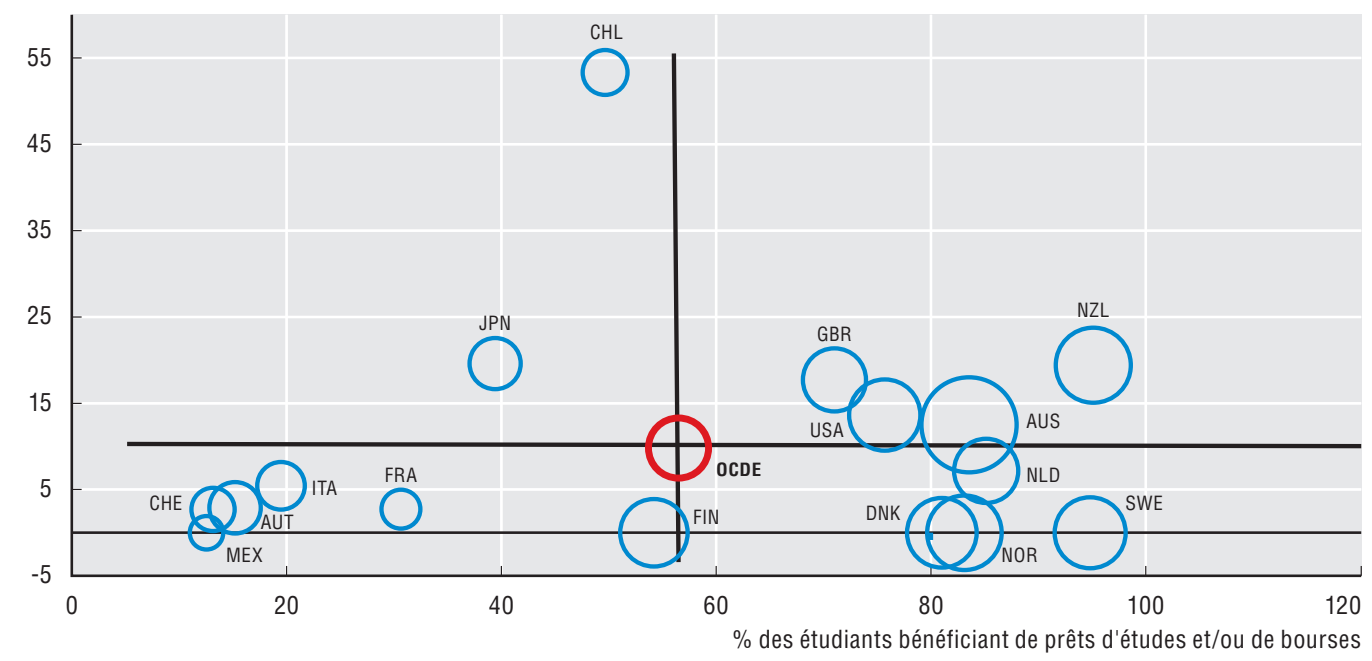

Source: OCDE (2013), Regards sur l'éducation 2013 : Les indicateurs de l'OCDE, Éditions OCDE, http://dx.doi: 10.1787/eag-2013fr (tableaux B5.1, B5.2 et C3.1a) et OCDE (2013), Panorama des comptes nationaux 2013, Éditions OCDE, http://dx.doi: 10.1787/ na_glance-2013-fr. 
Les particuliers comme les entreprises tendent de plus en plus à privilégier les canaux numériques dans leurs relations avec les administrations et pour accéder aux services publics. Les outils en ligne peuvent permettre à un éventail plus large d'utilisateurs d'accéder aux services publics, avec une commodité accrue et des coûts moindres pour toutes les parties prenantes, y compris l'administration. Si, de façon générale, l'adoption des services en ligne est en progression, des écarts de fréquentation persistent à différents égards dans de nombreux pays de l'OCDE. Les administrations publiques doivent poursuivre leurs efforts en vue de réduire ces disparités, tout en continuant à développer leur offre de services en ligne.

\section{La fréquentation des services publics en ligne par les entreprises}

Les services publics en ligne peuvent permettre aux entreprises de gagner du temps et d'alléger leurs contraintes administratives, tout en accroissant la probabilité qu'elles recourent aux TIC dans le cadre de leur exploitation dans un objectif d'amélioration de la productivité. Les entreprises étant considérées comme plus disposées que les particuliers à utiliser les services publics en ligne, les pays prennent des mesures visant à faire progresser encore leur niveau de fréquentation de ces services, notamment en rendant leur utilisation obligatoire pour certains types de formalités.

En Islande, en Finlande et en République slovaque, parmi d'autres pays, le niveau général de dialogue en ligne avec les autorités publiques est élevé, même si la situation semble assez différente en ce qui concerne l'utilisation des services réellement transactionnels, comme le traitement électronique des démarches administratives. Dans ce domaine, l'Australie, la France et la Slovénie arrivent en tête des pays membres. La législation propre à chaque pays, des différences quant à l'obligation faite aux usagers de passer par un service en ligne et le recours à des intermédiaires dans la prestation des services peuvent contribuer à expliquer les disparités entre les pays.

De manière générale, le niveau de fréquentation des services administratifs en ligne par les grandes entreprises (de 250 salariés ou plus) est très élevé ; cependant, ces entreprises ne représentent en moyenne que moins de $1 \%$ du nombre total d'entreprises de la zone OCDE. Le niveau de fréquentation des entreprises de taille moyenne (de 50 à 249 salariés) est assez proche de celui des grandes entreprises ; en revanche, il existe un écart considérable de fréquentation entre les grandes et les petites entreprises (de 10 à 49 salariés), dans tous les pays. Ce sont les pays comme l'Australie, la Corée, l'Espagne, la Hongrie et l'Italie qui présentent certains des écarts de fréquentation les plus importants entre grandes et petites entreprises.

Faire progresser la fréquentation des services en ligne par les entreprises présente un double avantage. Premièrement, orienter les entreprises vers les services publics assurés en ligne peut permettre aux administrations de réduire leurs dépenses - c'est pourquoi les pouvoirs publics se tournent de plus en plus vers des services où il est possible d'obliger les entreprises à utiliser les outils en ligne (par exemple pour les déclarations de TVA, l'octroi de licences ou des inscriptions). Deuxièmement, alors que le niveau de fréquentation des services publics en ligne par les petites entreprises est plus faible, les petites et moyennes entreprises restent un moteur important pour la croissance dans les pays de l'OCDE. Par conséquent, accorder une attention particulière à l'amélioration de leur accès aux services en ligne et de leurs capacités en matière de TIC peut alimenter la croissance économique de manière générale, et non pas exclusivement dans les domaines touchant à Internet.

\section{La fréquentation des services publics en ligne par les particuliers}

Les particuliers ont moins recours à Internet que les entreprises pour accéder aux services publics. Les pays nordiques montrent la voie dans ce domaine, tant en termes d'utilisation d'Internet par les citoyens pour dialoguer avec les administrations de manière générale que pour des démarches plus poussées telles que la télétransmission de formulaires remplis. En France, en Irlande et aux Pays-Bas, le niveau d'utilisation d'Internet par les citoyens pour la télétransmission de formulaires remplis aux autorités est lui aussi élevé.

Les taux moyens d'utilisation des services administratifs en ligne par les particuliers dissimulent des disparités importantes selon l'âge, le niveau d'études ou le niveau de revenus. Par exemple, la classe d'âge qui a le plus recours à Internet dans ses relations avec l'administration est celle des personnes de 25 à 34 ans. Une comparaison avec la population des 16 à 24 ans semble révéler l'existence d'un déficit de fréquentation des services administratifs en ligne chez cette dernière. Plusieurs motifs peuvent être avancés pour expliquer ce phénomène : ainsi, de manière générale, les 16-24 ans utilisent peut-être moins les services publics car ils restent rattachés au foyer de leurs parents. Dans une partie des pays, certains des services administratifs en ligne les plus communément déployés, comme les déclarations d'impôt en ligne, ne concernent pas les classes d'âge les plus jeunes, ou correspondent à des démarches que les jeunes n'effectuent pas nécessairement eux-mêmes. En revanche, dans de nombreux pays, des questions telles que l'inscription sur les listes électorales, le permis de conduire, les demandes de prestations, l'éducation ou le choix d'une profession obligent les plus jeunes à effectuer des démarches administratives ; le problème se pose donc bel et bien.

Les personnes âgées ont elles aussi moins tendance à recourir à Internet pour accéder aux services publics. Dans un certain nombre de pays, on constate des disparités considérables entre l'utilisation des services administratifs en ligne par la tranche d'âge des 25 à 34 ans et celle des 65 à 74 ans. Là encore, plusieurs explications peuvent être avancées. Le manque de compétences numériques représente un obstacle majeur pour les seniors, avec les inconvénients que cela entraîne à court et moyen terme pour les administrations qui s'efforcent de faire face aux évolutions démographiques en recourant davantage aux services sociaux numériques, et notamment aux services de santé en ligne comme la télémédecine.

Les administrations mettent en place un certain nombre de mesures visant à accroître la fréquentation des services en ligne par les particuliers. Par exemple, elles recensent les services pour lesquels les usagers sont prêts à accepter la suppression des canaux matériels; ou elles les incitent à recourir aux canaux numériques en offrant moins de rapidité s'ils optent pour un formulaire papier, voire en percevant des frais pour un accueil classique par un représentant de l'administration. Certains groupes de population, notamment les étudiants, font une utilisation intensive d'Internet, et il est plus facile de leur imposer le recours aux échanges numériques, comme cela a été fait au Danemark. Au Canada, une aide à la transition vers l'administration numérique, par exemple à 
travers l'utilisation des médias sociaux et grâce à des vidéos, contribue à faire progresser la fréquentation des services en ligne. Alors que certains pays mettent en place une approche visant à instaurer le "numérique par défaut » en imposant l'utilisation des canaux de communication électroniques, d'autres, tels que le Royaume-Uni, s'efforcent de créer une dynamique en faveur de l'administration numérique en s'appuyant sur la qualité des services en ligne fournis.

Les contacts à sens unique avec l'administration (par exemple, les demandes de renseignements) demeurent plus courants pour les particuliers comme pour les entreprises que les échanges plus complexes et de nature transactionnelle (par exemple, le traitement intégral de dossiers par voie électronique ou la télétransmission de formulaires remplis). La mise en place d'infrastructures adéquates et l'adoption de mesures visant à aider les usagers à s'acheminer vers ce dernier stade des échanges en ligne avec les administrations sont cruciales pour permettre aux administrations de tirer pleinement parti des gains d'efficience que peuvent autoriser les TIC.

\section{Méthodologie et définitions}

Les indicateurs relatifs aux particuliers et aux entreprises sont tirés des Statistiques sur la société de l'information d'Eurostat (base de données) et de la base de données de l'OCDE sur les TIC. Ils évaluent la proportion des particuliers et des entreprises qui utilisent Internet dans leurs relations avec les administrations publiques. Les deux sources sont comparables sur le plan méthodologique. Les données sont recueillies par les offices statistiques nationaux à partir de l'enquête type annuelle d'Eurostat sur l'utilisation des TIC et le commerce électronique dans les entreprises et de l'enquête communautaire annuelle d'Eurostat sur l'utilisation des TIC par les ménages et les particuliers.

Les données illustrent le recours à Internet pour les relations avec les autorités publiques. La notion d'autorités publiques désigne tant les services publics que les activités administratives, à tous les niveaux d'administration. Les relations avec l'administration représentent un indicateur dérivé qui englobe des souscatégories d'actions diverses réalisées par le biais d'Internet, comme les demandes de renseignements, le téléchargement et la télétransmission de formulaires ou la réalisation de démarches administratives et le traitement de dossiers. Bien que le recours à des intermédiaires soit largement répandu dans un certain nombre de pays, il n'est pas pris en considération dans ces données.

L'indicateur de fréquentation de l'administration électronique mesure le pourcentage d'entreprises ou de particuliers ayant utilisé Internet dans leurs relations avec les autorités publiques dans les douze mois précédant l'enquête. Le choix d'une période de douze mois permet d'effectuer une comparaison entre des pays ayant un calendrier de prestation de services variable selon les secteurs. Sont pris en considération : les entreprises de dix salariés ou plus, à l'exclusion du secteur financier; et les individus âgés de 16 à 74 ans.

\section{Pour en savoir plus}

OCDE/Union internationale des télécommunications (2011), MGovernment: Mobile Technologies for Responsive Governments and Connected Societies, Éditions OCDE, Paris, http://dx.doi.org/ 10.1787/9789264118706-en.

OCDE (2009), Rethinking e-Government Services: User-Centred Approaches, Éditions OCDE, Paris, http://dx.doi.org/10.1787/ 9789264059412-en.

\section{Notes relatives aux graphiques}

9.4 : On ne dispose pas de données pour le Canada, le Chili, les États-Unis, Israël, le Japon, le Mexique et la Turquie. On ne dispose pas de données, pour la Suisse, sur les échanges passant intégralement par la voie électronique et, pour l'Australie, sur les demandes de renseignements. Les données relatives à l'Australie, au Chili, à la Corée, à la NouvelleZélande et à la Suisse portent uniquement sur les demandes de renseignements, et non sur les relations avec l'administration de manière générale, et englobent le secteur financier. Les données relatives à l'Australie portent sur 2010 ; les données relatives à l'Islande, à la Nouvelle-Zélande et au Royaume-Uni portent sur 2012. Les données relatives à la Belgique et à la Finlande concernant les échanges par Internet portent sur 2012 ; les données relatives à la Belgique, à la Finlande, à l'Islande et au Royaume-Uni concernant les démarches administratives électroniques portent sur le traitement intégral de dossiers par voie électronique en 2010

9.5 : On ne dispose pas de données pour le Canada, le Chili, les États-Unis, Israël, le Japon, le Mexique et la Turquie. Les données relatives à l'Australie, à la Corée, à la Nouvelle-Zélande et à la Suisse portent uniquement sur les demandes de renseignements, et non sur les relations avec l'administration de manière générale, et englobent le secteur financier. Les données relatives à la Corée et à la Suisse portent sur 2011. Les données relatives à l'Australie portent sur 2010 et englobent l'utilisation d'Internet pour remplir des formulaires électroniques.

9.6 : On ne dispose pas de données pour la Corée, les États-Unis, le Mexique et la Turquie. On ne dispose pas de données sur la télétransmission de formulaires remplis pour l'Australie, Israël et la Suisse, ni sur les relations avec l'administration concernant le Japon. Les données relatives au Canada, à la Nouvelle-Zélande et à la Suisse portent exclusivement sur les demandes de renseignements, et non sur les relations avec l'administration de manière générale. Les données relatives à Israël portent sur les citoyens âgés de 20 ans et plus et englobent tant les demandes de renseignements que l'utilisation d'Internet pour remplir des formulaires électroniques. Les données relatives au Canada portent sur 2009 ; les données relatives à l'Australie, à Israël et à la Suisse portent sur 2010. Les données relatives à la Nouvelle-Zélande portent sur le téléchargement de formulaires, et non sur la télétransmission de formulaires remplis.

9.7 : On ne dispose pas de données pour la Corée, les États-Unis, le Japon, le Mexique et la Turquie. Les données relatives à l'Australie, au Canada, à Israël, à la Nouvelle-Zélande et à la Suisse portent exclusivement sur les demandes de renseignements, et non sur les relations avec l'administration de manière générale. Pour ces pays, la tranche d'âge des « 25-34 ans » désigne plutôt des individus âgés de 25 à 44 ans. Les données relatives au Chili portent sur le groupe des 25 à 64 ans. Les données relatives à Israël portent sur les citoyens âgés de 20 ans et plus et englobent tant les demandes de renseignements que l'utilisation d'Internet pour remplir des formulaires électroniques. Les données relatives au Canada, à Israël et à la Suisse portent sur 2010.

Informations sur les données concernant Israël : http://dx.doi.org/10.1787/ 888932315602 


\section{9. ÉCLAIRAGE - AU SERVICE DES CITOYENS : L'ACCESSIBILITÉ ET LA QUALITÉ}

\section{La fréquentation des services publics en ligne}

9.4. Entreprises utilisant Internet dans leurs relations avec les autorités publiques, selon le type d'activité (2011)

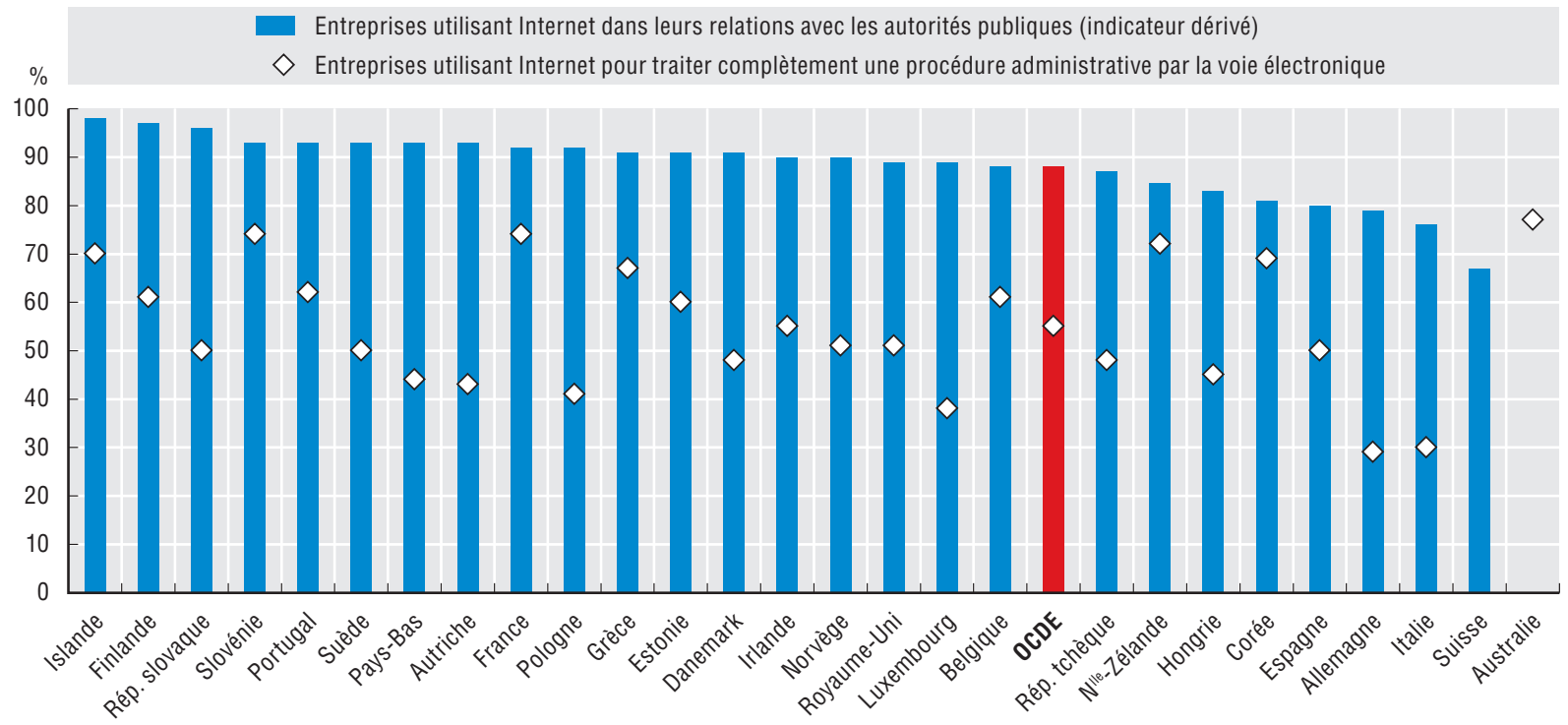

Source: Base de données de l'OCDE sur les TIC et Eurostat, Statistiques sur la société de l'information (base de données).

9.5. Entreprises utilisant Internet dans leurs relations avec les autorités publiques, selon la taille de l'entreprise (2012)

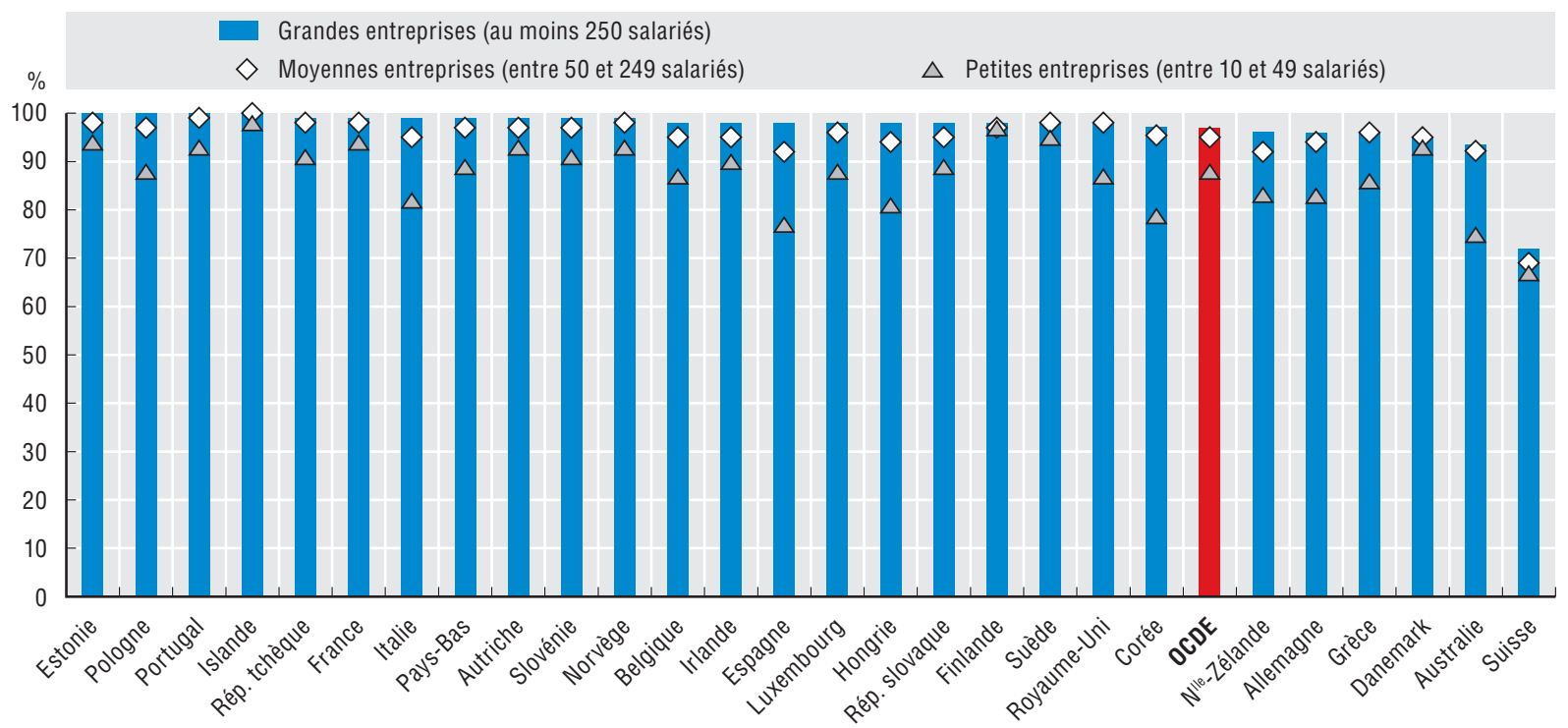

Source: Base de données de l'OCDE sur les TIC et Eurostat, Statistiques sur la société de l'information (base de données).

StatLink त्ञाज http://dx.doi.org/10.1787/888933159987 


\section{9. ÉCLAIRAGE - AU SERVICE DES CITOYENS : L'ACCESSIBILITÉ ET LA QUALITÉ}

9.6. Particuliers utilisant Internet dans leurs relations avec les autorités publiques, selon le type d'activité (2012)

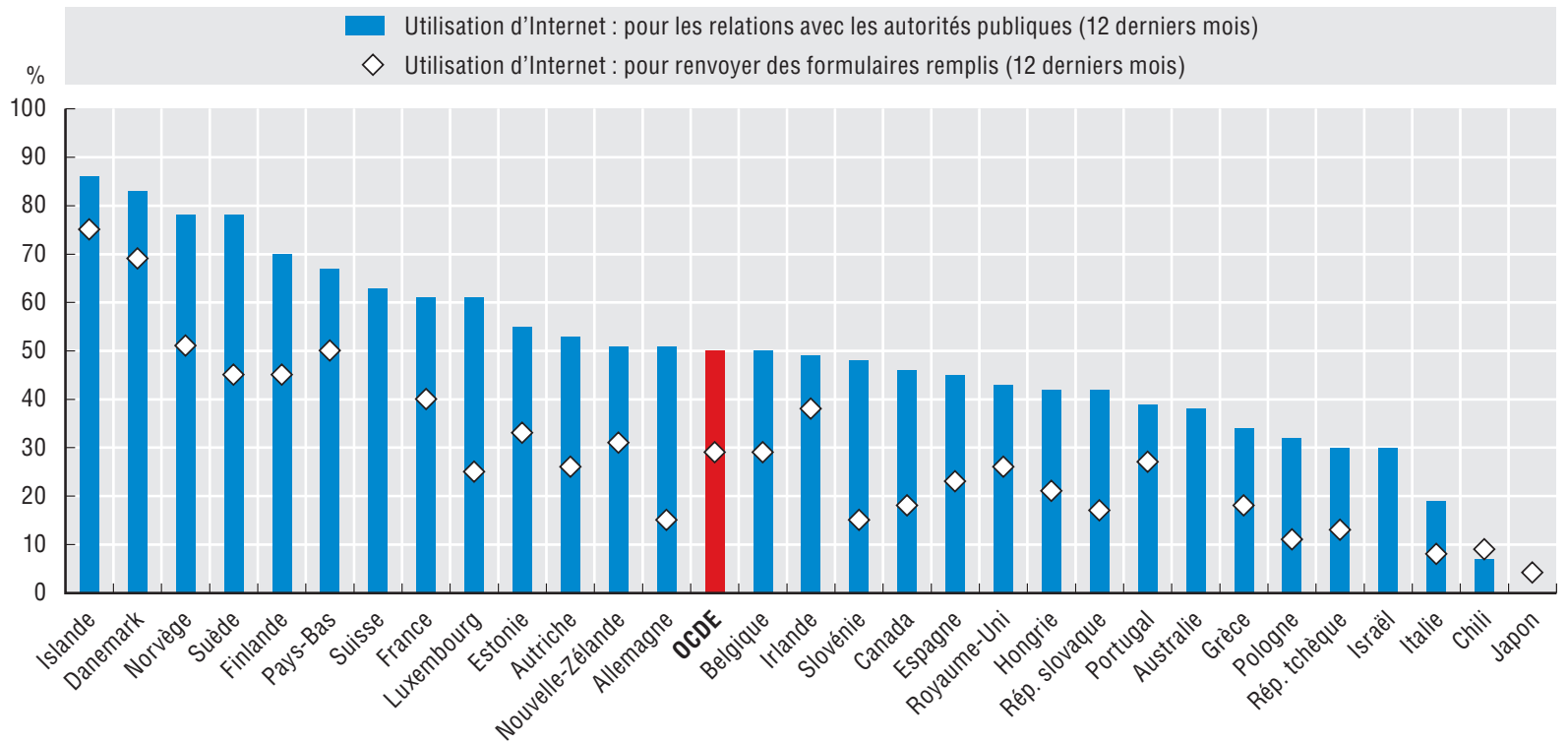

Source: Base de données de l'OCDE sur les TIC et Eurostat, Statistiques sur la société de l'information (base de données).

9.7. Particuliers utilisant Internet dans leurs relations avec les autorités publiques, par tranche d'âge (2012)

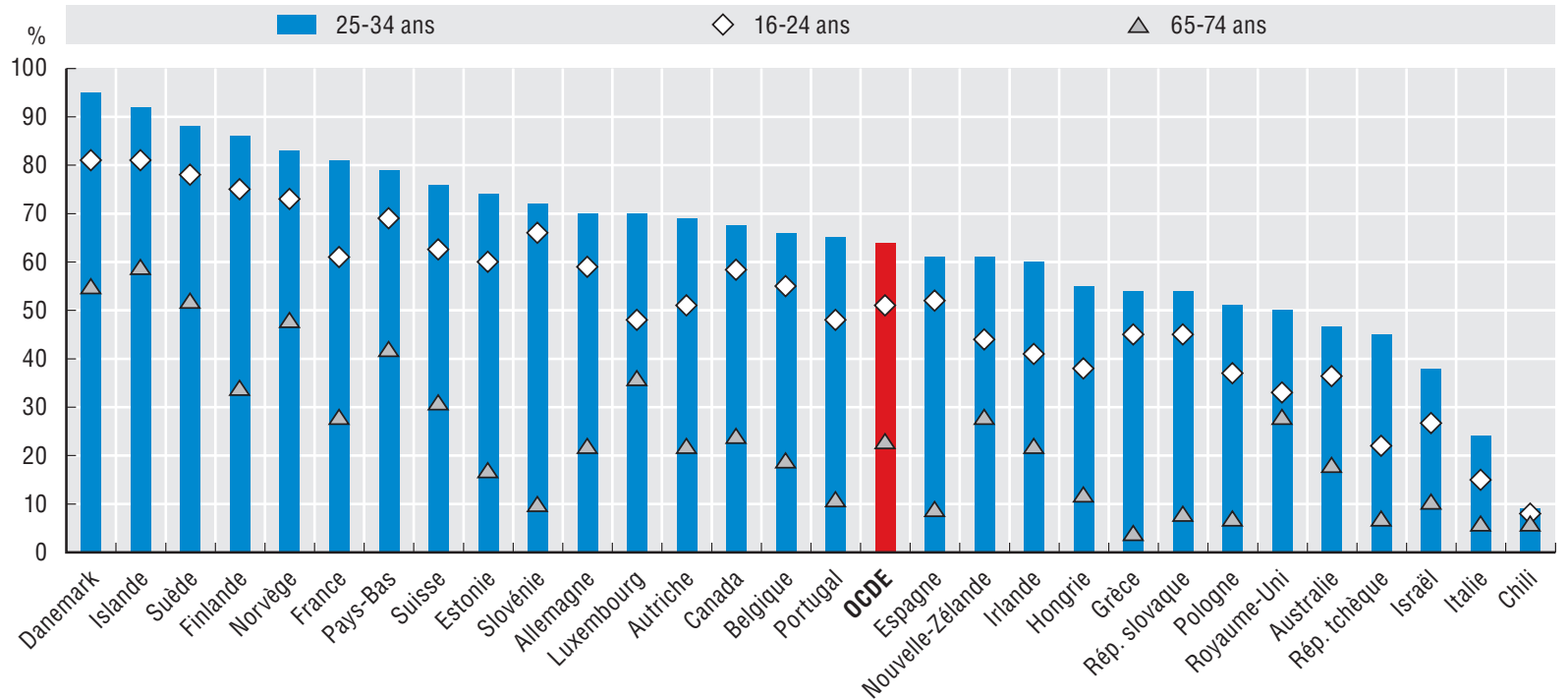

Source: Base de données de l'OCDE sur les TIC et Eurostat, Statistiques sur la société de l'information (base de données). 
Des biens et services publics réactifs tiennent explicitement compte de l'hétérogénéité des besoins des citoyens et s'y adaptent. Plutôt que d'adopter des solutions toutes faites, les prestataires de services réactifs mettent en place des stratégies de segmentation des usagers ainsi que des dispositifs proactifs permettant de recueillir et de prendre en compte leurs réactions ou leurs réclamations. De plus, des biens et services publics réactifs visent à s'adapter aux besoins, en y répondant aussi rapidement que possible et en évitant les retards. La rapidité d'exécution des services apparaît donc comme un indicateur de réactivité doté d'un impact particulièrement important sur la confiance des citoyens dans la capacité des services publics à répondre à leurs besoins.

\section{Soins de santé}

Le temps d'attente est l'un des critères qui permettent de mesurer la rapidité d'exécution des services. Des temps d'attente excessifs sont susceptibles d'affecter non seulement la perception de la qualité du service, mais également son impact probable. Par exemple, le fait de différer un traitement médical peut parfois entraîner une détérioration de la santé du patient et déboucher sur une hospitalisation qui aurait pu être évitée. Ces retards peuvent en outre compromettre la relation entre le médecin et le patient et amoindrir la confiance des citoyens dans le système de santé. Cependant, le délai d'attente peut également refléter le fait que, en l'absence d'autre critère de répartition, lorsque les services fournis sont entièrement gratuits, le temps peut devenir une variable d'ajustement en cas de ressources limitées. Parmi les pays de l'OCDE pour lesquels des données sont disponibles, en 2010 , en moyenne près de $40 \%$ des citoyens auxquels la consultation d'un spécialiste avait été conseillée ont dû patienter plus de quatre semaines pour le voir (graphique 9.8). Il existe cependant des différences importantes d'un pays à l'autre. La proportion de citoyens ayant dû attendre plus de quatre semaines s'élevait à près de $60 \%$ au Canada, contre moins de $20 \%$ en Allemagne.

Le pourcentage de citoyens ayant dû attendre plus de quatre mois pour une intervention chirurgicale non vitale était, en moyenne, moins élevé (environ $10 \%$ ). Environ $20 \%$ des citoyens ont fait état de délais d'attente longs au Canada, en Suède, en Norvège et au Royaume-Uni. Aucun n'a rapporté de délai excédant quatre mois en Allemagne. Le manque de personnel, une mauvaise organisation ou une pénurie de lits d'hôpitaux représentent autant de facteurs susceptibles de contribuer à créer de longs délais d'attente pour les interventions chirurgicales.

\section{Administration fiscale}

Un certain nombre de pays membres de l'OCDE font figurer la rapidité d'exécution parmi les principaux indicateurs de performance de certains services publics, notamment en matière d'administration fiscale. Parmi les pays ayant fixé des délais de traitement des déclarations d'impôt, le délai de traitement moyen ne dépasse pas 40 jours pour les déclarations sur papier et 35 jours pour les déclarations électroniques (graphique 9.9). Dans deux pays - les Pays-Bas et la Pologne - les déclarations sont traitées dans les trois mois. Pour tous les autres pays, les délais de traitement des déclarations sur papier vont de 10 jours en Irlande à 42 jours en Australie, au Danemark et au Japon. Dans la majorité des pays, l'instauration de déclarations en ligne n'a pas débouché sur une réduction sensible des délais de traitement, excepté en Australie, au Canada et en Irlande.
Dans ces pays, les déclarations d'impôt remplies par les citoyens sous forme électronique bénéficient d'un traitement trois à quatre fois plus rapide que les déclarations sur papier.

\section{Justice}

La rapidité d'exécution peut également revêtir une importance cruciale pour déterminer la qualité des systèmes judiciaires. Les retards peuvent avoir des conséquences néfastes sur la capacité de ces derniers à faire respecter le droit et à créer des conditions réellement équitables de résolution des litiges économiques, sapant ainsi la confiance des citoyens dans les institutions judiciaires. Les retards peuvent également entraîner des coûts supplémentaires : quand une affaire reste en suspens, la situation économique demeure incertaine, ce qui entrave les investissements futurs. À l'inverse, un délai de traitement des affaires trop court peut porter atteinte aux garanties d'une procédure régulière.

La durée des procès est un indicateur courant de la rapidité d'exécution dans le domaine de la justice. Parmi les 31 pays membres de l'OCDE pour lesquels des données sont disponibles, la durée moyenne de traitement d'une affaire civile de première instance oscille entre plus de 550 jours en Italie et 100 jours environ au Japon, avec une moyenne OCDE s'établissant à 242 jours environ (graphique 9.10). Ce sont les pays dont le système juridique s'inspire du modèle français qui enregistrent les délais les plus longs. Cependant, au-delà des différences de fond et de procédure entre les systèmes juridiques, les modalités d'organisation des systèmes judicaires $-\mathrm{y}$ compris les politiques de recrutement et de gestion des ressources humaines, le recours à l'informatique et les capacités d'absorption de la charge de travail - peuvent influer sur les délais nécessaires à la résolution des affaires devant les tribunaux.

\section{Méthodologie et définitions}

Les données relatives aux délais d'attente pour la consultation de spécialistes et les interventions chirurgicales non vitales proviennent des Statistiques de l'OCDE sur la santé 2011. Pour la consultation d'un spécialiste ou une intervention chirurgicale non vitale, le délai d'attente désigne le temps s'écoulant entre le moment où il est conseillé au patient de se faire soigner et la date du rendez-vous. Seuls les répondants ayant consulté un spécialiste ou subi une intervention chirurgicale non vitale au cours des deux années précédentes ont été priés de renseigner les délais d'attente. Les données relatives au délai de traitement des déclarations d'impôt des particuliers proviennent de l'étude Tax Administration 2013: Comparative Information on OECD and other Advanced and Emerging Economies. Le délai de traitement s'entend du délai s'écoulant entre le dépôt par un citoyen de sa déclaration de revenus et la décision de l'administration fiscale sur un remboursement éventuel. Les déclarations d'impôt sont les formulaires sur lesquels les citoyens déclarent leurs revenus imposables aux autorités compétentes. Les remboursements d'impôt désignent le remboursement reçu par les citoyens lorsque la somme qu'ils ont acquittée est plus élevée que celle dont ils sont redevables au titre de l'impôt. 
Les données relatives à la durée moyenne des procès civils de première instance proviennent de l'étude de l'OCDE «Judicial Performance and its Determinants: A Cross-Country Perspective ". La durée des procès est estimée à l'aide d'une formule utilisée couramment dans les ouvrages sur cette question: [ (affaires en suspens au cours de la période $t-1+$ affaires en suspens au cours de la période $\left.{ }_{t}\right) /\left(\right.$ nouvelles affaires au cours de la période ${ }_{t}+$ affaires résolues au cours de la période $t)]^{*} 365$. Lorsqu'on ne disposait pas de renseignements sur le nombre d'affaires en suspens, mais que le pays était en mesure de fournir des renseignements sur la durée effective des procès, cette dernière information a été utilisée (Angleterre et pays de Galles, Mexique, Nouvelle-Zélande et Pays-Bas). S'agissant des pays pour lesquels on ne disposait de données ni sur la durée estimative des procès, ni sur leur durée effective, on a imputé la valeur prévue de la régression de la durée estimative des procédures telle qu'elle figure dans la Base de données Doing Business du Groupe de la Banque mondiale.

\section{Pour en savoir plus}

OCDE (2013a), Tax Administration 2013: Comparative Information on OECD and other Advanced and Emerging Economies, Éditions OCDE, Paris, $h t t p: / / d x . d o i . o r g / 10.1787 / 9789264200814-e n$.

OCDE (2013b, à paraître), Panorama de la santé 2013 : Les indicateurs de l'OCDE, Éditions OCDE, Paris.
Palumbo, G. et al. (2013), "Judicial Performance and its Determinants: A Cross-Country Perspective », OECD Economic Policy Papers, $n^{\circ} 5$, Éditions OCDE, Paris, http://dx.doi.org/10.1787/ 5k44x00md5\g8-0en.

\section{Notes relatives aux graphiques}

9.9: Ces chiffres ne portent que sur les pays dans lesquels une norme administrative est appliquée en pratique. S'agissant des Pays-Bas, le nombre de jours n'est pas indiqué, car il n'a pas pu être estimé de façon suffisamment précise. Données relatives à l'Autriche : la même norme s'applique aux déclarations sur papier et par voie électronique. Données relatives au Chili : pour les déclarations renvoyées entre le $1^{\mathrm{er}}$ et le 19 avril, l'échéance des remboursements par virement est fixée au 10 mai et celle des remboursements par chèque au 30 mai ; pour les déclarations renvoyées entre le 20 et le 27 avril, l'échéance des remboursements par virement est fixée au 17 mai et celle des remboursements par chèque au 30 mai ; pour les déclarations présentées entre le 28 avril et le $9 \mathrm{mai}$, l'échéance des remboursements par virement est fixée au 26 mai et celle des remboursements par chèque au 30 mai. Les données relatives à la Hongrie reflètent la norme fixée par les autorités fiscales, et non les résultats effectifs. Données relatives aux États-Unis : la norme ne concerne que les déclarations individuelles sur papier. Aucune norme distincte ne s'applique aux déclarations remplies par voie électronique. S'agissant de ces dernières, l'objectif est d'émettre les remboursements dans un délai de 5 à 21 jours, et le fisc tient cet objectif dans la majorité des cas. L'Estonie et le Portugal ne sont pas concernés par les données relatives aux déclarations sur papier.

9.10 : Les données relatives au Royaume-Uni portent sur l'Angleterre et le pays de Galles.

Informations sur les données concernant Israël : http://dx.doi.org/10.1787/ 888932315602 . 


\section{9. ÉCLAIRAGE - AU SERVICE DES CITOYENS : L'ACCESSIBILITÉ ET LA QUALITÉ}

\section{La réactivité des services publics : la rapidité d'exécution}

\subsection{Délais d'attente pour un rendez-vous chez un spécialiste et une intervention chirurgicale non vitale (2010)}

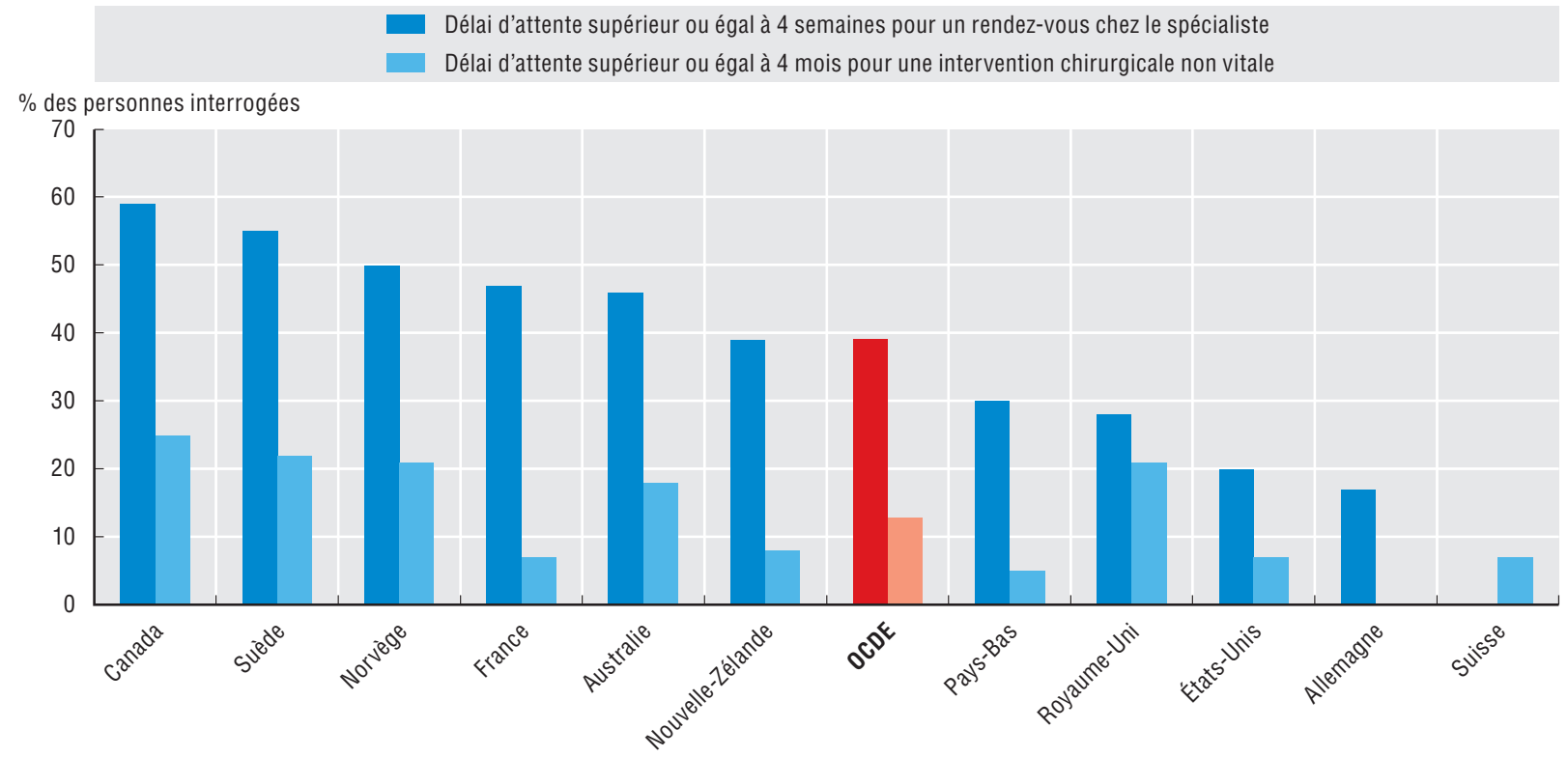

Source: Enquête internationale 2010 du Commonwealth Fund sur les politiques de santé.

StatLink क्ञाs $h$ ttp://dx.doi.org/10.1787/888933160010

9.9. Délai de traitement des déclarations d'impôt individuelles lorsqu'un remboursement est attendu (2011) Nombre moyen de jours de traitement pour au moins $80 \%$ des déclarations

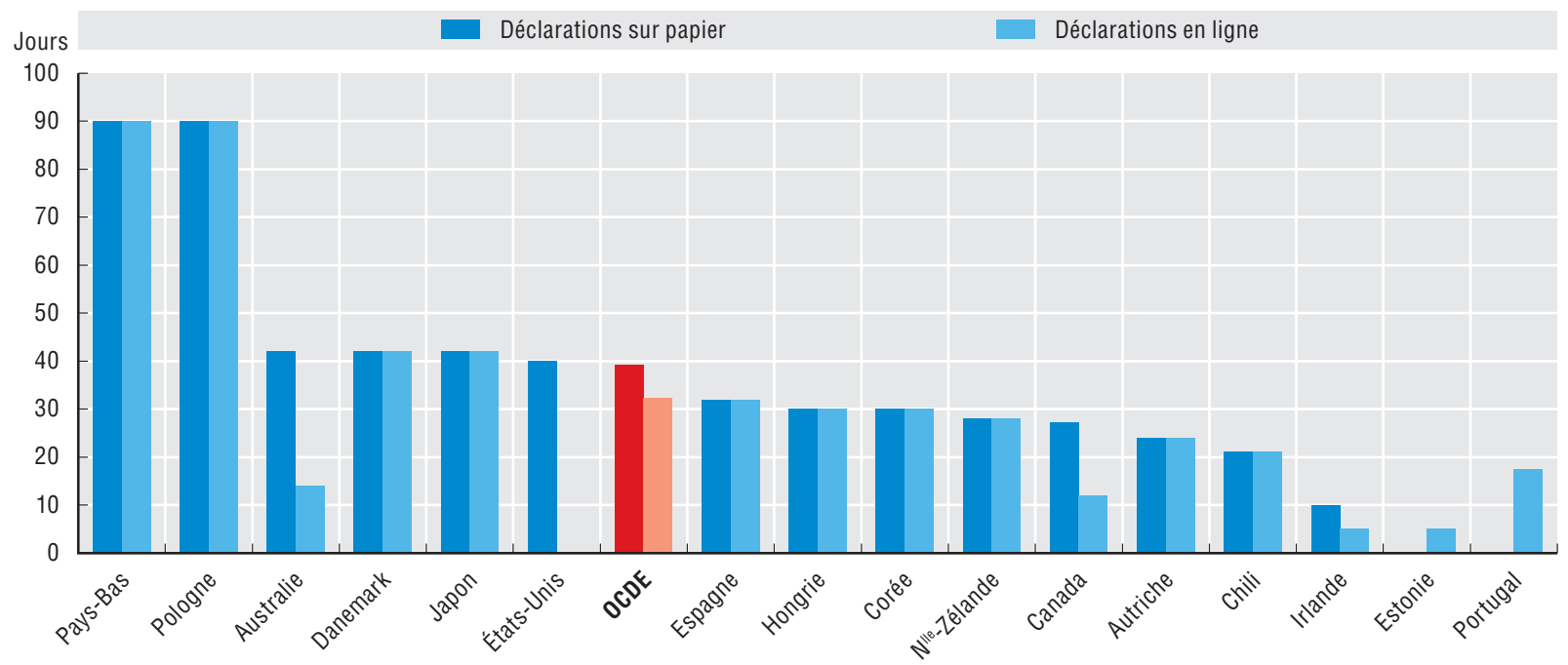

Source: OCDE (2013), Tax Administration 2013: Comparative Information on OECD and other Advanced and Emerging Economies, Éditions OCDE, Paris, $h$ ttp://dx.doi.org/10.1787/9789264200814-en. 


\section{9. ÉCLAIRAGE - AU SERVICE DES CITOYENS : L'ACCESSIBILITÉ ET LA QUALITÉ}

9.10. Durée des procès pour les affaires de première instance, en nombre de jours (2012)

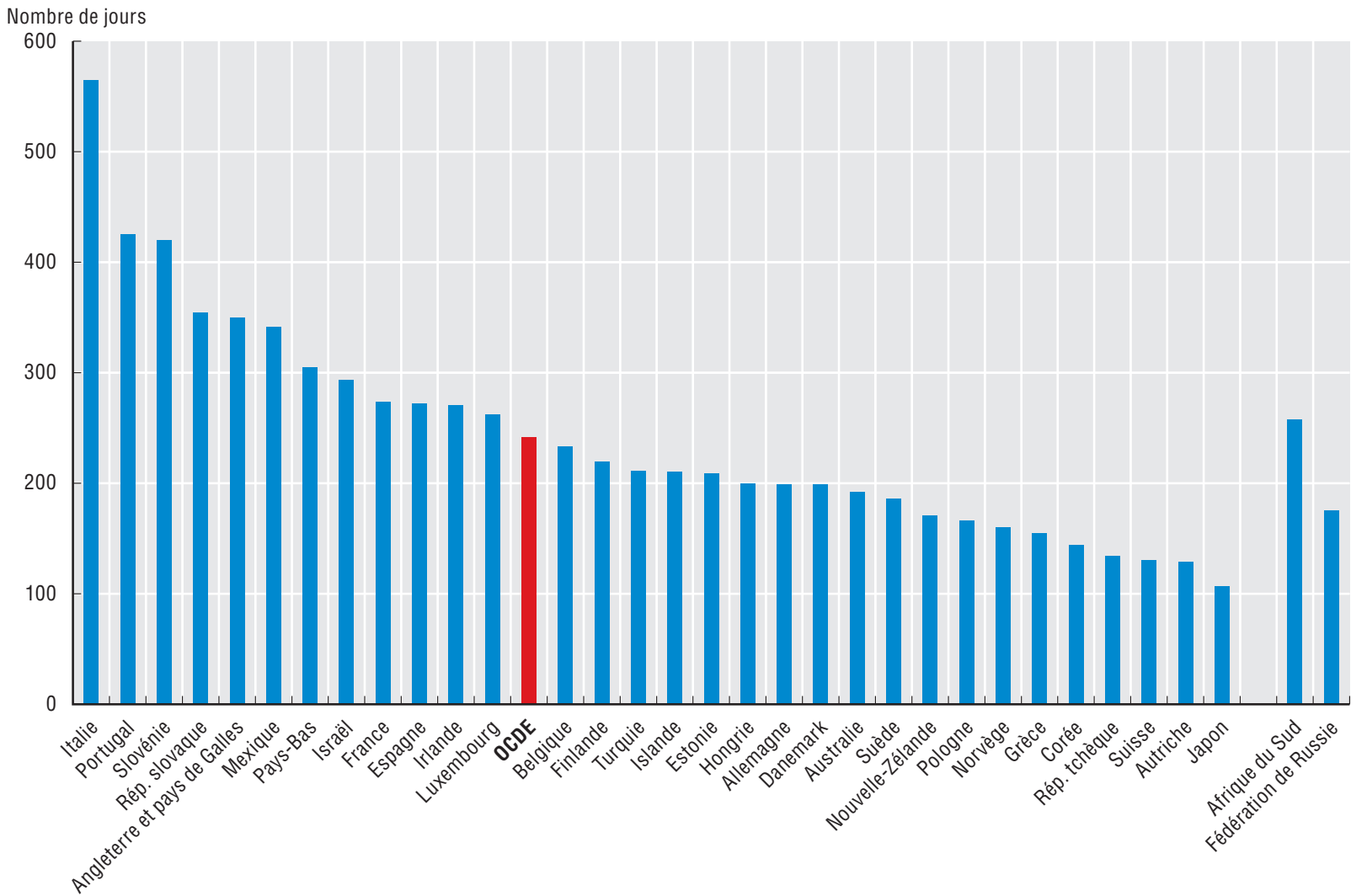

Source : OCDE, CEPEJ, Banque mondiale ; et Palumbo, G. et al. (2013), "Judicial Performance and its Determinants: A Cross-Country Perspective ", OECD Economic Policy Papers, $n^{\circ}$ 5, Éditions OCDE, Paris, http://dx.doi.org/10.1787/5k44x00md5 \g8-0en. 
Dans toute société démocratique, les citoyens sont titulaires d'un certain nombre de droits fondamentaux, ainsi que d'obligations à l'égard du gouvernement et de ses institutions. Alors que l'on considère la satisfaction des usagers à l'égard des biens et services assurés par le secteur public comme un indicateur important pour évaluer les performances du secteur public dans les pays de l'OCDE, peu d'indicateurs ont été définis de façon consensuelle pour mesurer la prise en compte des droits des citoyens dans le cadre institutionnel. Or, un cadre institutionnel tenant compte de ces droits contribue à garantir la fiabilité des services publics, en permettant aux citoyens d'être informés de leurs droits et en leur offrant des voies de recours et une assurance-qualité. Des déclarations relatives aux droits des citoyens, tels que la Citizen's Charter (Charte du citoyen) britannique, peuvent également consacrer l'existence de normes minimales en matière de service et de procédure.

La nécessité d'évaluer le degré de reconnaissance des droits des citoyens est devenue encore plus impérieuse dans un contexte de perte de confiance dans les autorités nationales et dans les dirigeants. Peu de pays possèdent une définition commune des droits des patients ou des contribuables, et encore moins de cadres réglementaires uniformes concernant les pratiques relatives au traitement des réclamations.

\section{Administration fiscale}

Dans le domaine de l'administration fiscale, la disparité des politiques fiscales nationales induit une diversité des environnements dans lesquels opèrent les organismes fiscaux, en conséquence de quoi le détail des droits concrets des contribuables varie quelque peu selon les pays. Il est néanmoins possible de discerner et donc d'analyser plusieurs tendances communes. Au début de la dernière décennie, le Groupe de travail $n^{\circ} 8$ du Comité des affaires fiscales de l'OCDE a publié un document faisant état de ce que, si la plupart des pays n'avaient pas, à cette époque, de " charte du contribuable " à proprement parler, tous les cadres administratifs garantissaient au contribuable les droits fondamentaux suivants : "le droit d'être informé, assisté et entendu; le droit d'exercer un recours; le droit de ne pas payer plus que le juste montant de l'impôt; le droit à la certitude ; le droit au respect de la vie privée ; et le droit à la confidentialité et au secret ".

Ces dernières années, de nombreux pays membres de l'OCDE ont établi des chartes du contribuable ou des chartes de service afin d'organiser ces droits fondamentaux. Une charte du contribuable ou charte de service consiste en une série de documents énonçant les comportements attendus tant des agents publics que des contribuables. Différents modèles de chartes existent selon les pays de l'OCDE. Dans certains cas, des mesures réglementaires prises en vue de garantir la protection des contribuables sont regroupées dans une " charte du contribuable " qui fait par la suite l'objet d'une large diffusion, mais d'autres solutions existent également. Par conséquent, les comparaisons internationales doivent tenir compte du fait qu'un pays dépourvu de charte du contribuable peut néanmoins attacher autant d'importance aux droits de ce dernier qu'un pays disposant d'une charte en bonne et due forme.

Comme le montre le tableau 9.11, en 2012, presque toutes les administrations fiscales chargées de tâches opérationnelles avaient formalisé un ensemble de droits des contribuables énoncés sous forme législative et/ou sous forme de documents administratifs. Sur les 33 pays membres de l'OCDE analysés, 30 avaient codifié ces droits (en tout ou partie) dans le cadre de leur législation fiscale ou d'autres branches de leur législation, les seules exceptions étant l'Irlande, la Nouvelle-Zélande et la Turquie. D'autre part, 26 administrations fiscales menaient une action encadrée par un ensemble de droits et d'obligations consacrées par des documents administratifs (désignés parfois sous le nom de "charte du contribuable " ou de " charte de service »). Au-delà des questions culturelles et juridiques, différents facteurs semblent influer sur la décision d'un pays d'opter, en pratique, pour la codification ou pour l'adoption d'une approche administrative. D'un côté, une approche administrative présente les avantages suivants : la rapidité de mise en œuvre, un langage plus accessible aux destinataires des documents, une portée plus large (notamment l'énonciation de droits plus étendus qui ne pourraient pas faire l'objet d'un processus législatif), une souplesse accrue et des recours faciles à exercer. De l'autre, la codification offre les avantages ci-après : une amélioration de l'opinion des contribuables à l'égard des autorités fiscales, car cette solution témoigne d'un engagement plus fort de la part de ces dernières ; une adhésion rapide du personnel à ces dispositions ; la stabilité du document adopté face aux changements résultant d'intérêts politiques divergents (pérennité) ; et la possibilité de soumettre le document à des mécanismes existants de recours et de contestation.

\section{Soins de santé}

Dans le secteur de la santé, les droits des patients et l'implication de ces derniers - notamment la possibilité de porter plainte - sont considérés comme des indicateurs de base pour évaluer l'attention accordée aux usagers dans le cadre de la fourniture des services publics. L'analyse menée dans ce domaine s'est fondée sur l'examen de cinq critères : l'existence au niveau national d'une définition officielle ou d'une charte des droits du patient; la possibilité offerte aux patients de demander réparation devant les tribunaux en cas d'erreur médicale ; l'obligation faite aux hôpitaux de disposer d'un bureau chargé de recueillir les réclamations des patients et de les régler; l'existence d'un service de médiation chargé d'enquêter sur et de régler les réclamations au sujet des services de santé ; et la possibilité d'engager des actions collectives contre les prestataires de services de santé et les laboratoires pharmaceutiques.

La grande majorité des pays de l'OCDE fait état de l'existence au niveau national de documents définissant officiellement les droits des patients (26 pays sur 33 ). Seuls le Canada, Israël, le Luxembourg, la Suède et la Suisse indiquent qu'ils ne disposent pas de tels documents. Dans tous les pays, à l'exception de la Finlande, de l'Islande, de la Nouvelle-Zélande et de la République slovaque, les patients peuvent demander réparation devant les tribunaux en cas d'erreur médicale. En outre, les hôpitaux sont tenus de disposer d'un bureau chargé d'enregistrer les réclamations des patients dans la majeure partie des pays (19 d'entre eux). La grande majorité des pays de l'OCDE signale la présence d'un médiateur chargé d'enquêter sur et de régler les réclamations des patients au sujet des services de santé. Seuls la Corée, le Danemark, le Japon, les Pays-Bas et la Turquie ne disposent pas de ce type de dispositif de médiation.

\section{Pour en savoir plus}

OCDE (2013), Tax Administration 2013: Comparative Information on OECD and other Advanced and Emerging Economies, Éditions OCDE, Paris, http://dx.doi.org/10.1787/9789264200814-en. 


\section{Méthodologie et définitions}

Les données sur les droits des contribuables proviennent de l'étude Tax Administration 2013: Comparative Information on OECD and other Advanced and Emerging Economies (OCDE, 2013). Ces informations ont été recueillies dans le cadre de deux enquêtes menées dans les pays membres de l'OCDE et un groupe d'autres économies majeures: l'Enquête fiscale de l'IBFD et l'Enquête de la série "Informations comparatives ". Les expressions " charte du contribuable » et " charte de service " désignent un ensemble de documents énonçant les comportements attendus tant des agents que des contribuables.

Les données sur les droits des patients proviennent de l'étude "Health Systems Institutional Characteristics: A Survey of 29 OECD Countries " (Paris et al., 2010). Ces informations ont été recueillies dans le cadre de l'Enquête de l'OCDE sur les caractéristiques des systèmes de santé (2008-2009 et 2012). S'agissant de l'enquête de 2012, on s'est fondé sur la question suivante : "Question 90 : Les droits des patients sont-ils définis officiellement au niveau national (par exemple, dans le cadre d'une loi, d'une charte) ? ". S'agissant de l'enquête 2008-2009, on s'est fondé sur les questions ci-après : "Question 76 : Les hôpitaux sont-ils tenus de disposer d'un bureau chargé de recueillir les réclamations des patients et de les régler ? "; "Question 78: Quel est le système de responsabilité civile en vigueur dans le pays ? "; " Question 80 : Est-il possible d'engager des actions collectives contre les prestataires de services de santé, les laboratoires pharmaceutiques, etc. ? »; et " Question 81 : Existe-t-il un médiateur chargé d'enquêter sur et de régler les réclamations des patients au sujet des services de santé ? ". On peut définir une action collective comme une action judiciaire introduite par une ou plusieurs personnes, en son/leur nom propre et au nom d'un groupe beaucoup plus vaste de personnes ayant toutes le même intérêt à agir.
Paris, V., M. Devaux et L. Wei (2010), "Health Systems Institutional Characteristics: A Survey of 29 OECD Countries ", OECD Health Working Papers, $n^{\circ} 50$, Éditions OCDE, $h$ ttp://dx.doi.org/10.1787/5kmfxfq9qbnr-en.

\section{Notes relatives aux graphiques}

9.12 : Canada : la Charte des droits des contribuables énonce quinze droits, qui représentent une combinaison de droits reconnus par la loi et par des documents administratifs. Les droits garantis par la loi sont codifiés dans la législation fiscale et généralement assortis d'un droit de recours. Les droits consacrés par des documents administratifs régissent la relation entre les autorités fiscales et les contribuables et sont assortis d'une procédure administrative de recours. Cette procédure débute par une tentative de règlement de la question avec l'agent compétent dans le dossier en cause; si le contribuable n'obtient pas satisfaction, il peut déposer une réclamation officielle par l'intermédiaire d'un programme "Plaintes liées au service "; s'il est toujours insatisfait, il peut déposer plainte auprès du Bureau de l'ombudsman des contribuables, qui l'examinera de manière indépendante et impartiale. Chili : en février 2010, un nouvel article énonçant de manière détaillée les droits minimaux reconnus et garantis aux contribuables par la loi a été ajouté au Code des impôts. Irlande : bien qu'il existe un mécanisme interne de traitement des réclamations, les contribuables peuvent également introduire un recours fondé sur des dispositions légales auprès des commissaires des recours, du Bureau de l'ombudsman ou du Tribunal de l'égalité. Luxembourg : les droits des contribuables énoncés de façon officielle dans des documents administratifs ne concernent que les impôts directs.

Informations sur les données concernant Israël : http://dx.doi.org/10.1787/ 888932315602 . 
9. ÉCLAIRAGE - AU SERVICE DES CITOYENS : L'ACCESSIBILITÉ ET LA QUALITÉ

La fiabilité des services publics : garantir les droits des citoyens

9.11. Droits des citoyens dans le cadre du système national de santé (2009-2012)

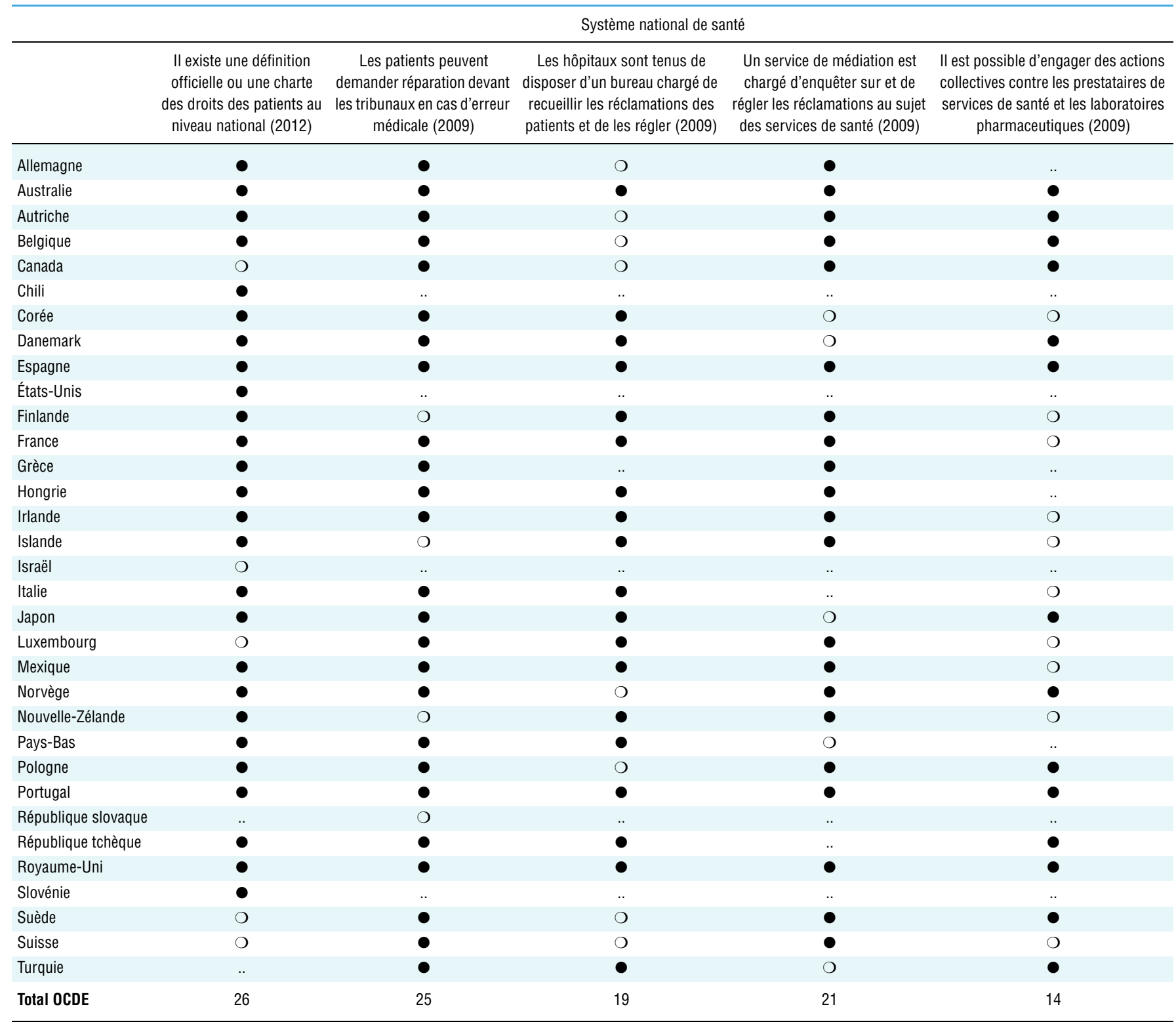

Oui

Non

Source : Paris, V., M. Devaux et L. Wei (2010), "Health Systems Institutional Characteristics: A Survey of 29 OECD Countries ", OECD Health Working Papers, $n^{\circ}$ 50, Éditions OCDE, Paris, http://dx.doi.org/10.1787/5kmfxfq9qbnr-en. 


\section{9. ÉCLAIRAGE - AU SERVICE DES CITOYENS : L'ACCESSIBILITÉ ET LA QUALITÉ}

La fiabilité des services publics : garantir les droits des citoyens

9.12. Droits des citoyens face à l'administration fiscale (2013)

\begin{tabular}{|c|c|c|}
\hline & $\begin{array}{l}\text { Les droits du contribuable sont officiellement définis } \\
\text { dans des lois fiscales ou d'autres textes de loi }\end{array}$ & $\begin{array}{l}\text { Les droits du contribuable sont officiellement définis } \\
\text { dans des documents administratifs }\end{array}$ \\
\hline Allemagne & $\bullet$ & O \\
\hline Australie & $\bullet$ & $\bullet$ \\
\hline Autriche & $\bullet$ & $\bullet$ \\
\hline Belgique & $\bullet$ & $\bullet$ \\
\hline Canada & $\bullet$ & • \\
\hline Chili & $\bullet$ & $\bullet$ \\
\hline Corée & $\bullet$ & $\bullet$ \\
\hline Danemark & $\bullet$ & $\bullet$ \\
\hline Espagne & $\bullet$ & $\bullet$ \\
\hline États-Unis & $\bullet$ & $\bullet$ \\
\hline Finlande & - & • \\
\hline France & $\bullet$ & $\bullet$ \\
\hline Grèce & $\bullet$ & $\bullet$ \\
\hline Hongrie & $\bullet$ & $\bullet$ \\
\hline Irlande & O & - \\
\hline Islande & $\bullet$ & O \\
\hline Israël & $\bullet$ & $\bullet$ \\
\hline Italie & $\bullet$ & $\bullet$ \\
\hline Japon & $\bullet$ & O \\
\hline Luxembourg & $\bullet$ & • \\
\hline Mexique & $\bullet$ & $\bullet$ \\
\hline Norvège & $\bullet$ & • \\
\hline Nouvelle-Zélande & O & $\bullet$ \\
\hline Pays-Bas & $\bullet$ & $\bullet$ \\
\hline Pologne & $\bullet$ & O \\
\hline Portugal & $\bullet$ & • \\
\hline République slovaque & $\bullet$ & O \\
\hline République tchèque & $\bullet$ & O \\
\hline Royaume-Uni & $\bullet$ & $\bullet$ \\
\hline Slovénie & $\bullet$ & $\bullet$ \\
\hline Suède & $\bullet$ & • \\
\hline Suisse & - & O \\
\hline Turquie & O & $\bullet$ \\
\hline Total OCDE & 30 & 26 \\
\hline
\end{tabular}

- Oui

O Non

Source: OCDE (2013), Tax Administration 2013: Comparative Information on OECD and other Advanced and Emerging Economies, Éditions OCDE, Paris, http://dx.doi.org/10.1787/9789264200814-en. 
L'évaluation de la satisfaction des usagers à l'égard des biens et des services publics est au cœur de toute démarche de service public axée sur le citoyen, et c'est aussi un volet important des stratégies d'amélioration continue de leurs performances mises en place par les administrations. On a généralement recours à des enquêtes d'opinion pour évaluer les expériences des citoyens dans leurs relations avec les administrations publiques et recueillir leur avis sur les prestations reçues. Les informations ainsi recueillies peuvent aider les gestionnaires publics à identifier les éléments qui, dans la fourniture des services, suscitent la satisfaction, ainsi qu'à contrôler l'impact des réformes sur les utilisateurs finals. Mesurer la satisfaction des citoyens est aussi un moyen de permettre aux responsables et gestionnaires publics de mieux comprendre leurs usagers, en contribuant à l'identification de sous-groupes d'usagers et de besoins ou d'inégalités en matière d'accessibilité. De plus, la satisfaction des citoyens peut constituer un important indicateur de réalisations permettant d'évaluer les performances globales des administrations publiques.

Dans le cadre des efforts consentis par les administrations publiques en vue d'améliorer la réactivité et la qualité des services publics, un nombre croissant d'entre elles prennent l'initiative de recueillir les réactions des citoyens et d'en tenir compte. L' " outil de mesure commune » du Canada et les "baromètres qualité " de la France et de l'Italie, par exemple, sont conçus de manière à permettre à différentes administrations publiques de mesurer et de suivre leur qualité de service dans le temps et de prendre en compte les données d'opinion recueillies auprès des citoyens. L'administration française dispose d'un panel de répondants fournissant en permanence un retour d'information sur les services dispensés, notamment pour les prestations correspondant aux évènements importants de la vie.

De manière générale, dans les pays membres de l'OCDE, la majorité des citoyens se déclare satisfaite des services publics. Ainsi, en 2012, 72 \% des citoyens de la zone OCDE, en moyenne, disaient faire confiance à leur police locale. Presque autant considéraient qu'ils avaient accès à des soins de qualité et $66 \%$ jugeaient satisfaisants le système éducatif et les établissements d'enseignement de leur ville ou de leur zone d'habitation.

En moyenne, les niveaux de satisfaction sont restés relativement stables pendant et immédiatement après la crise économique et sociale. Entre 2007 et 2012, les taux de satisfaction des citoyens n'ont connu qu'une augmentation minime, de moins de 2 points de pourcentage, dans les trois secteurs analysés ici. De grandes disparités existent cependant entre les pays. Les pays de l'OCDE ayant enregistré une augmentation de la confiance dans la police locale (15 pays) sont moins nombreux que ceux faisant état d'une satisfaction accrue en matière d'éducation (19 pays) et de soins de santé (19 pays). C'est en Estonie (14 points de pourcentage) et en République slovaque (13 p.p.) que l'augmentation de la confiance dans la police locale a été la plus marquée, tandis que cet indicateur reculait nettement au Mexique (15 p.p.) et en Hongrie (7 p.p.). La satisfaction à l'égard du système éducatif a connu sa progression la plus forte en Israël et au Royaume-Uni (9 p.p. dans les deux cas) et son recul le plus sensible au Chile (17 p.p.) et au Mexique (11 p.p.). Tous les pays, à neuf exceptions près, ont enregistré des variations du niveau de satisfaction à l'égard des soins de santé situées dans une fourchette de 5 points de pourcentage. Ce sont la Hongrie (10 p.p.) et le Royaume-Uni (8 p.p.) qui ont connu les progressions les plus fortes, et la Grèce (23 p.p.) et le Japon (10 p.p.) qui ont enregistré les reculs les plus nets.

De manière générale, la satisfaction à l'égard des services publics est supérieure à la confiance dans les autorités natio- nales. En 2012, en moyenne de la zone OCDE, la confiance dans la police locale et la satisfaction à l'égard des soins de santé étaient supérieures de 30 points de pourcentage à la confiance dans les pouvoirs publics, et la satisfaction à l'égard du système éducatif lui était supérieure de 26 points de pourcentage.

\section{Méthodologie et définitions}

Les données sont tirées de l'enquête Gallup World Poll, qui est réalisée dans environ 140 pays au moyen d'un questionnaire commun traduit dans les principales langues de chaque pays. À quelques exceptions près, tous les échantillons sont établis selon la méthode probabiliste et sont représentatifs de la population résidente âgée de 15 ans et plus de l'ensemble du pays (y compris les zones rurales). Néanmoins, les résultats peuvent être entachés d'erreurs d'échantillonnage ou autres. La taille des échantillons est au minimum de 1000 personnes par pays. Voir le chapitre 1 pour une analyse plus complète de l'évaluation de la confiance dans les pouvoirs publics.

Les données relatives à la confiance dans la police locale correspondent au pourcentage de réponses positives à la question "Dans la ville ou la zone que vous habitez, avez-vous confiance dans la police locale? ".

Les données relatives à la satisfaction à l'égard du système éducatif et des établissements d'enseignement correspondent au pourcentage de réponses "Satisfait(e) " à la question " Dans la ville ou la zone que vous habitez, êtes-vous satisfait(e) du système éducatif ou des établissements d'enseignement? ".

Les données relatives à l'accès à des soins de santé de qualité correspondent au pourcentage de réponses "Satisfait(e) « à la question «Dans la ville ou la zone que vous habitez, êtes-vous satisfait(e) de l'accès à des soins de santé de qualité ? ".

Le graphique 9.14, "Comparaison entre les niveaux de satisfaction et de confiance pour une sélection de services publics et la confiance dans les autorités nationales (2012) ", peut être consulté en ligne à l'adresse http://dx.doi.org/10.1787/888932943153.

\section{Pour en savoir plus}

OCDE (2012), Measuring Regulatory Performance: A Practitioner's Guide to Perception Surveys, Éditions OCDE, Paris, http:// dx.doi.org/10.1787/9789264167179-en.

\section{Notes relatives aux graphiques}

Les données relatives à l'Autriche, à la Finlande, à la France, à l'Irlande, au Portugal, à la République slovaque, à la Slovénie et à la Suisse portent sur 2006 et non 2007. Les données relatives à l'Allemagne, au Chili et au Royaume-Uni portent sur 2011 et non 2012. S'agissant de la confiance dans la police locale et de la satisfaction à l'égard des soins de santé, les données relatives à la Corée, au Japon et au Mexique portent sur 2011 et non 2012.

Informations sur les données concernant Israël : http://dx.doi.org/10.1787/ 888932315602 . 


\section{9. ÉCLAIRAGE - AU SERVICE DES CITOYENS : L'ACCESSIBILITÉ ET LA QUALITÉ}

La satisfaction des citoyens à l'égard des services publics

9.13. Niveaux de satisfaction et de confiance pour une sélection de services publics (2007 et 2012) 2012 $\diamond 2007$
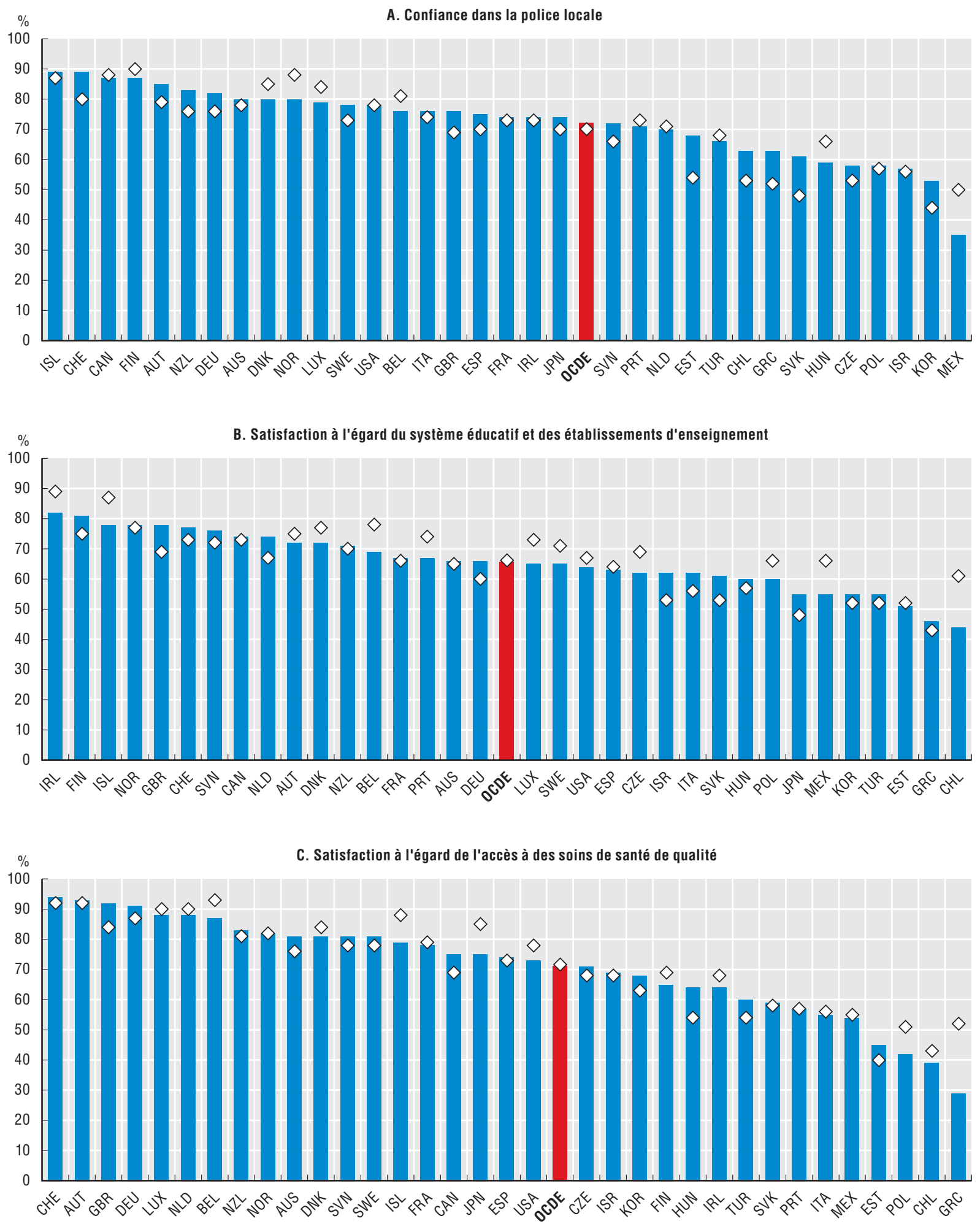

Source : Gallup World Poll. 



\section{ANNEXE A \\ Méthodologie utilisée pour les agrégats relatifs aux recettes}

Le tableau ci-dessous fournit des données détaillées sur la façon dont les agrégats relatifs aux impôts, aux cotisations sociales et aux aides et autres recettes présentés au chapitre 3, "Les finances et l'économie publiques ", ont été construits à partir des données issues des statistiques de l'OCDE relatives aux comptes nationaux.

\section{Tableau A.1. Agrégats relatifs aux recettes}

\begin{tabular}{llc}
\hline $\begin{array}{l}\text { Intitulé dans le Panorama } \\
\text { des administrations publiques }\end{array}$ & $\begin{array}{l}\text { Intitulé dans le Système } \\
\text { de comptabilité nationale }\end{array}$ & $\begin{array}{c}\text { Code dans la Base de données } \\
\text { de l'OCDE relative aux comptes } \\
\text { nationaux (Principaux agrégats } \\
\text { des administrations publiques) }\end{array}$ \\
\hline $\begin{array}{l}\text { Impôts } \\
\text { Impôts indirects }\end{array}$ & Impôts sur la production et les importations, à recevoir & GD2R \\
Impôts directs & Impôts courants sur le revenu et le patrimoine, à recevoir & GD5R \\
Impôts en capital & Impôts en capital & GD91R \\
Cotisations sociales & Cotisations sociales & GD61R \\
Aides et autres recettes & & Autres transferts courants, à recevoir \\
Aides courantes et aides à l'équipement & Autres transferts en capital et aides à l'investissement, à recevoir & GD7R \\
Ventes et redevances & Production marchande et production pour usage final propre & GP11_P12R \\
& Paiements au titre de la production non marchande & GP131R \\
Revenus de la propriété & Revenus de la propriété, à recevoir & GD4R \\
Subventions & Autres subventions sur la production, à recevoir & GD39R \\
Total des recettes & Total des recettes & GTR \\
\hline
\end{tabular}




\section{ANNEXE B \\ Classification des fonctions des administrations publiques (CFAP/COFOG)}

Conçue par l'OCDE, la Classification des fonctions des administrations publiques (CFAP ou COFOG) classe les données sur les dépenses des administrations publiques provenant du Système de comptabilité nationale selon l'objet auquel les fonds sont consacrés. Comme le montre le tableau B.1, la CFAP de niveau 1 répartit les données sur les dépenses selon 10 groupes ou sous-secteurs "fonctionnels " de dépenses (comme la défense, l'enseignement ou la protection sociale), et la CFAP de niveau 2 subdivise chaque groupe du niveau 1 en neuf sous-groupes au maximum. On dispose de données correspondant au niveau 1 de la CFAP pour 32 des 34 pays membres de l'OCDE mais, s'agissant du niveau 2, on ne dispose actuellement de données que pour 21 pays de l'OCDE membres de l'Union européenne, plus le Japon*.

Tableau B.1. Niveaux 1 et 2 de la CFAP (COFOG)

\begin{tabular}{ll}
\hline Niveau 1 & Niveau 2 \\
\hline Services généraux des administrations publiques & - Fonctionnement des organes exécutifs et législatifs, affaires financières et fiscales, \\
& affaires étrangères \\
& - Aide économique extérieure \\
& - Services généraux \\
& - Recherche fondamentale \\
& - R-D concernant des services généraux des administrations publiques \\
& - Services généraux des administrations publiques n.c.a. \\
& - Opérations concernant la dette publique \\
& - Transferts de caractère général entre administrations publiques \\
& - Défense militaire \\
& - Défense civile \\
- Aide militaire à des pays étrangers & - R-D concernant la défense \\
Défense & Défense n.c.a. \\
\hline
\end{tabular}

* On ne dispose pas de données correspondant au niveau 1 de la CFAP pour le Chili et le Mexique. Jusqu'à une époque récente, les données correspondant au niveau 2 de la CFAP étaient disponibles dans certains offices statistiques nationaux, mais n'étaient pas recueillies par les organisations internationales. De plus, les données correspondant à ce niveau n'étaient pas comparables d'un pays à l'autre, car le guide relatif au SCN des Nations Unies et le Manuel de statistiques de finances publiques du Fonds monétaire international ne donnaient que peu d'informations pratiques sur l'application des concepts de la CFAP. Toutefois, en 2005, Eurostat a mis en place un groupe de travail chargé de rédiger un manuel sur l'application de la CFAP aux données relatives aux dépenses figurant dans les comptes nationaux et de débattre du recueil de données correspondant au niveau 2 de la CFAP pour les pays européens. On ne dispose pas de données correspondant au niveau 2 de la CFAP pour la Suisse, ni pour les membres de l'OCDE non européens (à l'exception du Japon) : l'Australie, le Canada, le Chili, la Corée, les États-Unis, Israël, le Mexique et la Nouvelle-Zélande. Par ailleurs, dans certains pays membres de l'UE, ces données sont disponibles seulement pour certaines divisions de la CFAP. Des efforts sont en cours afin de parvenir à un accord avec ces pays sur la communication de ces données à l'OCDE. 
Tableau B.1. Niveaux 1 et $\mathbf{2}$ de la CFAP (COFOG) (suite)

\begin{tabular}{|c|c|}
\hline Niveau 1 & Niveau 2 \\
\hline Ordre et sécurité publics & $\begin{array}{l}\text { - Services de police } \\
\text { - Services de protection civile } \\
\text { - Tribunaux } \\
\text { - Administration pénitentiaire } \\
\text { - R-D concernant l'ordre et la sécurité publics } \\
\text { - Ordre et sécurité publics n.c.a. }\end{array}$ \\
\hline Affaires économiques & $\begin{array}{l}\text { - Tutelle de l'économie générale, des échanges et de l'emploi } \\
\text { - Agriculture, sylviculture, pêche et chasse } \\
\text { - Combustibles et énergie } \\
\text { - Industries extractives et manufacturières, construction } \\
\text { - Transports } \\
\text { - Communications } \\
\text { - Autres branches d'activité } \\
\text { - R-D concernant les affaires économiques } \\
\text { - Affaires économiques n.c.a. }\end{array}$ \\
\hline Protection de l'environnement & $\begin{array}{l}\text { - Gestion des déchets } \\
\text { - Gestion des eaux usées } \\
\text { - Lutte contre la pollution } \\
\text { - Préservation de la diversité biologique et protection de la nature } \\
\text { - R-D dans le domaine de la protection de l'environnement } \\
\text { - Protection de l'environnement n.c.a. }\end{array}$ \\
\hline Logements et équipements collectifs & $\begin{array}{l}\text { - Logements } \\
\text { - Équipements collectifs } \\
\text { - Alimentation en eau } \\
\text { - Éclairage public } \\
\text { - R-D dans le domaine du logement et des équipements collectifs } \\
\text { - Logements et équipements collectifs n.c.a. }\end{array}$ \\
\hline Santé & $\begin{array}{l}\text { - Produits, appareils et matériels médicaux } \\
\text { - Services ambulatoires } \\
\text { - Services hospitaliers } \\
\text { - Services de santé publique } \\
\text { - R-D dans le domaine de la santé } \\
\text { - Santé n.c.a. }\end{array}$ \\
\hline Loisirs, culture et culte & $\begin{array}{l}\text { - Services récréatifs et sportifs } \\
\text { - Services culturels } \\
\text { - Services de radiodiffusion, de télévision et d'édition } \\
\text { - Culte et autres services communautaires } \\
\text { - R-D dans le domaine des loisirs, de la culture et du culte } \\
\text { - Loisirs, culture et culte n.c.a. }\end{array}$ \\
\hline Enseignement & $\begin{array}{l}\text { - Enseignement préélémentaire et primaire } \\
\text { - Enseignement secondaire } \\
\text { - Enseignement postsecondaire non supérieur } \\
\text { - Enseignement supérieur } \\
\text { - Enseignement non défini par niveau } \\
\text { - Services annexes à l'enseignement } \\
\text { - R-D dans le domaine de l'enseignement } \\
\text { - Enseignement n.c.a. }\end{array}$ \\
\hline Protection sociale & $\begin{array}{l}\text { - Maladie et invalidité } \\
\text { - Vieillesse } \\
\text { - Survivants } \\
\text { - Famille et enfants } \\
\text { - Chômage } \\
\text { - Logement } \\
\text { - Exclusion sociale n.c.a. } \\
\text { - R-D dans le domaine de la protection sociale } \\
\text { - Protection sociale n.c.a. }\end{array}$ \\
\hline
\end{tabular}

n.c.a. : Éléments non classés ailleurs. 


\section{ANNEXE C}

\section{Indices composites sur les pratiques budgétaires}

On trouvera dans cette édition du Panorama des administrations publiques deux indices composites en matière de pratiques budgétaires : le recours à une perspective de moyen terme dans le processus budgétaire et le recours à un système de budgétisation axée sur la performance. Les données utilisées pour créer les composites ont été tirées de l'Enquête 2012 de l'OCDE sur les pratiques et procédures budgétaires et de l'Enquête 2011 de l'OCDE sur la budgétisation axée sur la performance. Les répondants étaient principalement de hauts responsables de ministères des Finances.

Les indices composites étroitement définis présentés dans le Panorama des administrations publiques constituent le meilleur moyen de rassembler des informations d'ordre qualitatif éparses sur les principaux aspects de pratiques budgétaires telles que les cadres de dépenses à moyen terme ou la budgétisation axée sur la performance. "Il est beaucoup plus facile d'interpréter des indices composites que d'essayer de dégager une tendance commune à partir de nombreux indicateurs distincts " (Nardo et al., 2004). Toutefois, leur conception et leur utilisation peuvent prêter à controverse. De tels indices sont facilement et fréquemment interprétés de façon erronée par les utilisateurs : faute de transparence suffisante sur la façon dont ils sont créés, il est difficile de déterminer ce qu'ils mesurent réellement.

L'OCDE a pris plusieurs dispositions pour éviter ou prendre en compte les problèmes couramment posés par les indices composites. Les indices composites présentés dans la présente publication suivent les étapes définies dans le Handbook on Constructing Composite Indicators (Nardo et al., 2008), qui sont nécessaires à la bonne construction d'indices composites ou synthétiques.

Chaque indice composite repose sur un cadre théorique représentant un concept reconnu dans le domaine traité. Les variables composant les indices ont été sélectionnées en fonction de leur pertinence à l'égard de ce concept par un groupe d'experts de l'OCDE, en concertation avec les délégués des pays auprès des groupes de travail compétents.

- Divers outils statistiques - comme l'analyse factorielle - ont été utilisés pour garantir que les variables composant chaque indice soient corrélées et représentent le même concept sous-jacent.

- Différentes méthodes visant à imputer les valeurs manquantes ont été examinées.

- Tous les sous-indicateurs et variables ont été normalisés pour assurer la comparabilité.

- Pour la construction des composites, tous les sous-indices ont été agrégés suivant une méthode linéaire, selon la méthodologie acceptée. 
- Une analyse de sensibilité basée sur des simulations Monte-Carlo a été menée afin d'établir la solidité des indices selon différentes options de pondération (par exemple, équipondérations, pondérations à l'aide des coefficients factoriels et pondération reposant sur l'avis d'experts). C'est la pondération reposant sur l'avis d'experts qui s'est avérée la plus adaptée.

Les indices n'ont pas vocation à mesurer la qualité globale des systèmes budgétaires. Il faudrait, pour cela, des fondements conceptuels et des hypothèses normatives bien plus solides. Les indices composites présentés dans le Panorama des administrations publiques sont plutôt de nature descriptive, et leur intitulé reflète cette nature. Les questions des enquêtes utilisées pour élaborer les indices sont les mêmes pour tous les pays, et les indices sont donc comparables.

Les indices composites ont été élaborés en coopération avec les pays membres, à la lumière de bonnes pratiques et/ou de théories bien établies. En revanche, le débat reste ouvert en ce qui concerne les variables qui composent ces indices et leur pondération, et elles pourront donc évoluer par la suite. L'OCDE est en train de redéfinir les bonnes pratiques en matière de transparence budgétaire et de réexaminer la notion de flexibilité budgétaire ; par conséquent, aucun indice composite relatif à ces thèmes n'est proposé au sein de la présente édition. Il n'est pas possible de comparer les indices composites présentés dans cette édition 2013 à ceux qui figuraient dans l'édition 2009 du Panorama des administrations publiques, car ils ne sont pas fondés sur la même version des enquêtes. Les nouvelles versions des enquêtes comportent des questions supplémentaires ou formulées différemment, ainsi que des pondérations différentes.

Pour construire les composites, on a suivi la méthodologie suivante : chaque thème a été divisé en grandes catégories couvrant les aspects théoriquement pertinents pour chacun des deux sujets (cadres de dépenses à moyen terme et budgétisation axée sur la performance), et une pondération a été attribuée à chacune de ces grandes catégories. Ensuite, au sein de chaque catégorie, les questions pertinentes ont été formulées, une sous-pondération a été attribuée à chacune de ces questions, et un score a été donné à chaque réponse. Le score obtenu par un pays pour chaque question est égal à la pondération de la grande catégorie et à la sous-pondération de la question, multipliées par la réponse fournie par le pays (1 ou 0). Le composite est égal à la somme de ces scores pour chaque pays. Les deux composites se situent entre 0 et 1 ; un score égal à 1 signifie que des pratiques satisfaisantes sont suivies pour un sujet donné. 


\section{Cadres de dépenses à moyen terme (CDMT) : variables, pondérations et score}

Pour construire l'indice composite relatif aux CDMT, on s'est basé sur les variables et les pondérations suivantes :

\section{Graphique C.1. Variables et pondérations utilisées pour l'indice relatif aux CDMT}

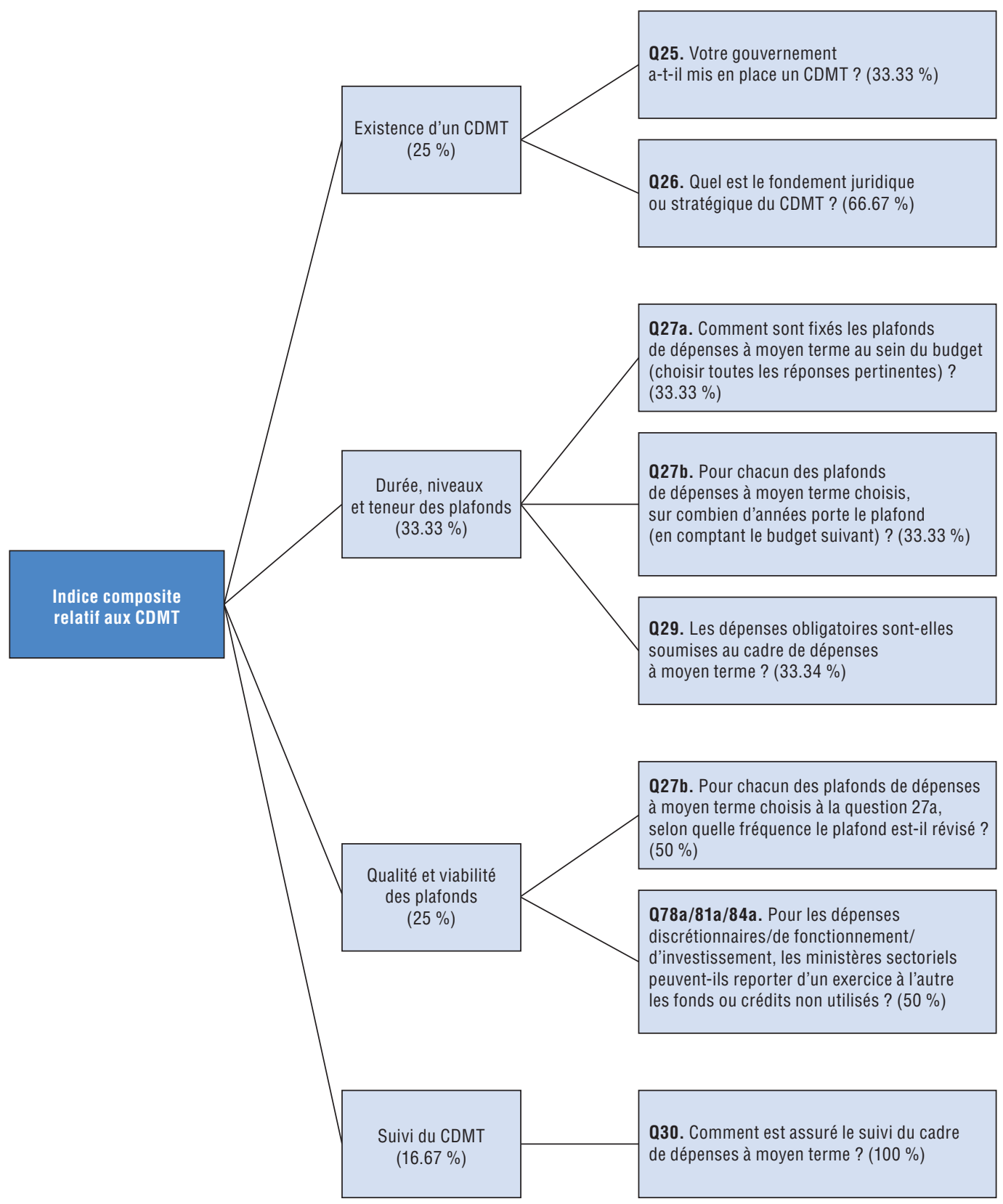

Note: On trouvera des précisions supplémentaires concernant le cadre théorique, la construction et les pondérations de chaque indice composite à l'adresse suivante : www.oecd.org/fr/gov/panorama-des-administrations-publiques.htm. 


\section{Recours à un système de budgétisation axée sur la performance au niveau de l'administration centrale : variables, pondérations et scores}

Pour construire l'indice composite relatif à la budgétisation axée sur la performance, on s'est basé sur les variables et les pondérations suivantes :

\section{Graphique C.2. Variables et pondérations utilisées pour l'indice relatif à la budgétisation axée sur la performance}

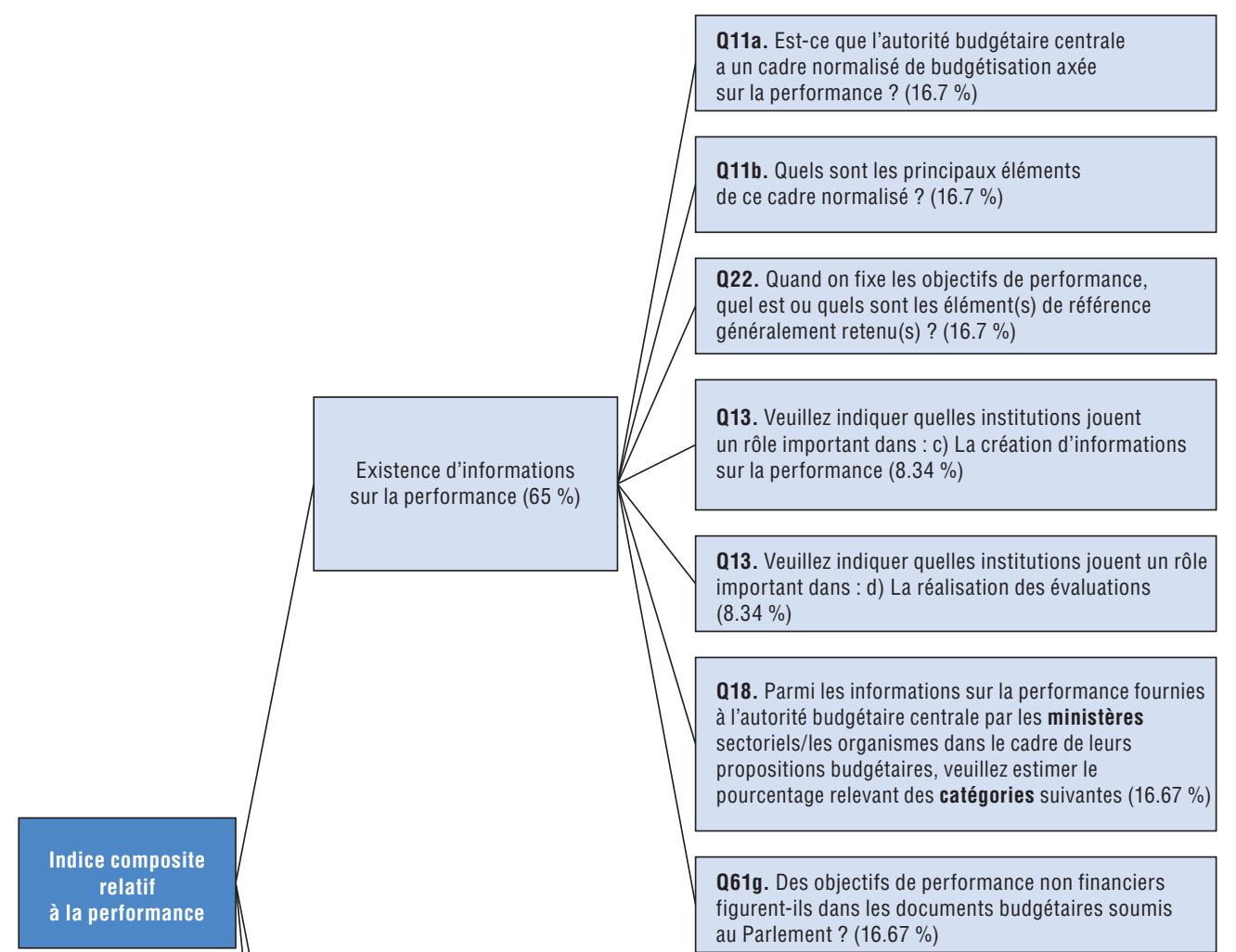

au Parlement? $(16.67 \%)$

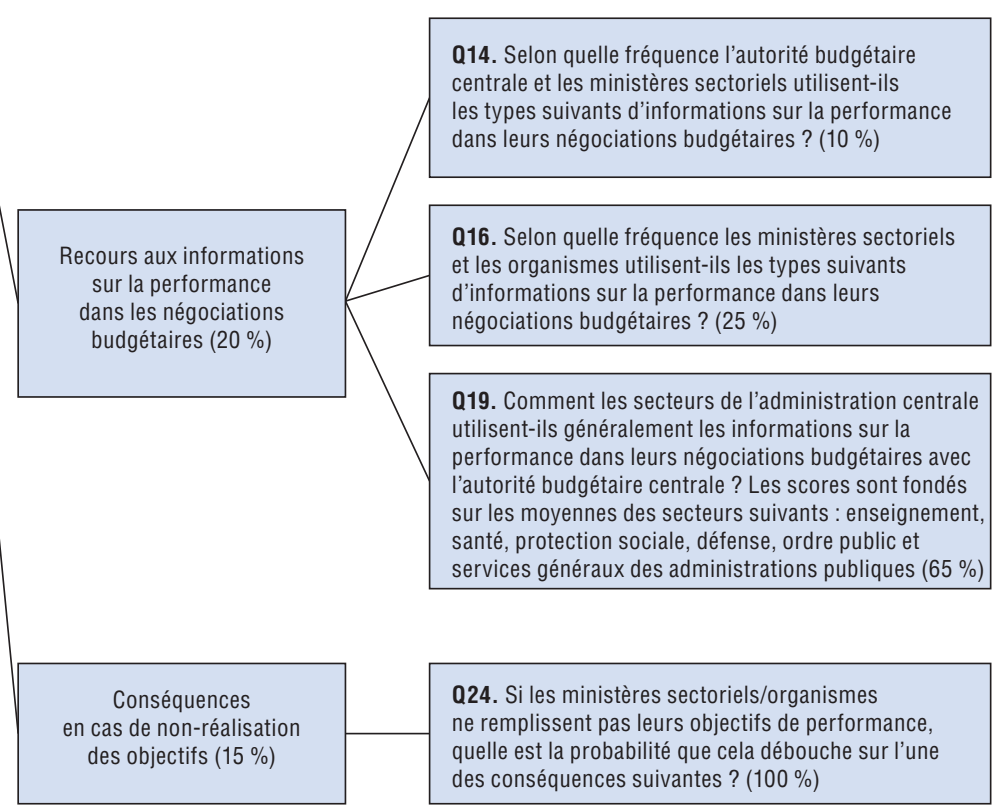

Note : On trouvera des précisions supplémentaires concernant le cadre théorique, la construction et la pondération de chaque indice composite à l'adresse suivante : www.oecd.org/fr/gov/panorama-des-administrations-publiques.htm. 


\section{Méthodologie et notes supplémentaires concernant la rémunération des agents publics*}

La rémunération joue un rôle important aussi bien pour attirer des agents qualifiés vers le secteur public que pour les motiver. Dans le cadre du processus d'assainissement des finances publiques lancé par de nombreux pays de l'OCDE, la rémunération des fonctionnaires est actuellement remise en cause avec, dans certains cas, une réduction des salaires et/ou des avantages non salariaux ou un gel des rémunérations. L'OCDE a créé en 2010 puis mis à jour en 2012 une base de données consacrée aux niveaux de rémunération pour certaines fonctions types exercées au sein de ministères régaliens. Cette base de données permet de mieux comprendre comment les rémunérations sont structurées au sein du secteur public et à quels niveaux elles se situent. Étant donné qu'il n'existe pas de définition commune des postes d'encadrement et que le nombre de niveaux hiérarchiques varie selon les pays et les ministères, cette enquête sur les rémunérations propose une typologie commune pour des professions précises au sein de l'administration centrale. La comparaison des rémunérations moyennes pratiquées dans le secteur public peut être trompeuse, car la fonction publique recouvre des professions diverses et hétérogènes selon les pays. En ciblant les rémunérations proposées pour des professions comparables, cette enquête nous permet d'affiner notre connaissance du secteur public.

La comparaison des niveaux de rémunération des cadres supérieurs, des cadres moyens, des spécialistes et des secrétaires porte sur leur rémunération totale relative à l'échelle des pays de l'OCDE, en tenant compte non seulement de leur salaire, mais aussi des contributions aux régimes d'assurance santé et retraite. Elle prend donc en compte à peu près l'ensemble des coûts, ce qui permet de faire des comparaisons internationales pertinentes.

On a également comparé, au sein des divers pays, les rémunérations des agents publics à la rémunération moyenne des diplômés de l'enseignement supérieur, afin d'évaluer l'attractivité relative des professions examinées par rapport à d'autres emplois exigeant des niveaux d'éducation similaires. Il faut également tenir compte, dans les comparaisons, du fait que les pays n'en sont pas tous au même stade de développement économique, d'où une correction en fonction du PIB par habitant. Cependant, toute comparaison internationale se doit d'être prudente, en raison des différences qui existent

* Les données statistiques concernant Israël sont fournies par et sous la responsabilité des autorités israéliennes compétentes. L'utilisation de ces données par l'OCDE est sans préjudice du statut des hauteurs du Golan, de Jérusalem-Est et des colonies de peuplement israéliennes en Cisjordanie aux termes du droit international. 
au niveau des marchés du travail, du consensus culturel et politique et, éventuellement, des critères qui déterminent les salaires, y compris au sein d'une même catégorie professionnelle, et la présente analyse ne tient pas compte de ces différences.

\section{Les professions}

L'enquête 2012 sur la rémunération des fonctionnaires des administrations centrales/ fédérales fait suite à une enquête de 2010 sur le même sujet. Les données recueillies dans le cadre de cette nouvelle enquête permettront de procéder à une analyse comparative et ouvrent la voie à d'autres travaux sur les politiques et pratiques en matière de rémunération. L'enquête a pour objet de recueillir des informations sur la rémunération annuelle des agents des administrations centrales/fédérales/nationales pour un échantillon de professions. L'objectif est de créer une base de données sur la rémunération de professions types de l'administration centrale, afin de mieux comprendre comment les rémunérations sont structurées au sein de la fonction publique et à quels niveaux elles se situent.

L'enquête porte uniquement sur les administrations centrales/fédérales, à l'exclusion des administrations d'États fédérés, des autorités régionales et locales et des administrations de sécurité sociale. Elle exclut également l'ensemble des entreprises publiques et quasi publiques, à tous les niveaux d'administration. De même, elle exclut les bureaux/organismes rattachés aux ministères, et que l'on désigne souvent sous l'appellation « agences ». Enfin, elle ne porte que sur les agents travaillant à temps plein, à l'exclusion des consultants et du personnel recruté pour une courte durée.

Le questionnaire a pour objet de recueillir des données pour quatre catégories professionnelles types de l'administration centrale/fédérale : les cadres supérieurs, les cadres moyens, les spécialistes et les secrétaires. On considère que ces professions sont relativement représentatives et comparables d'un pays à l'autre. Les informations relatives à ces professions (mais pas aux professions de service public) proviennent de trois ministères régaliens (Intérieur, Finances et Justice) et de trois ministères sectoriels (Éducation, Santé et Environnement). L'encadré D.1 décrit les responsabilités typiques des ministères couverts par l'enquête.

\section{Encadré D.1. Responsabilités typiques des ministères couverts par l'enquête}

Les descriptifs ci-après des activités ou des fonctions des ministères couverts par l'enquête sont à visée purement indicative. Dans certains pays, le ministère peut porter une appellation différente, ou même avoir le statut de département ou de secrétariat.

\section{Ministère de l'Intérieur}

- Assure la représentation de l'État dans l'ensemble du territoire

- Assure le respect des droits des citoyens en général grâce au suffrage universel

- Assure le respect des compétences des autorités locales dans le cadre de la décentralisation

- Définit la politique de l'immigration

- Définit et coordonne la politique de sécurité nationale

- Assure le maintien de la paix et de la sécurité au sein de la société

- Assure la préservation de la sécurité intérieure et la protection de l'ordre constitutionnel

\section{Ministère des Finances}

- Planifie et établit le budget de l'État

- Analyse et conçoit les politiques fiscales 
Encadré D.1. Responsabilités typiques des ministères couverts par l'enquête (suite)

- Élabore et met en œuvre la réglementation applicable aux établissements financiers

- Suit l'évolution de la situation économique et financière

- Administre les transferts financiers entre les autorités nationales/centrales/fédérales et les autorités infranationales

\section{Ministère de la Justice}

- Assure le bon fonctionnement du système judiciaire

- Prépare le texte des lois et des règlements régissant certains domaines

- Définit les principales orientations de l'action publique en matière de Justice et veille à sa mise en œuvre

- Assure un soutien aux victimes d'infractions

- Assure la mise à exécution juste, cohérente et efficace des peines et des autres sanctions

\section{Ministère de l'Éducation}

- Réglemente, coordonne et organise le système éducatif national, généralement de l'enseignement primaire à l'enseignement secondaire

- Assure l'égalité d'accès à l'enseignement public

- Contrôle et évalue les écoles et les établissements d'enseignement secondaire tant publics que privés

- Assure une bonne gestion des enseignants et du personnel administratif

\section{Ministère de la Santé}

- Conçoit et met en œuvre la politique de santé publique (prévention, organisation sanitaire et formation des professionnels)

- Définit la politique en matière de sports et de lutte contre la toxicomanie

- En collaboration avec les autres ministères, définit la réglementation en matière de sécurité sur le lieu de travail et de sécurité sociale

\section{Ministère de l'Environnement}

- Définit et gère le cadre législatif et réglementaire relatif à la protection de l'environnement et à l'utilisation efficiente des ressources naturelles

- Certifie, à travers l'ensemble du pays, les installations, l'occupation des sols et les industries pour s'assurer que les risques pour l'environnement, la santé humaine, la sécurité et les biens sont réduits au minimum

L'encadré D.2 présente la classification et les définitions des professions couvertes par l'enquête, qui sont considérées comme présentes de façon relativement classique dans toutes les administrations. L'accent est mis sur le personnel d'encadrement, selon une acception relativement large, puisqu'on considère comme un cadre tout agent supervisant et dirigeant le travail d'au moins trois personnes. Parce qu'il est extrêmement difficile de fournir des descriptifs plus détaillés des responsabilités permettant de faire la distinction entre les diverses strates d'encadrement, on a choisi une démarche axée sur la différentiation hiérarchique plutôt que sur une description plus précise des fonctions.

La classification et la définition des professions sont une adaptation de la Classification internationale type des professions (CITP-08) établie par l'Organisation internationale du travail (OIT). En effet, peu de pays suivent le modèle de la CITP pour classer les professions de leur fonction publique. 


\section{Encadré D.2. Classification et définition des professions}

\section{Les cadres de haut niveau}

Les cadres de niveau D1 (qui relèvent de la catégorie 1112 de la CITP-08) sont des fonctionnaires de très haut rang situés juste en dessous du ministre ou du secrétaire d'État. lls peuvent faire partie de la haute fonction publique et/ou être nommés par le gouvernement ou le chef du gouvernement. Ils conseillent le gouvernement en matière d'action publique, supervisent l'interprétation et la mise en œuvre des politiques publiques et, dans certains pays, ont des pouvoirs exécutifs. Les cadres D1 peuvent être autorisés à assister à certaines réunions du Cabinet ou du Conseil des ministres, mais ils n'appartiennent pas à ces instances. Ils assurent une direction et une gestion globales auprès du ministre/secrétaire d'État ou pour un domaine administratif particulier, dans des pays ayant un système d'agences autonomes, des pouvoirs décentralisés, des organisations dans lesquelles le nombre de niveaux hiérarchiques est réduit et des cadres jouissant d'une certaine autonomie. L'intitulé précis du poste peut varier d'un pays à l'autre.

Les cadres de niveau D2 (qui relèvent des catégories 11 et 12 de la CITP-08) sont situés immédiatement en dessous des cadres D1. Ils formulent et révisent les politiques et planifient, dirigent, coordonnent et évaluent les activités générales du ministère ou d'une direction/d'une unité, avec l'aide d'autres cadres. Ils peuvent faire partie de la haute fonction publique. Ils donnent des orientations en matière de coordination et de gestion du programme de travail et des directives aux équipes de spécialistes de différents domaines. Ils fixent les objectifs, stratégies et programmes pour l'unité administrative/le département qu'ils supervisent.

\section{Les cadres moyens (qui encadrent au moins 3 personnes)}

Les cadres de niveau D3 (qui relèvent de la catégorie 12 de la CITP-08) sont situés immédiatement en dessous des cadres D2. Ils planifient, dirigent et coordonnent le fonctionnement général d'une direction/d'une unité administrative au sein du ministère, avec l'aide d'autres cadres, en suivant généralement les lignes directrices établies par un conseil d'administration ou un organe de direction. Ils dirigent et encadrent des équipes de spécialistes dans leur domaine spécifique. Ces agents élaborent et conduisent le programme de travail d'unités, de divisions ou de domaines d'action et gèrent le personnel correspondant. Ils établissent et gèrent les budgets, contrôlent les dépenses et veillent à l'efficience dans l'utilisation des ressources. Ils suivent et évaluent les performances des différentes équipes de spécialistes.

Les cadres de niveau D4 (qui relèvent de la catégorie 121 de la CITP-08) sont situés immédiatement en dessous des cadres D3. Ils formulent et prodiguent des conseils d'action et assurent une planification stratégique et financière. Ils établissent et dirigent des procédures opérationnelles et administratives et adressent des avis aux cadres supérieurs. Ils supervisent la sélection, la formation et les performances du personnel, établissent les budgets et supervisent les opérations financières, contrôlent les dépenses et veillent à l'efficience dans l'utilisation des ressources. Ils dirigent des équipes spécifiques de spécialistes au sein d'une unité.

\section{Les spécialistes}

Les économistes/analystes des politiques senior (qui relèvent des catégories 242 et 2422 de la CITP-08) n'exercent pas de fonction de direction (ils encadrent trois personnes au maximum) et occupent un rang supérieur à celui des analystes junior et du personnel administratif/de secrétariat. Ils sont généralement tenus d'avoir un diplôme universitaire. Ils assurent certaines fonctions de pilotage pour un domaine de travail ou divers projets et conçoivent et analysent des politiques pour guider l'élaboration, la mise en œuvre et la modification des actions et programmes officiels. Ces spécialistes examinent les politiques et textes existants afin de détecter les anomalies et les dispositions dépassées. Ils analysent et formulent des options d'action et ils établissent des documents d'information et des recommandations en vue de faire évoluer les politiques existantes. Par ailleurs, ils évaluent l'impact, les conséquences financières et la faisabilité politique et administrative des politiques publiques. Les agents de cette catégorie ont la possibilité de progresser dans leur carrière jusqu'au rang de cadres. Leurs domaines de compétence peuvent aller du droit, de l'économie, de la politique, de l'administration publique ou des relations internationales à l'ingénierie, à l'environnement, à la pédagogie, à l'économie de la santé, etc. Les économistes/ analystes des politiques senior ont au moins cinq années d'expérience professionnelle. 


\section{Encadré D.2. Classification et définition des professions (suite)}

Les économistes/analystes des politiques junior (qui relèvent des catégories 242 et 2422 de la CITP-08) occupent un rang supérieur à celui du personnel administratif/de secrétariat. Ils sont généralement tenus d'avoir un diplôme universitaire. Ils n'exercent aucune fonction de direction. Ils conçoivent et analysent des politiques pour guider l'élaboration, la mise en œuvre et la modification des actions et programmes officiels. Ces spécialistes examinent les politiques et textes existants afin de détecter les anomalies et les dispositions dépassées. Ils analysent et formulent des options d'action et ils établissent des documents d'information et des recommandations en vue de faire évoluer les politiques existantes. Par ailleurs, ils évaluent l'impact, les conséquences financières et la faisabilité politique et administrative des politiques publiques. Leurs domaines de compétence peuvent aller du droit, de l'économie, de la politique, de l'administration publique ou des relations internationales à l'ingénierie, à l'environnement, à la pédagogie, à l'économie de la santé, etc. Les économistes/analystes des politiques junior ont moins de cinq années d'expérience professionnelle.

\section{Personnel de secrétariat}

Les secrétaires (employés de bureau, fonctions générales) (qui relèvent des catégories 411 et 4110 de la CITP-08) ne sont généralement pas tenus d'avoir un diplôme universitaire, même s'ils sont nombreux à être diplômés de l'enseignement supérieur. Ils assurent un large éventail de tâches administratives en rapport avec des opérations de trésorerie, l'organisation de déplacements, des demandes d'information et l'organisation de rendez-vous. Ils enregistrent, préparent, trient, classent et saisissent des informations ; trient, ouvrent et envoient le courrier ; préparent des rapports et des courriers ; enregistrent la remise de matériel au personnel ; répondent aux demandes de renseignement par téléphone ou en ligne ou les transmettent à qui de droit ; vérifient des chiffres; établissent des factures et enregistrent les détails des opérations financières réalisées ; saisissent des informations sur ordinateur et relisent et corrigent des épreuves. Certains aident à la préparation des budgets, au suivi des dépenses et à la rédaction de contrats et de bons de commande. Ceux qui occupent les rangs hiérarchiques les plus élevés, et qui supervisent les travaux de membres du personnel administratif, sont exclus de cette catégorie.

En l'absence d'une définition commune des postes d'encadrement, et le nombre de niveaux d'encadrement variant selon les pays et les ministères, le niveau D1 correspond, aux fins de l'enquête, à celui des agents situés immédiatement en dessous des ministères et des secrétaires d'État (lesquels sont désignés par le président ou par le premier ministre) et nommés par le ministre (ou, parfois, par le président ou le premier ministre). L'enquête va jusqu'au niveau d'encadrement D4. Les postes D1 et D2 sont considérés comme des postes de cadre supérieur, et les postes $\mathrm{D} 3$ et $\mathrm{D} 4$ comme des postes de cadre moyen.

La catégorie des "spécialistes " a été subdivisée entre "spécialistes junior » et "spécialistes senior ». En effet, ce groupe rassemble des agents dotés de niveaux très divers d'expérience professionnelle.

De plus, l'enquête de 2012 rassemble des informations sur un certain nombre de professions au contact direct des usagers (enquêteurs/inspecteurs de police, agents de police, agents de l'immigration, inspecteurs des douanes et inspecteurs des impôts). Ces fonctions sont organisées au niveau de l'administration centrale et peuvent être exercées, soit au sein de ministères, soit au sein d'organismes publics. Il faut noter que, dans certains pays, la profession d'agent de l'immigration n'existe pas, car les activités correspondantes sont menées à bien par la police. Dans d'autres pays, certaines des fonctions susmentionnées sont à la charge des États fédérés et/ou des administrations locales. 


\section{Encadré D.3. Agents exerçant des professions de service public-description des professions}

Les inspecteurs et enquêteurs de police (qui relèvent de la catégorie 3355 de la CITP-08) enquêtent sur les faits des infractions commises, et sur les circonstances qui les entourent, afin d'identifier des suspects. De plus, ils recherchent des informations peu accessibles ou apparentes concernant des établissements ou la situation et le comportement d'individus, en général pour prévenir des infractions. Leurs tâches consistent à établir des contacts et à trouver des sources d'information sur des infractions projetées ou commises afin de les prévenir ou d'identifier des suspects ; à obtenir, à vérifier et à analyser des éléments de preuve afin d'identifier les auteurs d'infractions ; à procéder à des arrestations ; et à témoigner devant les tribunaux, entre autres. Ils exercent généralement des fonctions d'encadrement. Les inspecteurs et enquêteurs de police doivent généralement être titulaires d'un diplôme universitaire et/ou sont recrutés par promotion interne à l'issue d'un certain nombre d'années d'expérience en qualité d'agent de police (généralement plus de cinq ans).

Les agents de police (qui relèvent de la catégorie 5412 de la CITP-08) assurent le maintien de l'ordre, patrouillent au sein des lieux publics, veillent au respect des textes législatifs et réglementaires et procèdent à l'arrestation de suspects. En outre, ils règlent la circulation, interviennent en cas d'accident de circulation et assurent une aide d'urgence aux victimes d'accidents, d'infractions et de catastrophes naturelles, entre autres. Les agents de police n'ont généralement pas à encadrer plus de trois personnes. Ils ne sont généralement pas tenus d'avoir un diplôme universitaire.

Les agents de l'immigration (qui relèvent de la catégorie 3351 de la CITP-08) contrôlent les personnes qui passent les frontières nationales afin de faire respecter les règles applicables. Dans le cadre de leurs attributions, ils patrouillent au niveau des frontières nationales et des eaux côtières pour empêcher les entrées illégales sur le territoire, de même que les sorties illégales du territoire ; ils vérifient les documents de voyage des personnes qui traversent les frontières nationales pour s'assurer qu'elles détiennent les autorisations et attestations nécessaires ; et ils assurent une coordination et une coopération avec les autres autorités chargées du maintien de l'ordre, des expulsions et des poursuites, entre autres. Les agents de l'immigration n'ont généralement pas à encadrer plus de trois personnes, quand ils exercent des fonctions d'encadrement.

Les agents des douanes (qui relèvent de la catégorie 3351 de la CITP-08) contrôlent les véhicules qui traversent les frontières nationales afin de faire respecter les règles applicables. Dans le cadre de leurs attributions, ils inspectent les bagages des personnes qui traversent les frontières nationales pour veiller au respect des règles officielles relatives aux importations et aux exportations de biens et de devises ; ils examinent les documents de transport et la cargaison des véhicules qui traversent les frontières nationales pour veiller au respect des règles officielles; et ils procèdent à des arrestations et à des saisies de biens interdits ou non déclarés en cas d'infraction aux règles relatives à l'immigration et aux douanes, entre autres. Les agents des douanes n'ont généralement pas à encadrer plus de trois personnes, quand ils exercent des fonctions d'encadrement.

Les inspecteurs des impôts (qui relèvent de la catégorie 3352 de la CITP-08) examinent les déclarations d'impôt et les actes de vente, entre autres documents, pour déterminer le type et le montant des impôts, taxes et autres types de redevances devant être acquittés par les individus et les entreprises, en transmettant les dossiers exceptionnels ou importants à des comptables ou à de hauts fonctionnaires. Ils conseillent les organisations, les entreprises et le public sur les règles applicables en matière de détermination et de versement des impôts, des taxes et des autres types de redevances ainsi que sur les droits et les obligations des contribuables; ils examinent les déclarations d'impôt et les actes de vente, entre autres documents pertinents ; et ils contrôlent les déclarations effectuées et les documents et systèmes comptables et dispositifs de contrôle interne des organisations afin de veiller au respect des textes législatifs et réglementaires applicables en matière d'imposition, entre autres. Les inspecteurs des impôts n'ont généralement pas à encadrer plus de trois personnes, quand ils exercent des fonctions d'encadrement. 


\section{La rémunération}

L'enquête porte sur la rémunération totale, qui compte deux grandes composantes : 1) les salaires et traitements bruts ; et 2) les cotisations sociales de l'employeur. Les questions relatives aux niveaux de rémunération étaient posées pour des emplois à temps plein.

1. Les salaires et traitements bruts comprennent le montant des éventuels impôts sur le revenu, cotisations sociales, etc., payables au salarié, même s'ils sont en réalité retenus à la source par l'employeur pour des raisons de commodité administrative ou autres, et versés directement aux régimes de sécurité sociale, aux autorités fiscales, etc. pour le compte du salarié. Les cotisations sociales de l'employeur ne sont pas comprises dans les salaires et traitements bruts. Toute rémunération en nature est exclue de l'enquête. Les salaires et traitements bruts englobent:

- Les salaires et traitements de base, qui correspondent aux paiements annuels réguliers faits aux agents en contrepartie de leur temps de travail et des services rendus à l'administration. Bien que les salaires et traitements soient versés selon un rythme hebdomadaire, mensuel ou autre, la rémunération retenue pour les besoins de l'enquête est la rémunération annuelle. Les paiements au titre des heures supplémentaires sont exclus des données.

- Les paiements supplémentaires - parce qu'il est difficile d'obtenir des données exhaustives et comparables à l'échelle internationale, la catégorie des paiements supplémentaires a été réduite à ses principaux éléments, à savoir :

* La rémunération des heures de travail non effectuées, qui correspond uniquement à la rémunération versée au titre des congés annuels et des jours fériés.

* Les primes et gratifications régulières, qui correspondent aux primes de fin d'année et aux primes saisonnières ; aux primes d'intéressement aux résultats ; et aux paiements supplémentaires au titre des vacances, qui s'ajoutent à la rémunération normale afférente aux congés et aux autres primes et gratifications.

* Les primes et gratifications exceptionnelles (liées à la performance), qui correspondent aux primes et aux autres paiements exceptionnels liés à la performance globale de l'employé.

2. Les cotisations sociales de l'employeur sont les cotisations sociales payables par les employeurs aux caisses de sécurité sociale ou à d'autres régimes d'assurance sociale liés à l'emploi afin d'assurer à leurs employés le droit à des prestations sociales (assurance santé, retraite) :

- Les cotisations de l'employeur aux régimes obligatoires de sécurité sociale ou à des systèmes privés d'assurance sociale couvrant la vieillesse, la retraite, l'invalidité et la maladie. Les cotisations sociales de l'employeur correspondent aux cotisations sociales payables par les employeurs à des caisses de sécurité sociale ou à d'autres régimes d'assurance sociale liés à l'emploi afin d'assurer à leurs employés le droit à des prestations sociales (assurance santé, retraite). Dans certains pays, ces cotisations sociales financent des régimes publics. Dans d'autres, elles financent des systèmes privés. Les cotisations sociales de l'employeur englobent parfois des fonds spécifiques créés, par exemple, dans le cadre d'accords sociaux. Dans l'enquête de 2011, on a uniquement recueilli des données sur les cotisations sociales versées par les employeurs à des fins d'assurance santé et d'assurance retraite, ce qui correspond à la majorité de leurs cotisations sociales.

- Les avantages sociaux non capitalisés destinés aux employés et financés par les employeurs (uniquement pour des prestations de santé et de retraite). Le terme « non 
capitalisé » désigne des avantages sociaux pour lesquels il n'existe pas de caisse de sécurité sociale ni de suivi officiel des cotisations sociales. Il existe des régimes de santé et de retraite de cet ordre dans de nombreux pays; dans ce cas de figure, les pensions et les prestations de santé destinées aux fonctionnaires sont financées à partir du budget des administrations publiques. Dans un certain nombre de pays, les cotisations de l'employeur et de l'employé ne couvrent pas l'intégralité des avantages sociaux des fonctionnaires. Dans ces cas de figure, des postes budgétaires spéciaux sont souvent consacrés au financement de la partie non capitalisée de ces avantages sociaux.

Tous les pays n'ont pas été en mesure d'intégrer les cotisations sociales à leurs réponses à l'enquête (principalement en raison des systèmes de retraite non capitalisés). Il a donc fallu estimer cette composante à l'aide d'autres sources de données pour ces pays. Dans la base de données de l'OCDE relative aux comptes nationaux, les imputations au titre des régimes de retraite non capitalisés sont rendues conceptuellement cohérentes pour l'ensemble des pays. Par conséquent, à partir des données figurant dans cette base de données, il a été possible d'estimer le taux global des cotisations sociales de l'employeur figurant dans les différentes bases de données existantes concernant la rémunération des agents publics. Le taux retenu pour calculer les coûts de rémunération dans les données figurant dans la présente publication a été choisi après enquête et discussion avec les pays. On a pris comme source les Comptes nationaux pour les pays suivants : l'Allemagne, la Finlande, la Grèce, le Japon et la Norvège. De plus, pour la Belgique, on a estimé ce taux en utilisant à la fois des informations tirées de l'enquête et des données tirées des Comptes nationaux.

Il convient de noter qu'alors que l'enquête sur la rémunération des fonctionnaires tient uniquement compte des cotisations de l'employeur en matière de santé et de retraite, les données tirées des Comptes nationaux prennent en compte l'ensemble des cotisations sociales versées par l'employeur. Par conséquent, les cotisations sociales sont, dans une certaine mesure, surestimées quand les données proviennent de cette source. De plus, les données tirées des Comptes nationaux fournissent un taux de cotisations sociales de l'employeur pour l'ensemble des agents publics. Recourir à ce taux ne permet pas de tenir compte des différences qui peuvent exister au niveau des taux de cotisations sociales en fonction des professions, par exemple. En revanche, pour les pays qui ont fourni des données relatives aux cotisations sociales de l'employeur dans le cadre de l'enquête, ce sont les chiffres exacts des cotisations (lesquels peuvent varier selon les professions) qui ont été utilisés.

Le niveau des cotisations sociales n'est qu'un indicateur indirect. La quantité et la qualité des avantages sociaux dont les salariés bénéficient grâce à leurs cotisations sociales et à celles de leur employeur dépendent de nombreuses variables telles que la qualité et de l'efficience de la gestion des ressources financières et des services dans chaque pays.

\section{Le recours à des comparateurs}

On a effectué les calculs en convertissant les chiffres relatifs aux rémunérations en USD au moyen des PPA. Cela permet de compenser les écarts de taux de change et de niveaux de prix relatifs. Les PPA ne tiennent pas compte des écarts de coût de la vie liés au fait de vivre dans la capitale de son pays, par rapport au reste du pays et par rapport aux autres pays. Or, dans de nombreux pays, la majorité des agents de l'administration centrale travaillent dans la capitale. Les salaires et traitements versés à ces agents peuvent avoir tendance à compenser le surcoût que cela implique.

L'OCDE a également procédé à des comparaisons entre les pays à la lumière de données normalisées grâce au PIB par habitant figurant dans les Statistiques de l'OCDE sur 
les comptes nationaux (base de données). Cette normalisation constitue un moyen de corriger les écarts qui existent entre les pays au niveau de la richesse moyenne.

L'OCDE a également calculé des ratios permettant de comparer la rémunération totale moyenne des agents publics à la rémunération moyenne des diplômés de l'enseignement supérieur (salaires bruts plus cotisations sociales de l'employeur). Étant donné que les postes au sein de l'administration centrale couverts par l'enquête impliquent généralement un niveau éducatif élevé, le calcul de ces ratios permet de comparer les rémunérations pratiquées au sein du secteur public et celles proposées au sein de l'ensemble de l'économie. Les données relatives à la rémunération des diplômés de l'enseignement supérieur sont tirées d'un ensemble de sources, comme c'est indiqué dans la publication Regards sur l'éducation (édition 2013 ou éditions antérieures). Si on tient ainsi compte du niveau éducatif, les données ne sont, en revanche, pas corrigées en fonction des autres caractéristiques individuelles des personnes occupant ces fonctions : ancienneté, âge, sexe, etc.

Les comparaisons entre la rémunération des agents publics et le PIB par habitant et entre la rémunération des agents publics et celle des diplômés de l'enseignement supérieur n'ont pas été corrigées pour tenir compte du temps de travail. Ce choix a été fait afin d'assurer une cohérence entre les trois valeurs.

\section{Les ajustements liés au temps de travail}

Pour calculer la rémunération moyenne annuelle ajustée en fonction du temps de travail, on rapproche le temps effectivement travaillé de la rémunération moyenne annuelle (rémunération brute moyenne annuelle plus cotisations sociales de l'employeur). En l'espèce, pour pouvoir effectuer des comparaisons internationales des niveaux de rémunération des agents publics, on tient compte des différences au niveau du temps de travail (nombre d'heures travaillées par semaine dans la fonction publique, durée légale ou moyenne des congés annuels et nombre de jours fériés dans l'administration) pour calculer une rémunération moyenne annuelle ajustée.

Pour tous les cadres (c'est-à-dire les postes D1, D2, D3 et D4), étant donné que la durée hebdomadaire du travail s'applique de façon très inégale à cette catégorie d'agents, les données ont uniquement été ajustées pour les congés.

Les ajustements liés au temps de travail sont indiqués dans le tableau D.1.

Calcul de la rémunération annuelle comparative moyenne :

$\mathrm{W}_{\mathrm{co}}^{a^{*}}=\frac{\left(\frac{\mathrm{W}_{\mathrm{co}}^{a}}{\mathrm{P}_{\mathrm{c}}}\right)}{\mathrm{H}_{\mathrm{c}}^{a^{*}}}$

où :

$\mathrm{W}_{c 0}^{a^{*}}=$ rémunération annuelle moyenne des employés en PPA dans un pays c pour une catégorie professionnelle 0 , après ajustement lié au temps de travail.

$\mathrm{W}_{\mathrm{co}}^{a}=$ rémunération annuelle moyenne en monnaie nationale dans un pays $c$ pour une catégorie professionnelle 0 .

$P_{c}=$ parité de pouvoir d'achat du pays $c$.

$H_{c}^{a^{*}}=$ ratio de temps de travail moyen dans le pays $c$. Il correspond au nombre moyen d'heures de travail par an dans le pays c (à la lumière des données fournies dans l'enquête), divisé par 2 088. Le nombre 2088 est égal au nombre théorique d'heures de travail par an, à raison de 40 heures de travail par semaine, sans jours fériés ni congés d'aucune sorte. Cela donne aussi une moyenne de 261 jours de travail par an, à raison de huit heures de travail par jour. 
Tableau D.1. Ajustements liés au temps de travail

\begin{tabular}{|c|c|c|c|c|c|c|c|c|}
\hline & $\begin{array}{c}\text { Temps de travail } \\
\text { contractuel } \\
\text { (nb. d'heures } \\
\text { par semaine) }\end{array}$ & $\begin{array}{l}\text { Nombre } \\
\text { moyen de jours } \\
\text { de congé }\end{array}$ & $\begin{array}{c}\text { Nombre moyen } \\
\text { de jours fériés } \\
\text { dans la fonction } \\
\text { publique }\end{array}$ & $\begin{array}{c}\text { Nombre moyen } \\
\text { de jours de travail } \\
\text { par an dans } \\
\text { le pays }\end{array}$ & $\begin{array}{l}\text { Nombre moyen } \\
\text { d'heures de } \\
\text { travail par an } \\
\text { dans le pays }\end{array}$ & $\begin{array}{l}\text { Coefficient pour } \\
\text { les ajustements } \\
\text { liés au temps } \\
\text { de travail - } \\
\text { heures de travail } \\
\text { hebdomadaires } \\
\text { et congés }\end{array}$ & $\begin{array}{l}\text { Coefficient } \\
\text { pour l'ajustement } \\
\text { lié au temps } \\
\text { de travail - } \\
\text { congés }\end{array}$ & $\begin{array}{c}\text { Coefficient } \\
\text { pour l'ajustement } \\
\text { lié au temps } \\
\text { de travail - pas } \\
\text { d'ajustement }\end{array}$ \\
\hline Allemagne & 41 & 29.5 & 10 & 221 & 1814 & 0.870 & 0.848 & 1.000 \\
\hline Australie & 37.5 & 20 & 10 & 231 & 1730 & 0.830 & 0,885 & 1.000 \\
\hline Autriche & 40 & 25 & 10 & 226 & 1806 & 0.866 & 0,866 & 1.000 \\
\hline Belgique & 38 & 26 & 10 & 225 & 1708 & 0.819 & 0,862 & 1.000 \\
\hline Chili & 44 & 15 & 9 & 237 & 2083 & 0.999 & 0,908 & 1.000 \\
\hline Corée & 40 & 20 & 14 & 227 & 1814 & 0.870 & 0.870 & 1.000 \\
\hline Danemark & 37 & 30 & 9 & 222 & 1641 & 0.787 & 0,850 & 1.000 \\
\hline Espagne & 37.5 & 22 & 14 & 225 & 1685 & 0.808 & 0.862 & 1.000 \\
\hline Estonie & 40 & 34 & 5 & 222 & 1774 & 0.850 & 0.850 & 1.000 \\
\hline États-Unis & 40 & 20 & 10 & 231 & 1846 & 0.885 & 0.885 & 1.000 \\
\hline Finlande & 36.3 & 32 & 7 & 222 & 1607 & 0.771 & 0.850 & 1.000 \\
\hline France & 35.5 & 25 & 7.5 & 228 & 1620 & 0.777 & 0.875 & 1.000 \\
\hline Grèce & 40 & 25 & 12 & 224 & 1790 & 0.858 & 0.858 & 1.000 \\
\hline Islande & 40 & 30 & 14 & 217 & 1734 & 0.831 & 0.831 & 1.000 \\
\hline Israël & 42.5 & 24 & 9 & 228 & 1936 & 0.928 & 0.873 & 1.000 \\
\hline Italie & 36 & 32.0 & 8 & 221 & 1589 & 0.762 & 0.847 & 1.000 \\
\hline Japon & 38.8 & 20 & 17 & 224 & 1734 & 0.831 & 0.858 & 1.000 \\
\hline Nouvelle-Zélande & 40 & 20 & 11 & 230 & 1838 & 0.881 & 0.881 & 1.000 \\
\hline Norvège & 37.5 & 25 & 10 & 226 & 1693 & 0.812 & 0.866 & 1.000 \\
\hline Pays-Bas & 36 & 23 & 8 & 230 & 1654 & 0.793 & 0.881 & 1.000 \\
\hline Pologne & 40 & 26 & 11 & 224 & 1790 & 0.858 & 0.858 & 1.000 \\
\hline Portugal & 35 & 25 & 11 & 225 & 1573 & 0.754 & 0.862 & 1.000 \\
\hline République slovaque & 37.5 & 25 & 15 & 221 & 1655 & 0.794 & 0.847 & 1.000 \\
\hline Royaume-Uni & 37 & 25 & 8 & 228 & 1685 & 0.808 & 0.873 & 1.000 \\
\hline Slovénie & 40 & 29.9 & 15 & 216 & 1727 & 0.828 & 0.828 & 1.000 \\
\hline Suède & 39.8 & 33 & 9 & 219 & 1739 & 0.834 & 0.839 & 1.000 \\
\hline
\end{tabular}

Notes : Les chiffres du tableau sont arrondis.

Nombre maximum de jours de travail par an à raison de 5 jours sur 7 de travail hebdomadaire : 261. Nombre maximum d'heures de travail par an à raison de 8 heures de travail par jour : 2088.

Allemagne : Le temps de travail contractuel est différent entre les agents publics et les fonctionnaires.

Autriche : Depuis le $1^{\text {er }}$ janvier 2011, la durée des congés dépend de l'âge : un employé à temps plein a droit à 240 heures de congé (30 jours/6 semaines) à partir de l'année de ses 43 ans, s'il est né avant le $1^{\text {er }}$ juillet. S'il est né après le 30 juin, il aura droit à ces 240 heures de congé à partir de l'année suivante.

Corée : Les fonctionnaires ont droit à des congés annuels d'une durée comprise entre 3 et 21 jours, en fonction de leur ancienneté.

Estonie : La durée des congés annuels dépend de l'ancienneté. À la base, elle est de 35 jours civils, soit 28 jours ouvrés, mais les agents dotés d'une ancienneté supérieure à trois ans bénéficient d'une journée de congé supplémentaire par an, avec un plafond de 10 jours de congé supplémentaires par an.

Finlande : Les congés annuels durent 30 ou 38 jours, en fonction de l'ancienneté.

Italie : La durée légale des congés varie, passant de 30 jours pendant les trois premières années de service à 32 jours à partir de la quatrième année. Pour la police, il existe deux sous-catégories en fonction de l'ancienneté : les policiers ayant entre 15 et 25 années d'ancienneté ont droit à 36 jours de congé, et les policiers ayant plus de 25 années d'ancienneté ont droit à 45 jours de congé.

République slovaque : Le temps de travail contractuel est de 40 ou 37.5 heures par semaine, en fonction du contrat. Le nombre de jours de congé dépend de l'âge et s'élève à 25 jours pour les moins de 33 ans et à 30 jours pour les plus de 33 ans.

Royaume-Uni : Le temps de travail est de 37 heures par semaine en dehors de Londres, où la plupart des fonctionnaires travaillent, et de 36 heures à Londres. Le nombre moyen de jours de congé annuel varie d'un service à l'autre.

Slovénie : Le nombre moyen de jours de congé annuel est une estimation. Chaque agent a droit à au moins quatre semaines de congé annuel. Il a droit, en plus, à un jour de congé annuel supplémentaire par enfant de moins de 15 ans. De plus, chaque agent peut avoir droit à un maximum de trois jours de congé supplémentaires, en fonction de ses résultats. Les congés annuels peuvent être prolongés de trois jours au maximum en cas de conditions de travail difficiles (bruit, chaleur...) ou de problèmes de santé, ou si le fonctionnaire dirige une unité opérationnelle.

Suède : Le nombre de jours de congé varie en fonction de l'âge, aux termes de la convention collective centrale. Les agents de moins de 30 ans ont 28 jours de congé ; entre 30 et 39 ans, les agents ont 31 jours de congé ; à partir de 40 ans, ils ont 35 jours de congé.

Source : Enquête de l'OCDE sur la rémunération des fonctionnaires des administrations centrales/fédérales (2011). 


\section{ANNEXE E \\ Données détaillées sur les déclarations de conflits d'intérêts*}

Cette annexe fournit des données pour chaque pays ayant répondu à l'enquête sur les types d'intérêts privés que les décideurs publics doivent divulguer ainsi que sur le degré de transparence de ces divulgations. Ces données ont servi à établir le récapitulatif présenté dans le graphique 8.4.

\section{Notes relatives au tableau E.1}

Les données reflètent les pratiques des pays membres. Pour calculer des agrégats à partir des données nationales fournies sur les déclarations d'intérêts obligatoires au sein des trois pouvoirs et sur leur niveau de divulgation au grand public, tous les intérêts privés et toutes les positions ont été considérés comme d'importance égale et, par conséquent, ont reçu la même pondération. Pour traduire les réponses des pays en points, on a procédé comme suit : les catégories « Interdit » et « Les informations sont divulguées et disponibles publiquement au format imprimé ou électronique » ont été créditées de 100 points, soit le maximum possible. La catégorie "Les informations sont divulguées et disponibles publiquement sur demande " a reçu 67 points (soit le deuxième score possible), et la catégorie "Les informations sont divulguées mais pas disponibles publiquement" 33 points (troisième valeur possible). Aucun point n'a été attribué pour la catégorie "La divulgation n'est pas obligatoire ». Tous les intérêts privés examinés ont reçu la même pondération.

Les données relatives au Brésil, à la Fédération de Russie, à la Grèce, à Israël et à la République tchèque portent sur 2010. De même, les données relatives aux pouvoirs législatif et judiciaire espagnols portent sur 2010.

Au Luxembourg, il n'existe aucune obligation de déclaration des intérêts privés. Aux États-Unis, les procureurs appartiennent au pouvoir exécutif. Les hauts fonctionnaires du ministère de la Justice sont tenus de faire des déclarations de patrimoine au même titre que tous les autres agents du pouvoir exécutif, et ces déclarations sont rendues publiques. Pour les procureurs fédéraux de moindre rang, un dispositif distinct et non public de déclaration des conflits d'intérêts s'applique. Les données concernant les juges norvégiens excluent les juges non professionnels et les juges des commissions de conciliation. En

* Les données statistiques concernant Israël sont fournies par et sous la responsabilité des autorités israéliennes compétentes. L'utilisation de ces données par l'OCDE est sans préjudice du statut des hauteurs du Golan, de Jérusalem-Est et des colonies de peuplement israéliennes en Cisjordanie aux termes du droit international. 
Nouvelle-Zélande, les juges et les procureurs ne sont soumis à aucune obligation de déclaration de leurs conflits d'intérêts. En revanche, des règles de conduite à l'usage des magistrats ont été formulées et peuvent être consultées sur le site des tribunaux néozélandais (www.courtsofnz.gout.nz/business/guidelines/guidelines-for-judicial-conduct). De plus, les procureurs sont souvent également avocats et, à ce titre, sont soumis à des obligations relevant de l'éthique professionnelle (en cas de manquement, ils peuvent, selon les circonstances, être sanctionnés ou même se voir retirer leur autorisation d'exercer). Au Brésil, une nouvelle loi sur les conflits d'intérêts pour les agents du pouvoir exécutif et les interdictions pesant sur les anciens agents publics (loi 12.813) est entrée en vigueur en juillet 2013.

Postes extérieurs rémunérés : dans le cadre de règles qui s'appliquent à tous les fonctionnaires en Autriche et en Belgique et aux juges en Islande et en Suisse, tout fonctionnaire titularisé doit se soumettre à la décision des autorités si un poste extérieur rémunéré est susceptible de donner lieu à un conflit d'intérêts. Au Danemark, les juges ne peuvent occuper un poste extérieur rémunéré (à condition de le déclarer) que si ce poste est réservé à un juge aux termes de la loi ou si une commission spéciale donne son autorisation. En Estonie, la loi interdit au Premier ministre, aux ministres, aux juges et aux procureurs d'occuper des postes extérieurs rémunérés, en dehors des activités de recherche et d'enseignement, qui doivent être déclarées. Au Japon, les juges ne peuvent pas occuper de poste extérieur rémunéré tant qu'ils restent en fonctions, sauf autorisation de la Cour suprême. En principe, les juges doivent également obtenir la permission de la Cour suprême ou de leur hiérarchie pour les postes extérieurs non rémunérés. Toutefois, il est déjà arrivé que des juges occupent des postes extérieurs non rémunérés tels que, par exemple, la présidence d'une association de parents d'élèves sans l'autorisation de la Cour suprême ou de leur hiérarchie. Si des informations sur les postes extérieurs des juges sont demandées, aucune information permettant l'identification des juges ne peut être rendue publique. En Pologne, le Premier ministre et les autres ministres n'ont pas le droit d'exercer certaines activités susceptibles d'aboutir à des conflits d'intérêts. De plus, ils sont tenus de signaler leur appartenance à des fondations, des sociétés commerciales ou des coopératives, même s'il s'agit de postes non rémunérés.

Emplois antérieurs : en Estonie, aucune règle n'oblige les membres des pouvoirs exécutif et législatif à publier des informations sur leurs emplois antérieurs ; toutefois, en pratique, ils prennent les devants et fournissent ces informations.

Actifs, dettes, montant et source des revenus, cadeaux : en Islande, le Premier ministre est uniquement tenu de divulguer les prêts qui ont été annulés ou modifiés à son avantage. En Irlande, les salaires et indemnités des parlementaires sont rendus publics. De plus, aux termes de lois sur l'éthique, tous les parlementaires et les titulaires de certaines fonctions publiques doivent déclarer leurs intérêts personnels - revenus provenant d'autres sources (postes extérieurs rémunérés), actions, appartenance à des conseils d'administration, terrains, cadeaux, prestations ou voyages à conditions préférentielles, activités de consultant et tout intérêt dans un marché public - dans des déclarations annuelles d'intérêts. Ces renseignements sont mis à la disposition du public au sein d'un registre à cet effet. Au Mexique, les cadeaux doivent être déclarés si leur valeur est égale ou supérieure à dix fois le salaire minimum. Des informations sur les fonctionnaires sont publiées en ligne si les fonctionnaires concernés l'autorisent. En pratique, 66 \% environ des fonctionnaires diffusent publiquement ces informations. En Pologne, le Premier ministre et les autres ministres sont tenus de faire des déclarations précisant, par exemple, leurs actifs, leurs dettes et la source et le montant de leurs revenus. La loi ne prévoit pas que ces 
déclarations soient rendues publiques, mais presque tous les ministres acceptent de les publier en ligne.

En Irlande et en Italie, la remise des formulaires de déclaration n'est suivie d'aucune autre étape, mais la plupart des informations fournies sont mises à la disposition du public, ce qui permet aux citoyens eux-mêmes de les examiner. 
Tableau E.1. Divulgation de certains intérêts privés et accessibilité des informations fournies au sein des trois pouvoirs, par pays (2012)

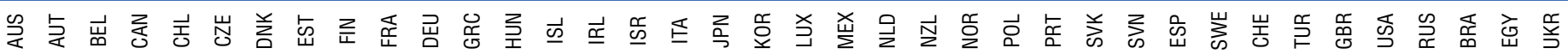



au sein des trois pouvoirs, par pays (2012) (suite)

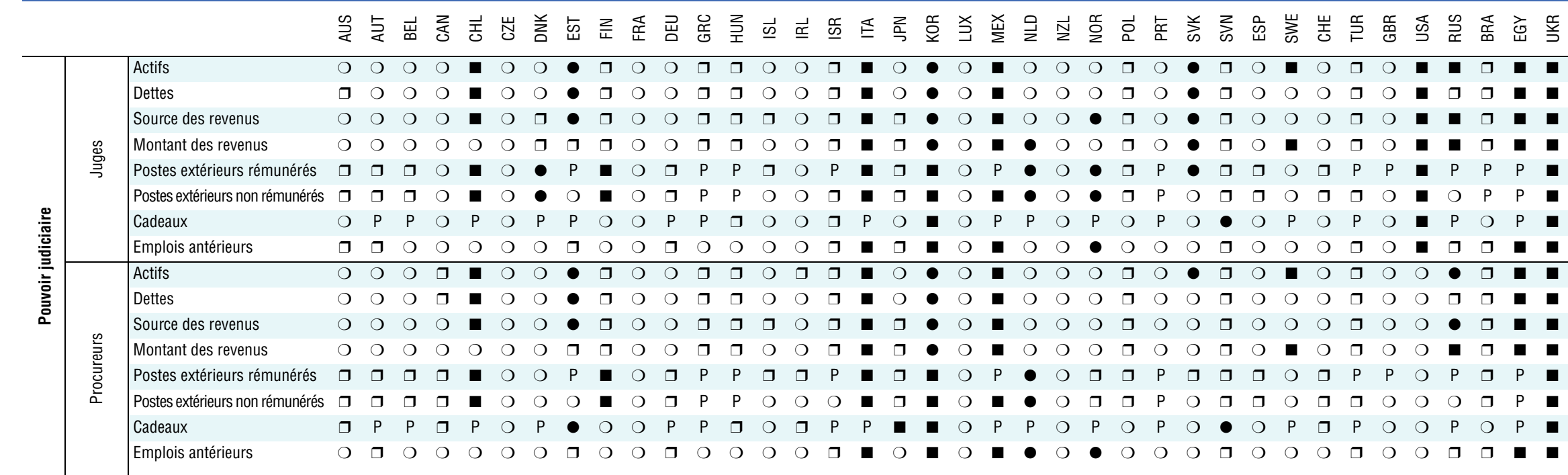

- Informations divulguées et librement accessibles en ligne ou sur papier

$\checkmark$ Informations divulguées mais non librement accessibles

- Informations divulguées et librement accessibles sur demande

O Divulgation non requise

P Prohibé

x Sans objet (le pays n'a pas de président par exemple)

“.." Données manquantes

Source: Enquête de l'OCDE sur la gestion des conflits d'intérêts (2012). 
ANNEXE F

\section{Membres du Groupe de direction}

\begin{tabular}{|c|c|c|c|}
\hline & Nom & Titre/fonction & Ministère \\
\hline Australie & Mme Kathie Potts & Commissaire adjointe aux services publics & Commission australienne des services publics \\
\hline Autriche & M. Michel Kallinger & Directeur général & $\begin{array}{l}\text { Chancellerie fédérale, Service public et } \\
\text { innovation administrative }\end{array}$ \\
\hline Belgique & M. Jacques Druart & Chef de la coordination internationale & $\begin{array}{l}\text { Service public fédéral } \\
\text { Personnel et organisation }\end{array}$ \\
\hline Canada & Nicholas Wise & Directeur exécutif & Secrétariat du Conseil du Trésor \\
\hline Corée & M. Seong Ju Kang & $\begin{array}{l}\text { Directeur général de la politique de sécurité } \\
\text { de l'information }\end{array}$ & $\begin{array}{l}\text { Bureau de la stratégie de l'information, } \\
\text { ministère de l'Administration publique et de la } \\
\text { Sécurité }\end{array}$ \\
\hline Danemark & M. David Fjord Nielson & Conseiller spécial & Ministère des Finances \\
\hline Finlande & Mme Katju Holkeri & Chef de l'Unité de la gouvernance & Ministère des Finances \\
\hline France & M. Daniel Aunay & Chef de la mission des relations internationales & $\begin{array}{l}\text { Ministère du Budget, des Comptes publics, de } \\
\text { la Fonction publique et de la Réformede l'État }\end{array}$ \\
\hline Italie & Dr Pia Marconi & Directrice générale & Département de l'administration publique \\
\hline Japon & M. Irie Akifumi & Premier secrétaire & $\begin{array}{l}\text { Délégation permanente du Japon auprès de } \\
\text { I'OCDE }\end{array}$ \\
\hline Norvège & M. Kleng Bratveit & & $\begin{array}{l}\text { Ministère de l'Administration publique et de la } \\
\text { Réforme, Département de la politique des TIC } \\
\text { et de la réforme du secteur public }\end{array}$ \\
\hline Pays-Bas & M. Peter van Der Gaast & $\begin{array}{l}\text { Chef du Département de l'analyse, du marché } \\
\text { du travail et des conseils macroéconomiques }\end{array}$ & $\begin{array}{l}\text { Ministère de l'Intérieur et des Relations } \\
\text { extérieures }\end{array}$ \\
\hline Royaume-Uni & Mme Liz McKeown & Directrice adjointe, Analyses et éclairages & Secrétariat du Cabinet \\
\hline Suède & Mme. Susanne Johansson & Conseillère & Statskontoret \\
\hline
\end{tabular}




\section{Glossaire}

\section{Terme ou expression}

Administrations publiques

Ajustement lié
au temps de travail

Autorité budgétaire centrale (ABC)

\section{Acception dans le Panorama des administrations publiques}

Le secteur des administrations publiques se compose : a) de toutes les unités appartenant à l'administration centrale, aux administrations d'États fédérés et aux administrations locales ; b) de toutes les administrations de sécurité sociale, à tous les niveaux d'administration; et c) de toutes les institutions sans but lucratif non marchandes contrôlées et financées à titre principal par des administrations publiques. Le secteur ne comprend pas les sociétés publiques, même quand la totalité du capital de ces sociétés est détenue par des administrations. Il ne comprend pas non plus les quasi-sociétés qui sont possédées et contrôlées par des administrations publiques. En revanche, les entreprises non constituées en sociétés qui appartiennent à des administrations publiques et qui ne sont pas des quasi-sociétés restent partie intégrante de ces administrations, et elles doivent donc être incluses dans le secteur des administrations publiques (Système de comptabilité nationale 1993)

Ajustement appliqué à la rémunération annuelle moyenne des agents publics qui compense les différences au niveau de la durée du travail en tenant compte, selon les cas, du nombre moyen de jours de travail et du nombre moyen d'heures de travail par semaine.

L'Autorité budgétaire centrale $(A B C)$ est une entité publique (ou plusieurs entités coordonnées) située au niveau de l'administration centrale/nationale/fédérale et chargée de tenir et de gérer le budget national/fédéral. Dans de nombreux pays, l'ABC est rattachée au ministère des Finances. Ses responsabilités précises peuvent varier selon les pays mais, de façon générale, l'ABC est chargée de formuler les propositions budgétaires, de mener les négociations budgétaires, d'affecter ou de réaffecter les ressources, de veiller au respect des textes budgétaires et de procéder à des évaluations des performances et/ou à des examens de l'efficience. L'Autorité régule l'exécution du budget mais n'assume pas nécessairement de fonction de gestion de la trésorerie l'amenant à procéder à des paiements à partir de fonds publics. Enfin, elle joue un rôle très important en suivant et en contrôlant la discipline budgétaire d'ensemble/nationale et en assurant le contrôle des dépenses effectuées à partir du budget. 
Biens et services collectifs

Biens et services individuels

Budget

Budget du citoyen

Cadre général d'emploi de la fonction publique

Efficacité

Efficience

Ensemble de données

Équivalent temps

plein (ETP)

État fédéral

États unitaires
Biens et services qui bénéficient à la communauté dans son ensemble. Les dépenses publiques consacrées à la défense et au maintien de l'ordre relèvent des biens et services collectifs.

Biens et services bénéficiant avant tout aux individus. Tel est, par exemple, le cas des programmes d'éducation, de santé ou d'assurance sociale.

État exhaustif des plans financiers de l'État présentant les dépenses, les recettes, le déficit ou l'excédent et la dette. Le budget constitue le principal document de politique économique du gouvernement, illustre comment le gouvernement prévoit de mettre les ressources publiques au service de ses objectifs et, dans une certaine mesure, traduit ses priorités.

Un "budget du citoyen » est défini ici comme une synthèse en langage simple des principaux volets du budget annuel présenté au parlement. Ce document devrait se suffire à lui-même et expliquer la teneur du projet de budget annuel et ses effets escomptés. Même si ce guide comporte des liens vers ou des références à des documents plus détaillés, le lecteur devrait pouvoir le comprendre sans être obligé de se référer à ces autres documents ou d'en connaître la teneur.

Il énonce généralement les conditions d'emploi de la plupart des agents publics et, en tout état de cause, de la plupart des agents statutaires. Selon cette définition, les agents occasionnels ne relèvent pas du Cadre général d'emploi de la fonction publique. Il convient de noter que, dans un certain nombre de pays, tous les agents, y compris ceux qui sont recrutés pour une courte durée, relèvent du Cadre général d'emploi de la fonction publique, à quelques exceptions près (il y a peu, voire pas d'agents occasionnels dans ces cas).

Degré de réalisation des objectifs définis pour une activité donnée (OECD Glossary of Statistical Terms, en anglais uniquement)

Obtention d'un produit maximum à partir d'un niveau déterminé de ressources affecté à l'exercice d'une activité (OECD Glossary of Statistical Terms, en anglais uniquement)

Ensemble d'indicateurs ou de variables concernant un sujet unique (la qualité de la réglementation, par exemple).

Nombre d'emplois en équivalent temps plein, défini comme le nombre total d'heures travaillées, divisé par la moyenne annuelle des heures travaillées dans des emplois à temps plein (OECD Glossary of Statistical Terms, en anglais uniquement).

Pays ayant délimité, dans sa constitution, une division de l'autorité politique entre une administration centrale et plusieurs administrations régionales ou d'États fédérés autonomes.

Pays n'ayant pas délimité, dans leur constitution, de division de l'autorité politique entre une administration centrale et plusieurs administrations régionales ou d'États fédérés autonomes. 
Genre

Gouvernance

Indicateur

Indice composite

Informations

sur la performance

Institution budgétaire indépendante (IBI)
Cependant, les États unitaires peuvent comporter des divisions administratives incluant des niveaux d'administration locaux, provinciaux ou régionaux.

Comportements et attentes résultant d'une construction et d'un apprentissage d'ordre social associés aux personnes de sexe féminin et de sexe masculin. Toutes les cultures interprètent et élaborent les différences biologiques entre femmes et hommes au sein d'un ensemble d'attentes sociales quant aux comportements et aux activités considérés comme acceptables et quant aux droits, aux ressources et au pouvoir attribués aux femmes et aux hommes. Tout comme la race, l'ethnicité et la classe, le genre est une catégorie sociale qui détermine en grande partie les possibilités offertes à l'individu. Il influe sur sa participation à la vie sociale et économique.

L'exercice de l'autorité politique, économique et administrative.

«(...) mesure quantitative ou qualitative dérivée d'une série de faits observés pouvant indiquer des positions relatives (par exemple, d'un pays) dans un domaine donné. Évalué à des intervalles réguliers, un indicateur peut indiquer le sens d'une évolution à travers différentes unités et au cours du temps" (Nardo et al., 2005).

Indicateur formé par la compilation d'indicateurs individuels en un indice unique, sur la base d'un modèle sous-jacent (Nardo et al., 2005).

Les informations sur la performance peuvent provenir aussi bien d'entités publiques que d'entités non publiques et être d'ordre tant qualitatif que quantitatif. Les informations sur la performance consistent en des éléments de mesure/des indicateurs/des données d'ordre général sur les intrants, les processus, les produits et les réalisations des politiques/programmes/organismes publics, et elles peuvent être utilisées, in fine, pour évaluer leur efficacité, leur rapport coût-efficacité et leur efficience. Les informations sur la performance peuvent être tirées : de statistiques; d'états financiers/de rapports d'activité d'organismes publics ; de rapports sur la performance publiés par des organismes publics; d'évaluations de politiques, de programmes ou d'organismes; ou d'examens des dépenses, par exemple.

Organe indépendant financé sur fonds publics et placé sous l'autorité du pouvoir exécutif ou du pouvoir législatif qui contrôle et analyse de manière non partisane les politiques et résultats budgétaires et, dans certains cas, formule des conseils en la matière. Les IBI jouent un rôle prospectif en matière de diagnostic ex ante (à la différence des institutions de contrôle des finances publiques, qui jouent un rôle tout aussi indispensable de contrôle ex post).

Intrants
Unités de travail, de capital, de biens et de services utilisées dans la production de biens et de services. 
Main-d'œuvre

Processus de secteur public

Productivité

Produit (" output ")

Produit intérieur brut (PIB)

Règle budgétaire
« En prenant l'exemple des services de santé, les intrants sont définis comme le temps de l'équipe médicale et non médicale, les médicaments, l'électricité et les autres intrants achetés, et l'utilisation de capital relative à l'équipement et aux locaux utilisés. " (Lequiller, 2005)

La main-d'œuvre ou population active du moment comprend toutes les personnes qui remplissent les conditions requises pour être incluses parmi les personnes pourvues d'un emploi ou les chômeurs durant une brève période de référence spécifiée (OECD Glossary of Statistical Terms, en anglais uniquement).

Structures, procédures et dispositifs de gestion largement appliqués au sein du secteur public.

La productivité est généralement définie comme le ratio entre une mesure en volume de produit et une mesure en volume d'utilisation d'intrants (OECD Glossary of Statistical Terms, en anglais uniquement). Les économistes distinguent la productivité totale, c'est-à-dire les produits totaux divisés par le ou les intrants (pondérés), de la productivité marginale, c'est-àdire l'évolution des produits divisée par l'évolution du ou des intrants (pondérés) (Coelli et al., 1999).

Aux fins de l'évaluation des performances des administrations publiques, les produits sont définis comme les biens ou services fournis par celles-ci (par exemple, les heures d'enseignement assurées, les allocations sociales calculées et versées) (OECD Glossary of Statistical Terms, en anglais uniquement)

Instrument classique de mesure de la valeur des biens et services produits par un pays au cours d'une période donnée. Plus précisément, le PIB peut être défini comme la somme des valeurs ajoutées brutes de toutes les unités institutionnelles résidentes qui exercent des activités de production (augmentée de la partie des impôts moins les subventions sur les produits qui n'est pas déjà incluse dans l'évaluation de la production); comme la somme des emplois finals de biens et de services (c'est-à-dire de tous les emplois à l'exception de la consommation intermédiaire), mesurés aux prix d'acquisition, diminuée de la valeur des importations de biens et de services; ou comme la somme des revenus primaires distribués par les unités productrices résidentes (OECD Glossary of Statistical Terms, en anglais uniquement).

Réalisation (" outcome ") Fait référence à ce qui est obtenu, en dernière analyse, grâce à une activité. Les réalisations reflètent les résultats, souhaités ou non, de l'action des administrations, mais des facteurs extérieurs à l'action des administrations entrent également en jeu (OECD Glossary of Statistical Terms, en anglais uniquement).

Aux fins du présent ouvrage, l'OCDE emploie une définition similaire à celle de la Commission européenne. Une règle budgétaire numérique consiste en une contrainte pesant à titre 
Secteur public

Services publics

Système de comptabilité nationale

Système européen des comptes

Transferts monétaires permanent sur les agrégats des finances publiques (par ex., les règles en cours d'exercice sont exclues).

Le secteur des administrations publiques, plus toutes les sociétés (quasi) publiques (Système de comptabilité nationale 1993).

Services réalisés au bénéfice de la population ou des institutions publiques. Les administrations assurent des services publics aux administrés, soit directement (par le truchement du secteur public), soit en finançant la prestation des services en question par le secteur privé. La notion de service public est associée à un consensus social selon lequel certains services doivent être accessibles à tous les citoyens, indépendamment de leurs ressources financières. Même quand des services publics ne sont ni assurés, ni financés par des acteurs publics, ils sont généralement assujettis, pour des raisons d'ordre social et politique, à une réglementation plus poussée que la plupart des secteurs économiques.

Le Système de comptabilité nationale (SCN) se compose d'une série cohérente de comptes macro-économiques, de comptes de patrimoine et de tableaux articulés et coordonnés qui s'appuient sur un ensemble de concepts, définitions, nomenclatures et règles de comptabilisation approuvés au plan international (paragraphe $1.1 \mathrm{du}$ SCN 1993).

Le Système de comptabilité nationale 1993 (SCN 1993) a été établi sous la responsabilité conjointe des Nations Unies, du Fonds monétaire international, de la Commission des communautés européennes, de l'OCDE et de la Banque mondiale (OECD Glossary of Statistical Terms, en anglais uniquement).

Le SCN 2008 a été récemment achevé. Il intègre un certain nombre de changements par rapport au SCN 1993. Pour l'ensemble des pays de l'OCDE, sauf l'Australie (et le Canada s'agissant de la dette publique), les indicateurs présentés au titre du SCN sont basés sur le SCN 1993. Il est important de noter qu'il faudra attendre un certain nombre d'années (jusqu'en 2014 pour la plupart des pays) pour que les comptes nationaux reflètent les changements intervenus (ce qui aura, dans une certaine mesure, une incidence sur la sélection d'indicateurs proposée dans la présente publication).

Cadre comptable compatible au plan international utilisé par les membres de l'Union européenne pour décrire de façon systématique et détaillée une économie totale (c'est-à-dire une région, un pays ou un groupe de pays), ses composantes et ses relations avec d'autres économies totales (OECD Glossary of Statistical Terms, en anglais uniquement). Ce cadre comptable est pleinement compatible avec le Système de comptabilité nationale (SCN).

Prestations versées par les administrations aux individus qui y ont droit et n'ayant pas à être consacrées à un bien ou à un service 
spécifique. Les transferts monétaires englobent, par exemple, les pensions, les allocations chômage et l'aide au développement.

Variable

Caractéristique d'une unité observée pouvant revêtir diverses valeurs et à laquelle peut être assignée une mesure numérique ou une catégorie relevant d'une classification (par exemple, revenu, âge, poids, etc., et " profession ", " secteur ", " maladie ", etc.) (OECD Glossary of Statistical Terms, en anglais uniquement). 


\section{ORGANISATION DE COOPÉRATION ET DE DÉVELOPPEMENT ÉCONOMIQUES}

L'OCDE est un forum unique en son genre où les gouvernements œuvrent ensemble pour relever les défis économiques, sociaux et environnementaux liés à la mondialisation. À l'avant-garde des efforts engagés pour comprendre les évolutions du monde actuel et les préoccupations qu'elles suscitent, l'OCDE aide les gouvernements à y faire face en menant une réflexion sur des thèmes tels que le gouvernement d'entreprise, l'économie de l'information et la problématique du vieillissement démographique. L'Organisation offre aux gouvernements un cadre leur permettant de confronter leurs expériences en matière d'action publique, de chercher des réponses à des problèmes communs, de recenser les bonnes pratiques et de travailler à la coordination des politiques nationales et internationales.

Les pays membres de l'OCDE sont : l'Allemagne, l'Australie, l'Autriche, la Belgique, le Canada, le Chili, la Corée, le Danemark, l'Espagne, l'Estonie, les États-Unis, la Finlande, la France, la Grèce, la Hongrie, l'Irlande, l'Islande, Israël, l'Italie, le Japon, le Luxembourg, le Mexique, la Norvège, la Nouvelle-Zélande, les Pays-Bas, la Pologne, le Portugal, la République slovaque, la République tchèque, le Royaume-Uni, la Slovénie, la Suède, la Suisse et la Turquie. L'Union européenne participe aux travaux de l'OCDE.

Les Éditions OCDE assurent une large diffusion aux travaux de l'Organisation. Ces derniers comprennent les résultats de l'activité de collecte de statistiques, les travaux de recherche menés sur des questions économiques, sociales et environnementales, ainsi que les conventions, les principes directeurs et les modèles développés par les pays membres. 


\section{Panorama des administrations publiques 2013}

Bien que la mesure de la performance des gouvernements soit reconnue depuis longtemps comme ayant un rôle important à jouer pour accroître l'efficacité et l'efficience du secteur public, la récente crise économique et les contraintes fiscales grandissantes dans plusieurs pays membres augmentent la nécessité de développer des indicateurs robustes pour accompagner les prises de décisions des gouvernements.

Le Panorama des administrations publiques 2013 jette un regard plus spécifique à la confiance des citoyens envers leurs gouvernements dans le chapitre d'ouverture et fournit ensuite aux lecteurs un vaste éventail d'indicateurs qui couvrent :

- La gouvernance stratégique

- Les finances et l'économie publiques

- Les pratiques et les procédures budgétaires

- L'emploi et la rémunération dans le secteur public

- Les femmes dans la fonction publique

- Les marchés publics

- Une administration ouverte et inclusive

- Au service des citoyens : accessibilité et qualité des services publics

Les fichiers Excel ${ }^{\mathrm{TM}}$ utilisés pour créer les tableaux et graphiques dans le Panorama des administrations publiques 2013 sont disponibles via les StatLinks fournis à travers la publication. Les fiches par pays et autres suppléments en ligne sont disponibles via le site Internet de l'OCDE au www.oecd.org/gov/govataglance.htm.

Veuillez consulter cet ouvrage en ligne : $h$ ttp://dx.doi.org/10.1787/gov_glance-2013-fr.

Cet ouvrage est publié sur OECD iLibrary, la bibliothèque en ligne de l'OCDE, qui regroupe tous les livres, périodiques et bases de données statistiques de l'Organisation.

Rendez-vous sur le site www.oecd-ilibrary.org pour plus d'informations.

\section{3}

